\title{
Rhodium-Catalyzed Regioselective Synthesis of Isocoumarins through Benzothiadiazine-Fused Frameworks
}

Prashant B. Dalvi, ${ }^{a}$ Kuang-Ling Lin, ${ }^{a b}$ Manohar V. Kulkarni ${ }^{a}$ and Chung-Ming Sun* $a b$

a Department of Applied Chemistry, 1001 Ta-Hseuh Road, National Chiao-Tung University, Hsinchu 300-10, Taiwan, ROC

${ }^{b}$ Department of Medicinal and Applied Chemistry, Kaohsiung Medical University, 100, Shih-Chuan $1^{\text {st }}$ Road, Kaohsiung 807-08, Taiwan, ROC.

E-mail: $\underline{\text { cmsun@mail.nctu.edu.tw }}$

\section{Contents}

Page

1. General remarks

S3

2. General schemes for the synthesis of intermediates $\mathbf{5}, \mathbf{1 5}, \mathbf{1 6}, \quad \mathrm{S} 4$ 17, 18 and 19

3. Proposed mechanism for formation of product $\mathbf{8}$ and 10, S5-S6 control experiment and proposed mechanism for the catalytic system forming compounds $\mathbf{2 1}$ and $\mathbf{2 3}$.

4. Optimization of suitable reaction condition: Table $\mathrm{S} 1$

5. Experimental procedures and spectra $\left({ }^{1} \mathrm{H}\right.$ and ${ }^{13} \mathrm{C}$ NMR, S10-S23 HRMS) of compounds 8a, 10a, 12a-19a, 21c and 23e

6. Data interpretation for compounds $8,10,21,22,23$ and 24

S24-S41

7. Spectra $\left({ }^{1} \mathrm{H}\right.$ and ${ }^{13} \mathrm{C}$ NMR, HRMS $)$ of compounds 12a-19a

S42-S66

8. Spectra $\left({ }^{1} \mathrm{H}\right.$ and ${ }^{13} \mathrm{C}$ NMR, HRMS $)$ of compounds 8a-8j, 10a- $\quad$ S67-S126 $10 \mathbf{j}$

9. Spectra $\left({ }^{1} \mathrm{H}\right.$ and ${ }^{13} \mathrm{C}$ NMR, HRMS $)$ of compounds 21a-k, 22a $\quad$ S127-S168 and 22b

10. Spectra $\left({ }^{1} \mathrm{H}\right.$ and ${ }^{13} \mathrm{C}$ NMR, HRMS $)$ of compounds 23a-j and $\quad$ S169-S198 24a 


\section{General Remarks}

All solvents were distilled from appropriate drying agents prior to use. All reactions were performed under an inert atmosphere with unpurified reagents and dry solvents.Reaction progress was monitored by thin layer chromatography (TLC). Flash chromatography was performed using the indicated solvent and silica gel 60 (230-400 mesh). ${ }^{1} \mathrm{H}$ NMR (300 MHz and $\left.400 \mathrm{MHz}\right)$ and ${ }^{13} \mathrm{C}$ NMR (75 MHz and $101 \mathrm{MHz}$ ) spectra were recorded on a $300 \mathrm{MHz}$ and $400 \mathrm{MHz}$ spectrometer. Chemical shifts are reported in parts per million (ppm) on the scale from an internal standard. 
<smiles>[R20]C(=O)c1ccc(F)c([N+](=O)[O-])c1</smiles>

$\mathrm{R}^{1}=\mathrm{H}$

1

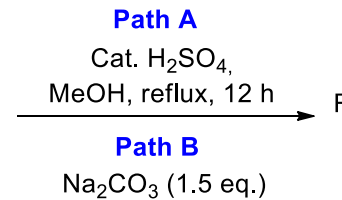

DMF, rt, 30 min

$\mathrm{BnBr}$ (1.1 eq.)

$45^{\circ} \mathrm{C}, 2 \mathrm{~h}$

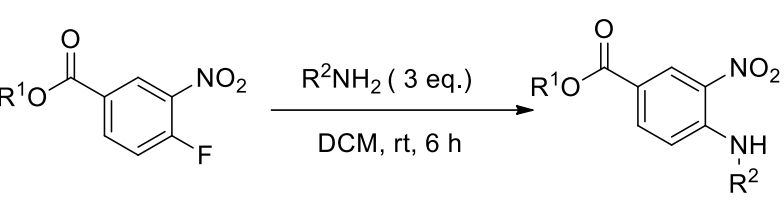

2; $R^{1}=\mathrm{Me}($ Path $A)$

12; $R^{1}=B n($ Path $B)$
3; $\mathrm{R}^{1}=\mathrm{Me}$

13; $R^{1}=B n$

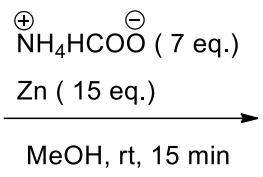<smiles>[R20]C(=O)c1ccc(NP)c(N)c1</smiles>

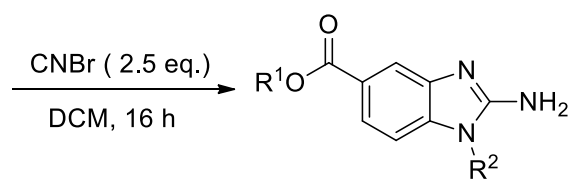

4; $\mathrm{R}^{1}=\mathrm{Me}$

14; $R^{1}=B n$

5; $\mathrm{R}^{1}=\mathrm{Me}$

15; $\mathrm{R}^{1}=\mathrm{Bn}$

Scheme S1. Synthesis of intermediates 2-aminobenzimidazole 5 and $\mathbf{1 5}$.

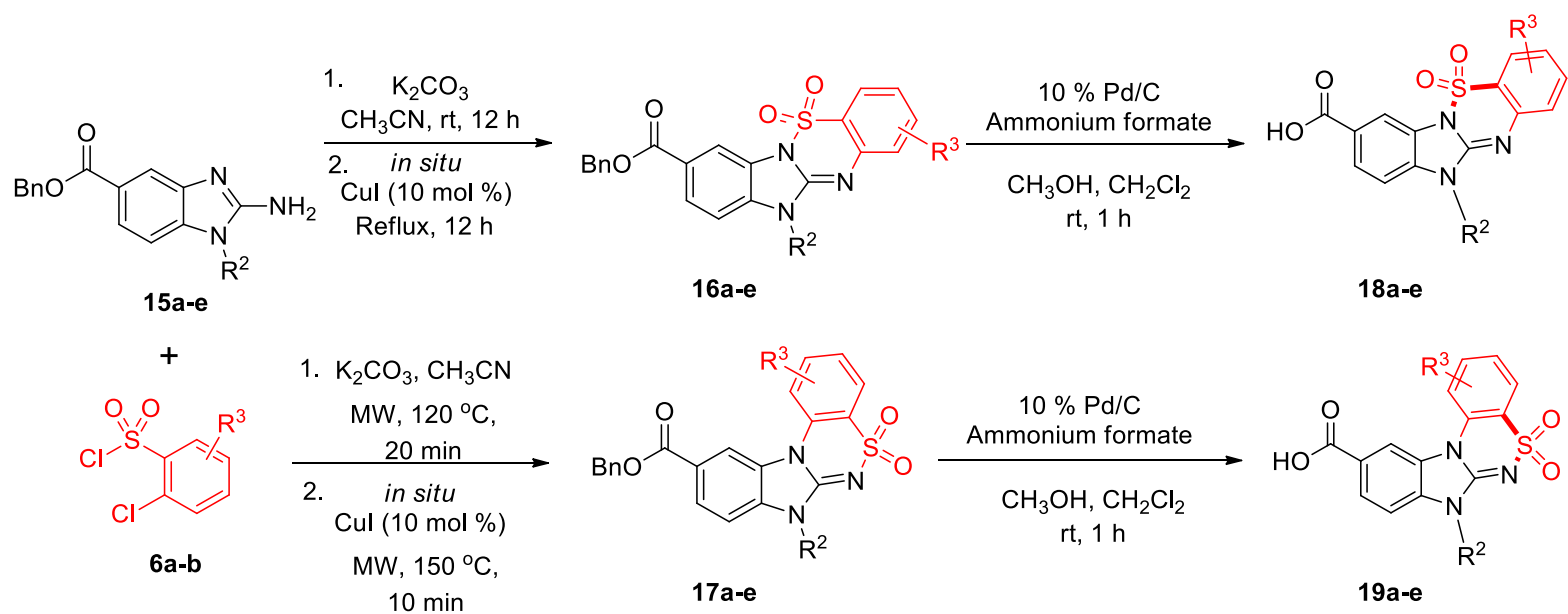

Scheme S2. Regioselective synthesis of benzothiadiazines 16 and $\mathbf{1 7}$ and their acid forms 18 and 19. 


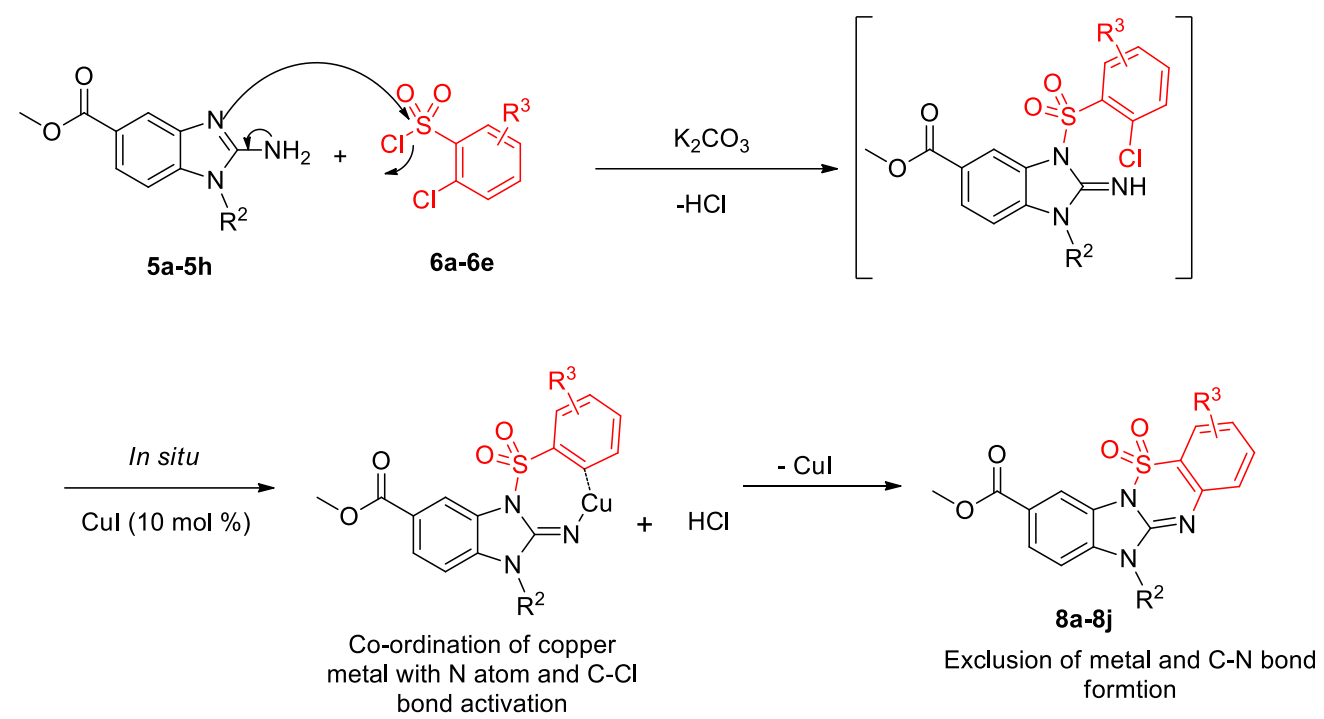

Scheme S3. Proposed mechanism for the formation of benzimidazothiadiazine5, 5- dioxides 8 .

\section{Control experiment:}

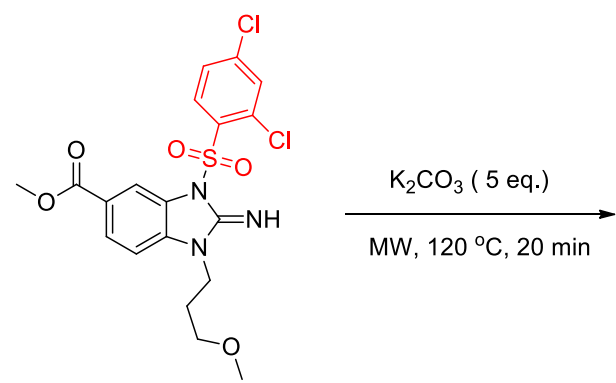

$7 a$

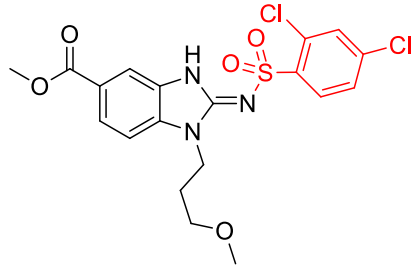

$9 \mathrm{a}$

Scheme S4. Inter conversion of intermediate sulfonamide 7a to 9a was studies under MW irradiation.

In our assumption, intermediate sulphonamide 9a could be a more stable under thermodynamic conditions because it was formed under MW irradiation. Therefore inter conversion of these two intermediates was studied to observe the formation of sulphonamide 9a via compound 7a. This assumption was failed in our study, otherwise we could have been in a better position to support the mechanism of this reaction. 


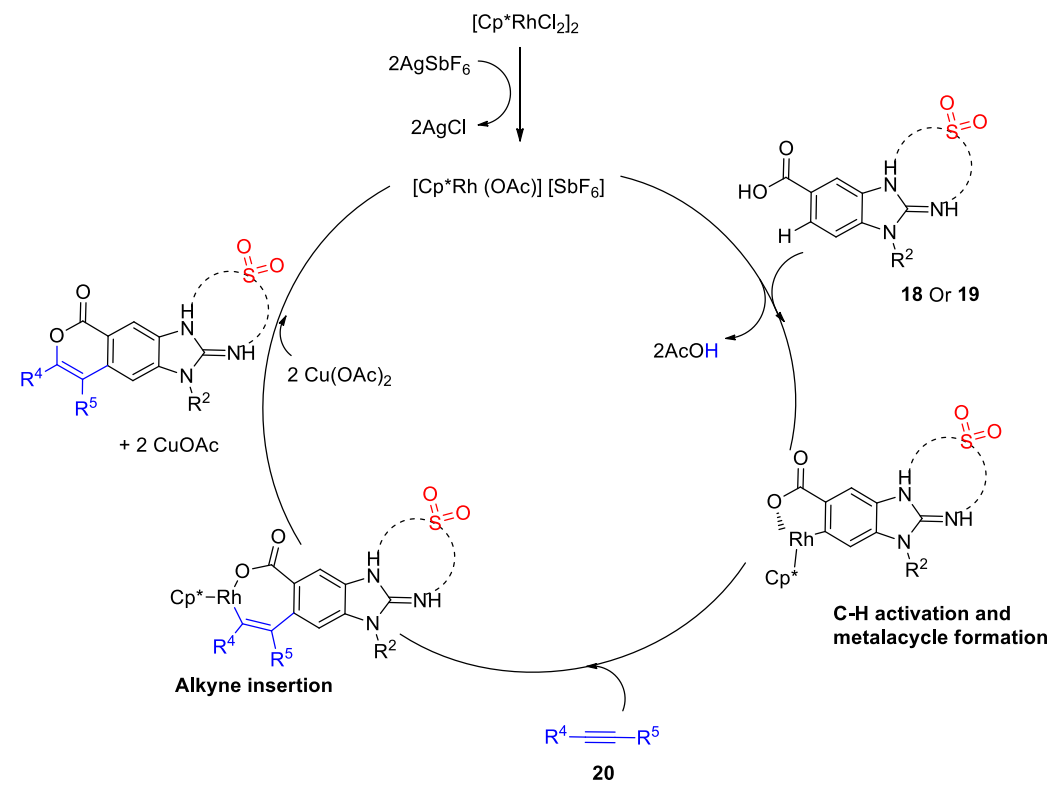

Scheme S5. Plausible reaction mechanism for an oxidative annulation. 
Table S1: Optimization of suitable reaction condition for the preparation of isocoumarins. ${ }^{\mathrm{a}}$

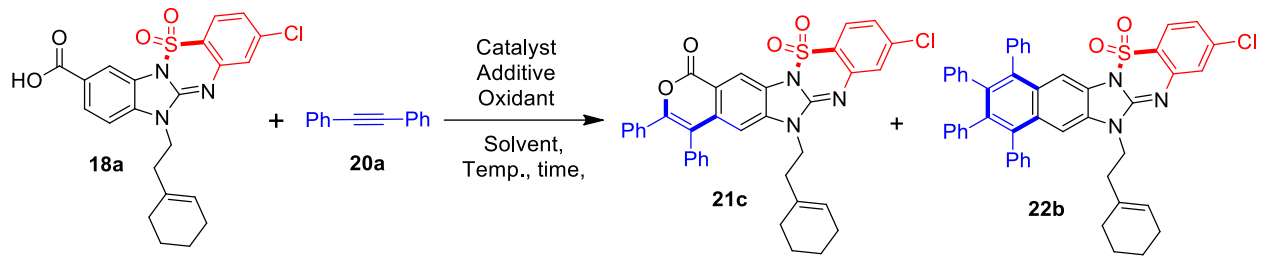

\begin{tabular}{|c|c|c|c|c|c|c|}
\hline Entry & Catalyst (mol \%) & Oxidant (mol \%) & Solvent & Temp $\left({ }^{\circ} \mathrm{C}\right)$ & Time $(h)$ & $\begin{array}{l}\left.\text { Yield }(\%)^{b}\right) \\
(21 c: 22 b)\end{array}$ \\
\hline 1 & Rh III (1) & $\mathrm{Cu}(\mathrm{OAc})_{2} \cdot \mathrm{H}_{2} \mathrm{O}(5)$ & DMF & 120 & 2 & $\mathrm{NR}^{\mathrm{c}}$ \\
\hline 2 & Rh III (1) & $\mathrm{Cu}(\mathrm{OAc})_{2} \cdot \mathrm{H}_{2} \mathrm{O}(5)$ & DMF & 150 & 2 & $\mathrm{NR}^{\mathrm{c}}$ \\
\hline $3^{d}$ & Rh III (5) & $\mathrm{Cu}(\mathrm{OAc})_{2} \cdot \mathrm{H}_{2} \mathrm{O}$ (2 eq.) & $\mathrm{H}_{2} \mathrm{O}$ & 110 & $30+15 \min$ & $36: 00$ \\
\hline $4^{d}$ & Rh III (5) & $\mathrm{Cu}(\mathrm{OAc})_{2} \cdot \mathrm{H}_{2} \mathrm{O}$ (2 eq.) & $\mathrm{H}_{2} \mathrm{O}$ & 120 & $30 \mathrm{~min}$ & Messy \\
\hline $5^{d}$ & Rh III (10) & $\mathrm{Cu}(\mathrm{OAc})_{2} \cdot \mathrm{H}_{2} \mathrm{O}$ (2 eq.) & $\mathrm{H}_{2} \mathrm{O}$ & 110 & $30 \mathrm{~min}$ & $41: 00$ \\
\hline 6 & Rh III (5) & $\mathrm{Cu}(\mathrm{OAc})_{2} \cdot \mathrm{H}_{2} \mathrm{O}$ (2 eq.) & $\mathrm{H}_{2} \mathrm{O}$ & 110 & 2 & 61:00 \\
\hline 7 & Rh III (5) & $\mathrm{CuCl}_{2}$ (2 eq.) & $\mathrm{H}_{2} \mathrm{O}$ & 110 & 2 & 20:00 \\
\hline 8 & $\mathrm{Ru}$ II (5) & $\mathrm{Cu}(\mathrm{OAc})_{2} \cdot \mathrm{H}_{2} \mathrm{O}$ (2 eq.) & $\mathrm{H}_{2} \mathrm{O}$ & 110 & $30 \mathrm{~min}$ & NR \\
\hline $\begin{array}{c}9 \\
10^{\mathrm{e}}\end{array}$ & $\begin{array}{l}\text { Rh III (5) } \\
\text { Ru II (5) }\end{array}$ & $\begin{array}{l}\mathrm{Cu}(\mathrm{OAc})_{2} \cdot \mathrm{H}_{2} \mathrm{O}(10) \\
\mathrm{Cu}(\mathrm{OAc})_{2} \cdot \mathrm{H}_{2} \mathrm{O}(20)\end{array}$ & $\begin{array}{l}\text { DMF } \\
\text { DMF }\end{array}$ & $\begin{array}{c}65 \\
120\end{array}$ & $\begin{array}{l}6 \\
2\end{array}$ & $\begin{array}{l}8: 72 \\
\text { NR }\end{array}$ \\
\hline $11^{\mathrm{e}}$ & Ru II (5) & $\mathrm{Cu}(\mathrm{OAc})_{2} \cdot \mathrm{H}_{2} \mathrm{O}(20)$. & EDC & 100 & 12 & NR \\
\hline $12^{f}$ & Ru II (5) & $\mathrm{Cu}(\mathrm{OAc})_{2} \cdot \mathrm{H}_{2} \mathrm{O}(20)$ & EDC & reflux & 12 & $16: 00$ \\
\hline $13^{f}$ & Rh III (5) & $\mathrm{Cu}(\mathrm{OAc})_{2} \cdot \mathrm{H}_{2} \mathrm{O}(20)$ & DMF & 65 & 6 & 76:00 \\
\hline 14 & Rh III (5) & $\mathrm{Cu}(\mathrm{OAc})_{2} \cdot \mathrm{H}_{2} \mathrm{O}$ (2 eq.) & GAA & RT & 1 & $N R^{c}$ \\
\hline 15 & Rh III (5) & $\mathrm{Cu}(\mathrm{OAc})_{2} \cdot \mathrm{H}_{2} \mathrm{O}$ (2 eq.) & GAA & reflux & 2 & $\mathrm{NR}^{\mathrm{c}}$ \\
\hline 16 & Rh III (5) & $\mathrm{Cu}(\mathrm{OAc})_{2} \cdot \mathrm{H}_{2} \mathrm{O}$ (2 eq.) & DMF & 150 & 2 & $\mathrm{NR}^{\mathrm{c}}$ \\
\hline 17 & Rh III (5) & $\mathrm{Cu}(\mathrm{OAc})_{2} \cdot \mathrm{H}_{2} \mathrm{O}$ (2 eq.) & Toluene & reflux & 2 & $N R^{c}$ \\
\hline 18 & Ru II (5) & $\mathrm{Cu}(\mathrm{OAc})_{2} \cdot \mathrm{H}_{2} \mathrm{O}$ (2 eq.) & DMF & 120 & 2 & $\mathrm{NR}^{\mathrm{c}}$ \\
\hline $19^{g}$ & $\mathrm{Ru}$ II (5) & $\mathrm{Cu}(\mathrm{OAc})_{2} \cdot \mathrm{H}_{2} \mathrm{O}$ (2 eq.) & DMF & 120 & 2 & $\mathrm{NR}^{\mathrm{c}}$ \\
\hline $20^{g}$ & Ru II (5) & $\mathrm{Cu}(\mathrm{OAc})_{2} \cdot \mathrm{H}_{2} \mathrm{O}$ (2 eq.) & $t-\mathrm{BuOH}$ & reflux & 12 & $6: 00$ \\
\hline $21^{\mathrm{h}}$ & Ru II (5) & $\mathrm{Cu}(\mathrm{OAc})_{2} \cdot \mathrm{H}_{2} \mathrm{O}$ (2 eq.) & $t-\mathrm{BuOH}$ & reflux & 12 & $N R^{c}$ \\
\hline 22 & Rh III (5) & $\mathrm{Cu}(\mathrm{OAc})_{2} \cdot \mathrm{H}_{2} \mathrm{O}(10)$ & $t-\mathrm{BuOH}$ & 65 & $6 h$ & $N R^{c}$ \\
\hline $23^{d}$ & Rh III (5) & $\mathrm{Cu}(\mathrm{OAc})_{2} \cdot \mathrm{H}_{2} \mathrm{O}(10)$ & $t-\mathrm{BuOH}$ & 120 & $15 \min$ & $N R^{c}$ \\
\hline 24 & Rh III (5) & $\mathrm{Cu}(\mathrm{OAc})_{2} \cdot \mathrm{H}_{2} \mathrm{O}(10)$ & DMSO & 65 & 6 & $N R^{c}$ \\
\hline 25 & Rh III (5) & $\mathrm{Cu}(\mathrm{OAc})_{2} \cdot \mathrm{H}_{2} \mathrm{O}(10)$ & DMSO & 160 & 3 & $\mathrm{NR}^{\mathrm{c}}$ \\
\hline
\end{tabular}

${ }^{a}$ Reaction was performed using $18 \mathrm{a}$ (1 equiv), 20a (1.2 equiv) with variable mol \% of catalyst, additive and oxidant in different solvents; ${ }^{b}$ yield of isolated product; ${ }^{\mathrm{C}} \mathrm{NR}=\mathrm{No}$ reaction; ${ }^{\mathrm{d}}$ Reaction was performed in microwave; ${ }^{\mathrm{e}} 10 \mathrm{~mol} \%$ AgOTf was used as additive; ${ }^{\mathrm{f}} 10 \mathrm{~mol} \%$ of $\mathrm{AgSbF}_{6}$ was used as additive; g $20 \mathrm{~mol} \%$ of AgOTf was used as additive; ${ }^{\mathrm{h}} 20 \mathrm{~mol} \%$ of $\mathrm{Ag}_{2} \mathrm{CO}_{3}$ was used as additive.

Initially, the treatment of acid 18a with diphenyl acetylene 20a in the presence of catalyst $\left[\mathrm{Cp}^{*} \mathrm{RhCl}_{2}\right]_{2}(1 \mathrm{~mol} \%)\left(\mathrm{Cp}^{*}=\right.$ pentamethylcyclopentadiene $)$ and oxidant 
$\mathrm{Cu}(\mathrm{OAc})_{2} \cdot \mathrm{H}_{2} \mathrm{O}(5 \mathrm{~mol} \%)$ was found ineffective at either $120{ }^{\circ} \mathrm{C}$ or $150{ }^{\circ} \mathrm{C}$ for 2 h (Table S1, entries 1 and 2). Furthermore, slight increase in the catalyst loading $(5 \mathrm{~mol} \%)$ and use oxidant $\mathrm{Cu}(\mathrm{OAc})_{2} \cdot \mathrm{H}_{2} \mathrm{O}$ delivered product $21 \mathrm{c}$ in $36 \%$ yield under microwave heating $\left(110^{\circ} \mathrm{C}\right)$ for $45 \mathrm{~min}$ (Table $\mathrm{S} 1$, entry 3$)$. If same reaction mixture was further heated, the decomposition of starting material was observed (Table S1, entry 4). When catalyst was increased to $10 \mathrm{~mol} \%$, the product was isolated in $41 \%$ yield. Change the catalyst to $\left[\mathrm{RuCl}_{2}(p \text {-cymene) }]_{2}\right.$ did not obtain any product (Table S1, entries 5 and 8). The significant improvement was noted when reaction mixture was heated at $110^{\circ} \mathrm{C}$ for $2 \mathrm{~h}$, resulted into $61 \%$ of product (Table S1, entry 6) and the isolated $20 \%$ yield was observed when oxidant was changed to $\mathrm{CuCl}_{2}$ (Table S1, entry 7).

The use of different polar and nonpolar solvents did not give satisfied results and several changes of catalysts and additives were also failed to deliver satisfactory results (Table S1, entries 14-25). Decarboxylative naphthalene type product 22b was yielded as a major product (90\%) along with desired product 21c (10\%) when reaction mixture was heated at $65{ }^{\circ} \mathrm{C}$ for $6 \mathrm{~h}$ in $\mathrm{DMF}$ with catalyst $\left[\mathrm{Cp}^{*} \mathrm{RhCl}_{2}\right]_{2}(5 \mathrm{~mol} \%)$ and $\mathrm{Cu}(\mathrm{OAc})_{2} \cdot \mathrm{H}_{2} \mathrm{O}(10 \mathrm{~mol} \%)$ (Table 1, entry 9). Next, we studied DMF and EDC with Ru (II) dimer (5 mol \%) and AgOTf as an additive (10 mol \%), but failed to give product 21c (Table S1, entries 10 and 11). However, $16 \%$ of product was isolated when $\mathrm{AgSbF}_{6}(10 \mathrm{~mol} \%)$ was used (Table S1, entry 12). Finally, use of catalyst $\left[\mathrm{Cp} * \mathrm{RhCl}_{2}\right]_{2}(5 \mathrm{~mol} \%)$, an oxidant $\mathrm{Cu}(\mathrm{OAc})_{2} \cdot \mathrm{H}_{2} \mathrm{O}$ 
(20 mol \%) and an additive $\mathrm{AgSbF}_{6}(10 \mathrm{~mol} \%)$ in $\mathrm{DMF}$ at $65^{\circ} \mathrm{C}$ for $6 \mathrm{~h}$ resulted into $76 \%$ yield of desired product regioselectively and decarboxylative naphthalene 22b was not observed (Table S1, entry 13). 
General Procedure for the synthesis of Methyl 4-chloro-11-(3methoxypropyl)-11H-benzo[e]benzo[4,5] imidazo[1,2-b][1,2,4]thiadiazine8-carboxylate 5,5-dioxide (8a)

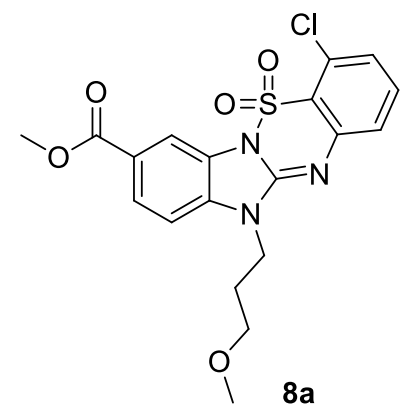

To a solution of methyl 2-amino-1-(3-methoxypropyl)-1H-benzo[d]imidazole-5carboxylate 5a $(0.100 \mathrm{~g}, 0.379 \mathrm{mmol})$ in $\mathrm{ACN}(10 \mathrm{~mL})$ was added 2-6 dichloro benzene sulfonyl chloride $(0.111 \mathrm{~g}, 0.455 \mathrm{mmol})$ and anhydrous potassium carboanate $(0.261 \mathrm{~g}, 1.89 \mathrm{mmol})$. The resulting reaction mixture was allowed to stir at room temperature. In situ addition of $\mathrm{CuI}(0.0072 \mathrm{~g}, 0.0379 \mathrm{mmol})$ was done to the same reaction mass after $12 \mathrm{~h}$ and it was heated at reflux temperature for additional $12 \mathrm{~h}$. Upon completion of reaction, the residue was filtered through pad of celite and filtrate was concentrated in vacuo. The crude mass was partitioned between aqueous and organic layers. Aqueous layer was extracted with ehyl acetate (20 mL x 2). Combined organic layer was dried over $\mathrm{MgSO}_{4}$ and concentrated under reduced pressure to give the crude product, which was purified by column chromatography (15\% Ethyl acetate in Hexane) on silica gel to afford compound 8a as a brown solid $(0.143 \mathrm{~g}, 87 \%)$. ${ }^{1} \mathrm{H}$ NMR (300 MHz, $\left.\mathrm{CDCl}_{3}\right) \delta 8.47(\mathrm{~d}, J=1.3 \mathrm{~Hz}, 1 \mathrm{H}), 8.09(\mathrm{dd}, J=8.5,1.3 \mathrm{~Hz}, 1 \mathrm{H}), 7.46(\mathrm{~d}, J=8.4$ 
$\mathrm{Hz}, 1 \mathrm{H}), 7.34(\mathrm{dd}, J=8.5,1.1 \mathrm{~Hz}, 1 \mathrm{H}), 7.27(\mathrm{~d}, J=8.4 \mathrm{~Hz}, 1 \mathrm{H}), 7.22(\mathrm{dd}, J=$ 7.8, 1.1 Hz, 1H), $4.23(\mathrm{t}, J=6.7 \mathrm{~Hz}, 2 \mathrm{H}), 3.95(\mathrm{~s}, 3 \mathrm{H}), 3.41(\mathrm{t}, J=5.6 \mathrm{~Hz}, 2 \mathrm{H})$, $3.32(\mathrm{~s}, 3 \mathrm{H}), 2.20-2.07(\mathrm{~m}, 2 \mathrm{H}) ;{ }^{13} \mathrm{C} \mathrm{NMR}\left(75 \mathrm{MHz}, \mathrm{CDCl}_{3}\right) \delta 166.4,146.6$, $146.5,135.2,134.2,130.1,128.4,126.1,125.7,125.0,124.5,121.3,114.3,108.7$, 69.2, 69.1, 52.7, 40.1, 28.3; HRMS : calcd for $\mathrm{C}_{19} \mathrm{H}_{18} \mathrm{ClN}_{3} \mathrm{O}_{5} \mathrm{~S} \mathrm{~m} / 2$ : 435.0656; found $435.0660(\mathrm{M})^{+}$.

General Procedure for the synthesis of methyl 4-chloro-7-(3methoxypropyl)-7H-benzo[e]benzo[4,5]imidazo $[2,1-c][1,2,4]$ thiadiazine-10carboxylate 5,5-dioxide (10a)

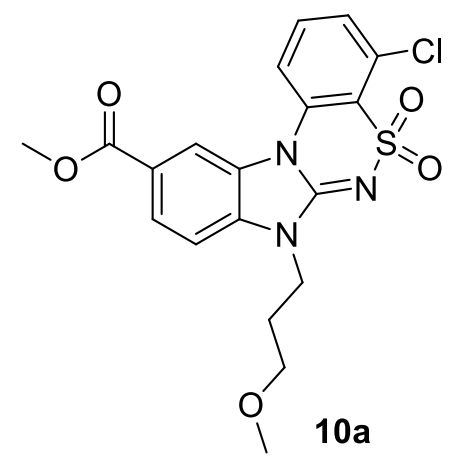

To a solution of methyl 2-amino-1-(3-methoxypropyl)-1H-benzo[d]imidazole-5carboxylate 5a $(0.100 \mathrm{~g}, 0.379 \mathrm{mmol})$ in $\mathrm{ACN}(3 \mathrm{~mL})$ was added 2-4 dichloro benzene sulfonyl chloride $(0.111 \mathrm{~g}, 0.455 \mathrm{mmol})$ and anhydrous potassium carboanate $(0.261 \mathrm{~g}, 1.89 \mathrm{mmol})$. The resulting reaction mixture was irradiated at $120{ }^{\circ} \mathrm{C}$ under microwave heating for $20 \mathrm{~min}$. In situ addition of $\mathrm{CuI}(0.0072 \mathrm{~g}$, $0.0379 \mathrm{mmol}$ ) was done to the same reaction mass and it was again irradiated at 
$150{ }^{\circ} \mathrm{C}$ for additional $15 \mathrm{~min}$. Upon completion of reaction, the residue was filtered through pad of celite and filtrate was concentrated in vacuo. The crude mass was partitioned between aqueous and organic layers. Aqueous layer was extracted with ethyl acetate $(20 \mathrm{~mL}$ x 2). Combined organic layer was dried over $\mathrm{MgSO}_{4}$ and concentrated under reduced pressure to give the crude product, which was purified by column chromatography ( $28 \%$ Ethyl acetate in Hexane) on silica gel to afford compound 10a as a off white solid (0.082 g, $63 \%) .{ }^{1} \mathrm{H}$ NMR (300 $\left.\mathrm{MHz}, \mathrm{CDCl}_{3}\right) \delta 8.58(\mathrm{~d}, J=1.1 \mathrm{~Hz}, 1 \mathrm{H}), 8.18(\mathrm{dd}, J=8.5,1.1 \mathrm{~Hz}, 1 \mathrm{H}), 8.09(\mathrm{dd}$, $J=8.5,0.9 \mathrm{~Hz}, 1 \mathrm{H}), 7.70(\mathrm{t}, J=8.0 \mathrm{~Hz}, 1 \mathrm{H}), 7.58(\mathrm{dd}, J=8.0,0.9 \mathrm{~Hz}, 1 \mathrm{H}), 7.48$ $(\mathrm{d}, J=8.5 \mathrm{~Hz}, 1 \mathrm{H}), 4.30(\mathrm{t}, J=6.8 \mathrm{~Hz}, 2 \mathrm{H}), 4.02(\mathrm{~s}, 3 \mathrm{H}), 3.42(\mathrm{t}, J=5.5 \mathrm{~Hz}, 2 \mathrm{H})$, $3.33(\mathrm{~s}, 3 \mathrm{H}), 2.21-2.09(\mathrm{~m}, 2 \mathrm{H}) ;{ }^{13} \mathrm{C} \mathrm{NMR}\left(75 \mathrm{MHz}, \mathrm{CDCl}_{3}\right) \delta 166.5,147.6$, $135.3,135.0,133.9,133.0,130.0,127.9,127.0,125.8,124.4,114.8,114.8,110.3$, 69.1, 59.1, 53.1, 40.5, 28.3; HRMS (ESI+) calcd for $\mathrm{C}_{19} \mathrm{H}_{18} \mathrm{ClN}_{3} \mathrm{O}_{5} \mathrm{SNa} \mathrm{m} / z$ : 458.0553; found 458.0550(M) ${ }^{+}$.

General Procedure for the synthesis of benzyl 4-fluoro-3-nitrobenzoate (12a)<smiles>O=C(OCc1ccccc1)c1ccc(F)c([N+](=O)[O-])c1</smiles>

To a solution of 4-fluoro-3-nitrobenzoic acid $1(0.100 \mathrm{~g}, 0.540 \mathrm{mmol})$ in DMF (5 $\mathrm{mL})$ was added sodium carbonate $(0.085 \mathrm{~g}, 0.810 \mathrm{mmol})$ and reaction mixture 
was allowed to stir at room temperature. A drop wise addition of benzyl bromide $(0.101 \mathrm{~g}, 0.594 \mathrm{mmol})$ to this solution was done after 30 minutes and resulting reaction mixture was heated at $45{ }^{\circ} \mathrm{C}$. Upon completion $(2 \mathrm{~h})$ of reaction, the mixture was filtered off and the filtrate was concentrated under reduced pressure. The residue was partitioned between aqueous and organic layer. The aqueous layer was extracted with ethyl acetate $(20 \mathrm{~mL}$ x 2$)$. Combined organic layer was dried over $\mathrm{MgSO}_{4}$ and concentrated under reduced pressure to give the crude product, which was purified by column chromatography (6\% Ethyl acetate in hexane) on silica gel to afford compound 12a as a yellow solid $(0.141 \mathrm{~g}, 95 \%)$. ${ }^{1} \mathrm{H}$ NMR (400 MHz, acetone-d $\left.{ }_{6}\right) \delta 8.68(\mathrm{dd}, J=7.3,2.2 \mathrm{~Hz}, 1 \mathrm{H}), 8.41$ (ddd, $J=$ 8.7, 4.3, $2.2 \mathrm{~Hz}, 1 \mathrm{H}), 7.64(\mathrm{dd}, J=10.8,8.7 \mathrm{~Hz}, 1 \mathrm{H}), 7.51(\mathrm{~m}, 2 \mathrm{H}), 7.38(\mathrm{~m}, 3 \mathrm{H})$, $5.42(\mathrm{~s}, 2 \mathrm{H}) ;{ }^{13} \mathrm{C}$ NMR $\left(101 \mathrm{MHz}\right.$, acetone- $\left.\mathrm{d}_{6}\right) \delta 163.2,159.2,156.5,136.7,136.6$, 135.8, 128.5, 128.4, 128.3, 127.3, 127.3, 127.3, 119.2, 118.9, 67.2; HRMS (EI, $\mathrm{m} / \mathrm{z}$ ) calcd for $\mathrm{C}_{14} \mathrm{H}_{10} \mathrm{NO}_{4} \mathrm{~F}(\mathrm{M})^{+} 275.0588$; found 275.0597 .

General Procedure for the synthesis of benzyl 4-((3-methoxypropyl)amino)3-nitrobenzoate (13a)<smiles>COCCCNc1ccc(C(=O)OCc2ccccc2)cc1[N+](=O)[O-]</smiles>
$13 a$

To a solution of benzyl 4-fluoro-3-nitrobenzoate 12a $(0.100 \mathrm{~g}, 0.363 \mathrm{mmol})$ in DCM (5 mL) was added 3-methoxy propylamine $(0.080 \mathrm{~g}, 0.908 \mathrm{mmol})$ and reaction mixture was allowed to stir at room temperature. Upon completion $(6 \mathrm{~h})$ 
of reaction, the reaction mass was partitioned between aqueous and organic layer. The aqueous layer was extracted with DCM (20 mL x 2). Combined organic layer was dried over $\mathrm{MgSO}_{4}$ and concentrated under reduced pressure to give the crude product, which was purified by column chromatography ( $8 \%$ Ethyl acetate in hexane) on silica gel to afford compound 13a yellowish semisolid (0.116 g, 93 \%). ${ }^{1} \mathrm{H}$ NMR (400 MHz, acetone-d $\left.{ }_{6}\right) \delta 8.74(\mathrm{~d}, J=1.9 \mathrm{~Hz}, 1 \mathrm{H}), 8.70(\mathrm{~s}, 1 \mathrm{H}), 8.01$ $(\mathrm{dd}, J=9.1,1.8 \mathrm{~Hz}, 1 \mathrm{H}), 7.49(\mathrm{~d}, J=7.5 \mathrm{~Hz}, 2 \mathrm{H}), 7.39(\mathrm{~d}, J=7.5 \mathrm{~Hz}, 2 \mathrm{H}), 7.34$ $(\mathrm{t}, J=7.1 \mathrm{~Hz}, 1 \mathrm{H}), 7.04(\mathrm{~d}, J=9.1 \mathrm{~Hz}, 1 \mathrm{H}), 5.34(\mathrm{~s}, 2 \mathrm{H}), 3.52(\mathrm{~m}, 5 \mathrm{H}), 3.32(\mathrm{~s}$, 3H), $1.97(\mathrm{~m}, 2 \mathrm{H}) ;{ }^{13} \mathrm{C}$ NMR (101 MHz, acetone-d6) $\delta 164.3,147.7,136.5,135.7$, 131.0, 128.7, 128.4, 128.1, 128.0, 116.2, 114.0, 70.4, 66.1, 57.9, 41.2, 28.4; HRMS (ESI+) calcd for $\mathrm{C}_{18} \mathrm{H}_{21} \mathrm{~N}_{2} \mathrm{O}_{5} \mathrm{~m} / \mathrm{z}$ : 345.1445; found 345.1449.

General Procedure for the synthesis of benzyl 3-amino4((3methoxypropyl)amino)benzoate (14a)

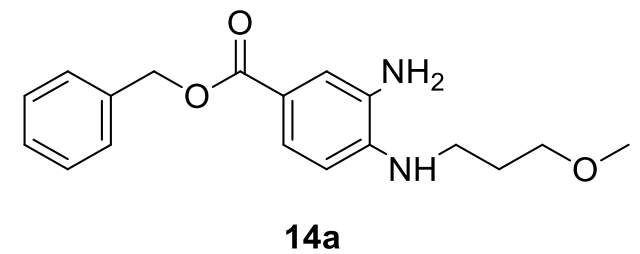

To a solution of benzyl 4-((3-methoxypropyl)amino)-3-nitrobenzoate 13a $(0.100$ $\mathrm{g}, 0.290 \mathrm{mmol})$ in $\mathrm{MeOH}(10 \mathrm{~mL})$ was added ammonium formate $(0.127 \mathrm{~g}, 2.03$ mmol) and zinc $(0.283 \mathrm{~g}, 4.355 \mathrm{mmol})$. The resulting reaction mixture was allowed to stir at room temperature. Upon completion (15 min.) of reaction, the solvent was evaporated under reduced pressure. The crude residue was washed 
with DCM (20 mL x 2) and filtered. Combined filtrate was dried over $\mathrm{MgSO}_{4}$ and concentrated under reduced pressure to give the crude product, which was purified by column chromatography (12\% Ethyl acetate in Hexane) on silica gel to afford compound $\mathbf{1 4 a}$ dark brownish semisolid (0.079 g, $87 \%) .{ }^{1} \mathrm{H}$ NMR (400 MHz, acetone- $\left.\mathrm{d}_{6}\right) \delta 7.53-7.27(\mathrm{~m}, 7 \mathrm{H}), 6.58(\mathrm{~d}, J=8.3 \mathrm{~Hz}, 1 \mathrm{H}), 5.28(\mathrm{~s}, 2 \mathrm{H})$, $4.79(\mathrm{~s}, 1 \mathrm{H}), 4.20(\mathrm{~s}, 2 \mathrm{H}), 3.49(\mathrm{t}, J=6.0 \mathrm{~Hz}, 2 \mathrm{H}), 3.28(\mathrm{~m}, 5 \mathrm{H}), 1.90(\mathrm{~m}, 2 \mathrm{H})$; ${ }^{13} \mathrm{C}$ NMR (101 MHz, acetone- $\left.\mathrm{d}_{6}\right) \delta 166.3,142.1,137.3,133.8,128.3,127.8,127.7$, 122.3, 117.8, 115.8, 108.2, 70.4, 65.1, 57.7, 40.8, 29.0; HRMS (ESI+) calcd for $\mathrm{C}_{18} \mathrm{H}_{23} \mathrm{~N}_{2} \mathrm{O}_{3} \mathrm{~m} / \mathrm{z}: 315.1703$; found 315.1710.

General Procedure for the synthesis of benzyl 2-amino-1-(3methoxypropyl)-1H-benzo[d]imidazole-5-carboxylate (15a)

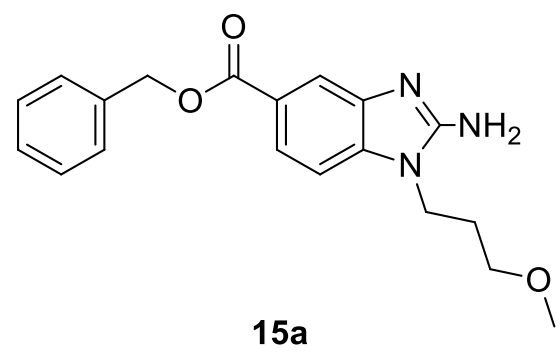

To a solution of benzyl 3-amino-4((3-methoxypropyl)amino)benzoate 14a $(0.100$ $\mathrm{g}, 0.318 \mathrm{mmol})$ in DCM $(5 \mathrm{~mL})$ was added cynogen bromide $(0.084 \mathrm{~g}, 0.795$ mmol) and reaction mixture was allowed to stir at room temperature. Upon completion $(16 \mathrm{~h})$ of reaction, $1 \mathrm{~N} \mathrm{NaOH}(10 \mathrm{~mL})$ was added to the reaction mixture. The aqueous layer was extracted with DCM (20 mL x 2). Combined organic layer was dried over $\mathrm{MgSO}_{4}$ and concentrated under reduced pressure to 
give the crude product, which was purified by column chromatography $(60 \%$ Ethyl acetate in Hexane) on silica gel to afford compound 15a as a brown solid (0.079 g, $78 \%) .{ }^{1} \mathrm{H}$ NMR (400 MHz, $\left.\mathrm{CD}_{3} \mathrm{OD}\right) \delta 7.92(\mathrm{~d}, J=1.4 \mathrm{~Hz}, 1 \mathrm{H}), 7.76$ $(\mathrm{dd}, J=8.4,1.5 \mathrm{~Hz}, 1 \mathrm{H}), 7.44(\mathrm{~d}, J=7.6 \mathrm{~Hz}, 2 \mathrm{H}), 7.36(\mathrm{t}, J=7.5 \mathrm{~Hz}, 2 \mathrm{H}), 7.31$ $(\mathrm{m}, 1 \mathrm{H}), 7.21(\mathrm{~d}, J=8.4 \mathrm{~Hz}, 1 \mathrm{H}), 5.33(\mathrm{~s}, 2 \mathrm{H}), 4.09(\mathrm{t}, J=6.7 \mathrm{~Hz}, 2 \mathrm{H}), 3.31(\mathrm{~m}$, 5H), $1.98(\mathrm{~m}, 2 \mathrm{H}) ;{ }^{13} \mathrm{C}$ NMR (101 MHz, $\left.\mathrm{CD}_{3} \mathrm{OD}\right) \delta 167.3,156.2,141.1,137.9$, $136.5,128.1,127.7,127.6,122.8,121.4,115.9,107.1,68.2,66.0,57.4,38.6,28.1$; HRMS (ESI+) calcd for $\mathrm{C}_{19} \mathrm{H}_{22} \mathrm{~N}_{3} \mathrm{O}_{3} \mathrm{~m} / \mathrm{z}$ : 340.1656; found 340.1658 .

General Procedure for the synthesis of benzyl 2-chloro-11-(3methoxypropyl)-11H-benzo $[e]$ benzo $[4,5]$ imidazo $[1,2-b][1,2,4]$ thiadiazine-8carboxylate 5,5-dioxide (16a)

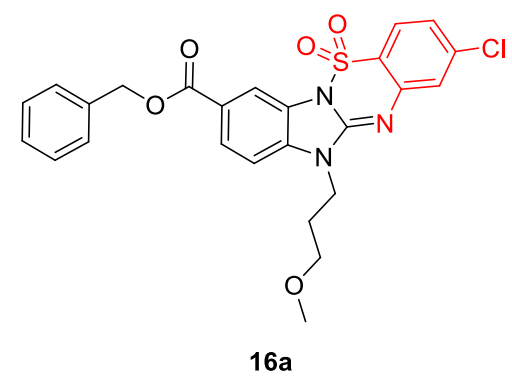

To a solution of benzyl 2-amino-1-(3-methoxypropyl)-1H-benzo[ $d]$ imidazole-5carboxylate 15a $(0.100 \mathrm{~g}, 0.294 \mathrm{mmol})$ in ACN $(10 \mathrm{~mL})$ was added 2-4 dichloro benzene sulfonyl chloride $(0.087 \mathrm{~g}, 0.353 \mathrm{mmol})$ and anhydrous potassium carboanate $(0.203 \mathrm{~g}, 1.47 \mathrm{mmol})$. The resulting reaction mixture was allowed to stir at room temperature. In situ addition of $\mathrm{CuI}(0.0055 \mathrm{~g}, 0.0294 \mathrm{mmol})$ was done to the same reaction mass after $12 \mathrm{~h}$ and it was heated at reflux temperature for additional $12 \mathrm{~h}$. Upon completion of reaction, the residue was filtered through 
pad of celite and filtrate was concentrated in vacuo. The crude mass was partitioned between aqueous and organic layers. Aqueous layer was extracted with ehyl acetate $(20 \mathrm{~mL} \times 2)$. Combined organic layer was dried over $\mathrm{MgSO}_{4}$ and concentrated under reduced pressure to give the crude product, which was purified by column chromatography (15\% ethyl acetate in Hexane) on silica gel to afford compound 16a as a light brown solid (0.125 g, $83 \%)$. ${ }^{1} \mathrm{H}$ NMR (400 MHz, acetone- $\left.\mathrm{d}_{6}\right) \delta 8.38(\mathrm{~d}, J=1.4 \mathrm{~Hz}, 1 \mathrm{H}), 8.17(\mathrm{dd}, J=8.6,1.4 \mathrm{~Hz}, 1 \mathrm{H}), 8.04$ $(\mathrm{d}, J=8.6 \mathrm{~Hz}, 1 \mathrm{H}), 7.59(\mathrm{~d}, J=8.5 \mathrm{~Hz}, 1 \mathrm{H}), 7.53(\mathrm{~d}, J=8.5 \mathrm{~Hz}, 1 \mathrm{H}), 7.48(\mathrm{~d}, J$ $=2.0 \mathrm{~Hz}, 1 \mathrm{H}), 7.39(\mathrm{~m}, 5 \mathrm{H}), 5.44(\mathrm{~s}, 2 \mathrm{H}), 4.34(\mathrm{t}, J=6.7 \mathrm{~Hz}, 3 \mathrm{H}), 3.44(\mathrm{t}, J=5.8$ $\mathrm{Hz}, 3 \mathrm{H}), 3.23(\mathrm{~s}, 3 \mathrm{H}), 2.15(\mathrm{~m}, 2 \mathrm{H}) ;{ }^{13} \mathrm{C} \mathrm{NMR}\left(101 \mathrm{MHz}\right.$, acetone-d $\left.\mathrm{d}_{6}\right) \delta 164.9$, $145.8,139.8,136.4,135.6,128.5,128.2,128.1,127.9,125.7,124.4,124.3,123.9$, 123.3, 121.2, 113.0, 109.5, 68.9, 66.5, 57.7, 40.1, 27.4; HRMS (ESI+) calcd for $\mathrm{C}_{25} \mathrm{H}_{23} \mathrm{ClN}_{3} \mathrm{O}_{5} \mathrm{~S} \mathrm{~m} / \mathrm{z}$ : 512.1041; found 512.1036.

General Procedure for the synthesis of benzyl 4-chloro-7-(3methoxypropyl)-7H-benzo $[e]$ benzo $[4,5]$ imidazo $[2,1-c][1,2,4]$ thiadiazine-10carboxylate 5,5-dioxide (17a)

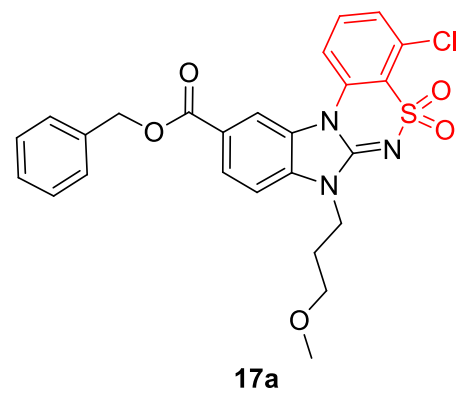


To a solution of benzyl 2-amino-1-(3-methoxypropyl)-1H-benzo[ $d]$ imidazole-5carboxylate 15a $(0.100 \mathrm{~g}, 0.294 \mathrm{mmol})$ in $\mathrm{ACN}(3 \mathrm{~mL})$ was added 2-4 dichloro benzene sulfonyl chloride $(0.087 \mathrm{~g}, 0.353 \mathrm{mmol})$ and anhydrous potassium carboanate $(0.203 \mathrm{~g}, 1.47 \mathrm{mmol})$. The resulting reaction mixture was irradiated at $120{ }^{\circ} \mathrm{C}$ under microwave heating for $20 \mathrm{~min}$. In situ addition of $\mathrm{CuI}(0.0055 \mathrm{~g}$, $0.0294 \mathrm{mmol}$ ) was done to the same reaction mass and it was again irradiated at $150{ }^{\circ} \mathrm{C}$ for additional $15 \mathrm{~min}$. Upon completion of reaction, the residue was filtered through pad of celite and filtrate was concentrated in vacuo. The crude mass was partitioned between aqueous and organic layers. Aqueous layer was extracted with ethyl acetate $(20 \mathrm{~mL}$ x 2$)$. Combined organic layer was dried over $\mathrm{MgSO}_{4}$ and concentrated under reduced pressure to give the crude product, which was purified by column chromatography ( $28 \%$ Ethyl acetate in Hexane) on silica gel to afford compound 17a as a off white $(0.117 \mathrm{~g}, 78 \%)$. ${ }^{1} \mathrm{H}$ NMR $(400 \mathrm{MHz}$, acetone- $\left._{6}\right) \delta 8.61(\mathrm{~s}, 1 \mathrm{H}), 8.28(\mathrm{~d}, J=8.3 \mathrm{~Hz}, 1 \mathrm{H}), 8.14(\mathrm{~d}, J=8.3 \mathrm{~Hz}, 1 \mathrm{H}), 7.81$ (t, $J=8.1 \mathrm{~Hz}, 1 \mathrm{H}), 7.66(\mathrm{~d}, J=8.3 \mathrm{~Hz}, 1 \mathrm{H}), 7.59(\mathrm{~d}, J=8.0 \mathrm{~Hz}, 1 \mathrm{H}), 7.51(\mathrm{~m}$, 2H), $7.37(\mathrm{~m}, 3 \mathrm{H}), 5.40(\mathrm{~s}, 2 \mathrm{H}), 4.27(\mathrm{t}, J=6.4 \mathrm{~Hz}, 2 \mathrm{H}), 3.41(\mathrm{t}, J=5.4 \mathrm{~Hz}, 2 \mathrm{H})$, $3.22(\mathrm{~s}, 3 \mathrm{H}), 2,09(\mathrm{~m}, 2 \mathrm{H}) ;{ }^{13} \mathrm{C}$ NMR $\left(101 \mathrm{MHz}\right.$, acetone- $\left.\mathrm{d}_{6}\right) \delta 165.1,147.3,136.4$, $135.1,134.9,132.9,131.8,129.0,128.5,128.1,128.0,127.0,126.8,124.8,123.9$, 115.4, 114.3, 110.1, 68.7, 66.5, 57.7, 39.8, 27.5; HRMS (ESI+) calcd for C25H23ClN3O5S m/z : 512.1041; found 512.1044. 
General Procedure for the synthesis of 2-chloro-11-(3-methoxypropyl)-11Hbenzo $[e]$ benzo $[4,5]$ imidazo $[1,2-b][1,2,4]$ thiadiazine-8-carboxylic acid $5,5-$ dioxide (18a)

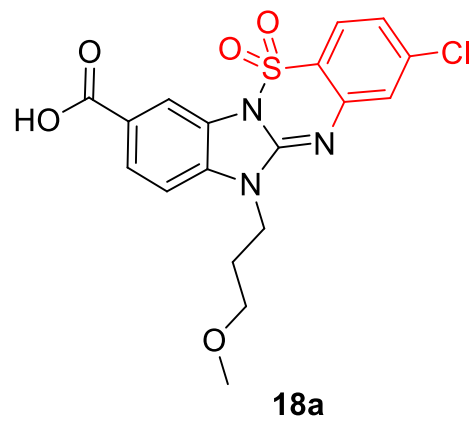

To a solution of benzyl 2-chloro-11-(3-methoxypropyl)-11Hbenzo[ $e]$ benzo[4,5]imidazo[1,2-b][1,2,4]thiadiazine-8-carboxylate 5,5 -dioxide 16a $(0.100 \mathrm{~g}, 0.195 \mathrm{mmol})$ in $\mathrm{MeOH}$ and $\mathrm{DCM}(7: 3,10 \mathrm{~mL})$ was added ammonium formate $(0.036 \mathrm{~g}, 0.585 \mathrm{mmol})$ and $10 \%$ palladium $(0.062 \mathrm{~g}, 0.585$ mmol). The resulting reaction mixture was allowed to stir at room temperature. Upon completion $(1 \mathrm{~h})$ of reaction, the reaction mass filtered and filtrate was evaporated under reduced pressure to give the crude product, which was purified by adjusting the $\mathrm{pH}$ to afford compound $\mathbf{1 8 a}$ as a white solid (0.063 gm, $76 \%)$. ${ }^{1} \mathrm{H}$ NMR (400 MHz, DMSO-d $\left.{ }_{6}\right) \delta 8.20(\mathrm{~s}, 1 \mathrm{H}), 8.07(\mathrm{~d}, J=7.9 \mathrm{~Hz}, 1 \mathrm{H}), 8.04(\mathrm{dd}$, $J=8.4,1.0 \mathrm{~Hz}, 1 \mathrm{H}), 7.75(\mathrm{t}, J=7.7 \mathrm{~Hz}, 1 \mathrm{H}), 7.58(\mathrm{~d}, J=8.4 \mathrm{~Hz}, 1 \mathrm{H}), 7.47(\mathrm{~d}, J$ $=8.2 \mathrm{~Hz}, 1 \mathrm{H}), 7.37(\mathrm{t}, J=7.7 \mathrm{~Hz}, 1 \mathrm{H}), 4.21(\mathrm{t}, J=6.6 \mathrm{~Hz}, 2 \mathrm{H}), 3.37(\mathrm{t}, J=5.8$ $\mathrm{Hz}, 3 \mathrm{H}), 3.14(\mathrm{~s}, 4 \mathrm{H}), 2.06-1.95(\mathrm{~m}, 2 \mathrm{H}) ;{ }^{13} \mathrm{C} \mathrm{NMR}\left(101 \mathrm{MHz}, \mathrm{DMSO}-\mathrm{d}_{6}\right) \delta$ $166.9,147.1,143.9,135.4,128.1,126.6,125.3,124.0,122.6,121.9,112.9,109.9$, 
69.3, 58.3, 40.3, 27.5; HRMS (ESI+) calcd for $\mathrm{C}_{18} \mathrm{H}_{17} \mathrm{ClN}_{3} \mathrm{O}_{5} \mathrm{~S} \mathrm{~m} / \mathrm{z}: 422.0572$; found 422.0578 .

General Procedure for the synthesis of 4-chloro-7-(3-methoxypropyl)-7Hbenzo $[e]$ benzo $[4,5]$ imidazo $[2,1-c][1,2,4]$ thiadiazine-10-carboxylic acid $5,5-$ dioxide (19a)

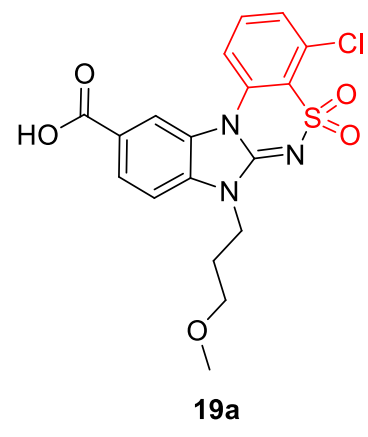

To a solution of benzyl 4-chloro-7-(3-methoxypropyl)-7Hbenzo[ $e]$ benzo[4,5]imidazo[2,1-c][1,2,4]thiadiazine-10-carboxylate 5,5-dioxide 17a $(0.100 \mathrm{~g}, 0.195 \mathrm{mmol})$ in $\mathrm{MeOH}$ and $\mathrm{DCM}(7: 3,10 \mathrm{~mL})$ was added ammonium formate $(0.036 \mathrm{gm}, 0.585 \mathrm{mmol})$ and $10 \%$ palladium on carbon $(0.062 \mathrm{~g}, 0.585 \mathrm{mmol})$. The resulting reaction mixture was allowed to stir at room temperature. Upon completion $(1 \mathrm{~h})$ of reaction, the reaction mass filtered and filtrate was evaporated under reduced pressure to give the crude product, which was purified by adjusting the $\mathrm{pH}$ to afford compound 19a as a white solid $(0.060$ g, $73 \%) .{ }^{1} \mathrm{H}$ NMR (400 MHz, DMSO-d 6 ) $\delta 8.51(\mathrm{~s}, 1 \mathrm{H}), 8.23(\mathrm{~d}, J=8.3 \mathrm{~Hz}, 1 \mathrm{H})$, $8.05(\mathrm{dd}, J=8.4,1.0 \mathrm{~Hz}, 1 \mathrm{H}), 7.88(\mathrm{t}, J=8.3 \mathrm{~Hz}, 1 \mathrm{H}), 7.69(\mathrm{~m}, 2 \mathrm{H}), 4.18(\mathrm{t}, J=$ $6.7 \mathrm{~Hz}, 2 \mathrm{H}), 3.37(\mathrm{t}, J=5.9 \mathrm{~Hz}, 2 \mathrm{H}), 3.17(\mathrm{~s}, 3 \mathrm{H}), 1.98(\mathrm{~m}, 2 \mathrm{H}) ;{ }^{13} \mathrm{C}$ NMR $(101$ MHz, DMSO-d $\left.\mathrm{d}_{6}\right) \delta 167.24,147.3,135.2,134.5,134.1,131.1,129.4,127.2,126.9$, 
126.1, 123.3, 116.4, 114.6, 110.8, 69.1, 58.3, 27.65; HRMS (ESI+) calcd for $\mathrm{C}_{18} \mathrm{H}_{17} \mathrm{ClN}_{3} \mathrm{O}_{5} \mathrm{~S} \mathrm{~m} / \mathrm{z}: 422.0572$; found 422.0572.

General procedure for the synthesis of 9-chloro-6-(2-(cyclohex-1-en-1yl)ethyl)-3,4diphenylbenzo[ $e] \quad$ isochromeno[6',7':4,5]imidazo[1,2-b][1,2,4] thiadiazin-1(6H)-one 12,12-dioxide (21c)

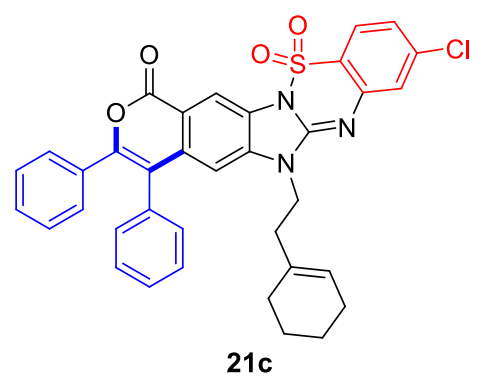

A oven dried seal tube was charged with 2-chloro-11-(2-(cyclohex-1-en-1yl)ethyl)-11Hbenzo[ $e$ ]benzo[4,5] imidazo[1,2- $b][1,2,4]$ thiadiazine-8-carboxylic acid 5,5-dioxide $(0.070 \mathrm{~g}, 0.152 \mathrm{mmol})$ and diphenyl acetylene $(0.032 \mathrm{~g}, 0.183$ mmol) under nitrogen atmosphere. $2 \mathrm{~mL}$ DMF was added to this mixture followed by addition of $\left[\mathrm{Cp}^{*} \mathrm{RhCl}_{2}\right]_{2}(4.65 \mathrm{mg}, 0.0076 \mathrm{mmol}), \mathrm{AgSbF}_{6}(5.22 \mathrm{mg}, 0.0152$ mmol) and $\mathrm{CuSO}_{4} \cdot \mathrm{H}_{2} \mathrm{O}(6.00 \mathrm{mg}, 0.0304 \mathrm{mmol})$. The resulting reaction mixture was sealed in a tube and further heated at $65{ }^{\circ} \mathrm{C}$. Upon completion of reaction ( 9 h), the residue was filtered through pad of celite and filtrate was concentrated in vacuo. The crude mass was partitioned between aqueous and organic layers. Aqueous layer was extracted with ethyl acetate $(20 \mathrm{~mL}$ x 2). Combined organic layer was dried over $\mathrm{MgSO}_{4}$ and concentrated under reduced pressure to give the crude product, which was purified by column chromatography (20\% Ethyl acetate in Hexane) on silica gel to afford compound 21c as light yellow solid 
$(0.073 \mathrm{~g}, 76 \%) .{ }^{1} \mathrm{H} \mathrm{NMR}\left(300 \mathrm{MHz}, \mathrm{CDCl}_{3}\right) \delta 8.70(\mathrm{~s}, 1 \mathrm{H}), 7.91(\mathrm{~d}, J=8.5 \mathrm{~Hz}$, 1H), $7.49(\mathrm{~m}, 3 \mathrm{H}), 7.44(\mathrm{~d}, J=1.8 \mathrm{~Hz}, 1 \mathrm{H}), 7.33(\mathrm{~m}, 4 \mathrm{H}), 7.23(\mathrm{~m}, 4 \mathrm{H}), 6.77(\mathrm{~s}$, 1H), $5.20(\mathrm{~s}, 1 \mathrm{H}), 4.02(\mathrm{t}, J=6.9 \mathrm{~Hz}, 2 \mathrm{H}), 2.30(\mathrm{t}, J=6.9 \mathrm{~Hz}, 2 \mathrm{H}), 1.82(\mathrm{~m}, 4 \mathrm{H})$, $1.54-1.35(\mathrm{~m}, 4 \mathrm{H}) ;{ }^{13} \mathrm{C}$ NMR $\left(75 \mathrm{MHz}, \mathrm{CDCl}_{3}\right) \delta 161.3,151.3,147.5,145.0$, $140.5,138.0,136.8,134.2,132.7,132.5,131.2,129.3,129.2,129.1,128.4,127.8$ $126.2,125.5,124.4,123.9,123.6,121.2,116.2,115.2,113.2,104.0,41.4,35.3$, 27.9, 25.0, 22.6, 21.9; HRMS (ESI+) calcd for $\mathrm{C}_{36} \mathrm{H}_{29} \mathrm{ClN}_{3} \mathrm{O}_{4} \mathrm{~S} \mathrm{~m} / \mathrm{z}: 634.1562$; found 634.1569 .

General procedure for the synthesis of 2-chloro-7-(2-(cyclohex-1-en-1yl)ethyl)-9,10-diphenylbenzo[e] isochromeno $\left[6^{\prime}, 7^{\prime}: 4,5\right]$ imidazo $[2,1-$ c] $][1,2,4]$ thiadiazin-12(7H)-one 5,5-dioxide (23e)

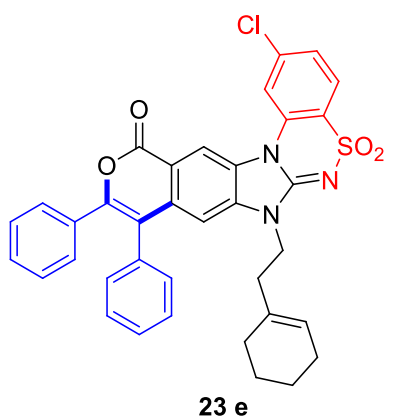

A oven dried seal tube was charged with 2-chloro-11-(2-(cyclohex-1-en-1yl)ethyl)-11H-benzo[ $e$ ]benzo[4,5] imidazo[1,2-b][1,2,4]thiadiazine-8-carboxylic acid 5,5-dioxide $(0.070 \mathrm{~g}, 0.152 \mathrm{mmol})$ and diphenyl acetylene $(0.032 \mathrm{~g}, 0.183$ mmol) under nitrogen atmosphere. $2 \mathrm{~mL}$ DMF was added to this mixture followed by addition of $\left[\mathrm{Cp}^{*} \mathrm{RhCl}_{2}\right]_{2}(4.65 \mathrm{mg}, 0.0076 \mathrm{mmol}), \mathrm{AgSbF}_{6}(5.22 \mathrm{mg}, 0.0152$ $\mathrm{mmol})$ and $\mathrm{CuSO}_{4} \cdot \mathrm{H}_{2} \mathrm{O}(6.00 \mathrm{mg}, 0.0304 \mathrm{mmol})$. The resulting reaction mixture was sealed in a tube and further heated at $65^{\circ} \mathrm{C}$. Upon completion of reaction $(11$ 
h), the residue was filtered through pad of celite and filtrate was concentrated in vacuo. The crude mass was partitioned between aqueous and organic layers. Aqueous layer was extracted with ethyl acetate $(20 \mathrm{~mL}$ x 2). Combined organic layer was dried over $\mathrm{MgSO}_{4}$ and concentrated under reduced pressure to give the crude product, which was purified by column chromatography (30-35 \% Ethyl acetate in Hexane) on silica gel to afford compound 23e as a brown solid (0.061 $\mathrm{g}, 63 \%) .{ }^{1} \mathrm{H}$ NMR $\left(400 \mathrm{MHz}, \mathrm{CDCl}_{3}\right) \delta 8.84(\mathrm{~s}, 1 \mathrm{H}), 8.17(\mathrm{~s}, 1 \mathrm{H}), 8.08(\mathrm{~d}, J=$ $8.4 \mathrm{~Hz}, 1 \mathrm{H}), 7.57(\mathrm{~d}, J=8.4 \mathrm{~Hz}, 1 \mathrm{H}), 7.45(\mathrm{~m}, 3 \mathrm{H}), 7.31(\mathrm{~m}, 4 \mathrm{H}), 7.23(\mathrm{~m}, 3 \mathrm{H})$, $7.16(\mathrm{~s}, 1 \mathrm{H}), 4.12(\mathrm{t}, J=6.2 \mathrm{~Hz}, 2 \mathrm{H}), 3.21(\mathrm{t}, J=5.4 \mathrm{~Hz}, 2 \mathrm{H}), 2.96(\mathrm{~s}, 3 \mathrm{H}), 2.01$ $(\mathrm{m}, 2 \mathrm{H}) ;{ }^{13} \mathrm{C} \mathrm{NMR}\left(101 \mathrm{MHz}, \mathrm{CDCl}_{3}\right) \delta 161.6,151.7,149.4,139.4,137.4,136.5$, 133.8, 133.6, 132.3, 131.2, 129.4, 129.3, 129.2, 128.6, 127.9, 127.8, 127.0, 126.3, 123.2, 116.2, 115.9, 115.6, 113.3, 105.7, 68.2, 58.4, 40.2, 27.3; HRMS (ESI+) calcd for $\mathrm{C}_{32} \mathrm{H}_{25} \mathrm{ClN}_{3} \mathrm{O}_{5} \mathrm{~S} \mathrm{~m} / \mathrm{z}$ : 598.1198; found 598.1176.

Methyl 2-chloro-11-(3-methoxypropyl)-3-methyl-11H-benzimidazo [1,2b][1,2,4]benzothiadiazine-8-carboxylate 5,5-dioxide (8b) Off white solid; $(0.145 \mathrm{~g}, 85 \%) ;{ }^{1} \mathrm{H} \mathrm{NMR}\left(300 \mathrm{MHz}, \mathrm{CDCl}_{3}\right) \delta 8.48(\mathrm{~d}, J=1.3 \mathrm{~Hz}, 1 \mathrm{H}), 8.12(\mathrm{dd}$, $J=8.4,1.3 \mathrm{~Hz}, 1 \mathrm{H}), 7.84(\mathrm{~s}, 1 \mathrm{H}), 7.49(\mathrm{~s}, 1 \mathrm{H}), 7.27(\mathrm{~d}, J=8.4 \mathrm{~Hz}, 1 \mathrm{H}), 4.23(\mathrm{t}$, 
$J=6.7 \mathrm{~Hz}, 2 \mathrm{H}), 3.98(\mathrm{~s}, 3 \mathrm{H}), 3.41(\mathrm{t}, J=6.7 \mathrm{~Hz}, 2 \mathrm{H}), 3.33(\mathrm{~s}, 3 \mathrm{H}), 2.48(\mathrm{~s}, 3 \mathrm{H})$, 2.25-2.05 (m, 2H); ${ }^{13} \mathrm{C}$ NMR (75 MHz, $\left.\mathrm{CDCl}_{3}\right) \delta 166.6,147.5,143.5,141.3$, 135.6, 132.2, 128.5, 126.8, 125.0, 124.7, 123.9, 121.0, 114.5, 108.6, 69.2, 59.1, 52.8, 40.22, 28.2, 20.2; HRMS : calcd for $\mathrm{C}_{20} \mathrm{H}_{20} \mathrm{ClN}_{3} \mathrm{O}_{5} \mathrm{~S} \mathrm{~m} / z$ : 449.0812; found $449.0817(\mathrm{M})^{+}$.

Methyl 4-chloro-11-[2-(cyclohex-1-en-1-yl)ethyl]-11H-benzimidazo $\quad[1,2-$ b][1,2,4]benzothiadiazine-8-carboxylate 5,5-dioxide (8c) Light brown solid; $(0.140 \mathrm{~g}, 89 \%) ;{ }^{1} \mathrm{H} \mathrm{NMR}\left(300 \mathrm{MHz}, \mathrm{CDCl}_{3}\right) \delta 8.48(\mathrm{~d}, J=1.4 \mathrm{~Hz}, 1 \mathrm{H}), 8.08(\mathrm{dd}$, $J=8.4,1.4 \mathrm{~Hz}, 1 \mathrm{H}), 7.48(\mathrm{t}, J=8.4 \mathrm{~Hz}, 1 \mathrm{H}), 7.33(\mathrm{dd}, J=8.4,1.2 \mathrm{~Hz}, 1 \mathrm{H}), 7.22$ $(\mathrm{dd}, J=7.8,1.2 \mathrm{~Hz}, 1 \mathrm{H}), 7.16(\mathrm{~d}, J=8.4 \mathrm{~Hz}, 1 \mathrm{H}), 5.26(\mathrm{~s}, 1 \mathrm{H}), 4.18(\mathrm{t}, J=7.2$ $\mathrm{Hz}, 2 \mathrm{H}), 3.95(\mathrm{~s}, 3 \mathrm{H}), 2.41(\mathrm{t}, J=7.2 \mathrm{~Hz}, 2 \mathrm{H}), 2.12-2.03(\mathrm{~m}, 2 \mathrm{H}), 1.91-1.76(\mathrm{~m}$, 2H), 1.65-1.48 (m, 2H), 1.49-1.34 (m, 2H); $\left.{ }^{13} \mathrm{C} \mathrm{NMR} \mathrm{(75} \mathrm{MHz,} \mathrm{CDCl}_{3}\right) \delta 166.4$, 146.6, 146.5, 134.8, 134.2, 133.6, 130.1, 128.2, 126.1, 125.6, 125.4, 124.9, 124.5, 121.2, 114.4, 108.8, 52.7, 41.5, 36.0, 28.6, 25.3, 23.1, 22.4; HRMS : calcd for $\mathrm{C}_{23} \mathrm{H}_{22} \mathrm{ClN}_{3} \mathrm{O}_{4} \mathrm{~S} m / z$ : 471.1020; found $471.1021(\mathrm{M})^{+}$.

Methyl 2-chloro-11-[2-(cyclohex-1-en-1-yl)ethyl]-3-methyl-11Hbenzimidazo[1,2-b][1,2,4]benzothiadiazine-8-carboxylate 5,5 -dioxide $(8 d)$ Off white solid; (0.140 g, $89 \%) ;{ }^{1} \mathrm{H}$ NMR (300 MHz, $\left.\mathrm{CDCl}_{3}\right) \delta 8.42(\mathrm{~s}, 1 \mathrm{H}), 8.06$ (d, $J=8.4 \mathrm{~Hz}, 1 \mathrm{H}), 7.80(\mathrm{~s}, 1 \mathrm{H}), 7.44(\mathrm{~s}, 1 \mathrm{H}), 7.12(\mathrm{~d}, J=8.4 \mathrm{~Hz}, 1 \mathrm{H}), 5.26(\mathrm{~s}$, $1 \mathrm{H}), 4.14(\mathrm{t}, J=7.1 \mathrm{~Hz}, 2 \mathrm{H}), 3.96(\mathrm{~s}, 3 \mathrm{H}), 2.45(\mathrm{~d}, J=6.6 \mathrm{~Hz}, 3 \mathrm{H}), 2.40(\mathrm{t}, J=$ $6.5 \mathrm{~Hz}, 2 \mathrm{H}), 2.10-2.02(\mathrm{~m}, 2 \mathrm{H}), 1.87-1.76(\mathrm{~m}, 2 \mathrm{H}), 1.61-1.50(\mathrm{~m}, 2 \mathrm{H}), 1.48-1.38$ 
(m, 2H); ${ }^{13} \mathrm{C} \mathrm{NMR}\left(75 \mathrm{MHz}, \mathrm{CDCl}_{3}\right) \delta 166.5,147.5,143.5,141.2,135.2,133.5$, 132.0, 128.3, 126.7, 125.4, 124.8, 124.7, 123.8, 121.0, 114.4, 108.6, 52.8, 41.6, 35.9, 28.6, 25.5, 23.1, 22.4, 20.1; HRMS : calcd for $\mathrm{C}_{24} \mathrm{H}_{22} \mathrm{ClN}_{3} \mathrm{O}_{4} \mathrm{~S} \mathrm{~m} / \mathrm{z}$ : 485.1176; found $485.1169(\mathrm{M})^{+}$.

Methyl 2-cyano-11-[2-(cyclohex-1-en-1-yl)ethyl]-11H-benzimidazo $\quad[1,2-$ b][1,2,4]benzothiadiazine-8-carboxylate 5,5-dioxide (8e) Off white solid; $(0.134 \mathrm{~g}, 87 \%) ;{ }^{1} \mathrm{H}$ NMR $\left(300 \mathrm{MHz}, \mathrm{CDCl}_{3}\right) \delta 8.49(\mathrm{~s}, 1 \mathrm{H}), 8.14(\mathrm{~d}, J=8.4 \mathrm{~Hz}$, 1H), $8.07(\mathrm{~d}, J=8.2 \mathrm{~Hz}, 1 \mathrm{H}), 7.75(\mathrm{~s}, 1 \mathrm{H}), 7.48(\mathrm{dd}, J=8.2,1.2 \mathrm{~Hz}, 1 \mathrm{H}), 7.22$ $(\mathrm{d}, J=8.4 \mathrm{~Hz}, 1 \mathrm{H}), 5.27(\mathrm{~s}, 1 \mathrm{H}), 4.23(\mathrm{t}, J=7.0 \mathrm{~Hz}, 2 \mathrm{H}), 3.98(\mathrm{~s}, 3 \mathrm{H}), 2.44(\mathrm{t}, J$ $=6.6 \mathrm{~Hz}, 2 \mathrm{H}), 2.18-2.02(\mathrm{~m}, 2 \mathrm{H}), 1.83-1.75(\mathrm{~m}, 2 \mathrm{H}), 1.64-1.52(\mathrm{~m}, 2 \mathrm{H})$, 1.49-1.36 (m, 2H); ${ }^{13} \mathrm{C}$ NMR (75 MHz, $\left.\mathrm{CDCl}_{3}\right) \delta 166.3,148.1,145.2,135.0$, $133.3,131.2,128.7,125.8,125.7,125.5,125.3,124.5,124.0,118.3,117.7,114.8$, 109.1, 52.9, 41.8, 36.0, 28.6, 25.5, 23.1, 22.4; HRMS : calcd for $\mathrm{C}_{24} \mathrm{H}_{22} \mathrm{~N}_{4} \mathrm{O}_{4} \mathrm{~S}$ $m / z: 462.1362$; found $462.1355(\mathrm{M})^{+}$.

Methyl 11-butyl-4-chloro-11H-benzimidazo[1,2-b][1,2,4]benzothia- diazine8-carboxylate 5,5-dioxide (8f) Light brown solid; (0.144 g, $85 \%)$; ${ }^{1} \mathrm{H}$ NMR (300 $\left.\mathrm{MHz}, \mathrm{CDCl}_{3}\right) \delta 8.43(\mathrm{~d}, J=1.4 \mathrm{~Hz}, 1 \mathrm{H}), 8.03(\mathrm{dd}, J=8.4,1.4 \mathrm{~Hz}, 1 \mathrm{H}), 7.46(\mathrm{t}, J$ $=8.4 \mathrm{~Hz}, 1 \mathrm{H}), 7.31(\mathrm{dd}, J=8.4,1.1 \mathrm{~Hz}, 1 \mathrm{H}), 7.19(\mathrm{dd}, J=7.8,1.1 \mathrm{~Hz}, 1 \mathrm{H}), 7.15$ (d, $J=8.4 \mathrm{~Hz}, 1 \mathrm{H}), 4.09(\mathrm{t}, J=7.3 \mathrm{~Hz}, 2 \mathrm{H}), 3.94(\mathrm{~s}, 3 \mathrm{H}), 1.95-1.71(\mathrm{~m}, 2 \mathrm{H})$, $1.57-1.33(\mathrm{~m}, 2 \mathrm{H}), 0.99(\mathrm{t}, J=7.3 \mathrm{~Hz}, 3 \mathrm{H}) ;{ }^{13} \mathrm{C} \mathrm{NMR}\left(75 \mathrm{MHz}, \mathrm{CDCl}_{3}\right) \delta 166.4$, $146.6,146.5,134.9,134.2,130.0,128.3,126.2,125.6,124.9,124.6,121.2,114.3$, 
108.6, 52.7, 42.7, 30.2, 20.4, 14.1; HRMS : calcd for $\mathrm{C}_{19} \mathrm{H}_{18} \mathrm{ClN}_{3} \mathrm{O}_{4} \mathrm{~S} \mathrm{~m} / z$ : 419.0707; found $419.0703(\mathrm{M})^{+}$.

Methyl 11-butyl-2-fluoro-11H-benzimidazo[1,2-b][1,2,4]benzothia- diazine-

8-carboxylate 5,5-dioxide (8g) Light brown solid; (0.148 g, $91 \%)$; ${ }^{1} \mathrm{H}$ NMR $\left(300 \mathrm{MHz}, \mathrm{CDCl}_{3}\right) \delta 8.45(\mathrm{~d}, J=1.4 \mathrm{~Hz}, 1 \mathrm{H}), 8.08(\mathrm{dd}, J=8.4,1.4 \mathrm{~Hz}, 1 \mathrm{H}), 7.97$ $(\mathrm{dd}, J=8.9,5.8 \mathrm{~Hz}, 1 \mathrm{H}), 7.18(\mathrm{~d}, J=8.4 \mathrm{~Hz}, 1 \mathrm{H}), 7.10(\mathrm{dd}, J=10.4,2.4 \mathrm{~Hz}, 1 \mathrm{H})$, 6.99 (ddd, $J=8.9,7.9,2.5 \mathrm{~Hz}, 1 \mathrm{H}), 4.11$ (t, $J=7.3 \mathrm{~Hz}, 2 \mathrm{H}), 3.96$ (s, 3H), $1.93-1.68(\mathrm{~m}, 2 \mathrm{H}), 1.54-1.31(\mathrm{~m}, 2 \mathrm{H}), 1.01(\mathrm{t}, J=7.3 \mathrm{~Hz}, 3 \mathrm{H}) ;{ }^{13} \mathrm{C}$ NMR $(75$ $\left.\mathrm{MHz}, \mathrm{CDCl}_{3}\right) \delta 168.2,166.4,164.8,147.9,147.4\left(J_{\mathrm{CF}}=13.5 \mathrm{~Hz}\right), 135.2,128.4$ $125.2\left(J_{\mathrm{CF}}=9.2 \mathrm{~Hz}\right), 124.9\left(J_{\mathrm{CF}}=25.9 \mathrm{~Hz}\right), 119.1,114.5,112.8\left(J_{\mathrm{CF}}=23.1 \mathrm{~Hz}\right)$, $111.8\left(J_{\mathrm{CF}}=24.1 \mathrm{~Hz}\right), 108.6,52.8,42.8,30.2,20.4,14.1$; HRMS : calcd for $\mathrm{C}_{19} \mathrm{H}_{18} \mathrm{FN}_{3} \mathrm{O}_{4} \mathrm{~S} m / z: 403.1002$; found $403.1010(\mathrm{M})^{+}$.

Methyl

4-chloro-11-cyclopentyl-11H-benzimidazo[1,2-b][1,2,4] benzothiadiazine-8-carboxylate 5,5-dioxide (8h) Light brown solid; (0.128 g, $77 \%) ;{ }^{1} \mathrm{H}$ NMR (300 MHz, $\left.\mathrm{CDCl}_{3}\right) \delta 8.56(\mathrm{~d}, J=1.5 \mathrm{~Hz}, 1 \mathrm{H}), 8.10(\mathrm{dd}, J=8.5$, $1.6 \mathrm{~Hz}, 1 \mathrm{H}), 7.50(\mathrm{t}, J=8.1 \mathrm{~Hz}, 1 \mathrm{H}), 7.35(\mathrm{dd}, J=8.4,1.1 \mathrm{~Hz}, 1 \mathrm{H}), 7.29-7.21$ (m, 2H), $5.21(\mathrm{p}, J=8.8 \mathrm{~Hz}, 1 \mathrm{H}), 3.97(\mathrm{~s}, 3 \mathrm{H}), 2.37-2.12(\mathrm{~m}, 4 \mathrm{H}), 2.12-1.95(\mathrm{~m}$, 2H), $1.90-1.71(\mathrm{~m}, 2 \mathrm{H}) ;{ }^{13} \mathrm{C}$ NMR $\left(75 \mathrm{MHz}, \mathrm{CDCl}_{3}\right) \delta 166.5,146.6,146.4$, 134.2, 133.6, 130.2, 128.0, 126.1, 125.6, 125.0, 124.8, 121.1, 114.6, 109.9, 55.3, 52.8, 28.9, 25.6; HRMS : calcd for $\mathrm{C}_{20} \mathrm{H}_{18}{ }^{37} \mathrm{ClN}_{3} \mathrm{O}_{4} \mathrm{~S} \mathrm{~m} / z$ : 433.0677; found $433.0679(\mathrm{M})^{+}$. 
diazine-8-carboxylate 5,5-dioxide (8i) White solid; (0.147 g, $89 \%)$; ${ }^{1} \mathrm{H}$ NMR $\left(300 \mathrm{MHz}, \mathrm{CDCl}_{3}\right) \delta 8.40(\mathrm{~d}, J=1.4 \mathrm{~Hz}, 1 \mathrm{H}), 8.01(\mathrm{dd}, J=8.4,1.4 \mathrm{~Hz}, 1 \mathrm{H}), 7.44$ (t, $J=8.4,1 \mathrm{H}), 7.30(\mathrm{dd}, J=8.4,1.2 \mathrm{~Hz}, 1 \mathrm{H}), 7.17(\mathrm{dd}, J=7.8,1.2 \mathrm{~Hz}, 1 \mathrm{H}), 7.14$ $(\mathrm{d}, J=8.4 \mathrm{~Hz}, 1 \mathrm{H}), 4.06(\mathrm{t}, J=7.4,2 \mathrm{H}), 3.93(\mathrm{~s}, 3 \mathrm{H}), 1.93-1.67(\mathrm{~m}, 2 \mathrm{H})$, $1.45-1.31(\mathrm{~m}, 4 \mathrm{H}), 0.90(\mathrm{t}, J=6.8 \mathrm{~Hz}, 3 \mathrm{H}) ;{ }^{13} \mathrm{C} \mathrm{NMR}\left(75 \mathrm{MHz}, \mathrm{CDCl}_{3}\right) \delta 166.3$, $146.6,146.4,134.9,134.1,129.9,128.5,126.2,125.5,124.8,124.5,121.1,114.2$, 108.6, 52.7, 42.9, 29.2, 27.8, 22.6, 14.3; HRMS : calcd for $\mathrm{C}_{20} \mathrm{H}_{20} \mathrm{ClN}_{3} \mathrm{O}_{4} \mathrm{~S} \mathrm{~m} / z$ : 433.0863; found $433.0858(\mathrm{M})^{+}$.

Methyl 4-chloro-11-cyclooctyl-11H-benzimidazo[1,2-b][1,2,4]benzothiadiazine-8-carboxylate 5,5-dioxide (8j) Off white solid; $(0.130 \mathrm{~g}, 83 \%) ;{ }^{1} \mathrm{H}$ $\operatorname{NMR}\left(300 \mathrm{MHz}, \mathrm{CDCl}_{3}\right) \delta 8.50(\mathrm{~s}, 1 \mathrm{H}), 8.05(\mathrm{~d}, J=8.5 \mathrm{~Hz}, 1 \mathrm{H}), 7.48(\mathrm{t}, J=8.0$ $\mathrm{Hz}, 1 \mathrm{H}), 7.37(\mathrm{~d}, J=8.0 \mathrm{~Hz}, 1 \mathrm{H}), 7.28(\mathrm{~d}, J=8.5 \mathrm{~Hz}, 1 \mathrm{H}), 7.20(\mathrm{~d}, J=7.6 \mathrm{~Hz}$, 1H), $5.00(\mathrm{~s}, 1 \mathrm{H}), 3.95(\mathrm{~s}, 3 \mathrm{H}), 2.03-1.87(\mathrm{~m}, 2 \mathrm{H}), 2.12-1.50(\mathrm{~m}, 12 \mathrm{H}) ;{ }^{13} \mathrm{C} \mathrm{NMR}$ $\left(75 \mathrm{MHz}, \mathrm{CDCl}_{3}\right) \delta 166.5,146.7,145.9,134.1,133.6,130.0,127.9,126.3,125.4$, 124.9, 124.6, 120.9, 114.4, 110.4, 55.7, 52.7, 32.2, 26.5, 25.7; HRMS : calcd for $\mathrm{C}_{23} \mathrm{H}_{24} \mathrm{ClN}_{3} \mathrm{O}_{4} \mathrm{~S} \mathrm{~m} / \mathrm{z}:$ : 473.1176; found $473.1173(\mathrm{M})^{+}$.

Methyl 2-chloro-7-(3-methoxypropyl)-7H-benzimidazo[2,1-c][1,2,4] benzothiadiazine-10-carboxylate 5,5-dioxide (10b) Off white solid; $(0.132 \mathrm{~g}$, $80 \%) ;{ }^{1} \mathrm{H}$ NMR $\left(300 \mathrm{MHz}, \mathrm{CDCl}_{3}\right) \delta 8.56(\mathrm{~s}, 1 \mathrm{H}), 8.19(\mathrm{dd}, J=8.5,1.0 \mathrm{~Hz}, 1 \mathrm{H})$, $8.10(\mathrm{~s}, 1 \mathrm{H}), 8.03(\mathrm{~s}, 1 \mathrm{H}), 7.49(\mathrm{~d}, J=8.5 \mathrm{~Hz}, 1 \mathrm{H}), 4.15(\mathrm{t}, J=6.8 \mathrm{~Hz}, 2 \mathrm{H}), 4.04$ 
(s, 3H), 2.29-2.06 (m, 2H), 1.45-1.34 (m, 4H), $0.89(\mathrm{t}, J=7.2 \mathrm{~Hz}, 3 \mathrm{H}) ;{ }^{13} \mathrm{C}$ NMR $\left(75 \mathrm{MHz}, \mathrm{CDCl}_{3}\right) \delta 166.5,149.6,139.5,136.5,134.9,132.2,127.8,127.6,126.8$ 125.9, 123.7, 116.5, 114.6, 110.4, 69.1, 59.1, 53.2, 40.7, 28.3, 20.3; HRMS : calcd for $\mathrm{C}_{19} \mathrm{H}_{18} \mathrm{ClN}_{3} \mathrm{O}_{5} \mathrm{~S} \mathrm{~m} / z$ : 435.0656; found $435.0660(\mathrm{M})^{+}$.

Methyl 2-chloro-7-(3-methoxypropyl)-3-methyl-7H-benzimidazo $\quad[2,1-$ c][1,2,4]benzothiadiazine-10-carboxylate 5,5-dioxide (10c) Light brown solid; $(0.122 \mathrm{~g}, 72 \%) ;{ }^{1} \mathrm{H}$ NMR $\left(300 \mathrm{MHz}, \mathrm{CDCl}_{3}\right) \delta 8.56(\mathrm{~s}, 1 \mathrm{H}), 8.19(\mathrm{dd}, J=8.5$, $1.0 \mathrm{~Hz}, 1 \mathrm{H}), 8.10(\mathrm{~s}, 1 \mathrm{H}), 8.03(\mathrm{~s}, 1 \mathrm{H}), 7.49(\mathrm{~d}, J=8.5 \mathrm{~Hz}, 1 \mathrm{H}), 4.31(\mathrm{t}, J=6.8$ $\mathrm{Hz}, 2 \mathrm{H}), 4.04$ (s, 3H), $3.41(\mathrm{~s}, 2 \mathrm{H}), 3.33(\mathrm{~s}, 3 \mathrm{H}), 2.53(\mathrm{~s}, 3 \mathrm{H}), 2.29-2.06(\mathrm{~m}, 2 \mathrm{H})$; ${ }^{13} \mathrm{C}$ NMR $\left(75 \mathrm{MHz}, \mathrm{CDCl}_{3}\right) \delta 166.5,149.6,139.5,136.5,134.9,132.2,127.8$, 127.6, 126.8, 125.9, 123.7, 116.5, 114.6, 110.4, 69.1, 59.1, 53.2, 40.7, 28.3, 20.3; HRMS : calcd for $\mathrm{C}_{20} \mathrm{H}_{20} \mathrm{ClN}_{3} \mathrm{O}_{5} \mathrm{SNa} m / z$ : 472.0710; found $472.0714(\mathrm{M})^{+}$.

Methyl 7-butyl-4-chloro-7H-benzimidazo[2,1-c][1,2,4]benzothia- diazine10-carboxylate 5,5-dioxide (10d) Light brown solid; (0.132 g, $78 \%) ;{ }^{1} \mathrm{H}$ NMR $\left(300 \mathrm{MHz}, \mathrm{CDCl}_{3}\right) \delta 8.57(\mathrm{~s}, 1 \mathrm{H}), 8.17(\mathrm{dd}, J=8.4,1.2 \mathrm{~Hz}, 1 \mathrm{H}), 8.08(\mathrm{~d}, J=8.4$ $\mathrm{Hz}, 1 \mathrm{H}), 7.70(\mathrm{t}, J=8.0 \mathrm{~Hz}, 1 \mathrm{H}), 7.58(\mathrm{~d}, J=8.0 \mathrm{~Hz},, 1 \mathrm{H}), 7.37(\mathrm{~d}, J=8.4 \mathrm{~Hz}$, $1 \mathrm{H}), 4.17(\mathrm{t}, J=7.3 \mathrm{~Hz}, 2 \mathrm{H}), 4.01(\mathrm{~s}, 3 \mathrm{H}), 1.94-1.78(\mathrm{~m}, 2 \mathrm{H}), 1.60-1.37(\mathrm{~m}, 2 \mathrm{H})$, $1.01(\mathrm{t}, J=7.3 \mathrm{~Hz}, 3 \mathrm{H}) ;{ }^{13} \mathrm{C} \mathrm{NMR}\left(75 \mathrm{MHz}, \mathrm{CDCl}_{3}\right) \delta 166.4,147.6,135.3,134.6$, 133.9, 133.0, 130.0, 127.8, 127.1, 125.8, 124.4, 114.9, 114.9, 110.1, 53.1, 43.2, 
30.2, 20.5, 14.1; HRMS : calcd for $\mathrm{C}_{19} \mathrm{H}_{18} \mathrm{ClN}_{3} \mathrm{O}_{4} \mathrm{~S} \mathrm{~m} / \mathrm{z}: 419.0707$; found $419.0696(\mathrm{M})^{+}$.

Methyl 4-chloro-7-cyclopentyl-7H-benzimidazo[2,1-c] $] 1,2,4]$ benzo

thiadiazine-10-carboxylate 5,5-dioxide (10e) Light brown solid; (0.116 g, 70 $\%) ;{ }^{1} \mathrm{H}$ NMR (300 MHz, $\left.\mathrm{CDCl}_{3}\right) \delta 8.58(\mathrm{~s}, 1 \mathrm{H}), 8.16(\mathrm{~d}, J=8.5 \mathrm{~Hz}, 1 \mathrm{H})$, 8.13-8.06 (m, 2H), $7.57(\mathrm{dd}, J=8.4,1.1 \mathrm{~Hz}, 1 \mathrm{H}), 7.46(\mathrm{~d}, J=8.5 \mathrm{~Hz}, 1 \mathrm{H}), 5.23$ (m, 1H), $4.03(\mathrm{~s}, 3 \mathrm{H}), 2.42-2.13(\mathrm{~m}, 2 \mathrm{H}), 2.12-1.99(\mathrm{~m}, 2 \mathrm{H}), 1.93-1.69(\mathrm{~m}, 2 \mathrm{H})$, $1.02-0.70(\mathrm{~m}, 2 \mathrm{H}) ;{ }^{13} \mathrm{C} \mathrm{NMR}\left(75 \mathrm{MHz}, \mathrm{CDCl}_{3}\right) \delta 166.3,149.8,139.4,134.6$, $133.4,128.0,127.5,127.3,127.3,125.7,124.0,116.3,115.0,111.5,56.1,53.2$, 29.1, 25.5; HRMS : calcd for $\mathrm{C}_{20} \mathrm{H}_{18} \mathrm{ClN}_{3} \mathrm{O}_{4} \mathrm{~S} \mathrm{~m} / 2$ : 431.0707 ; found 431.0700 (M) $+$

Methyl 4-chloro-7-pentyl-7H-benzimidazo[2,1-c][1,2,4]benzothia- diazine10-carboxylate 5,5-dioxide (10f) Off white solid; (0.107 g, $65 \%)$; ${ }^{1} \mathrm{H}$ NMR (300 $\left.\mathrm{MHz}, \mathrm{CDCl}_{3}\right) \delta 8.57(\mathrm{~s}, 1 \mathrm{H}), 8.16(\mathrm{~d}, J=8.4 \mathrm{~Hz}, 1 \mathrm{H}), 8.07(\mathrm{~d}, J=8.4 \mathrm{~Hz}, 1 \mathrm{H})$, $7.69(\mathrm{t}, J=8.0 \mathrm{~Hz}, 1 \mathrm{H}), 7.57(\mathrm{~d}, J=8.0 \mathrm{~Hz}, 1 \mathrm{H}), 7.35(\mathrm{~d}, J=8.5 \mathrm{~Hz}, 1 \mathrm{H}), 4.15$ $(\mathrm{t}, J=6.8 \mathrm{~Hz}, 2 \mathrm{H}), 4.00(\mathrm{~s}, 3 \mathrm{H}), 1.97-1.78(\mathrm{~m}, 2 \mathrm{H}), 1.48-1.33(\mathrm{~m}, 4 \mathrm{H}), 0.93(\mathrm{t}$, $J=6.7 \mathrm{~Hz}, 3 \mathrm{H}) ;{ }^{13} \mathrm{C} \mathrm{NMR}\left(75 \mathrm{MHz}, \mathrm{CDCl}_{3}\right) \delta 166.5,147.6,135.3,134.7,133.9$, 133.0, 130.0, 127.8, 127.2, 125.8, 124.4, 114.9, 114.9, 110.1, 53.1, 43.4, 29.2, 
27.9, 22.7, 14.3; HRMS : calcd for $\mathrm{C}_{20} \mathrm{H}_{20} \mathrm{ClN}_{3} \mathrm{O}_{4} \mathrm{~S} \mathrm{~m} / \mathrm{z}$ : 433.0863; found $433.0853(\mathrm{M})^{+}$.

Methyl 2-chloro-7-pentyl-7H-benzimidazo[2,1-c][1,2,4]benzothia- diazine10-carboxylate 5,5-dioxide (10g) Off white solid; (0.132 g, $80 \%) ;{ }^{1} \mathrm{H}$ NMR (300 $\left.\mathrm{MHz}, \mathrm{CDCl}_{3}\right) \delta 8.56(\mathrm{~d}, J=1.0 \mathrm{~Hz}, 1 \mathrm{H}), 8.19(\mathrm{dd}, J=8.5,1.0 \mathrm{~Hz}, 1 \mathrm{H}), 8.10(\mathrm{~d}$, $J=5.1 \mathrm{~Hz}, 1 \mathrm{H}), 8.08(\mathrm{~d}, J=1.7 \mathrm{~Hz}, 1 \mathrm{H}), 7.56(\mathrm{dd}, J=8.5,1.7 \mathrm{~Hz}, 1 \mathrm{H}), 7.39(\mathrm{~d}$, $J=8.5 \mathrm{~Hz}, 1 \mathrm{H}), 4.18(\mathrm{t}, J=7.5 \mathrm{~Hz}, 2 \mathrm{H}), 4.03(\mathrm{~s}, 3 \mathrm{H}), 1.96-1.74(\mathrm{~m}, 2 \mathrm{H})$, $1.51-1.34(\mathrm{~m}, 4 \mathrm{H}), 0.89(\mathrm{t}, \mathrm{J}=6.7 \mathrm{~Hz}, 3 \mathrm{H}) ;{ }^{13} \mathrm{C} \mathrm{NMR}\left(75 \mathrm{MHz}, \mathrm{CDCl}_{3}\right) \delta 166.4$, $149.8,139.5,134.6,134.5,128.0,128.0,127.3,126.9,126.0,124.0,116.2,114.8$, 110.3, 53.2, 43.7, 29.2, 27.9, 22.7, 14.3; HRMS : calcd for $\mathrm{C}_{20} \mathrm{H}_{20} \mathrm{ClN}_{3} \mathrm{O}_{4} \mathrm{~S} \mathrm{~m} / \mathrm{z}$ : 433.0863; found $433.0858(\mathrm{M})^{+}$.

Methyl 4-chloro-7-(2-methoxyethyl)-7H-benzimidazo $[2,1-c][1,2,4]$ benzothiadiazine-10-carboxylate 5,5-dioxide (10h) White solid; $(0.120$ g, 71 $\%) ;{ }^{1} \mathrm{H} \mathrm{NMR}\left(300 \mathrm{MHz}, \mathrm{CDCl}_{3}\right) \delta 8.57(\mathrm{~d}, J=1.1 \mathrm{~Hz}, 1 \mathrm{H}), 8.16(\mathrm{dd}, J=8.5,1.1$ $\mathrm{Hz}, 1 \mathrm{H}), 8.11(\mathrm{~d}, J=7.6 \mathrm{~Hz}, 1 \mathrm{H}), 7.71(\mathrm{t}, J=8.1 \mathrm{~Hz}, 1 \mathrm{H}), 7.60(\mathrm{dd}, J=8.1,0.9$ $\mathrm{Hz}, 1 \mathrm{H}), 7.55(\mathrm{~d}, J=8.5 \mathrm{~Hz}, 1 \mathrm{H}), 3.82(\mathrm{t}, J=5.0 \mathrm{~Hz}, 2 \mathrm{H}), 4.02(\mathrm{~s}, 3 \mathrm{H}), 3.82(\mathrm{t}, J$ $=5.0 \mathrm{~Hz}, 2 \mathrm{H}), 3.33(\mathrm{~s}, 3 \mathrm{H}) ;{ }^{13} \mathrm{C} \mathrm{NMR}\left(75 \mathrm{MHz}, \mathrm{CDCl}_{3}\right) \delta 166.5,147.6,135.3$, $134.7,133.9,133.0,130.0,127.8,127.2,125.8,124.4,114.9,114.9,110.1,70.4$, 59.5, 53.1, 44.1; HRMS : calcd for $\mathrm{C}_{18} \mathrm{H}_{16} \mathrm{ClN}_{3} \mathrm{O}_{5} \mathrm{~S} \mathrm{~m} / \mathrm{z}$ : 421.0499; found $421.0503(\mathrm{M})^{+}$. 
benzothiadiazine-10-carboxylate 5,5-dioxide (10i) White solid; (0.121 g, $72 \%)$; ${ }^{1} \mathrm{H}$ NMR (300 MHz, $\left.\mathrm{CDCl}_{3}\right) \delta 8.56(\mathrm{~s}, 1 \mathrm{H}), 8.18(\mathrm{dd}, J=8.5,1.3 \mathrm{~Hz}, 1 \mathrm{H})$, 8.13-7.97 (m, 2H), 7.63-7.53 (m, 2H), $4.37(\mathrm{t}, J=4.9 \mathrm{~Hz}, 2 \mathrm{H}), 4.04(\mathrm{~s}, 3 \mathrm{H}), 3.81$ $(\mathrm{t}, J=4.9 \mathrm{~Hz}, 2 \mathrm{H}), 3.32(\mathrm{~s}, 3 \mathrm{H}) ;{ }^{13} \mathrm{C} \mathrm{NMR}\left(75 \mathrm{MHz}, \mathrm{CDCl}_{3}\right) \delta 166.4,149.9,139.5$, 135.6, 134.6, 128.0, 127.9, 127.4, 126.7, 126.0, 123.9, 116.2, 114.5, 111.7, 70.4, 59.5, 53.1, 44.1; HRMS : calcd for $\mathrm{C}_{18} \mathrm{H}_{16} \mathrm{ClN}_{3} \mathrm{O}_{5} \mathrm{~S} \mathrm{~m} / z$ : 421.0499; found $421.0503(\mathrm{M})^{+}$.

Methyl 2-chloro-7-(2-methylpropyl)-7H-benzimidazo $[2,1-c][1,2,4]$ benzothiadiazine-10-carboxylate 5,5-dioxide (10j) Light brown solid; (0.127 g, $75 \%) ;{ }^{1} \mathrm{H}$ NMR $\left(300 \mathrm{MHz}, \mathrm{CDCl}_{3}\right) \delta 8.58(\mathrm{~s}, 1 \mathrm{H}), 8.19(\mathrm{dd}, J=8.5,1.2 \mathrm{~Hz}, 1 \mathrm{H})$, 8.16-8.08 (m, 2H), $7.58(\mathrm{dd}, J=8.5,1.2 \mathrm{~Hz}, 1 \mathrm{H}), 7.40(\mathrm{~d}, J=8.5 \mathrm{~Hz}, 1 \mathrm{H}), 4.04$ $(\mathrm{s}, 3 \mathrm{H}), 4.01(\mathrm{~d}, J=7.6 \mathrm{~Hz}, 2 \mathrm{H}), 2.39(\mathrm{~m}, 1 \mathrm{H}), 1.05(\mathrm{~d}, J=6.7 \mathrm{~Hz}, 6 \mathrm{H}) ;{ }^{13} \mathrm{C} \mathrm{NMR}$ $\left(75 \mathrm{MHz}, \mathrm{CDCl}_{3}\right) \delta 166.3,150.2,139.5,135.1,134.5,128.1,127.9,127.4,126.8$ 126.0, 124.0, 116.3, 114.8, 110.6, 53.2, 50.8, 28.1, 20.6; HRMS : calcd for $\mathrm{C}_{19} \mathrm{H}_{18} \mathrm{ClN}_{3} \mathrm{O}_{4} \mathrm{~S} \mathrm{~m} / \mathrm{z}: 419.0707$; found $419.0715(\mathrm{M})^{+}$.

6-(3-methoxypropyl)-3,4-

diphenylbenzo $[e]$ isochromeno $\left[6^{\prime}, 7^{\prime}: 4,5\right]$ imidazo $[1,2-b][1,2,4]$ thiadiazin1(6H)-one 12,12-dioxide (21a) 
Off white solid; (0.073 g, $78 \%)$; ${ }^{1} \mathrm{H}$ NMR $\left(400 \mathrm{MHz}, \mathrm{CDCl}_{3}\right) \delta 8.69(\mathrm{~s}, 1 \mathrm{H}), 7.96$ $(\mathrm{d}, J=8.0 \mathrm{~Hz}, 1 \mathrm{H}), 7.57(\mathrm{~m}, 1 \mathrm{H}), 7.44(\mathrm{~m}, 3 \mathrm{H}), 7.39(\mathrm{~d}, J=8.3 \mathrm{~Hz}, 1 \mathrm{H}), 7.28(\mathrm{~m}$, 5H), $7.16(\mathrm{~m}, 3 \mathrm{H}), 6.90(\mathrm{~s}, 1 \mathrm{H}), 4.05(\mathrm{t}, J=6.4 \mathrm{~Hz}, 2 \mathrm{H}), 3.22(\mathrm{t}, J=5.6 \mathrm{~Hz}, 2 \mathrm{H})$, $2.98(\mathrm{~s}, 3 \mathrm{H}), 1.99(\mathrm{~m}, 2 \mathrm{H}) ;{ }^{13} \mathrm{C} \mathrm{NMR}\left(101 \mathrm{MHz}, \mathrm{CDCl}_{3}\right) \delta 161.4,151.2,146.9$, $143.5,137.9,137.4,134.2,134.1,132.6,131.2,129.2,129.0,128.3,127.8,126.6$, $124.6,123.7,122.8,122.2,116.3,115.0,112.9,103.7,68.4,58.3,39.6,27.3$; HRMS (ESI+) calcd for $\mathrm{C}_{32} \mathrm{H}_{26} \mathrm{~N}_{3} \mathrm{O}_{5} \mathrm{~S}$ m/z : 564.1588; found 564.1590.

\section{9-chloro-6-(furan-2-ylmethyl)-3,4-diphenylbenzo[e]isochromeno[6 $\left.6^{\prime}, 7^{\prime}: 4,5\right]$}

\section{imidazo $[1,2-b][1,2,4]$ thiadiazin-1(6H)-one 12,12-dioxide (21b)}

Light brown solid; (0.057 g, $58 \%)$; ${ }^{1} \mathrm{H}$ NMR (400 MHz, $\left.\mathrm{CDCl}_{3}\right) \delta 8.68(\mathrm{~s}, 1 \mathrm{H})$, $7.90(\mathrm{~d}, J=8.5 \mathrm{~Hz}, 1 \mathrm{H}), 7.46(\mathrm{~m}, 4 \mathrm{H}), 7.30(\mathrm{~m}, 5 \mathrm{H}), 7.18(\mathrm{~m}, 4 \mathrm{H}), 6.92(\mathrm{~s}, 1 \mathrm{H})$, $6.27(\mathrm{~s}, 1 \mathrm{H}), 6.22(\mathrm{~d}, J=2.5 \mathrm{~Hz}, 1 \mathrm{H}), 5.14(\mathrm{~s}, 2 \mathrm{H}) ;{ }^{13} \mathrm{C} \mathrm{NMR}\left(101 \mathrm{MHz}, \mathrm{CDCl}_{3}\right)$ $\delta 161.2,151.1,147.1,146.7,144.7,143.1,140.5,138.0,136.1,134.1,132.4$, $131.2,129.1,129.1,129.0,128.3,127.8,126.4,124.3,124.0,123.5,121.1,116.3$, 115.3, 112.7, 110.5, 110.4, 105.1, 38.8; HRMS (ESI+) calcd for $\mathrm{C}_{33} \mathrm{H}_{21} \mathrm{ClN}_{3} \mathrm{O}_{5} \mathrm{~S}$ $\mathrm{m} / \mathrm{z}: 606.0885$; found 606.0888 .

\section{9-chloro-6-cyclooctyl-3,4-diphenylbenzo[e]isochromeno[6', $\left.7^{\prime}: 4,5\right]$}

\section{imidazo[1,2-b][1,2,4] thiadiazin-1(6H)-one 12,12-dioxide (21d)}

White solid; (0.059 g, $61 \%)$; ${ }^{1} \mathrm{H}$ NMR $\left(400 \mathrm{MHz}, \mathrm{CDCl}_{3}\right) \delta 8.71(\mathrm{~s}, 1 \mathrm{H}), 7.89$ (d, $J=8.6 \mathrm{~Hz}, 1 \mathrm{H}), 7.47(\mathrm{~m}, 4 \mathrm{H}), 7.36(\mathrm{~m}, 2 \mathrm{H}), 7.29(\mathrm{~m}, 2 \mathrm{H}), 7.19(\mathrm{~m}, 4 \mathrm{H}), 4.82(\mathrm{~m}$, $1 \mathrm{H}), 1.91(\mathrm{~m}, 2 \mathrm{H}), 1.77(\mathrm{~m}, 7 \mathrm{H}), 1.46(\mathrm{~m}, 5 \mathrm{H}) ;{ }^{13} \mathrm{C} \mathrm{NMR}\left(101 \mathrm{MHz}, \mathrm{CDCl}_{3}\right) \delta$ $161.3,150.9,146.9,145.0,140.3,137.7,135.4,134.3,132.5,131.1,129.2,129.1$, 
$128.4,127.8,126.3,124.7,123.6,123.5,120.8,116.2,114.7,112.9,105.9,55.6$, 30.6, 26.4, 25.1, 24.7; HRMS (ESI+) calcd for $\mathrm{C}_{36} \mathrm{H}_{31} \mathrm{ClN}_{3} \mathrm{O}_{4} \mathrm{~S} \mathrm{~m} / \mathrm{z}: 636.1718$; found 636.1708 .

\section{9-chloro-6-cyclooctyl-3,4-dipropylbenzo[e $]$ isochromeno $\left[6^{\prime}, 7^{\prime}: 4,5\right]$}

\section{imidazo[1,2-b][1,2,4] thiadiazin-1(6H)-one 12,12-dioxide (21e)}

Light brown solid; (0.063 g, $73 \%)$; ${ }^{1} \mathrm{H}$ NMR (400 MHz, $\left.\mathrm{CDCl}_{3}\right) \delta 8.69$ (s, 1H), $7.91(\mathrm{~d}, J=8.5,1 \mathrm{H}), 7.50(\mathrm{~d}, J=1.9 \mathrm{~Hz}, 1 \mathrm{H}), 7.21(\mathrm{~s}, 1 \mathrm{H}), 5.04(\mathrm{~m}, 1 \mathrm{H}), 2.68-$ $2.56(\mathrm{~m}, 4 \mathrm{H}), 2.31(\mathrm{~m}, 3 \mathrm{H}), 1.96(\mathrm{~m}, 5 \mathrm{H}), 1.86-1.72(\mathrm{~m}, 10 \mathrm{H}), 1.09(\mathrm{t}, J=7.4$ $\mathrm{Hz}, 3 \mathrm{H}), 1.01(\mathrm{t}, J=7.4 \mathrm{~Hz}, 3 \mathrm{H}) ;{ }^{13} \mathrm{C} \mathrm{NMR}\left(101 \mathrm{MHz}, \mathrm{CDCl}_{3}\right) \delta 161.9,154.6$, $140.3,136.5,126.3,124.1,123.7,123.6,121.0,115.3,113.6,111.4,55.4,32.8$ 31.4, 29.6, 28.5, 26.3, 25.8, 25.1, 22.6, 21.1, 14.1, 13.8; HRMS (ESI+) calcd for $\mathrm{C}_{30} \mathrm{H}_{35} \mathrm{ClN}_{3} \mathrm{O}_{4} \mathrm{~S} \mathrm{~m} / \mathrm{z}$ : 568.2031; found 568.2034.

\section{6-(3-methoxypropyl)-3,4-dipropylbenzo[e]isochromeno[6',7':4,5]}

\section{imidazo[1,2-b][1,2,4] thiadiazin-1(6H)-one 12,12-dioxide (21f)}

White solid; (0.062 g, $69 \%) ;{ }^{1} \mathrm{H}$ NMR (400 MHz, $\left.\mathrm{CDCl}_{3}\right) \delta 8.63$ (s, 1H), 7.97 $(\mathrm{dd}, J=8.0,1.1 \mathrm{~Hz}, 1 \mathrm{H}), 7.65-7.55(\mathrm{~m}, 1 \mathrm{H}), 7.42(\mathrm{~d}, J=8.2 \mathrm{~Hz}, 1 \mathrm{H}), 7.27(\mathrm{t}, J$ $=7.6 \mathrm{~Hz}, 1 \mathrm{H}), 7.19(\mathrm{~s}, 1 \mathrm{H}), 4.24(\mathrm{t}, J=6.6 \mathrm{~Hz}, 2 \mathrm{H}), 3.41(\mathrm{t}, J=5.5 \mathrm{~Hz}, 2 \mathrm{H}), 3.32$ (s, 3H), $2.59(\mathrm{~m}, 4 \mathrm{H}), 2.14(\mathrm{~m}, 2 \mathrm{H}), 1.73(\mathrm{~m}, 2 \mathrm{H}), 1.61(\mathrm{~m}, 2 \mathrm{H}), 1.06(\mathrm{t}, J=7.3$ $\mathrm{Hz}, 3 \mathrm{H}), 0.99(\mathrm{t}, J=7.4 \mathrm{~Hz}, 3 \mathrm{H}) ;{ }^{13} \mathrm{C} \mathrm{NMR}\left(101 \mathrm{MHz}, \mathrm{CDCl}_{3}\right) \delta 162.1,154.4$, 147.0, 143.6, 137.3, 137.1, 134.2, 126.6, 123.9, 123.6, 122.9, 122.3, 115.4, 113.4, 111.7, 101.1, 68.7, 58.7, 39.5, 32.8, 28.4, 27.6, 22.7, 21.1, 14.1, 13.8; HRMS (ESI+) calcd for $\mathrm{C}_{26} \mathrm{H}_{30} \mathrm{~N}_{3} \mathrm{O}_{5} \mathrm{~S} \mathrm{~m} / \mathrm{z}$ : 496.1901; found 496.1903 . 
9-chloro-6-cyclopentyl-4-methyl-3-phenylbenzo[e]isochromeno[6',7':4,5]

imidazo[1,2-b] $[1,2,4]$ thiadiazin-1(6H)-one 12,12-dioxide (21g)

Light brown solid; (0.066 g, 74\%); ${ }^{1} \mathrm{H}$ NMR (400 MHz, $\left.\mathrm{CDCl}_{3}\right) \delta 8.55(\mathrm{~s}, 1 \mathrm{H})$, $7.81(\mathrm{~d}, J=8.5 \mathrm{~Hz}, 1 \mathrm{H}), 7.54(\mathrm{~m}, 2 \mathrm{H}), 7.43(\mathrm{~m}, 4 \mathrm{H}), 7.27(\mathrm{~s}, 1 \mathrm{H}), 7.19(\mathrm{dd}, J=$ 8.5, 1.7 Hz, 1H), $5.21(\mathrm{p}, J=8.8 \mathrm{~Hz}, 1 \mathrm{H}), 2.34(\mathrm{~s}, 3 \mathrm{H}), 2.29(\mathrm{~m}, 2 \mathrm{H}), 2.20(\mathrm{~m}$, 2H), $2.10(\mathrm{~m}, 2 \mathrm{H}), 1.85(\mathrm{~m}, 2 \mathrm{H}) ;{ }^{13} \mathrm{C} \mathrm{NMR}\left(101 \mathrm{MHz}, \mathrm{CDCl}_{3}\right) \delta 161.5,151.5$, $147.2,144.9,140.4,137.4,135.8,132.8,129.4,128.2,126.3,124.5,123.7,123.4$, 120.8, 115.1, 113.0, 108.3, 103.0, 55.3, 28.4, 25.5, 13.9; HRMS (ESI+) calcd for $\mathrm{C}_{28} \mathrm{H}_{23} \mathrm{ClN}_{3} \mathrm{O}_{4} \mathrm{~S} \mathrm{~m} / \mathrm{z}$ : 532.1092; found 532.1087.

9-chloro-6-cyclopentyl-3,4-dipropylbenzo $[e]$ isochromeno $\left[6^{\prime}, 7^{\prime}: 4,5\right]$ imidazo $[1,2-b][1,2,4]$ thiadiazin-1(6H)-one 12,12-dioxide (21h)

White solid; (0.060 g, $69 \%) ;{ }^{1} \mathrm{H}$ NMR (400 MHz, $\left.\mathrm{CDCl}_{3}\right) \delta 8.66(\mathrm{~s}, 1 \mathrm{H}), 7.88(\mathrm{~d}$, $J=8.5 \mathrm{~Hz}, 1 \mathrm{H}), 7.43(\mathrm{~d}, J=1.6 \mathrm{~Hz}, 1 \mathrm{H}), 7.22(\mathrm{~d}, J=1.7 \mathrm{~Hz}, 1 \mathrm{H}), 7.17(\mathrm{~s}, 1 \mathrm{H})$, $5.26(\mathrm{p}, J=8.7 \mathrm{~Hz}, 1 \mathrm{H}), 2.60(\mathrm{~m}, 4 \mathrm{H}), 2.19(\mathrm{~m}, 4 \mathrm{H}), 2.04(\mathrm{~m}, 2 \mathrm{H}), 1.87(\mathrm{~m}, 2 \mathrm{H})$, $1.74(\mathrm{~m}, 2 \mathrm{H}), 1.62(\mathrm{~m}, 2 \mathrm{H}), 1.06(\mathrm{t}, J=7.3 \mathrm{~Hz}, 3 \mathrm{H}), 0.99(\mathrm{t}, J=7.3 \mathrm{~Hz}, 3 \mathrm{H}) ;{ }^{13} \mathrm{C}$ $\operatorname{NMR}\left(101 \mathrm{MHz}, \mathrm{CDCl}_{3}\right) \delta 161.9,154.7,147.4,145.0,140.3,136.5,135.3,126.2$, $124.1,123.7,123.5,121.0,115.3,113.5,111.4,102.5,54.7,34.0,32.8,28.6,28.4$, 25.7, 22.6, 22.3, 21.1, 14.1, 14.0, 13.7; HRMS (ESI+) calcd for $\mathrm{C}_{27} \mathrm{H}_{29} \mathrm{ClN}_{3} \mathrm{O}_{4} \mathrm{~S}$ $\mathrm{m} / \mathrm{z}: 526.1562 ;$ Found 526.1564

9-chloro-6-cyclopentyl-3,4-diethylbenzo $[e]$ isochromeno $\left[6^{\prime}, 7^{\prime}: 4,5\right]$ imidazo $[1,2-b][1,2,4]$ thiadiazin-1 $(6 H)$-one 12,12-dioxide (21i) 
Light brown solid; (0.059 g, $71 \%) ;{ }^{1} \mathrm{H}$ NMR (400 MHz, $\left.\mathrm{CDCl}_{3}\right) \delta 8.64(\mathrm{~s}, 1 \mathrm{H})$, $7.87(\mathrm{~d}, J=8.6 \mathrm{~Hz}, 1 \mathrm{H}), 7.42(\mathrm{~d}, J=1.9 \mathrm{~Hz}, 1 \mathrm{H}), 7.22(\mathrm{dd}, J=8.6,2.0 \mathrm{~Hz}, 1 \mathrm{H})$, $7.18(\mathrm{~s}, 1 \mathrm{H}), 5.20(\mathrm{p}, J=8.7 \mathrm{~Hz}, 1 \mathrm{H}), 2.64(\mathrm{~m}, 4 \mathrm{H}), 2.19(\mathrm{~m}, 4 \mathrm{H}), 2.05(\mathrm{~m}, 2 \mathrm{H})$, $1.76(\mathrm{~m}, 2 \mathrm{H}), 1.28(\mathrm{t}, J=7.5 \mathrm{~Hz}, 3 \mathrm{H}), 1.23(\mathrm{t}, J=7.5 \mathrm{~Hz}, 3 \mathrm{H}) ;{ }^{13} \mathrm{C}$ NMR $(101$ $\left.\mathrm{MHz}, \mathrm{CDCl}_{3}\right) \delta 161.9,155.5,147.3,145.0,140.3,136.3,135.5,126.2,124.0$ 123.7, 123.5, 121.0, 115.4, 113.6, 112.2, 102.2, 54.9, 28.5, 25.6, 24.2, 19.7, 13.9, 12.5; HRMS (ESI+) calcd for $\mathrm{C}_{25} \mathrm{H}_{25} \mathrm{ClN}_{3} \mathrm{O}_{4} \mathrm{~S}$ m/z : 498.1249; Found 498.1246. 3,4-diethyl-6-(3-methoxypropyl)benzo[e]isochromeno[6', $\left.7^{\prime}: 4,5\right]$ imidazo[1,2$b][1,2,4]$ thiadiazin-1(6H)-one 12,12-dioxide $(21 \mathrm{j})$

White solid; (0.058 g, $75 \%)$; ${ }^{1} \mathrm{H}$ NMR (400 MHz, $\left.\mathrm{CDCl}_{3}\right) \delta 8.62(\mathrm{~s}, 1 \mathrm{H}), 7.96$ $(\mathrm{dd}, J=8.0,1.0 \mathrm{~Hz}, 1 \mathrm{H}), 7.62-7.57(\mathrm{~m}, 1 \mathrm{H}), 7.42(\mathrm{~d}, J=8.2 \mathrm{~Hz}, 1 \mathrm{H}), 7.27(\mathrm{t}, J$ $=7.6 \mathrm{~Hz}, 1 \mathrm{H}), 7.20(\mathrm{~s}, 1 \mathrm{H}), 4.24(\mathrm{t}, J=6.6 \mathrm{~Hz}, 2 \mathrm{H}), 3.40(\mathrm{t}, J=5.6 \mathrm{~Hz}, 2 \mathrm{H}), 2.63$ (m, 4H), $2.19-2.10(\mathrm{~m}, 2 \mathrm{H}), 1.27(\mathrm{t}, J=7.5 \mathrm{~Hz}, 4 \mathrm{H}), 1.22(\mathrm{t}, J=7.5 \mathrm{~Hz}, 4 \mathrm{H})$; ${ }^{13} \mathrm{C}$ NMR $\left(101 \mathrm{MHz}, \mathrm{CDCl}_{3}\right) \delta 162.1,155.2,146.9,143.6,137.4,136.8,134.2$, $126.6,123.9,123.6,122.9,122.3,115.4,113.4,112.5,101.0,68.7,58.7,39.6$, 27.6, 24.1, 19.6, 14.1, 12.4; HRMS (ESI+) calcd for $\mathrm{C}_{24} \mathrm{H}_{26} \mathrm{~N}_{3} \mathrm{O}_{5} \mathrm{~S} \mathrm{~m} / \mathrm{z}$ : 468.1588 ; found 468.1595 .

\section{9-chloro-3,4-diethyl-6-(3-methoxypropyl)benzo[e]isochromeno[6', $\left.7^{\prime}: 4,5\right]$}

\section{imidazo[1,2-b][1,2,4]thiadiazin-1(6H)-one 12,12-dioxide (21k)}

White solid; (0.057 g, $69 \%) ;{ }^{1} \mathrm{H}$ NMR (400 MHz, $\left.\mathrm{CDCl}_{3}\right) \delta 8.69(\mathrm{~s}, 1 \mathrm{H}), 7.92(\mathrm{~d}$, $J=8.5 \mathrm{~Hz}, 1 \mathrm{H}), 7.53-7.51(\mathrm{~m}, 1 \mathrm{H}), 7.28(\mathrm{~s}, 2 \mathrm{H}), 4.31(\mathrm{t}, J=6.4 \mathrm{~Hz}, 2 \mathrm{H}), 3.43$ (t, $J=5.4 \mathrm{~Hz}, 2 \mathrm{H}), 3.32(\mathrm{~s}, 3 \mathrm{H}), 2.67(\mathrm{~m}, 4 \mathrm{H}), 2.21-2.12(\mathrm{~m}, 2 \mathrm{H}), 1.30(\mathrm{t}, J=$ 
$7.5 \mathrm{~Hz}, 3 \mathrm{H}), 1.25(\mathrm{t}, J=7.4 \mathrm{~Hz}, 3 \mathrm{H}) ;{ }^{13} \mathrm{C} \mathrm{NMR}\left(101 \mathrm{MHz}, \mathrm{CDCl}_{3}\right) \delta 162.0,155.5$, 147.6, 140.5, 137.1, 137.0, 126.1, 124.0, 123.7, 123.7, 121.3, 115.9, 113.8, 112.5, 101.4, 68.7, 58.7, 39.9, 27.6, 24.2, 19.6, 14.1, 12.4; HRMS (ESI+) calcd for $\mathrm{C}_{24} \mathrm{H}_{25} \mathrm{ClN}_{3} \mathrm{O}_{5} \mathrm{~S} \mathrm{~m} / \mathrm{z}:$ 502.1198; found 502.1206.

Tetraethyl 13-(3-methoxypropyl)-13H-benzo[e]naphtha[2',3':4,5] imidazo[1,2-b][1,2,4]thiadiazine-8,9,10,11-tetracarboxylate 5,5-dioxide (22a) Yellow solid; (0.026 g, $23 \%) ;{ }^{1} \mathrm{H}$ NMR (400 MHz, $\left.\mathrm{CDCl}_{3}\right) \delta 8.42(\mathrm{~s}, 1 \mathrm{H}), 7.96$ $(\mathrm{dd}, J=8.0,0.9 \mathrm{~Hz}, 1 \mathrm{H}), 7.76(\mathrm{~s}, 1 \mathrm{H}), 7.65-7.59(\mathrm{~m}, 1 \mathrm{H}), 7.45(\mathrm{~d}, J=8.0 \mathrm{~Hz}$, 1H), $7.30(\mathrm{t}, J=7.3 \mathrm{~Hz}, 1 \mathrm{H}), 4.56(\mathrm{q}, J=7.2 \mathrm{~Hz}, 3 \mathrm{H}), 4.49(\mathrm{q}, J=7.2 \mathrm{~Hz}, 3 \mathrm{H})$, $4.35(\mathrm{q}, J=7.2 \mathrm{~Hz}, 4 \mathrm{H}), 4.25(\mathrm{t}, J=6.6 \mathrm{~Hz}, 2 \mathrm{H}), 3.37(\mathrm{t}, J=5.6 \mathrm{~Hz}, 2 \mathrm{H}), 3.26$ (s, 3H), $2.15(\mathrm{~m}, 2 \mathrm{H}), 1.50(\mathrm{t}, J=7.2 \mathrm{~Hz}, 3 \mathrm{H}), 1.42(\mathrm{t}, J=7.2 \mathrm{~Hz}, 3 \mathrm{H}), 1.36(\mathrm{t}, J$ $=7.2 \mathrm{~Hz}, 6 \mathrm{H}) ;{ }^{13} \mathrm{C} \mathrm{NMR}\left(101 \mathrm{MHz}, \mathrm{CDCl}_{3}\right) \delta 166.7,166.6,166.3,166.1,147.1$, $143.5,134.4,134.1,132.1,128.7,128.5,127.6,126.8,126.6,126.3,123.9,123.2$, $122.1,108.3,102.6,68.7,62.6,62.3,62.2,58.7,39.7,36.4,27.5,14.1,13.9,13.9$; HRMS (ESI+) calcd for $\mathrm{C}_{33} \mathrm{H}_{36} \mathrm{~N}_{3} \mathrm{O}_{11} \mathrm{~S} \mathrm{~m} / \mathrm{z}$ : 682.2065; found 682.2066 .

\section{2-chloro-13-(2-(cyclohex-1-en-1-yl)ethyl)-8,9,10,11-tetraphenyl-13H-} benzo $[e]$ naphtha $\left[2^{\prime}, 3^{\prime}: 4,5\right]$ imidazo $[1,2-b][1,2,4]$ thiadiazine 5,5 -dioxide (22b) Light yellow solid; (0.084 g, $72 \%) ;{ }^{1} \mathrm{H}$ NMR $\left(300 \mathrm{MHz}, \mathrm{CDCl}_{3}\right) \delta 8.04(\mathrm{~s}, 1 \mathrm{H})$, $7.82(\mathrm{~d}, J=8.5 \mathrm{~Hz}, 1 \mathrm{H}), 7.44(\mathrm{~d}, J=1.4 \mathrm{~Hz}, 1 \mathrm{H}), 7.36-7.25(\mathrm{~m}, 11 \mathrm{H}), 7.21(\mathrm{~s}$, $1 \mathrm{H}), 6.89(\mathrm{~m}, 10 \mathrm{H}), 5.24(\mathrm{~s}, 1 \mathrm{H}), 4.05(\mathrm{t}, J=7.1 \mathrm{~Hz}, 2 \mathrm{H}), 2.34(\mathrm{t}, J=7.0 \mathrm{~Hz}, 2 \mathrm{H})$, $1.87(\mathrm{~m}, 4 \mathrm{H}), 1.45(\mathrm{~m}, 4 \mathrm{H}) ;{ }^{13} \mathrm{C} \mathrm{NMR}\left(75 \mathrm{MHz}, \mathrm{CDCl}_{3}\right) \delta 145.74,140.3,139.5$, 
$139.2,138.9,138.3,137.5,133.1,131.3,131.2,130.5,128.8,127.8,126.9,126.6$, $125.9,125.4,125.1,124.6,123.3,123.2,121.4,109.9,104.2,41.0,35.2,27.9$, 25.1, 22.7, 22.0; HRMS (ESI+) calcd for $\mathrm{C}_{49} \mathrm{H}_{39} \mathrm{ClN}_{3} \mathrm{O}_{2} \mathrm{~S} \mathrm{~m} / \mathrm{z}$ : 768.2446; found 768.2450 .

\section{2-chloro-7-(3-methoxypropyl)-9,10-diphenylbenzo[ $\left[\right.$ ]isochromeno $\left[6^{\prime}, 7^{\prime}: 4,5\right]$} imidazo[2,1-c] $[1,2,4]$ thiadiazin-12(7H)-one 5,5-dioxide (23a)

Off white solid; (0.072 g, $73 \%) ;{ }^{1} \mathrm{H}$ NMR (400 MHz, $\left.\mathrm{CDCl}_{3}\right) \delta 8.84(\mathrm{~s}, 1 \mathrm{H}), 8.17$ (s, 1H), $8.08(\mathrm{~d}, J=8.4 \mathrm{~Hz}, 1 \mathrm{H}), 7.57(\mathrm{~d}, J=8.4 \mathrm{~Hz}, 1 \mathrm{H}), 7.45(\mathrm{~m}, 3 \mathrm{H}), 7.31(\mathrm{~m}$, 4H), $7.23(\mathrm{~m}, 3 \mathrm{H}), 7.16(\mathrm{~s}, 1 \mathrm{H}), 4.12(\mathrm{t}, J=6.2 \mathrm{~Hz}, 2 \mathrm{H}), 3.21(\mathrm{t}, J=5.4 \mathrm{~Hz}, 2 \mathrm{H})$, $2.96(\mathrm{~s}, 3 \mathrm{H}), 2.01(\mathrm{~m}, 2 \mathrm{H}) ;{ }^{13} \mathrm{C} \mathrm{NMR}\left(101 \mathrm{MHz}, \mathrm{CDCl}_{3}\right) \delta 161.6,151.7,149.4$, 139.4, 137.4, 136.5, 133.8, 133.6, 132.3, 131.2, 129.4, 129.3, 129.2, 128.6, 127.9, $127.8,127.0,126.3,123.2,116.2,115.9,115.6,113.3,105.7,68.2,58.4,40.2$, 27.3; HRMS (ESI+) calcd for $\mathrm{C}_{32} \mathrm{H}_{25} \mathrm{ClN}_{3} \mathrm{O}_{5} \mathrm{~S} \mathrm{~m} / \mathrm{z}$ : 598.1198; found 598.1176.

\section{2-chloro-9,10-diethyl-7-(3-methoxypropyl)benzo[ $[e]$ isochromeno $\left[6^{\prime}, 7^{\prime}: 4,5\right]$}

\section{imidazo[2,1-c] $[1,2,4]$ thiadiazin-12(7H)-one 5,5-dioxide (23b)}

Brown solid; (0.060 g, $72 \%) ;{ }^{1} \mathrm{H}$ NMR (400 MHz, $\left.\mathrm{CDCl}_{3}\right) \delta 8.73(\mathrm{~s}, 1 \mathrm{H}), 8.10$ (s, 1H), $8.05(\mathrm{~d}, J=8.3 \mathrm{~Hz}, 1 \mathrm{H}), 7.53(\mathrm{~d}, J=8.2 \mathrm{~Hz}, 1 \mathrm{H}), 7.48(\mathrm{~s}, 1 \mathrm{H}), 4.32(\mathrm{t}, J$ $=6.2 \mathrm{~Hz}, 4 \mathrm{H}), 3.42(\mathrm{t}, J=5.4 \mathrm{~Hz}, 2 \mathrm{H}), 3.31(\mathrm{~s}, 3 \mathrm{H}), 2.68(\mathrm{~m}, 4 \mathrm{H}), 2.15(\mathrm{~m}, 2 \mathrm{H})$, $1.31(\mathrm{t}, J=7.3 \mathrm{~Hz}, 3 \mathrm{H}), 1.23(\mathrm{t}, J=7.4 \mathrm{~Hz}, 3 \mathrm{H}) ;{ }^{13} \mathrm{C} \mathrm{NMR}\left(101 \mathrm{MHz}, \mathrm{CDCl}_{3}\right) \delta$ $162.2,155.8,149.5,139.4,136.5,136.4,133.8,127.7,126.9,125.7,123.1,116.4$, 115.6, 113.7, 112.5, 103.0, 68.5, 58.7, 40.2, 27.6, 24.2, 19.6, 14.1, 12.4; HRMS (ESI+) calcd for $\mathrm{C}_{24} \mathrm{H}_{25} \mathrm{ClN}_{3} \mathrm{O}_{5} \mathrm{~S} \mathrm{~m} / \mathrm{z}: 502.1198$; found 502.1197 . 
2-chloro-7-(3-methoxypropyl)-9,10-dipropylbenzo[e]isochromeno[6',7':4,5] imidazo[2,1-c][1,2,4]thiadiazin-12(7H)-one 5,5-dioxide (23c)

White solid; (0.049 g, $56 \%) ;{ }^{1} \mathrm{H}$ NMR $\left(400 \mathrm{MHz}, \mathrm{CDCl}_{3}\right) \delta 8.71(\mathrm{~s}, 1 \mathrm{H}), 8.08(\mathrm{~s}$, $1 \mathrm{H}), 8.03(\mathrm{~d}, J=8.3 \mathrm{~Hz}, 1 \mathrm{H}), 7.52(\mathrm{~d}, J=8.2 \mathrm{~Hz}, 1 \mathrm{H}), 7.45(\mathrm{~s}, 1 \mathrm{H}), 4.30(\mathrm{t}, J=$ $5.7 \mathrm{~Hz}, 2 \mathrm{H}), 3.40(\mathrm{t}, J=4.4 \mathrm{~Hz} 2 \mathrm{H}), 3.32(\mathrm{~s}, 3 \mathrm{H}) 2.63(\mathrm{~m}, 4 \mathrm{H}), 2.12(\mathrm{~m}, 2 \mathrm{H}), 1.76$ $(\mathrm{m}, 2 \mathrm{H}), 1.63(\mathrm{~m}, 2 \mathrm{H}), 1.07(\mathrm{t}, J=6.8 \mathrm{~Hz}, 3 \mathrm{H}), 1.01(\mathrm{t}, J=7.2 \mathrm{~Hz}, 3 \mathrm{H}) ;{ }^{13} \mathrm{C} \mathrm{NMR}$ $\left(101 \mathrm{MHz}, \mathrm{CDCl}_{3}\right) \delta 162.2,155.0,149.5,139.3,136.6,136.5,133.8,127.6,126.9$, 125.6, 123.1, 116.3, 115.6, 113.6, 111.7, 103.3, 68.5, 58.7, 40.1, 32.8, 28.4, 27.5, 22.7, 21.1, 14.1, 13.8; HRMS (ESI+) calcd for $\mathrm{C}_{26} \mathrm{H}_{29} \mathrm{ClN}_{3} \mathrm{O}_{5} \mathrm{~S} \mathrm{~m} / \mathrm{z}: 530.1511$; found 530.1516 .

7-(3-methoxypropyl)-9,10-

dipropylbenzo $[e]$ isochromeno $\left[6^{\prime}, 7^{\prime}: 4,5\right]$ imidazo $[2,1-c][1,2,4]$ thiadiazin12(7H)-one 5,5-dioxide (23d)

White solid; (0.012 g, $15 \%)$; ${ }^{1} \mathrm{H}$ NMR (400 MHz, $\left.\mathrm{CDCl}_{3}\right) \delta 8.80$ (s, 1H), 8.17 (m, 2H), $7.80(\mathrm{t}, J=7.6 \mathrm{~Hz}, 1 \mathrm{H}), 7.60(\mathrm{t}, J=7.5 \mathrm{~Hz}, 1 \mathrm{H}), 7.47(\mathrm{~s}, 1 \mathrm{H}), 4.33(\mathrm{t}, J$ $=5.7 \mathrm{~Hz} 2 \mathrm{H}), 3.43(\mathrm{t}, J=4.8 \mathrm{~Hz}, 2 \mathrm{H}), 3.35(\mathrm{~s}, 3 \mathrm{H}), 2.65(\mathrm{~m}, 4 \mathrm{H}), 2.17(\mathrm{~m}, 2 \mathrm{H})$, $1.73(\mathrm{~m}, 4 \mathrm{H}), 1.10(\mathrm{t}, J=7.2 \mathrm{~Hz}, 3 \mathrm{H}), 1.03(\mathrm{t}, J=7.3 \mathrm{~Hz}, 3 \mathrm{H}) ;{ }^{13} \mathrm{C}$ NMR (101 $\left.\mathrm{MHz}, \mathrm{CDCl}_{3}\right) \delta 162.4,154.8,149.4,136.6,136.3,133.2,132.9,127.4,126.0$ 125.6, 124.7, 116.1, 115.6, 113.7, 111.8, 103.0, 68.6, 58.7, 40.0, 38.1, 32.8, 31.2, 28.4, 27.6, 22.7, 21.1, 14.1, 13.8; HRMS (ESI+) calcd for $\mathrm{C}_{26} \mathrm{H}_{30} \mathrm{~N}_{3} \mathrm{O}_{5} \mathrm{~S} \mathrm{~m} / \mathrm{z}$ : 496.1901; found 496.1900. 
2-chloro-7-(furan-2-ylmethyl)-9,10-diphenylbenzo[e]isochromeno[6',7':4,5] imidazo[2,1-c][1,2,4]thiadiazin-12(7H)-one 5,5-dioxide (23f)

Light yellow solid; (0.057 g, $58 \%) ;{ }^{1} \mathrm{H}$ NMR (400 MHz, $\left.\mathrm{CDCl}_{3}\right) \delta 8.79(\mathrm{~s}, 1 \mathrm{H})$, $8.14(\mathrm{~s}, 1 \mathrm{H}), 8.08(\mathrm{~d}, J=8.4 \mathrm{~Hz}, 1 \mathrm{H}), 7.56(\mathrm{dd}, J=8.4,1.2 \mathrm{~Hz}, 1 \mathrm{H}), 7.50(\mathrm{~m}$, 3H), $7.35(\mathrm{~m}, 2 \mathrm{H}), 7.27(\mathrm{~m}, 4 \mathrm{H}), 7.20(\mathrm{~m}, 3 \mathrm{H}), 6.33(\mathrm{~d}, J=3.0 \mathrm{~Hz}, 1 \mathrm{H}), 6.28(\mathrm{~d}$, $J=1.5 \mathrm{~Hz}, 1 \mathrm{H}), 5.19(\mathrm{~s}, 2 \mathrm{H}) ;{ }^{13} \mathrm{C} \mathrm{NMR}\left(101 \mathrm{MHz}, \mathrm{CDCl}_{3}\right) \delta 161.5,151.5,149.1$, $146.1,143.3,139.5,137.5,135.6,133.8,133.7,132.2,131.1,129.4,129.3,129.1$, $128.6,128.0,127.9,127.0,126.3,123.1,116.1,116.0,115.7,113.2,111.0,110.8$ 106.6, 39.3; HRMS (ESI+) calcd for $\mathrm{C}_{33} \mathrm{H}_{21} \mathrm{ClN}_{3} \mathrm{O}_{5} \mathrm{~S} \mathrm{~m} / \mathrm{z}$ : 606.0885; found 606.0882.

4-chloro-7-(3-methoxypropyl)-9,10-diphenylbenzo[e]isochromeno[6',7':4,5] imidazo[2,1-c][1,2,4]thiadiazin-12(7H)-one 5,5-dioxide (23g)

White solid; (0.071 g, $72 \%) ;{ }^{1} \mathrm{H}$ NMR $\left(400 \mathrm{MHz}, \mathrm{CDCl}_{3}\right) \delta 8.82(\mathrm{~s}, 1 \mathrm{H}), 8.16(\mathrm{~d}$, $J=8.4 \mathrm{~Hz}, 1 \mathrm{H}), 7.72(\mathrm{t}, J=8.3 \mathrm{~Hz}, 1 \mathrm{H}), 7.56(\mathrm{~d}, J=8.0 \mathrm{~Hz}, 1 \mathrm{H}), 7.47(\mathrm{~m}, 3 \mathrm{H})$, $7.27(\mathrm{~m}, 7 \mathrm{H}), 7.15(\mathrm{~s}, 1 \mathrm{H}), 4.11(\mathrm{t}, J=6.4 \mathrm{~Hz}, 2 \mathrm{H}), 3.24(\mathrm{t}, J=5.5 \mathrm{~Hz}, 2 \mathrm{H}), 2.98$ (s, 3H), $2.01(\mathrm{~m}, 2 \mathrm{H}) ;{ }^{13} \mathrm{C} \mathrm{NMR}\left(101 \mathrm{MHz}, \mathrm{CDCl}_{3}\right) \delta 161.8,151.6,147.1,137.3$, 136.6, 134.5, 133.6, 133.5, 132.9, 132.3, 131.1, 129.7, 129.4, 129.3, 129.2, 128.6, $127.9,126.6,123.5,116.2,115.7,114.4,113.2,105.5,68.3,58.4,39.9,27.3$; HRMS (ESI+) calcd for $\mathrm{C}_{32} \mathrm{H}_{25} \mathrm{ClN}_{3} \mathrm{O}_{5} \mathrm{~S} \mathrm{~m} / \mathrm{z}$ : 598.1198; found 598.1199 .

4-chloro-9,10-diethyl-7-(3-methoxypropyl)benzo[e]isochromeno[6', $\left.7^{\prime}: 4,5\right]$ imidazo[2,1-c][1,2,4]thiadiazin-12(7H)-one 5,5-dioxide (23h) 
Off white solid; (0.063 g, $76 \%) ;{ }^{1} \mathrm{H}$ NMR $\left(400 \mathrm{MHz}, \mathrm{CDCl}_{3}\right) \delta 8.75(\mathrm{~s}, 1 \mathrm{H}), 8.12$ $(\mathrm{d}, J=8.0 \mathrm{~Hz}, 1 \mathrm{H}), 7.69(\mathrm{t}, J=8.3 \mathrm{~Hz}, 1 \mathrm{H}), 7.56(\mathrm{~d}, J=8.0 \mathrm{~Hz}, 1 \mathrm{H}), 7.48(\mathrm{~s}, 1 \mathrm{H})$, $4.32(\mathrm{t}, J=6.6 \mathrm{~Hz}, 2 \mathrm{H}), 3.43(\mathrm{t}, J=5.4 \mathrm{~Hz}, 2 \mathrm{H}), 3.34(\mathrm{~s}, 3 \mathrm{H}), 2.74(\mathrm{q}, J=7.5 \mathrm{~Hz}$, 2H), $2.68(\mathrm{q}, J=7.5 \mathrm{~Hz}, 2 \mathrm{H}), 2.17(\mathrm{~m}, 2 \mathrm{H}), 1.33(\mathrm{t}, J=7.5 \mathrm{~Hz}, 3 \mathrm{H}), 1.27(\mathrm{t}, J=$ 7.5 Hz, 3H); ${ }^{13} \mathrm{C}$ NMR (101 MHz, $\left.\mathrm{CDCl}_{3}\right) \delta 162.4,155.8,147.1,136.6,136.3$, $134.5,133.5,132.8,129.6,125.9,123.5,116.1,114.3,113.6,112.6,102.9,68.6$, 58.8, 39.9, 27.6, 24.2, 19.6, 14.2, 12.4; HRMS (ESI+) calcd for $\mathrm{C}_{24} \mathrm{H}_{25} \mathrm{ClN}_{3} \mathrm{O}_{5} \mathrm{~S}$ $\mathrm{m} / \mathrm{z}:$ 502.1198; found 502.1195.

\section{4-chloro-7-cyclooctyl-9,10-diethylbenzo $[e]$ isochromeno $\left[6^{\prime}, 7^{\prime}: 4,5\right]$}

\section{imidazo[2,1-c] $[1,2,4]$ thiadiazin-12(7H)-one 5,5-dioxide (23j)}

White solid; (0.010 g, $13 \%) ;{ }^{1} \mathrm{H}$ NMR (400 MHz, $\left.\mathrm{CDCl}_{3}\right) \delta 8.76(\mathrm{~s}, 1 \mathrm{H}), 8.11$ $(\mathrm{dd}, J=8.5,0.8 \mathrm{~Hz}, 1 \mathrm{H}), 7.68(\mathrm{t}, J=8.3 \mathrm{~Hz}, 1 \mathrm{H}), 7.57(\mathrm{dd}, J=8.0,0.9 \mathrm{~Hz}, 1 \mathrm{H})$, $7.41(\mathrm{~s}, 1 \mathrm{H}), 2.73(\mathrm{q}, J=7.6 \mathrm{~Hz}, 2 \mathrm{H}), 2.68(\mathrm{q}, J=7.5 \mathrm{~Hz}, 2 \mathrm{H}), 2.31(\mathrm{~m}, 2 \mathrm{H}), 2.03$ (m, 2H), $1.91(\mathrm{~m}, 3 \mathrm{H}), 1.74(\mathrm{~m}, 11 \mathrm{H}), 1.33(\mathrm{t}, J=7.5 \mathrm{~Hz}, 3 \mathrm{H}), 1.28(\mathrm{t}, \mathrm{J}=7.6 \mathrm{~Hz}$ $3 \mathrm{H}) ;{ }^{13} \mathrm{C} \mathrm{NMR}\left(101 \mathrm{MHz}, \mathrm{CDCl}_{3}\right) \delta 162.3,155.8,146.6,135.6,134.9,134.6$, $133.4,132.7,129.6,126.4,123.7,115.7,114.4,113.7,112.3,104.3,55.9,31.6$, 26.1, 25.8, 25.1, 24.2, 19.7, 14.0, 12.5; HRMS (ESI+) calcd for $\mathrm{C}_{28} \mathrm{H}_{31} \mathrm{ClN}_{3} \mathrm{O}_{4} \mathrm{~S}$ $\mathrm{m} / \mathrm{z}: 540.1718$; found 540.1728 .

\section{4-chloro-7-(3-methoxypropyl)-9,10,11,12-tetraphenyl-7H-benzo[ $e]$ naphtha}

\section{$\left[2^{\prime}, 3^{\prime}: 4,5\right]$ imidazo[2,1-c] $][1,2,4]$ thiadiazine 5,5-dioxide (24a)}

White solid; (0.074 g, 61\%); ${ }^{1} \mathrm{H}$ NMR (400 MHz, $\left.\mathrm{CDCl}_{3}\right) \delta 8.00$ (s, 1H), 7.65 (dd, $J=8.2,1.1 \mathrm{~Hz}, 1 \mathrm{H}), 7.52(\mathrm{~s}, 1 \mathrm{H}), 7.41(\mathrm{~m}, 2 \mathrm{H}), 7.29(\mathrm{~m}, 10 \mathrm{H}), 6.87(\mathrm{~m}, 10 \mathrm{H})$ 
$4.13(\mathrm{t}, J=6.5 \mathrm{~Hz}, 2 \mathrm{H}), 3.29(\mathrm{t}, J=5.6 \mathrm{~Hz}, 2 \mathrm{H}), 3.06(\mathrm{~s}, 3 \mathrm{H}), 2.03(\mathrm{~m}, 2 \mathrm{H}) ;{ }^{13} \mathrm{C}$ $\mathrm{NMR}\left(101 \mathrm{MHz}, \mathrm{CDCl}_{3}\right) \delta 147.8,140.0,139.7,139.2,138.9,138.8,138.5,137.8$, 135.2, 133.3, 132.1, 131.1, 131.1, 131.0, 130.3, 129.9, 128.9, 128.8, 127.9, 127.9, $127.0,126.9,126.7,126.6,126.5,125.6,125.5,123.8,113.5,110.0,105.7,68.6$, 58.5, 39.6, 27.4; HRMS (ESI+) calcd for $\mathrm{C}_{45} \mathrm{H}_{35} \mathrm{ClN}_{3} \mathrm{O}_{3} \mathrm{~S} \mathrm{~m} / \mathrm{z}$ : 732.2082; found 732.2091 


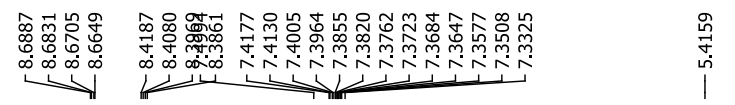

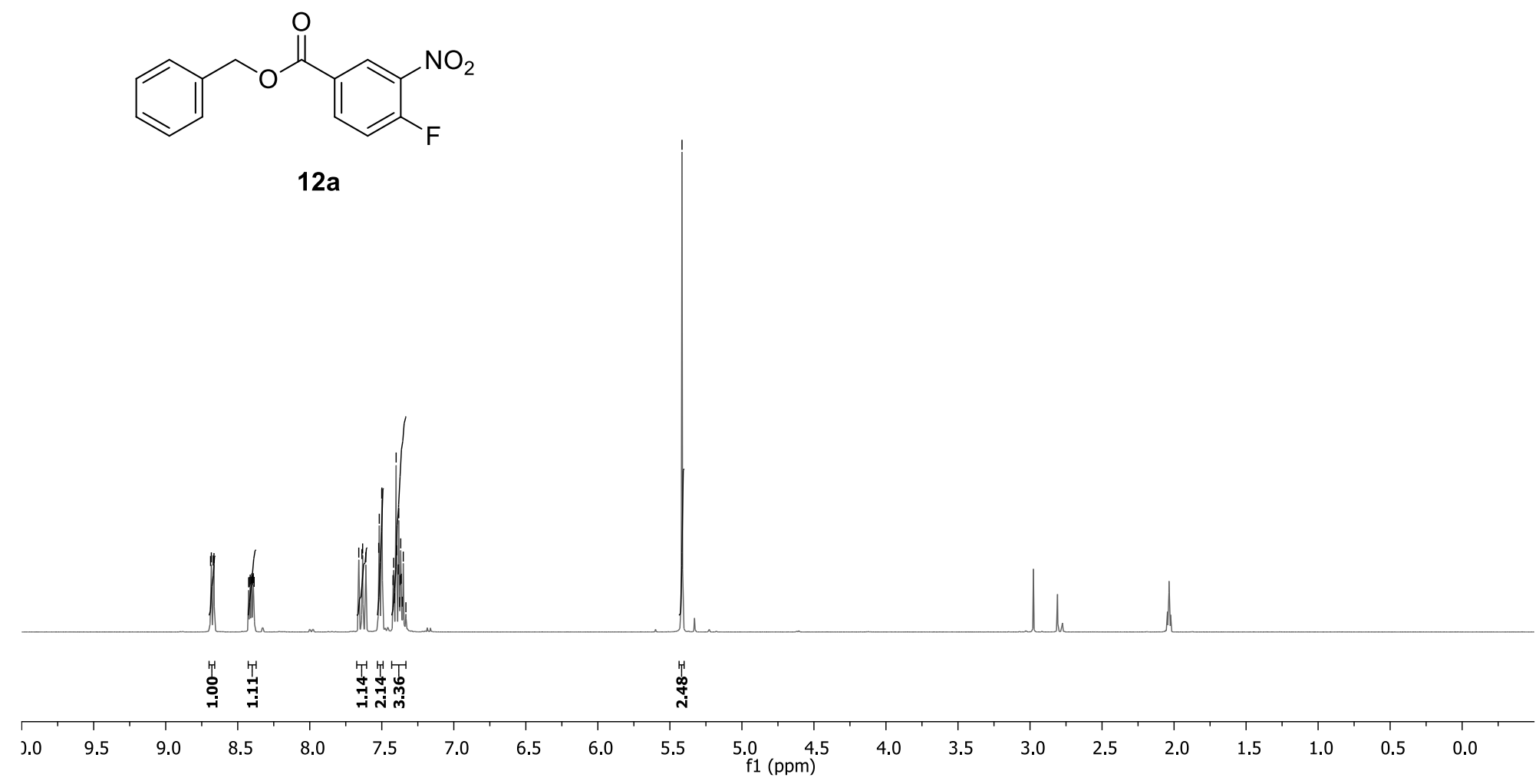

${ }^{1} \mathrm{H}$ NMR spectrum $(400 \mathrm{MHz})$ of compound 12a in acetone- $\mathrm{d}_{6}$ 


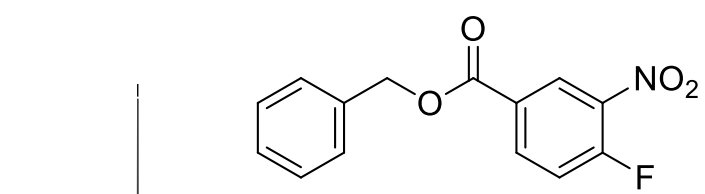

$12 a$

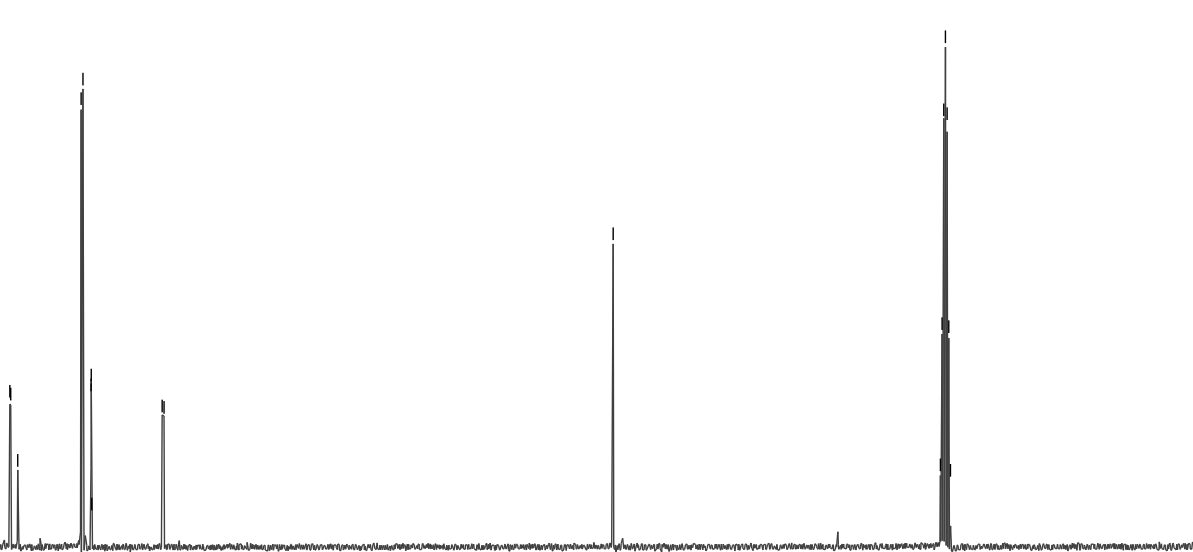

$20 \quad 210$

$180 \quad 170 \quad 160$

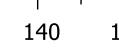

$\begin{array}{llll}130 & 120 & 110 & 100\end{array}$

70

50

${ }^{13} \mathrm{C}$ NMR spectrum $(101 \mathrm{MHz})$ of compound 12a in acetone- $\mathrm{d}_{6}$ 


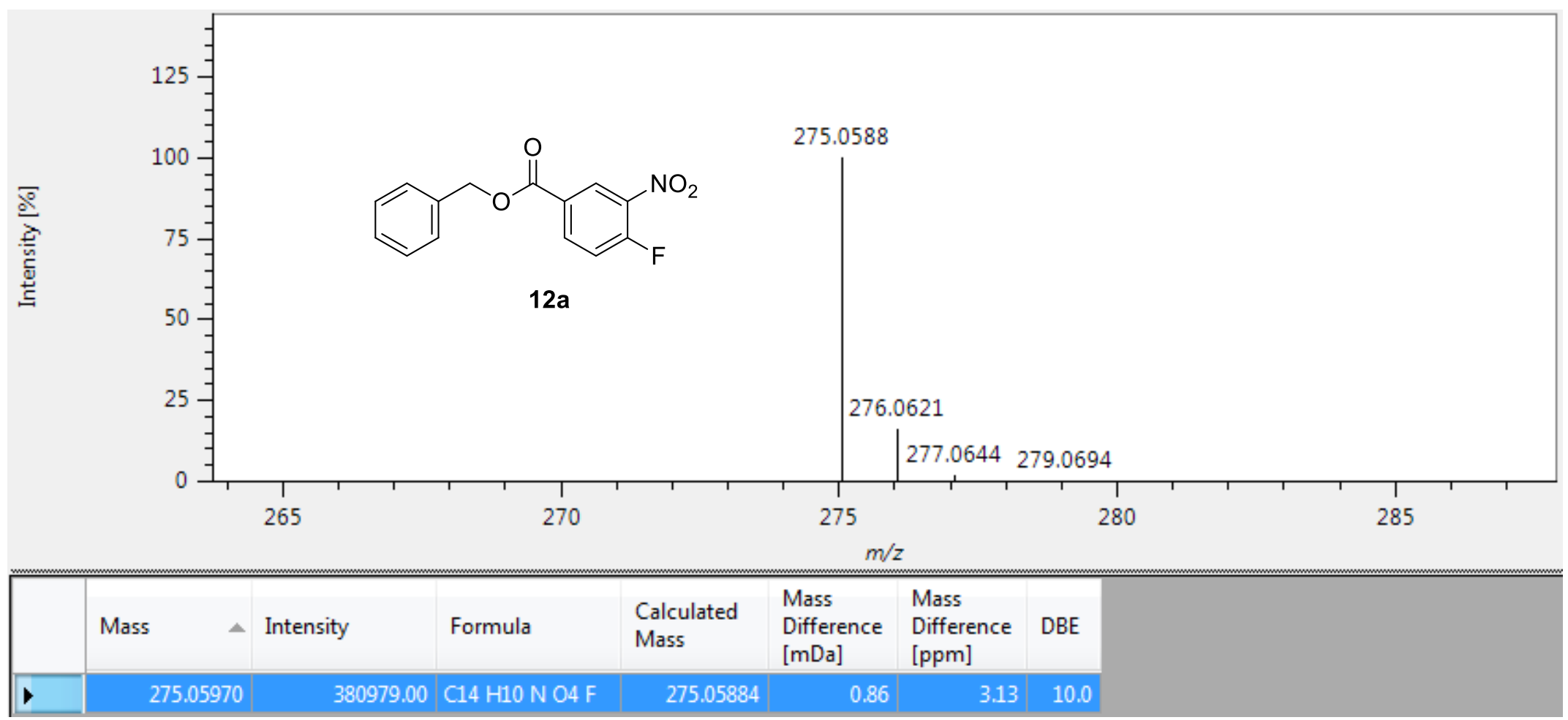

HRMS spectrum (EI) of compound 12a 


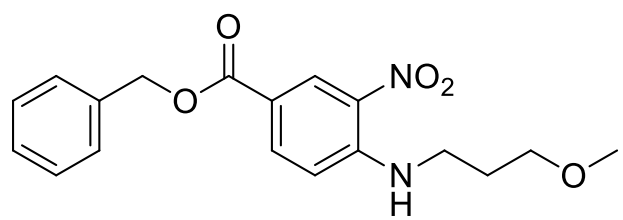

$13 a$

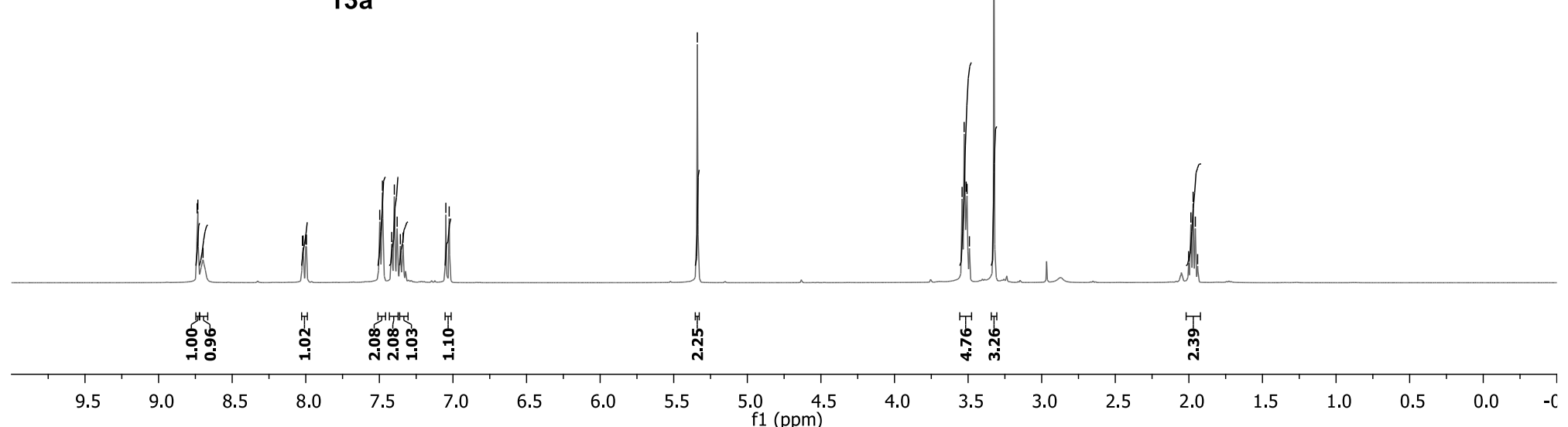

${ }^{1} \mathrm{H}$ NMR spectrum $\left(400 \mathrm{MHz}\right.$ ) of compound 13a in acetone- $\mathrm{d}_{6}$ 

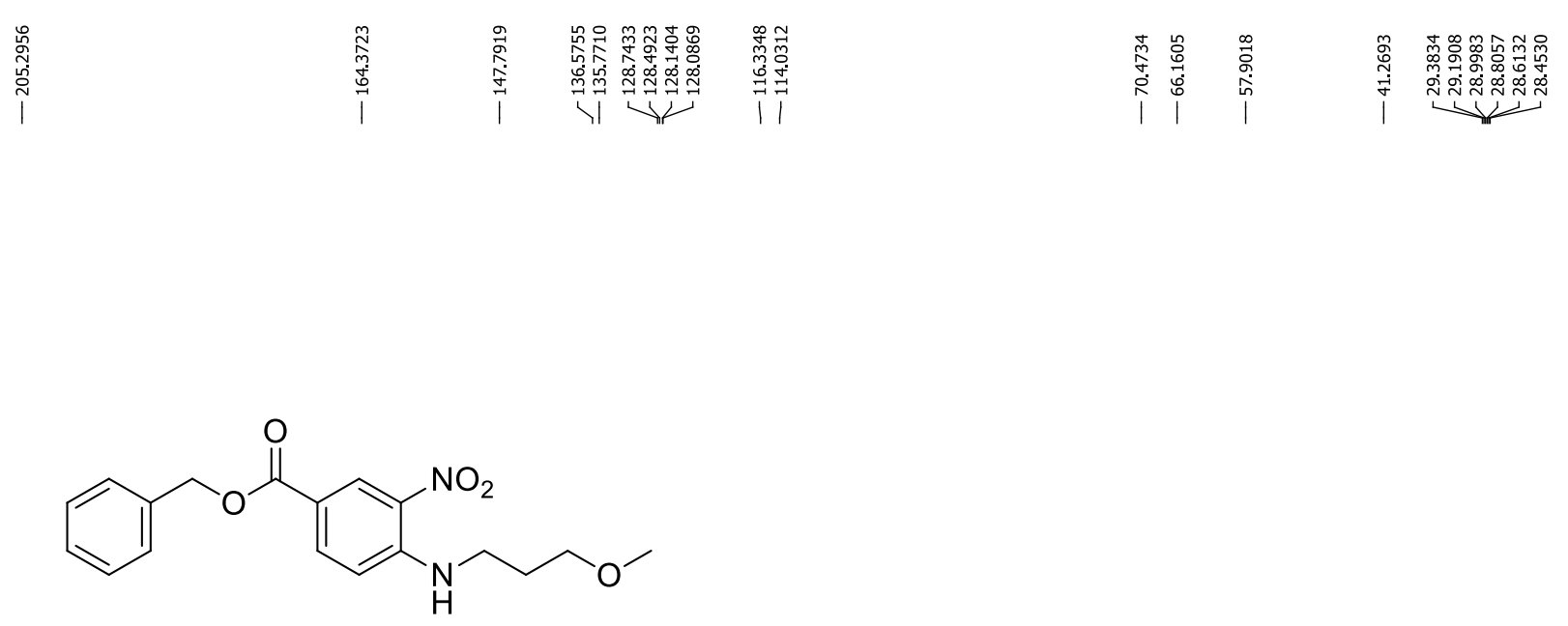

$13 a$
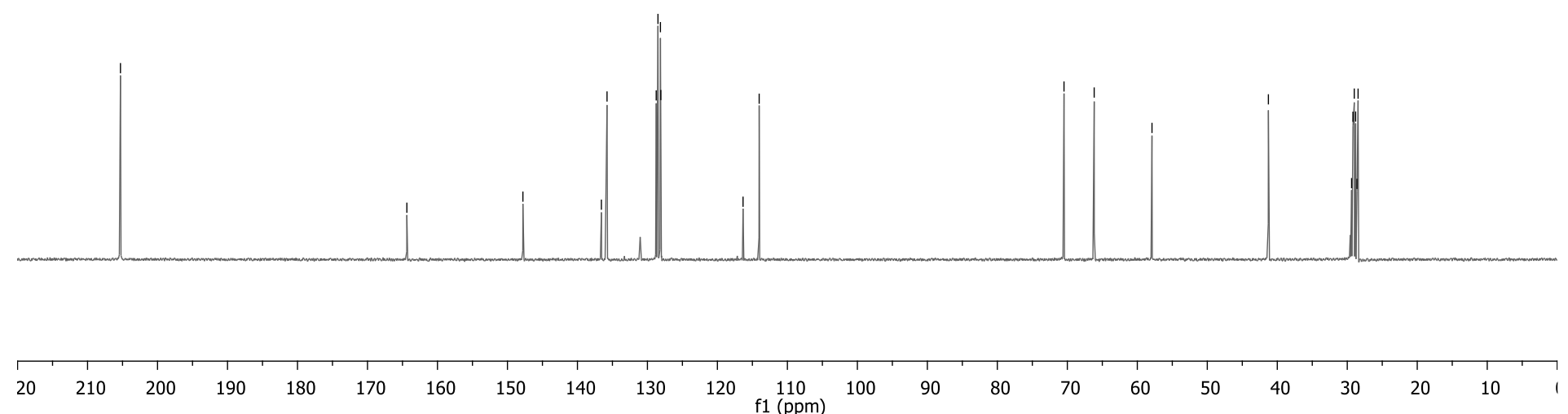

${ }^{13} \mathrm{C}$ NMR spectrum $(101 \mathrm{MHz})$ of compound 13a in acetone- $\mathrm{d}_{6}$ 


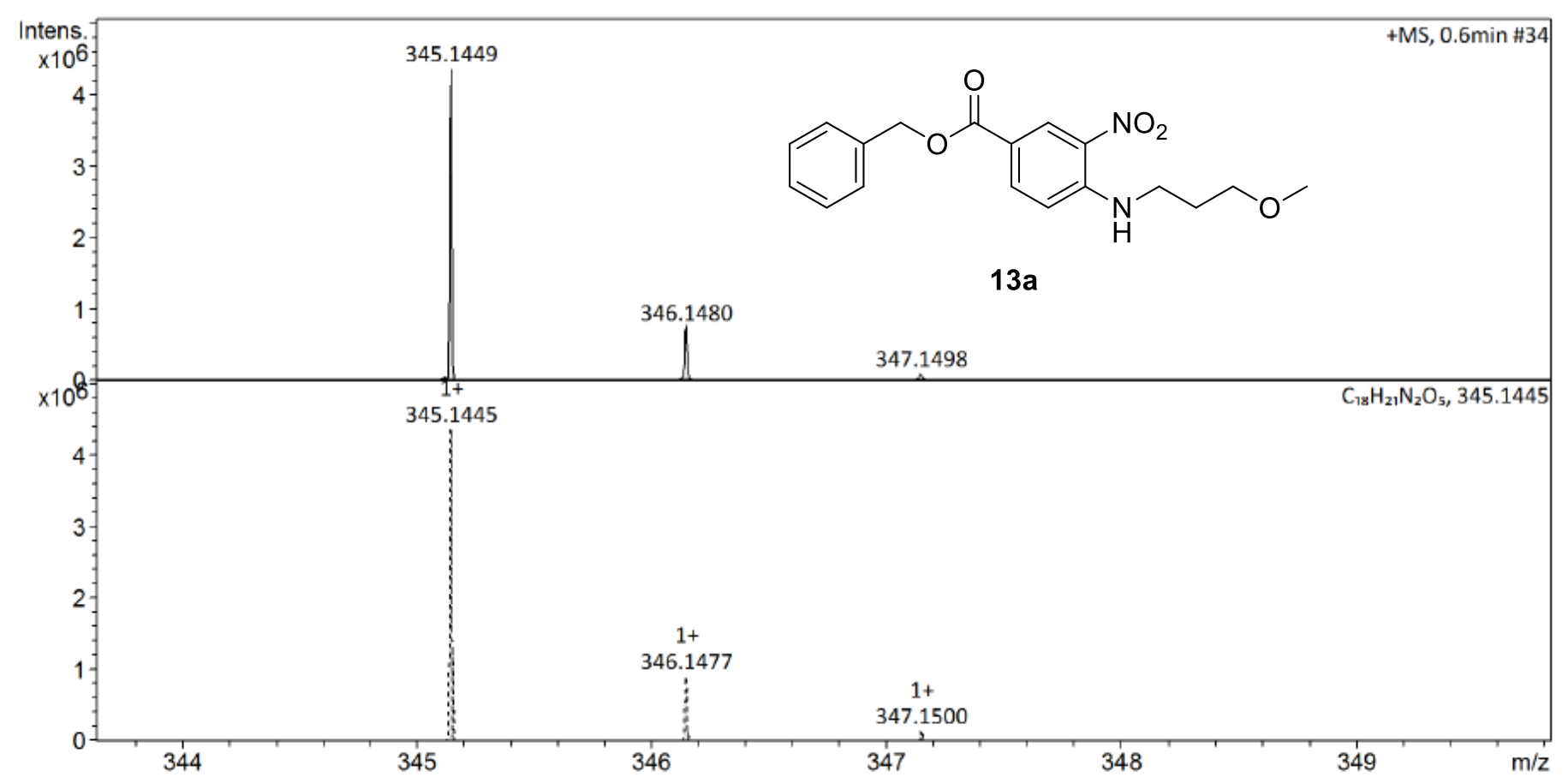

PD-N7-40 ESI+ RA5 01 8568.d

Bruker Compass DataAnalysis 4.1 printed: $1 / 13 / 20162: 31: 14 \mathrm{PM}$

\section{Display Report}

\begin{tabular}{|c|c|c|c|c|c|c|c|c|c|c|}
\hline Meas. $\mathrm{m} / \mathrm{z}$ & $\#$ & Ion Formula & $\mathrm{m} / \mathrm{z}$ & err [ppm] & mSigma & \# Sigma & Score & $\mathrm{rdb}$ & $e^{-}$Conf & N-Rule \\
\hline 345.1449 & 1 & $\mathrm{C} 18 \mathrm{H} 21 \mathrm{~N} 2 \mathrm{O} 5$ & 345.1445 & -1.3 & 22.6 & 1 & 100.00 & 9.5 & even & ok \\
\hline
\end{tabular}

HRMS spectrum (ESI) of compound 13a 

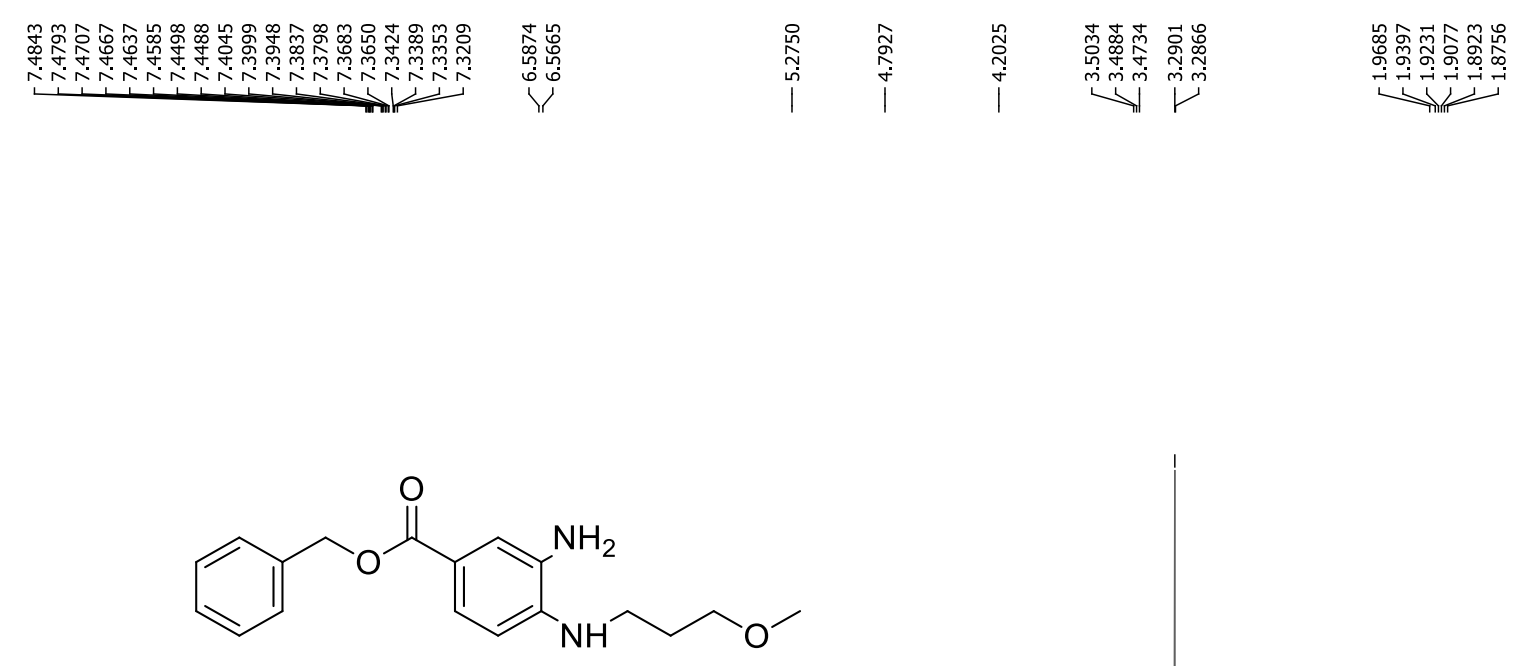

$14 a$

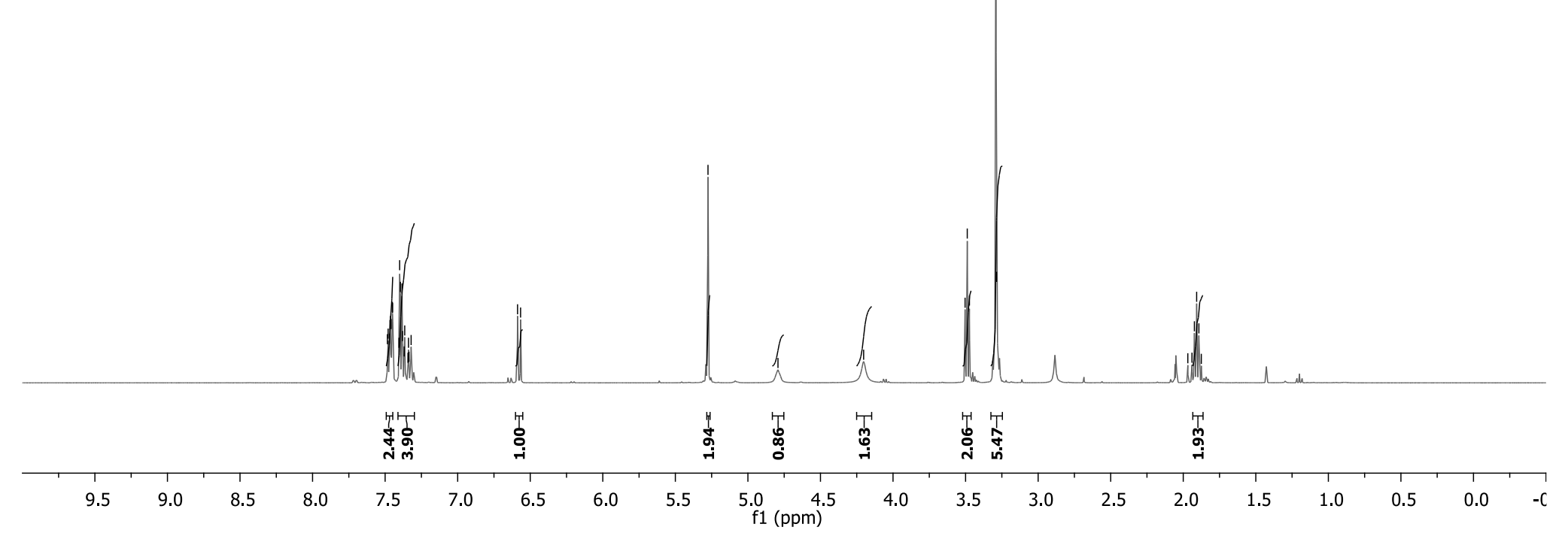

${ }^{1} \mathrm{H}$ NMR spectrum (400 MHz) of compound 14a in acetone- $\mathrm{d}_{6}$ 

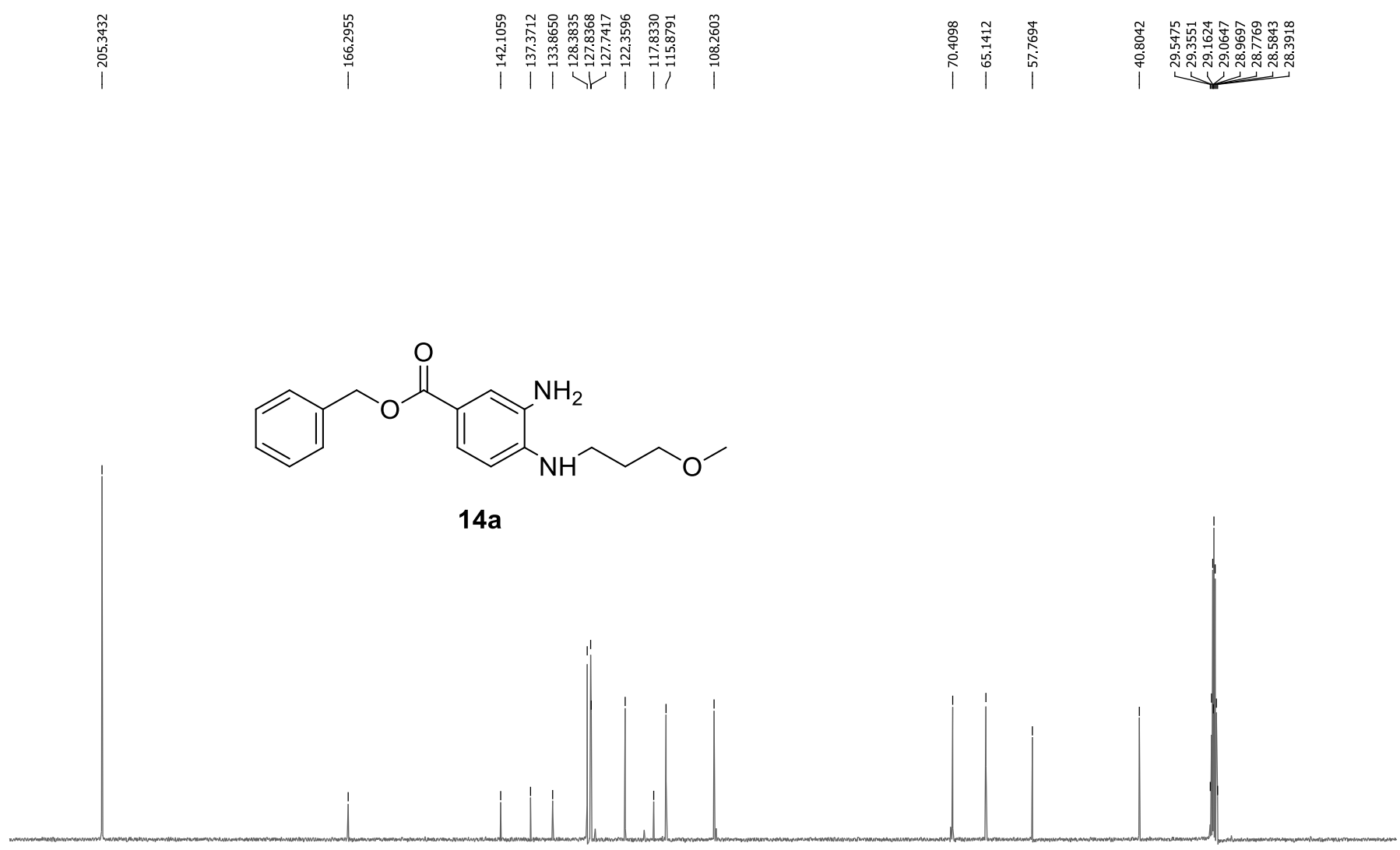

$\begin{array}{llllllllllll}20 & 210 & 200 & 190 & 180 & 170 & 160 & 150 & 140 & 130 & 120 & 110\end{array}$

${ }^{13} \mathrm{C}$ NMR spectrum $(101 \mathrm{MHz})$ of compound 14a in acetone- $\mathrm{d}_{6}$ 


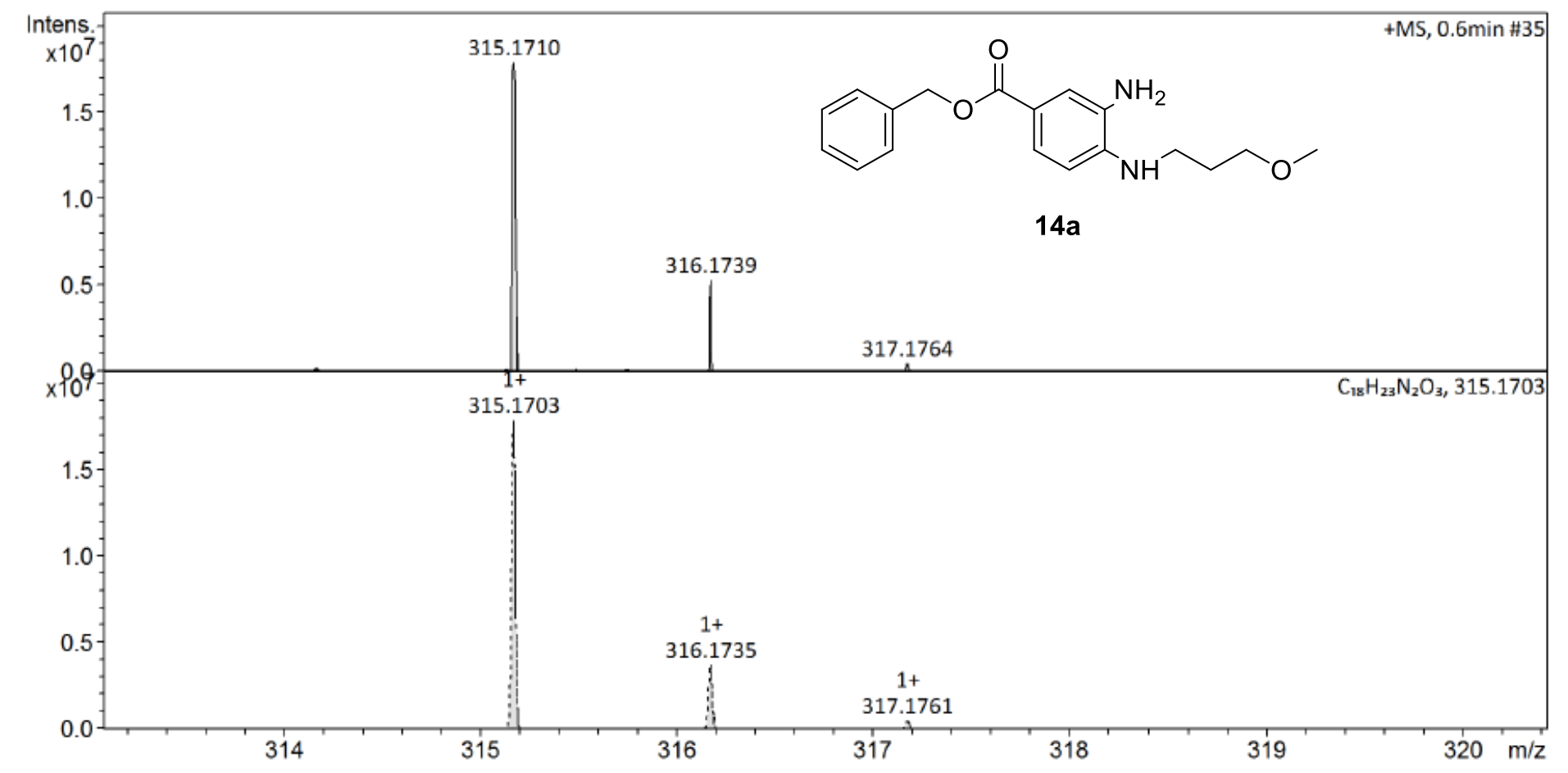

PD-N7-47 ESI+ RA6 01 8569.d

Bruker Compass DataAnalysis 4.1

printed: 1/13/2016 2:24:45 PM

by: NCTU

Page 1 of 2

eas. $\mathrm{m} / \mathrm{z}$ \# Ion Formula

Display Report

\# Sigma

Score
100.00

$e^{-}$Cont

N-Rule Adduct

HRMS spectrum (ESI) of compound 14a 


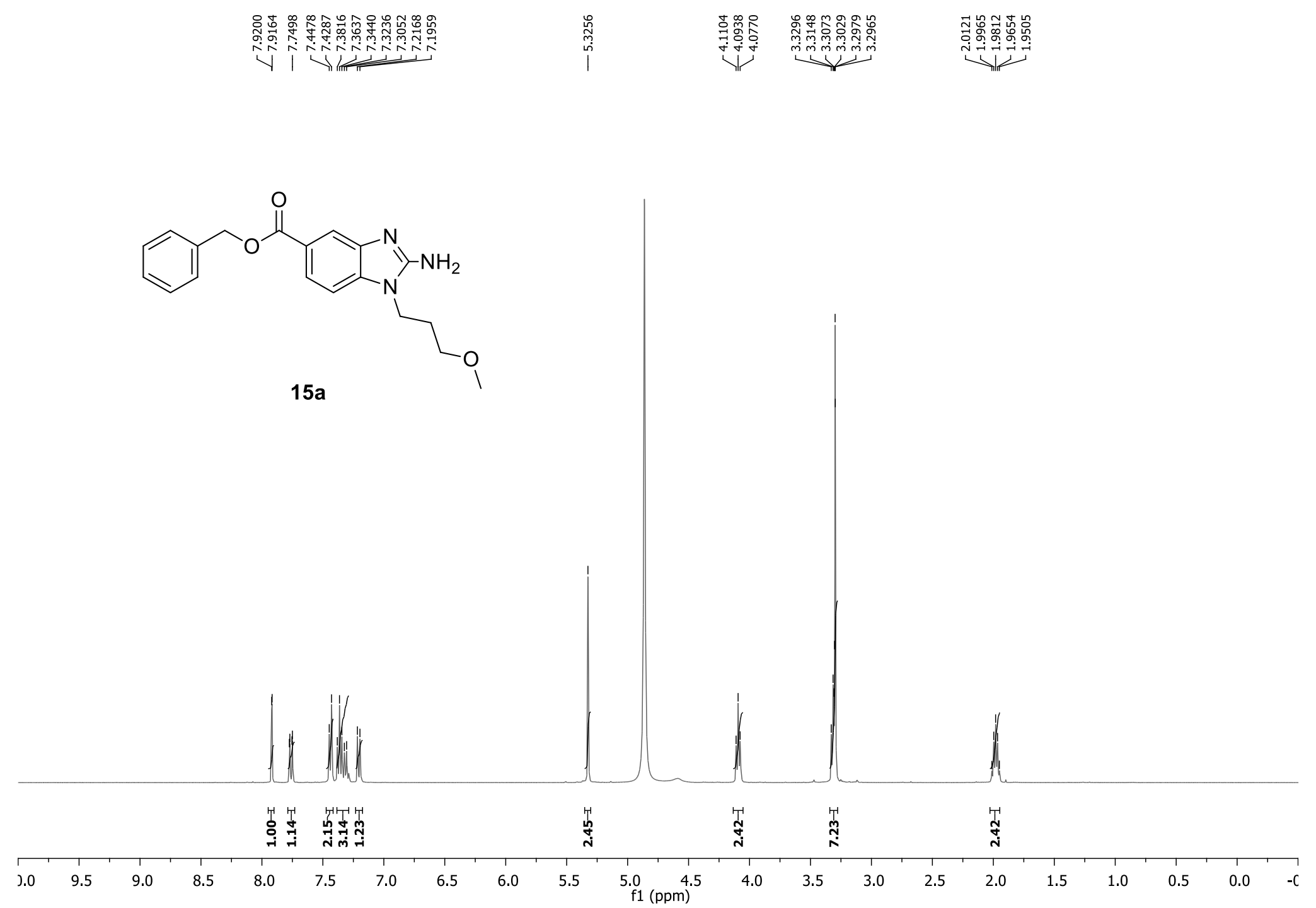

${ }^{1} \mathrm{H}$ NMR spectrum $\left(400 \mathrm{MHz}\right.$ ) of compound 15a in $\mathrm{CD}_{3} \mathrm{OD}$ 

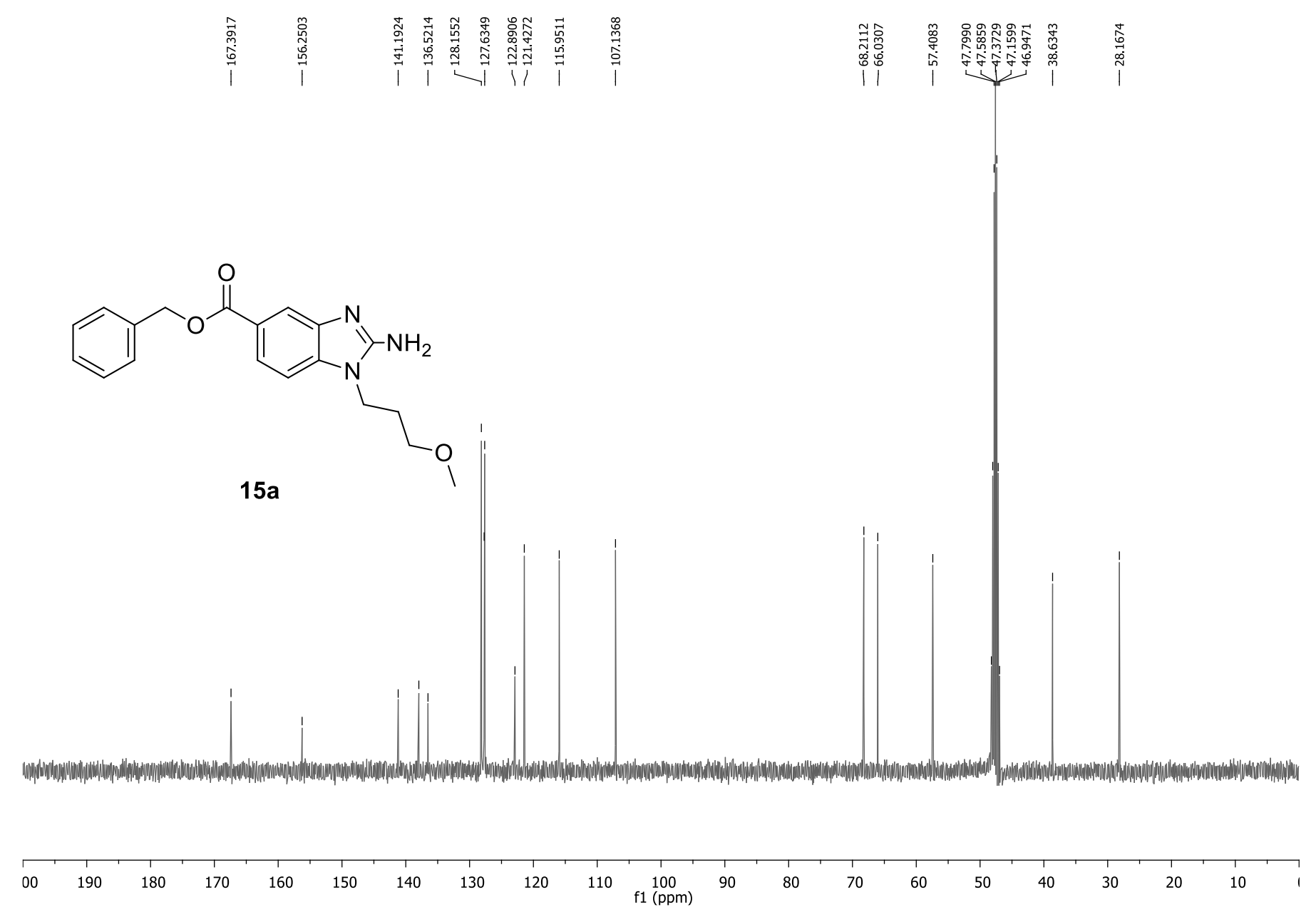

${ }^{13} \mathrm{C}$ NMR spectrum (101 MHz) of compound 15a in $\mathrm{CD}_{3} \mathrm{OD}$ 


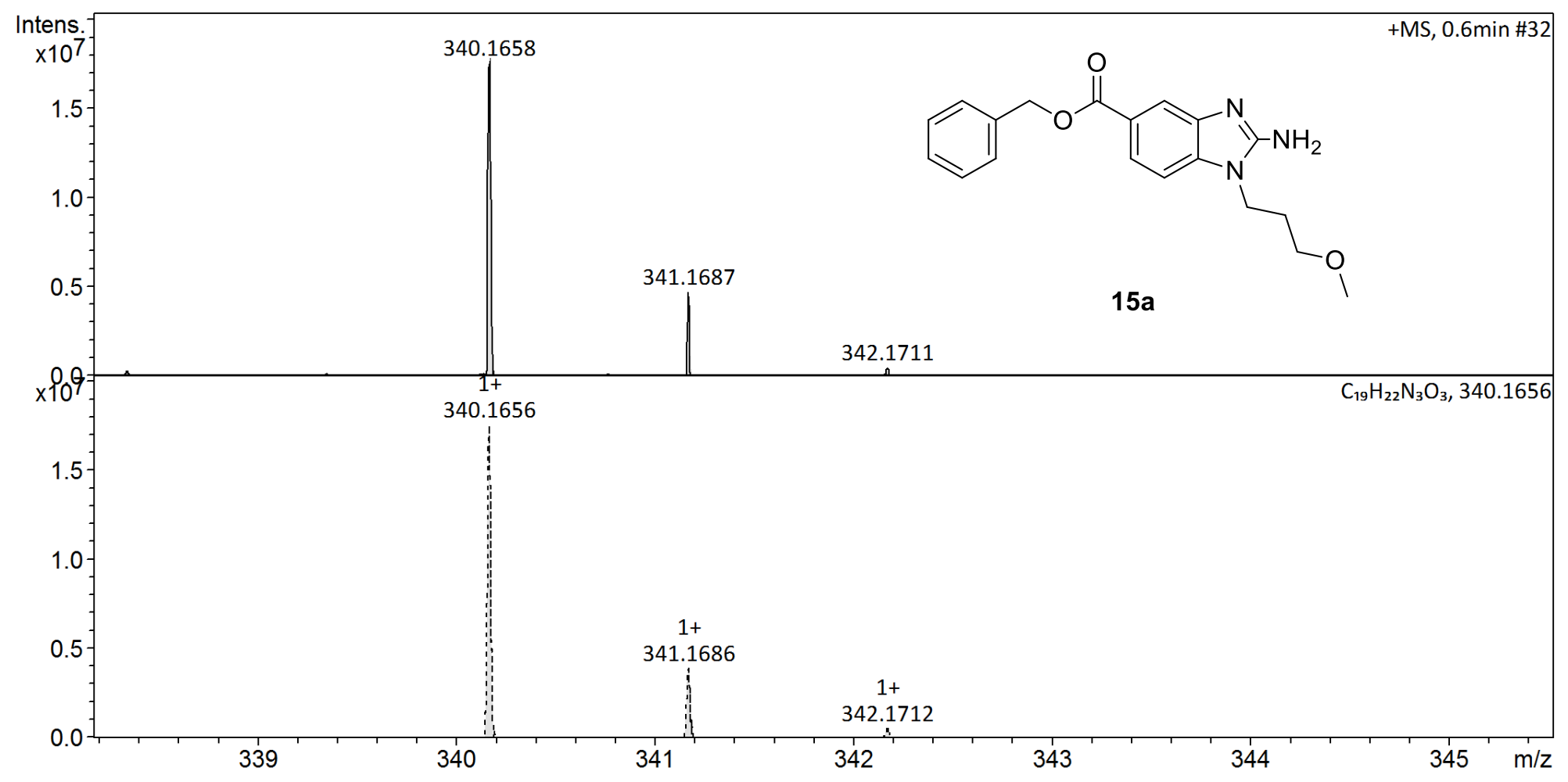

\section{Display Report}

Meas. $\mathrm{m} / \mathrm{z}$ \# Ion Formula $\mathrm{m} / \mathrm{z}$ err [ppm] mSigma \# Sigma Score $\mathrm{rdb} \mathrm{e}^{-}$Conf N-Rule Adduct $340.1658 \quad 1 \quad \mathrm{C} 19 \mathrm{H} 22 \mathrm{N3O3}$

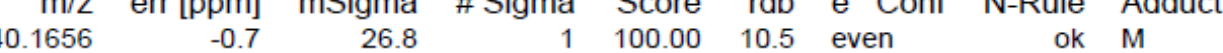

HRMS spectrum (ESI) of compound 15a 


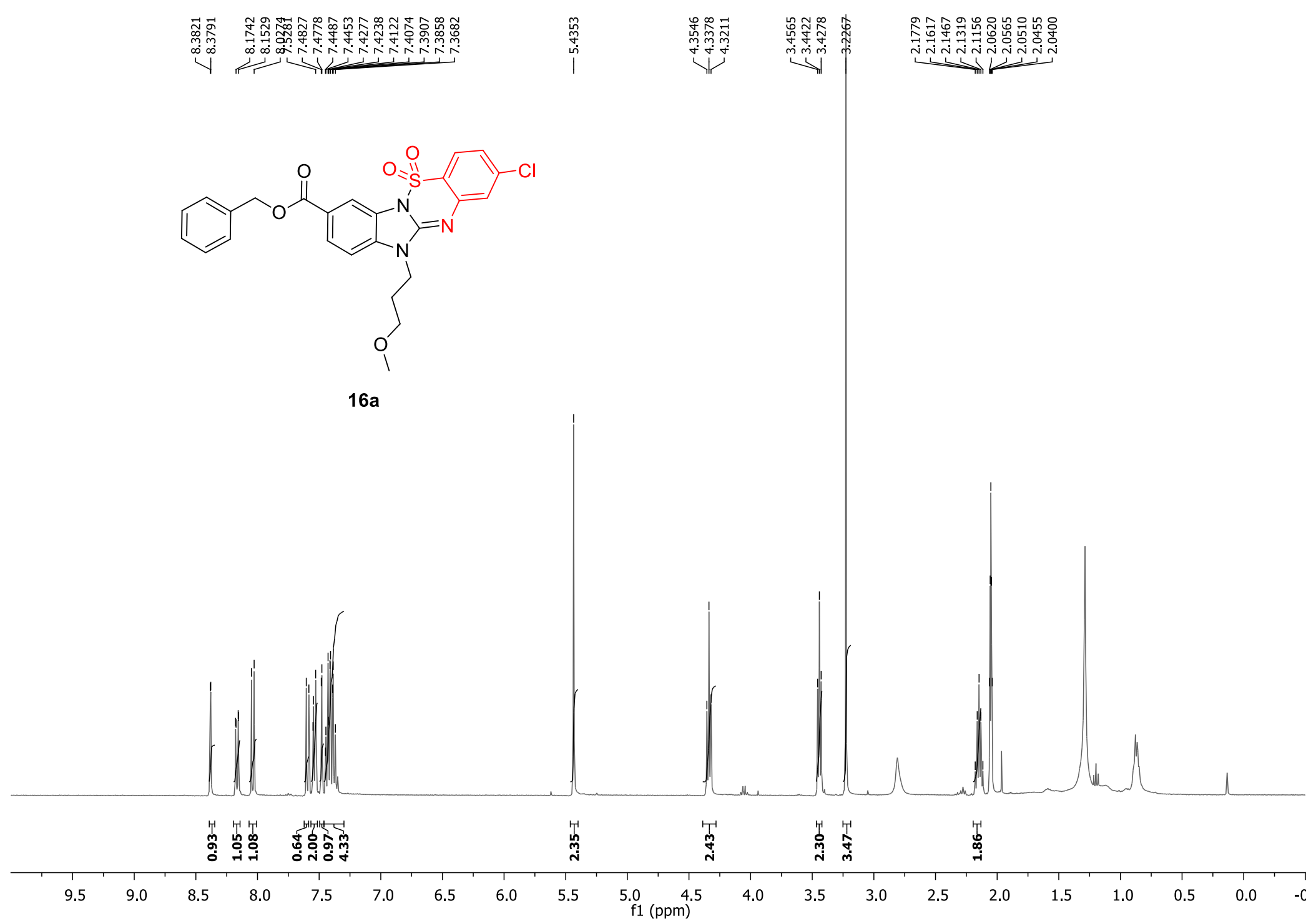

${ }^{1} \mathrm{H}$ NMR spectrum $(400 \mathrm{MHz})$ of compound 16a in acetone- $\mathrm{d}_{6}$ 

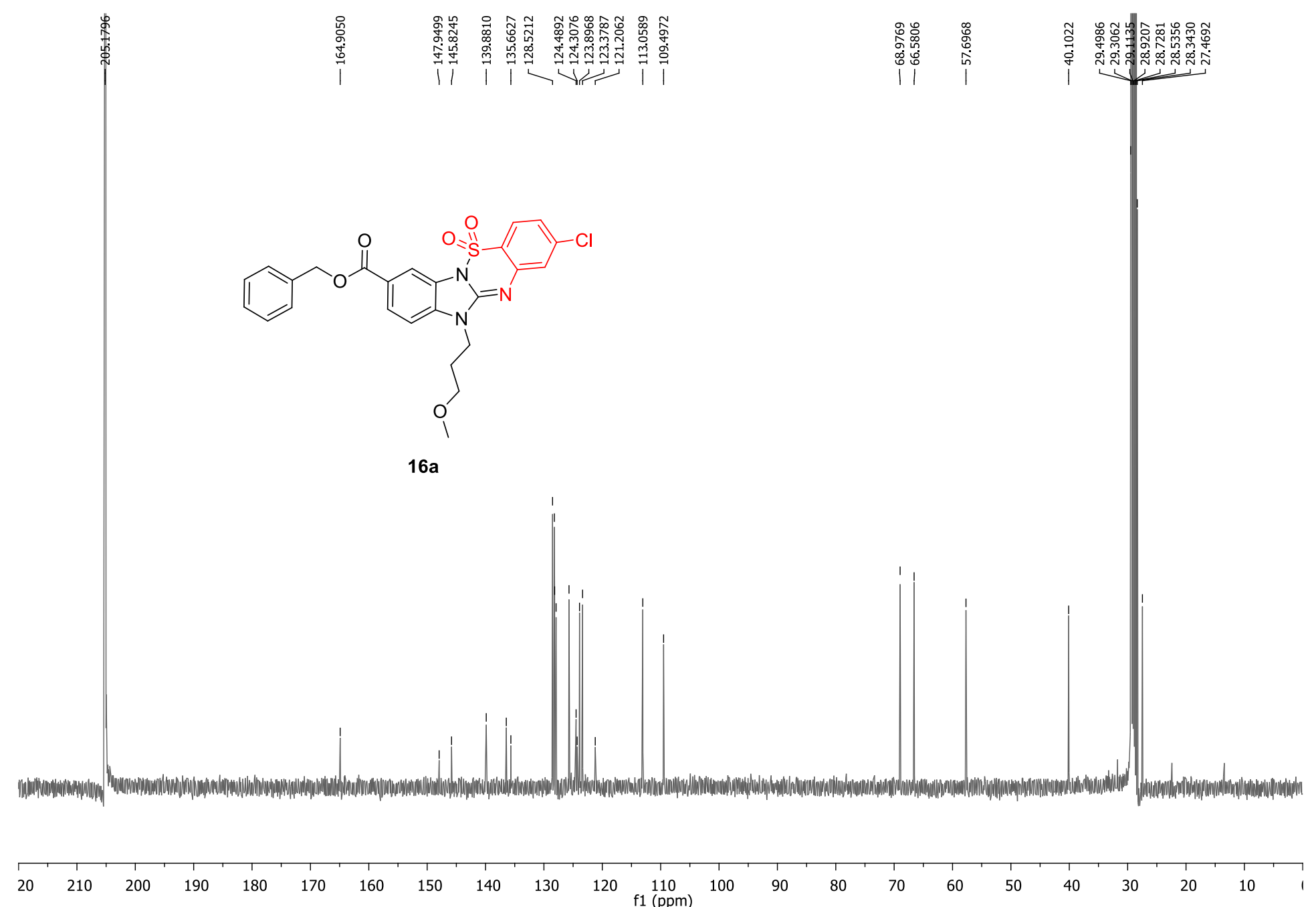

${ }^{13} \mathrm{C}$ NMR spectrum $(101 \mathrm{MHz})$ of compound 16a in acetone- $\mathrm{d}_{6}$ 


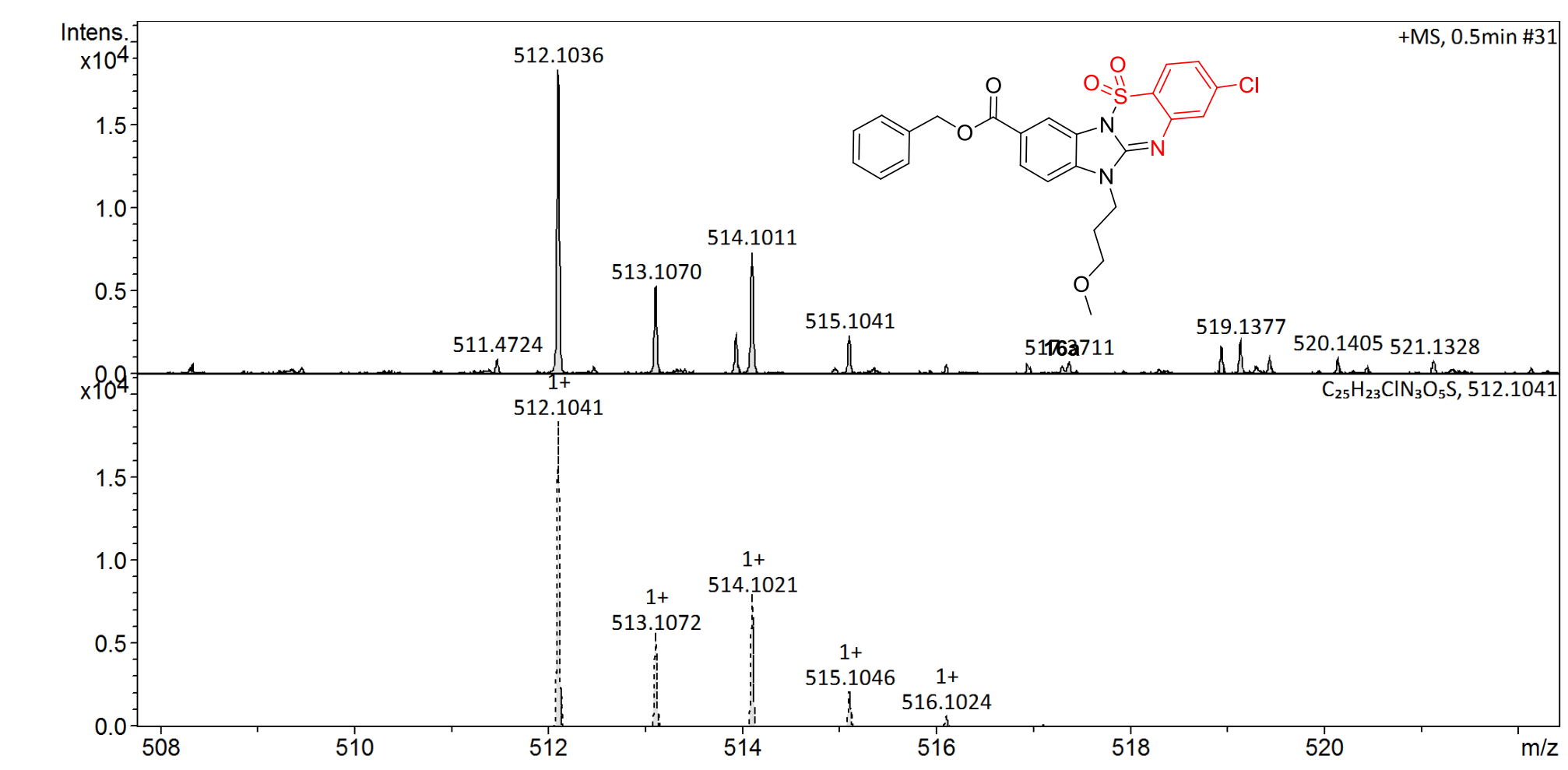

\section{Display Report}

$\begin{array}{rrlrrrrrrrrr}\text { Meas. } \mathrm{m} / \mathrm{z} & \# & \text { Ion Formula } & \mathrm{m} / \mathrm{z} & \text { err }[\mathrm{ppm}] & \mathrm{mSigma} & \text { \# Sigma } & \text { Score } & \text { rdb } & \mathrm{e}^{-} \text {Conf } & \mathrm{N}-\mathrm{Rule} & \text { Adduct } \\ 512.1036 & 1 & \text { C25H23CIN3O5S } & 512.1041 & 1.0 & 16.4 & 1 & 100.00 & 15.5 & \text { even } & \text { ok } & \mathrm{M}\end{array}$

HRMS spectrum (ESI) of compound 16a 


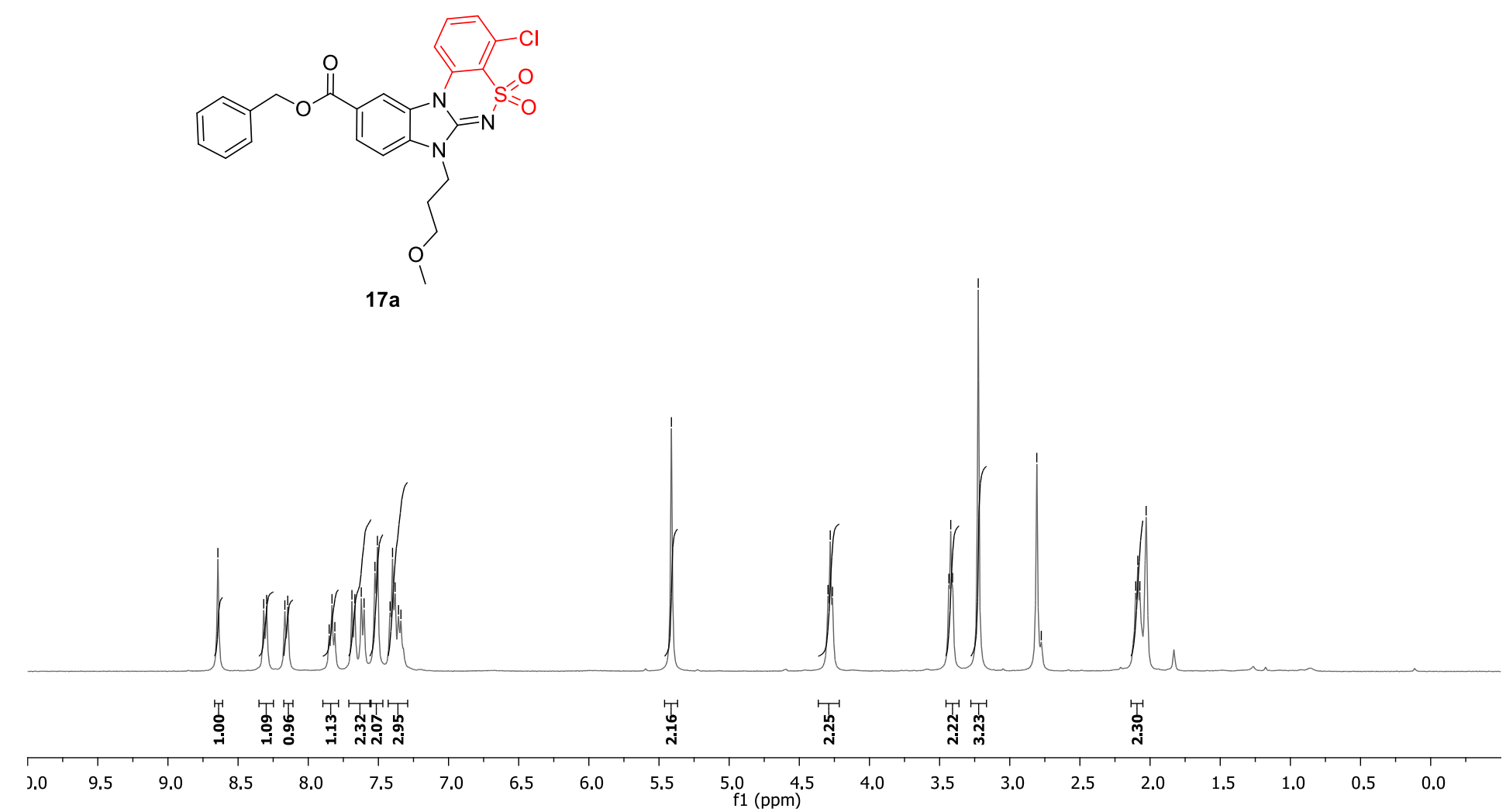

${ }^{1} \mathrm{H}$ NMR spectrum $\left(400 \mathrm{MHz}\right.$ ) of compound $\mathbf{1 7} \mathbf{a}$ in acetone- $\mathrm{d}_{6}$ 


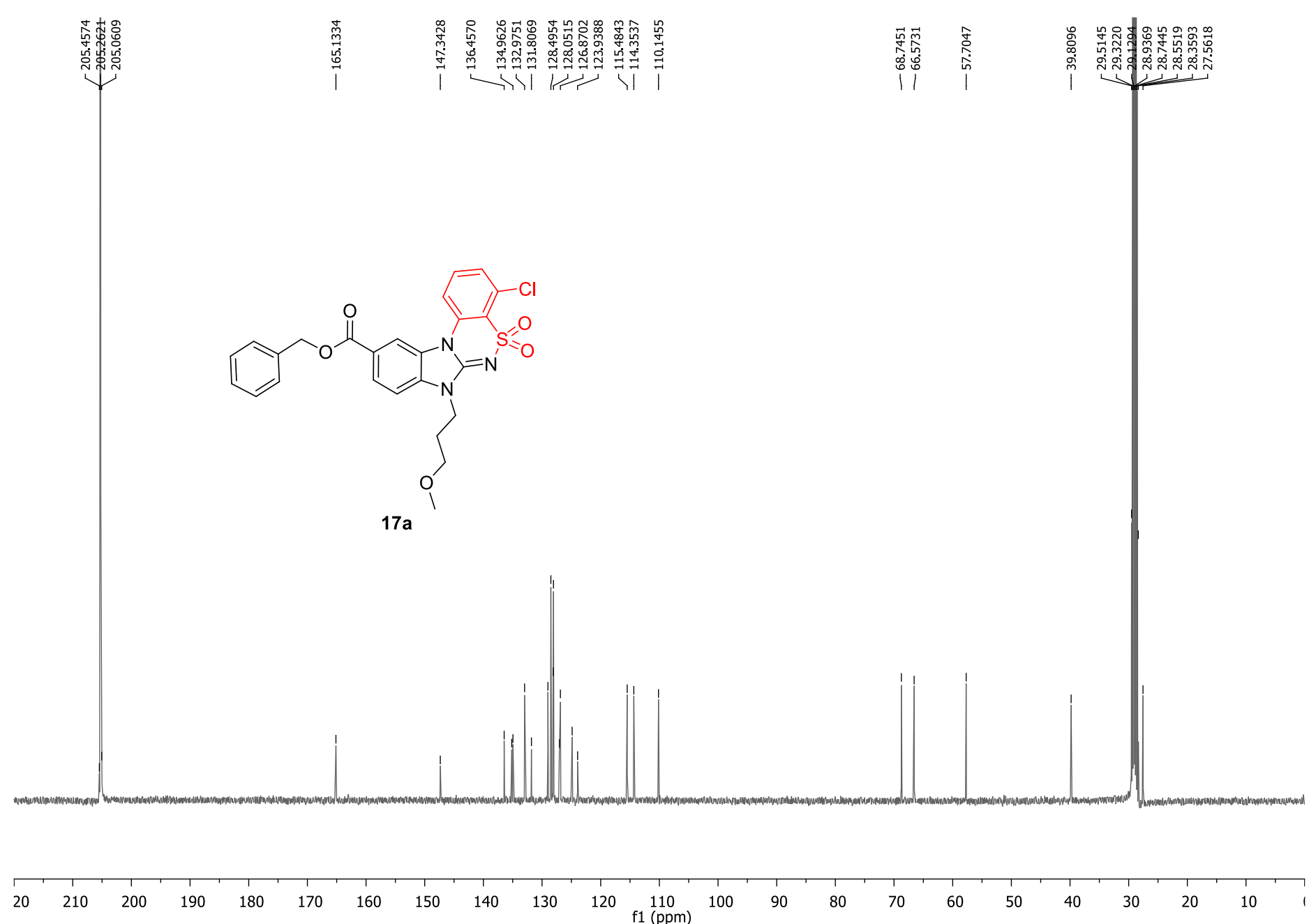

${ }^{13} \mathrm{C}$ NMR spectrum $(101 \mathrm{MHz})$ of compound $\mathbf{1 7} \mathbf{a}$ in acetone- $\mathrm{d}_{6}$ 


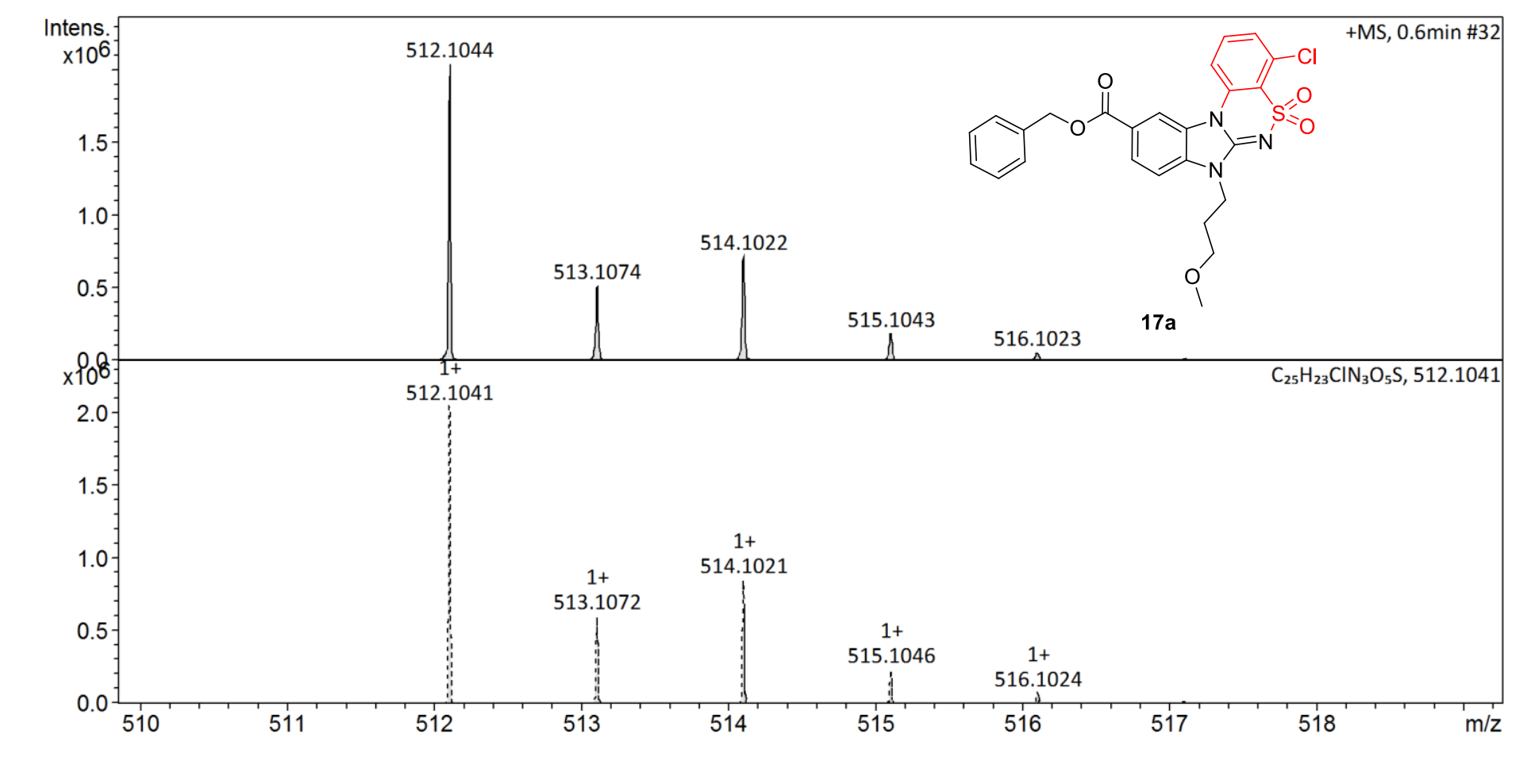

\section{Display Report}

Meas. $\mathrm{m} / \mathrm{z}$ \# Ion Formula

$512.1044 \quad 1 \quad \mathrm{C} 25 \mathrm{H} 23 \mathrm{CIN} 3 \mathrm{O} 5 \mathrm{~S}$

$\mathrm{m} / \mathrm{z}$ err [ppm] mSigma \# Sigma Score $\mathrm{rdb} \mathrm{e}^{-}$Conf N-Rule Adduct

512.1041

$1100.00 \quad 15.5$ even

ok M

HRMS spectrum (ESI) of compound 17a 


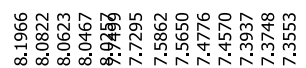

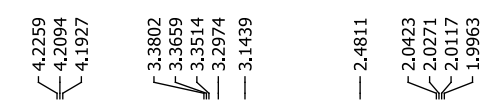

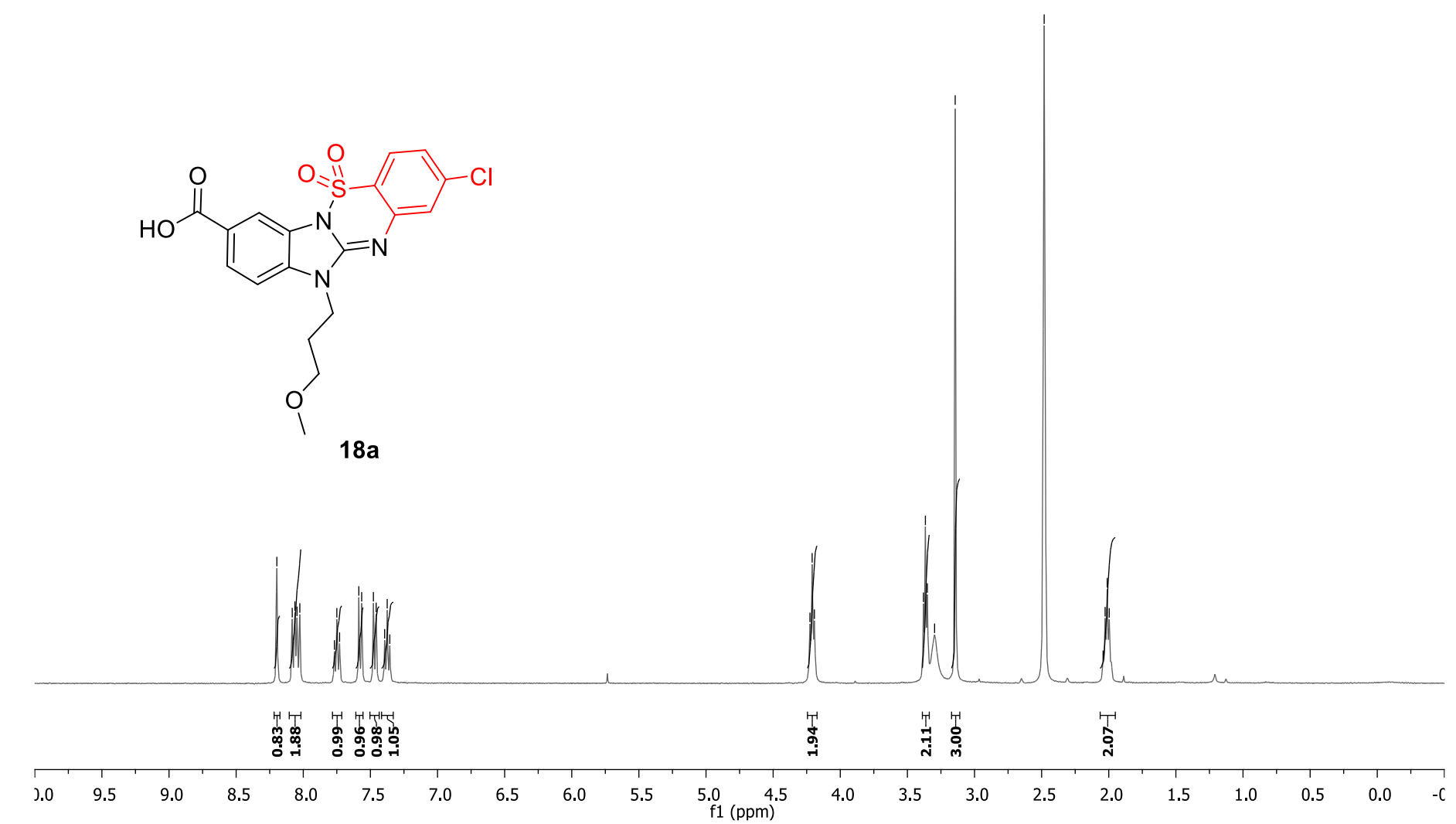

${ }^{1} \mathrm{H}$ NMR spectrum (400 MHz) of compound 18a in DMSO-d $\mathrm{d}_{6}$ 


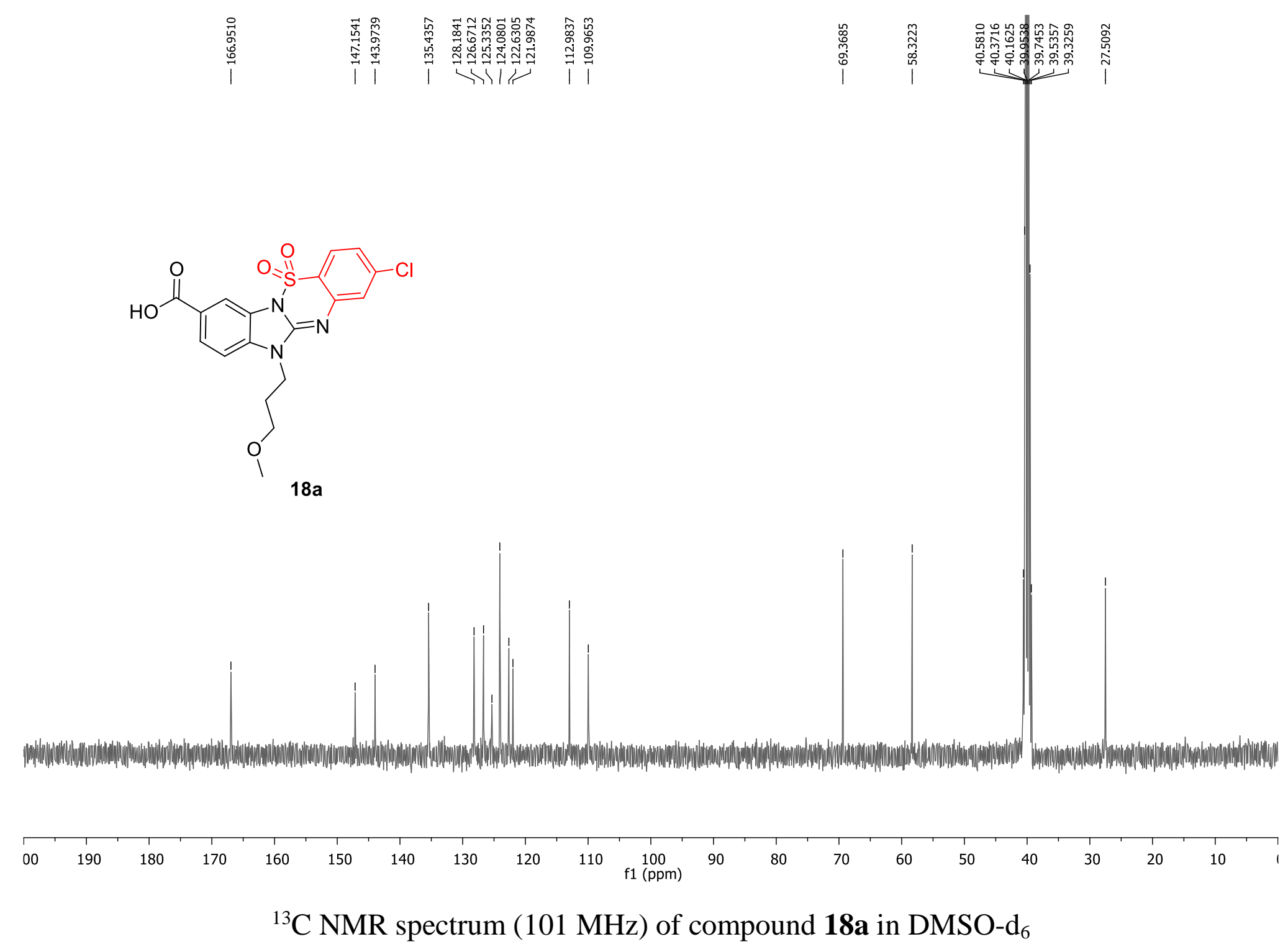



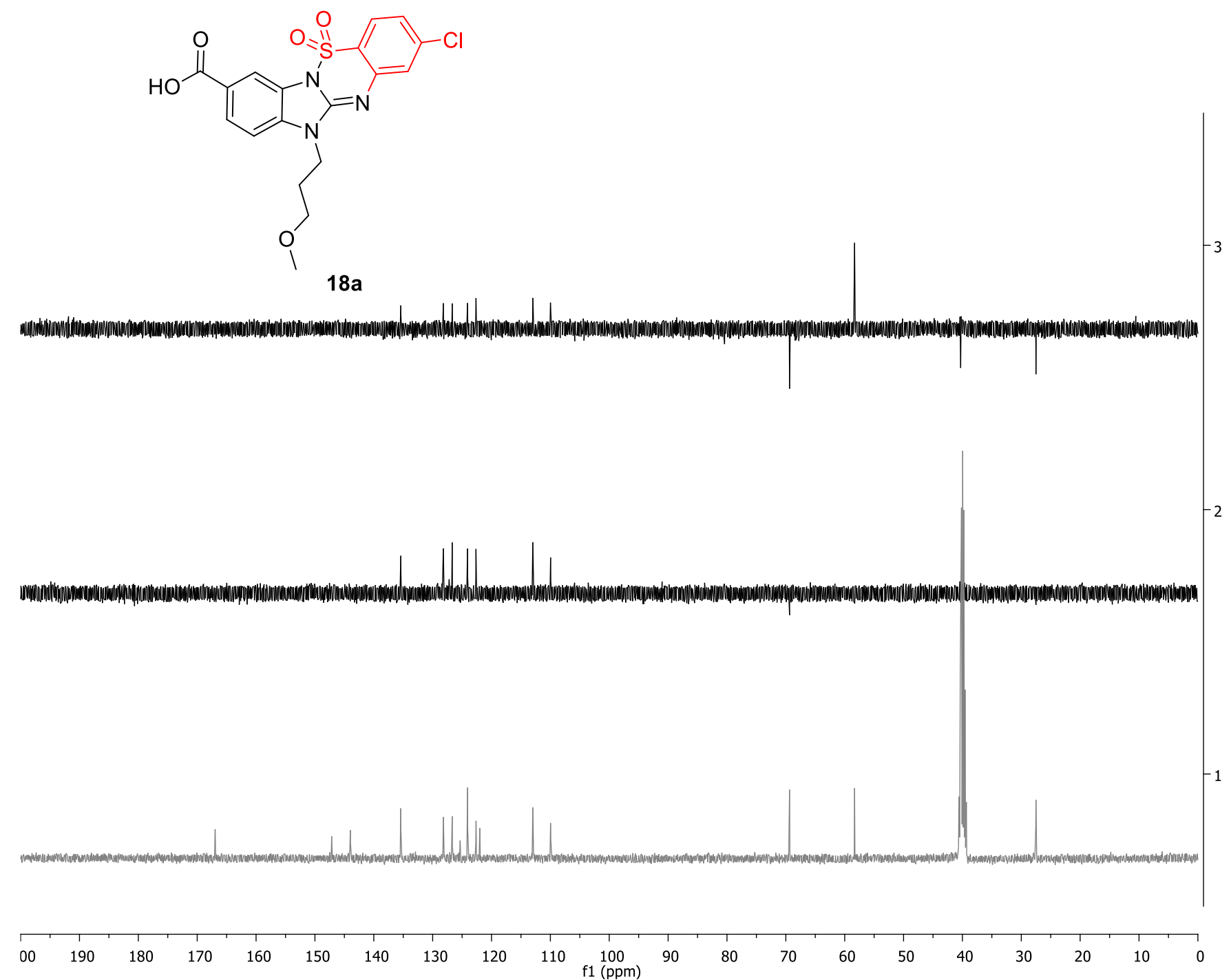

${ }^{13} \mathrm{C}$ NMR and DEPT spectrum (101 MHz) of compound 18a in DMSO-d 6 


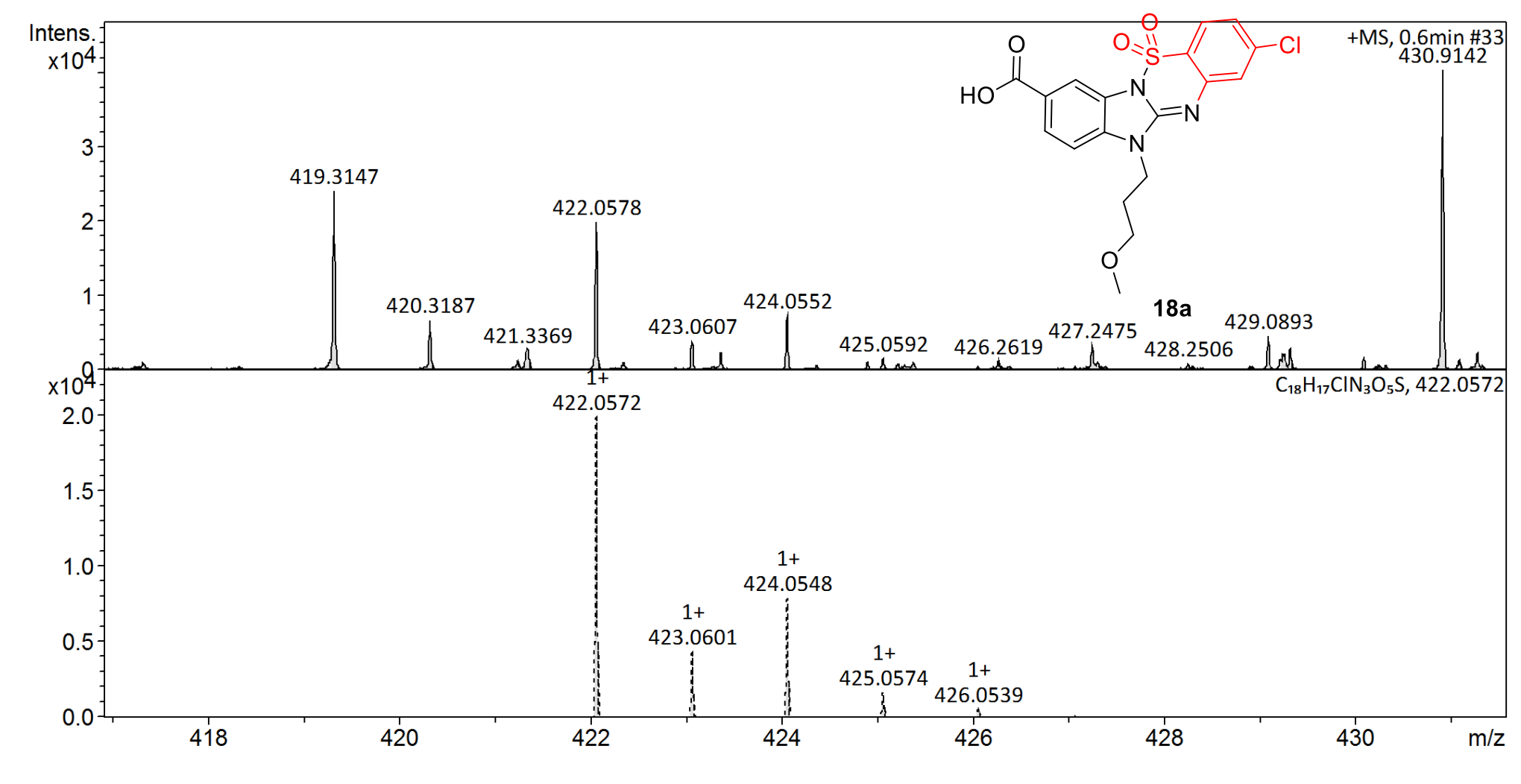

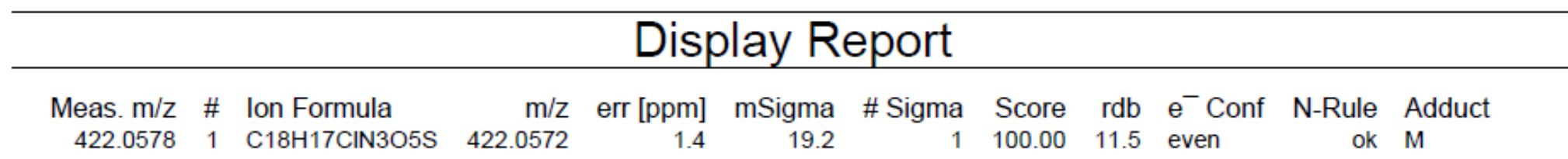

HRMS spectrum (ESI) of compound 18a 


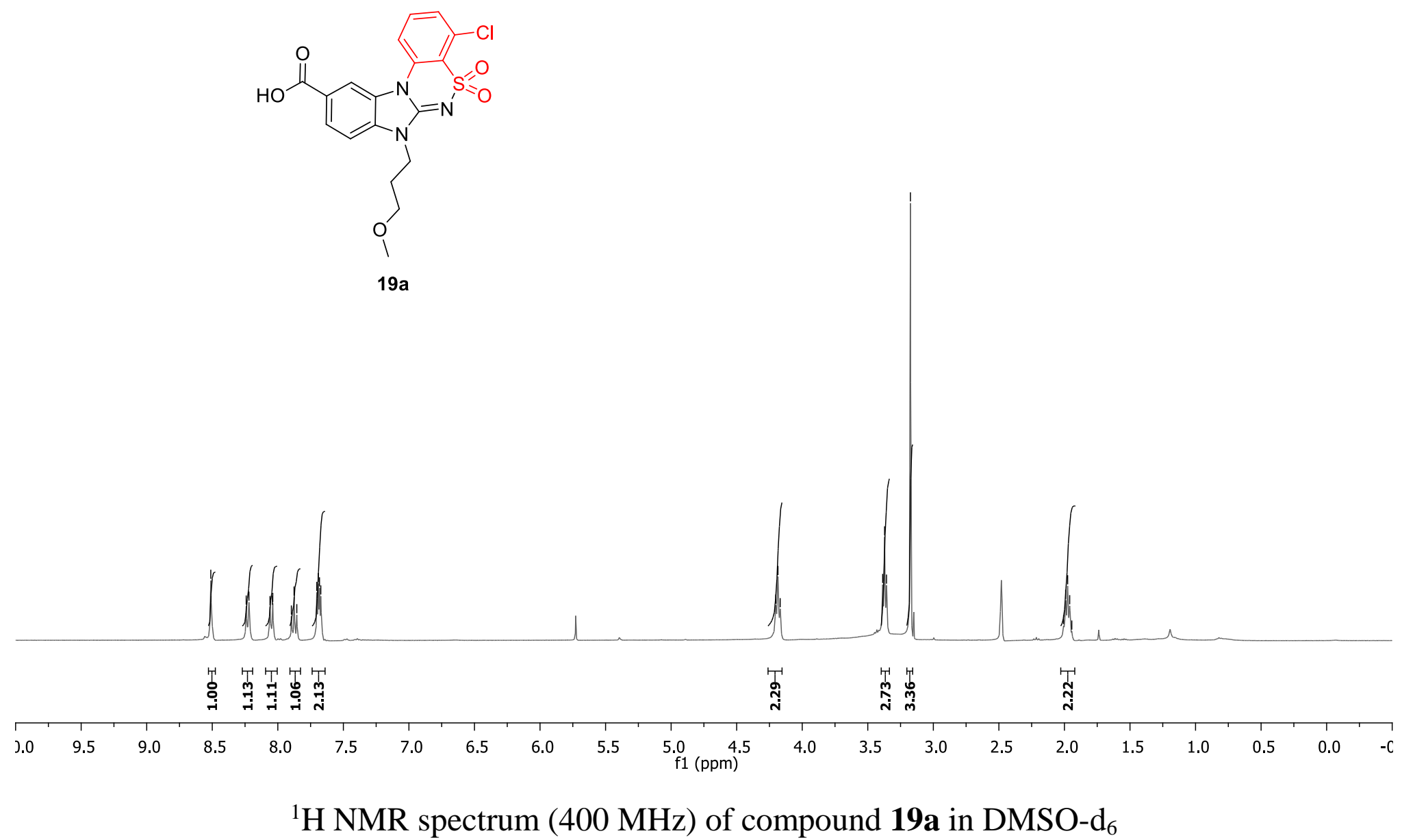




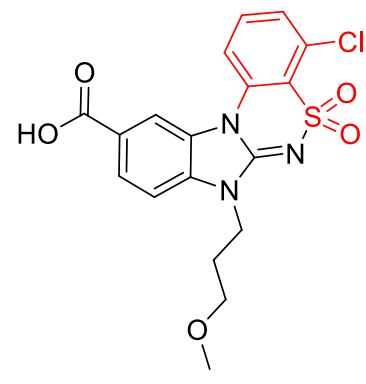

$19 a$

$00 \quad 190$ 180

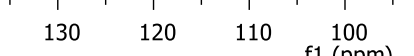

90

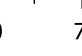

60

50

${ }^{13} \mathrm{C}$ NMR spectrum $(101 \mathrm{MHz})$ of compound 19a in DMSO-d $\mathrm{d}_{6}$ 


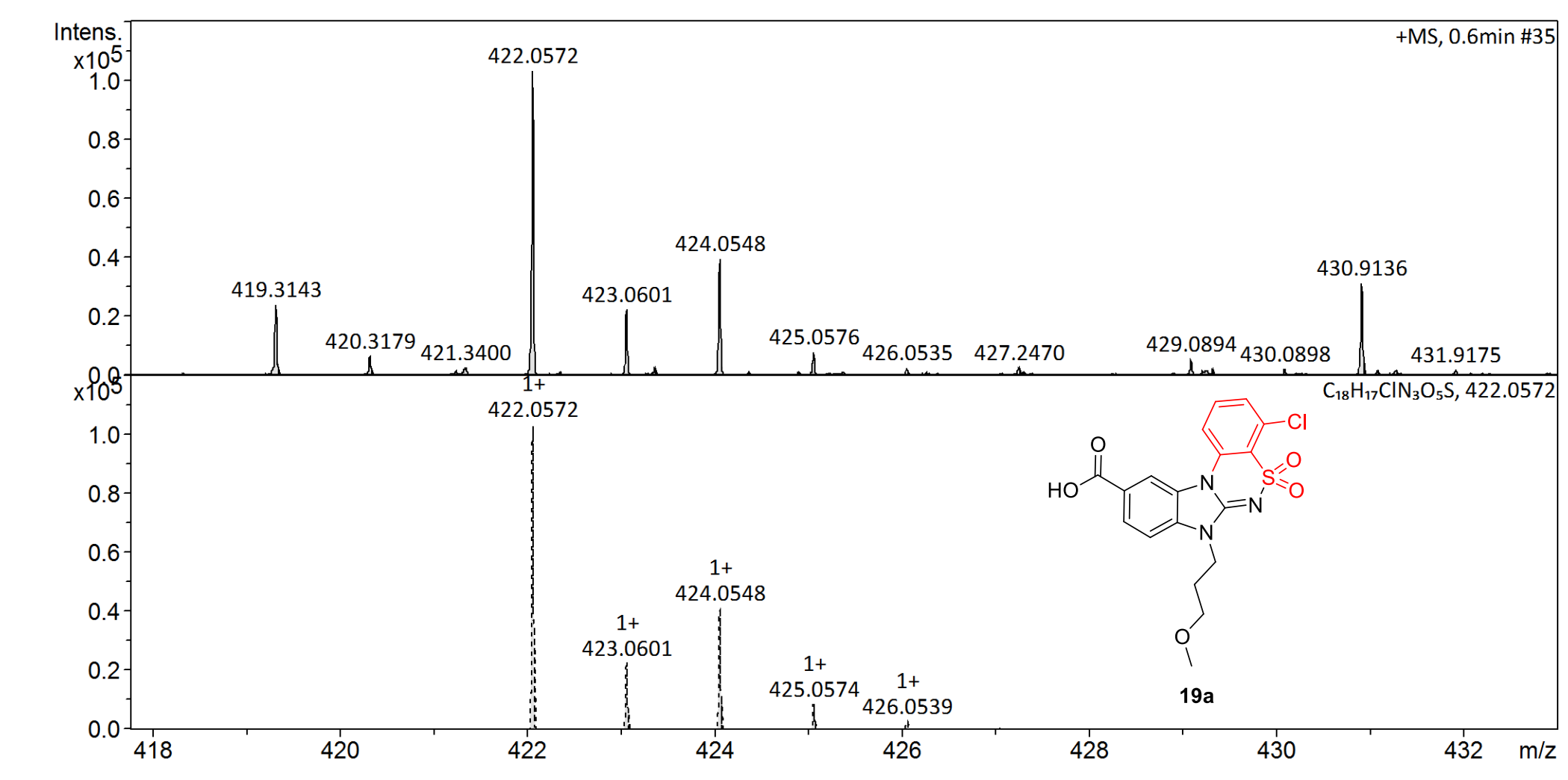

\section{Display Report}

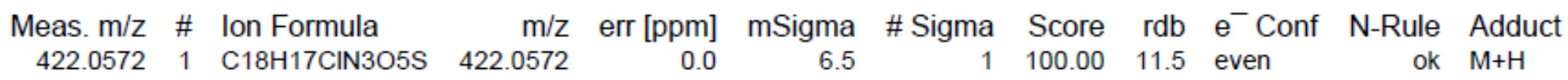

HRMS spectrum (ESI) of compound 19a 


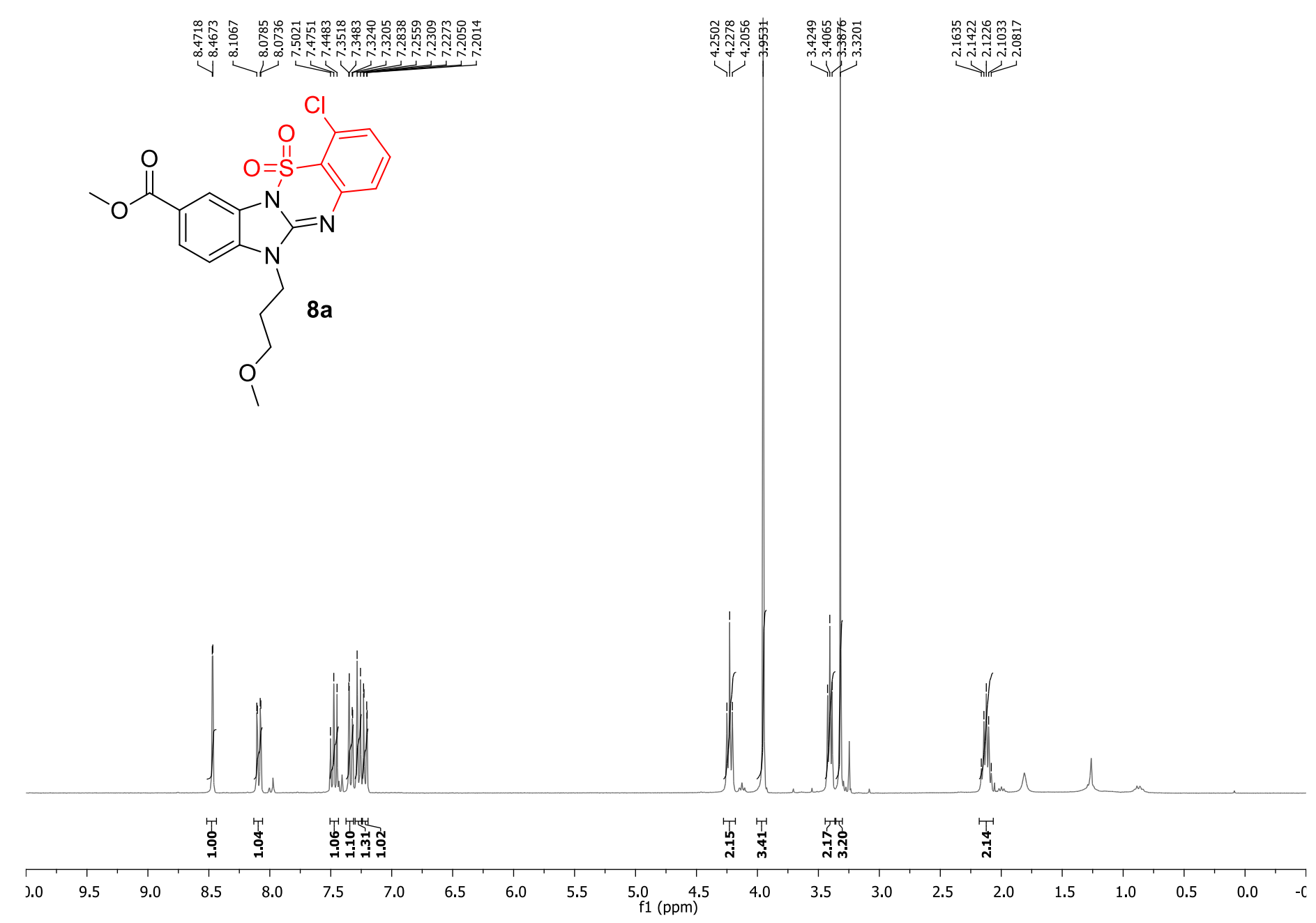

${ }^{1} \mathrm{H}$ NMR spectrum $(300 \mathrm{MHz})$ of compound $\mathbf{8 a}$ in $\mathrm{CDCl}_{3}$ 


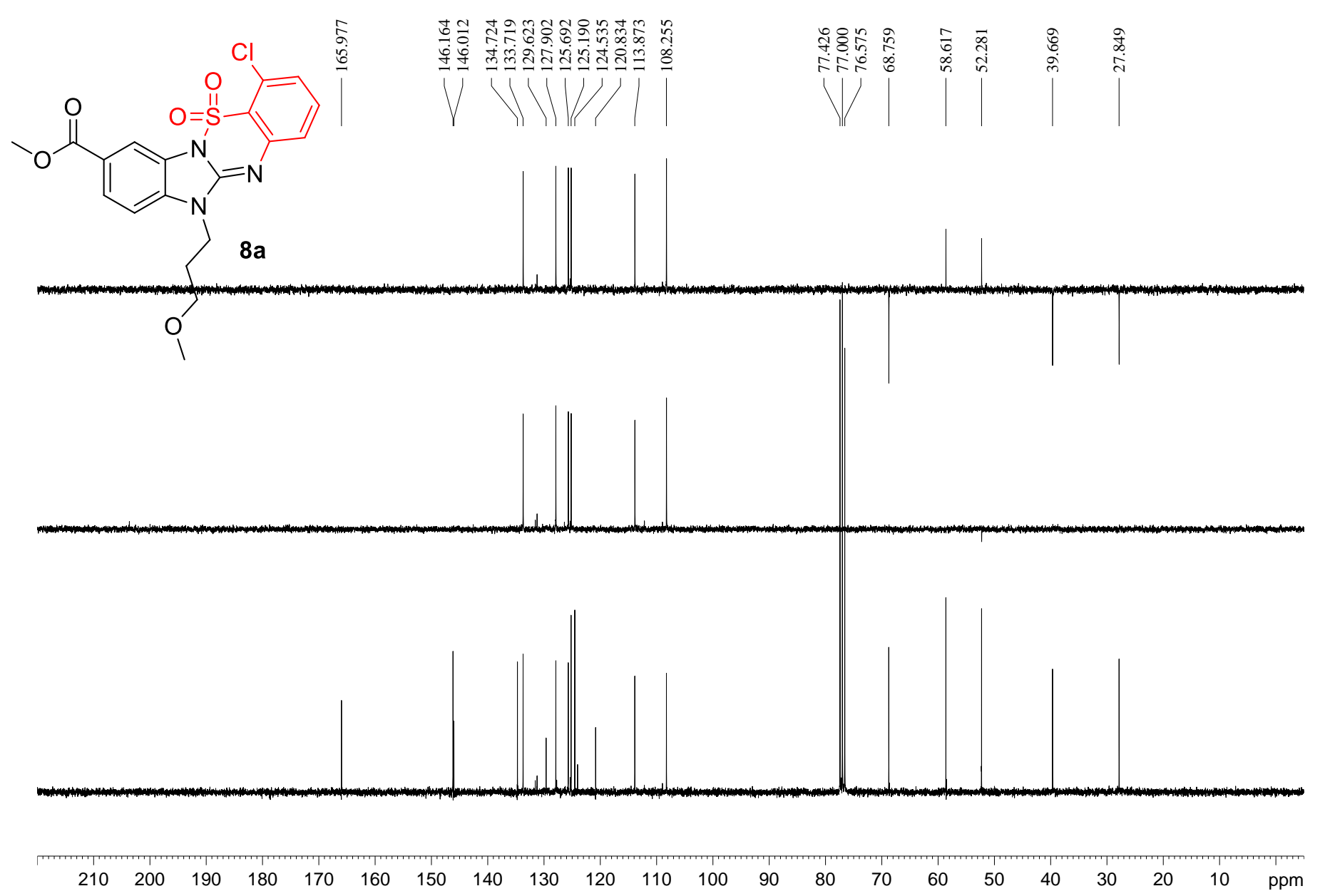

${ }^{13} \mathrm{C}$ and DEPT NMR spectrum (75 MHz) of compound $\mathbf{8 a}$ in $\mathrm{CDCl}_{3}$ 
LIST: hei1448-c1

Samp: 1201522 6la (S2)

Mode: EI +VE +LMR ESCAN (EXP) UP HR NRM

07-Mar-12 REG : 01:12.2 \#9

Oper:

Limt: $\left(\begin{array}{l}35) \\ \text { Oper: }\end{array}\right.$

(456) C20.H27. $\mathrm{R}+\mathrm{D}:-2.0>60.0$

Peak: 1000.00 mmu (CMASS : converted/CMASS : converted / CMASS : conve

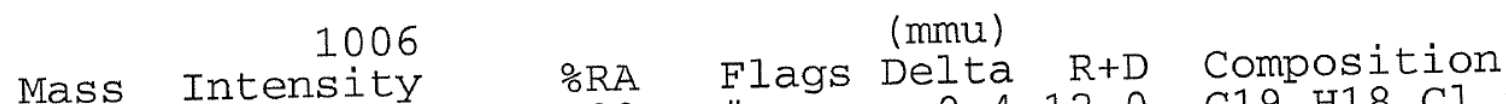

435.0660

$245547 \quad 100.00$

$\#$

-0.412 .0 C19.H18.C1.S.N3.05

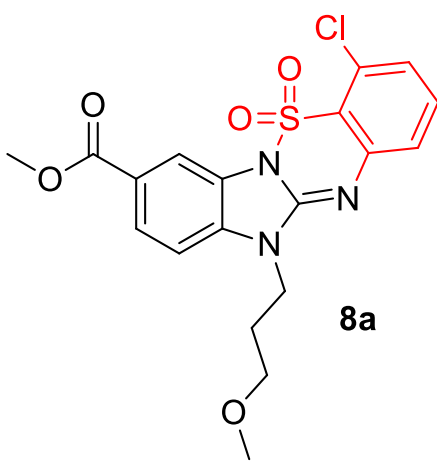

HRMS Mass (EI) spectrum of compound 8a 


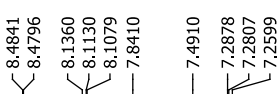

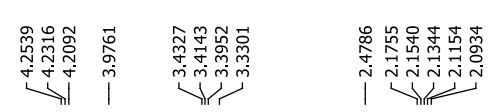

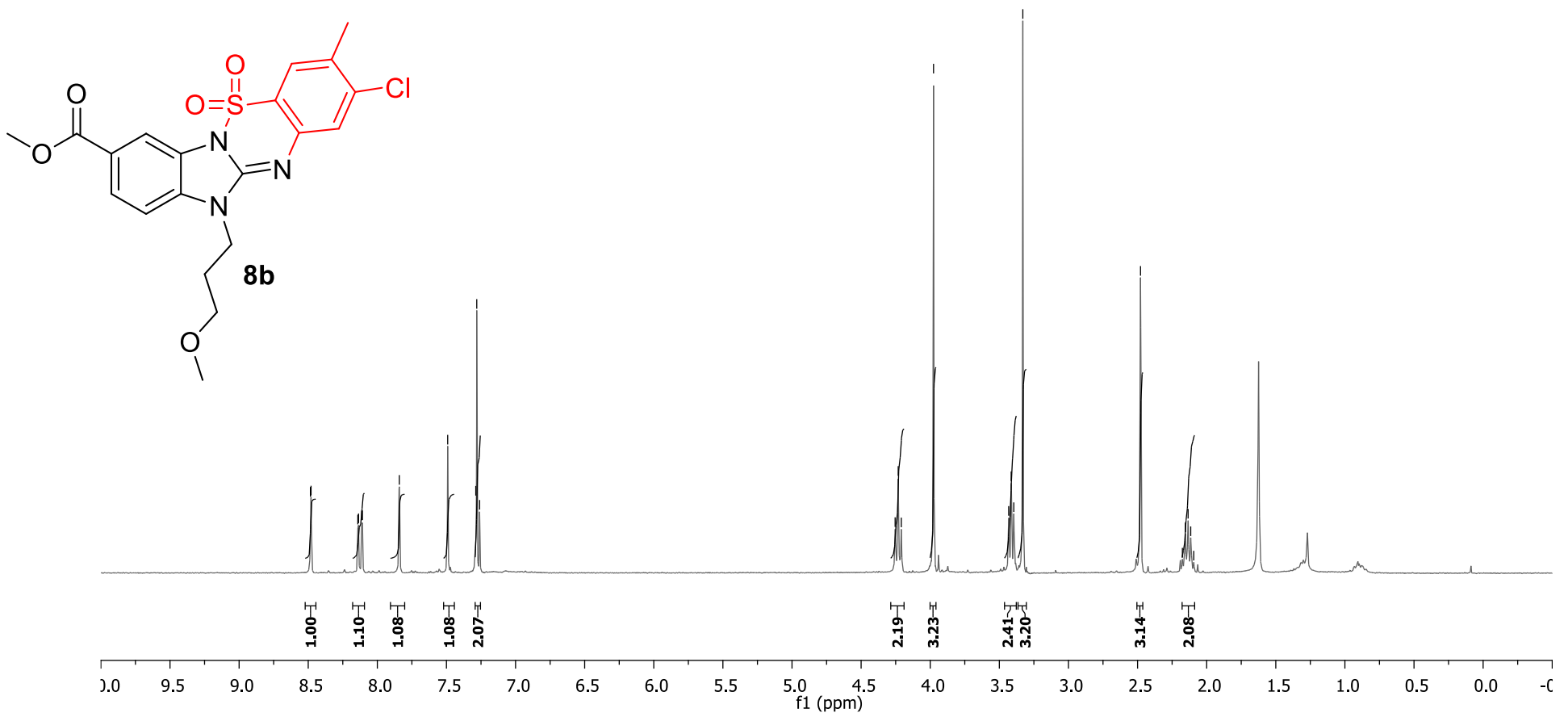

${ }^{1} \mathrm{H}$ NMR spectrum $\left(300 \mathrm{MHz}\right.$ ) of compound $\mathbf{8 b}$ in $\mathrm{CDCl}_{3}$ 


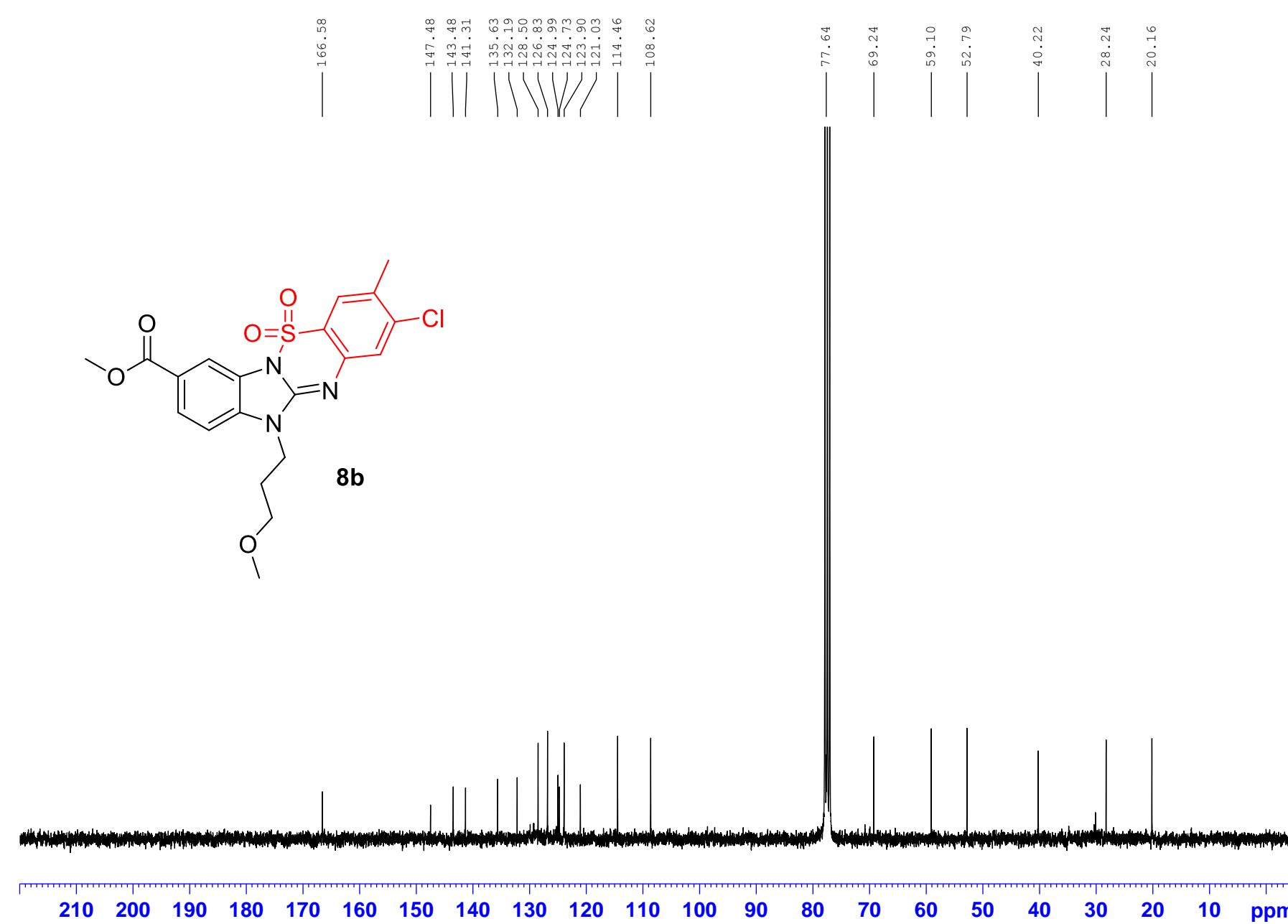

${ }^{13} \mathrm{C}$ NMR spectrum $\left(75 \mathrm{MHz}\right.$ ) of compound $\mathbf{8 b}$ in $\mathrm{CDCl}_{3}$ 


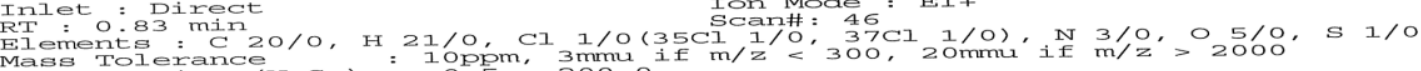

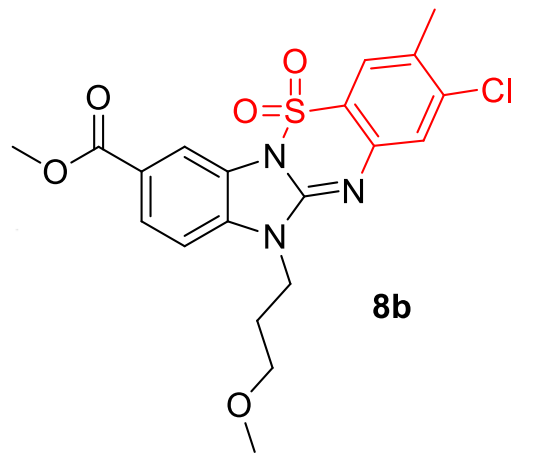

HRMS Mass (EI) spectrum of compound $\mathbf{8 b}$ 

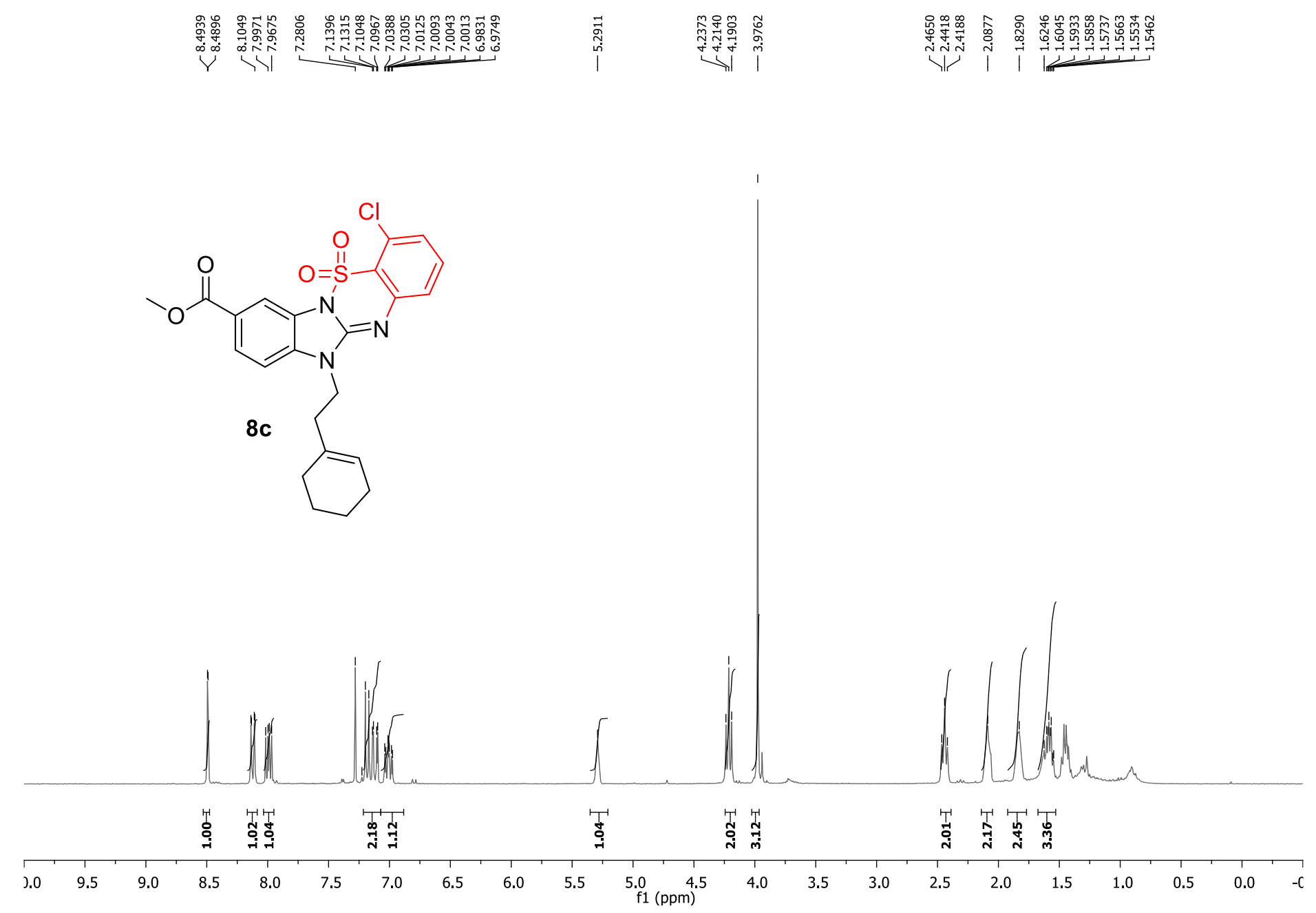

${ }^{1} \mathrm{H}$ NMR spectrum $\left(300 \mathrm{MHz}\right.$ ) of compound $\mathbf{8 c}$ in $\mathrm{CDCl}_{3}$ 


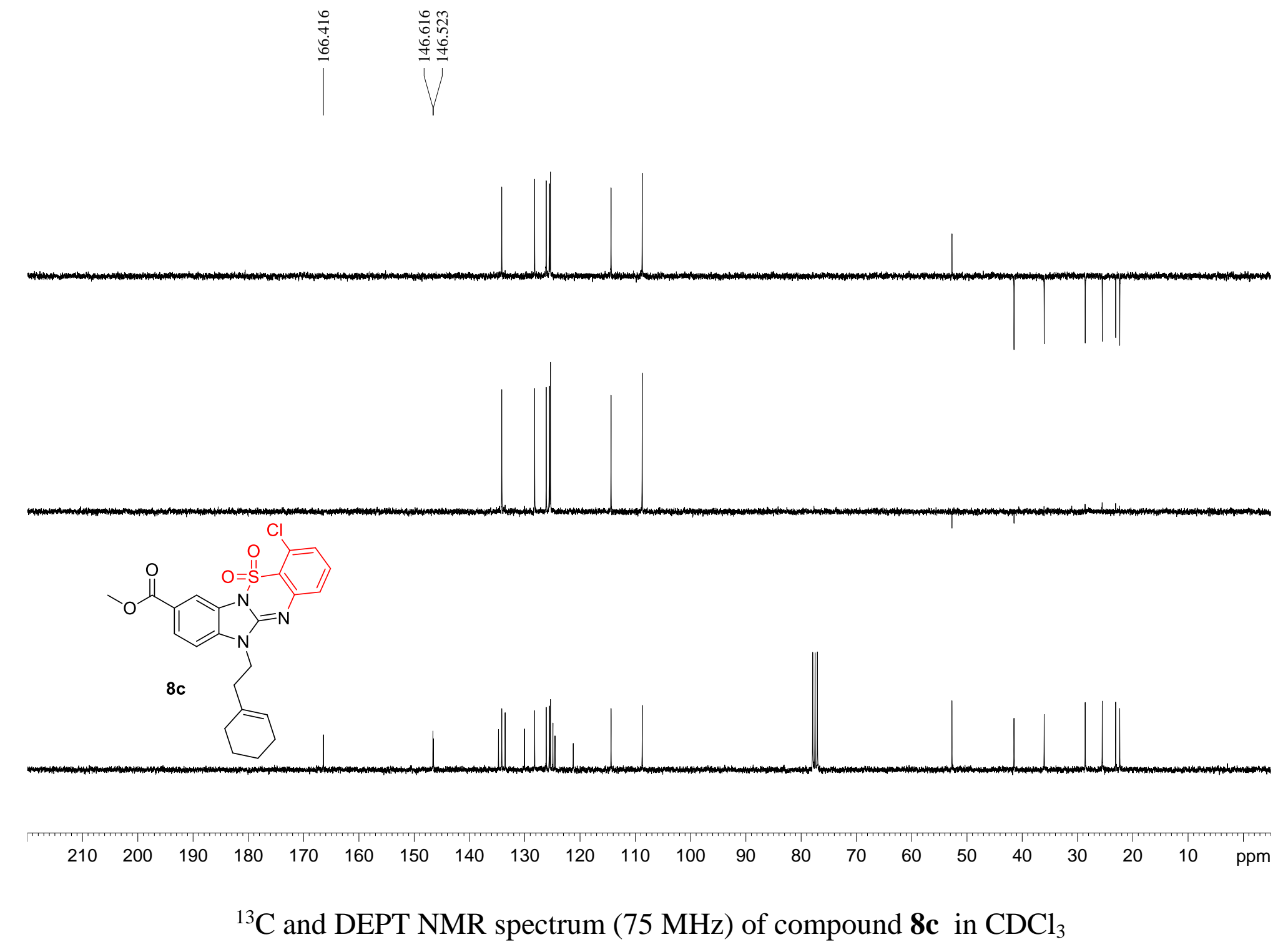


LIST: hei1471-c4

Samp: 1225 blb(S 3)

Mode: $\mathrm{EI}+\mathrm{VE}+\mathrm{LMR}$

Oper:

Limt :

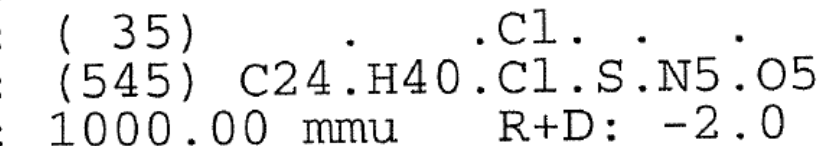

Peak: $1000.00 \mathrm{mmu} \mathrm{R}+\mathrm{D}:-2.0>60.0$

Data: +/487>1028 (CMASS : converted

$$
08-\operatorname{Mar}-12
$$

UP HR NRM

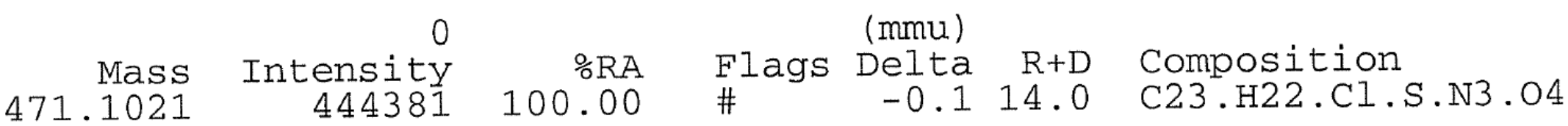

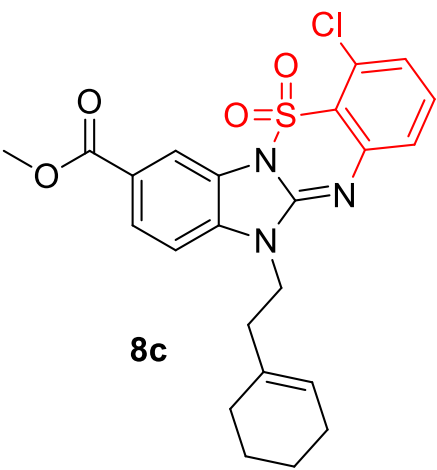

HRMS Mass (EI) spectrum of compound 8c 

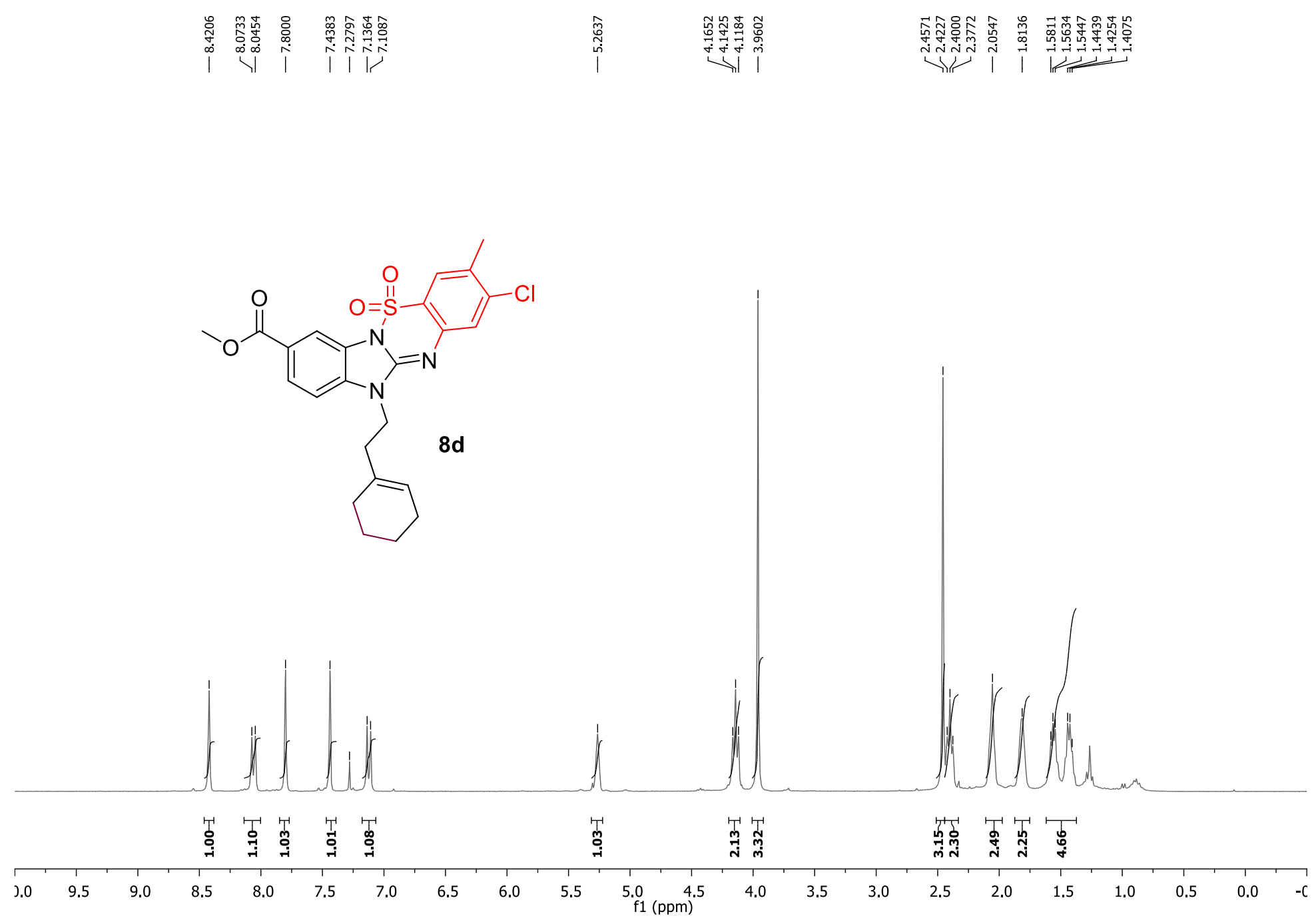

${ }^{1} \mathrm{H}$ NMR spectrum $\left(300 \mathrm{MHz}\right.$ ) of compound $\mathbf{8 d}$ in $\mathrm{CDCl}_{3}$ 

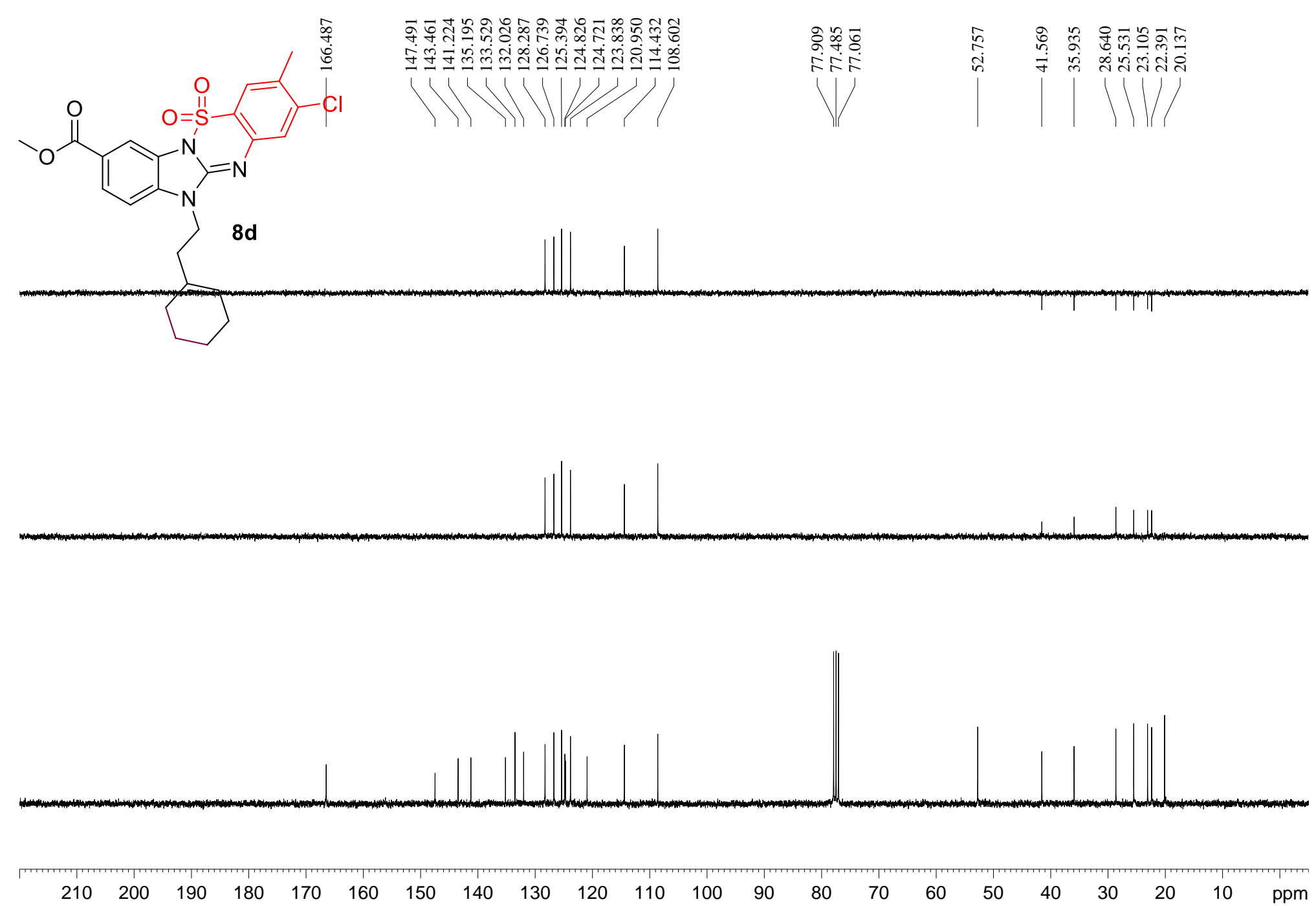

${ }^{13} \mathrm{C}$ and DEPT NMR spectrum (75 MHz) of compound $\mathbf{8 d}$ in $\mathrm{CDCl}_{3}$ 


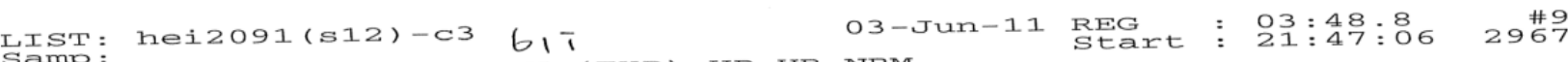
SOMP: EI +VE + IMR ESCAN ( $\mathrm{EXP}$ ) UP HR

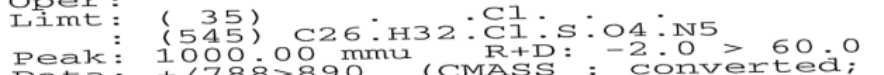
Mass Intensity
485.1169
100.00 Fly

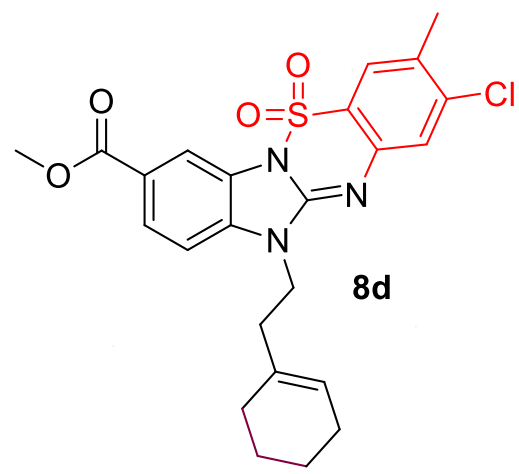

HRMS Mass (EI) spectrum of compound 8d 


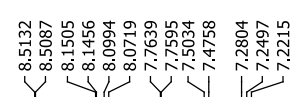

隹

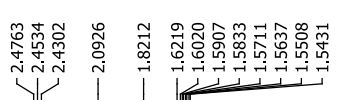

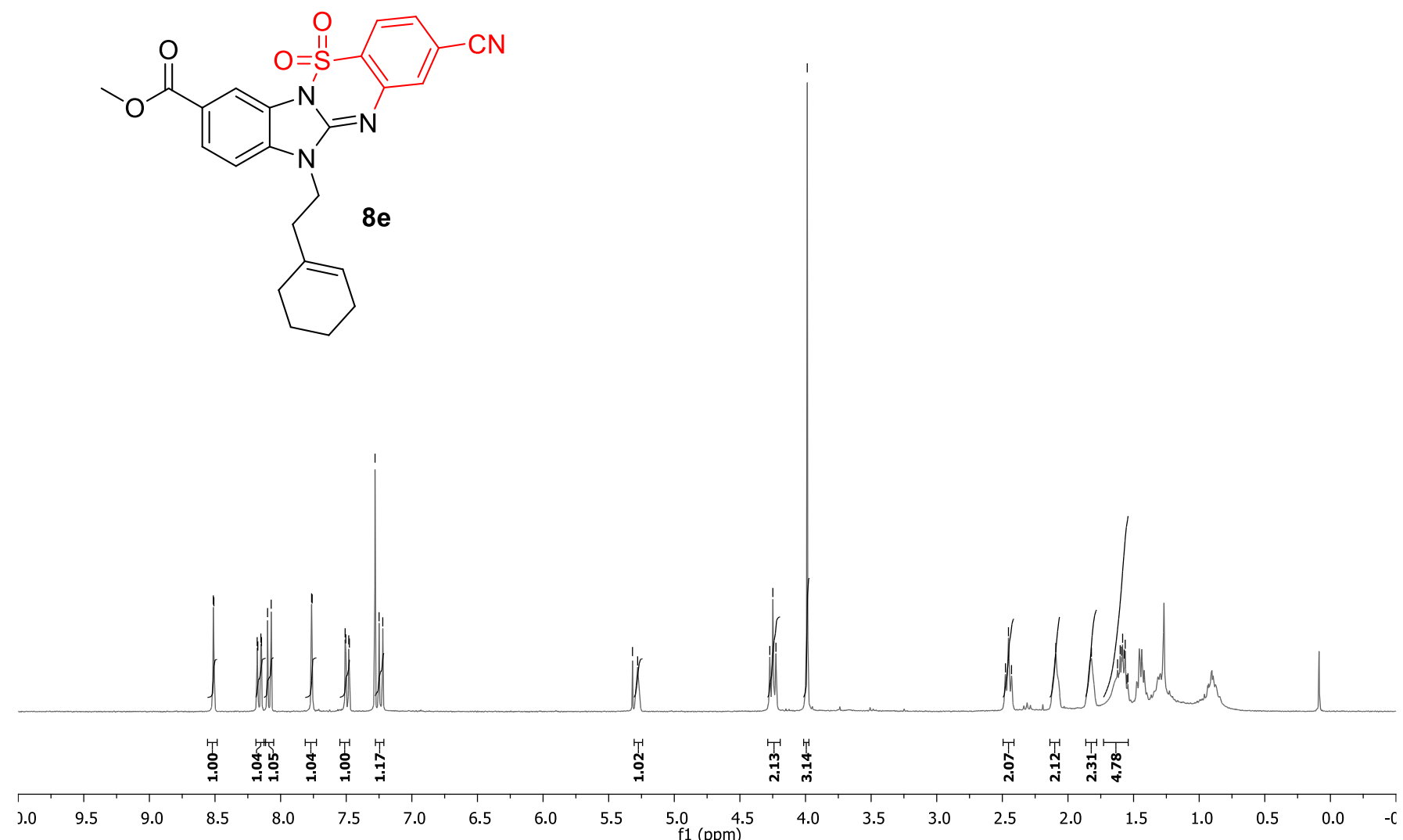

${ }^{1} \mathrm{H}$ NMR spectrum $\left(300 \mathrm{MHz}\right.$ ) of compound $\mathbf{8 e}$ in $\mathrm{CDCl}_{3}$ 

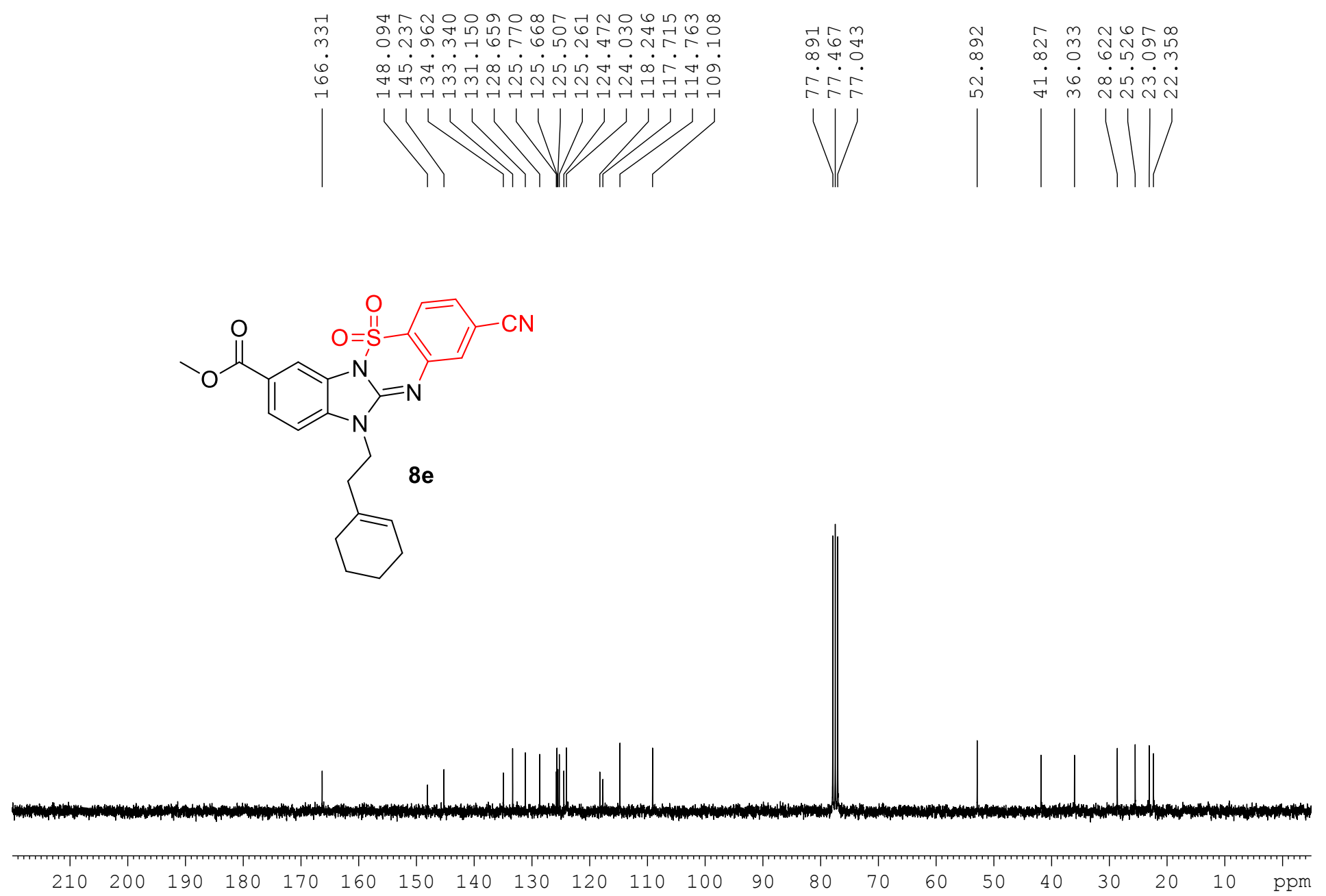

${ }^{13} \mathrm{C}$ NMR spectrum (75 MHz) of compound $8 \mathbf{e}$ in $\mathrm{CDCl}_{3}$ 


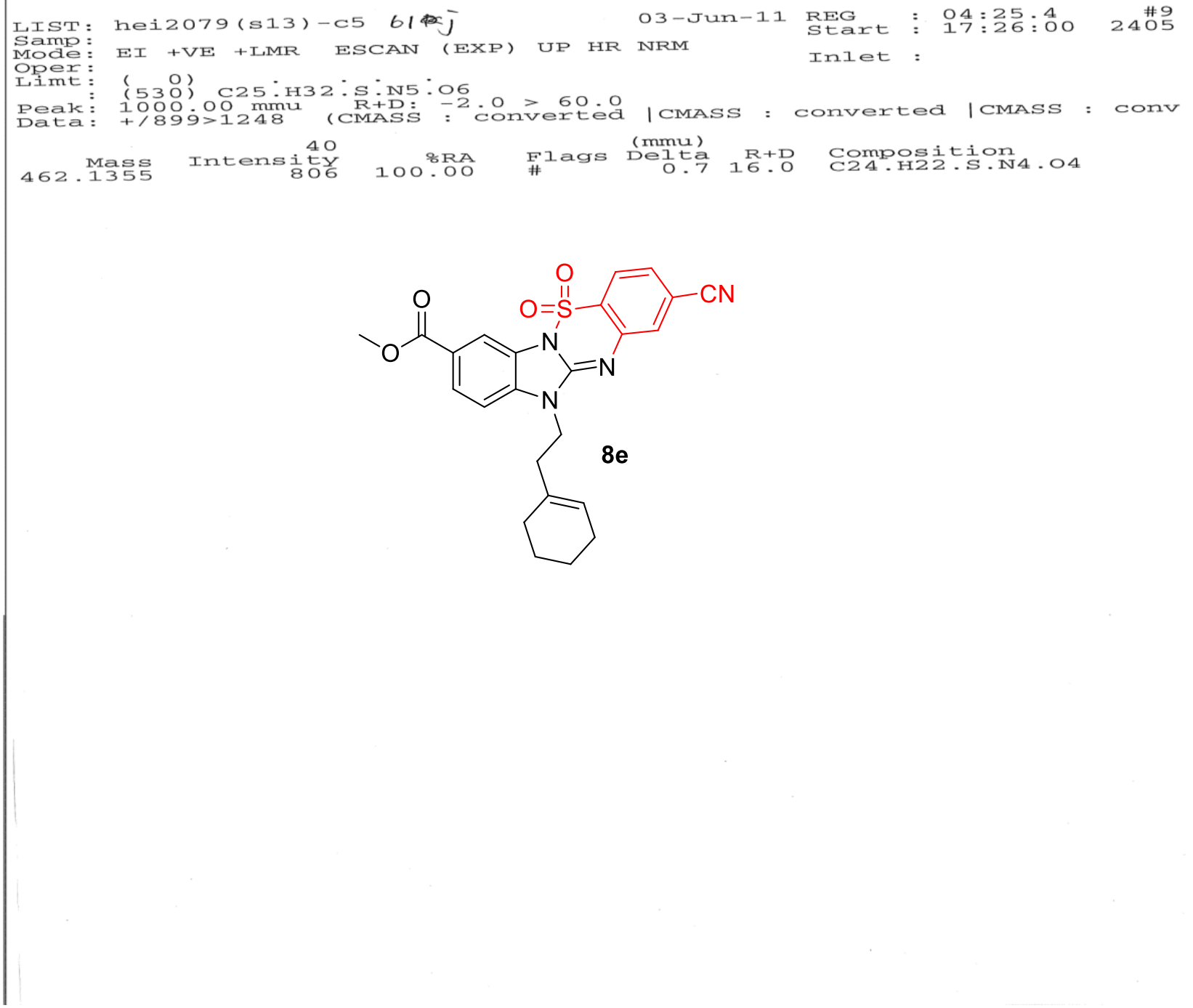

HRMS Mass (EI) spectrum of compound $\mathbf{8 e}$ 

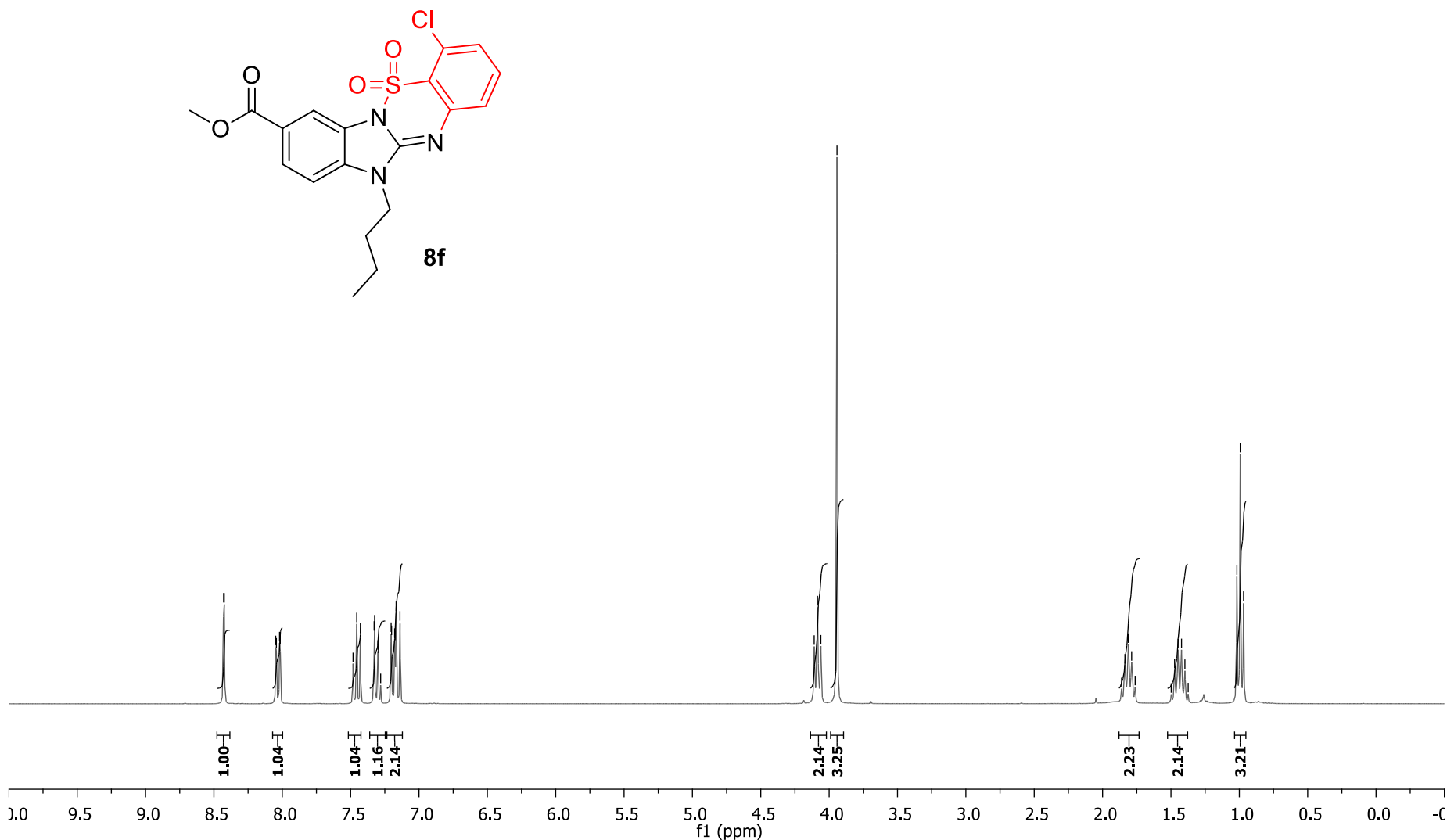

${ }^{1} \mathrm{H}$ NMR spectrum (300 MHz) of compound $\mathbf{8 f}$ in $\mathrm{CDCl}_{3}$ 


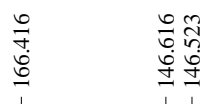
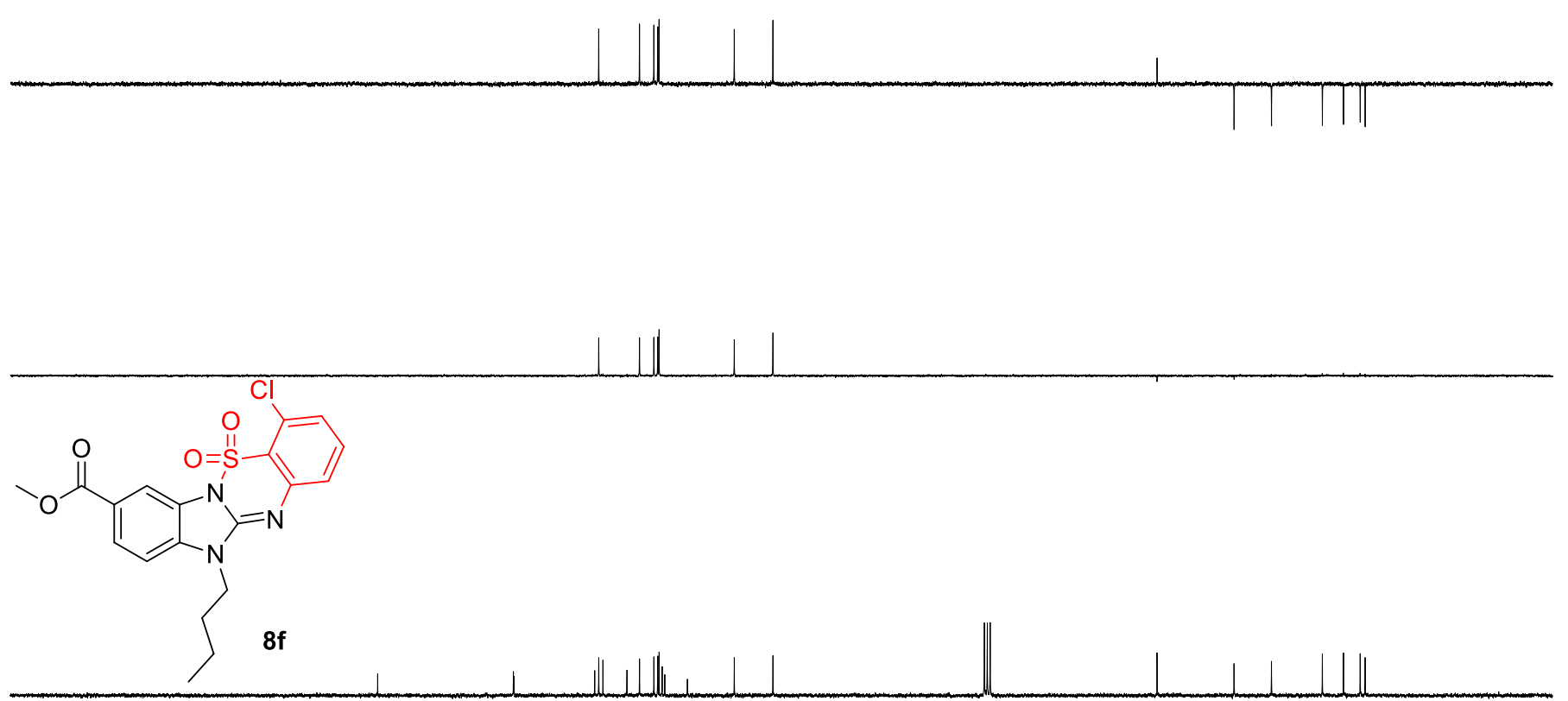

$\begin{array}{llllllllllllllllllllll}210 & 200 & 190 & 180 & 170 & 160 & 150 & 140 & 130 & 120 & 110 & 100 & 90 & 80 & 70 & 60 & 50 & 40 & 30 & 20 & 10 & \mathrm{ppm}\end{array}$

${ }^{13} \mathrm{C}$ and DEPT NMR spectrum (75 MHz) of compound $\mathbf{8 f}$ in $\mathrm{CDCl}_{3}$ 
LIST: hei1473-c3

Samp: 1226 blc (54)

Mode: EI +VE + LMR ESCAN (EXP) UP HR NRM

Oper:

Limt : ( 35$)$ : (497) C20.H40.C1.S.N5:05

Peak: $1000.00 \mathrm{mmu} \mathrm{R}+\mathrm{D}:-2.0>60.0$

Data: $+/ 264>645$ (CMASS : converted |CMASS : converted |CMASS : conve

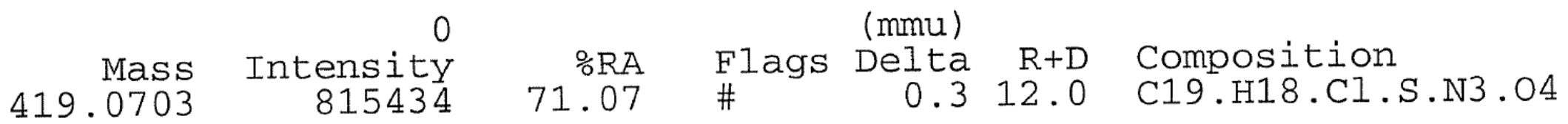

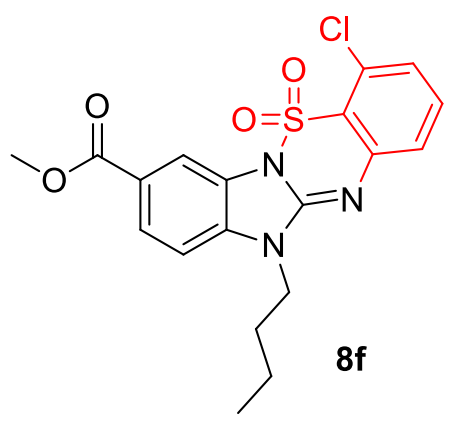

HRMS Mass (EI) spectrum of compound $\mathbf{8 f}$ 

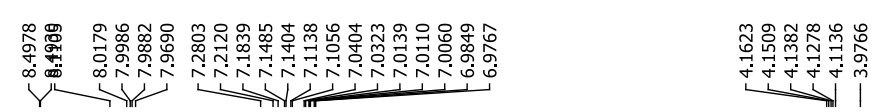

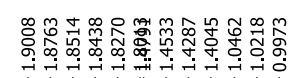
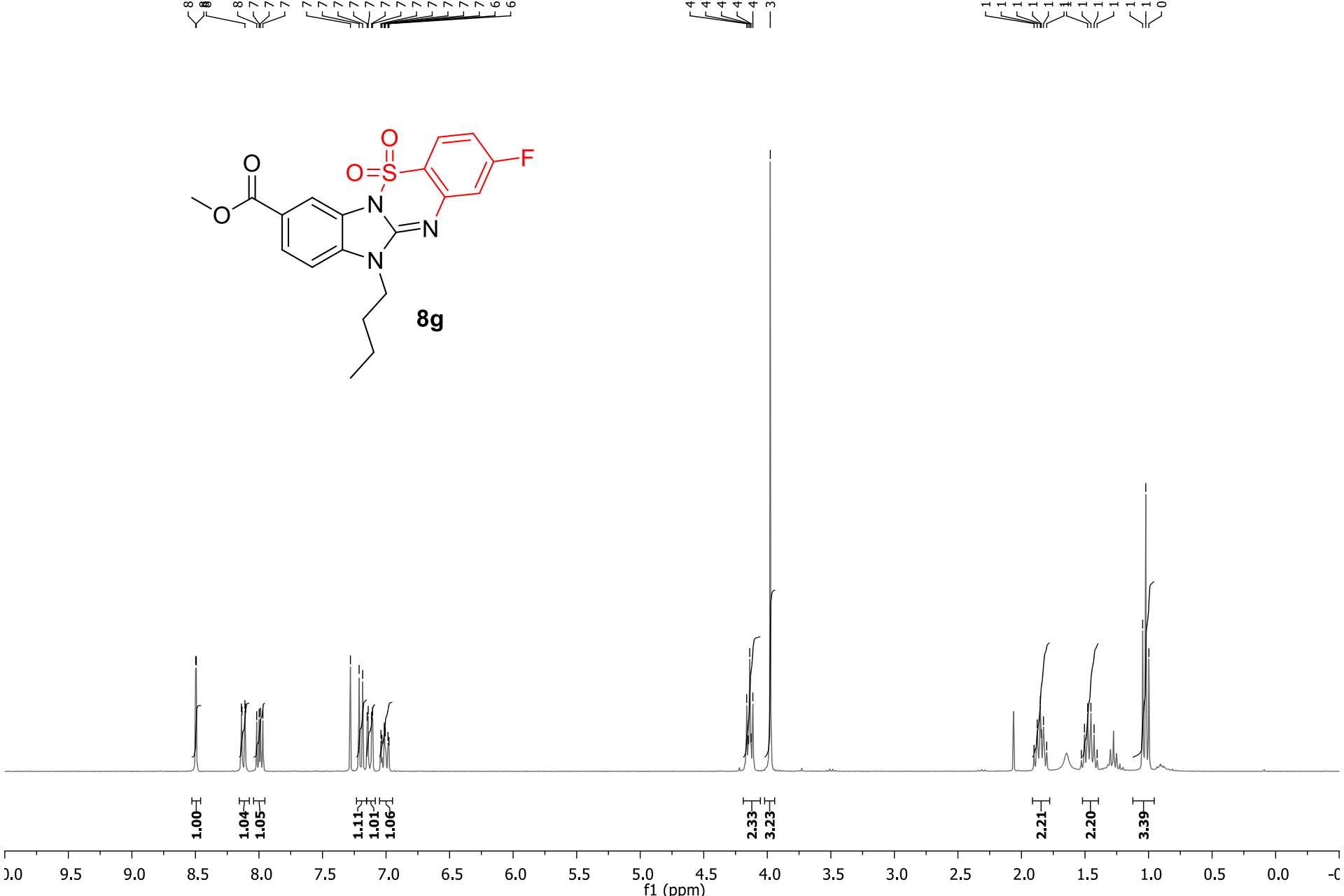

${ }^{1} \mathrm{H}$ NMR spectrum $\left(300 \mathrm{MHz}\right.$ ) of compound $\mathbf{8 g}$ in $\mathrm{CDCl}_{3}$ 

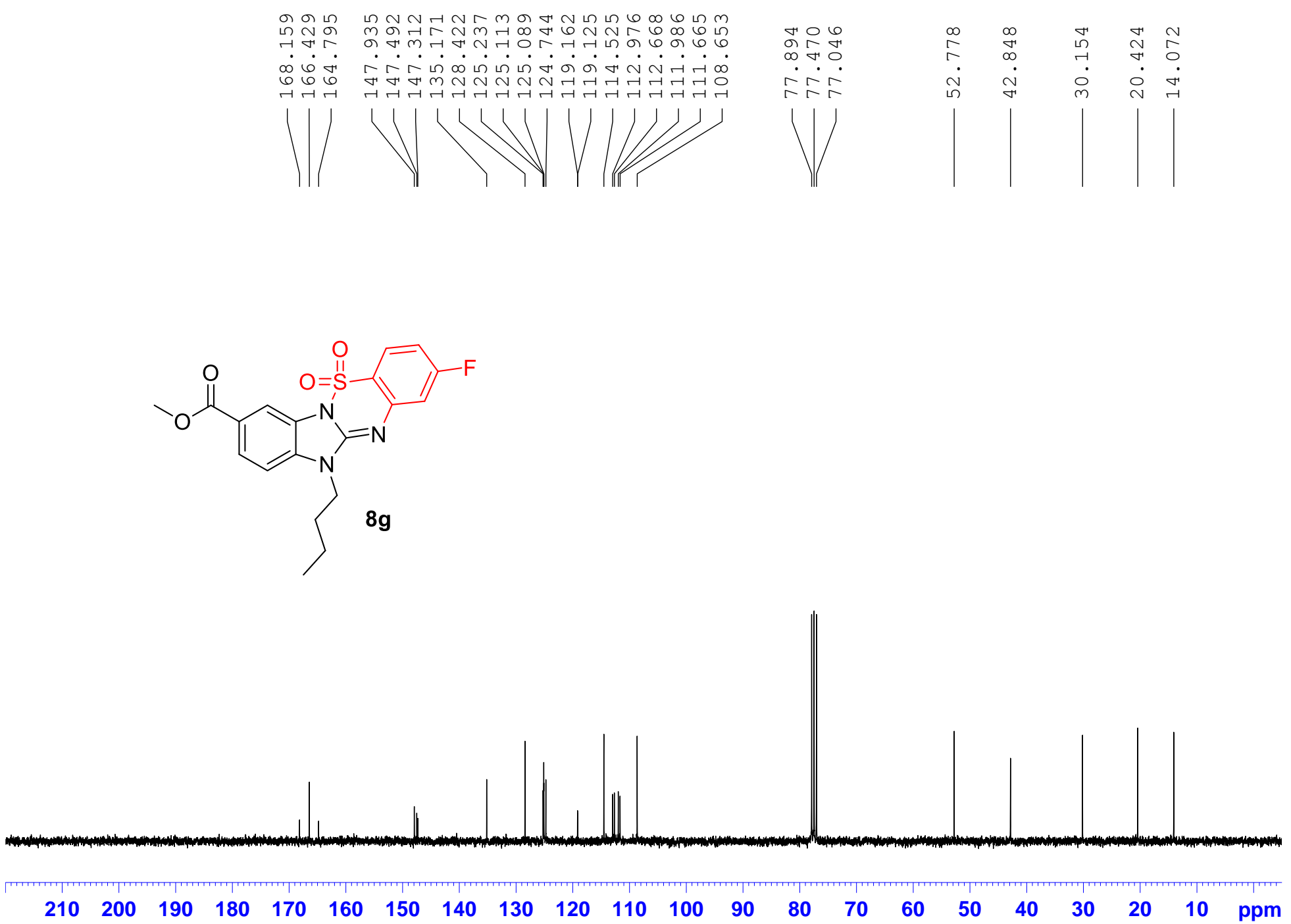

${ }^{13} \mathrm{C}$ NMR spectrum $\left(75 \mathrm{MHz}\right.$ ) of compound $\mathbf{8 g}$ in $\mathrm{CDCl}_{3}$ 
LIST: hei2062(s7)-c5 $61 \mathrm{f}$

$$
\text { 03-Jun-11 REG: } 03: 47.4
$$

Samp :

Mode: $\mathrm{EI}+\mathrm{VE}+\mathrm{LMR}$ ESCAN (EXP) UP HR NRM

Start : 09:08:36 1633

Oper:

Limt: $(0)$

: (492) C20.H51.F.S.N5.O5

Peak: $1000.00 \mathrm{mmu} \mathrm{R}+\mathrm{D}:-2.0>60.0$

Data: +/735>844 (CMASS: converted |CMASS : converted |CMASS : conve
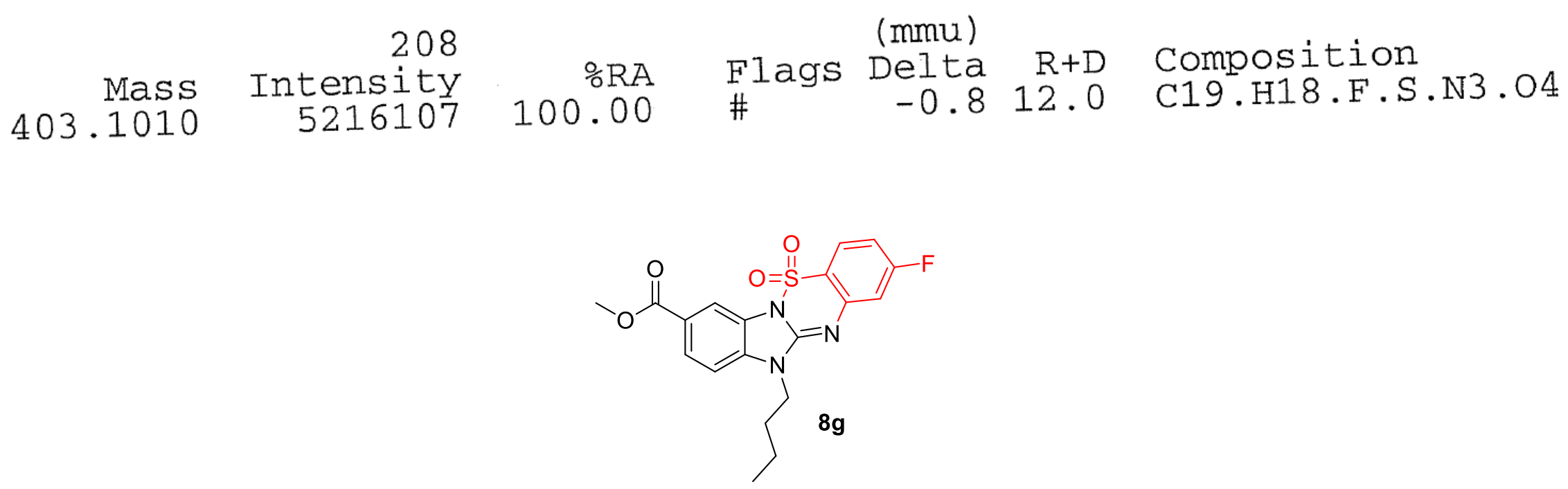

HRMS Mass (EI) spectrum of compound $\mathbf{8 g}$ 


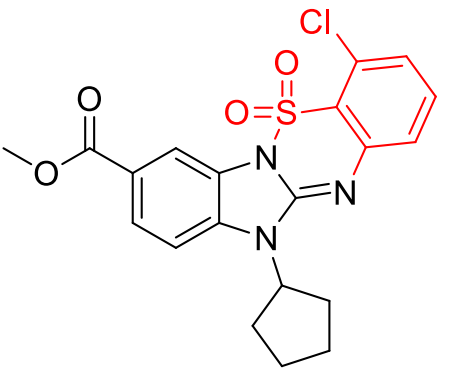

$8 h$

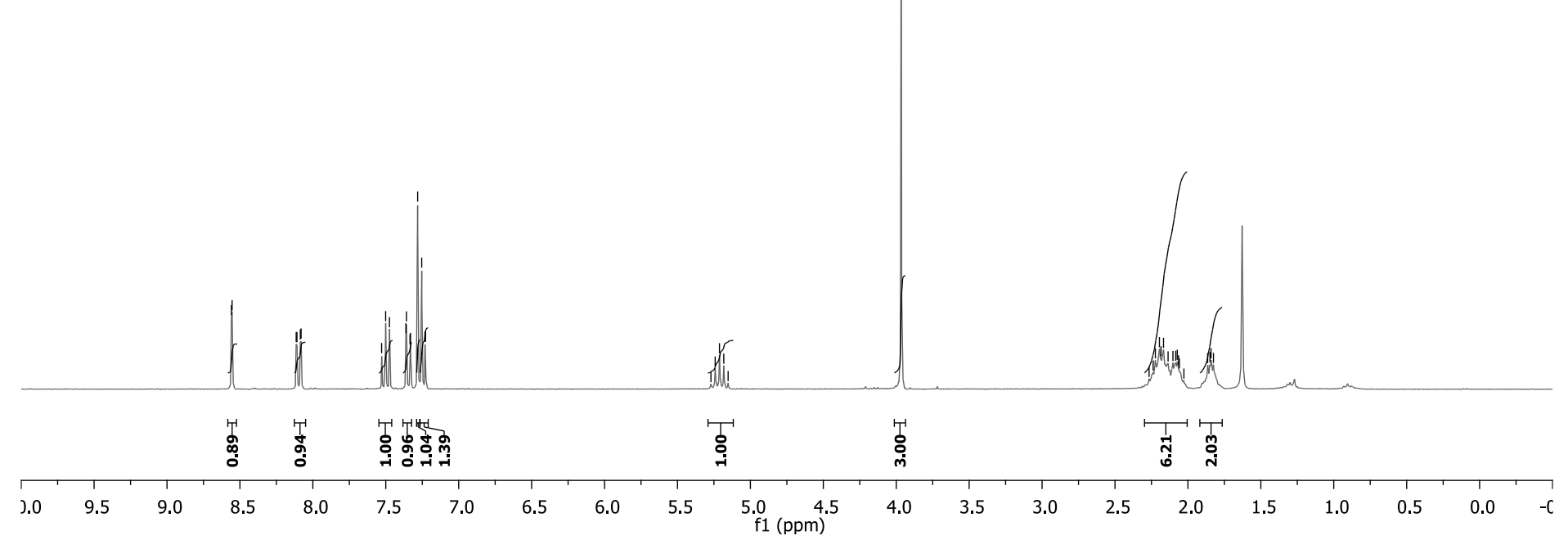

${ }^{1} \mathrm{H}$ NMR spectrum $\left(300 \mathrm{MHz}\right.$ ) of compound $\mathbf{8 h}$ in $\mathrm{CDCl}_{3}$ 

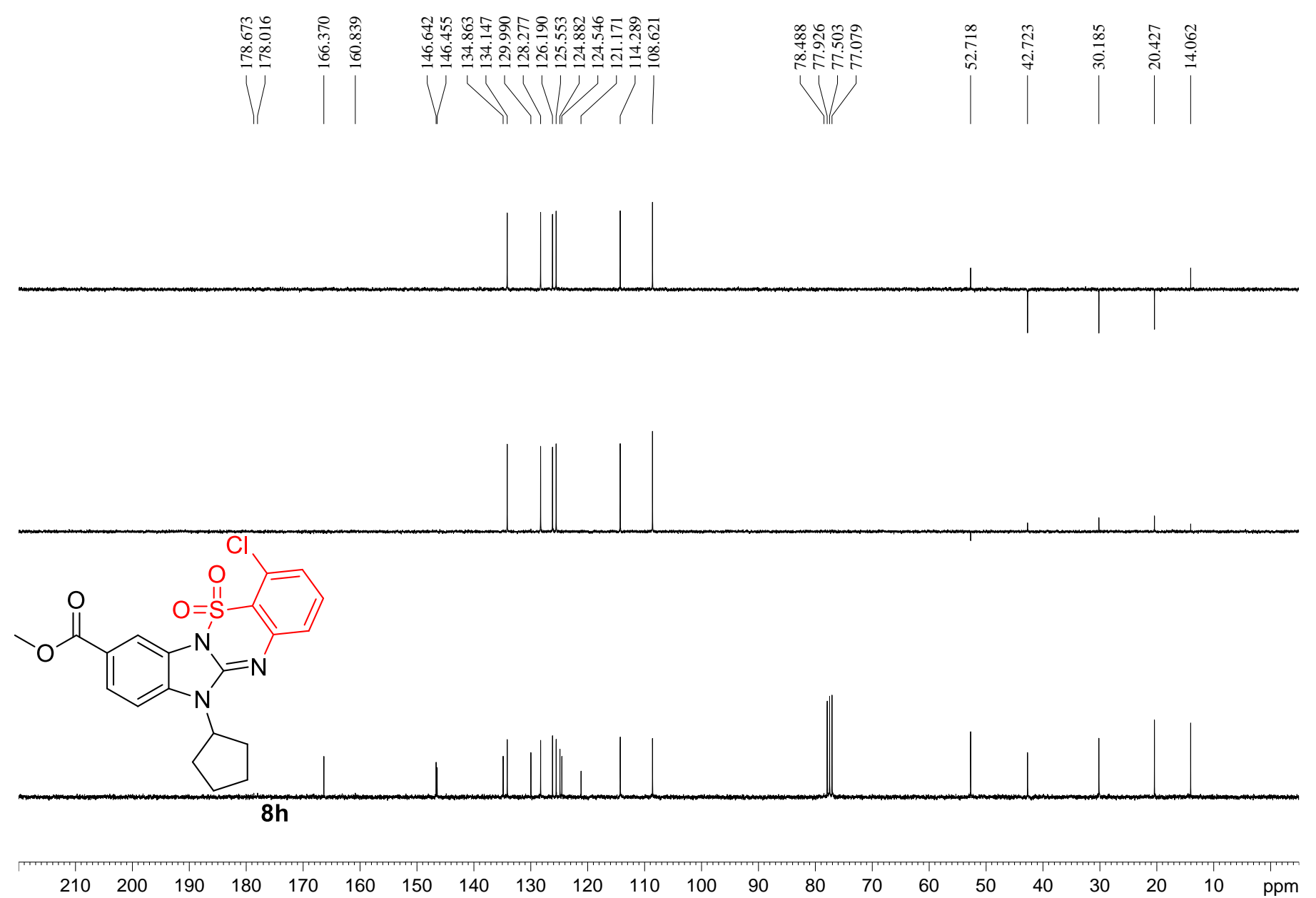

${ }^{13} \mathrm{C}$ and DEPT NMR spectrum (75 MHz) of compound $\mathbf{8 h}$ in $\mathrm{CDCl}_{3}$ 
[ Elemental Composition ]

Data : 1000512-002

Sample: S5 bld

Note : 431.07

Inlet : Direct

RT : $0.95 \mathrm{~min}$

Date : 12-May-2011 10:01

Page: 1

Elements : C 20/0, H 19/0, Cl 1/0(35Cl 1/0,37Cl 1/0), N 3/0, O 4/0, S 1/0

Mass Tolerance

Unsaturation (U.S.) : $-0.5-200.0$

Observed $\mathrm{m} / \mathrm{z}$ Int\% Err [ppm / mmu] U.S. Composition 433.0679

11.2

$+0.5 /+0.2$

14.0

C $20 \mathrm{H} 1837 \mathrm{Cl} N 3 \circ 4 \mathrm{~S}$

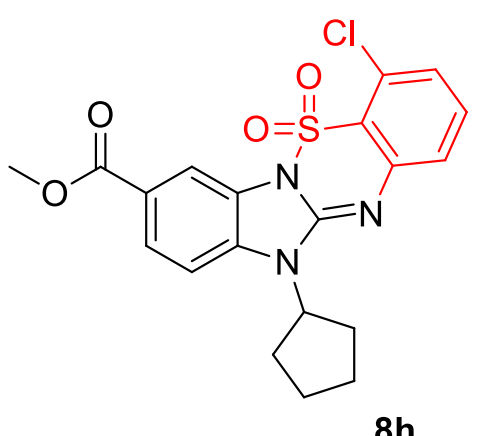

HRMS Mass (EI) spectrum of compound $\mathbf{8 h}$ 


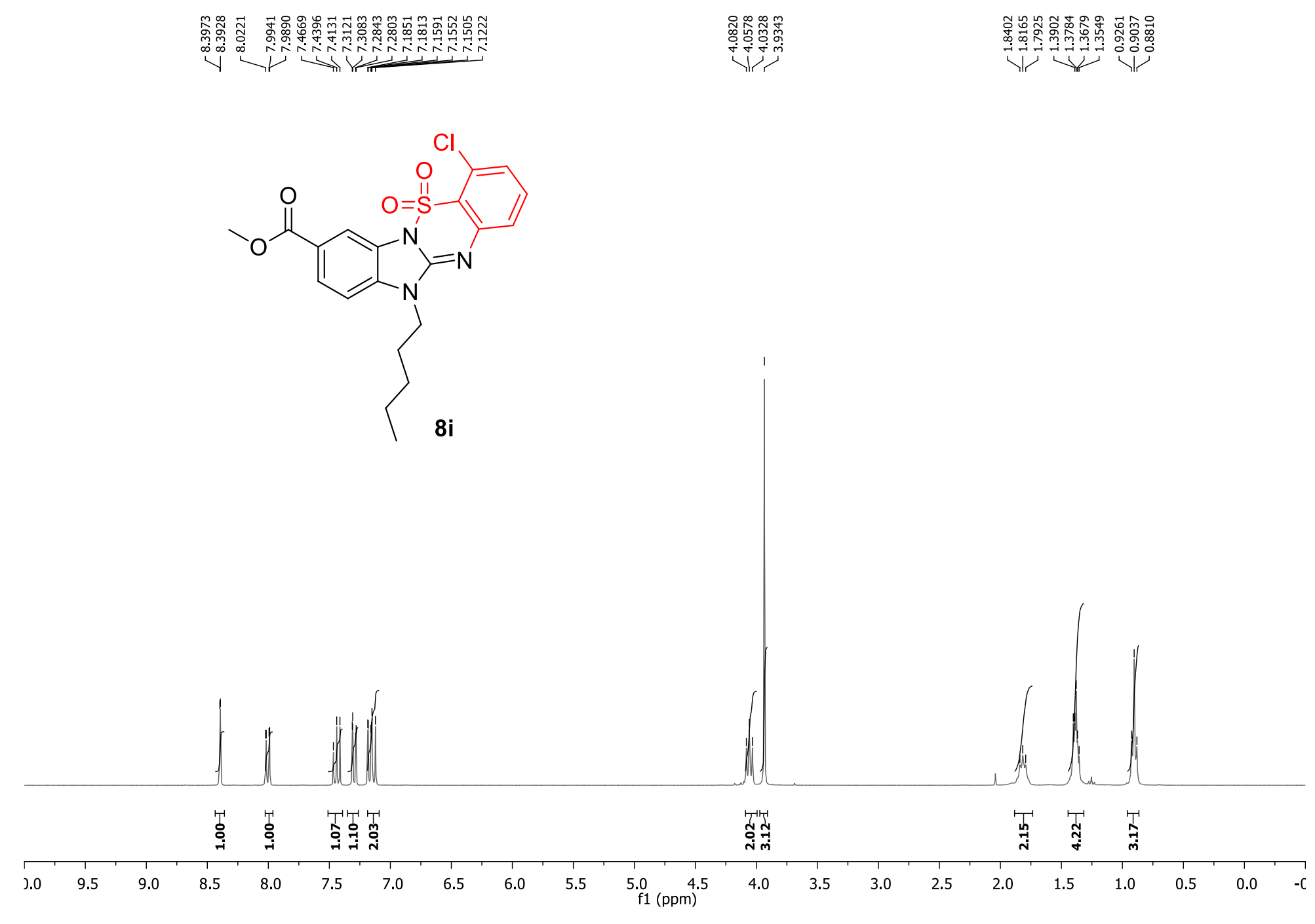

${ }^{1} \mathrm{H}$ NMR spectrum (300 MHz) of compound $\mathbf{8 i}$ in $\mathrm{CDCl}_{3}$ 

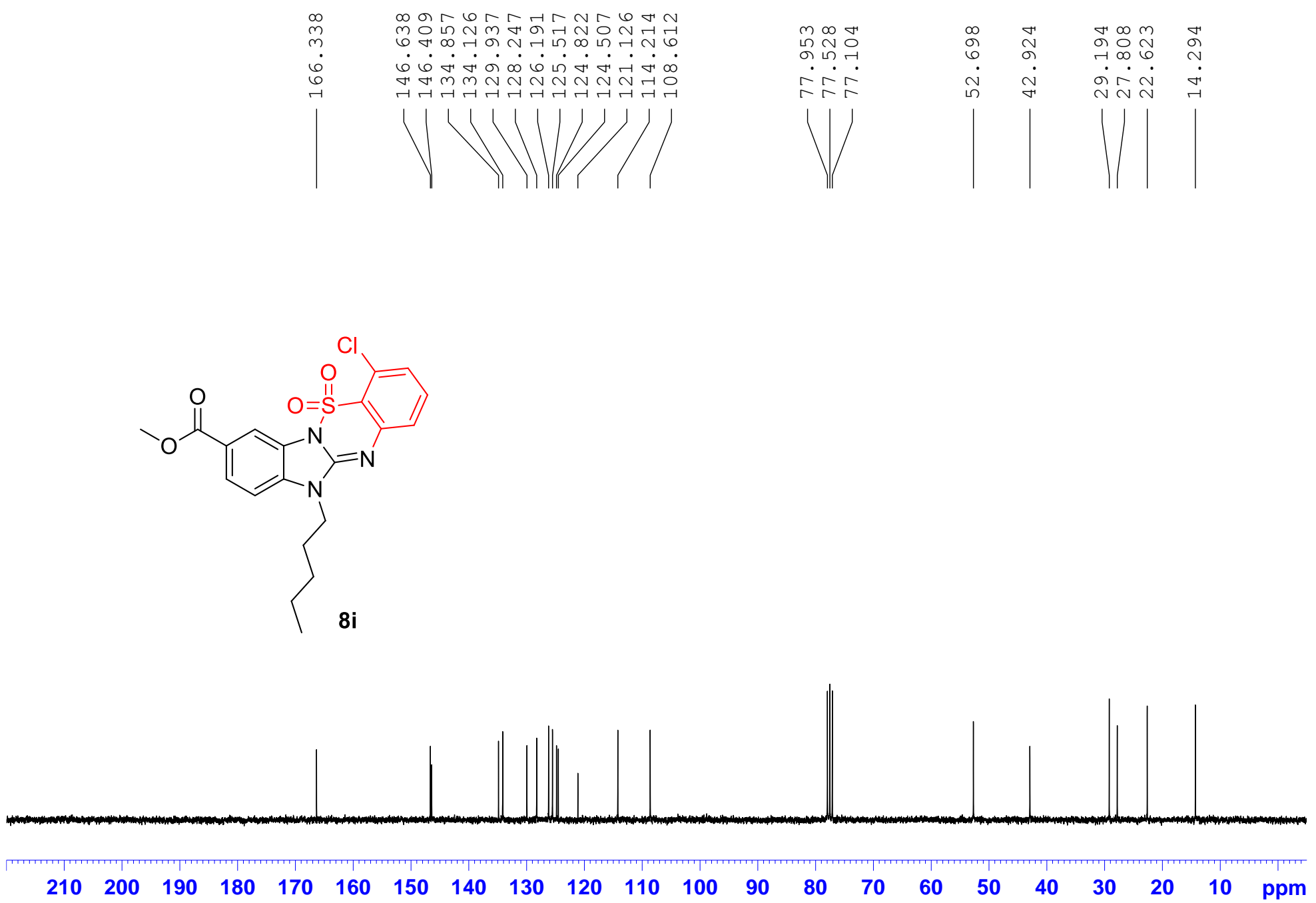

${ }^{13} \mathrm{C}$ NMR spectrum (75 MHz) of compound $8 \mathbf{i}$ in $\mathrm{CDCl}_{3}$ 
LIST: hei1474-C1

Samp: 1227 6le (56)

Mode: $\mathrm{EI}+\mathrm{VE}+\mathrm{LMR}$ ESCAN (EXP) UP HR NRM

Oper:

$\begin{array}{lllr}08-M a r-12 & \text { REG } & : 03: 53.9 & \# 9 \\ \text { Start }: & 16: 43: 57 & 1278\end{array}$

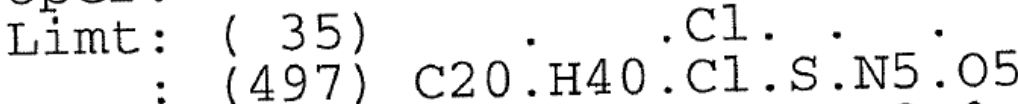

Peak: $1000.00 \mathrm{mmu} \mathrm{R}+\mathrm{D}:-2.0>60.0$

Data: + /790>1041 (CMASS : converted |CMASS : converted /CMASS : conv

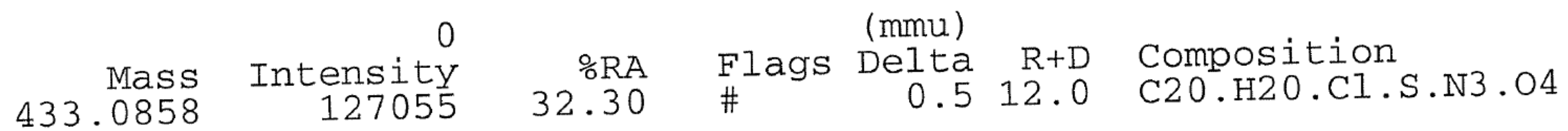

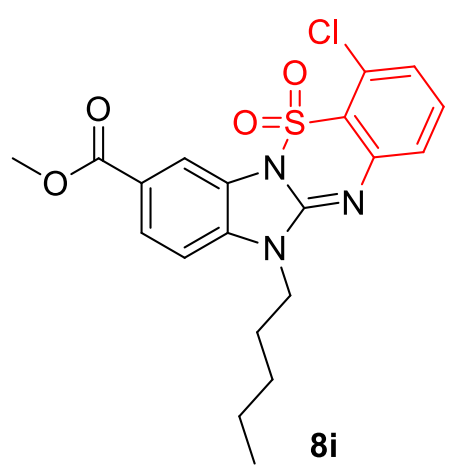

HRMS Mass (ESI) spectrum of compound $\mathbf{8 i}$ 

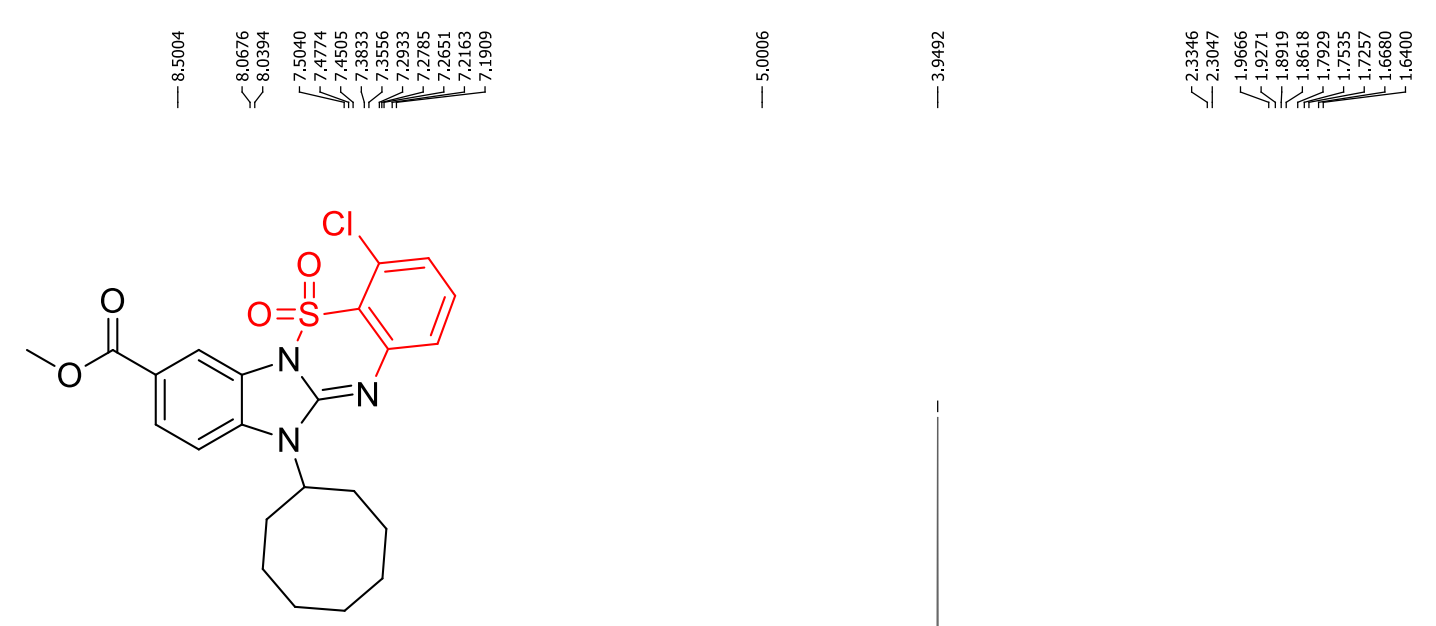

8j

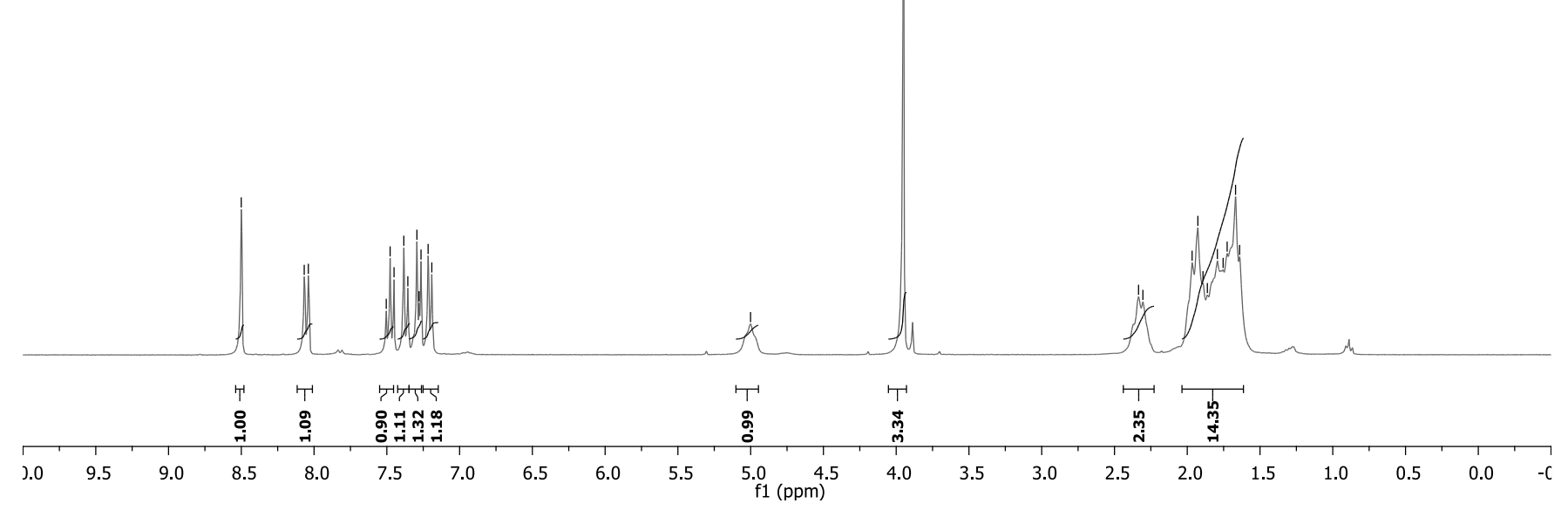

${ }^{1} \mathrm{H}$ NMR spectrum $\left(300 \mathrm{MHz}\right.$ ) of compound $\mathbf{8 j}$ in $\mathrm{CDCl}_{3}$ 


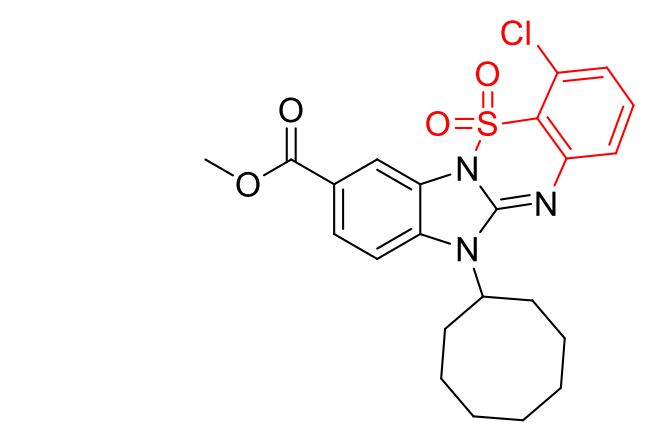

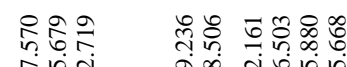

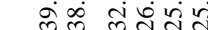

8j
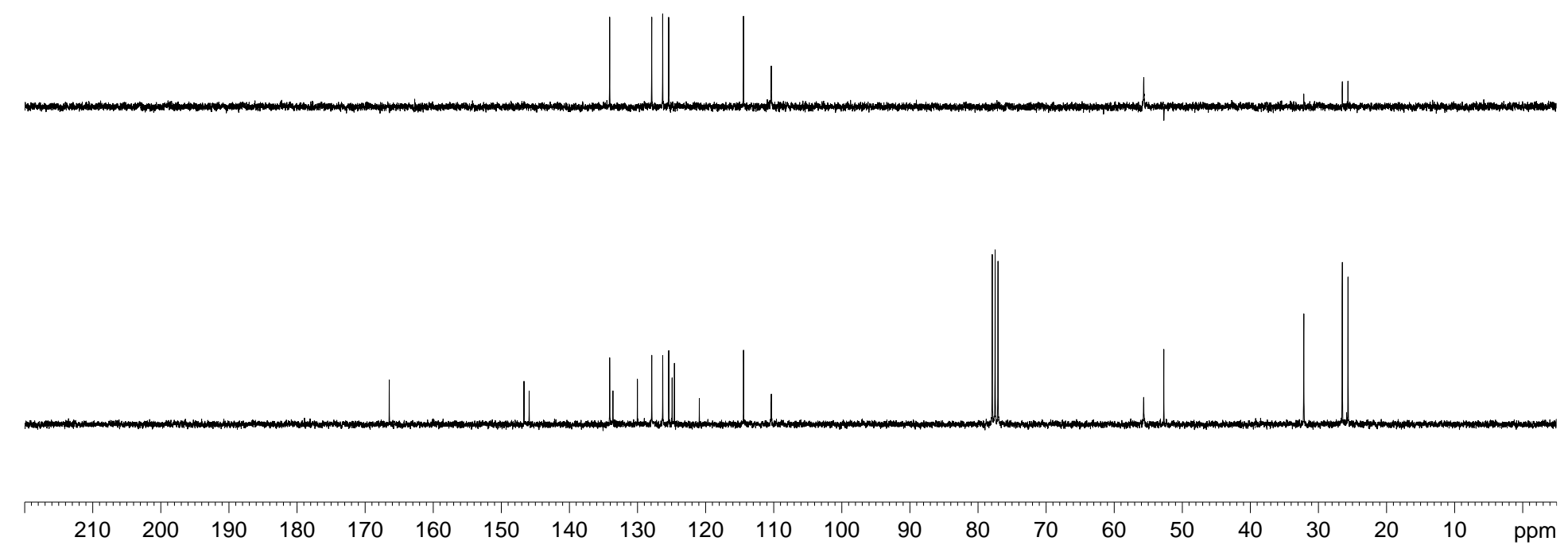

${ }^{13} \mathrm{C}$ NMR spectrum (75 MHz) of compound $\mathbf{8 j}$ in $\mathrm{CDCl}_{3}$ 


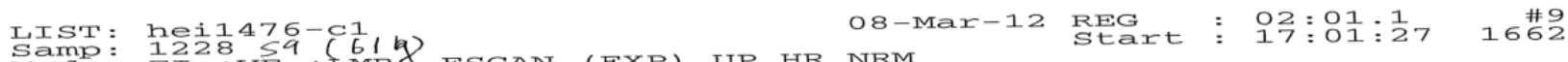
MOC: $12^{2}+V{ }^{4}+$ LMR 8 ESCAN (EXP) UP HR NRM Inlet :

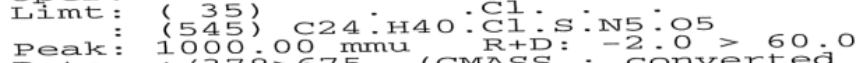

Paak 1 1000

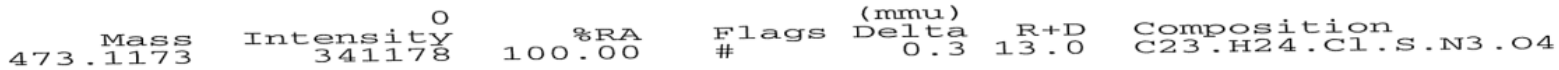

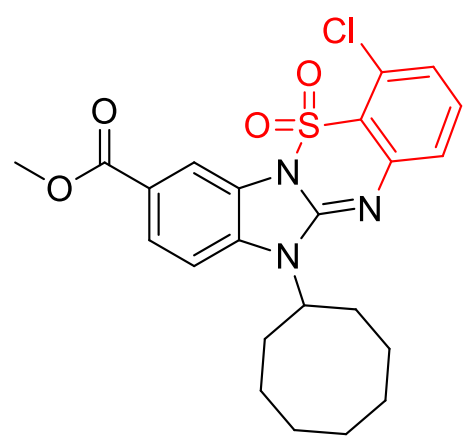

8j

HRMS Mass (ESI) spectrum of compound $\mathbf{8 j}$ 


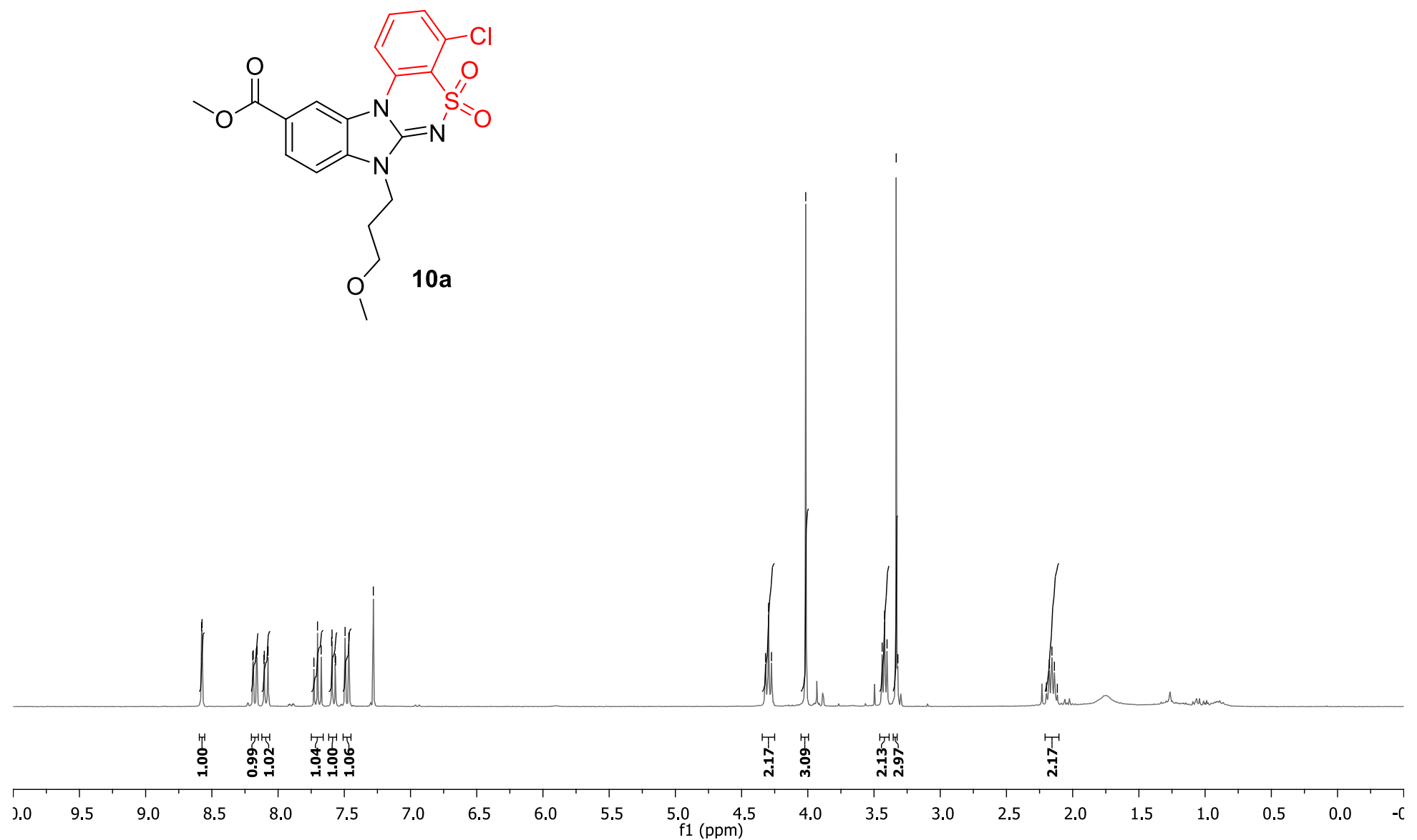

${ }^{1} \mathrm{H}$ NMR spectrum $\left(300 \mathrm{MHz}\right.$ ) of compound 10a in $\mathrm{CDCl}_{3}$ 

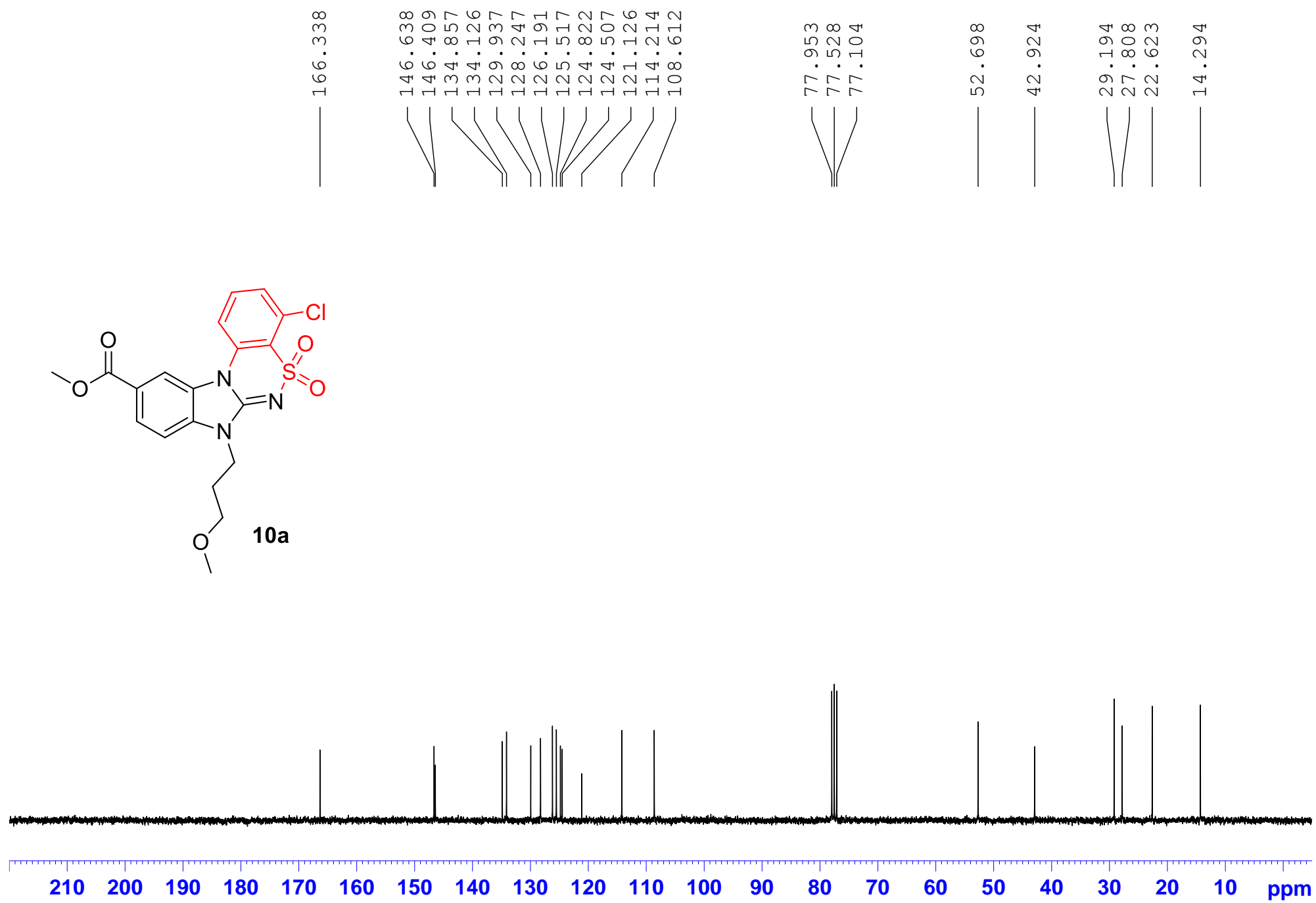

${ }^{13} \mathrm{C}$ NMR spectrum (75 MHz) of compound $\mathbf{1 0 a}$ in $\mathrm{CDCl}_{3}$ 


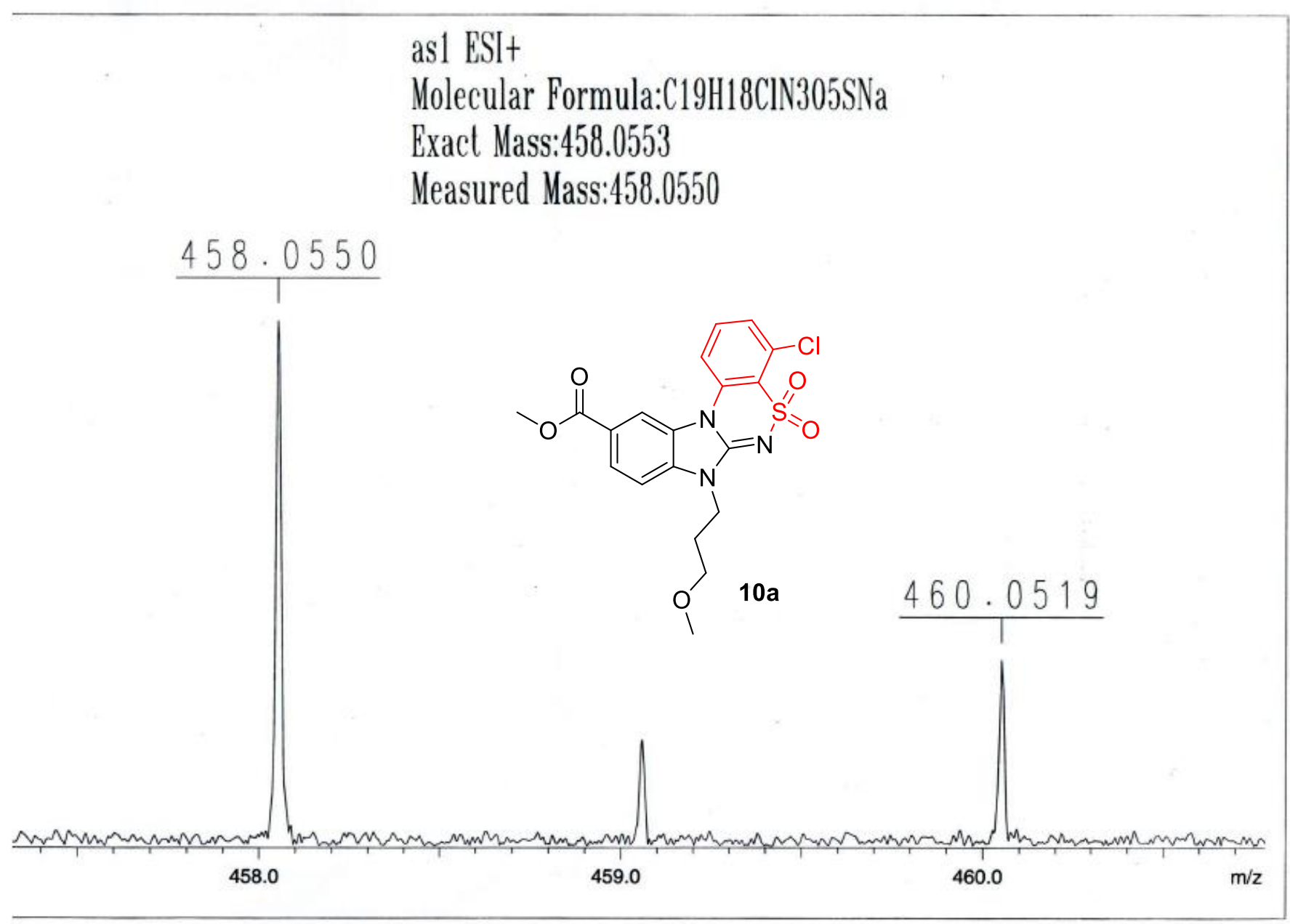

/d=/Data/yu/AS1/2/pdata/1 Administrator Fri Sep 30 15:40:27 2011

HRMS mass (ESI) spectrum of compound 10a 


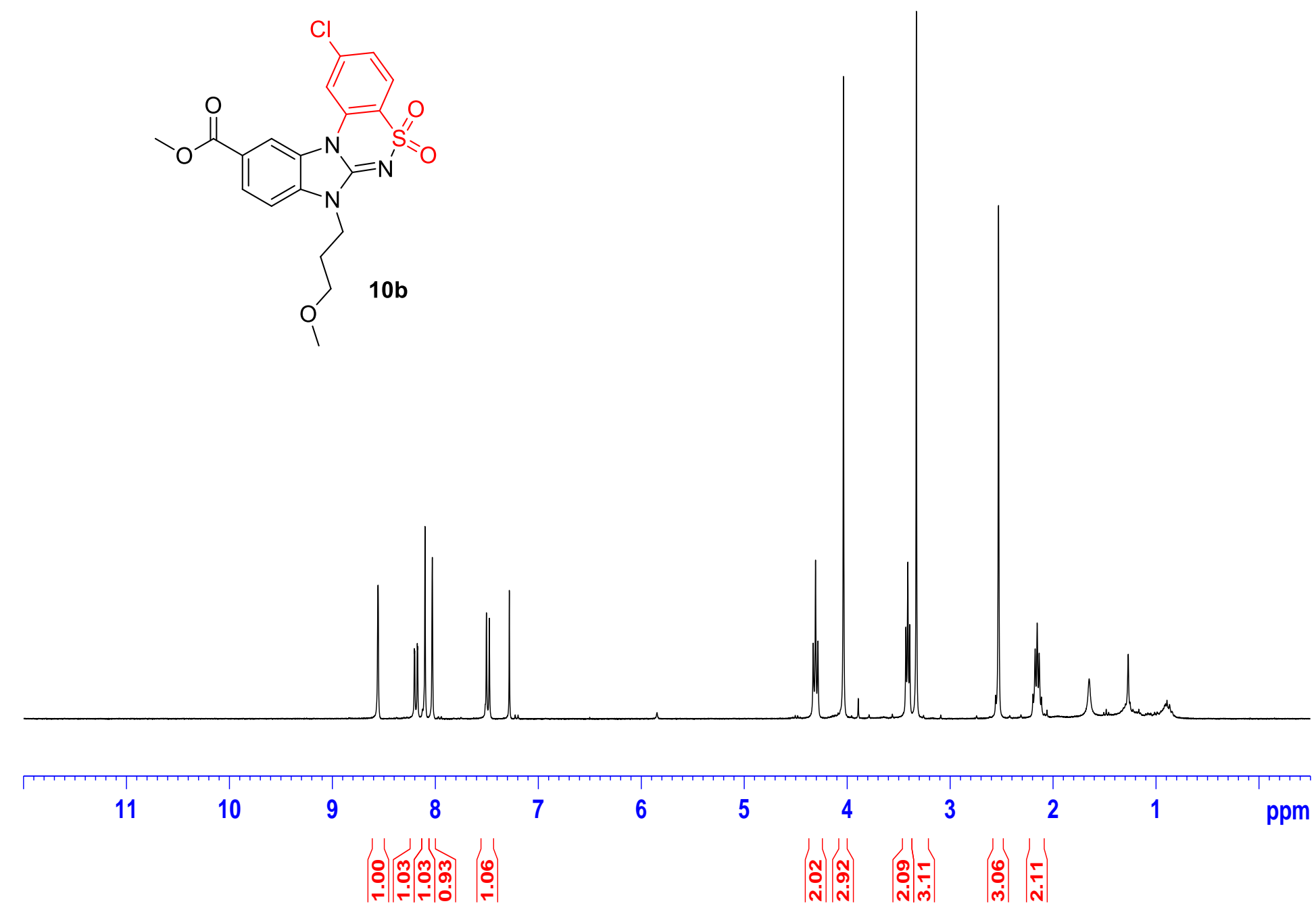

${ }^{1} \mathrm{H}$ NMR spectrum (300 MHz) of compound $\mathbf{1 0 b}$ in $\mathrm{CDCl}_{3}$ 

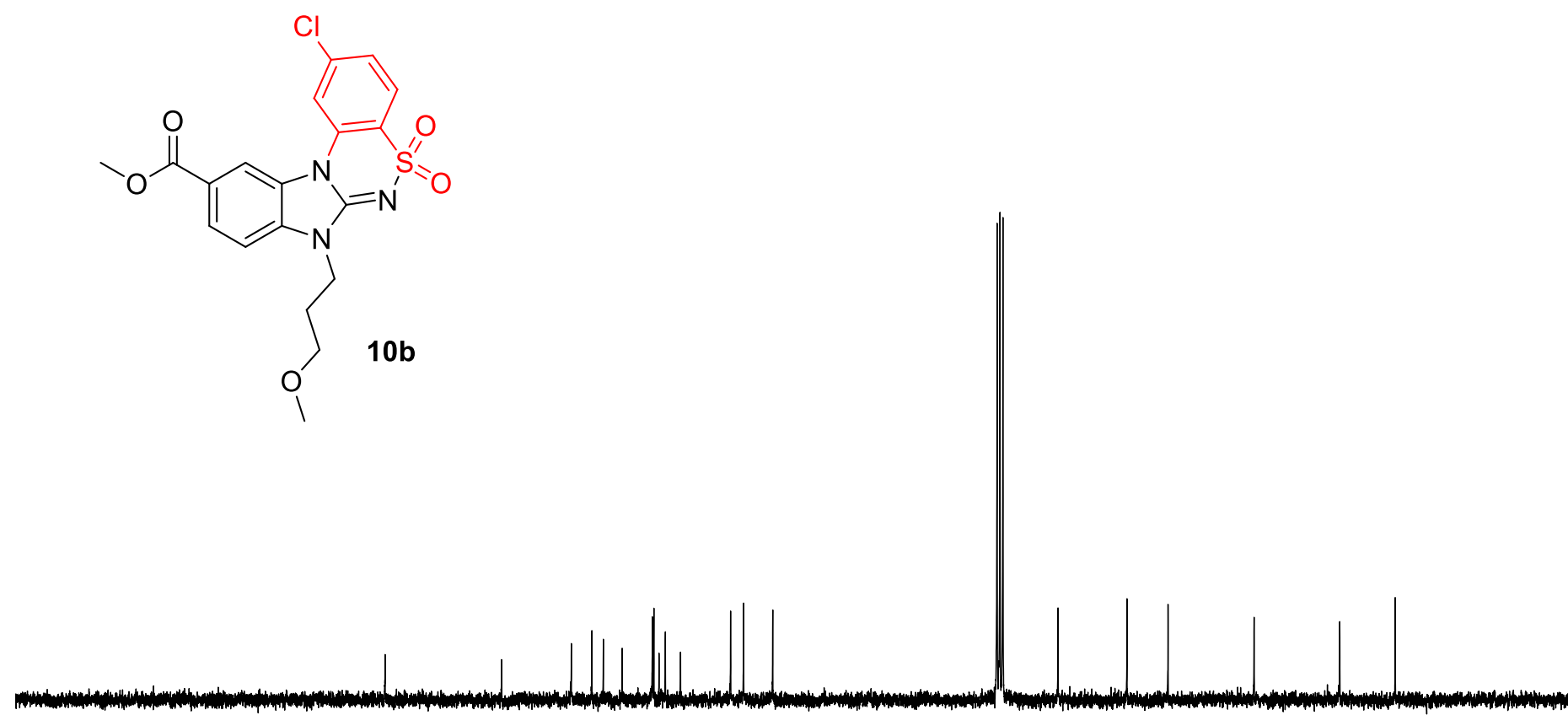

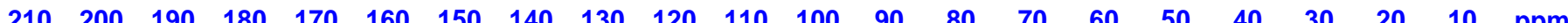

${ }^{13} \mathrm{C}$ NMR spectrum $\left(75 \mathrm{MHz}\right.$ ) of compound $\mathbf{1 0 b}$ in $\mathrm{CDCl}_{3}$ 


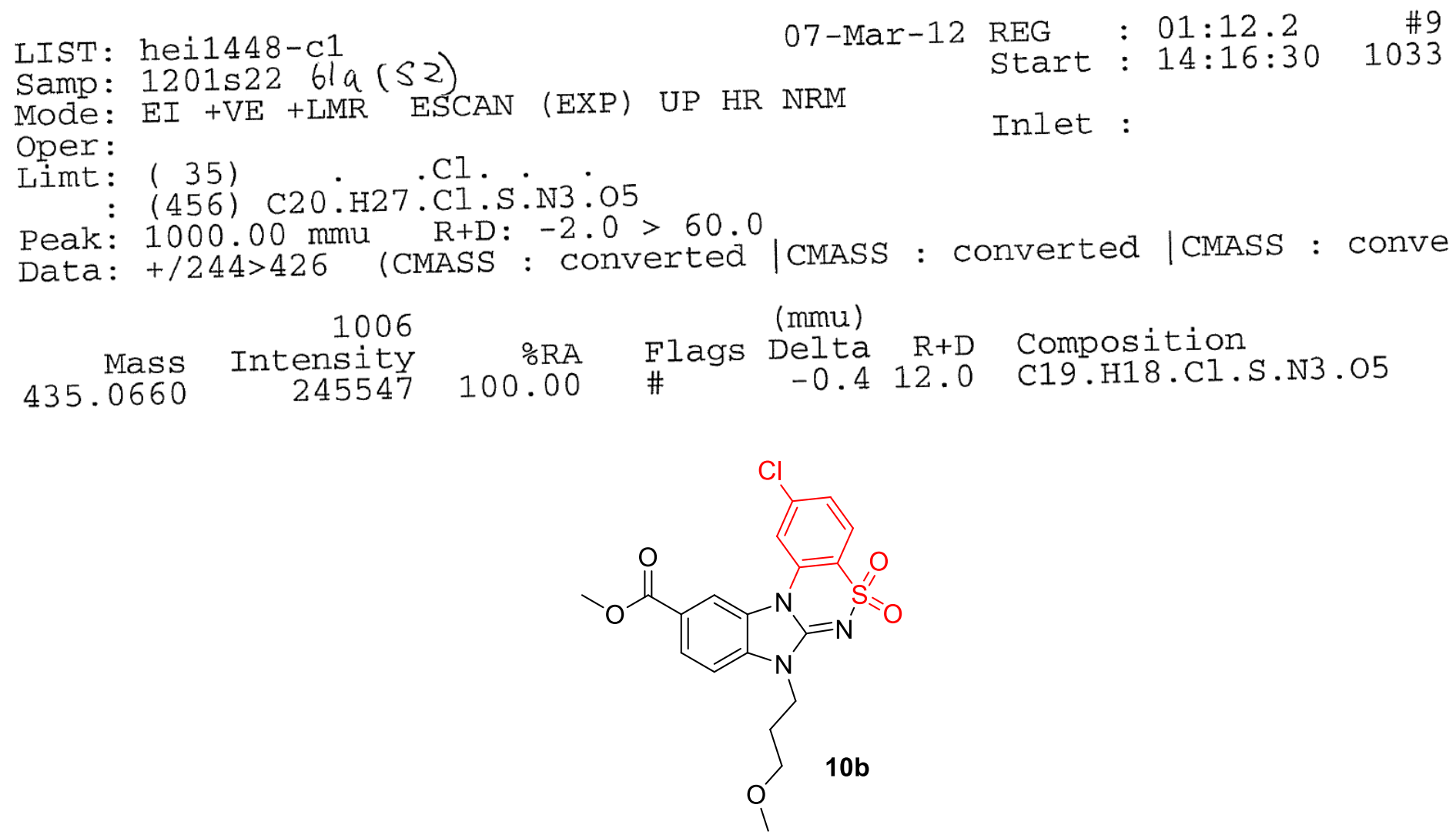

HRMS mass (ESI) spectrum of compound $\mathbf{1 0 b}$ 

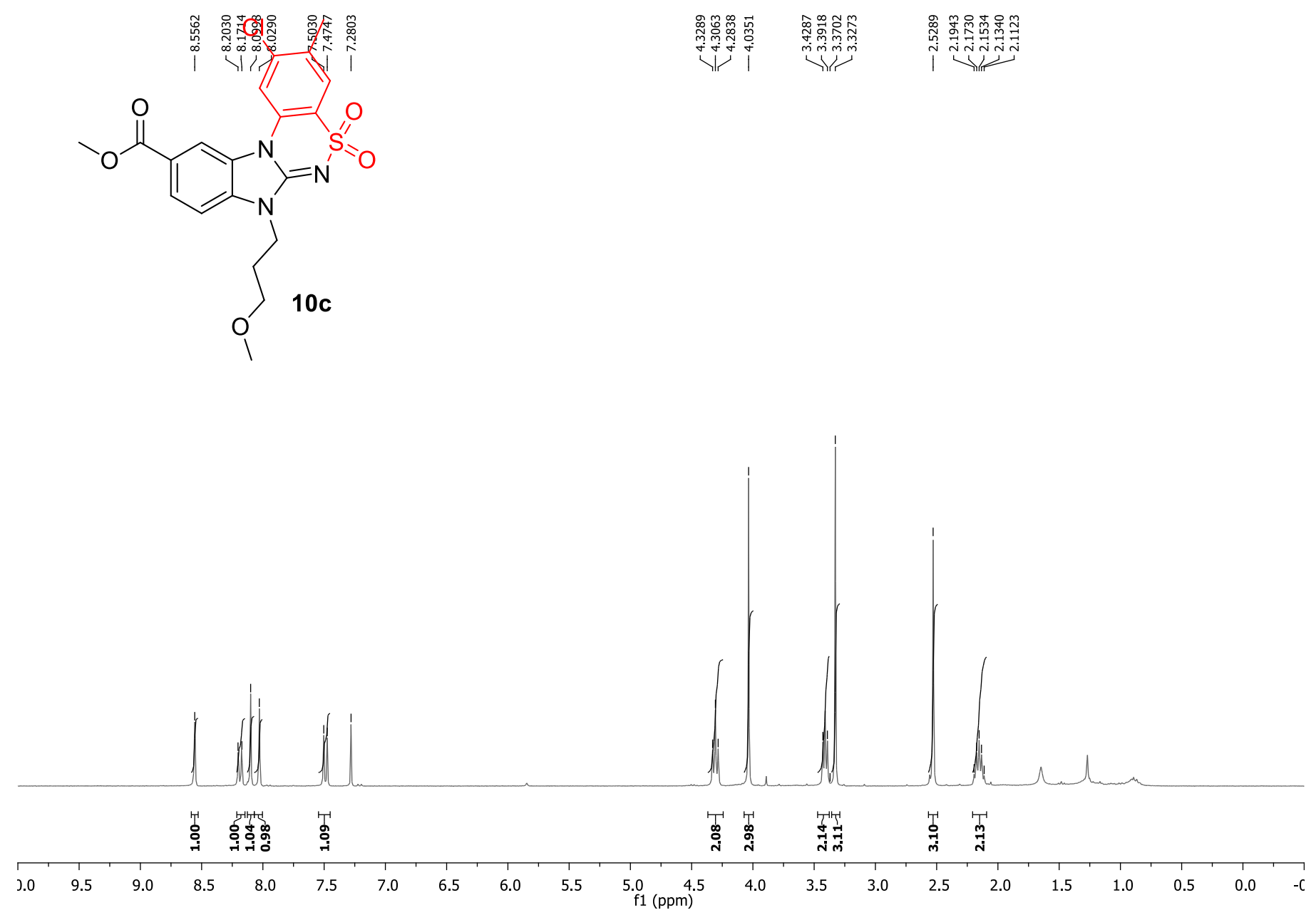

${ }^{1} \mathrm{H}$ NMR spectrum (300 MHz) of compound $10 \mathrm{c}$ in $\mathrm{CDCl}_{3}$ 


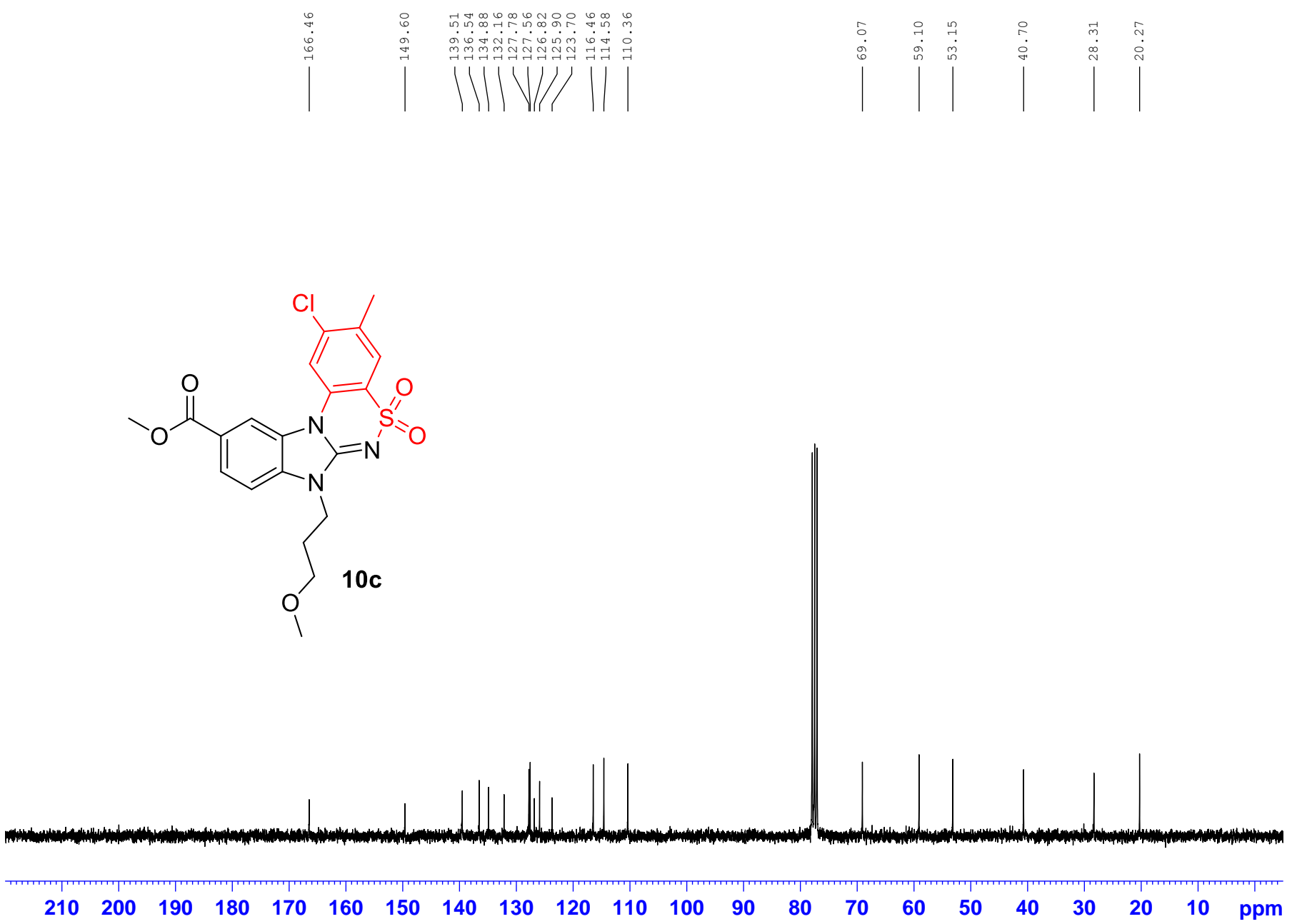

${ }^{13} \mathrm{C}$ NMR spectrum $(75 \mathrm{MHz})$ of compound 10c in $\mathrm{CDCl}_{3}$ 


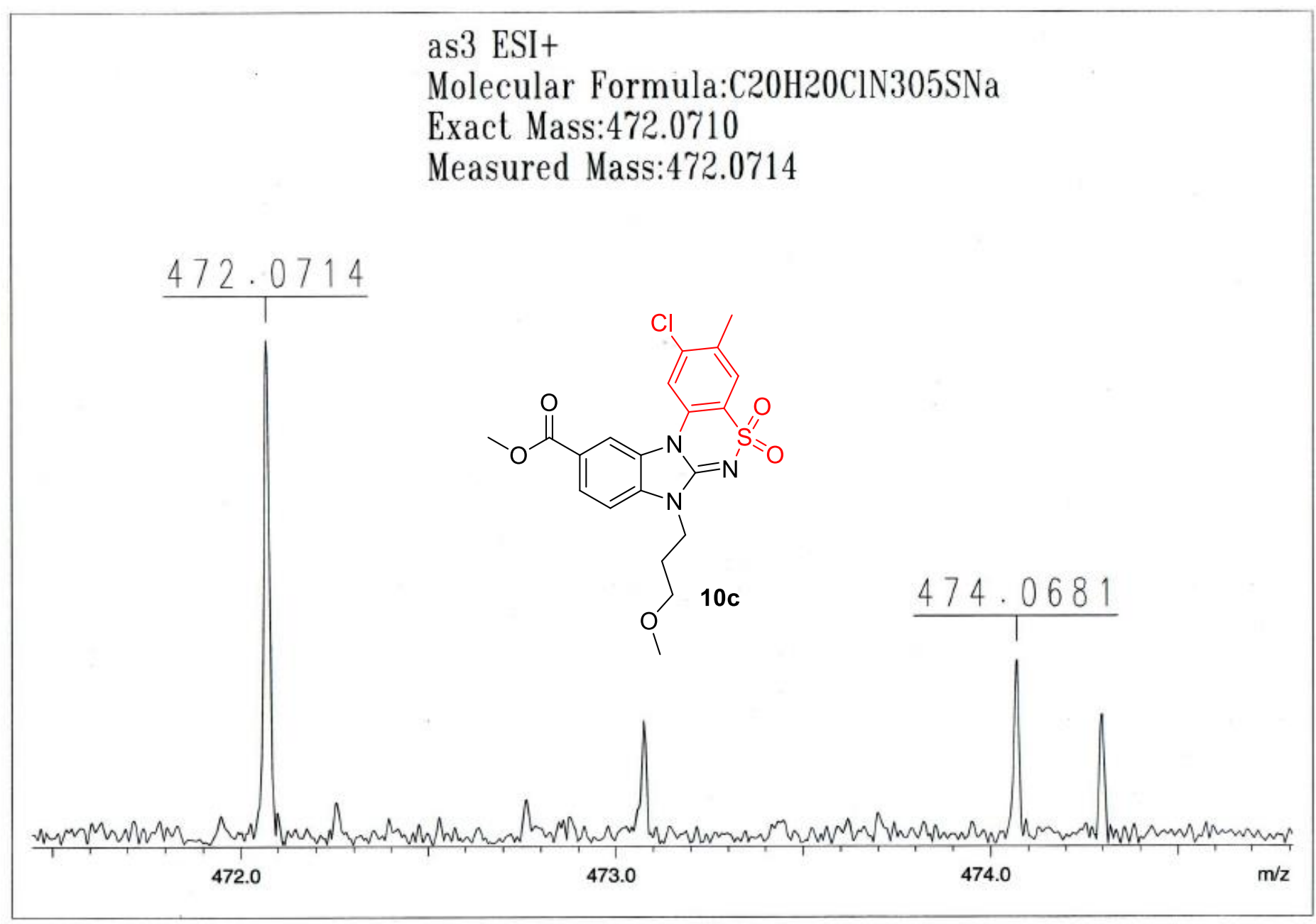

/d=/Data/yu/AS3/1/pdata/1 Administrator Fri Sep 30 16:06:25 2011

HRMS mass (ESI) spectrum of compound 10c 


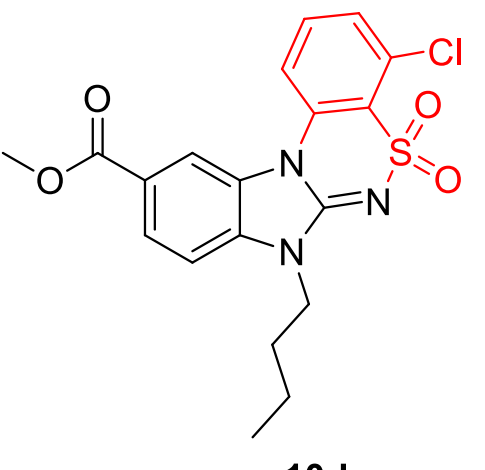

10d

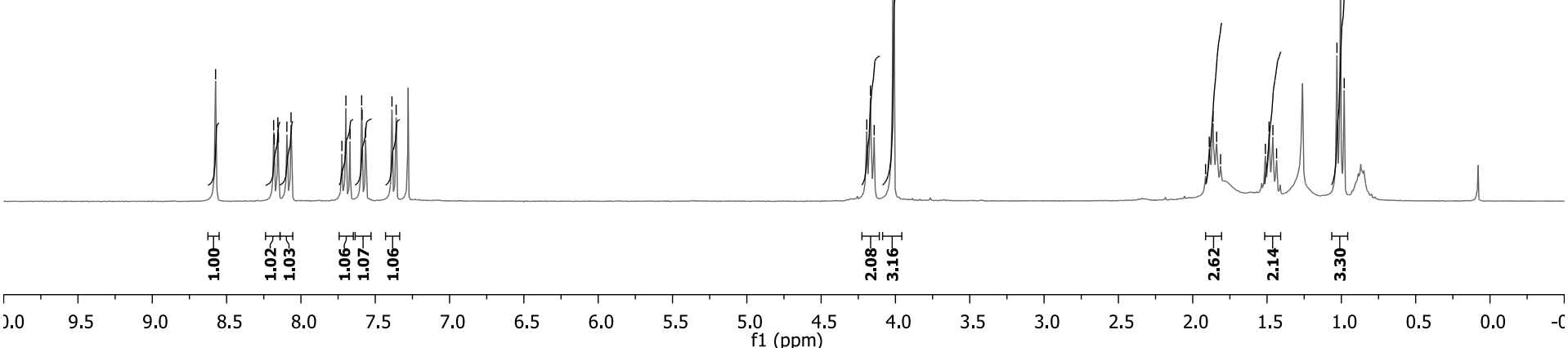

${ }^{1} \mathrm{H}$ NMR spectrum $(300 \mathrm{MHz})$ of compound $\mathbf{1 0 d}$ in $\mathrm{CDCl}_{3}$ 


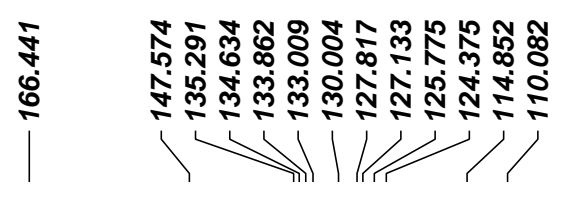

|

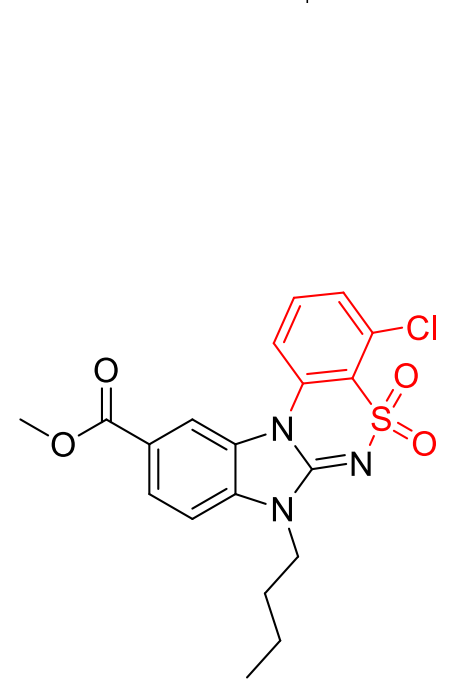

10d

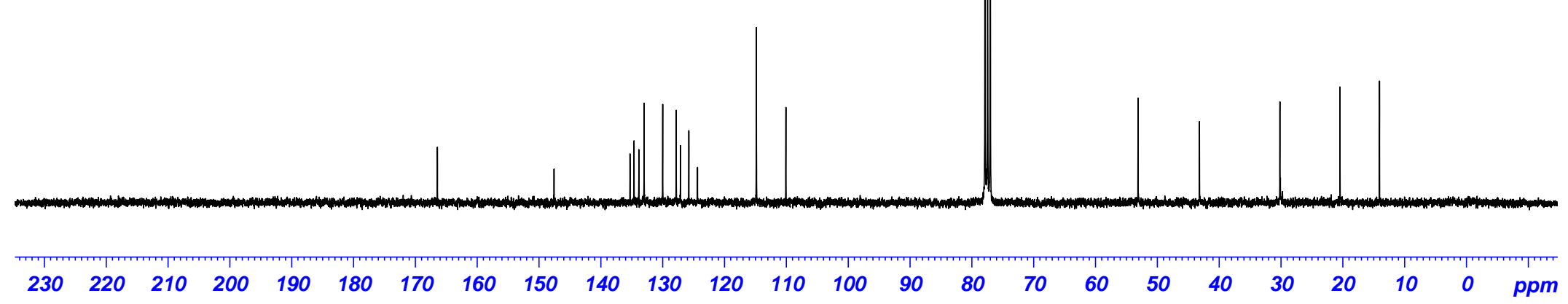

${ }^{13} \mathrm{C}$ NMR spectrum (75 MHz) of compound 10d in $\mathrm{CDCl}_{3}$ 

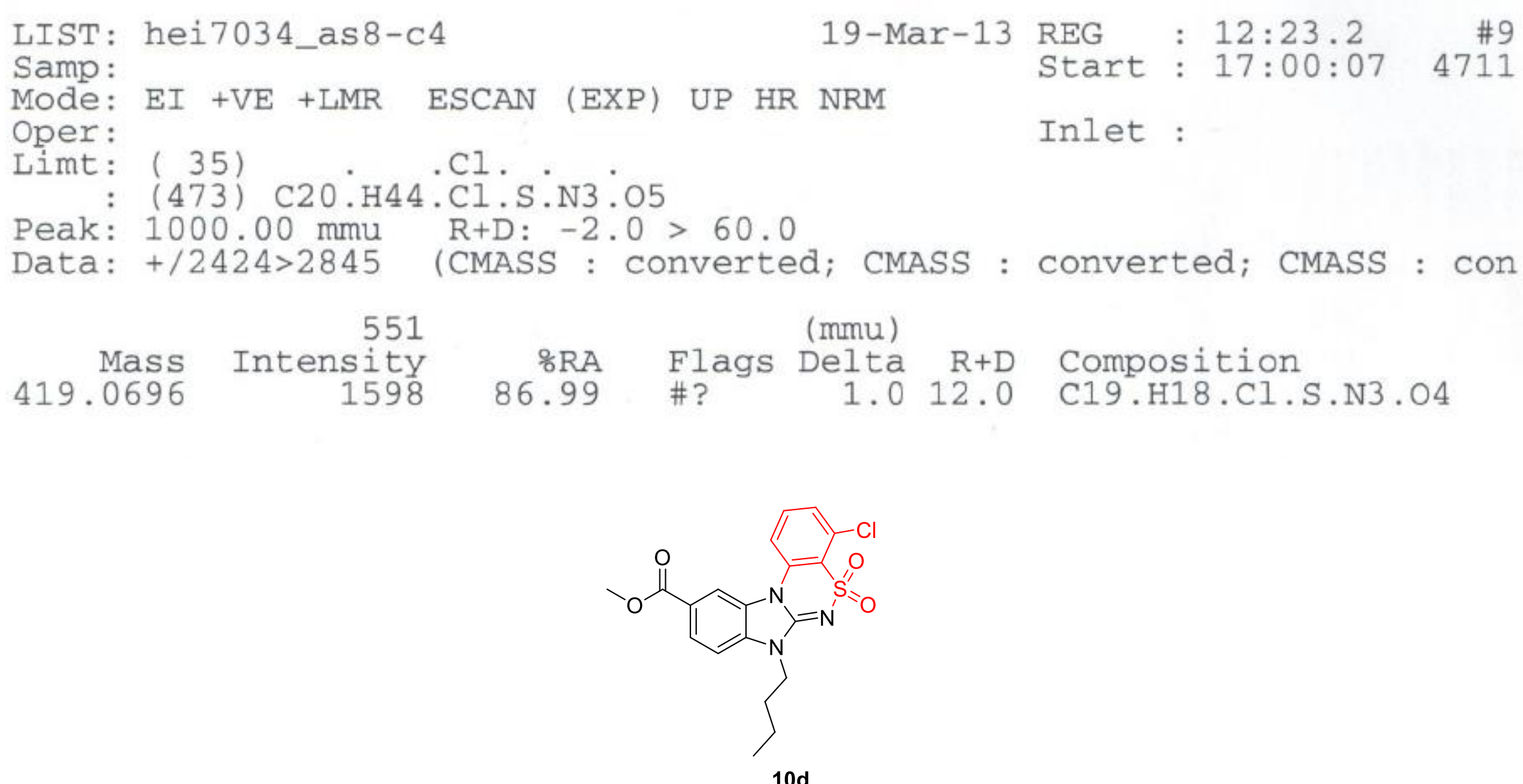

HRMS mass (EI) spectrum of compound 10d 


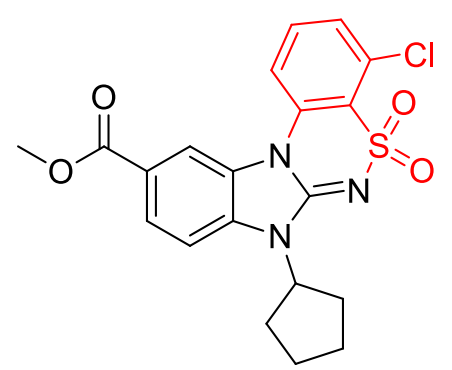

$10 e$

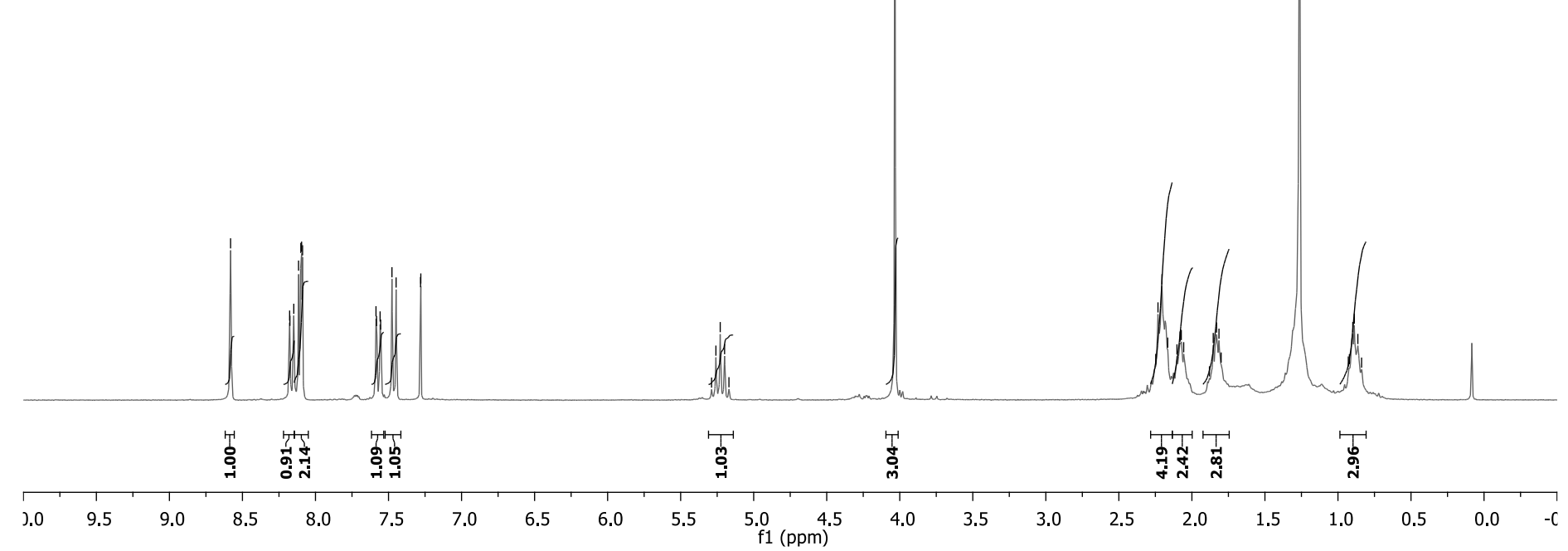

${ }^{1} \mathrm{H}$ NMR spectrum (300 MHz) of compound 10e in $\mathrm{CDCl}_{3}$ 


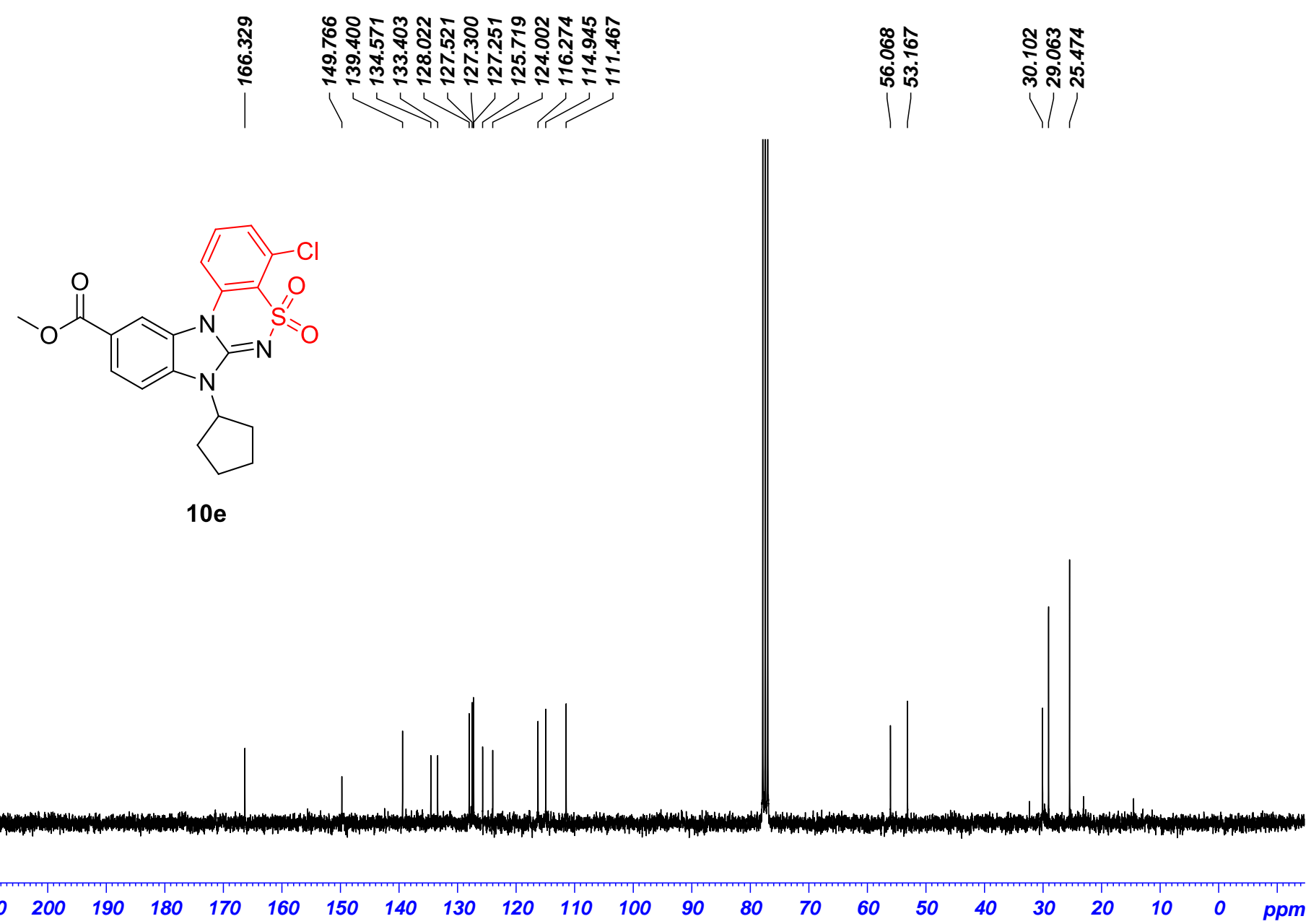

${ }^{13} \mathrm{C}$ NMR spectrum $(75 \mathrm{MHz})$ of compound $10 \mathrm{e}$ in $\mathrm{CDCl}_{3}$ 


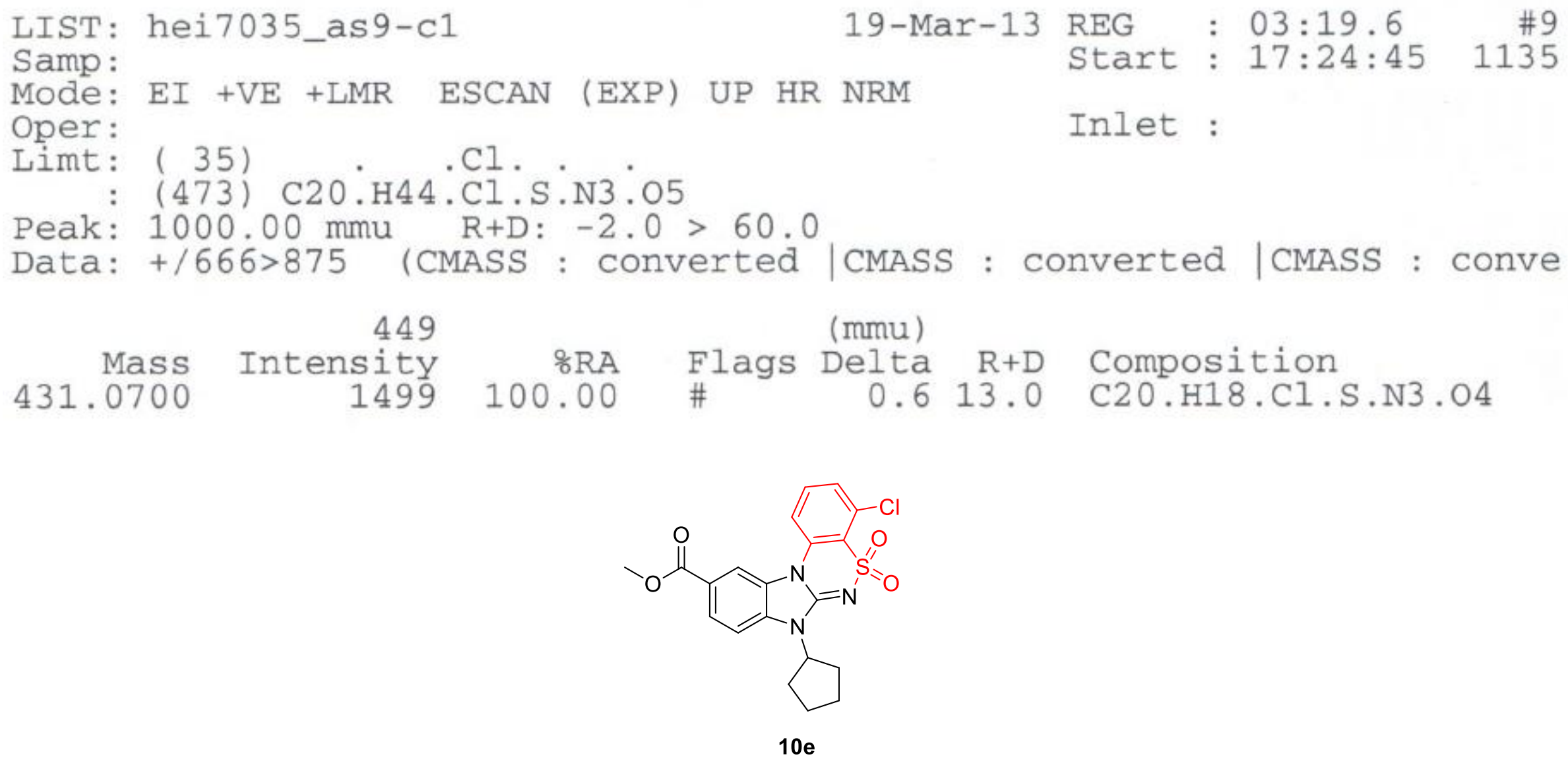

$10 \mathrm{e}$

HRMS mass (EI) spectrum of compound 10e 


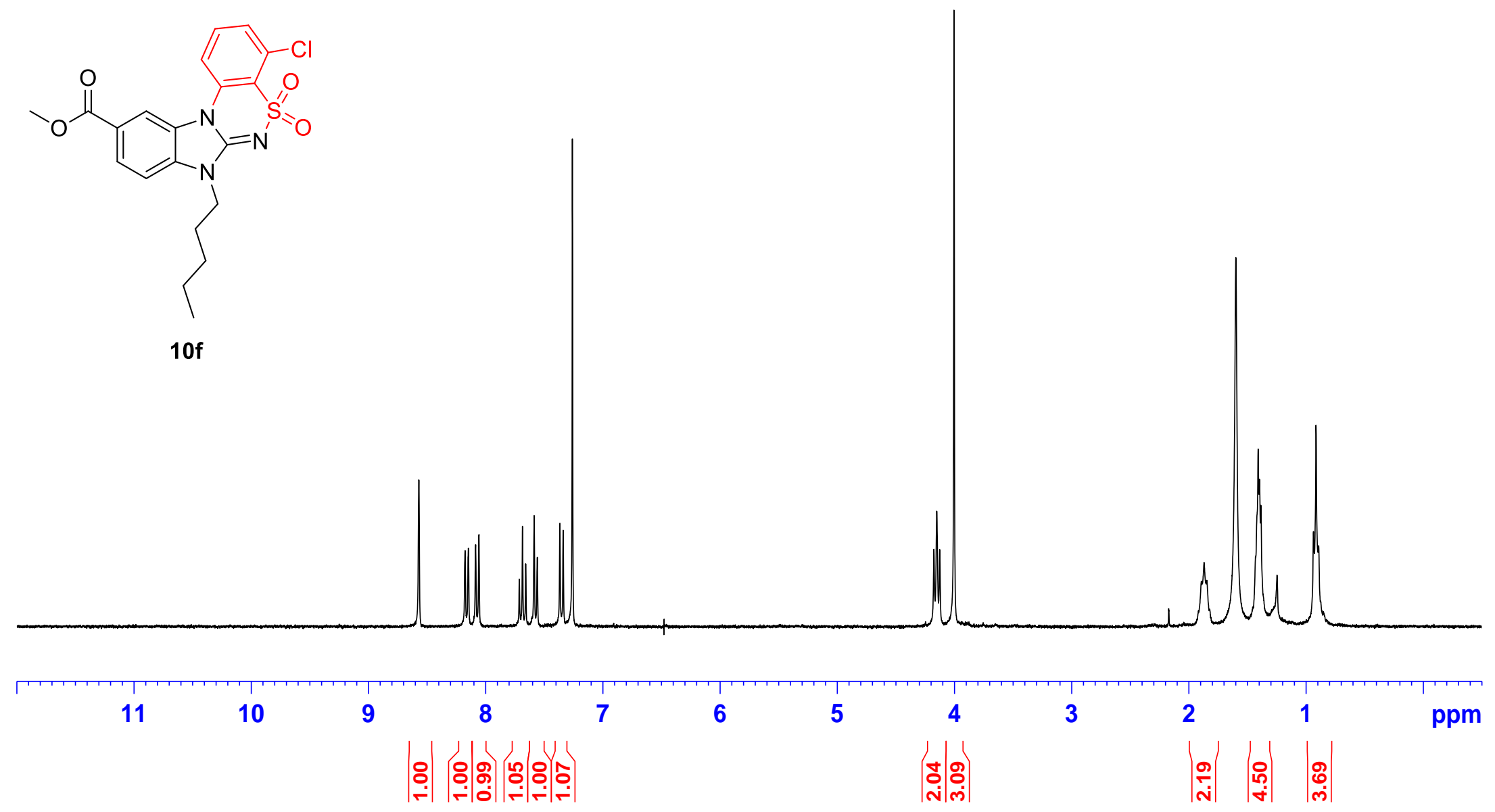

${ }^{1} \mathrm{H}$ NMR spectrum (300 MHz) of compound $\mathbf{1 0 f}$ in $\mathrm{CDCl}_{3}$ 


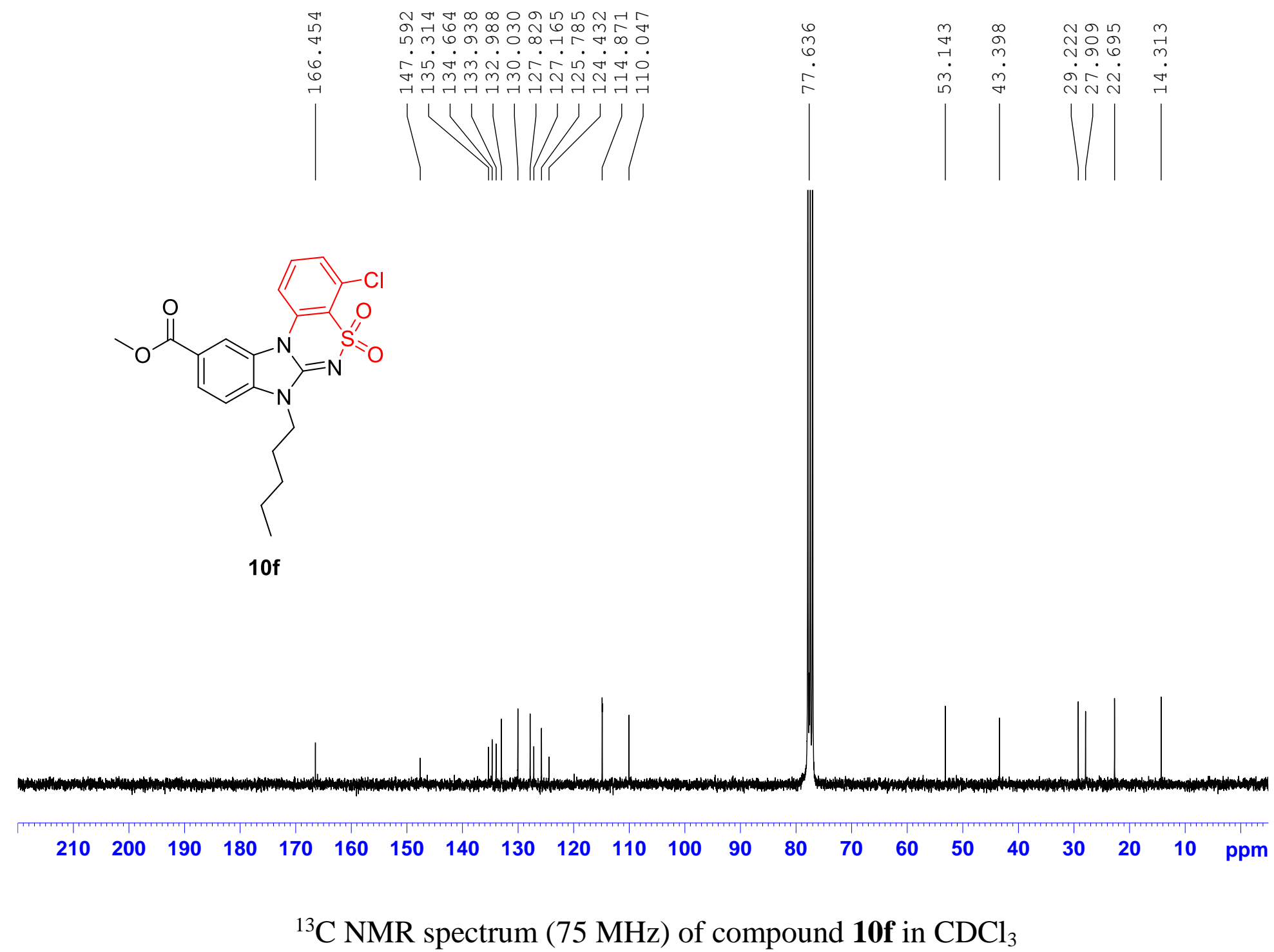


LIST: hei7033_as7-c1

Samp :

Mode: $\mathrm{EI}+\mathrm{VE}+\mathrm{LMR}$ ESCAN (EXP) UP HR NRM

Oper:

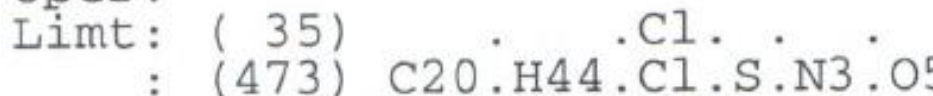

Peak: $1000.00 \mathrm{mmu} \mathrm{R}+\mathrm{D}:-2.0>60.0$

Data: +/888>979 (CMASS : converted |CMASS : converted |CMASS : conve

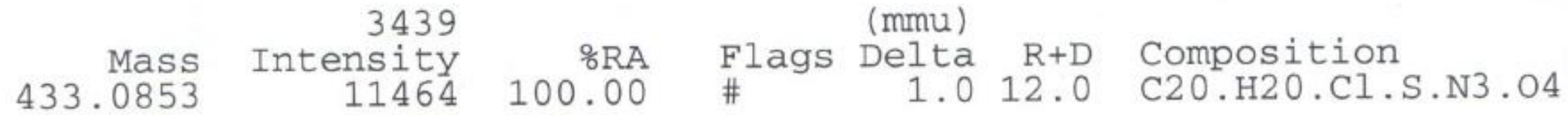

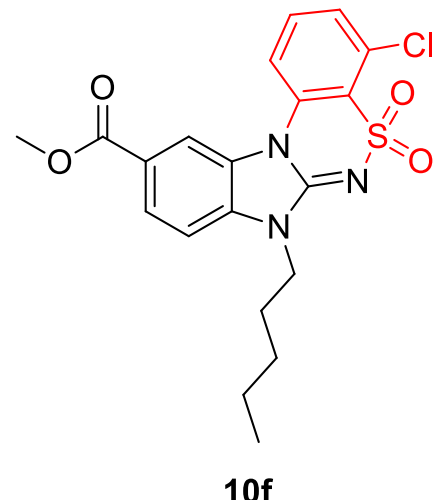

HRMS mass (ESI) spectrum of compound $\mathbf{1 0 f}$ 


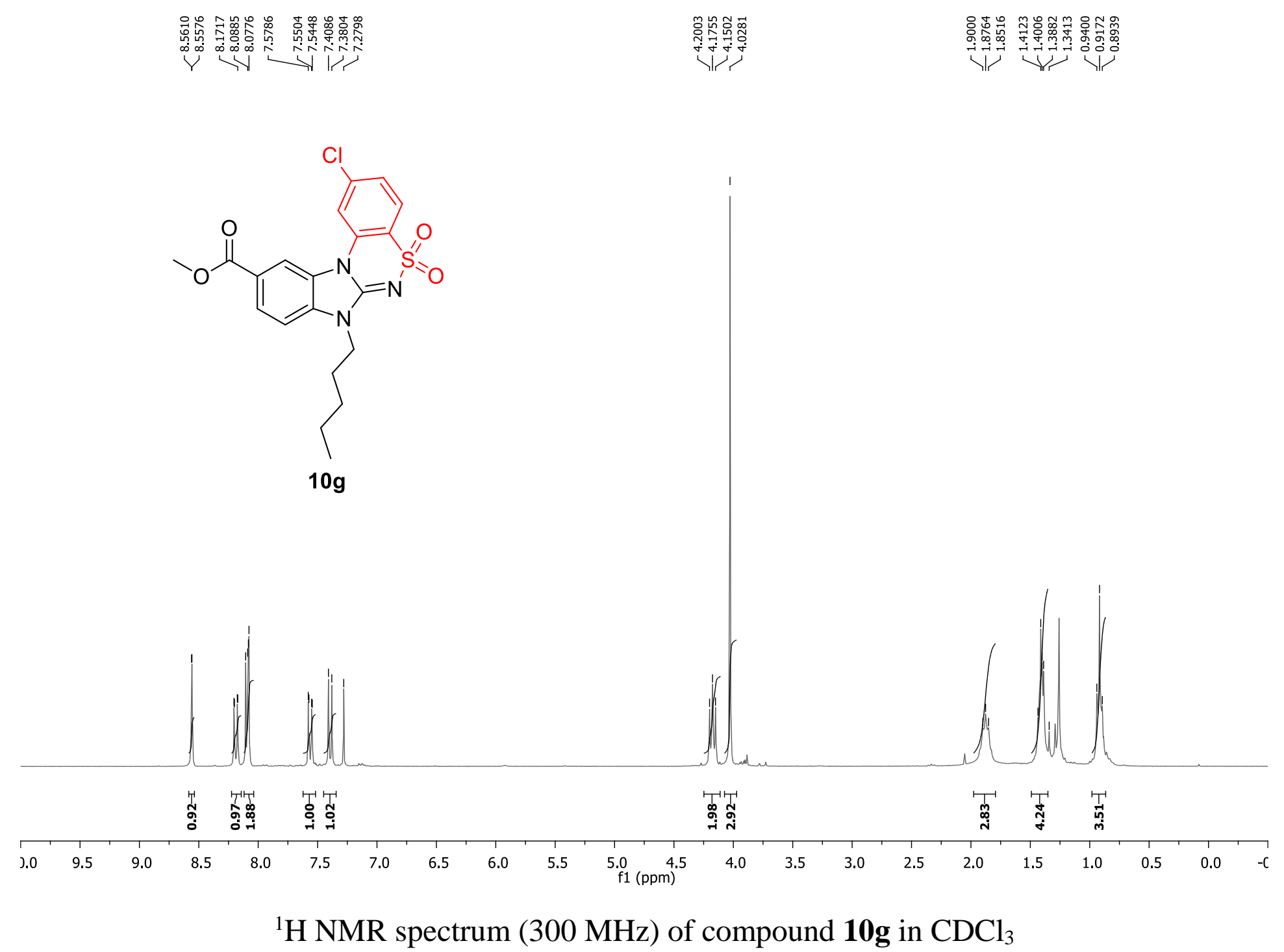




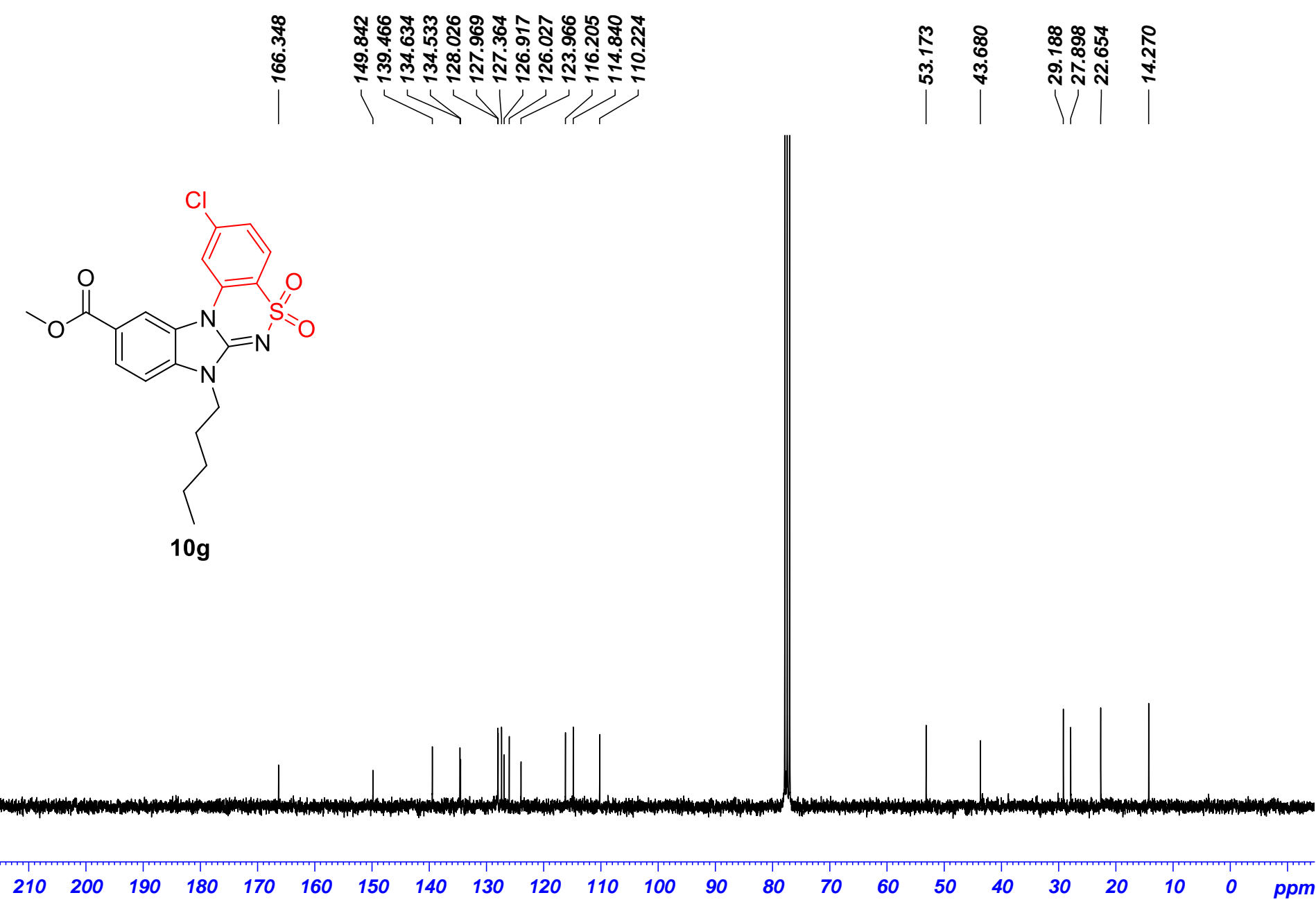

${ }^{13} \mathrm{C}$ NMR spectrum (75 MHz) of compound $\mathbf{1 0 g}$ in $\mathrm{CDCl}_{3}$ 
LIST: hei1474-c1

Samp: 1227 ble (56)

Mode: $\mathrm{EI}+\mathrm{VE}+\mathrm{LMR}$ ESCAN (EXP) UP HR NRM

Oper:

Limt: $(35)$

: (497) C20.H40.CI.S.N5.O5

Peak: $1000.00 \mathrm{mmu}$ R+D: $-2.0>60.0$

Data: +/790>1041 (CMASS : converted |CMASS : converted /CMASS : conv

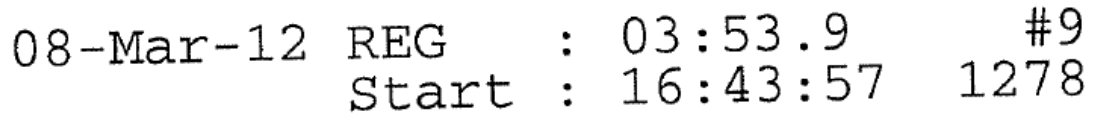

Inlet :

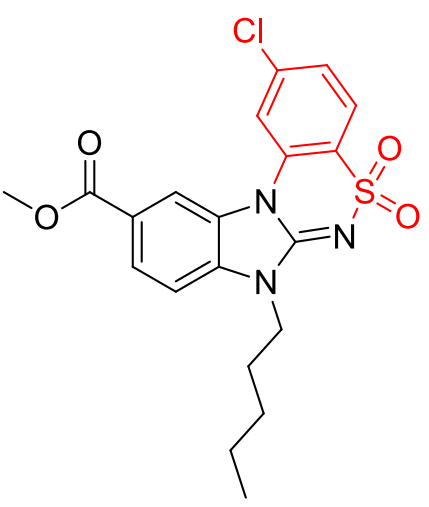

$10 \mathrm{~g}$

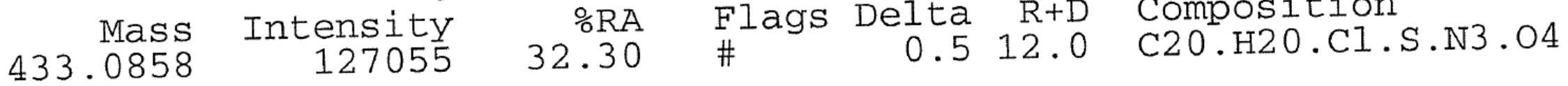

HRMS mass (ESI) spectrum of compound $\mathbf{1 0 g}$ 


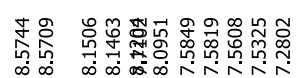

我

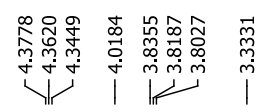
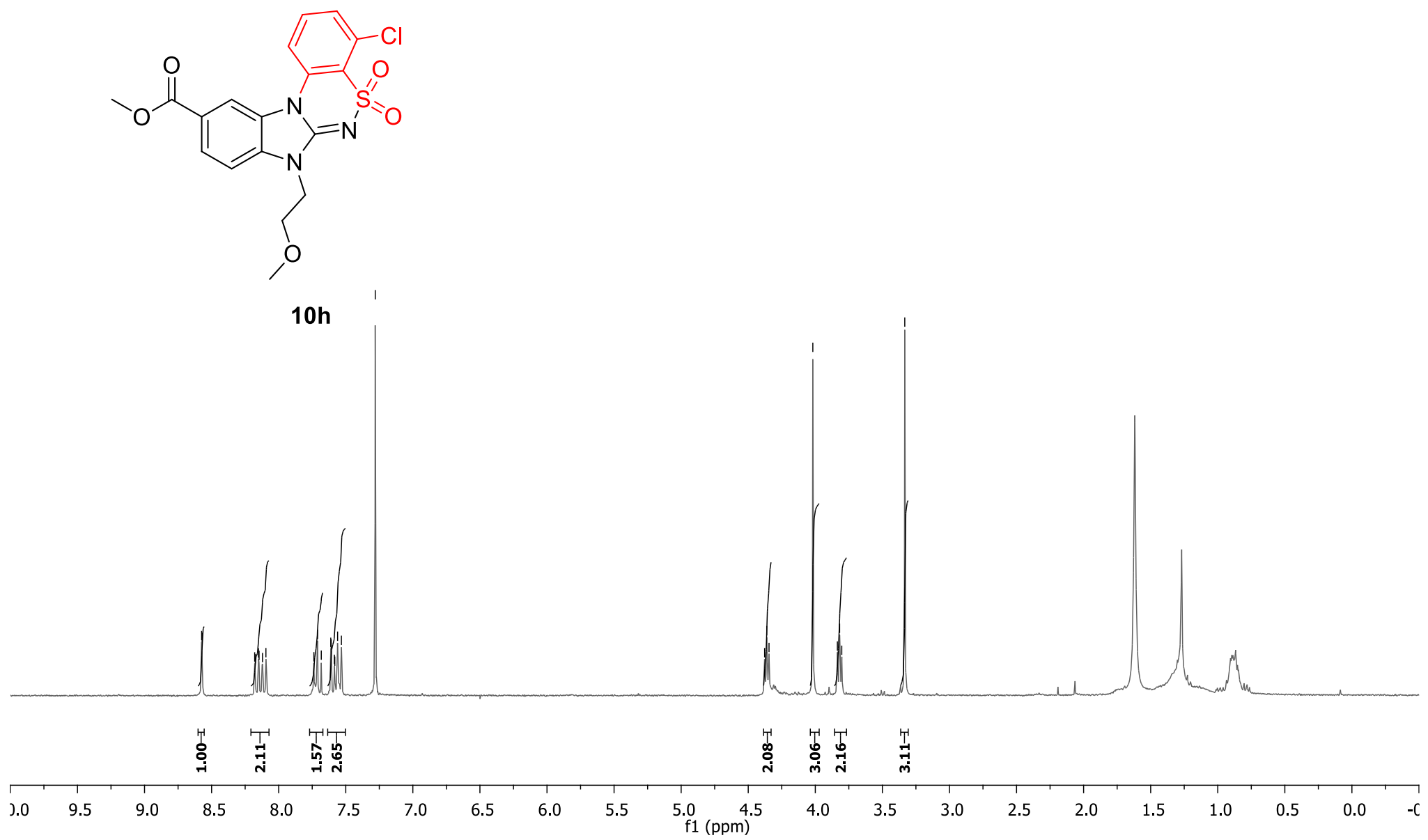

${ }^{1} \mathrm{H}$ NMR spectrum $(300 \mathrm{MHz})$ of compound $\mathbf{1 0 h}$ in $\mathrm{CDCl}_{3}$ 


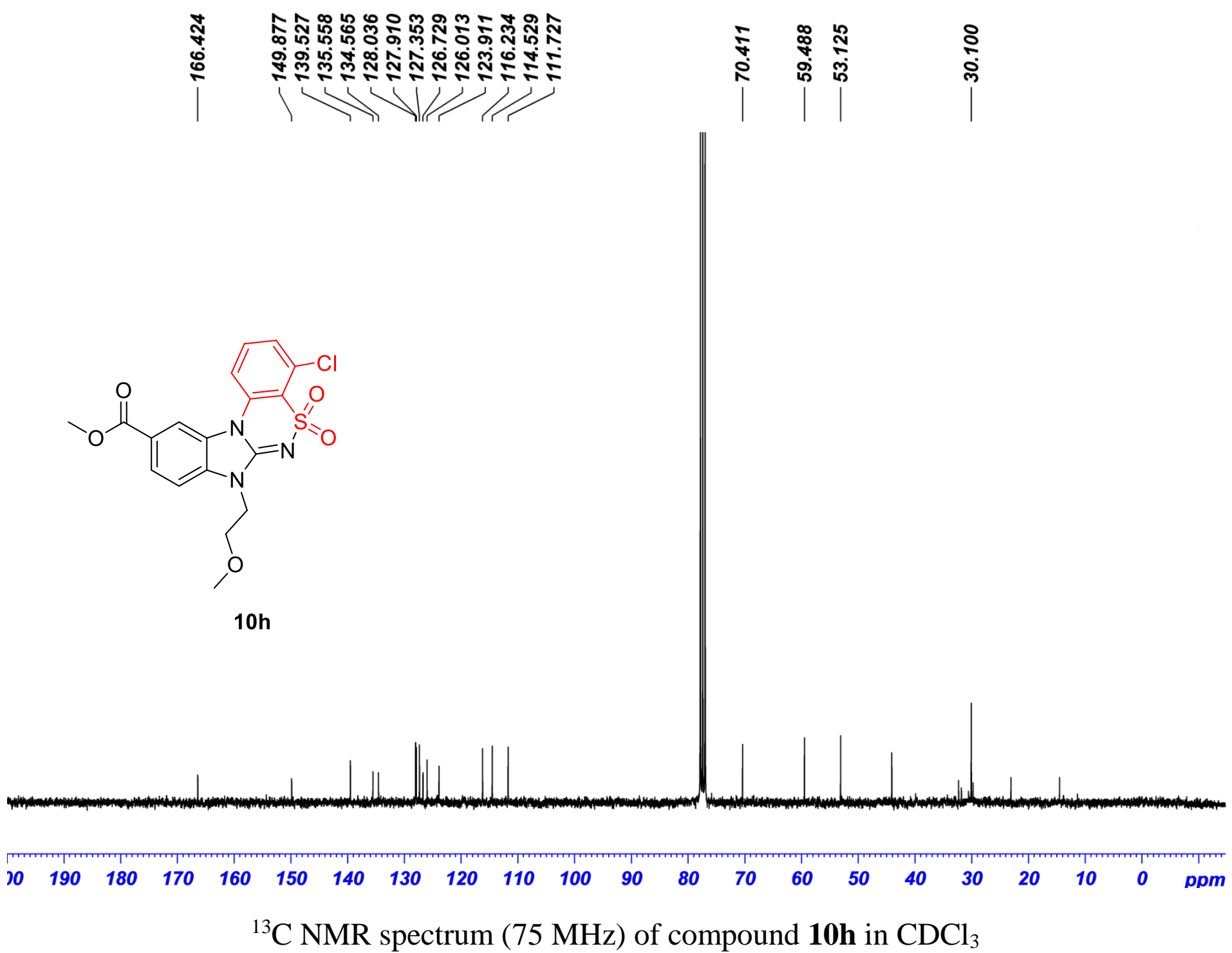


LIST: hei7032_as6-c3

Samp :

Mode: $\mathrm{EI}+\mathrm{VE}+\mathrm{LMR}$ ESCAN (EXP) UP HR NRM

Oper:

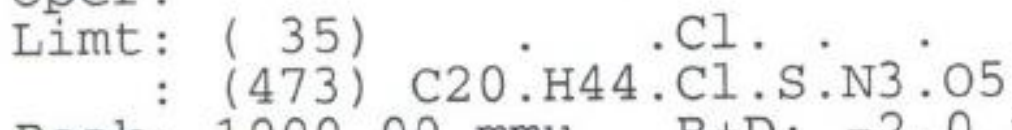

Peak: $1000.00 \mathrm{mmu} \mathrm{R}+\mathrm{D}:-2.0>60.0$

Data: +/1818>1964 (CMASS : converted; CMASS : converted |CMASS : Con

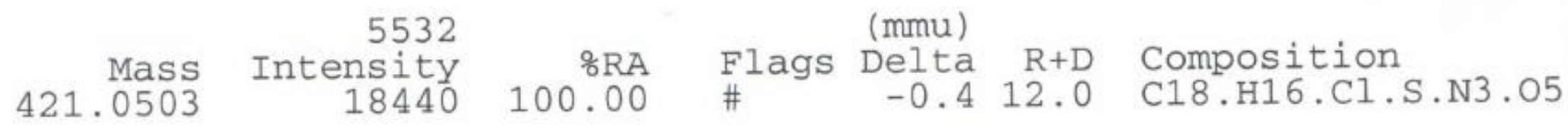

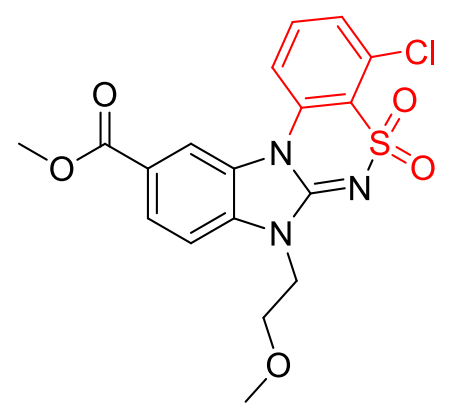

$10 \mathrm{~h}$

HRMS mass (ESI) spectrum of compound $\mathbf{1 0 h}$ 


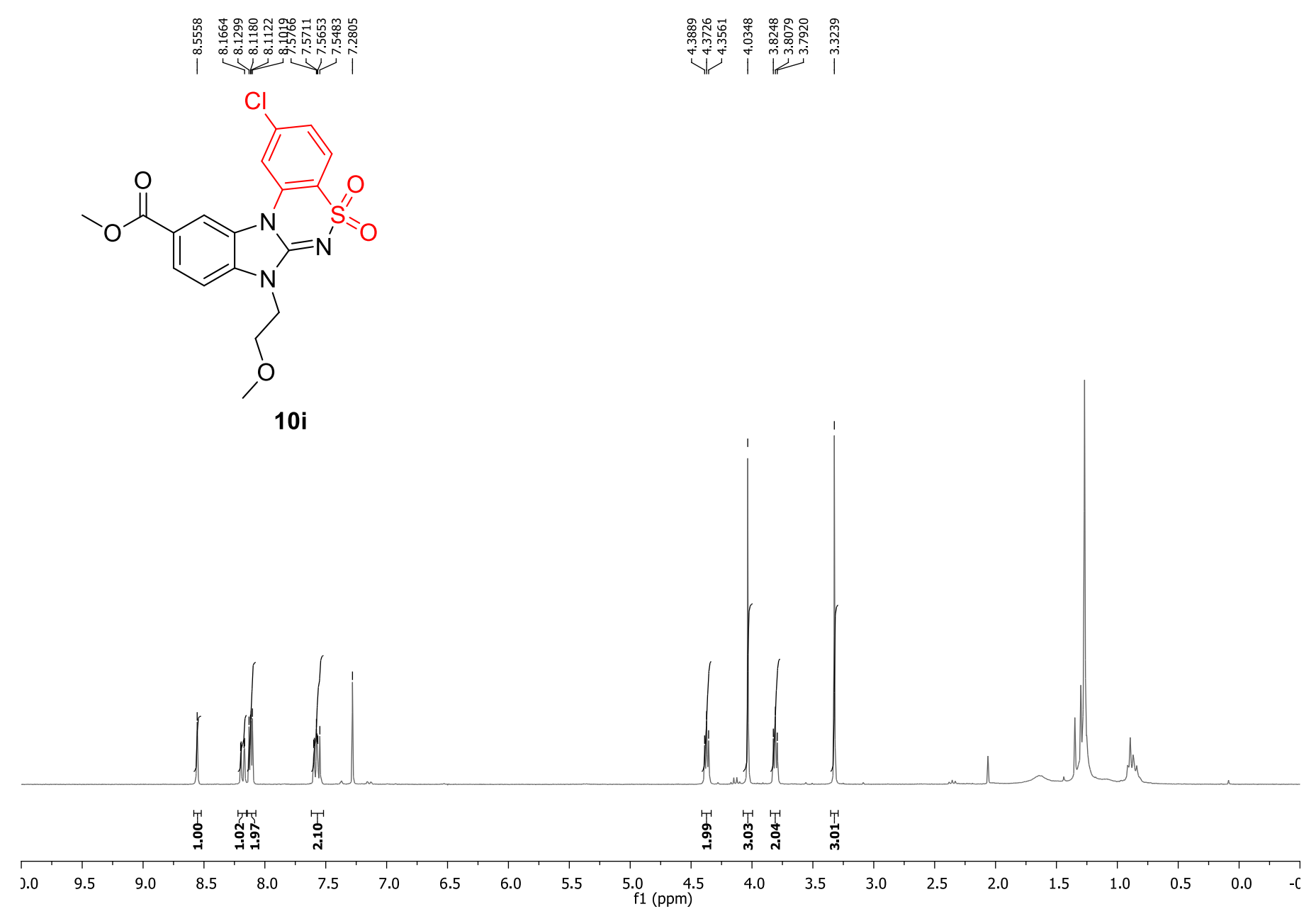

${ }^{1} \mathrm{H}$ NMR spectrum $\left(300 \mathrm{MHz}\right.$ ) of compound $\mathbf{1 0} \mathbf{i}$ in $\mathrm{CDCl}_{3}$ 


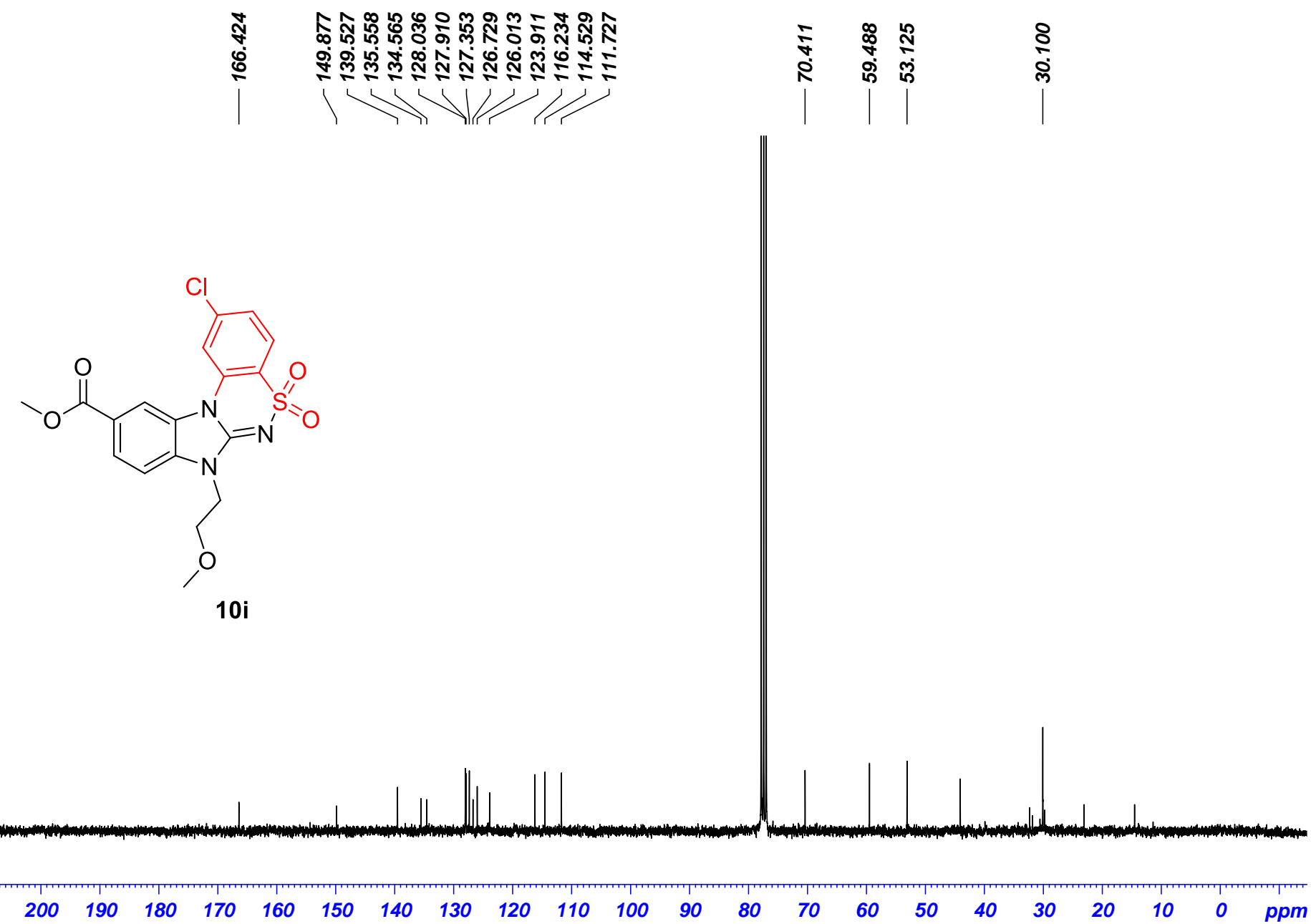

${ }^{13} \mathrm{C}$ NMR spectrum $\left(75 \mathrm{MHz}\right.$ ) of compound $10 \mathrm{i}$ in $\mathrm{CDCl}_{3}$ 
LIST: hei7031_as5-c3

Samp :

Mode:

Oper:

Limt : ( 35

$$
\text { : }(473) \text { C20.H44.Cl.S.N3.OS }
$$

Peak: $1000.00 \mathrm{mmu}$ R+D: $-2.0>60.0$

Data: +/1983>2327 (CMASS : converted; CMASS : converted |CMASS : con

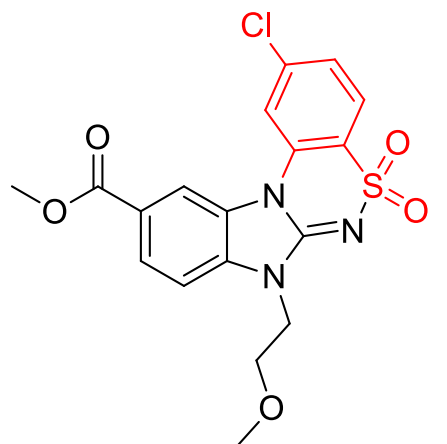

$10 i$

HRMS mass (ESI) spectrum of compound 10i 

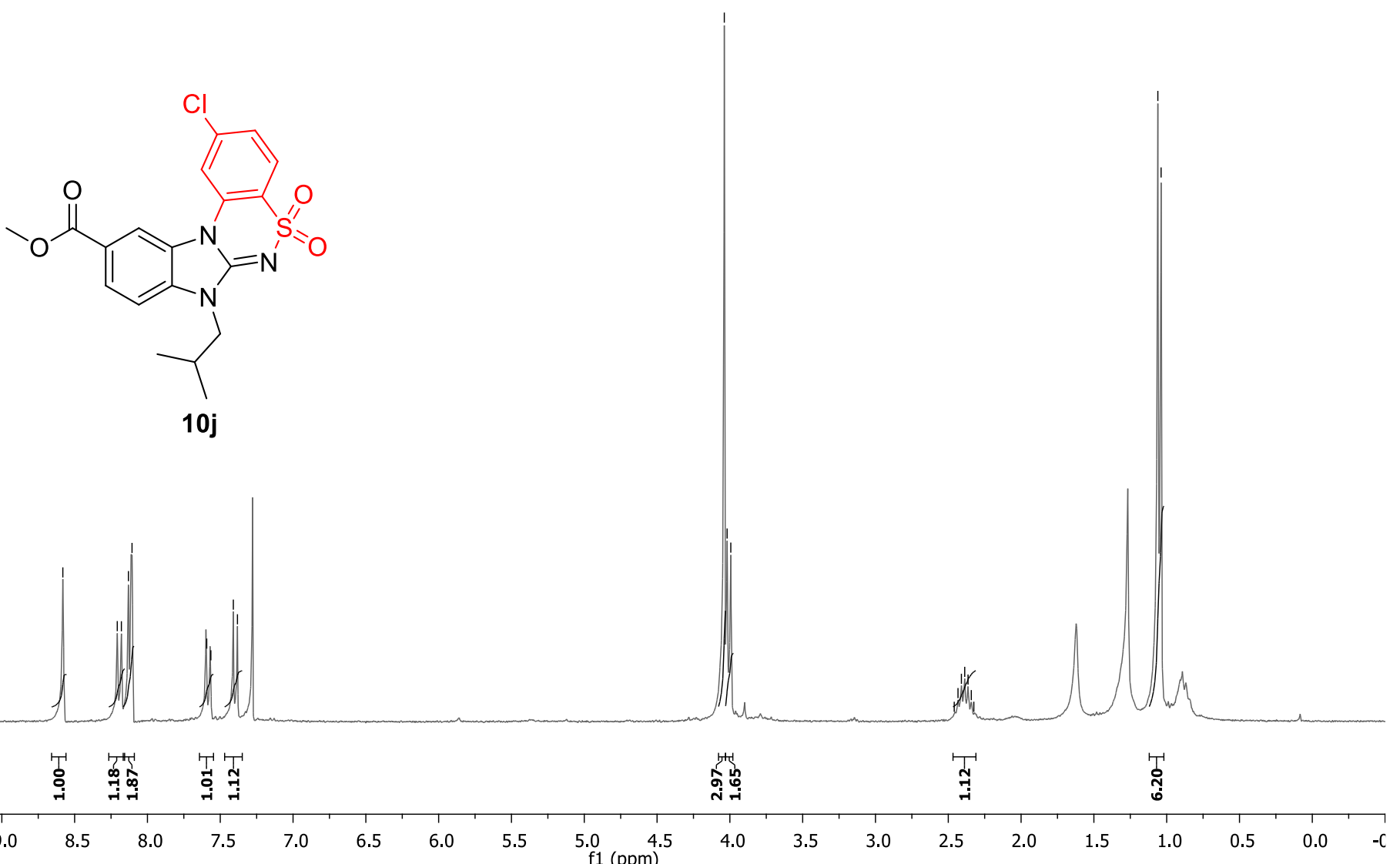

${ }^{1} \mathrm{H}$ NMR spectrum $\left(300 \mathrm{MHz}\right.$ ) of compound $\mathbf{1 0} \mathbf{j}$ in $\mathrm{CDCl}_{3}$ 


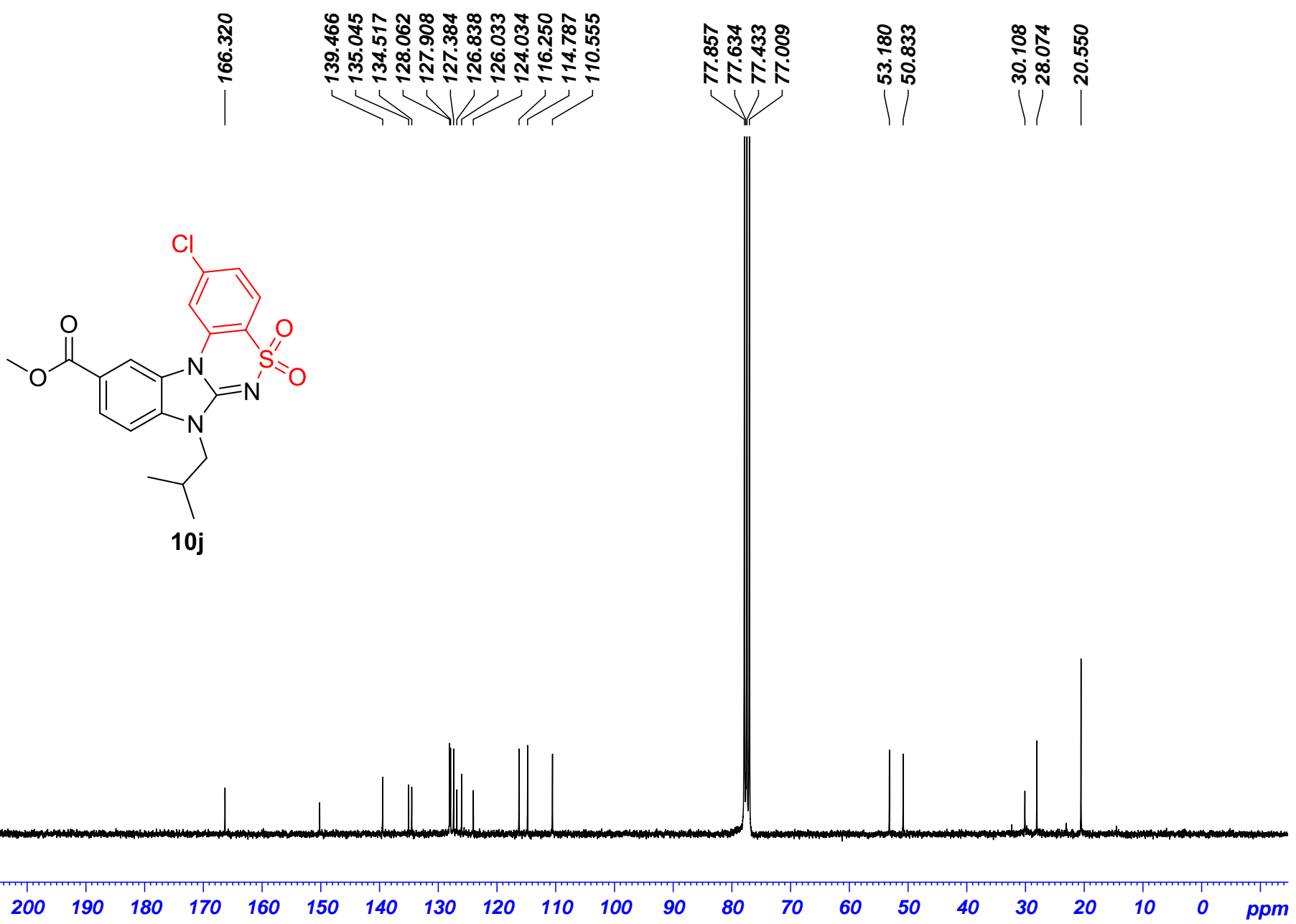

${ }^{13} \mathrm{C}$ NMR spectrum $\left(75 \mathrm{MHz}\right.$ ) of compound $\mathbf{1 0 j}$ in $\mathrm{CDCl}_{3}$ 


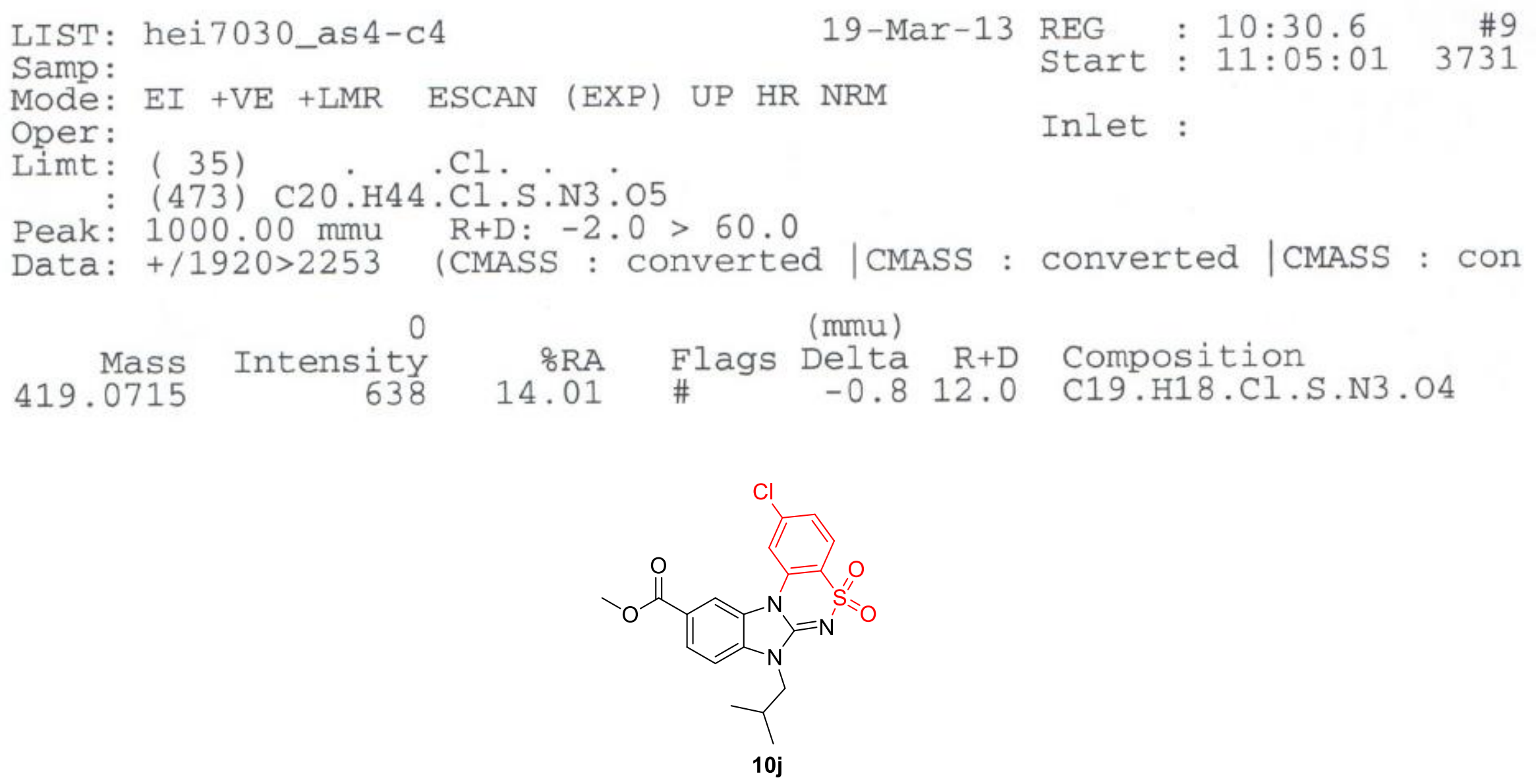

HRMS mass (ESI) spectrum of compound $\mathbf{1 0 j}$ 


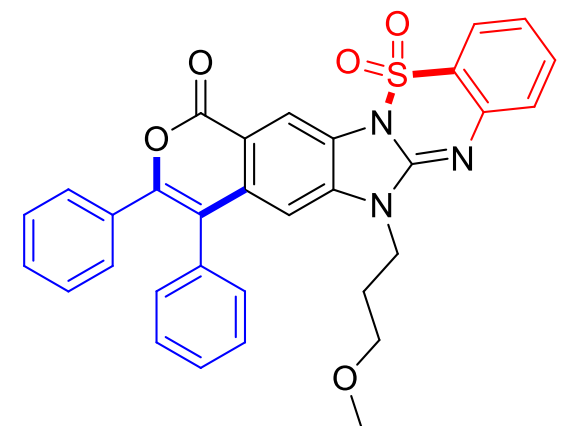

21a

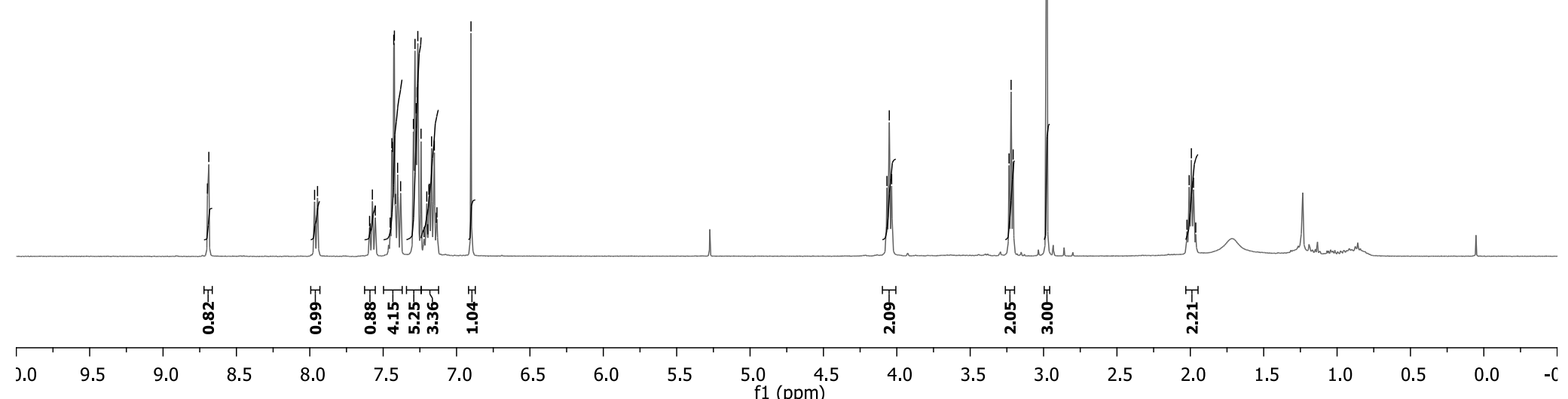

${ }^{1} \mathrm{H}$ NMR spectrum $\left(400 \mathrm{MHz}\right.$ ) of compound 21a in $\mathrm{CDCl}_{3}$ 

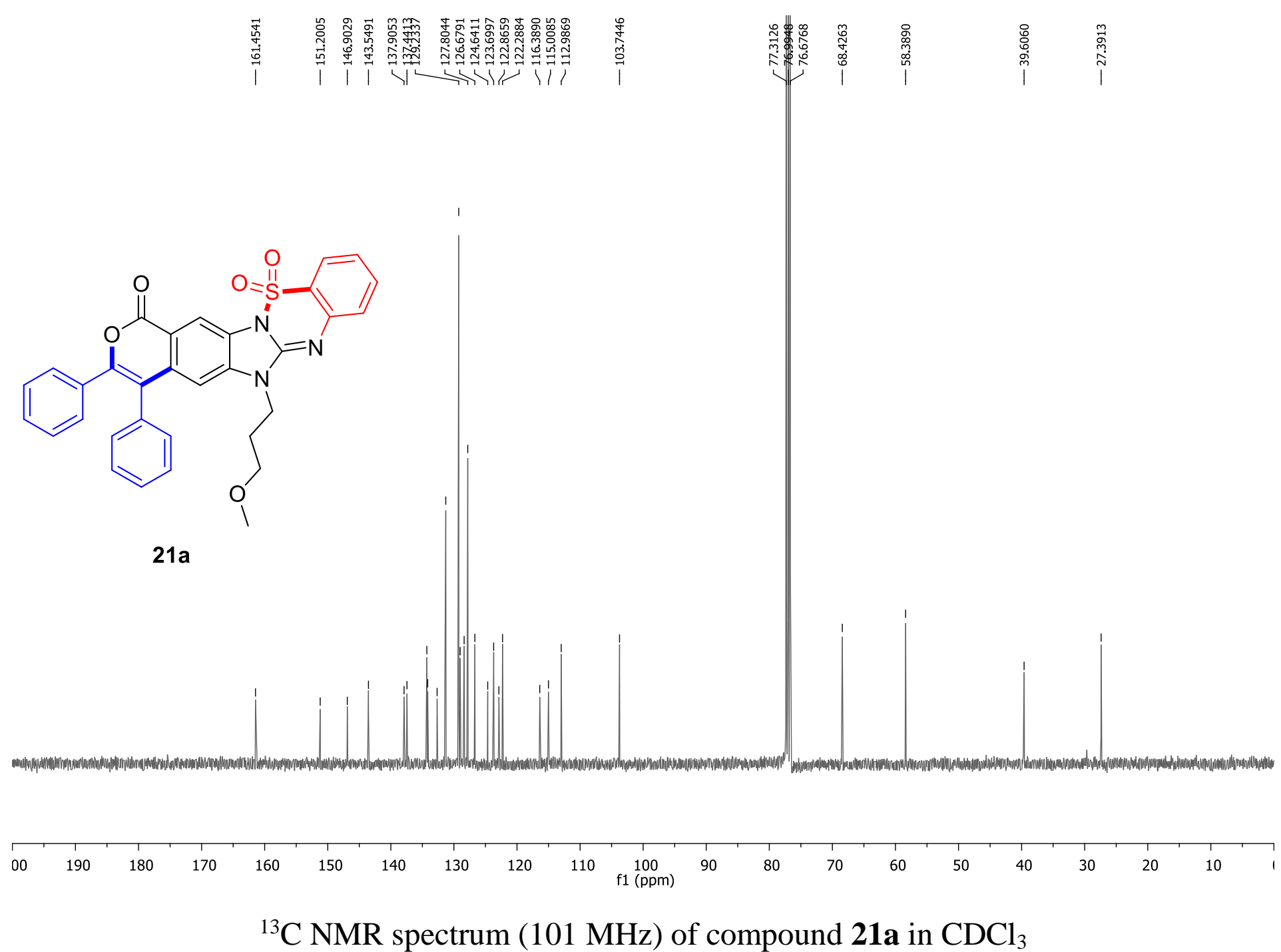


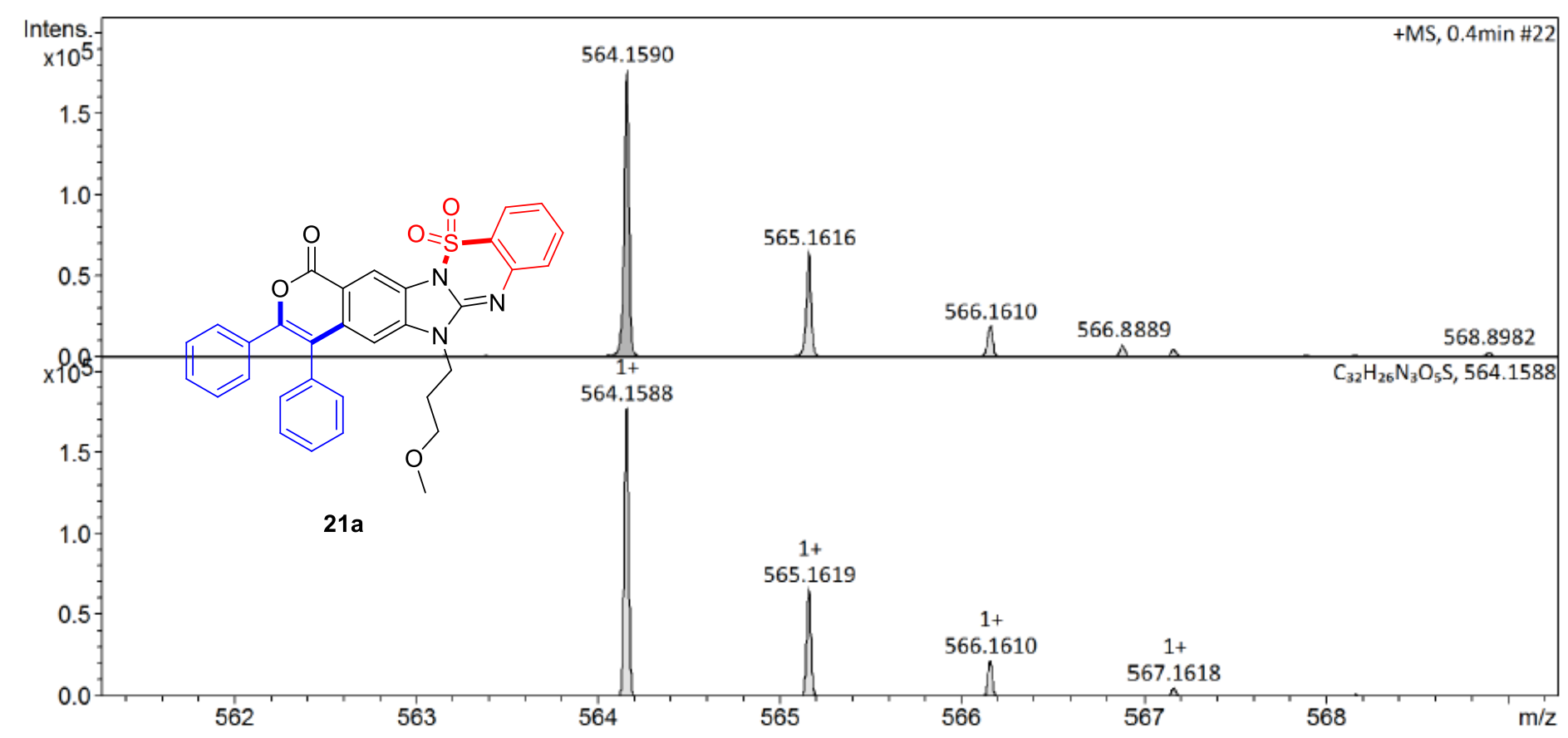

\section{Display Report}

Meas. $\mathrm{m} / \mathrm{z}$ \# Ion Formula $\mathrm{m} / \mathrm{z}$ err $[\mathrm{ppm}] \mathrm{mSigma}$ \# Sigma Score rdb $\mathrm{e}^{-}$Conf N-Rule Adduct $\begin{array}{llllllllllll}564.1590 & 1 & \mathrm{C} 32 \mathrm{H} 26 \mathrm{~N} 3 \mathrm{O} 5 \mathrm{~S} & 564.1588 & -0.3 & 6.6 & 1 & 100.00 & 21.5 & \text { even } & \mathrm{ok} & \mathrm{M}+\mathrm{H}\end{array}$

HRMS spectrum (ESI) of compound 21a 


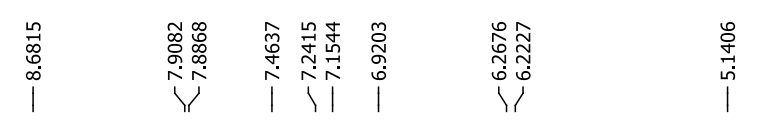

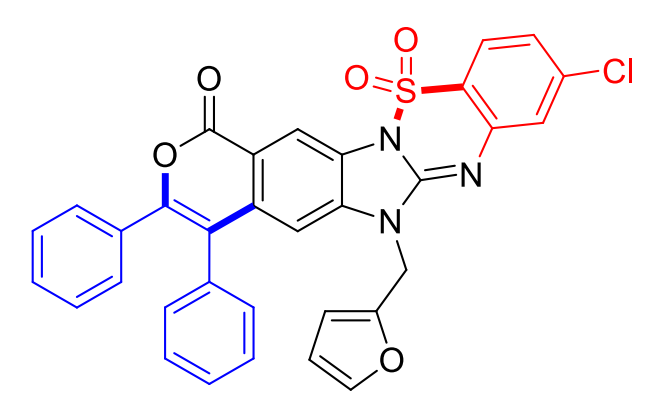

21b

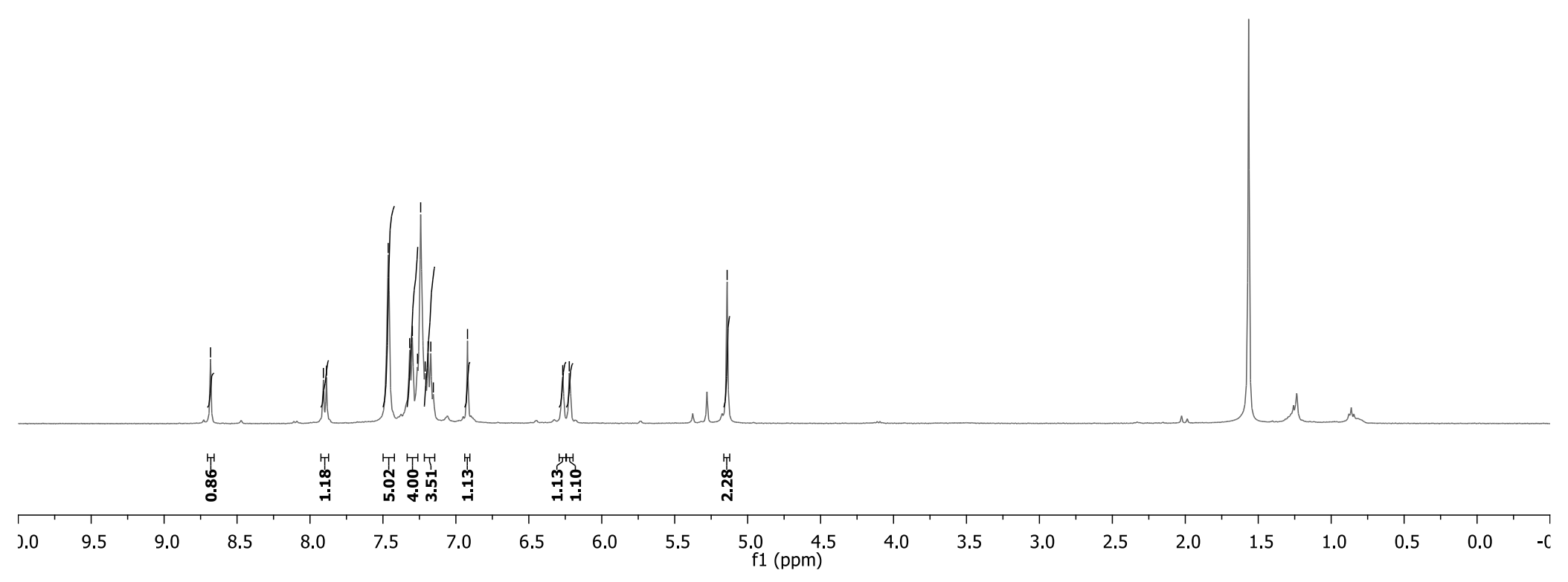

${ }^{1} \mathrm{H}$ NMR spectrum (400 MHz) of compound $\mathbf{2 1 b}$ in $\mathrm{CDCl}_{3}$ 


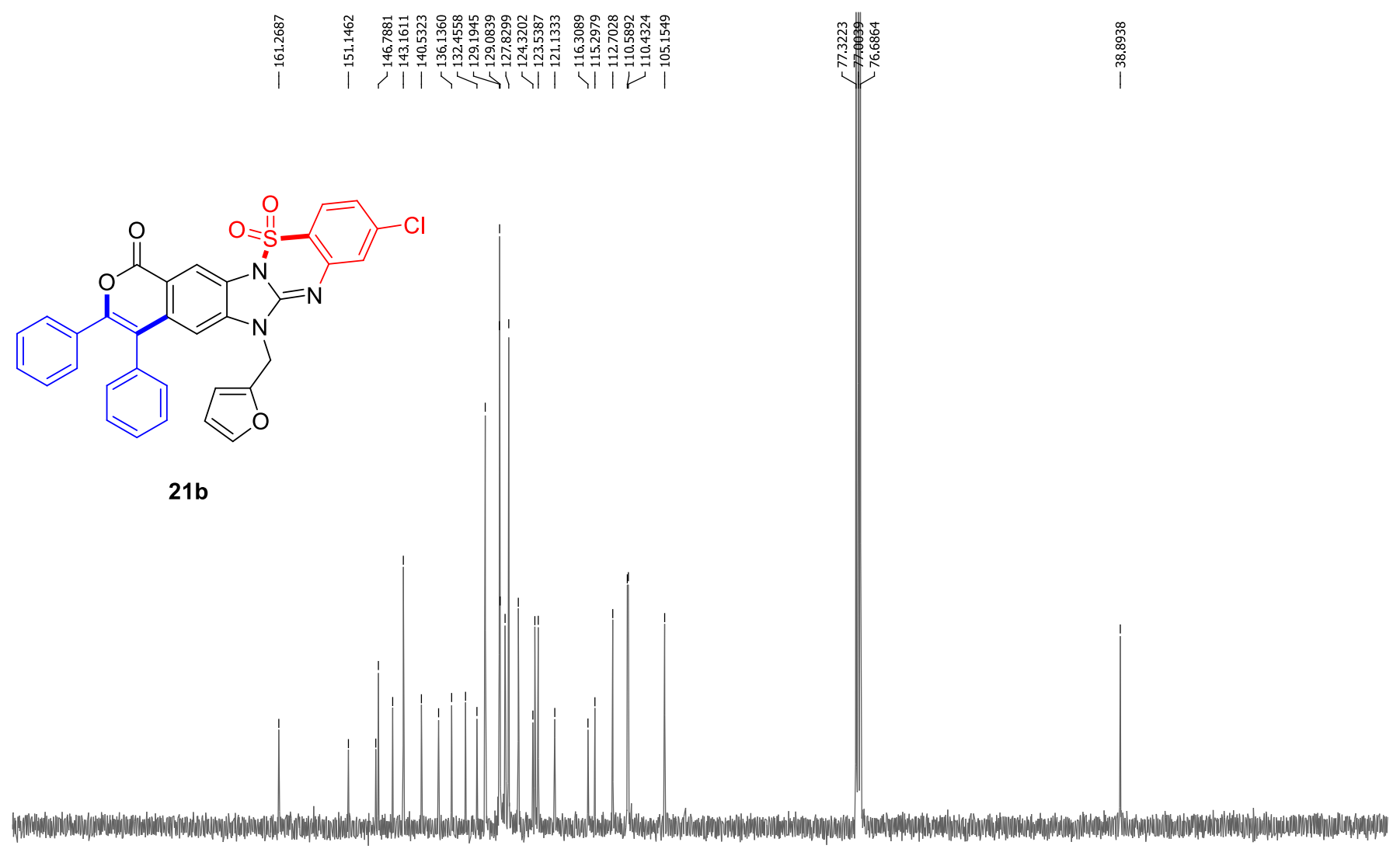

$\begin{array}{lllllllllll}00 & 190 & 180 & 170 & 160 & 150 & 140 & 130 & 120 & 110 & 100\end{array}$

${ }^{13} \mathrm{C}$ NMR spectrum (101 MHz) of compound $\mathbf{2 1 b}$ in $\mathrm{CDCl}_{3}$ 


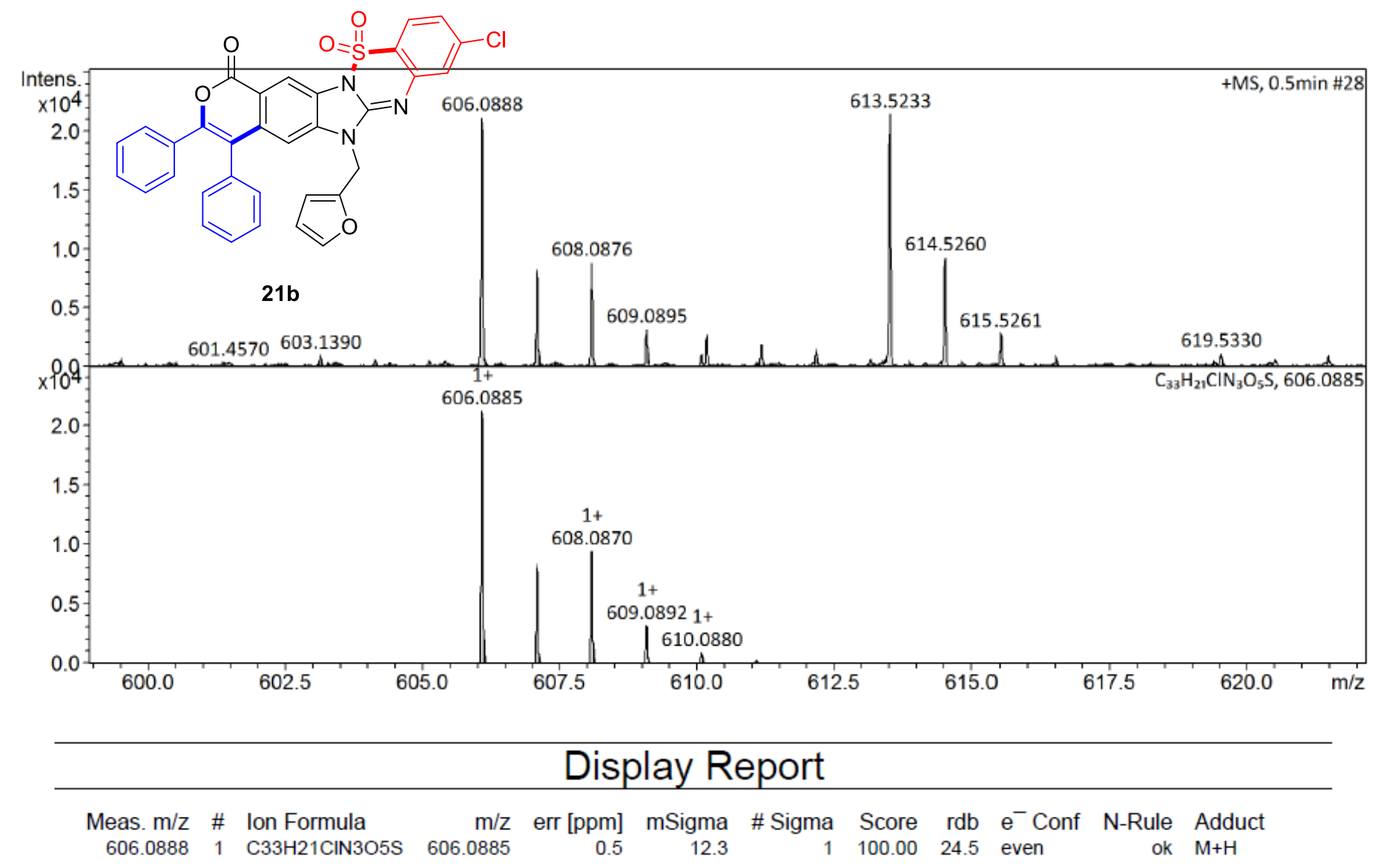

HRMS spectrum (ESI) of compound 21b 
|

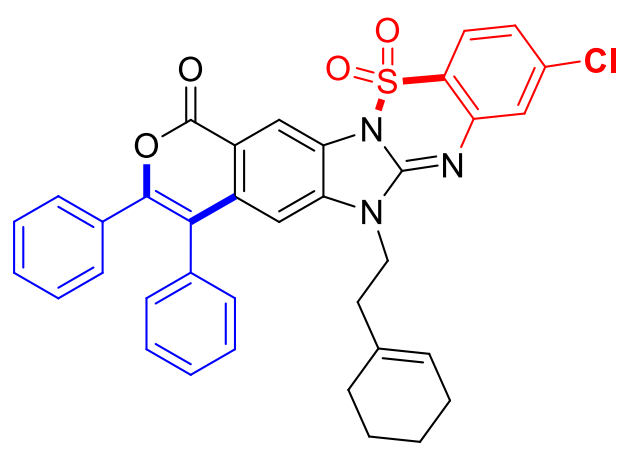

21c

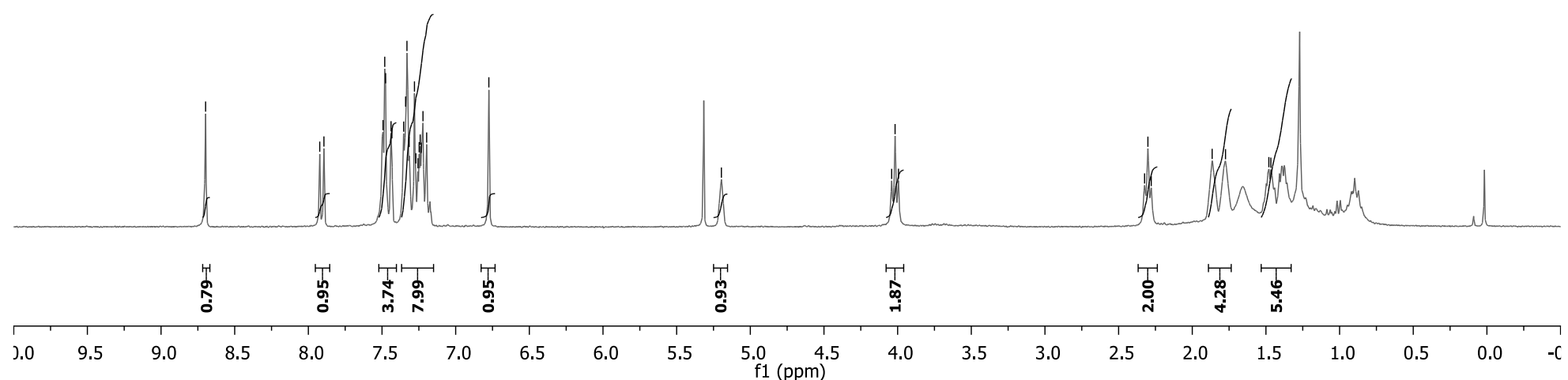

${ }^{1} \mathrm{H}$ NMR spectrum (400 MHz) of compound 21c in $\mathrm{CDCl}_{3}$ 


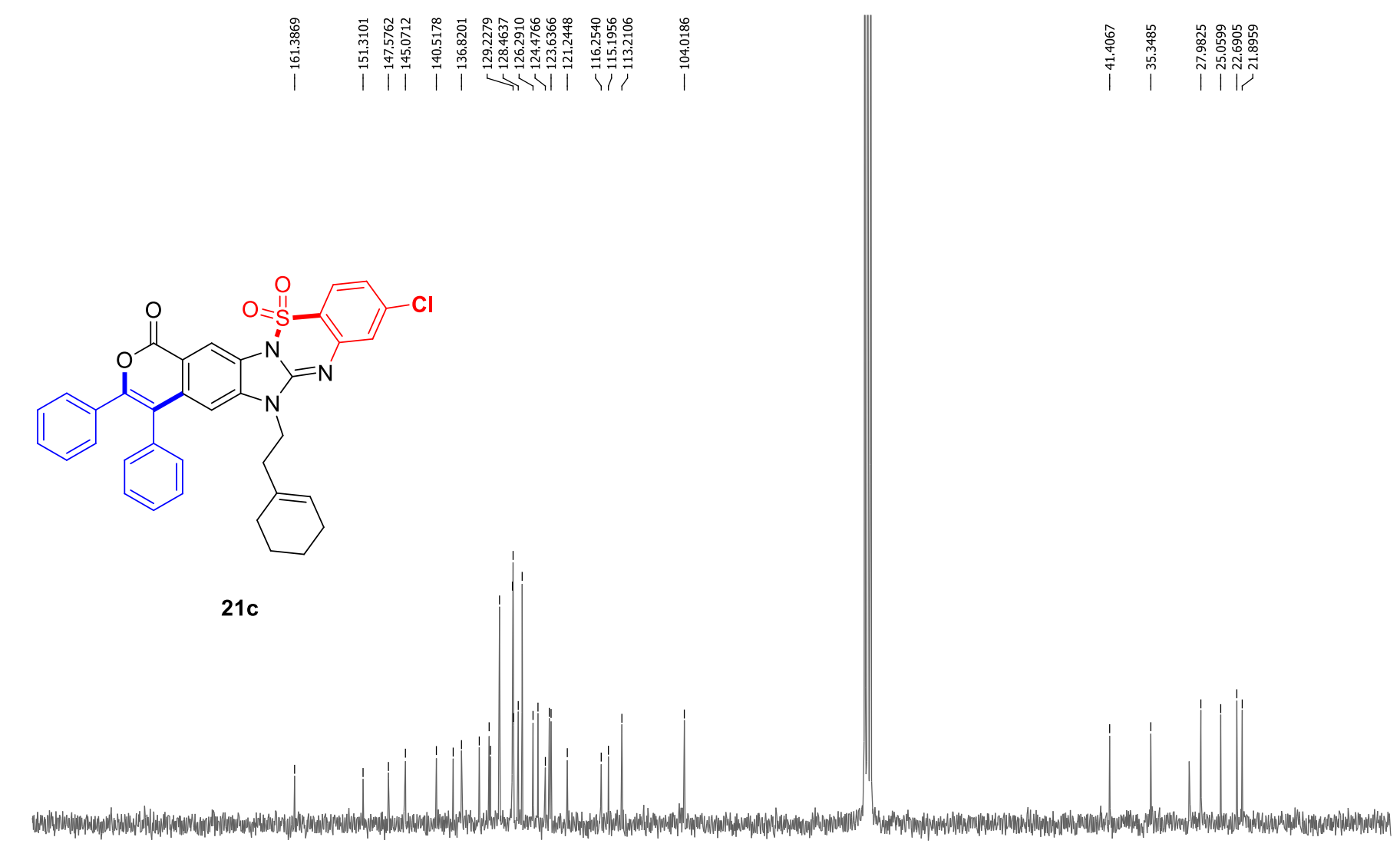

00

$190 \quad 180$

160

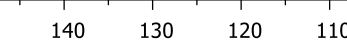

100
$\mathrm{f} 1(\mathrm{ppm})$

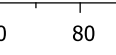

60

50

30 $20 \quad 10$

${ }^{13} \mathrm{C}$ NMR spectrum (101 MHz) of compound 21c in $\mathrm{CDCl}_{3}$ 


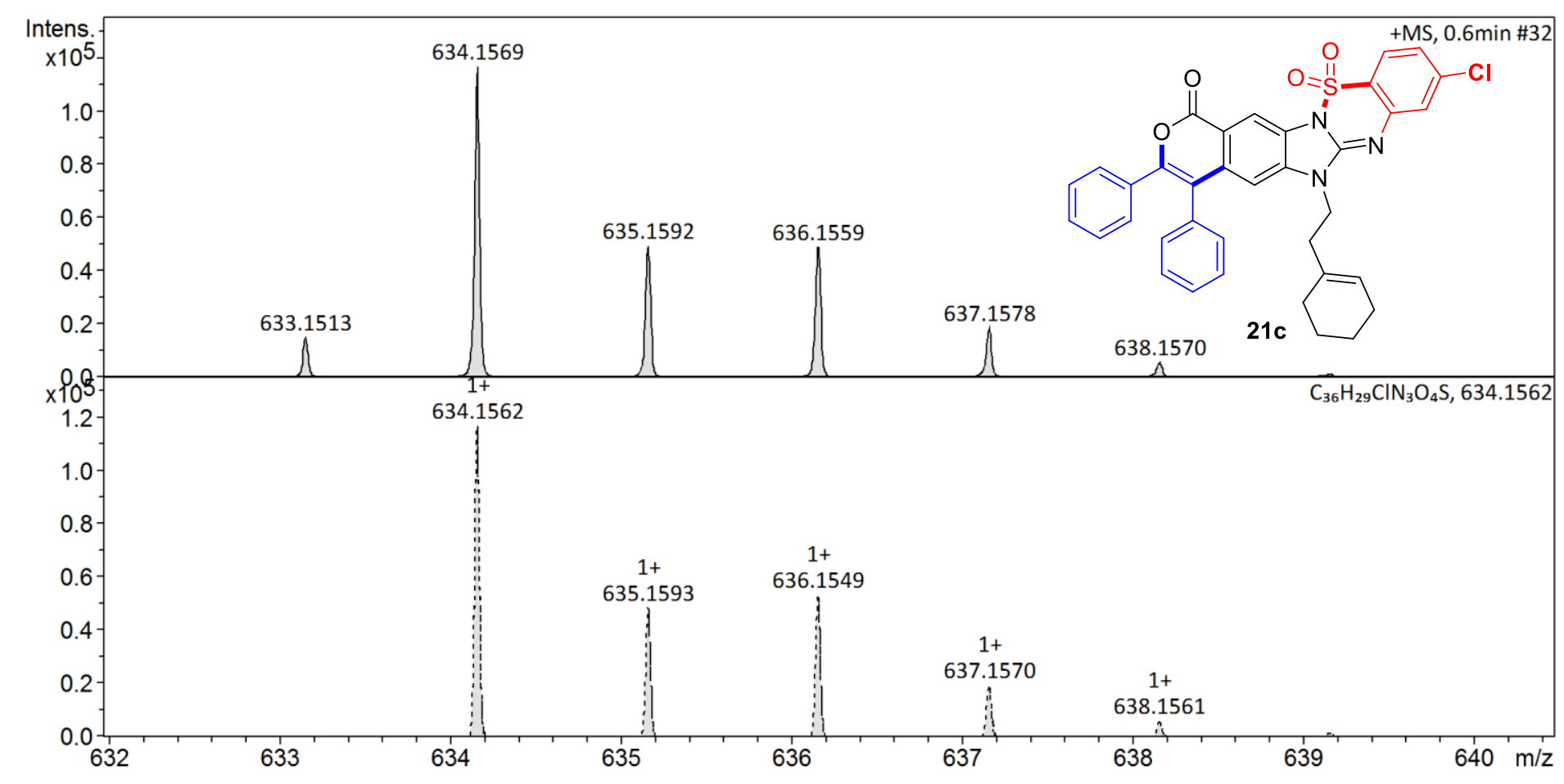

\section{Display Report}

Meas. $\mathrm{m} / \mathrm{z}$ \# Ion Formula $\mathrm{m} / \mathrm{z}$ err [ppm] mSigma \# Sigma Score $\mathrm{rdb} \mathrm{e}^{-}$Conf $\mathrm{N}$-Rule Adduct

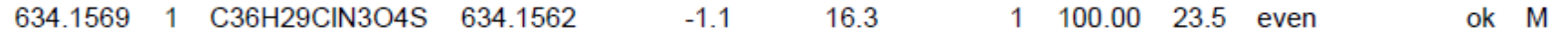

HRMS spectrum (ESI) of compound 21c 

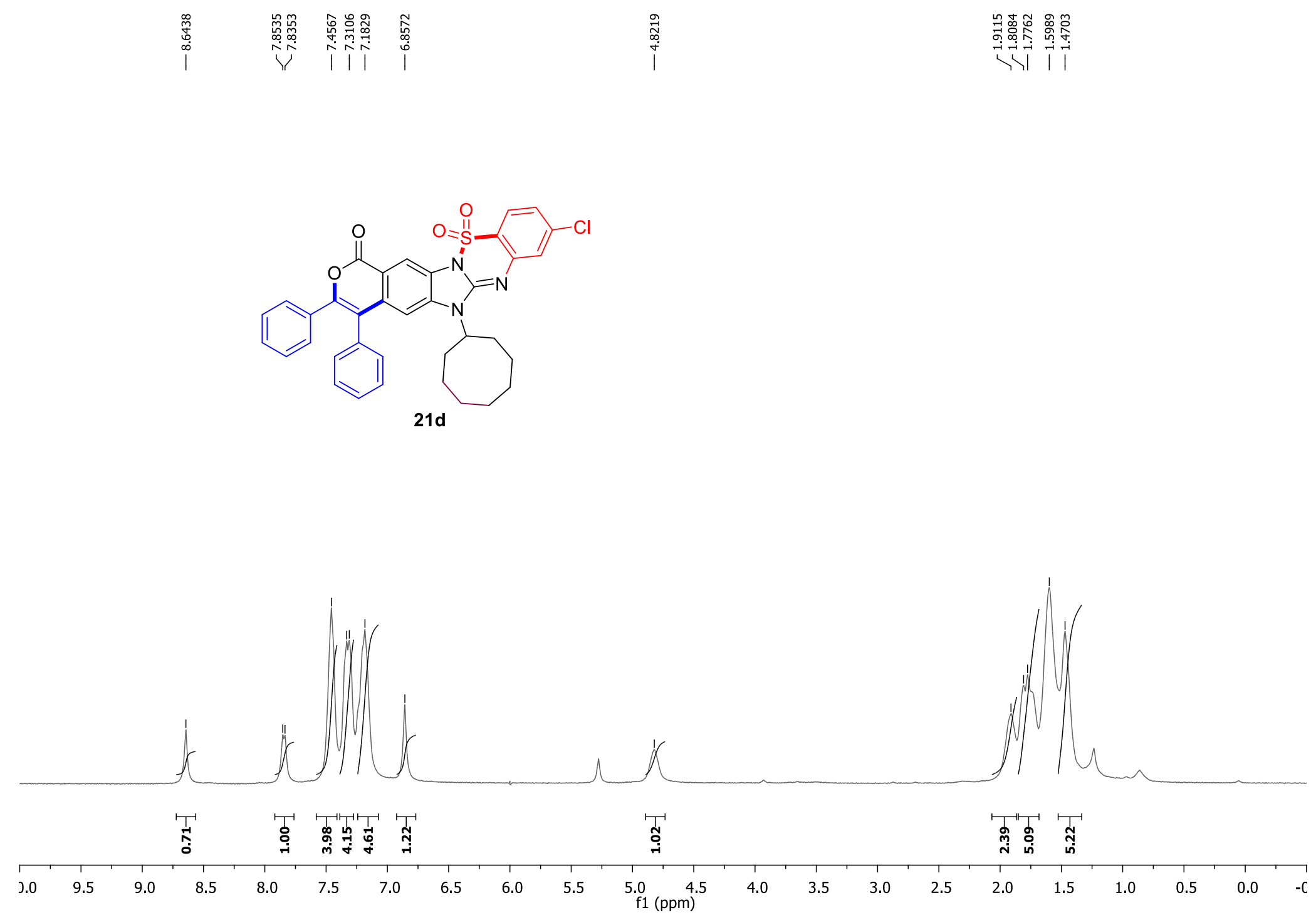

${ }^{1} \mathrm{H}$ NMR spectrum (400 MHz) of compound 21d in $\mathrm{CDCl}_{3}$ 

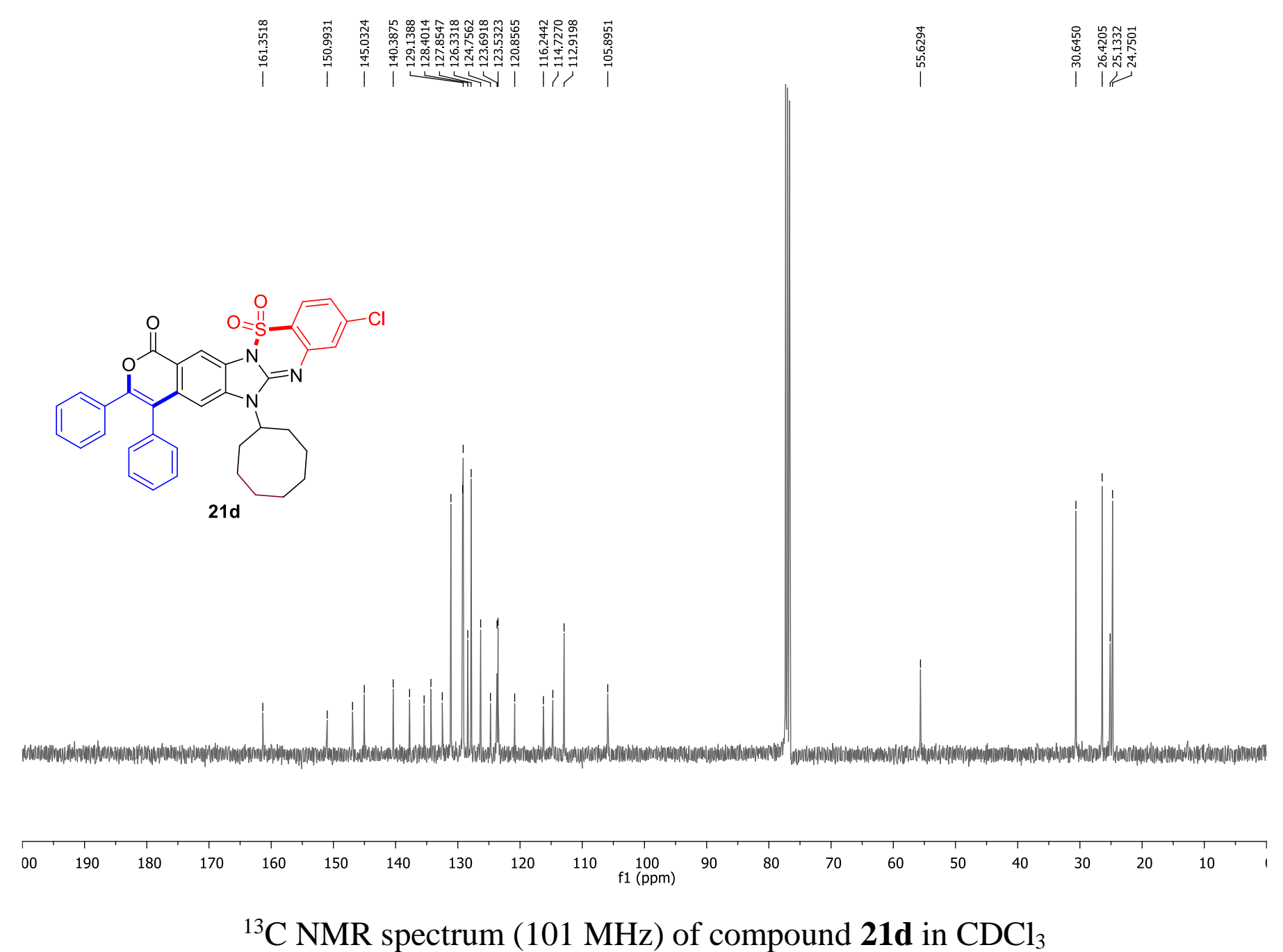


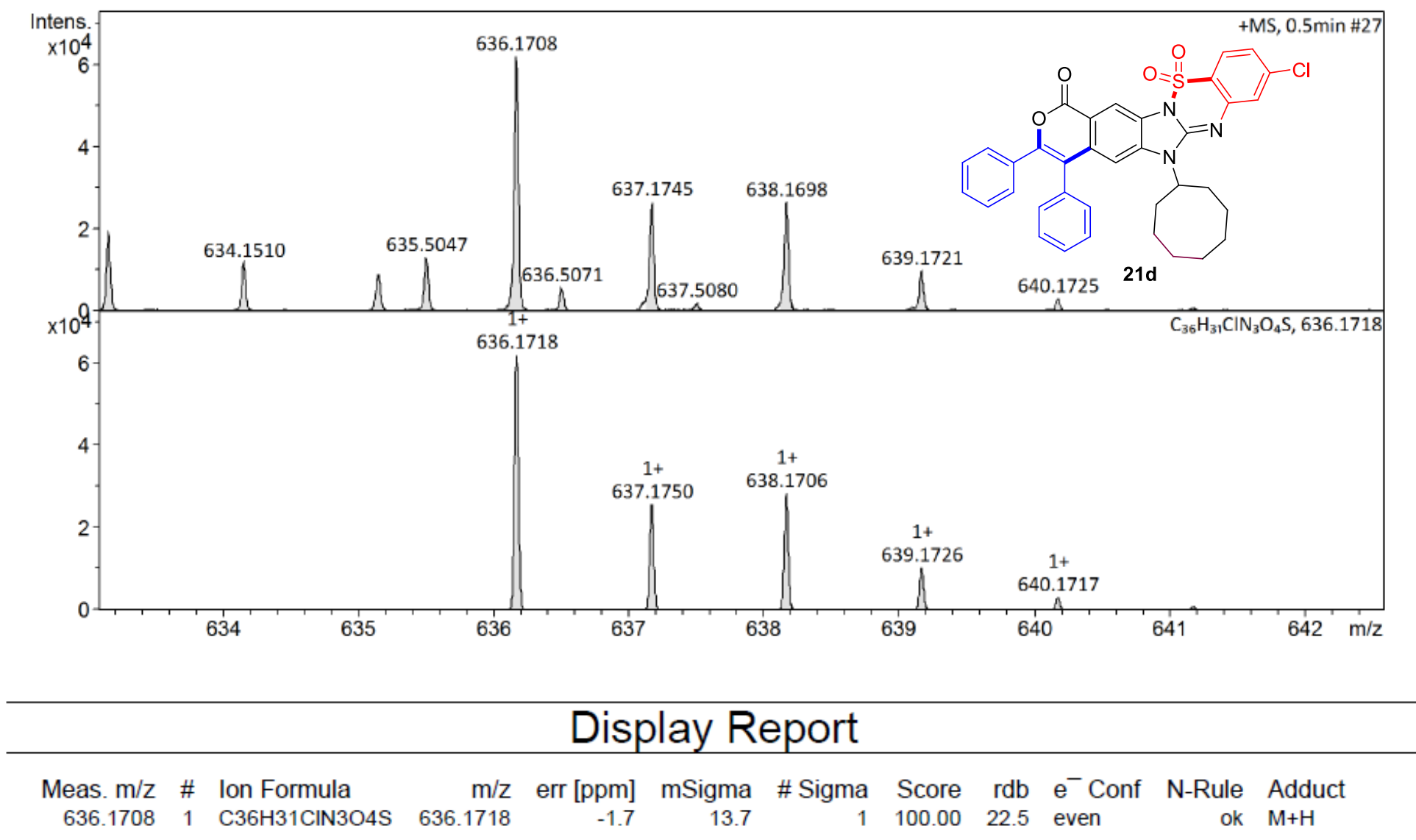

HRMS spectrum (ESI) of compound 21d 


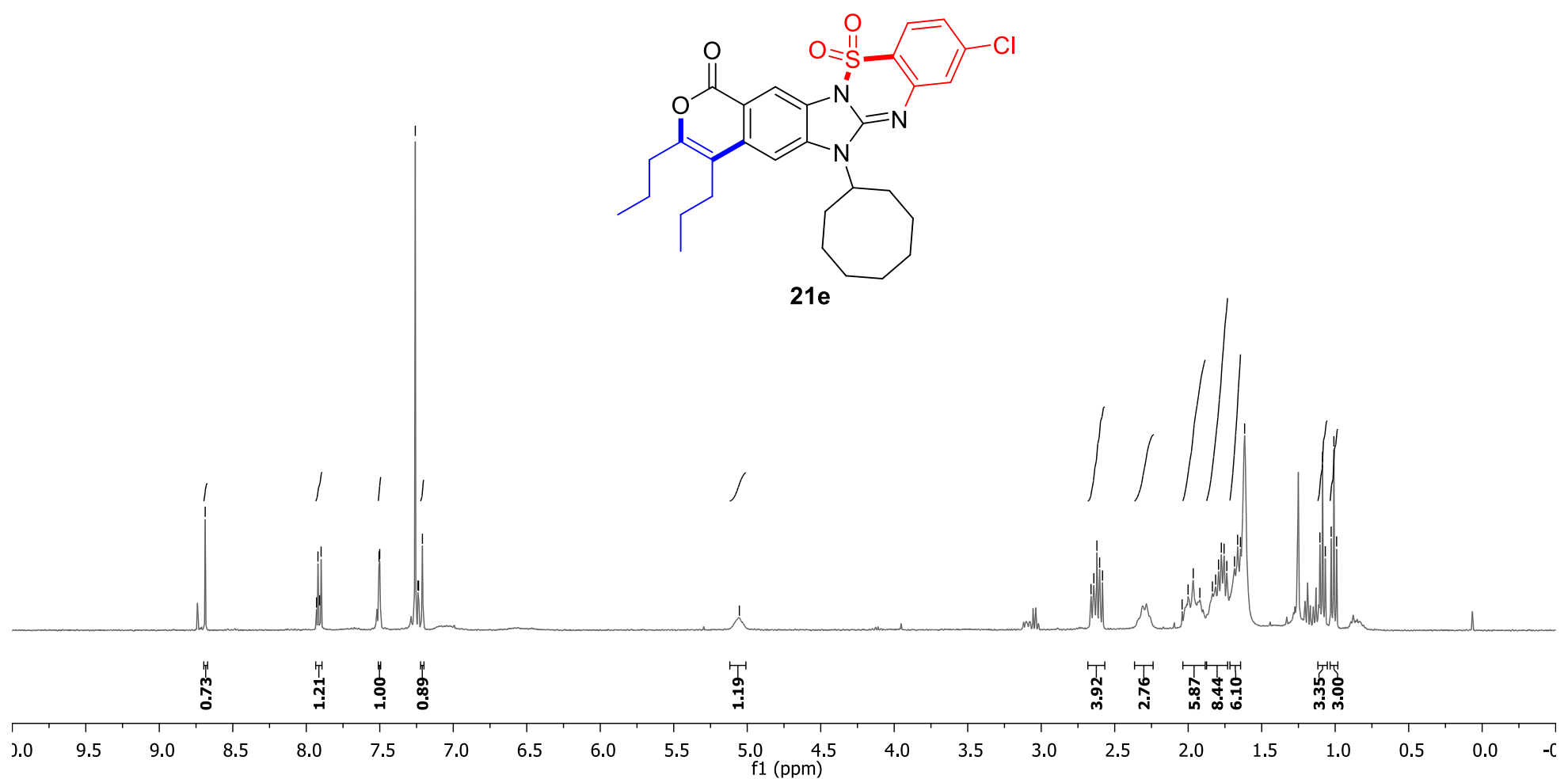

${ }^{1} \mathrm{H}$ NMR spectrum (400 MHz) of compound 21e in $\mathrm{CDCl}_{3}$ 

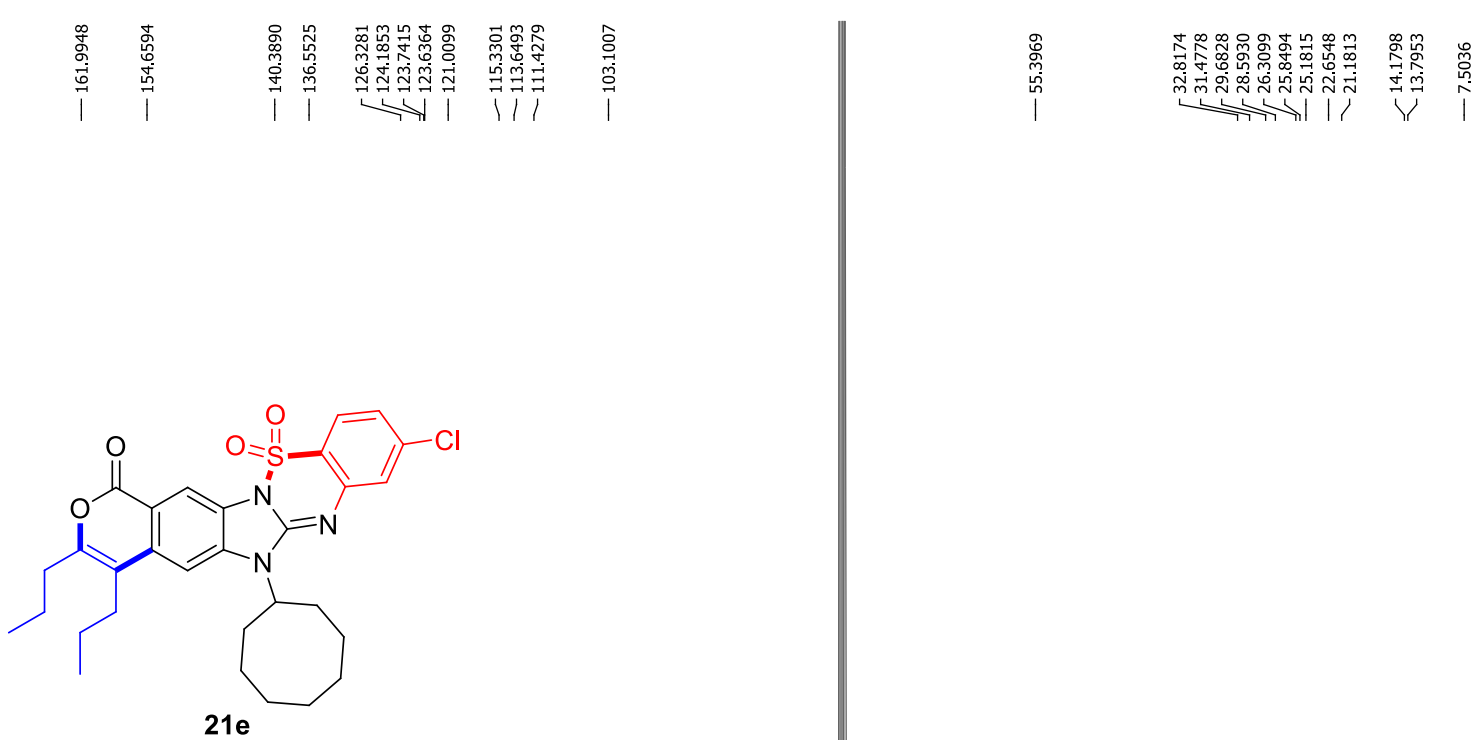

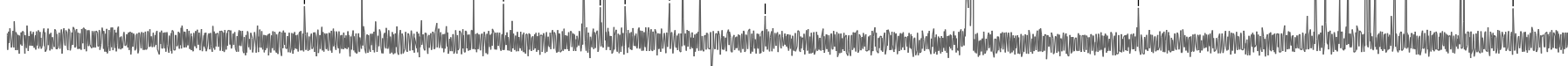

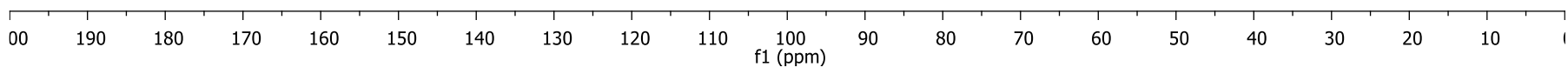

${ }^{13} \mathrm{C}$ NMR spectrum (101 MHz) of compound 21e in $\mathrm{CDCl}_{3}$ 


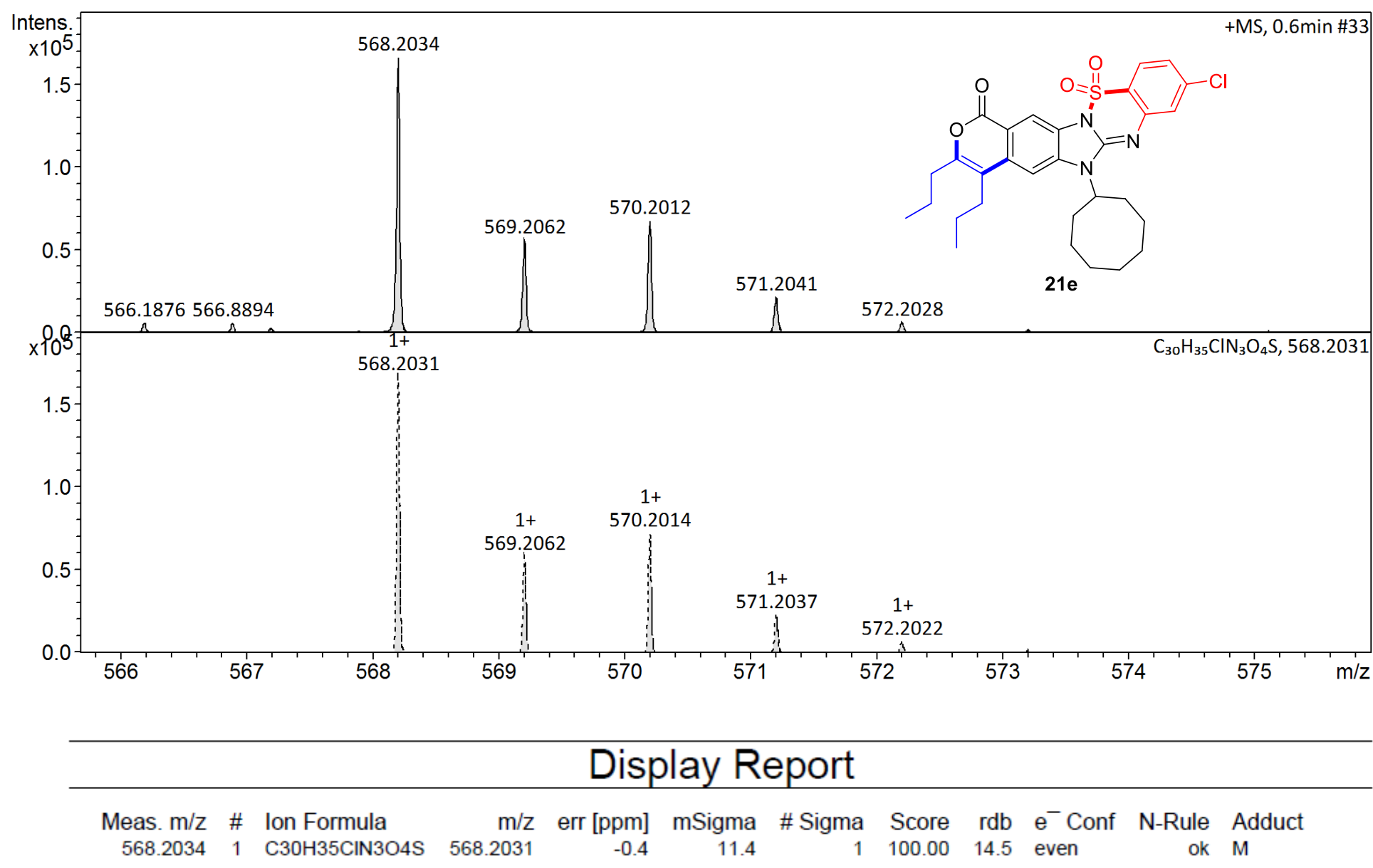

HRMS spectrum (ESI) of compound 21e 

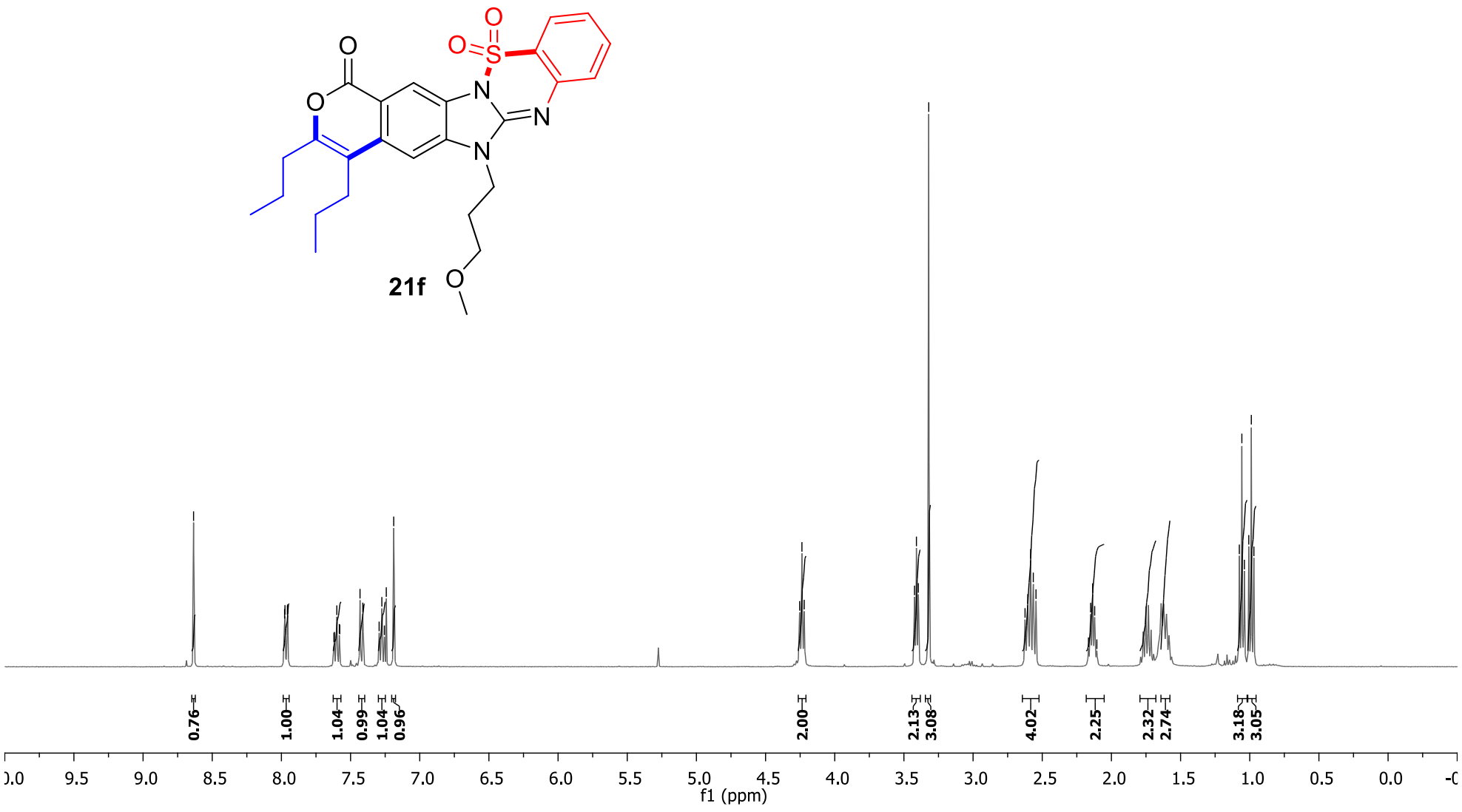

${ }^{1} \mathrm{H}$ NMR spectrum (400 MHz) of compound $21 f$ in $\mathrm{CDCl}_{3}$ 

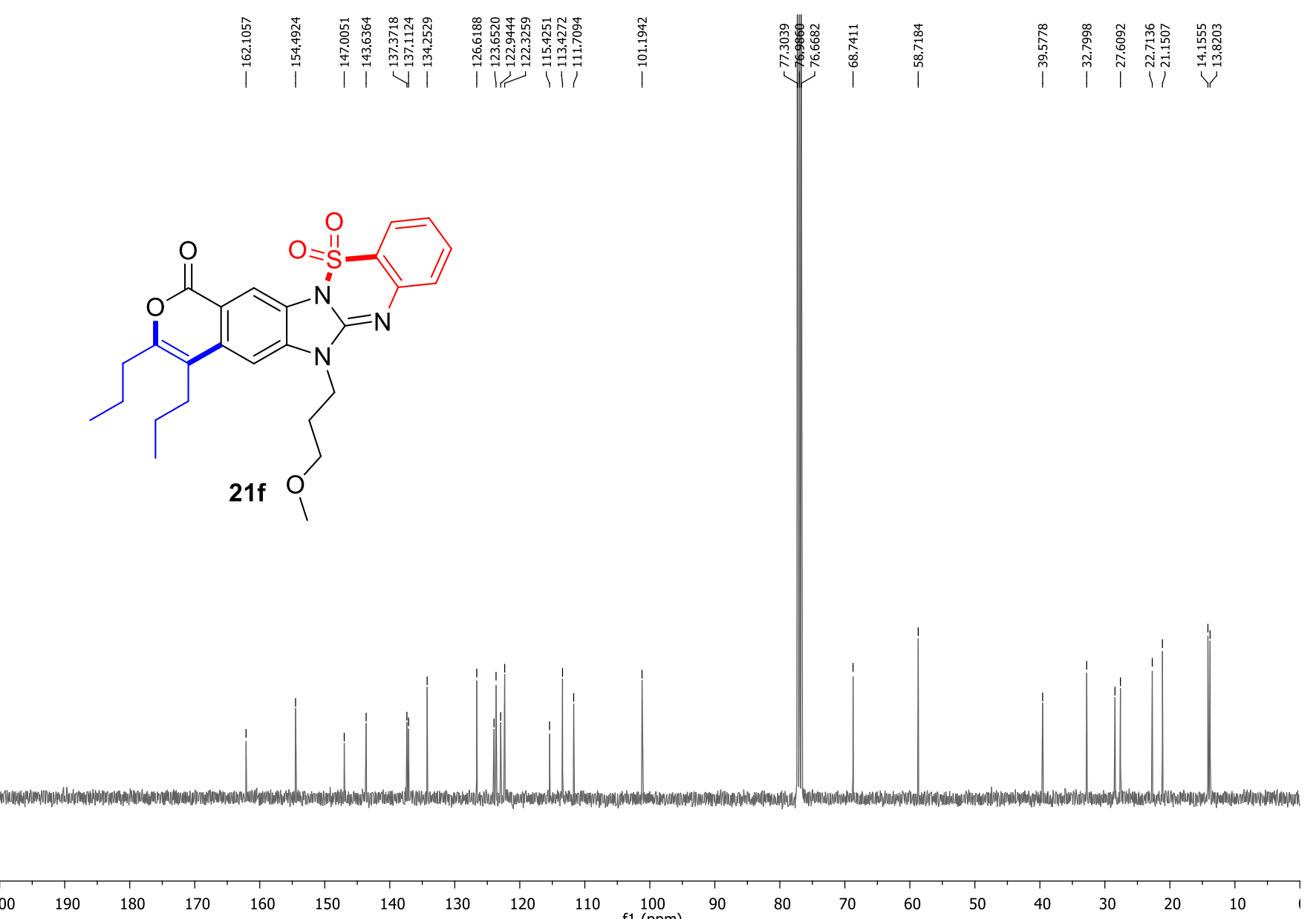

160

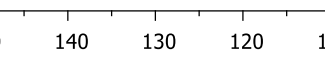

$10 \quad 100$

80

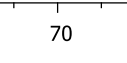

60

30

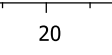

${ }^{13} \mathrm{C}$ NMR spectrum $(101 \mathrm{MHz})$ of compound $21 \mathrm{f}$ in $\mathrm{CDCl}_{3}$ 


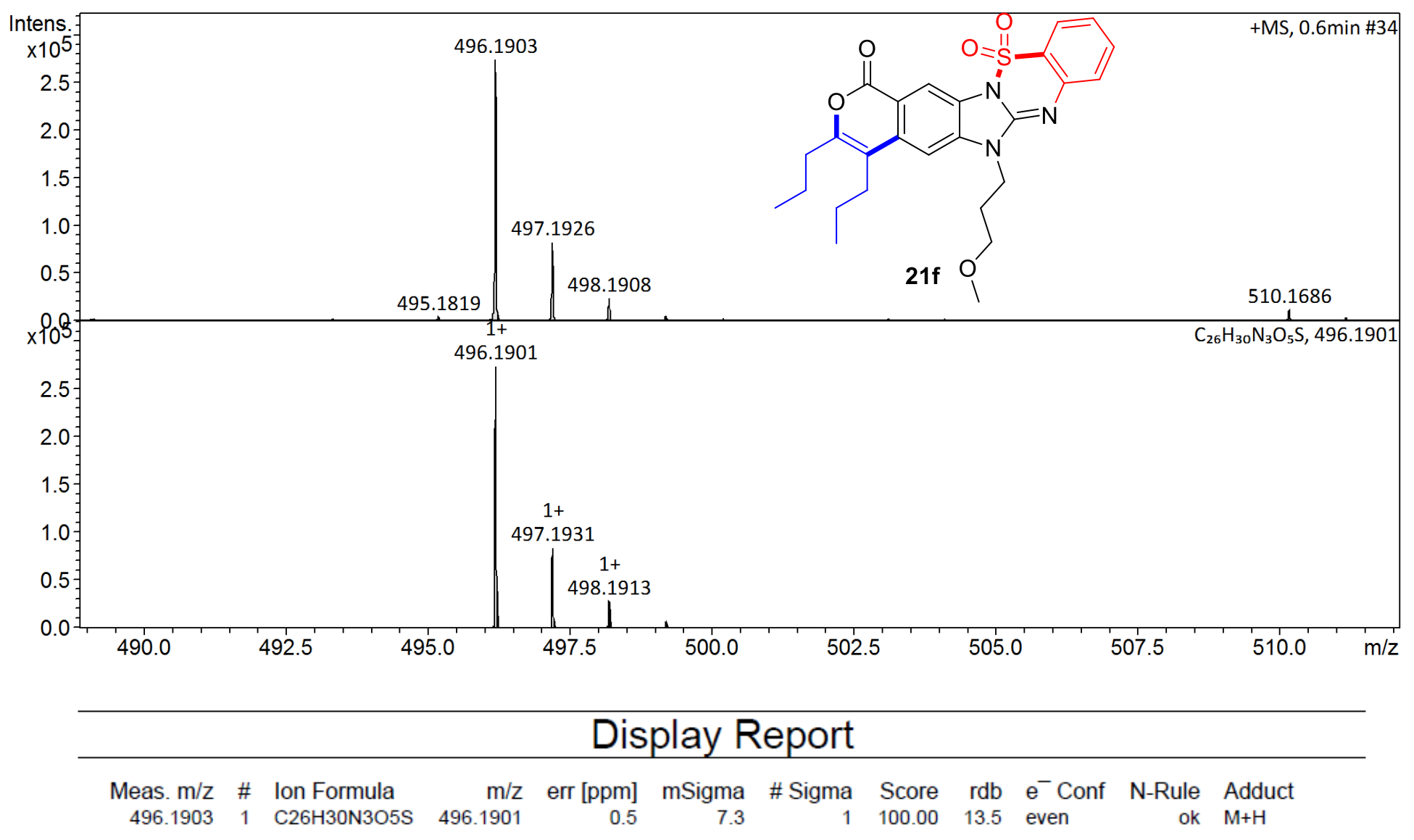

HRMS spectrum (ESI) of compound $21 \mathbf{f}$ 


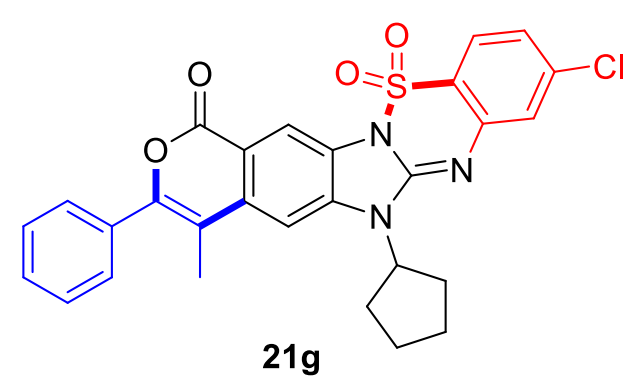

21g

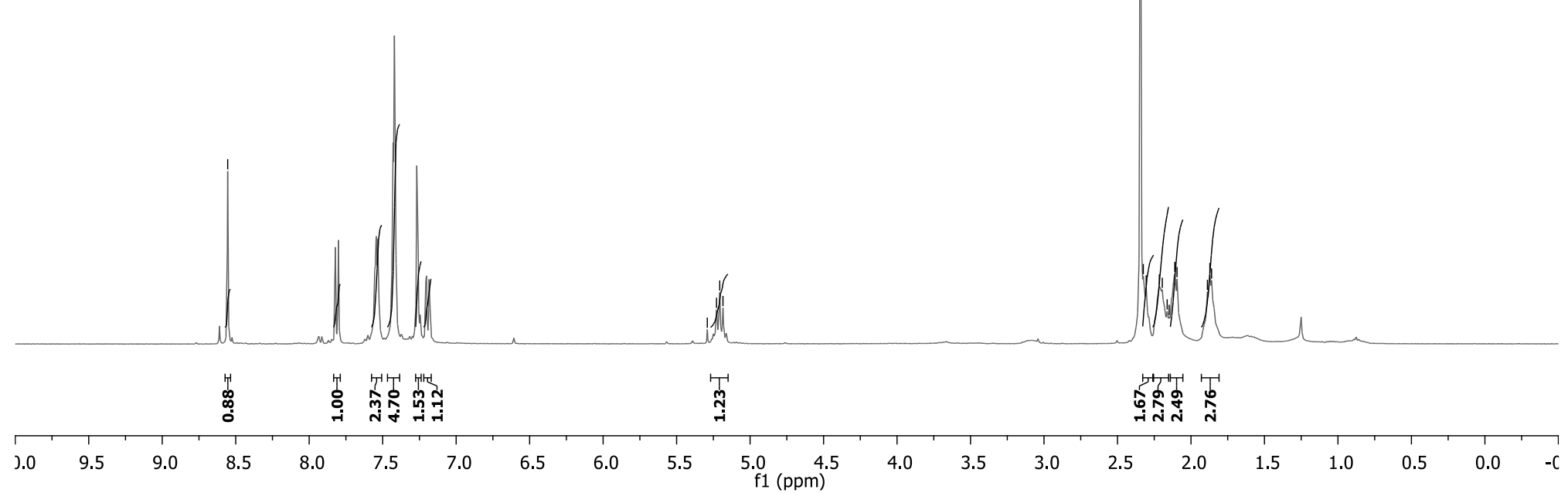

${ }^{1} \mathrm{H}$ NMR spectrum (400 MHz) of compound $21 \mathrm{~g}$ in $\mathrm{CDCl}_{3}$ 

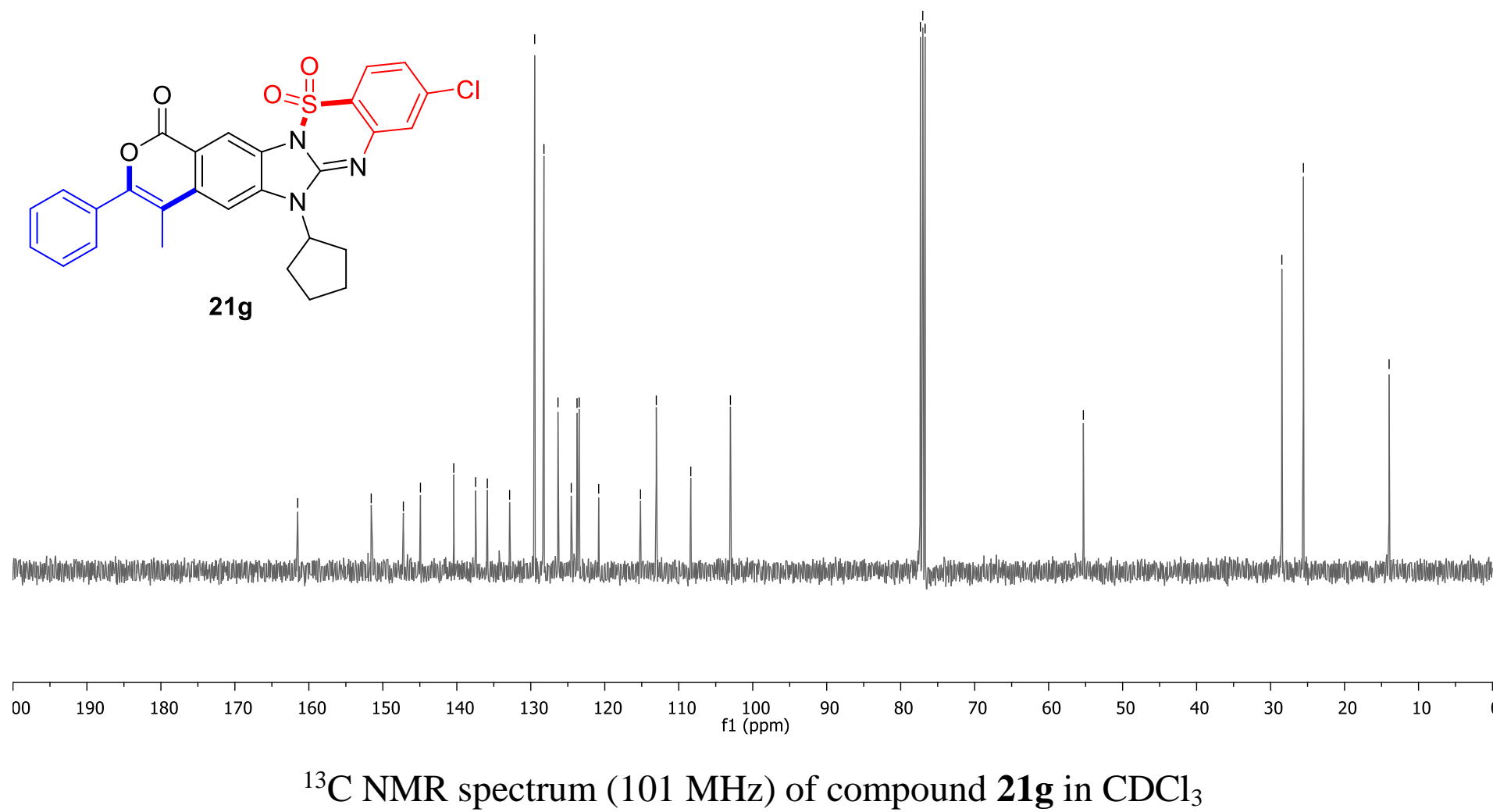


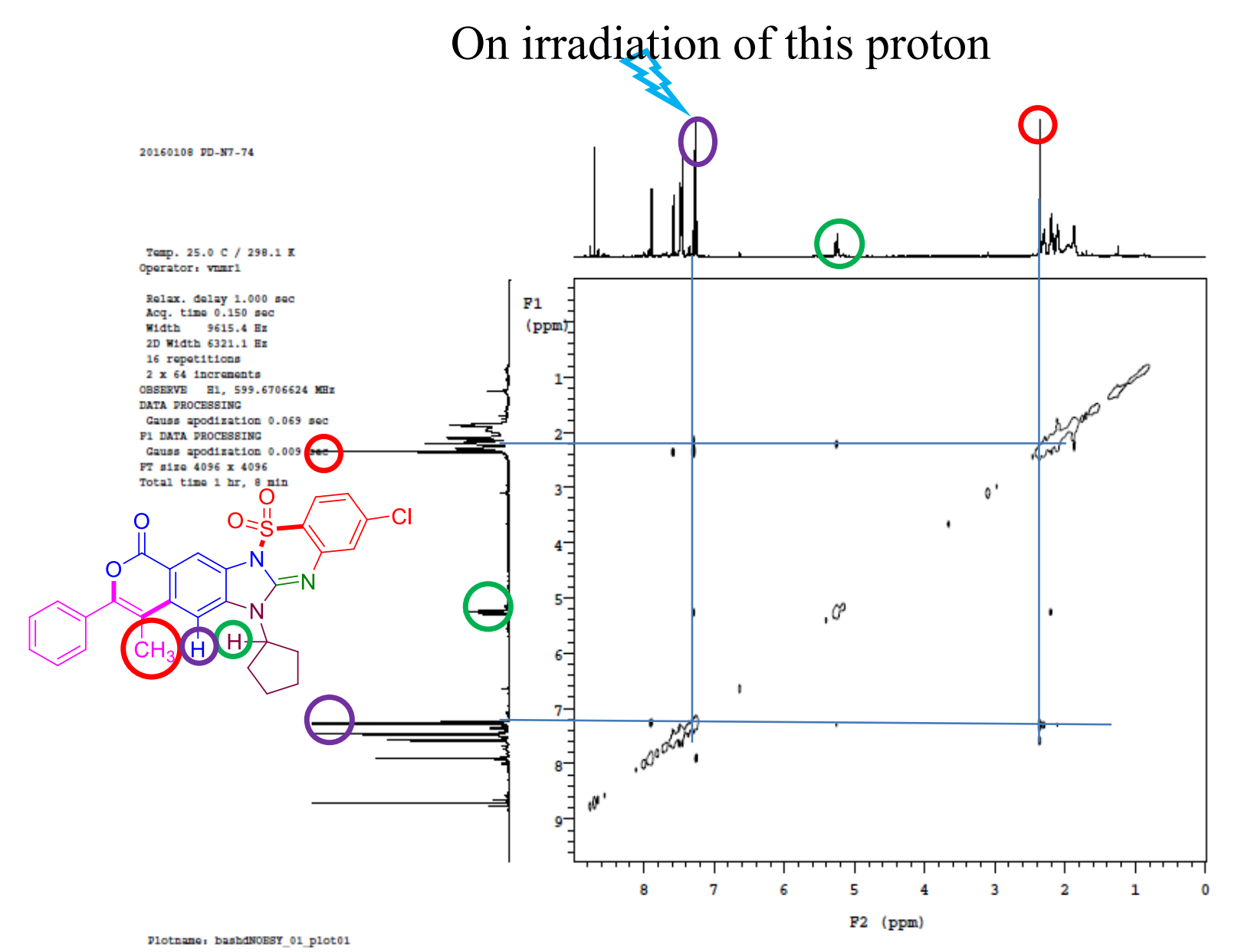

BashdNOESY spectrum $(500 \mathrm{MHz})$ of compound $\mathbf{2 1} \mathbf{g}$ in $\mathrm{CDCl}_{3}$ 
Band selective homonuclear 2D correlation experiment (bashdNOESY) showed a clear relation with methyl protons on irradiating a $\mathrm{C}^{6}-\mathrm{H}$ proton in compound $\mathbf{2 1 g}$. This suggested that these two protons are in close proximity as a result of sterically less crowded C-C bond formation. These results are also in good agreement with the available literature reports. ${ }^{1 \mathrm{a}, \mathrm{b}}$

\section{References:}

[1] (a) Q. Li, Y. Yan, X. Wang, B. Gong, X. Tang, J. Shi, H. E. Xu, W. Yi, RSC Adv. 2013, 3, 23402-23408. (b) J. Pospech, K. Graczyk, K. Rauch, L. Ackermann, Org. Lett. 2012, 14, 930-933 


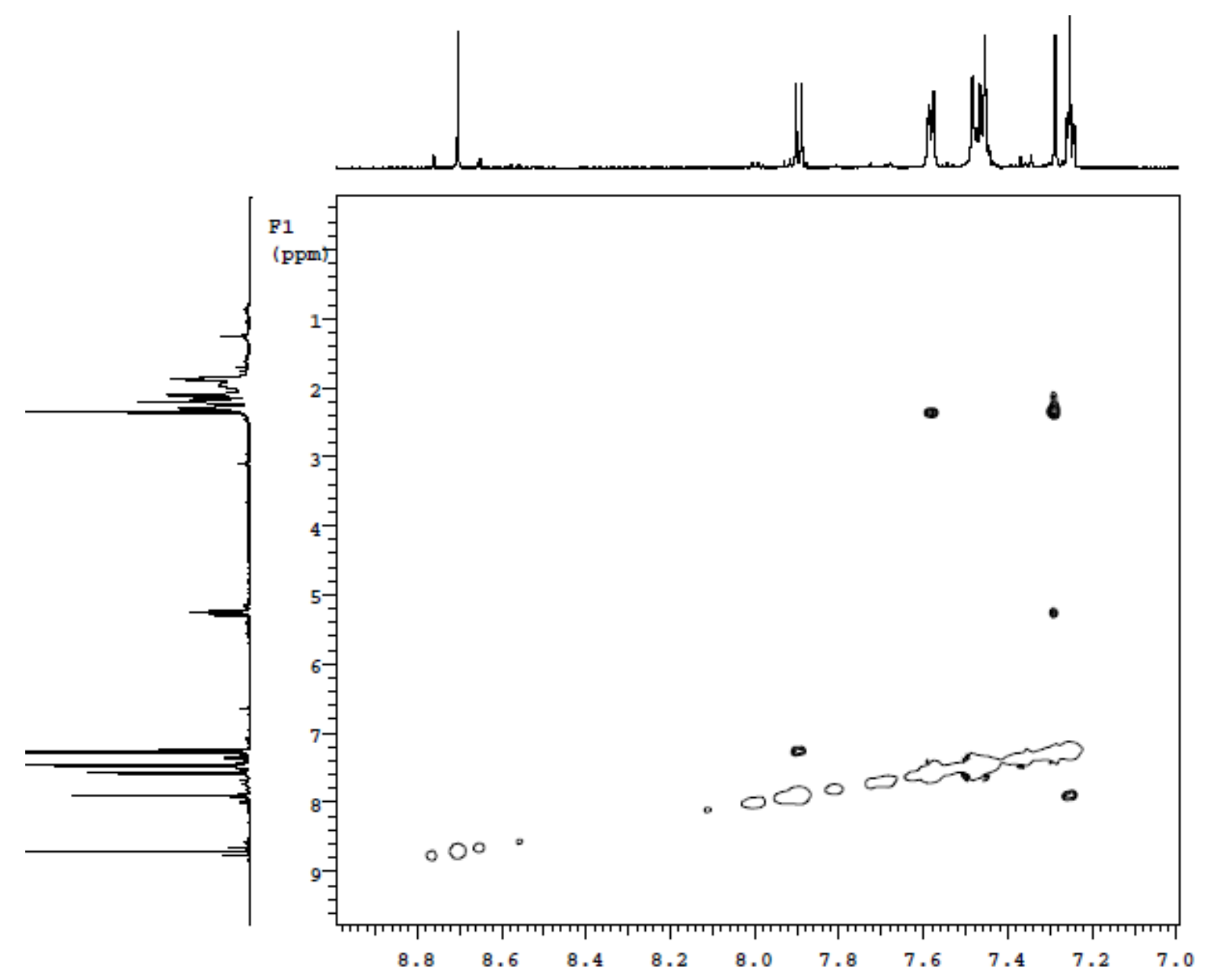

An expansion of bashdNOESY spectrum (500 MHz) of compound $21 \mathrm{~g}$ in $\mathrm{CDCl}_{3}$ 


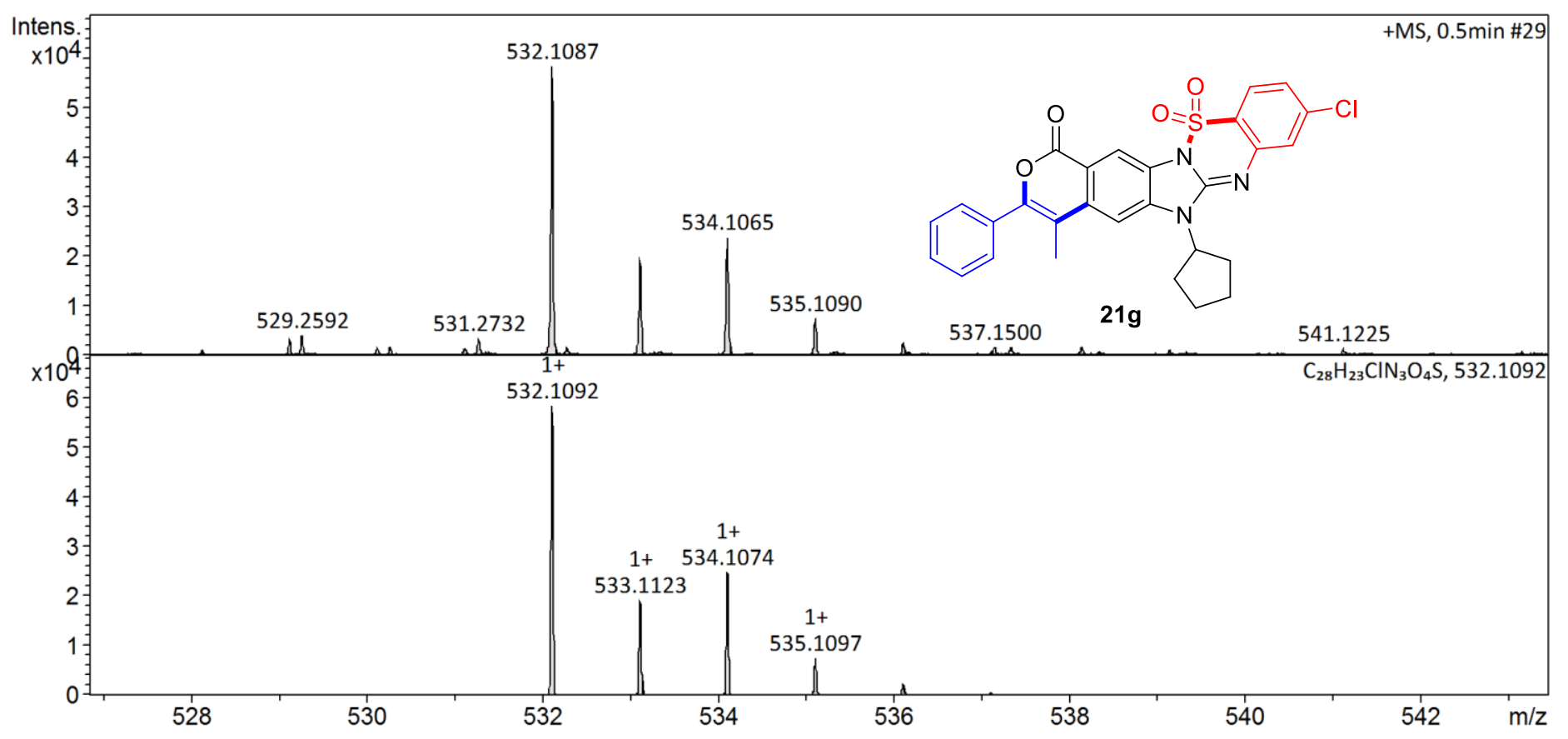

\section{Display Report}
Meas. $\mathrm{m} / \mathbf{z}$ \# Ion Formula
$\mathrm{m} / \mathbf{z}$ err [ppm] mSigma \# Sigma Score $\mathrm{rdb} e^{-}$Conf N-Rule Adduct $532.1087 \quad 1 \quad \mathrm{C} 28 \mathrm{H} 23 \mathrm{CIN} 3 \mathrm{O} 4 \mathrm{~S} \quad 532.1092$
1.0 Sigma

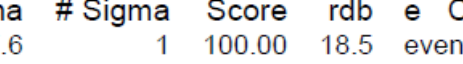
ok $\mathrm{M}+\mathrm{H}$

HRMS spectrum (ESI) of compound $\mathbf{2 1 g}$ 


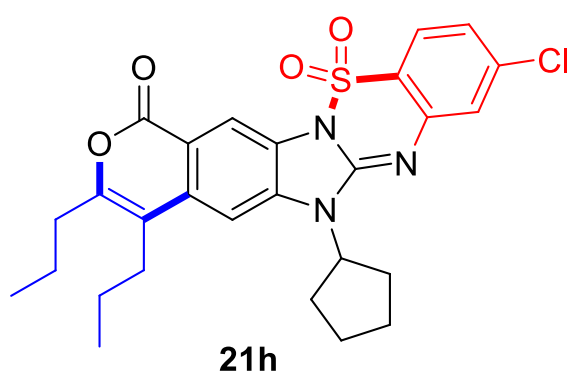

$21 \mathrm{~h}$

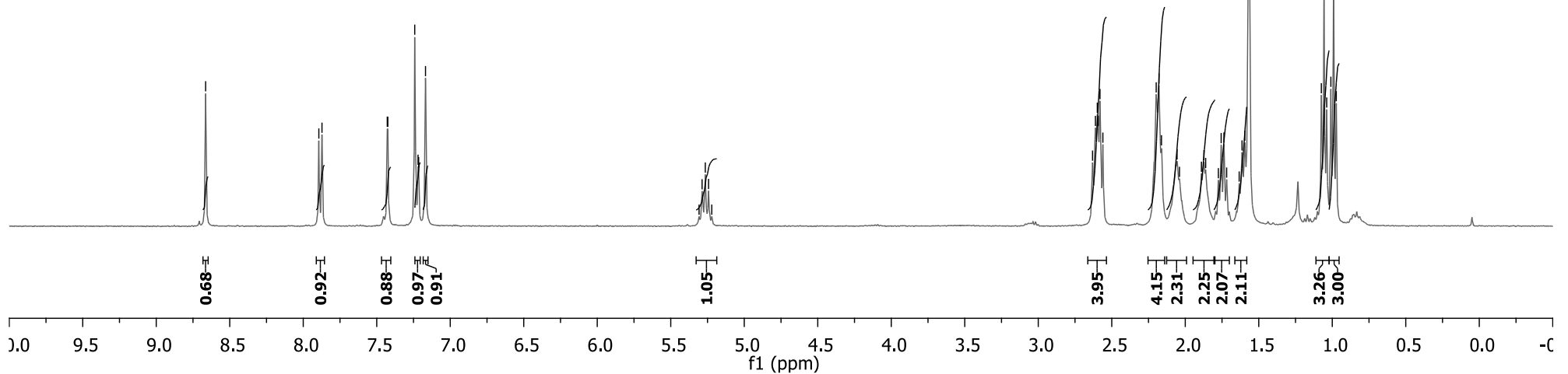

${ }^{1} \mathrm{H}$ NMR spectrum $\left(400 \mathrm{MHz}\right.$ ) of compound $21 \mathbf{h}$ in $\mathrm{CDCl}_{3}$ 


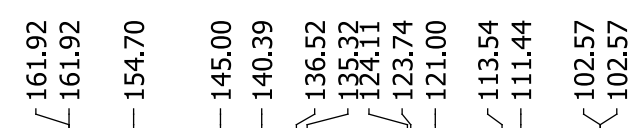

다

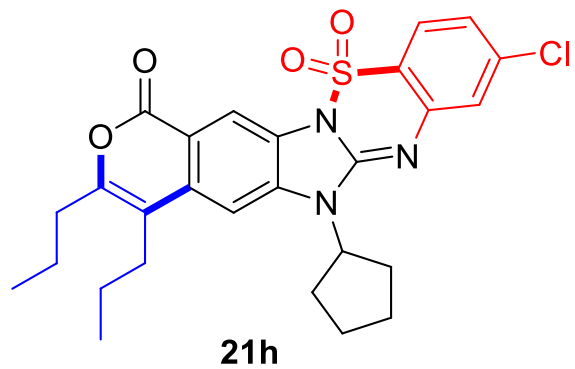

$21 \mathrm{~h}$

$\begin{array}{llllllllll}00 & 190 & 180 & 170 & 160 & 150 & 140 & 130 & 120 & 110 \begin{array}{l}100 \\ \mathrm{f} 1(\mathrm{ppm})\end{array}\end{array}$
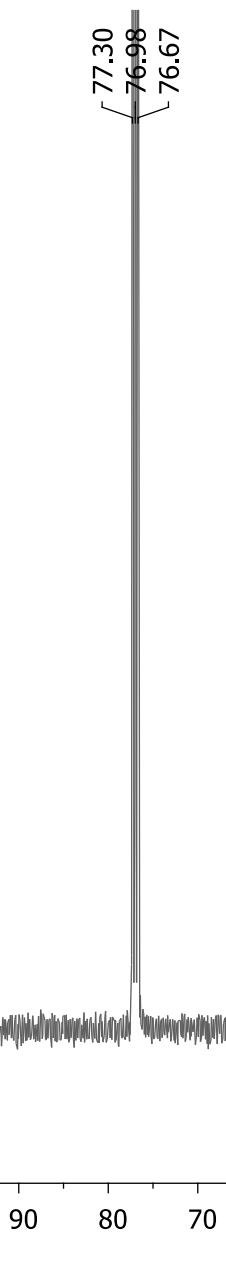

管
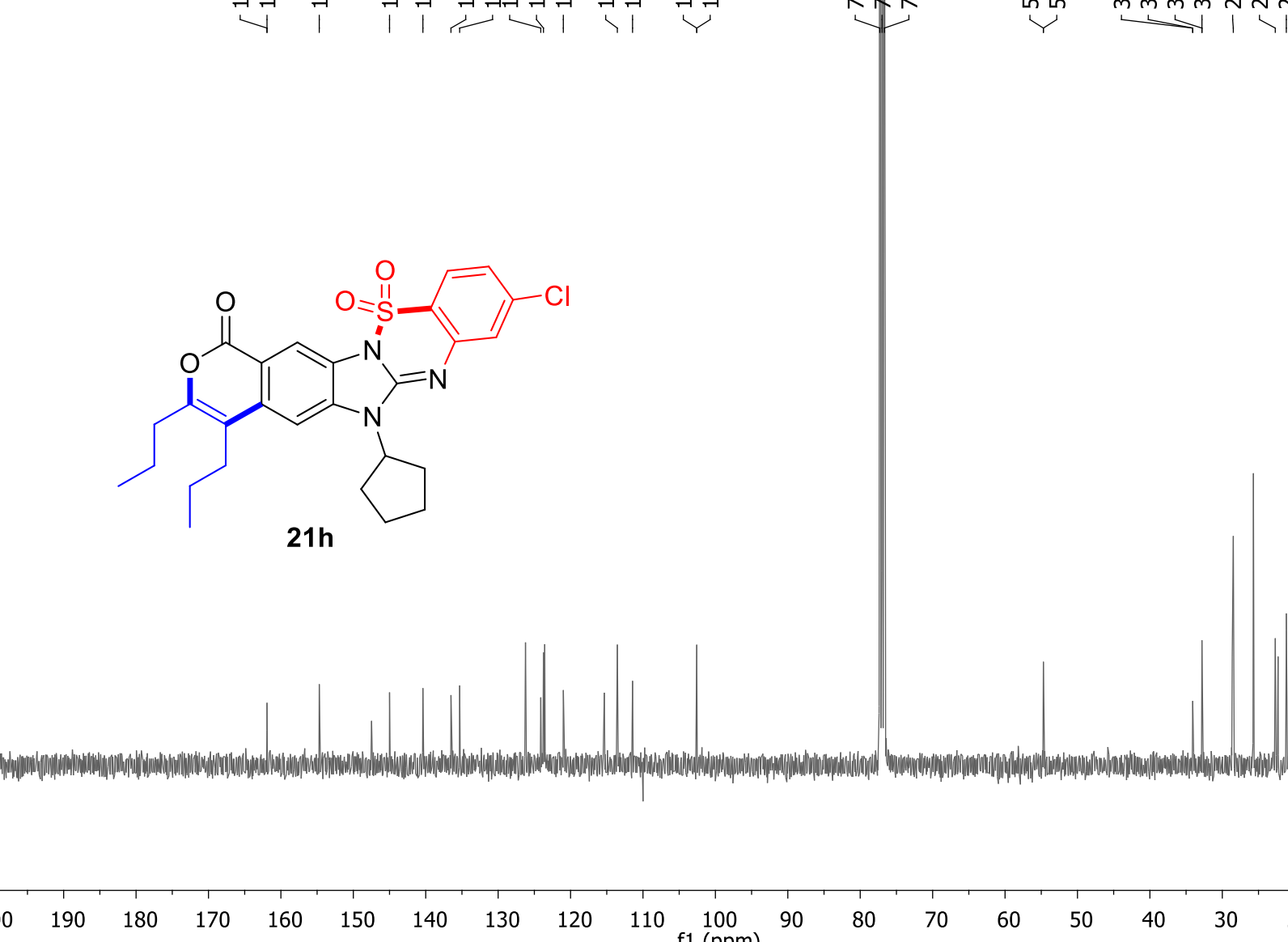

${ }^{13} \mathrm{C}$ NMR spectrum (101 MHz) of compound $21 \mathbf{h}$ in $\mathrm{CDCl}_{3}$ 


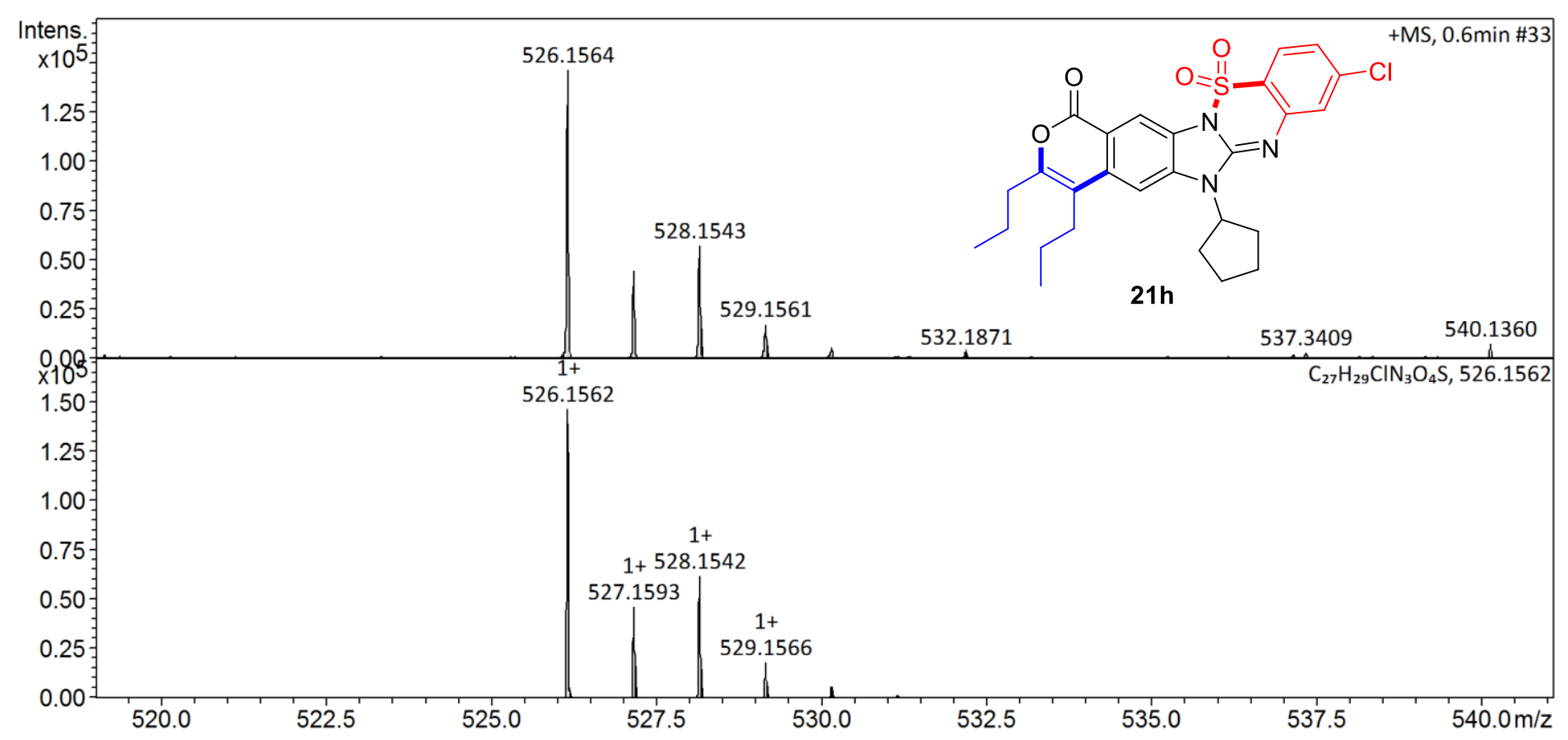

\section{Display Report}

Meas. $\mathrm{m} / \mathbf{z}$ \# Ion Formula $\mathrm{m} / \mathbf{z}$ err [ppm] mSigma \#Sigma Score $\mathrm{rdb} e^{-}$Conf N-Rule Adduct

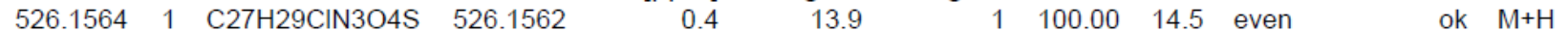

HRMS spectrum (ESI) of compound $\mathbf{2 1 h}$ 


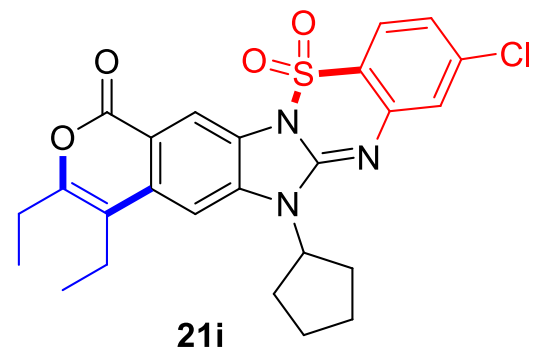

21

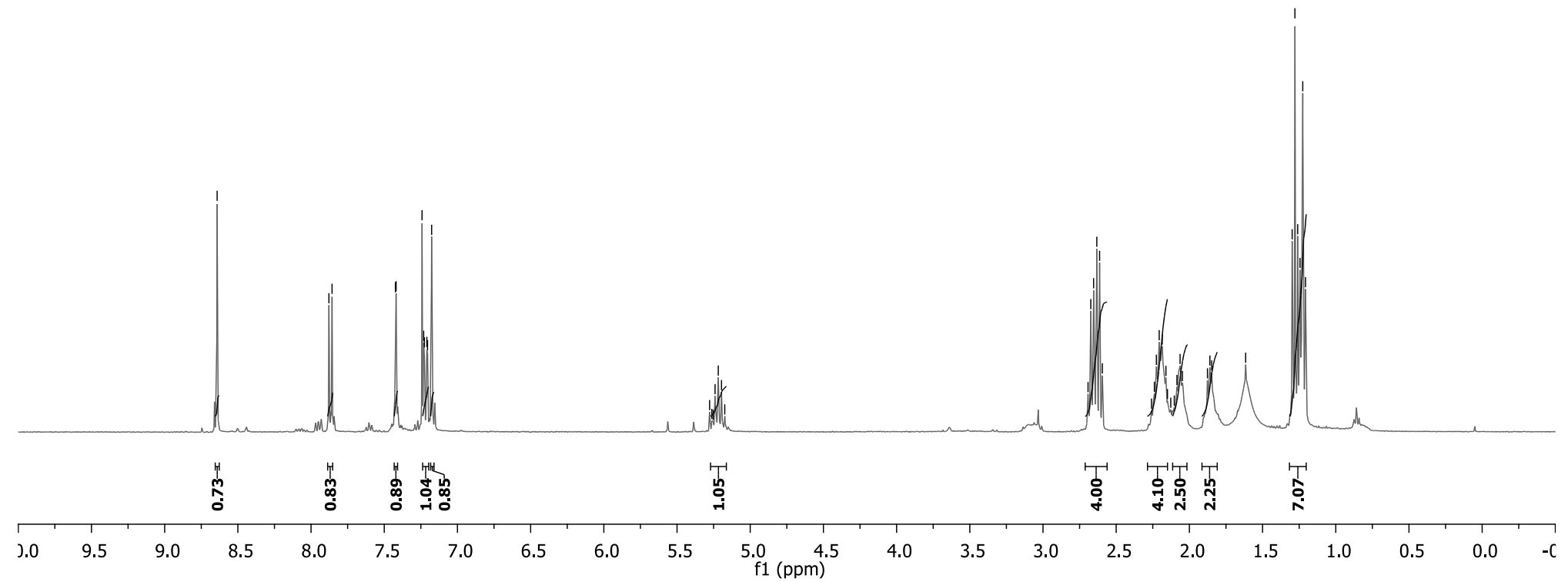

${ }^{1} \mathrm{H}$ NMR spectrum (400 MHz) of compound 21i in $\mathrm{CDCl}$ 


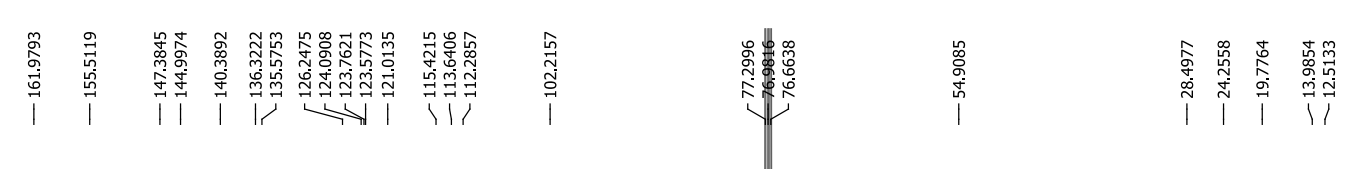

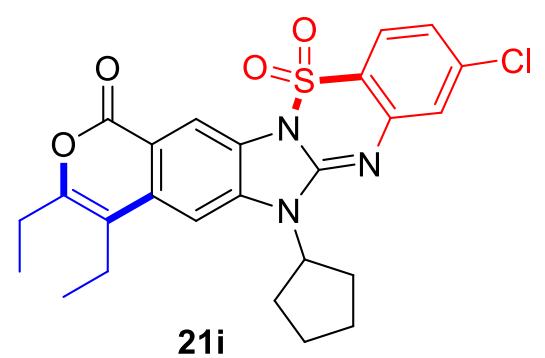

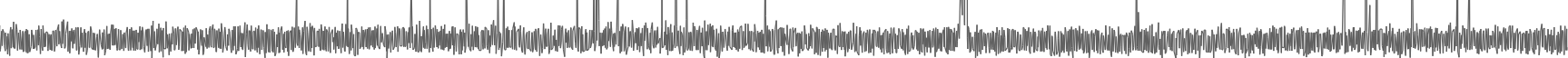

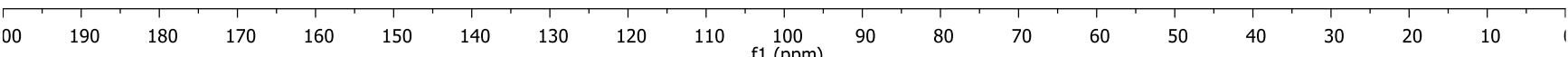

${ }^{13} \mathrm{C}$ NMR spectrum (101 MHz) of compound $21 \mathbf{i}$ in $\mathrm{CDCl}_{3}$ 


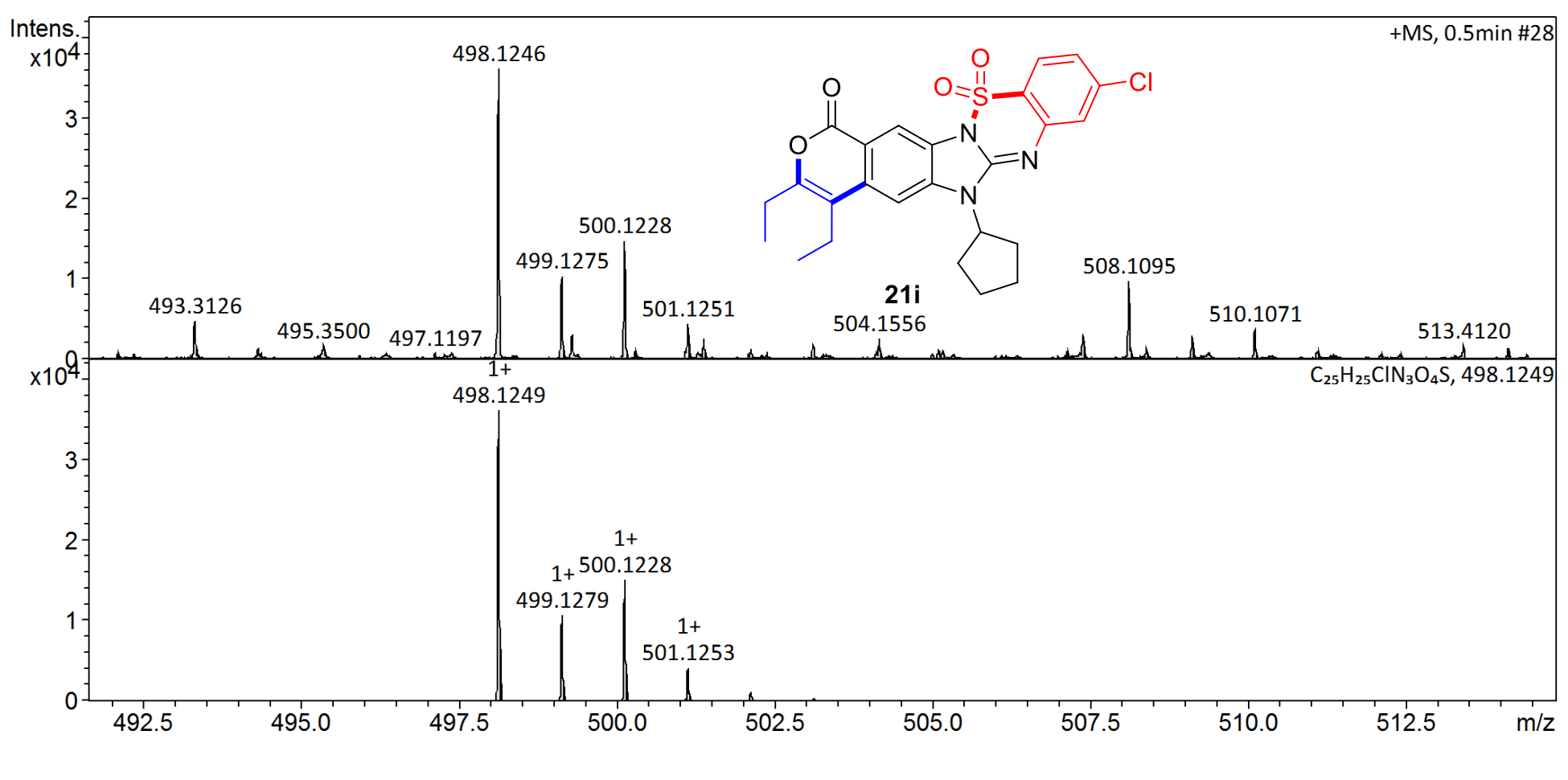

\section{Display Report}

Meas. $\mathrm{m} / \mathrm{z}$ \# Ion Formula $\mathrm{m} / \mathrm{z}$ err [ppm] mSigma \# Sigma Score $\mathrm{rdb} \mathrm{e}^{-}$Conf $\mathrm{N}$-Rule Adduct

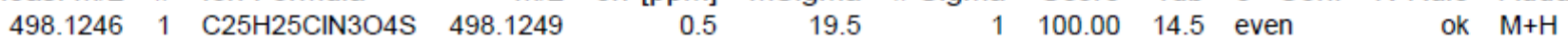

HRMS spectrum (ESI) of compound $\mathbf{2 1 i}$ 


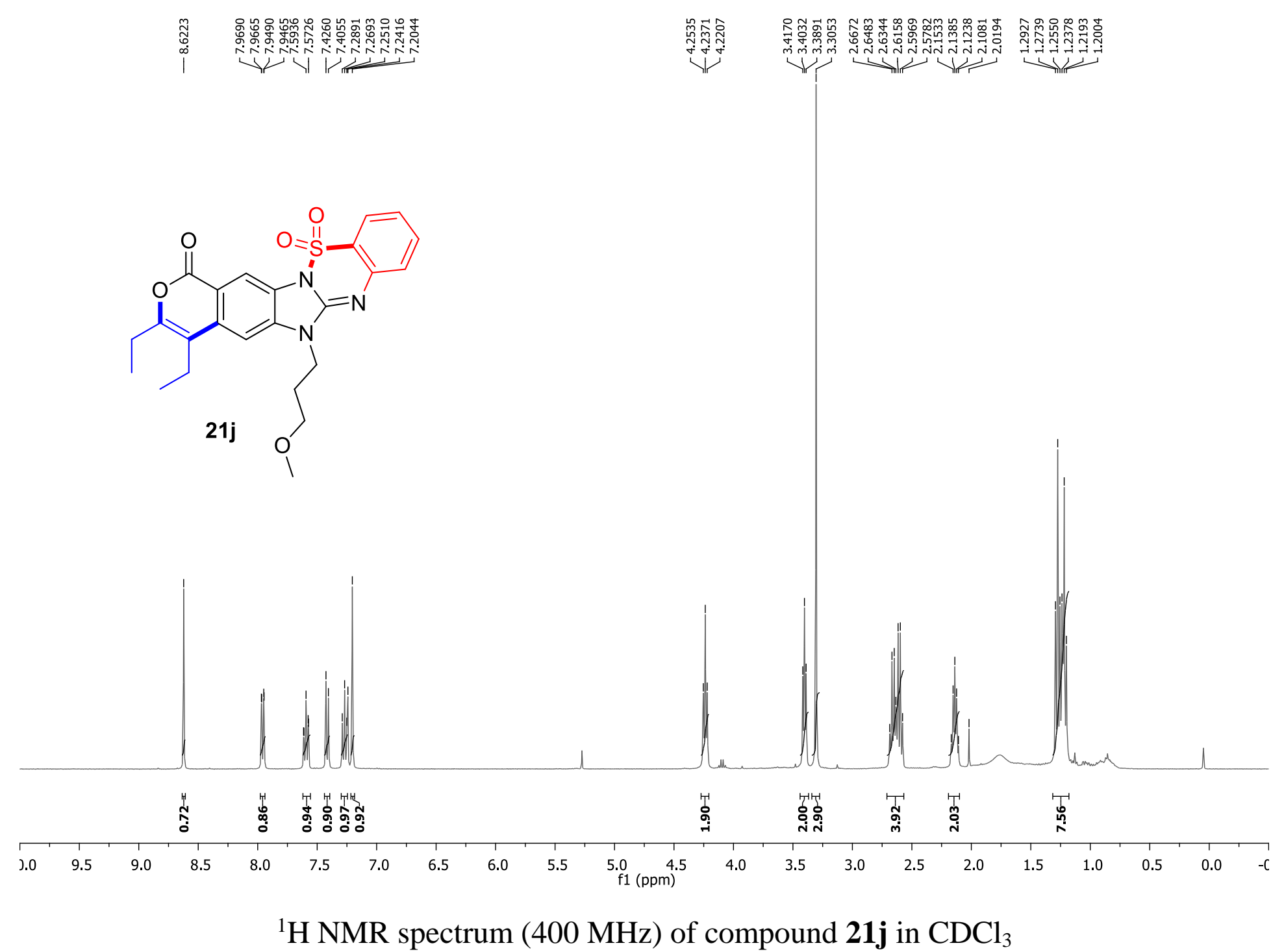




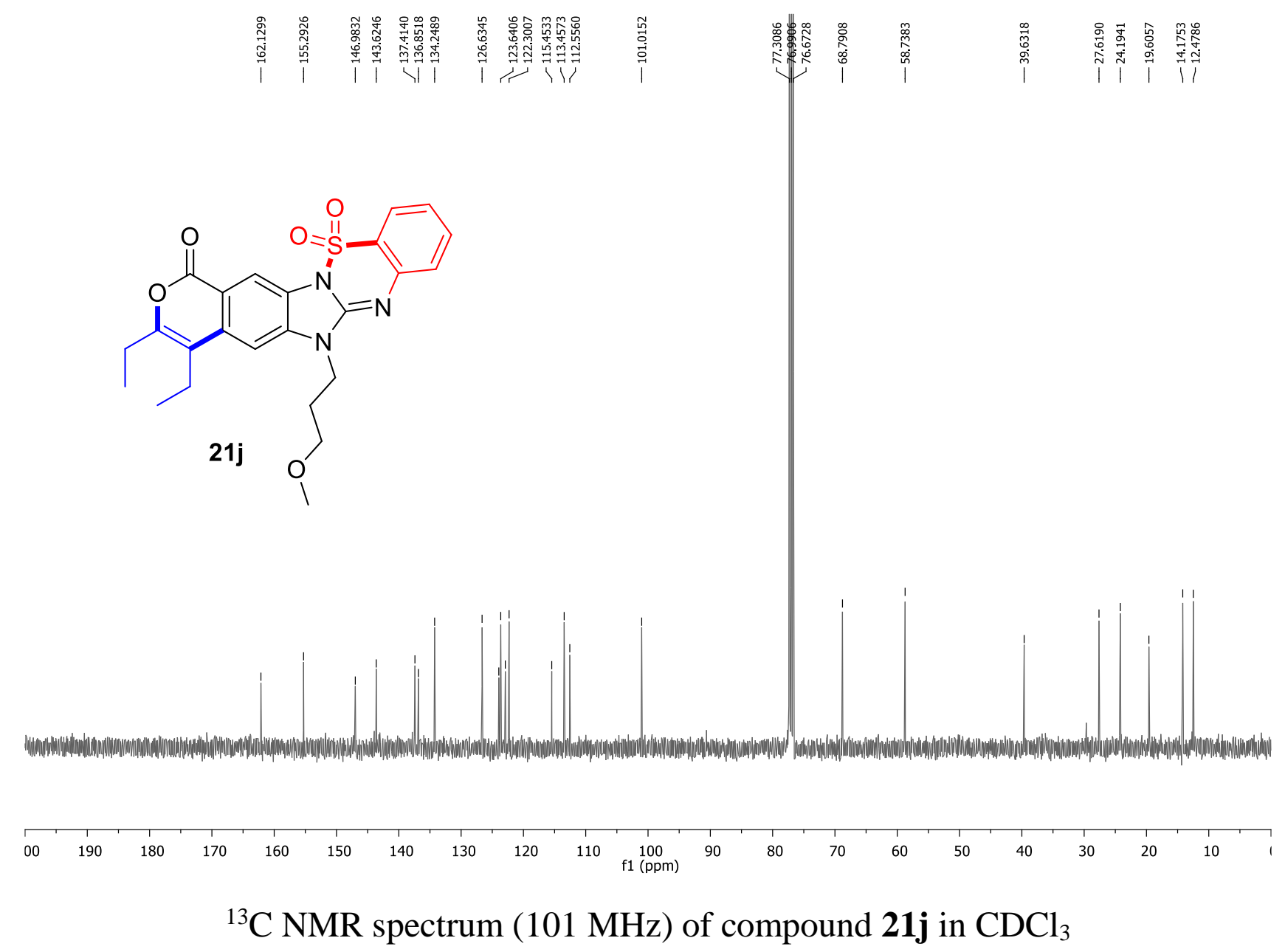




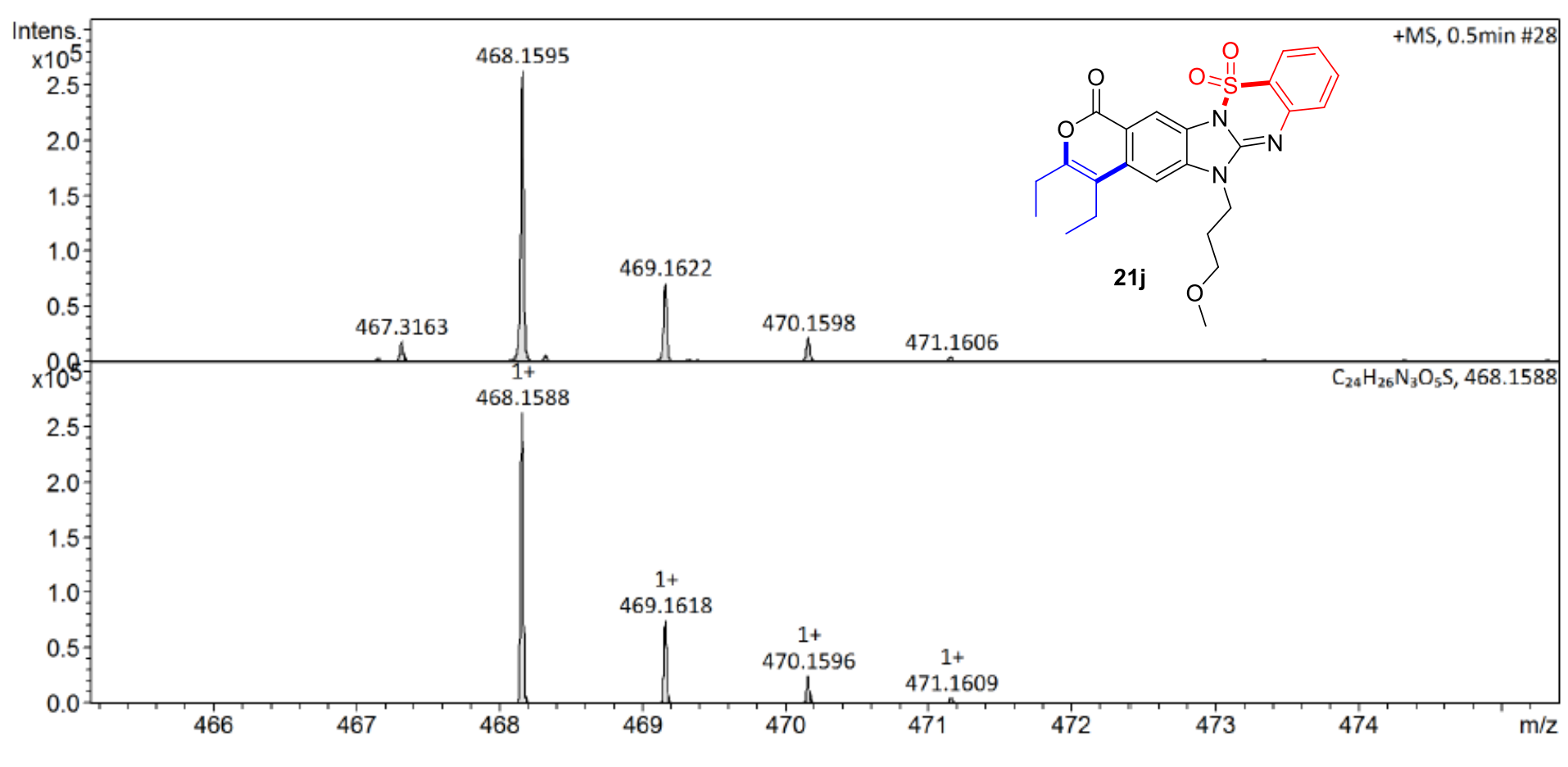

\section{Display Report}

Meas. $\mathrm{m} / \mathrm{z}$ \# Ion Formula $\mathrm{m} / \mathrm{z}$ err [ppm] mSigma \# Sigma Score $\mathrm{rdb} \mathrm{e}^{-}$Conf N-Rule Adduct

$\begin{array}{llllllllllll}468.1595 & 1 & \mathrm{C} 24 \mathrm{H} 26 \mathrm{~N} 3 \mathrm{O} 5 \mathrm{~S} & 468.1588 & 1.5 & 8.7 & 1 & 100.00 & 13.5 & \text { even } & \mathrm{ok} & \mathrm{M}+\mathrm{H}\end{array}$

HRMS spectrum (ESI) of compound $\mathbf{2 1 j}$ 


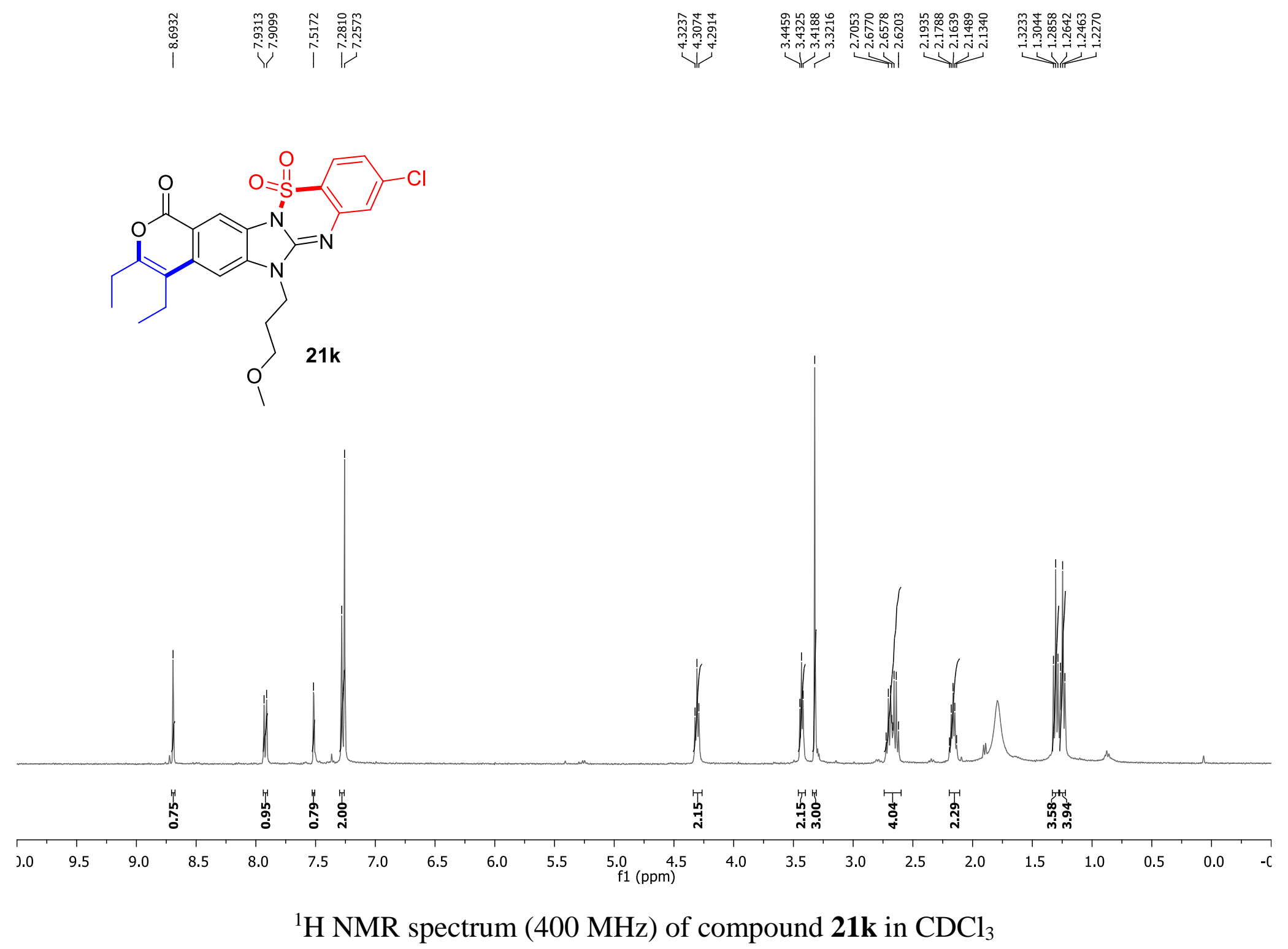




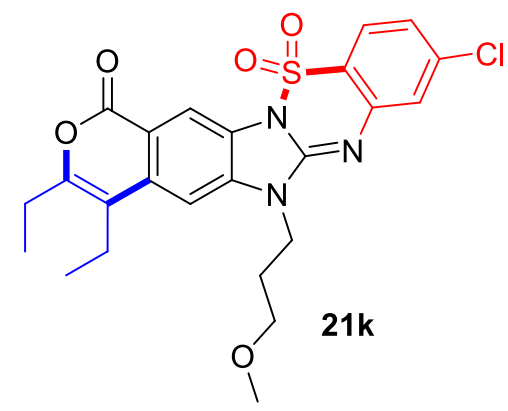

$\begin{array}{llll}00 & 190 & 180 & 170\end{array}$

${ }^{13} \mathrm{C}$ NMR spectrum (101 MHz) of compound $21 \mathbf{k}$ in $\mathrm{CDCl}_{3}$ 


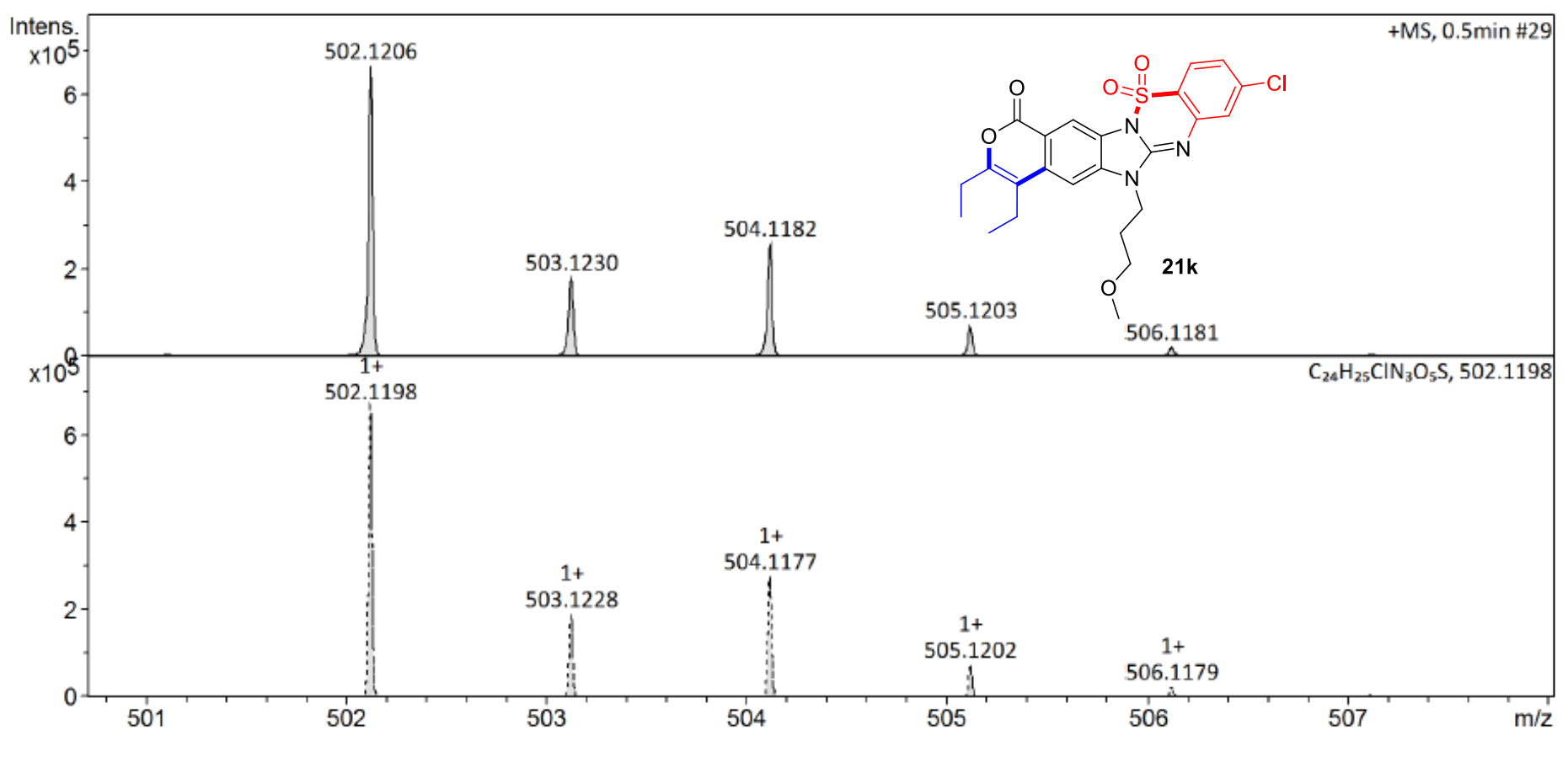

\section{Display Report}

Meas. $\mathrm{m} / \mathrm{z}$ \# Ion Formula $\mathrm{m} / \mathrm{z}$ err [ppm] mSigma \# Sigma Score $\mathrm{rdb} \mathrm{e}^{-}$Conf N-Rule Adduct

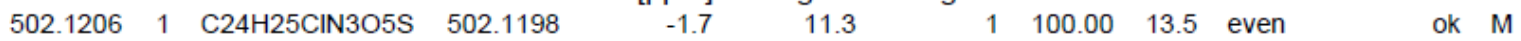

HRMS spectrum (ESI) of compound 21k 


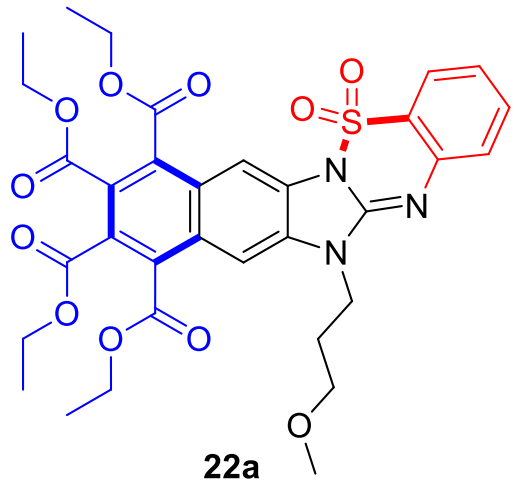

22a

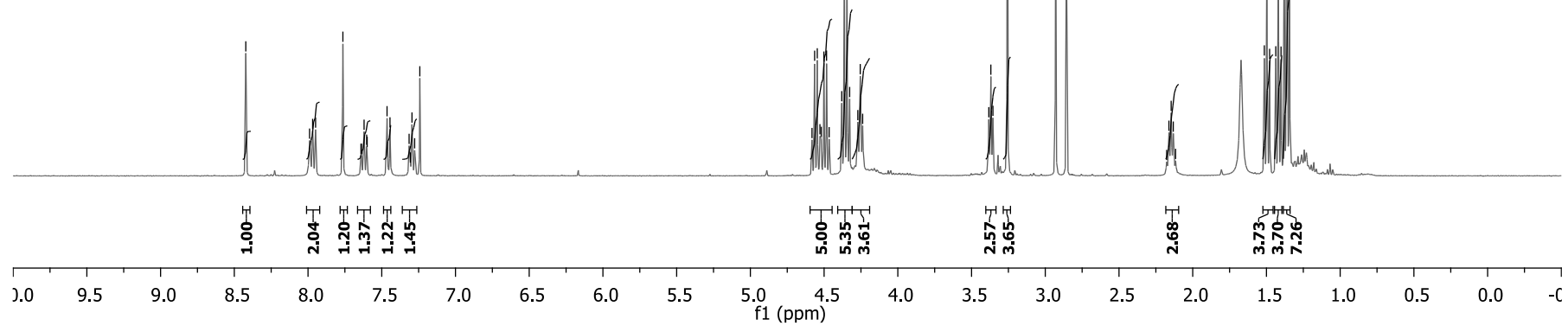

${ }^{1} \mathrm{H}$ NMR spectrum (400 MHz) of compound 22a in $\mathrm{CDCl}_{3}$ 


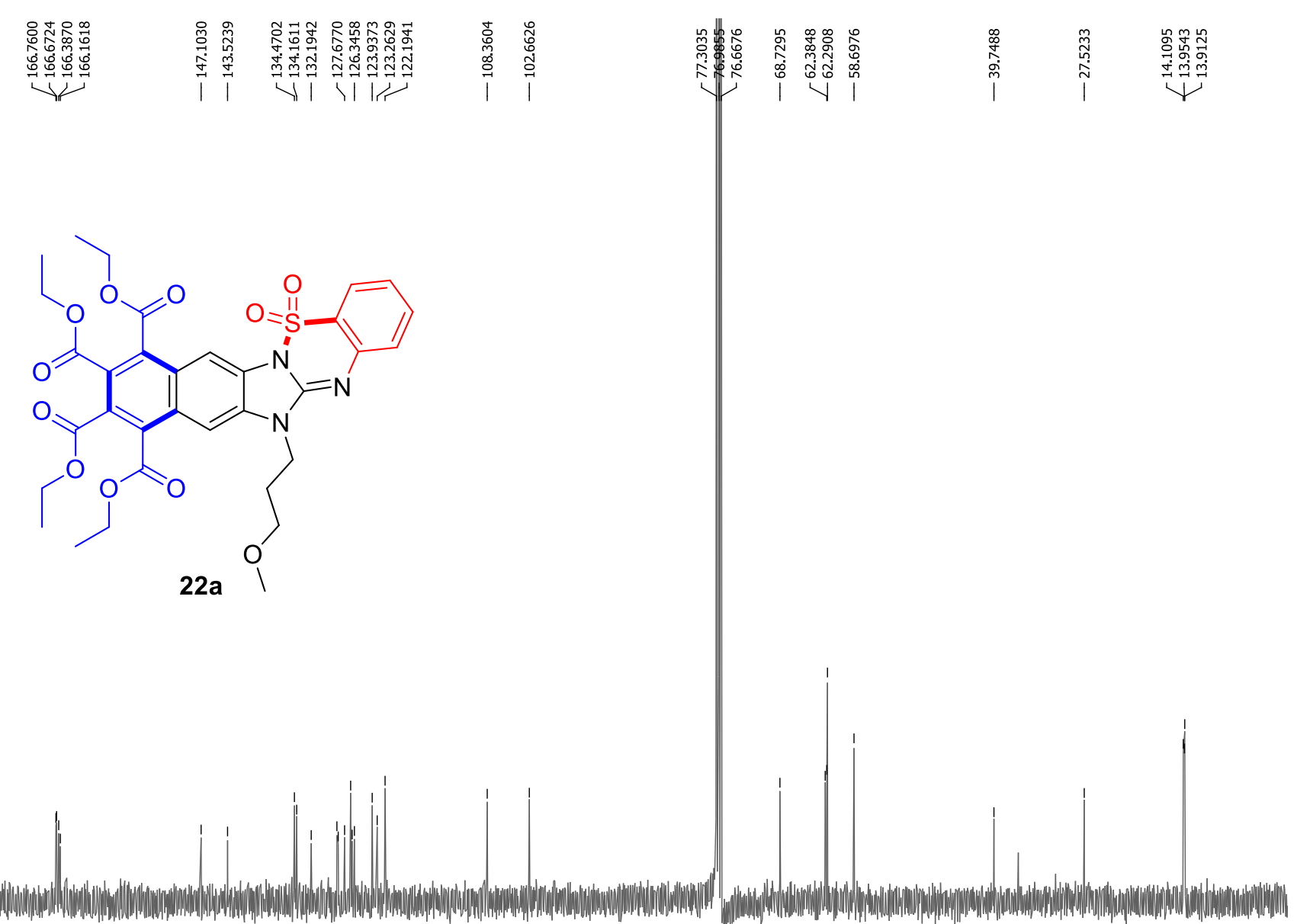

\begin{tabular}{lllllllllllllllllllll}
\hline 0 & 190 & 180 & 170 & 160 & 150 & 140 & 130 & 120 & 110 & 100 & 90 & 80 & 70 & 60 & 50 & 40 & 30 & 20 & 10 & 1
\end{tabular}

${ }^{13} \mathrm{C}$ NMR spectrum (101 MHz) of compound 22a in $\mathrm{CDCl}_{3}$ 


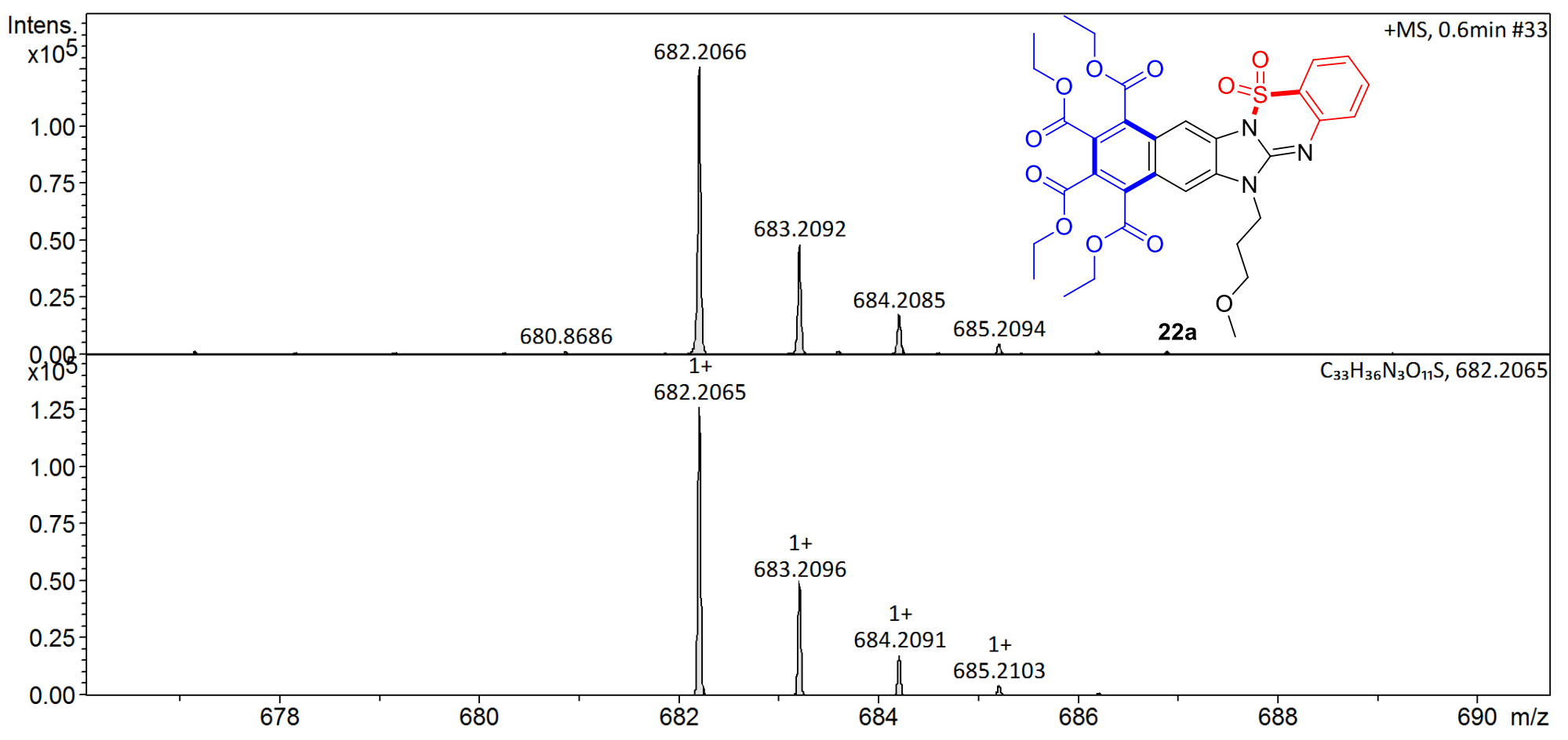

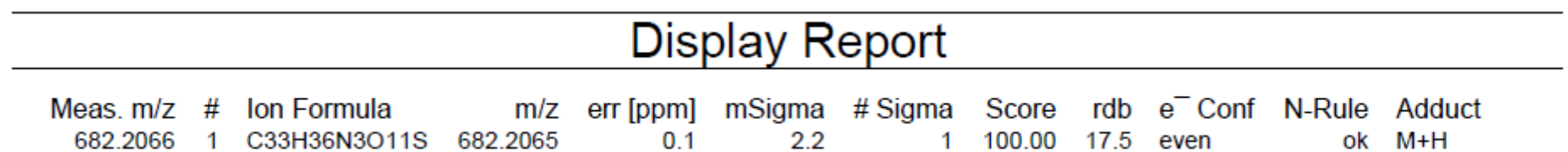

HRMS spectrum (ESI) of compound 22a 


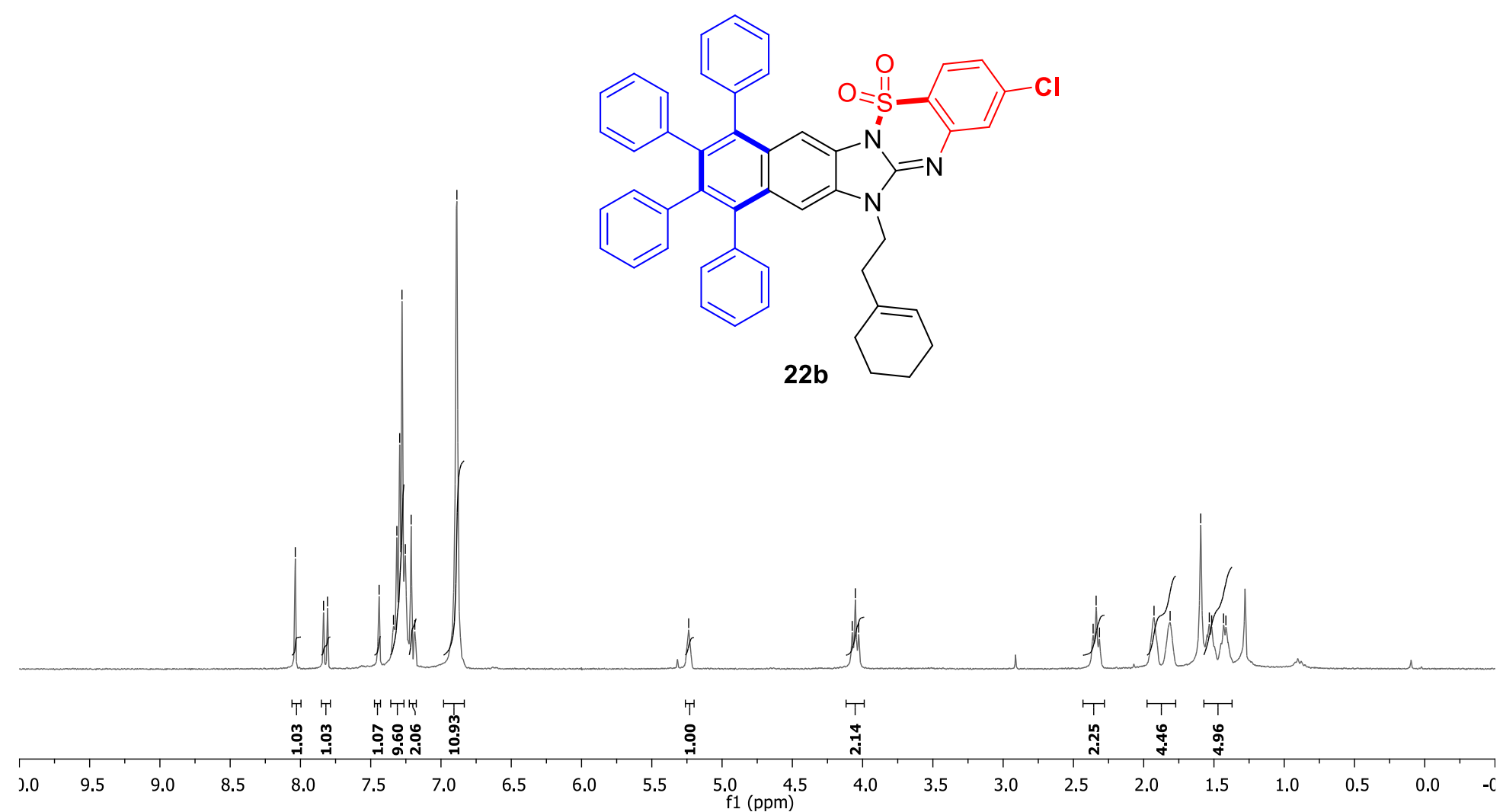

${ }^{1} \mathrm{H}$ NMR spectrum (400 MHz) of compound $22 \mathbf{b}$ in $\mathrm{CDCl}_{3}$ 


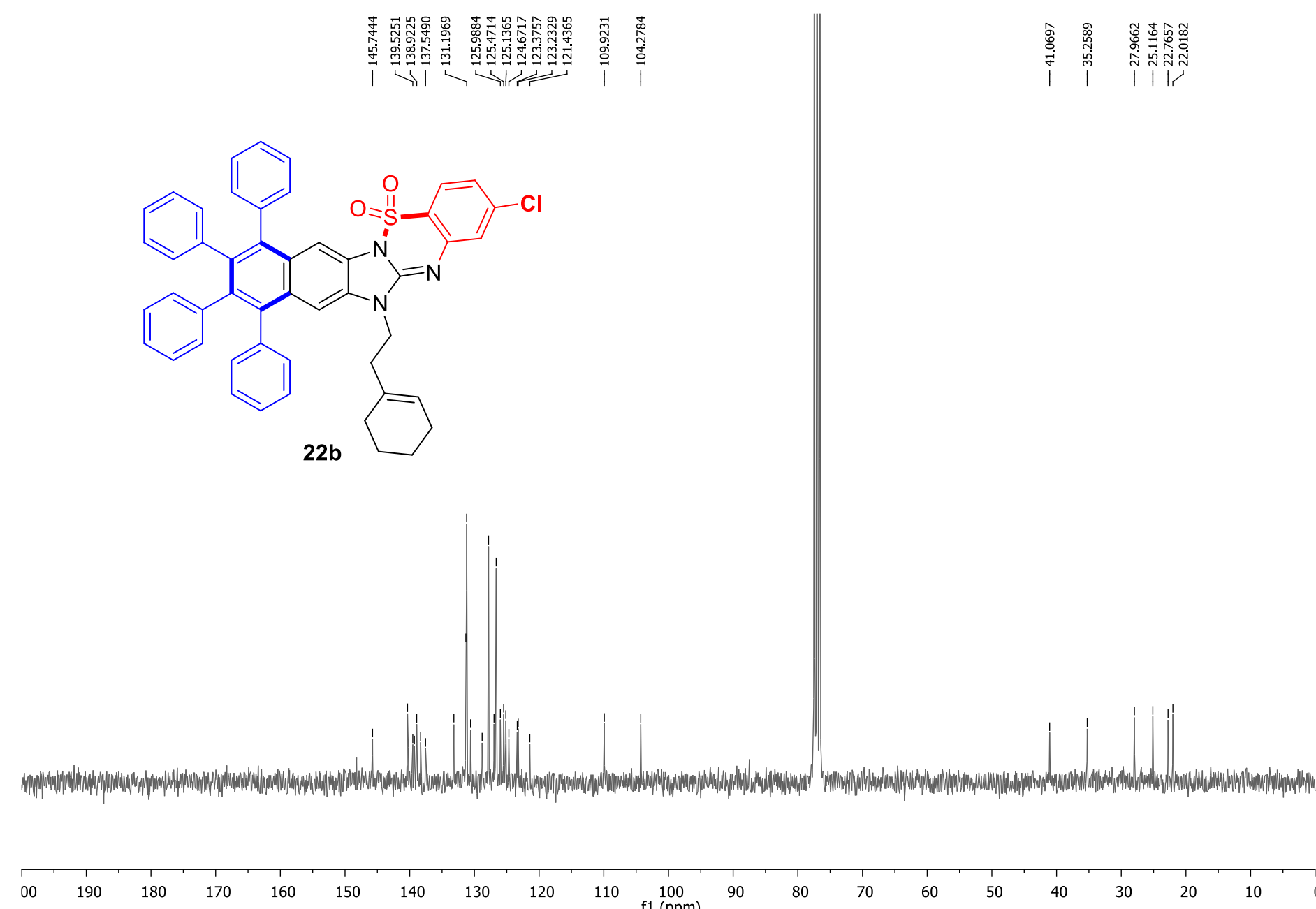

${ }^{13} \mathrm{C}$ NMR spectrum (101 MHz) of compound $\mathbf{2 2 b}$ in $\mathrm{CDCl}_{3}$ 


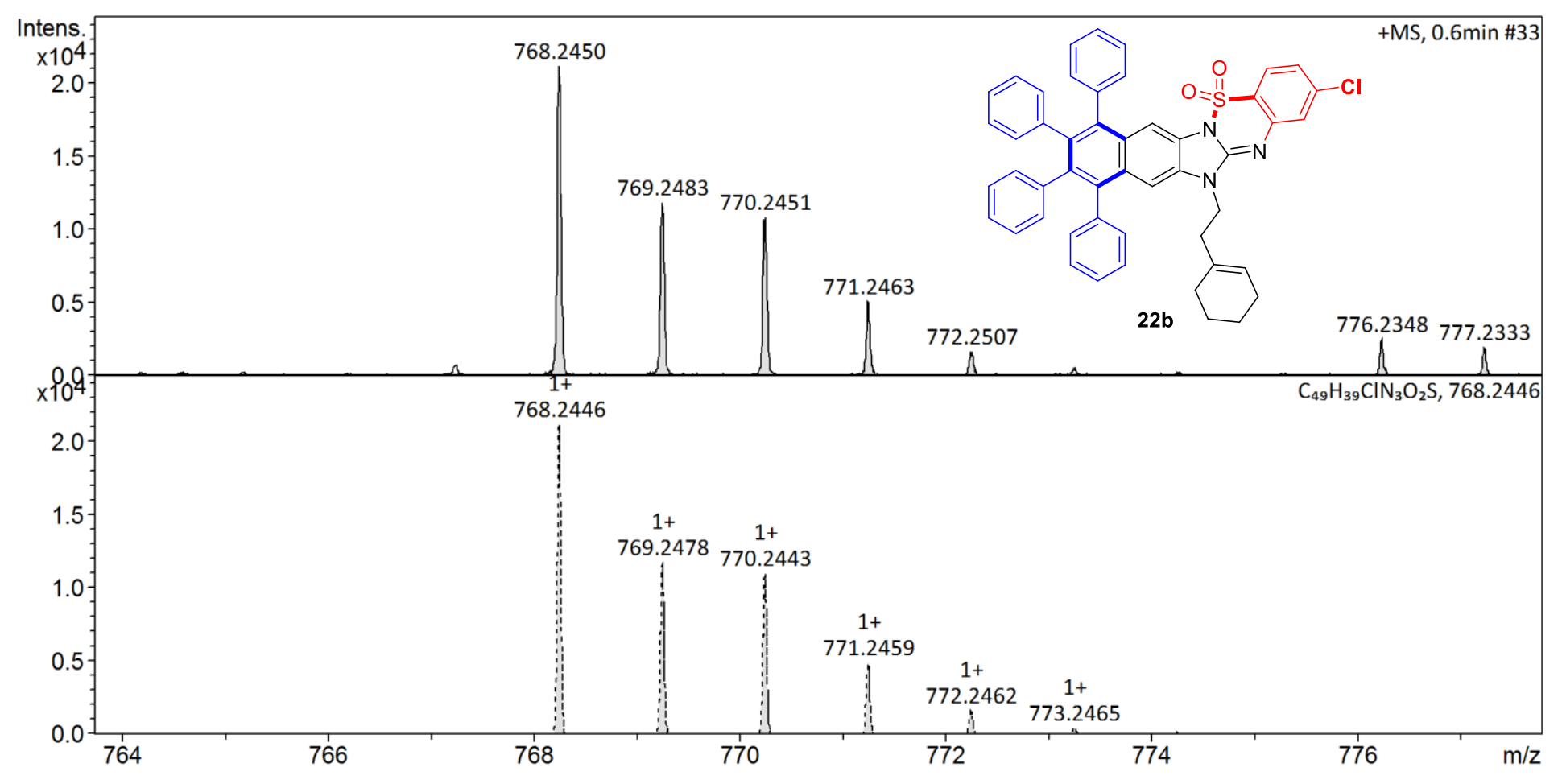

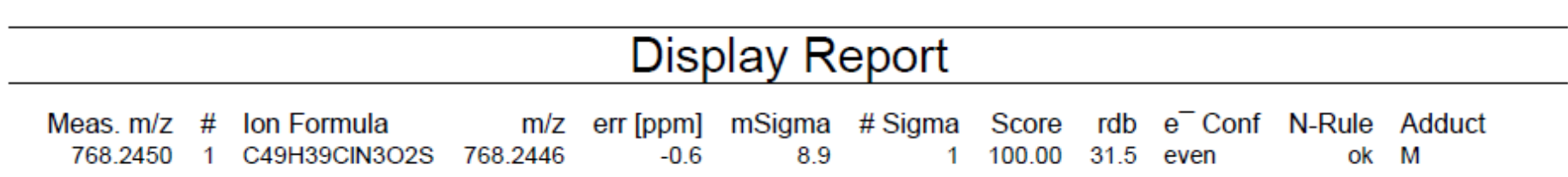

HRMS spectrum (ESI) of compound 22b 


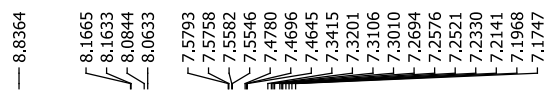

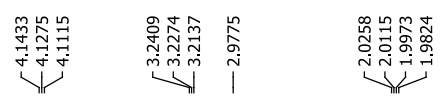

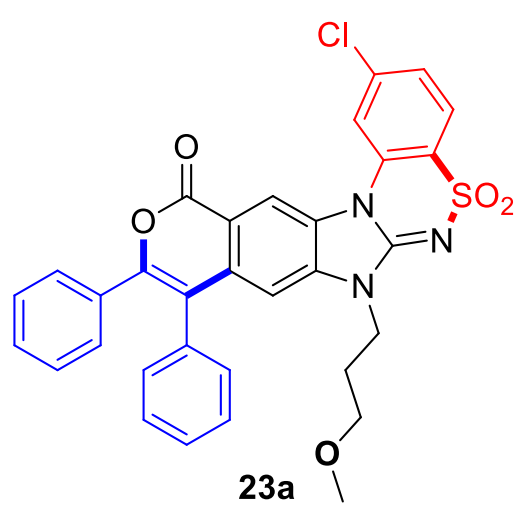

23a

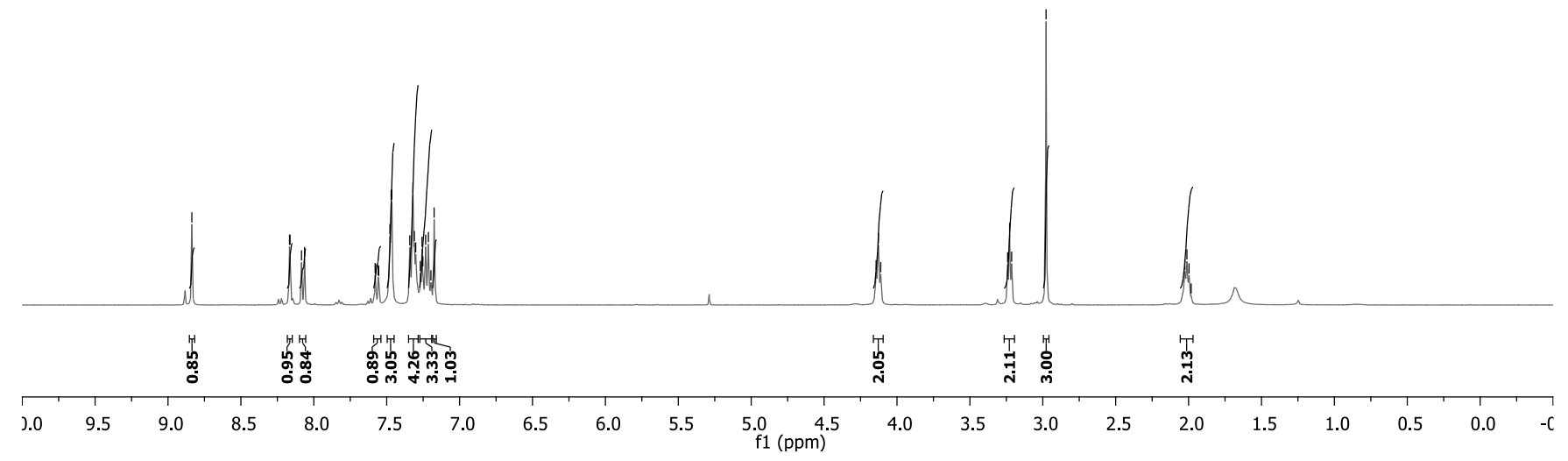

${ }^{1} \mathrm{H}$ NMR spectrum (400 MHz) of compound 23a in $\mathrm{CDCl}_{3}$ 


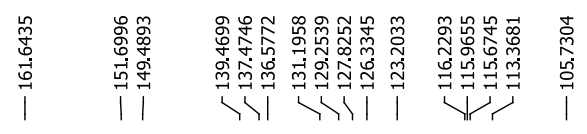

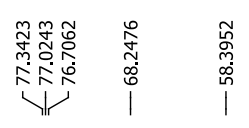

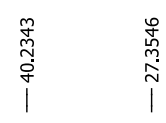
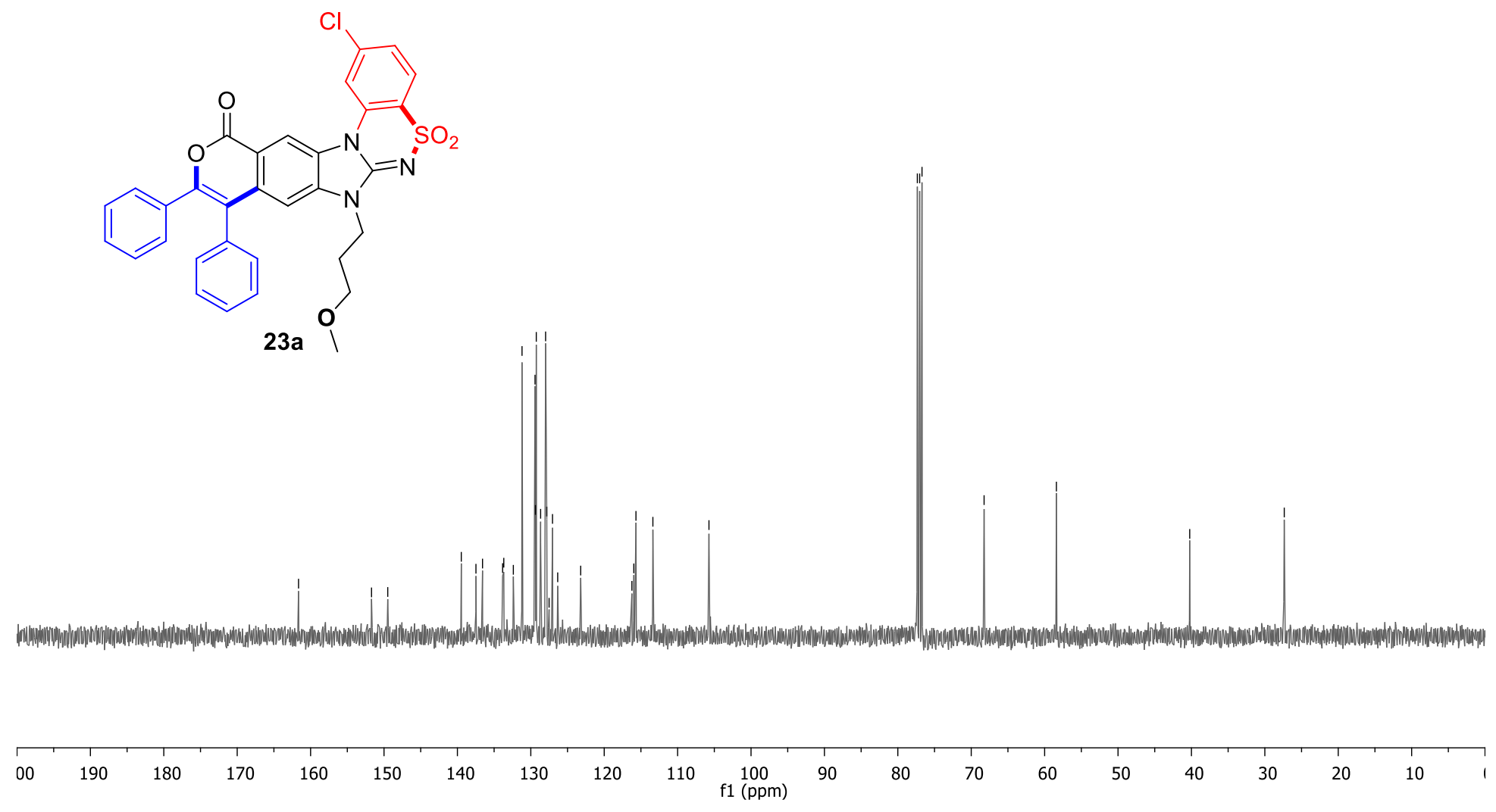

${ }^{13} \mathrm{C}$ NMR spectrum (101 MHz) of compound 23a in $\mathrm{CDCl}_{3}$

S170 


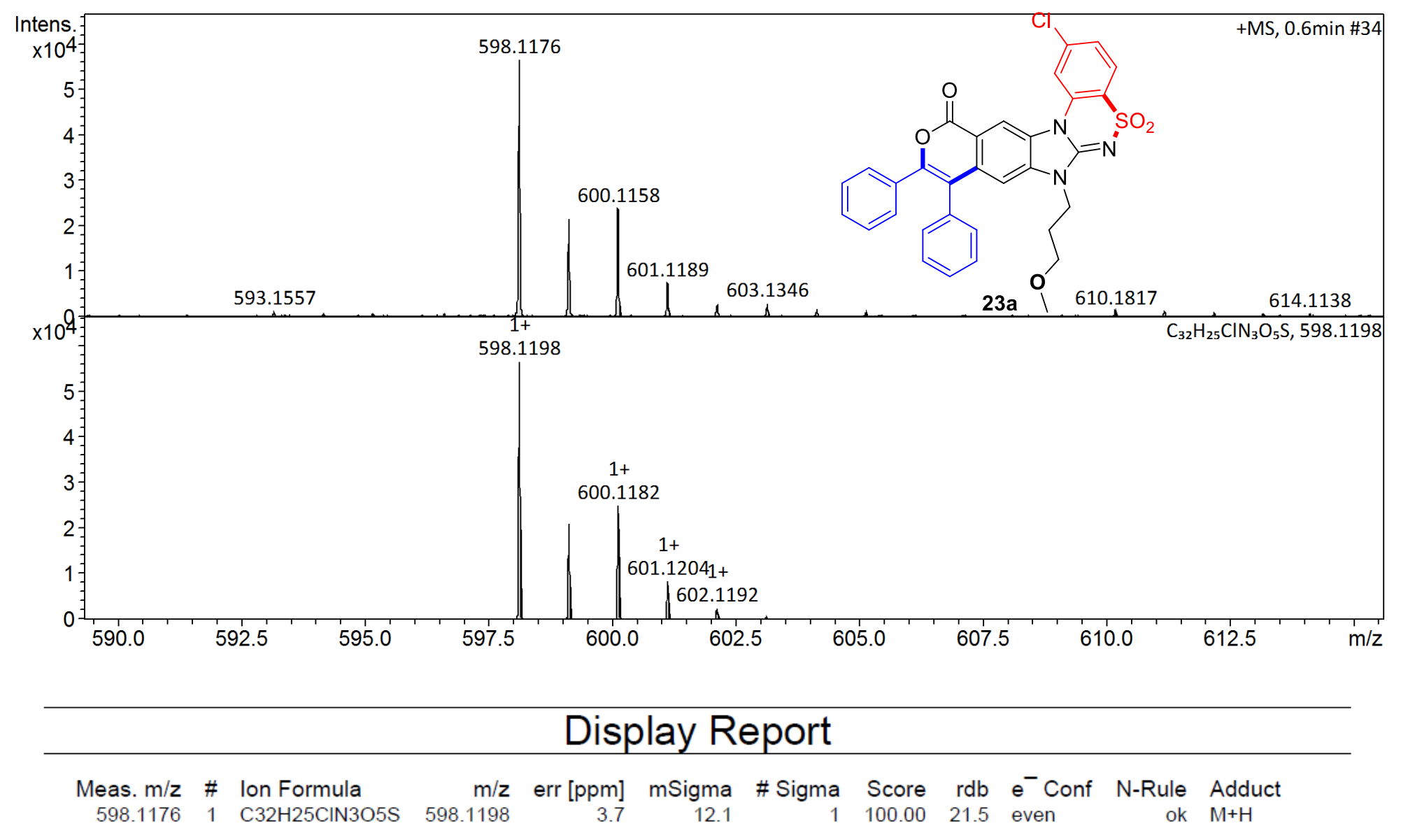

HRMS spectrum (ESI) of compound 23a 


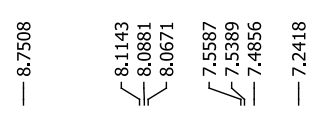

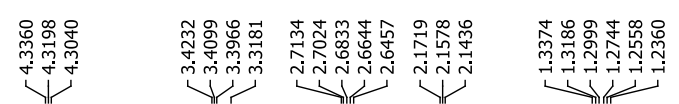

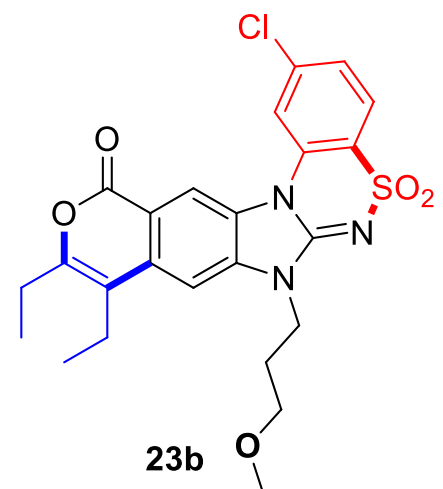

23b

$$
1
$$

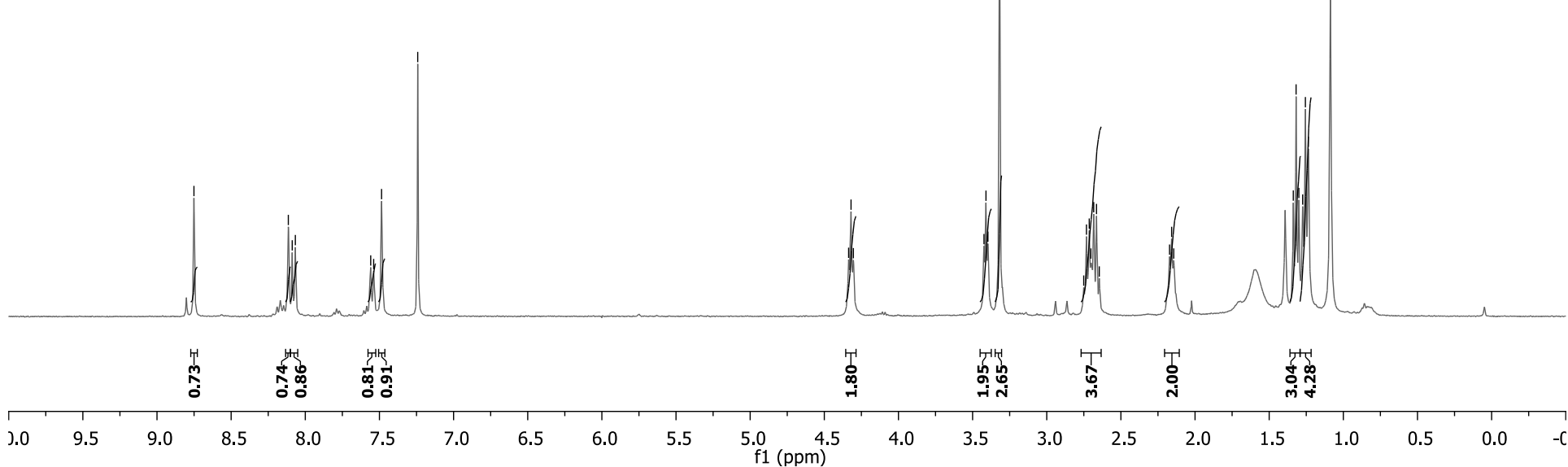

${ }^{1} \mathrm{H}$ NMR spectrum (400 MHz) of compound 23b in $\mathrm{CDCl}_{3}$ 


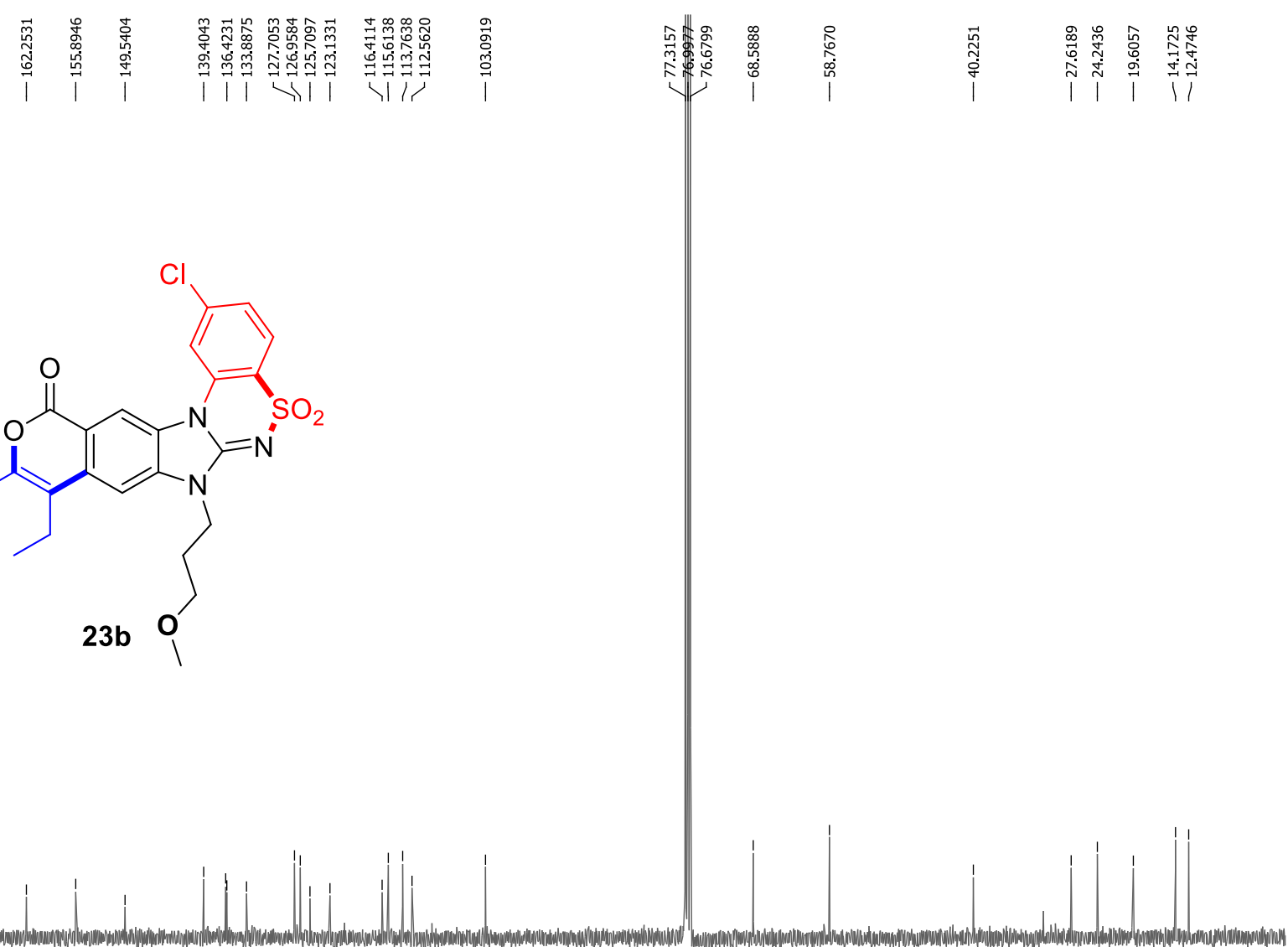

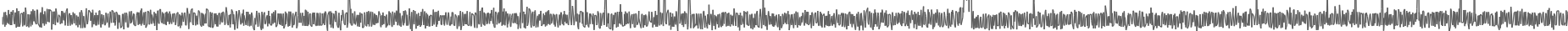

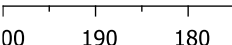

160

$150 \quad 140$

100
$\mathrm{f} 1(\mathrm{ppm})$

${ }^{13} \mathrm{C}$ NMR spectrum (101 MHz) of compound $\mathbf{2 3 b}$ in $\mathrm{CDCl}_{3}$ 


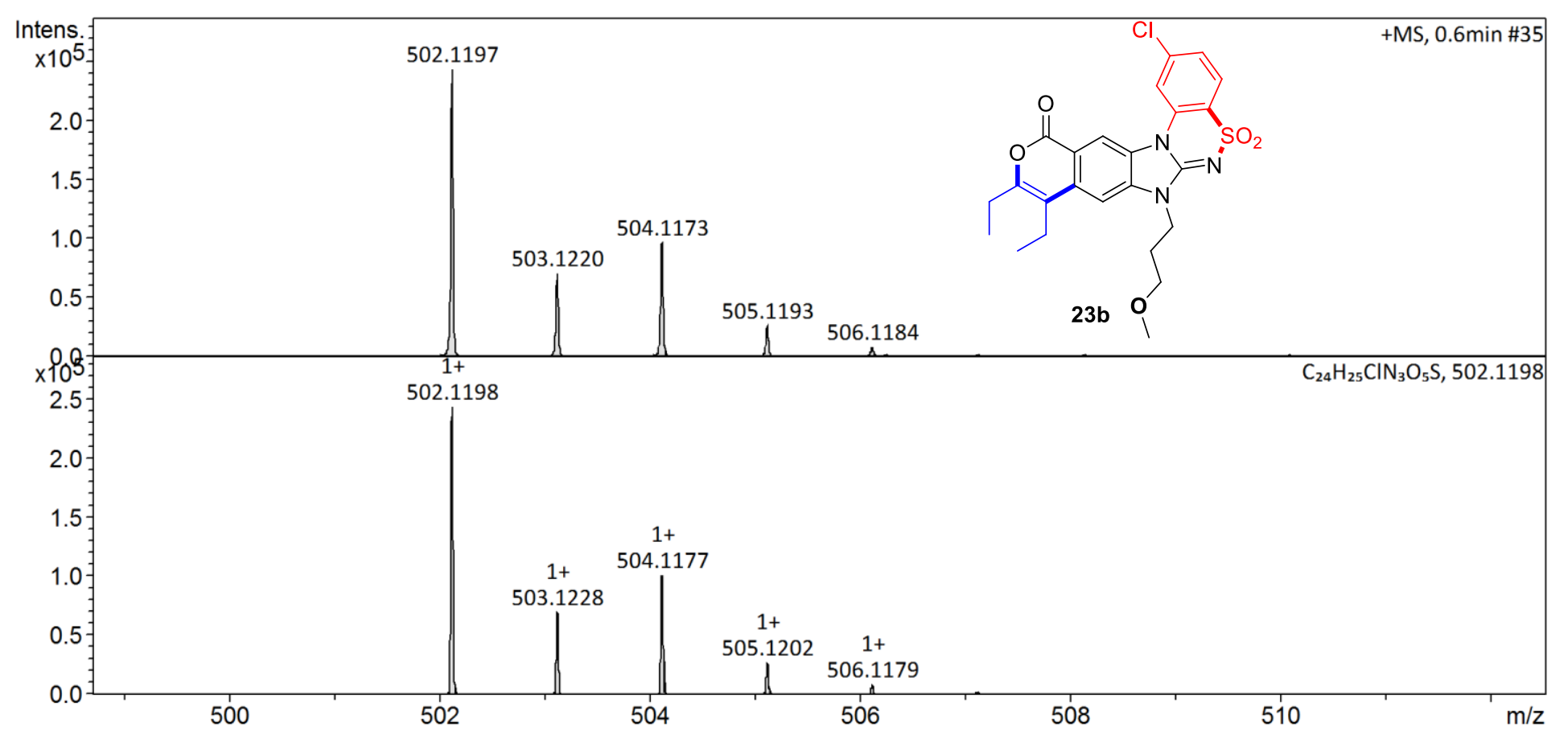

\section{Display Report}

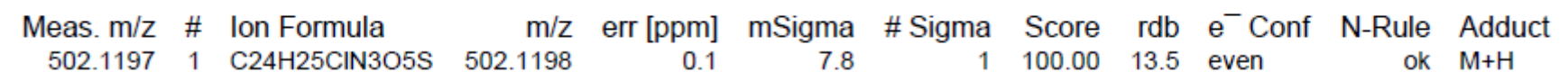

HRMS spectrum (ESI) of compound 23b 

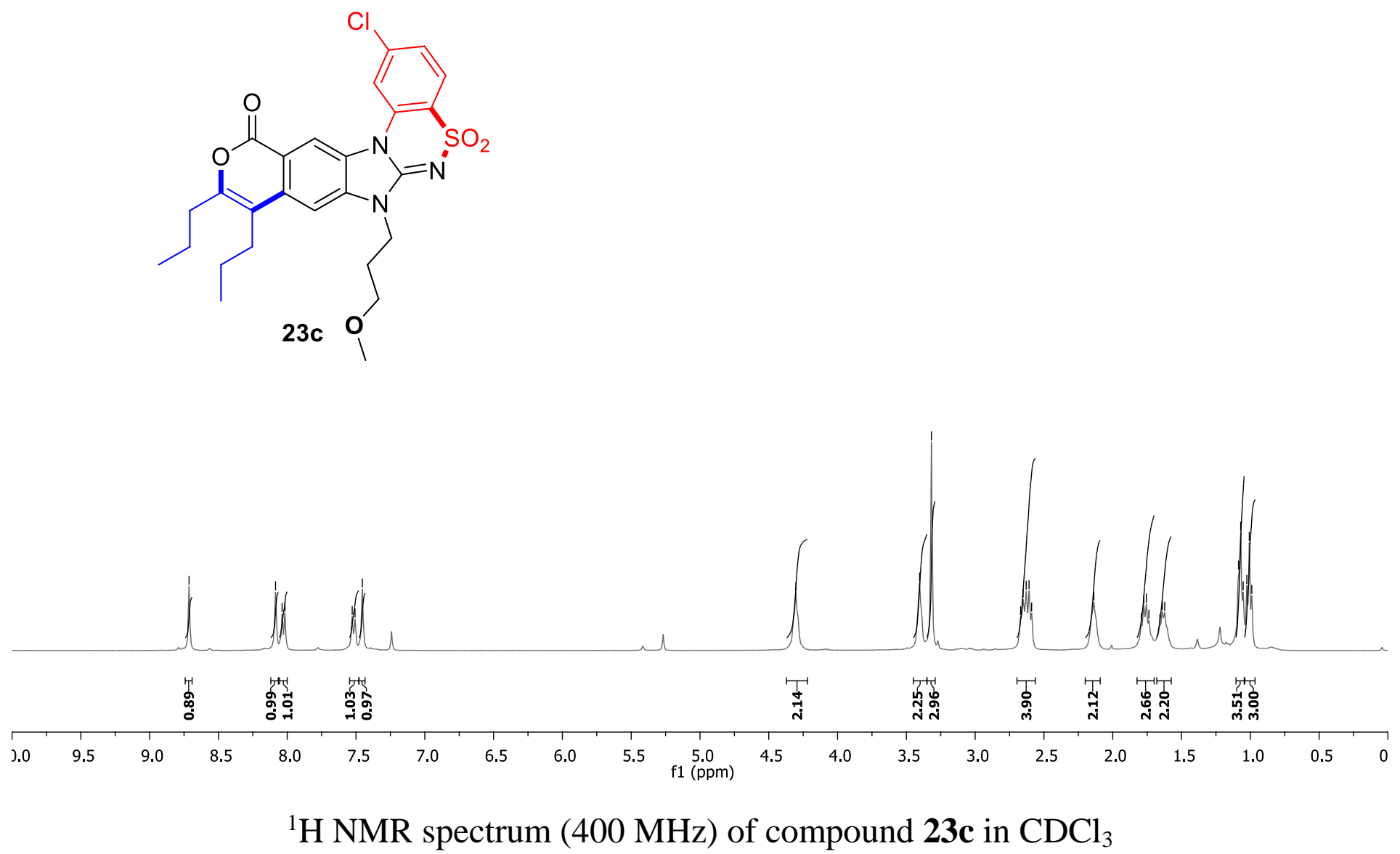


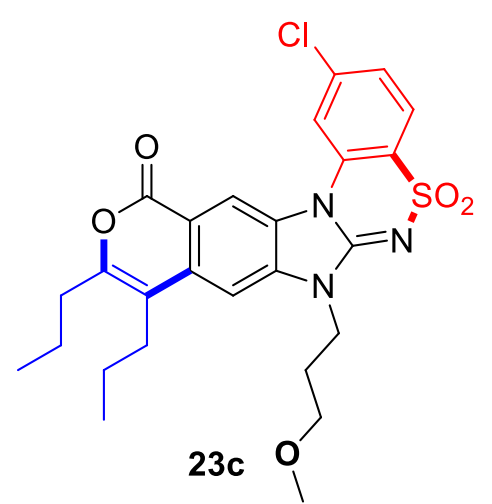

\begin{tabular}{lllll}
\hline & 190 & 180 & 170 & 160
\end{tabular}

${ }^{13} \mathrm{C}$ NMR spectrum (101 MHz) of compound $\mathbf{2 3 c}$ in $\mathrm{CDCl}_{3}$ 


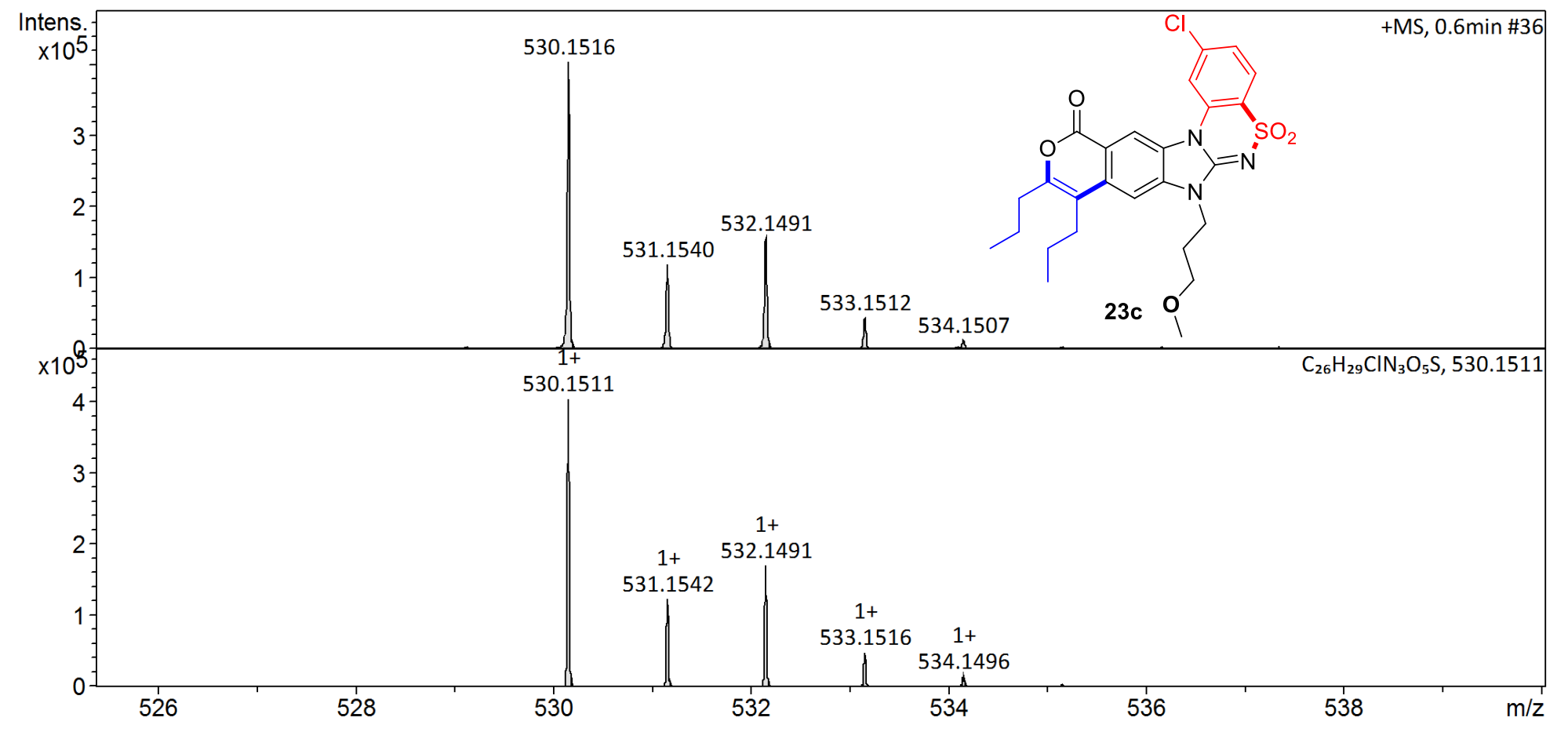

\section{Display Report}

Meas. $\mathrm{m} / \mathrm{z}$ \# Ion Formula

$\mathrm{m} / \mathrm{z}$ err $[\mathrm{ppm}]$ mSigma \# Sigma Score $\mathrm{rdb} \mathrm{e}^{-}$Conf $\mathrm{N}$-Rule Adduct

$530.1516 \quad 1 \quad \mathrm{C} 26 \mathrm{H} 29 \mathrm{CIN} 3 \mathrm{O} 5 \mathrm{~S} \quad 530.1511$

$0.9 \quad 14.9$

$1 \quad 100.00 \quad 13.5$ even

ok $\mathrm{M}+\mathrm{H}$

HRMS spectrum (ESI) of compound 23c 

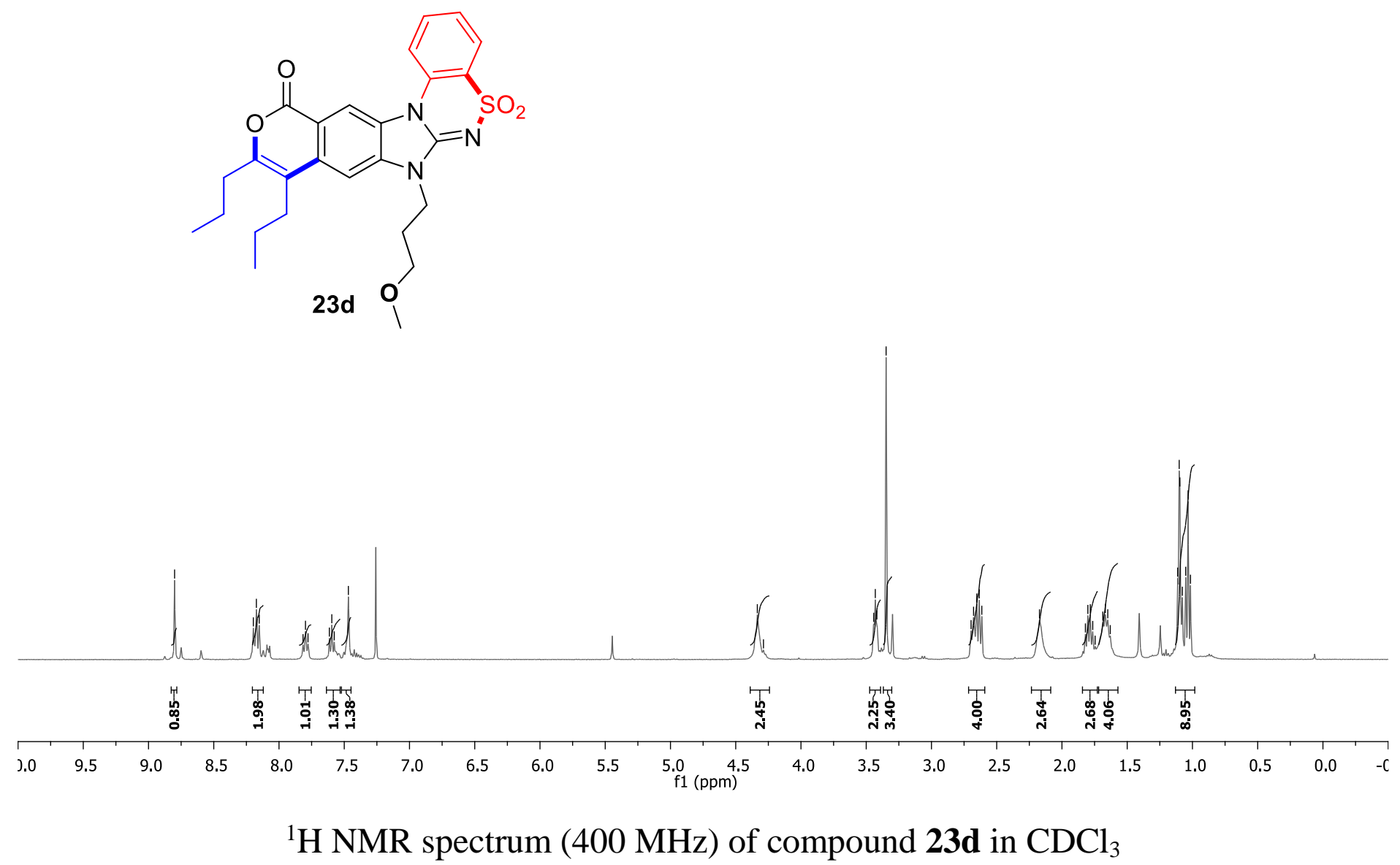


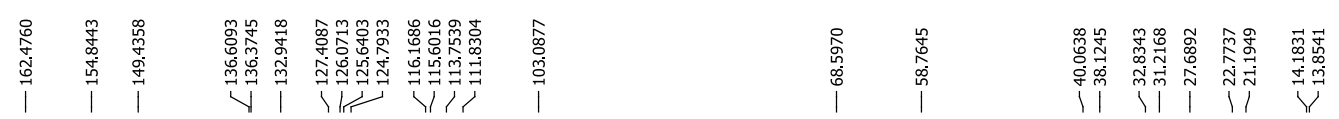

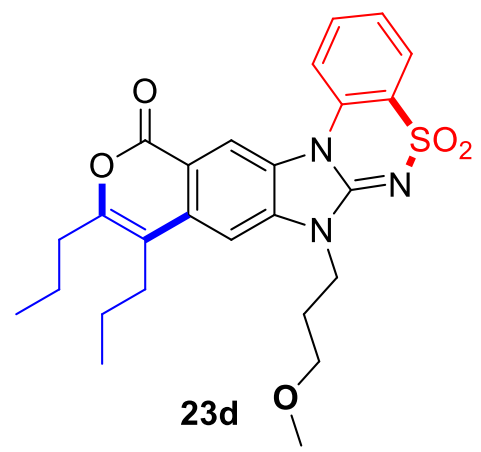

$00 \quad 1$

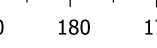

160
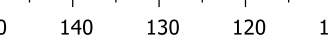

100
f1 $(\mathrm{ppm})$

${ }^{13} \mathrm{C}$ NMR spectrum (101 MHz) of compound 23d in $\mathrm{CDCl}_{3}$ 


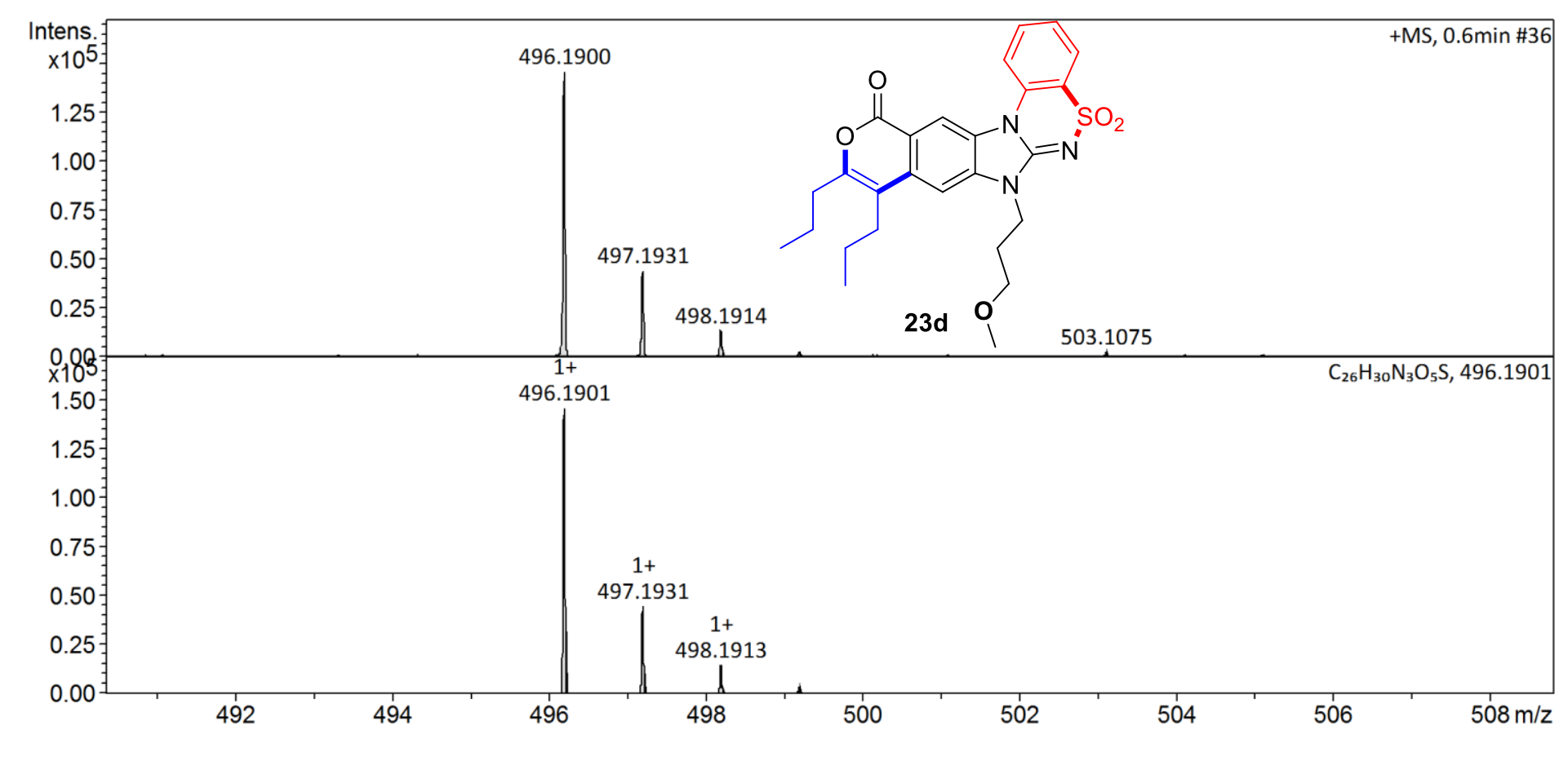

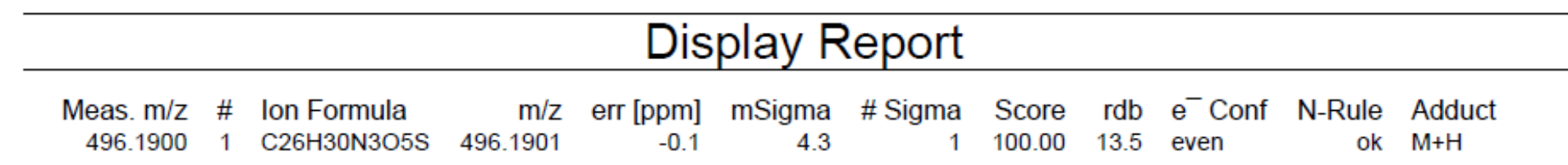

HRMS spectrum (ESI) of compound 23d 

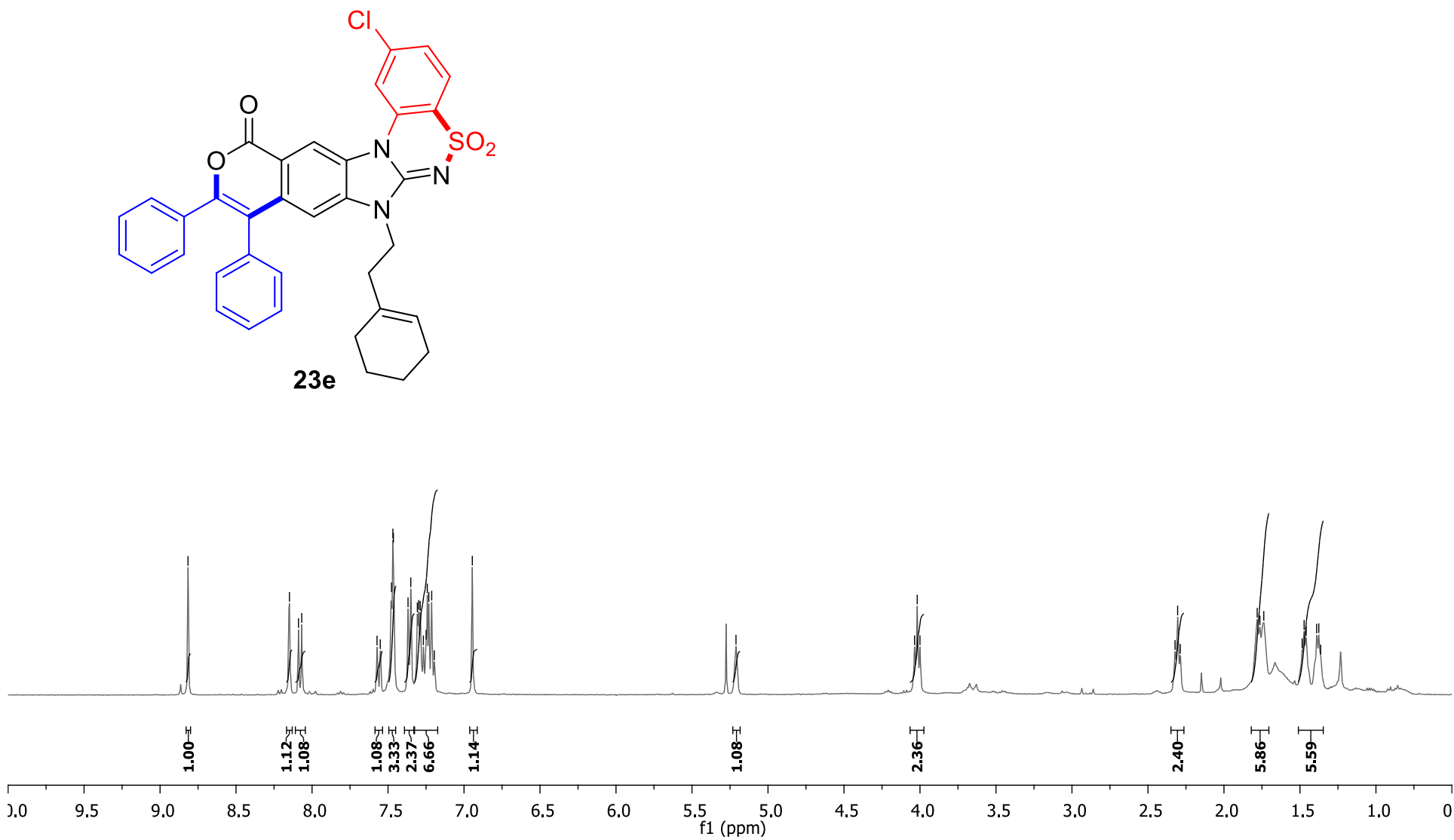

${ }^{1} \mathrm{H}$ NMR spectrum (400 MHz) of compound 23e in $\mathrm{CDCl}_{3}$ 


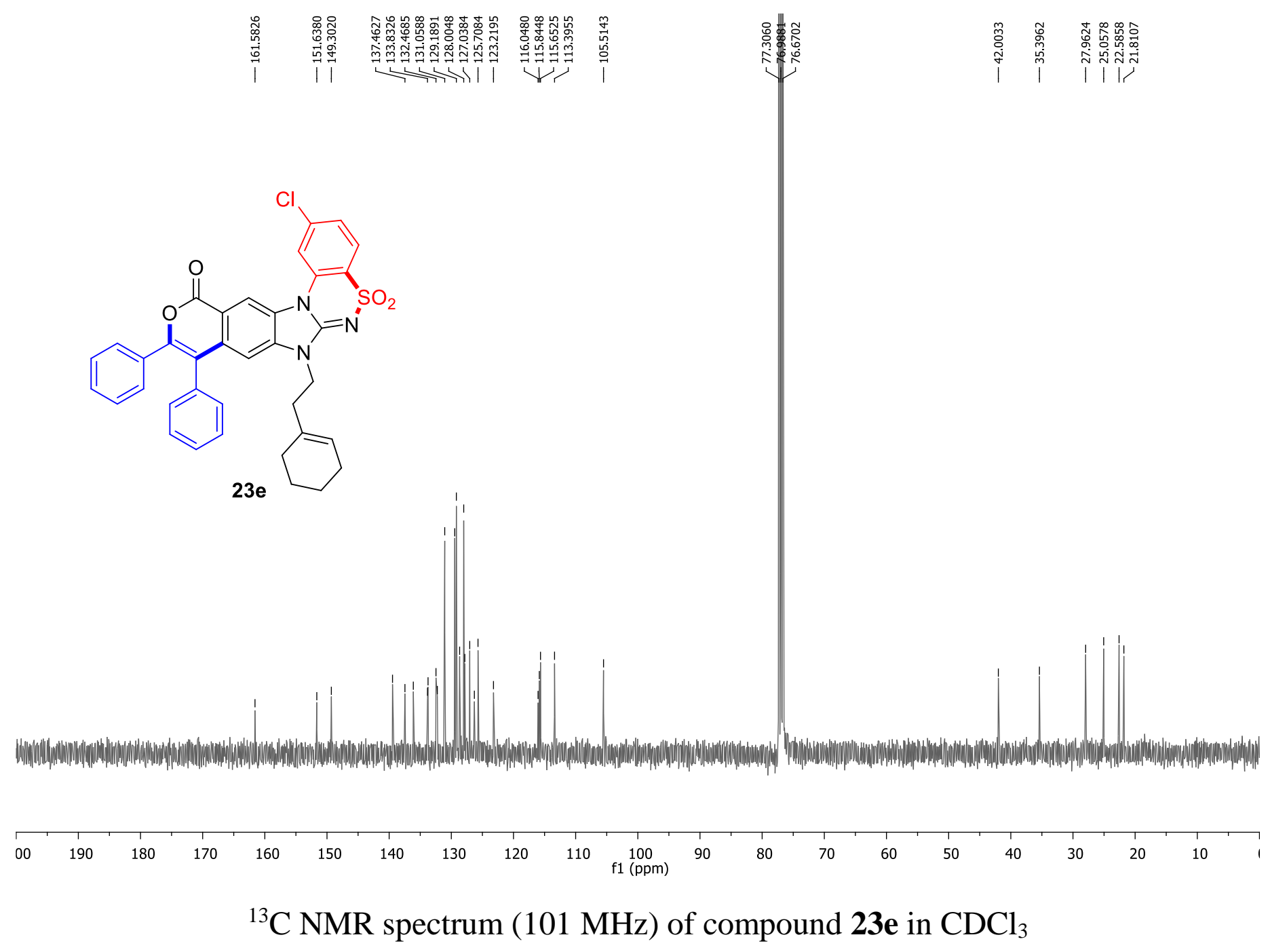




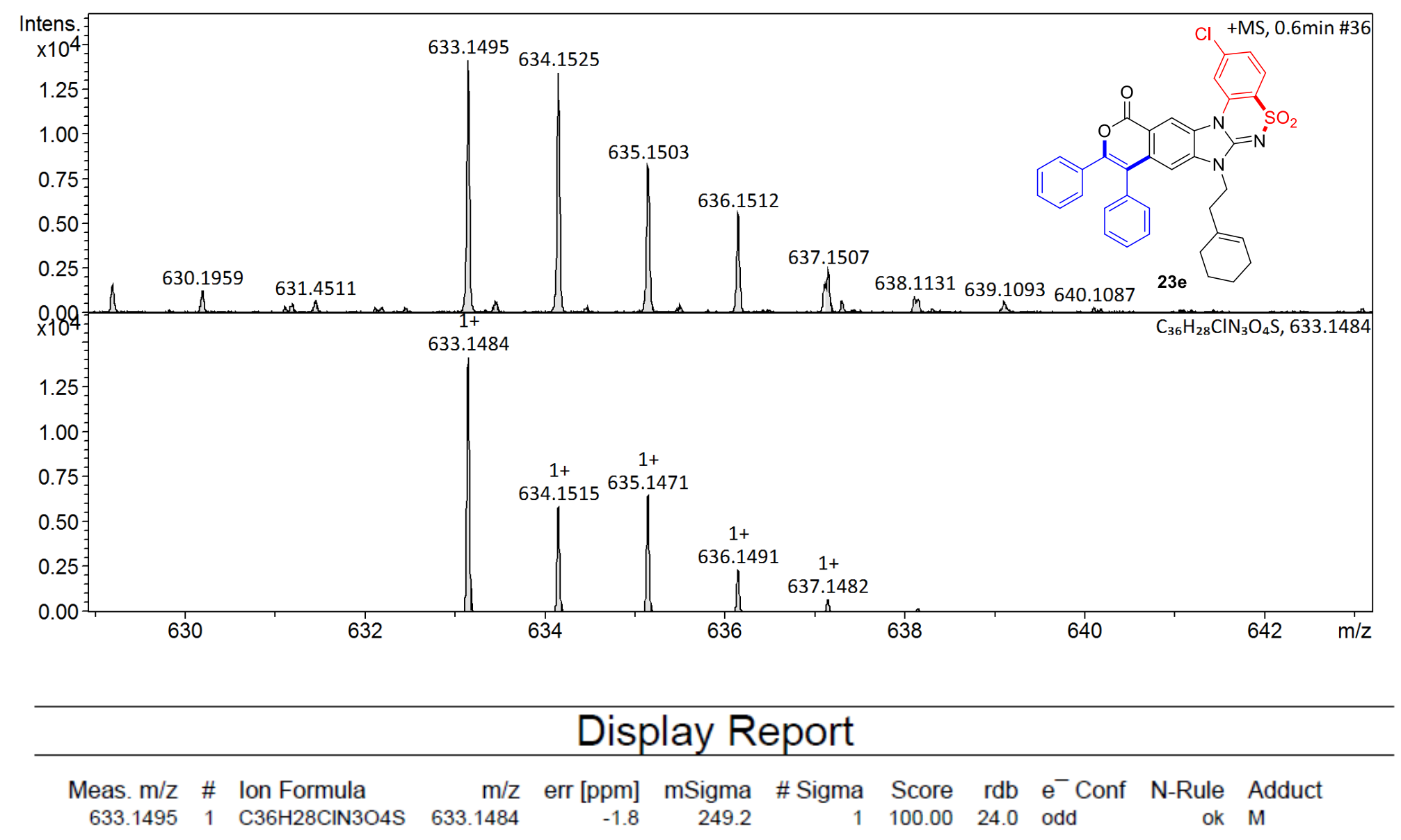

HRMS spectrum (ESI) of compound 23e 


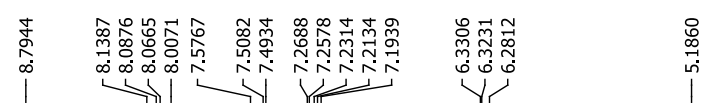
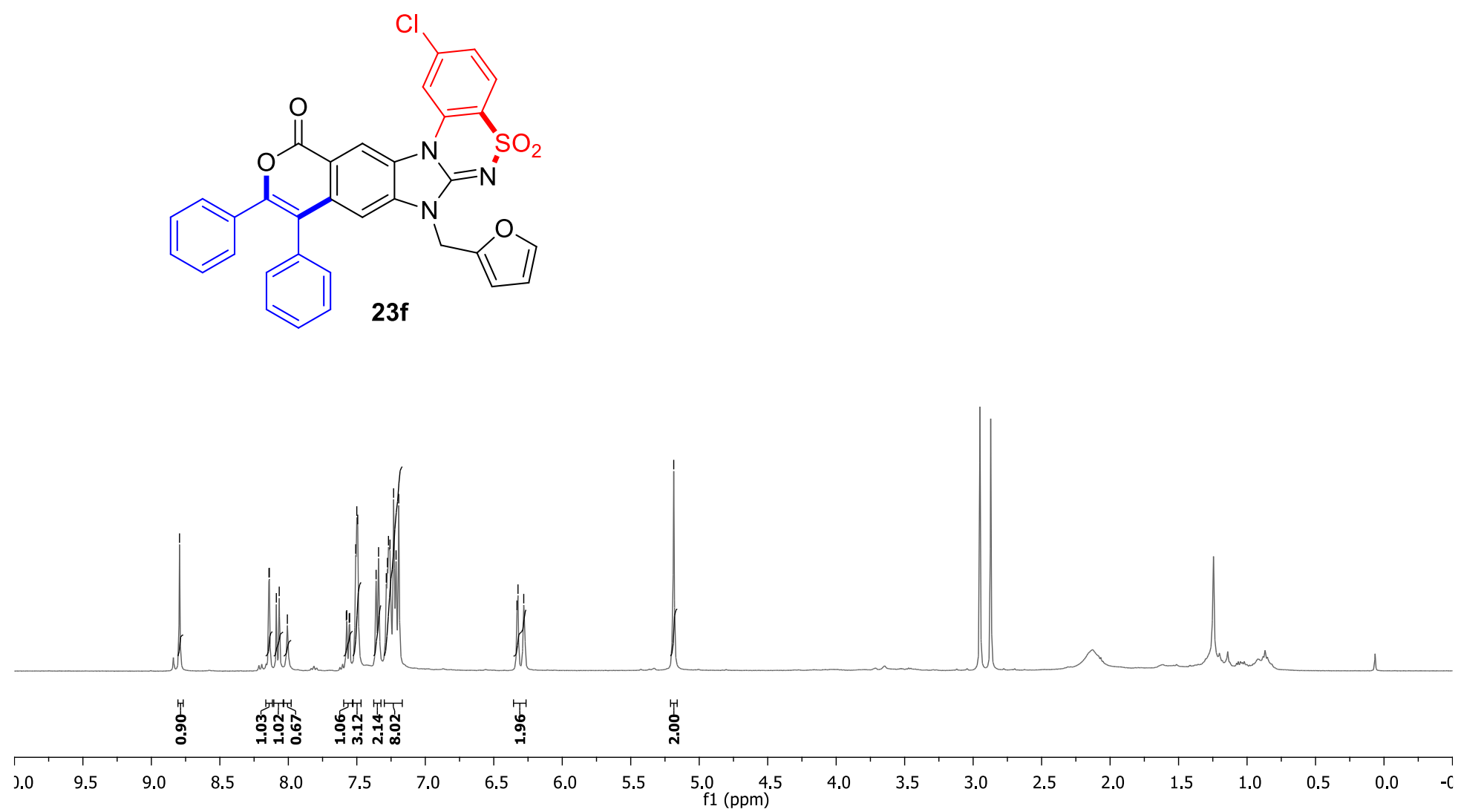

${ }^{1} \mathrm{H}$ NMR spectrum (400 MHz) of compound $23 f$ in $\mathrm{CDCl}_{3}$ 


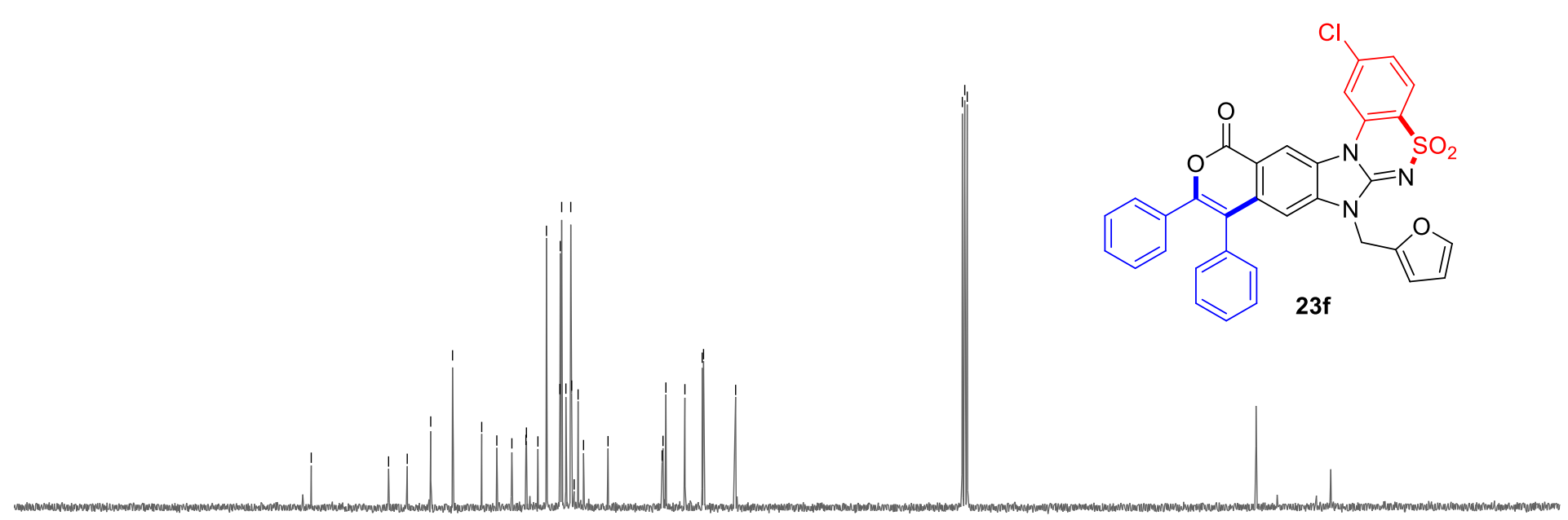

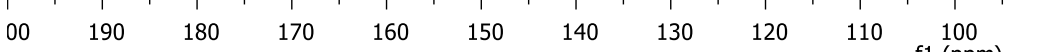

${ }^{13} \mathrm{C}$ NMR spectrum (101 MHz) of compound $23 \mathrm{f}$ in $\mathrm{CDCl}_{3}$ 


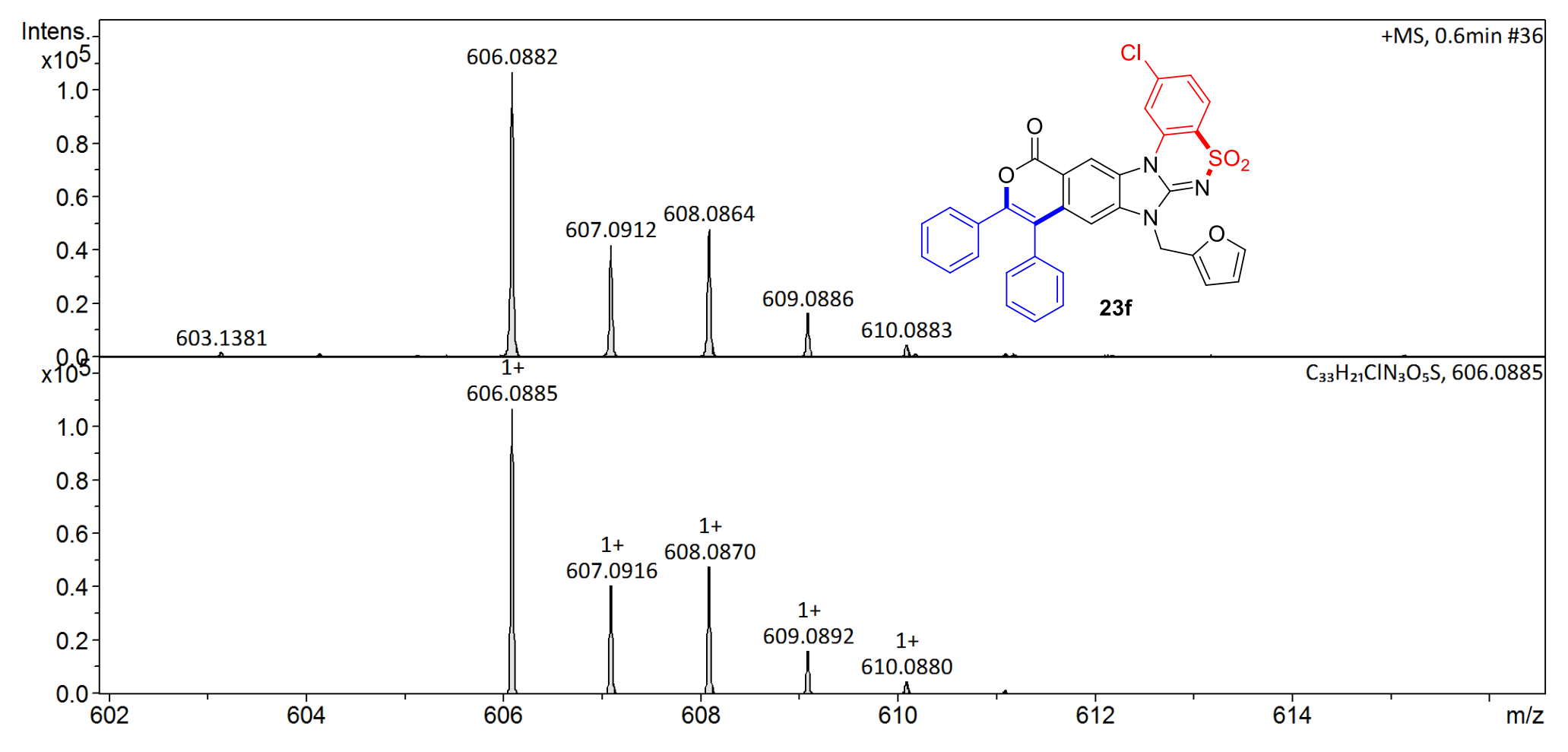

\section{Display Report}

$\begin{array}{rrlrrrrrrrr}\text { Meas. m/z } & \text { \# } & \text { Ion Formula } & \mathrm{m} / \mathrm{z} & \text { err [ppm] } & \text { mSigma } & \text { \# Sigma } & \text { Score } & \text { rdb } & \mathrm{e}^{-} \text {Conf } & \mathrm{N}-\mathrm{Rule} \\ 606.0882 & 1 & \text { C33H21CIN305S } & 606.0885 & 0.5 & 6.5 & 1 & 100.00 & 24.5 & \text { even } & \text { ok }\end{array}$

HRMS spectrum (ESI) of compound $\mathbf{2 3 f}$ 

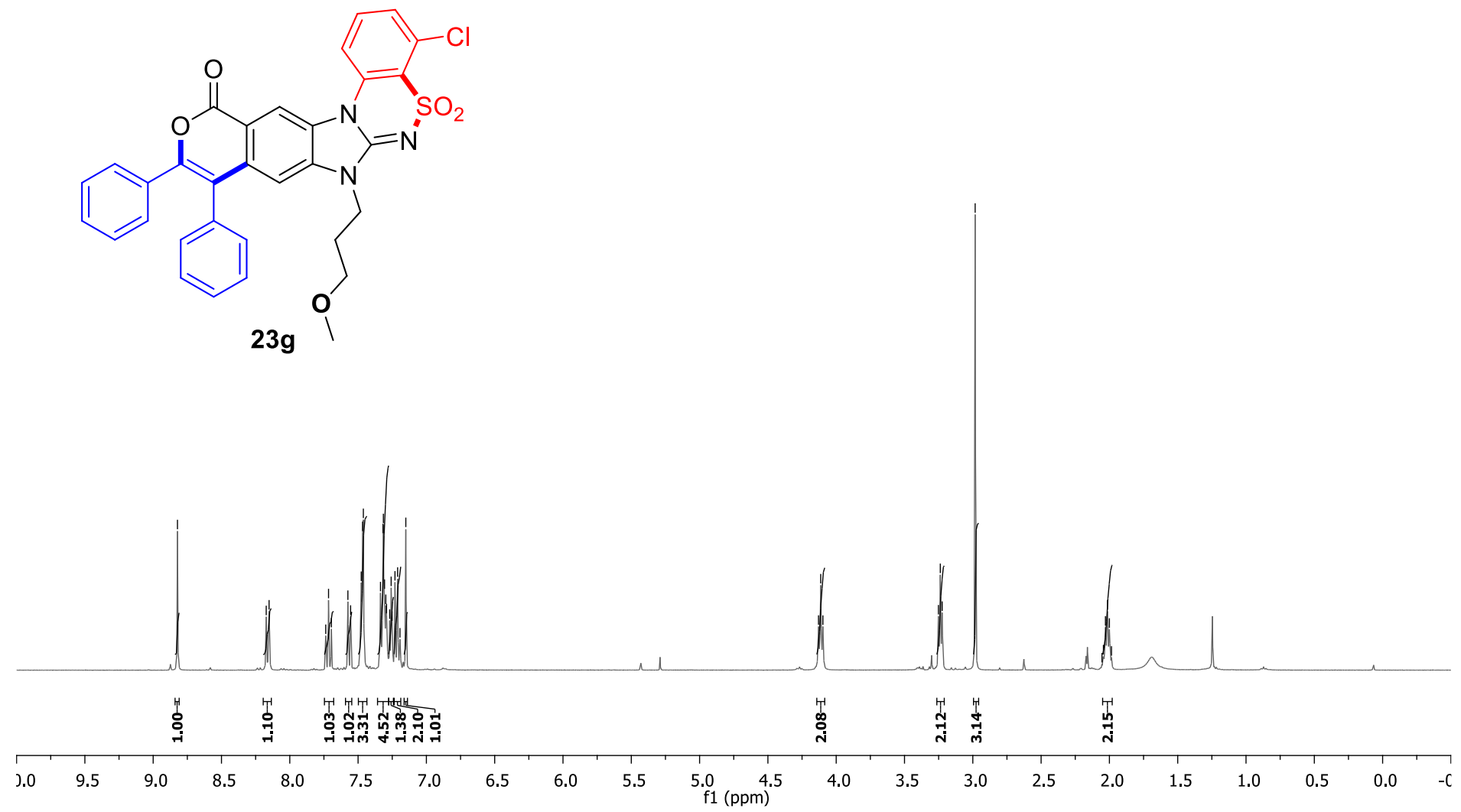

${ }^{1} \mathrm{H}$ NMR spectrum (400 MHz) of compound $\mathbf{2 3 g}$ in $\mathrm{CDCl}_{3}$ 

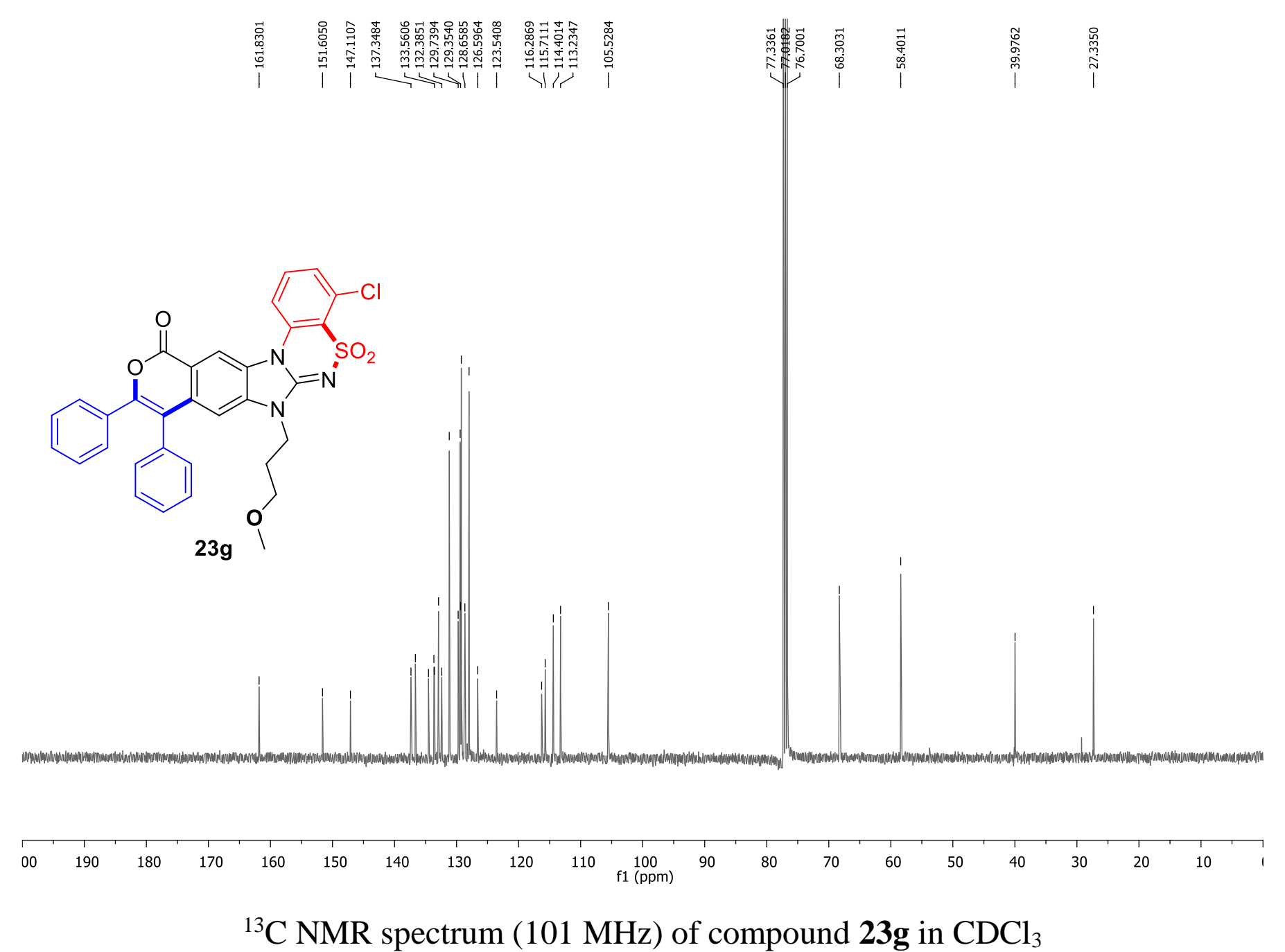


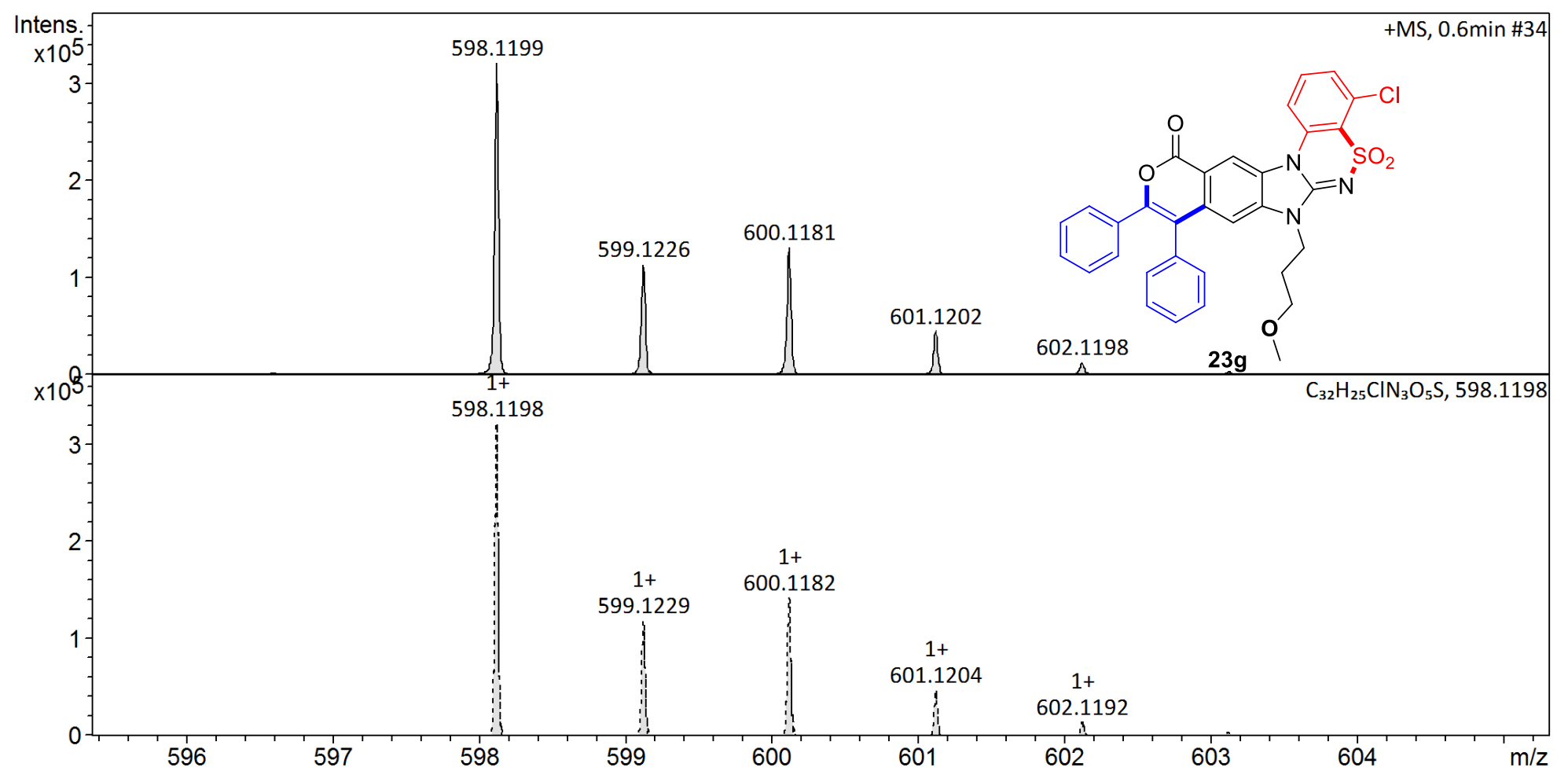

\section{Display Report}

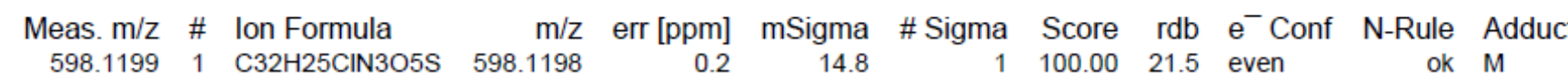

HRMS spectrum (ESI) of compound 23g 


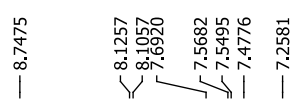

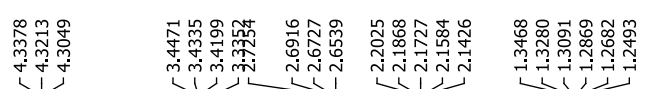
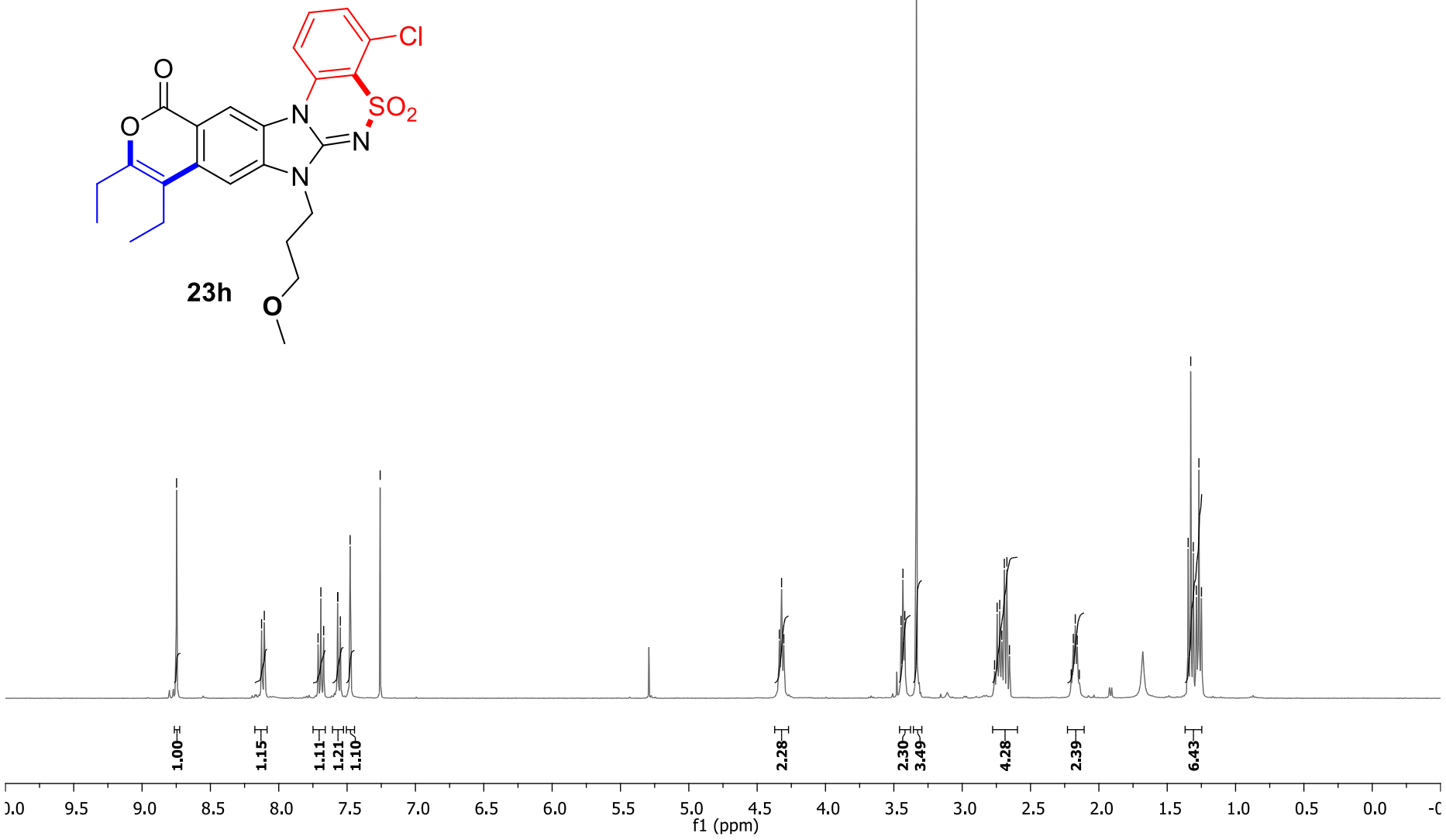

${ }^{1} \mathrm{H}$ NMR spectrum (400 MHz) of compound $\mathbf{2 3 h}$ in $\mathrm{CDCl}_{3}$ 


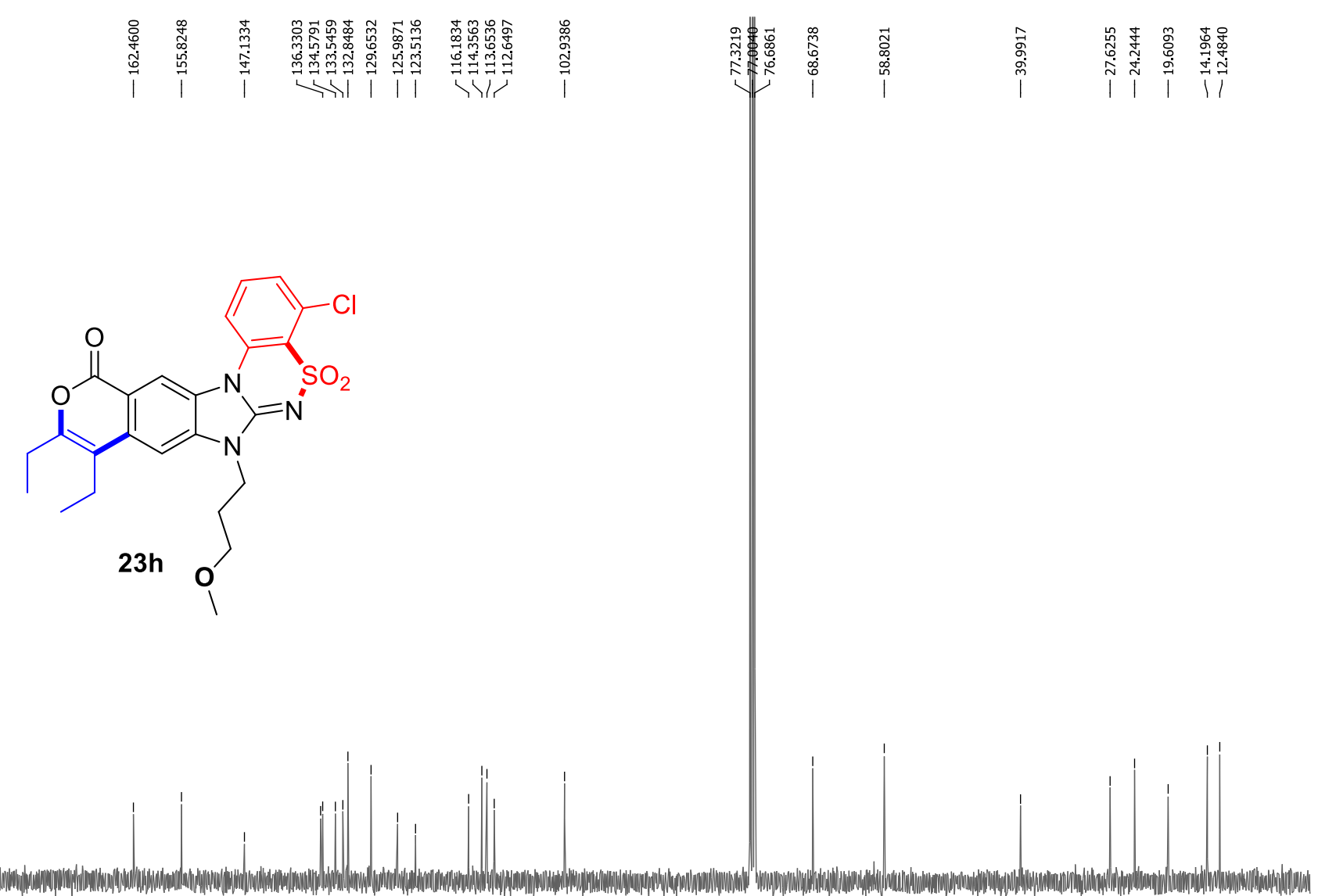

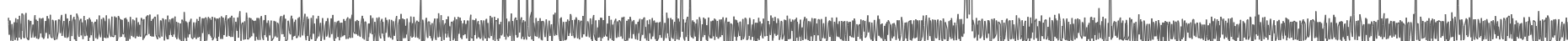
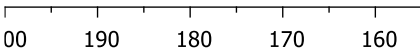

$150 \quad 140$

$130 \quad 120$

100
$\mathrm{f} 1(\mathrm{ppm})$

${ }^{13} \mathrm{C}$ NMR spectrum (101 MHz) of compound $\mathbf{2 3 h}$ in $\mathrm{CDCl}_{3}$ 


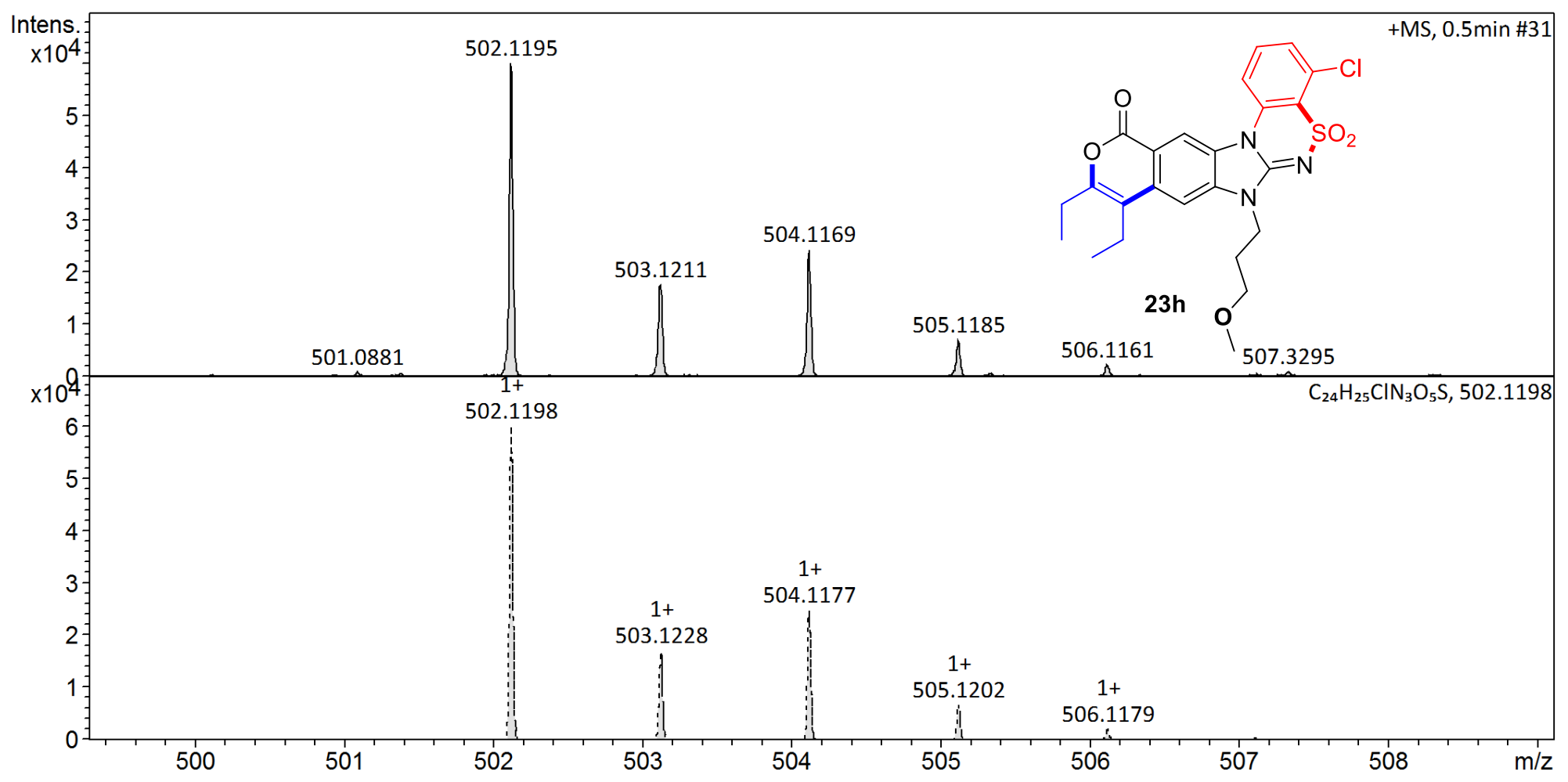

\section{Display Report}

Meas. $\mathrm{m} / \mathrm{z}$ \# Ion Formula $\mathrm{m} / \mathrm{z}$ err [ppm] mSigma \# Sigma Score rdb $\mathrm{e}^{-}$Conf $\mathrm{N}$-Rule Adduct

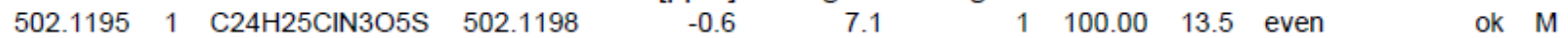

HRMS spectrum (ESI) of compound $\mathbf{2 3 h}$ 

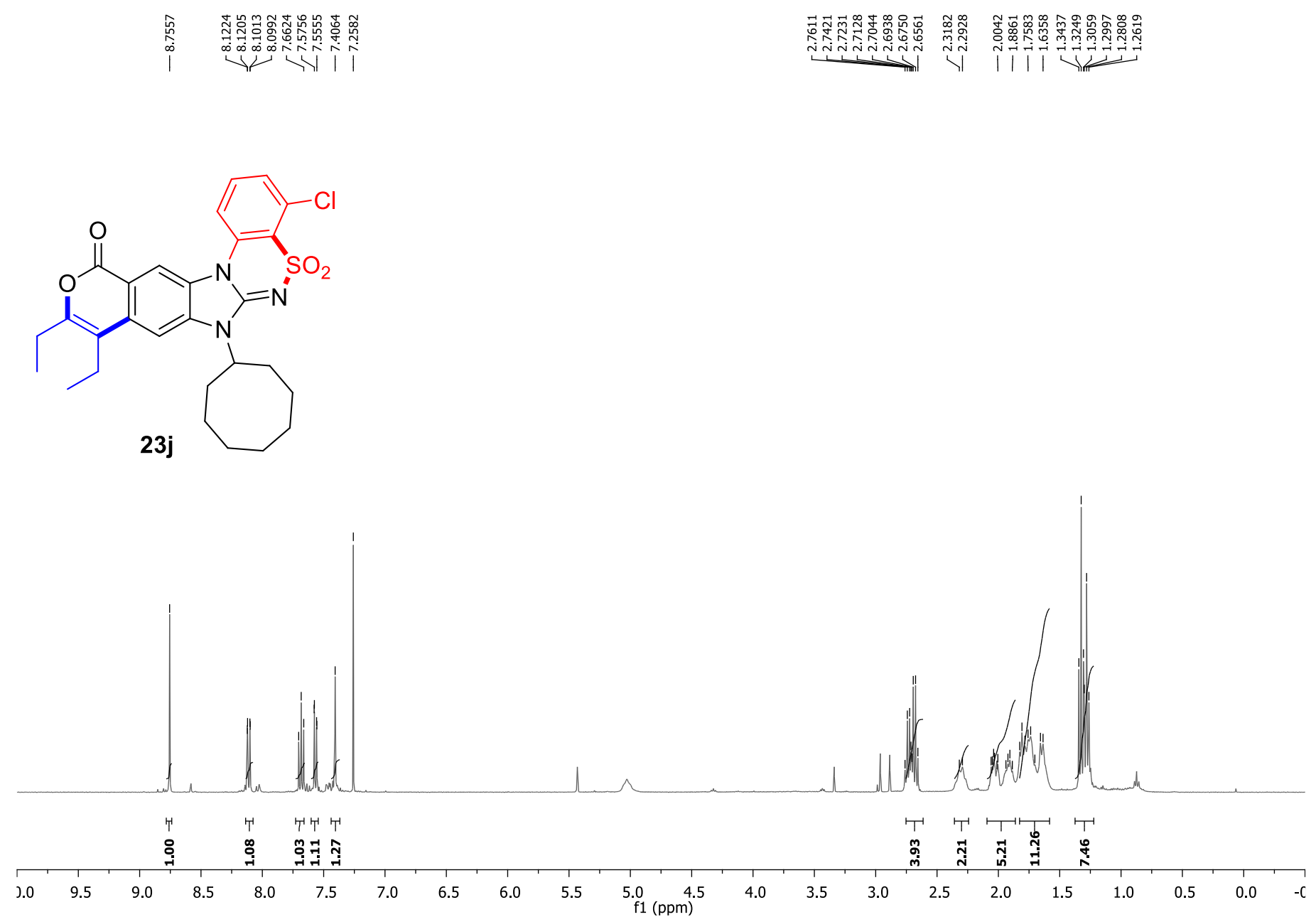

${ }^{1} \mathrm{H}$ NMR spectrum (400 MHz) of compound $\mathbf{2 3 j}$ in $\mathrm{CDCl}_{3}$ 


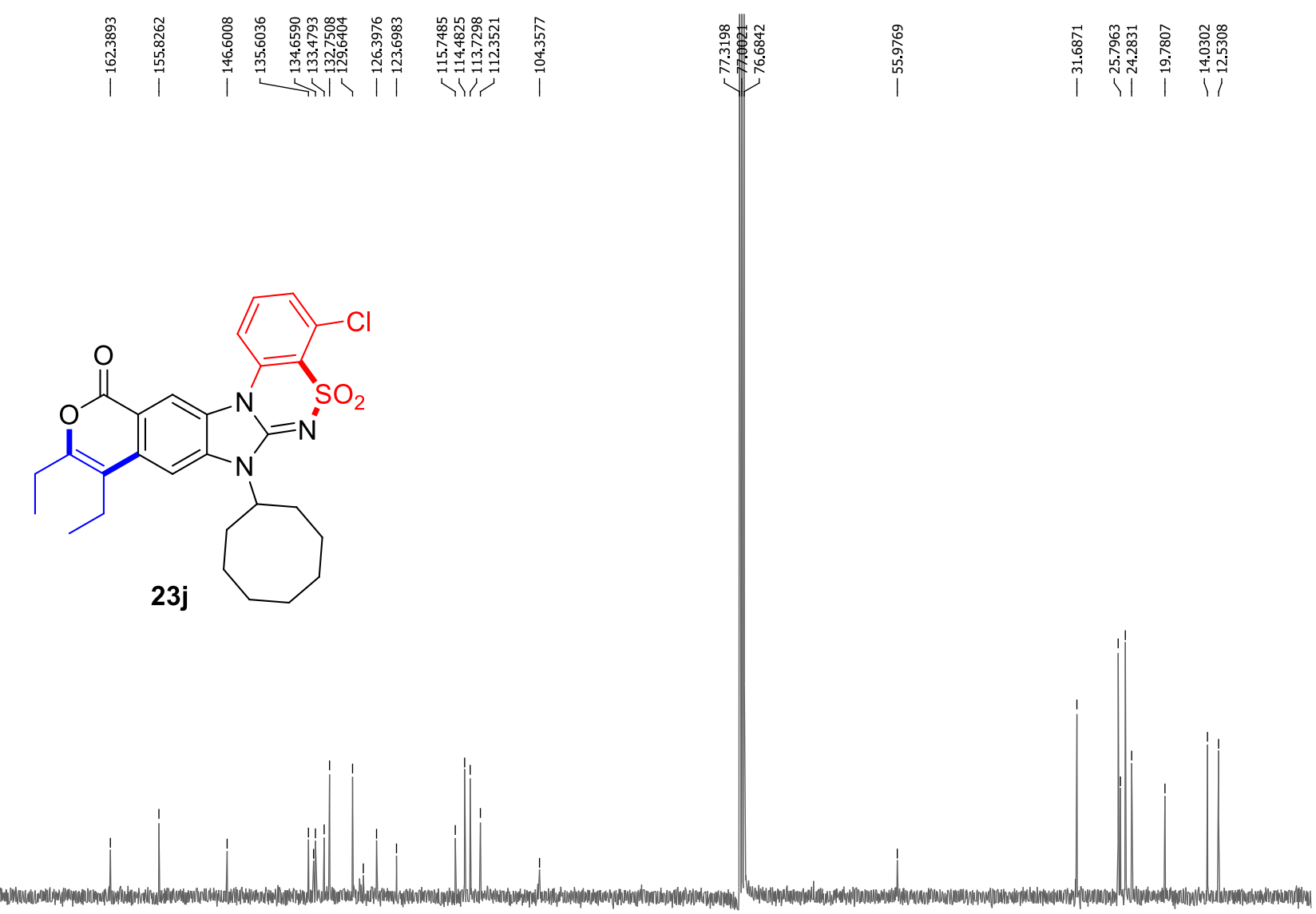

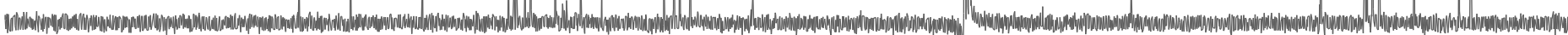
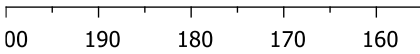

$150 \quad 140$

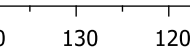

$10 \quad \begin{gathered}100 \\ \mathrm{f} 1(\mathrm{ppm})\end{gathered}$
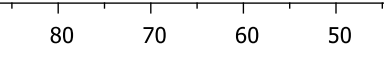

3 $\begin{array}{lll}30 & 20 & 10\end{array}$

${ }^{13} \mathrm{C}$ NMR spectrum (101 MHz) of compound $\mathbf{2 3} \mathbf{j}$ in $\mathrm{CDCl}_{3}$ 


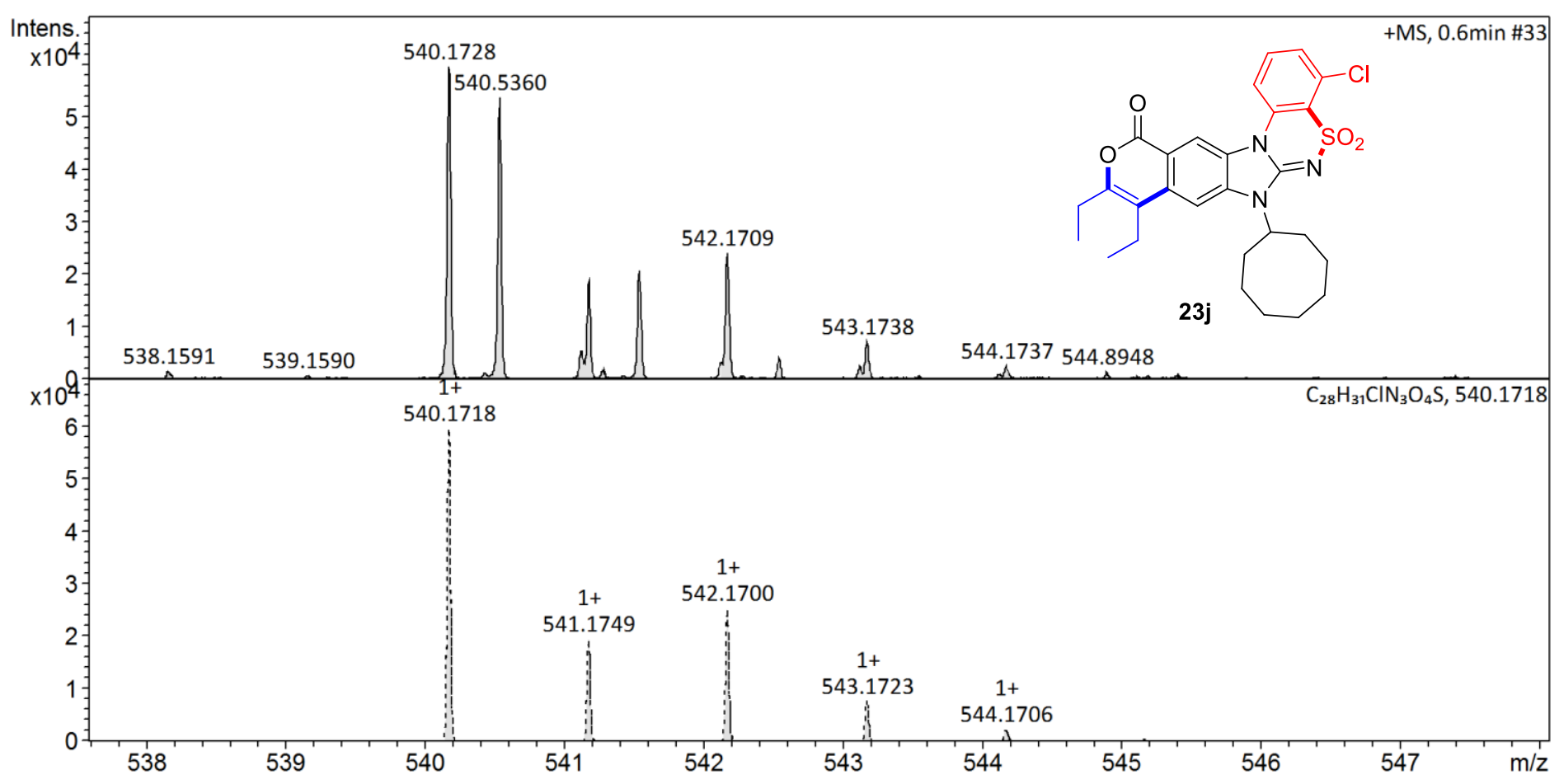

\section{Display Report}

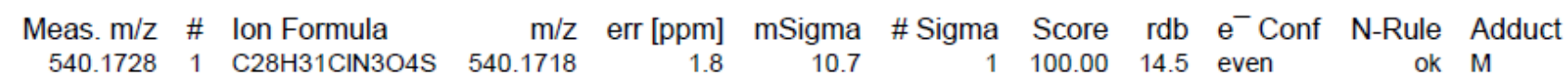

HRMS spectrum (ESI) of compound $\mathbf{2 3 j}$ 


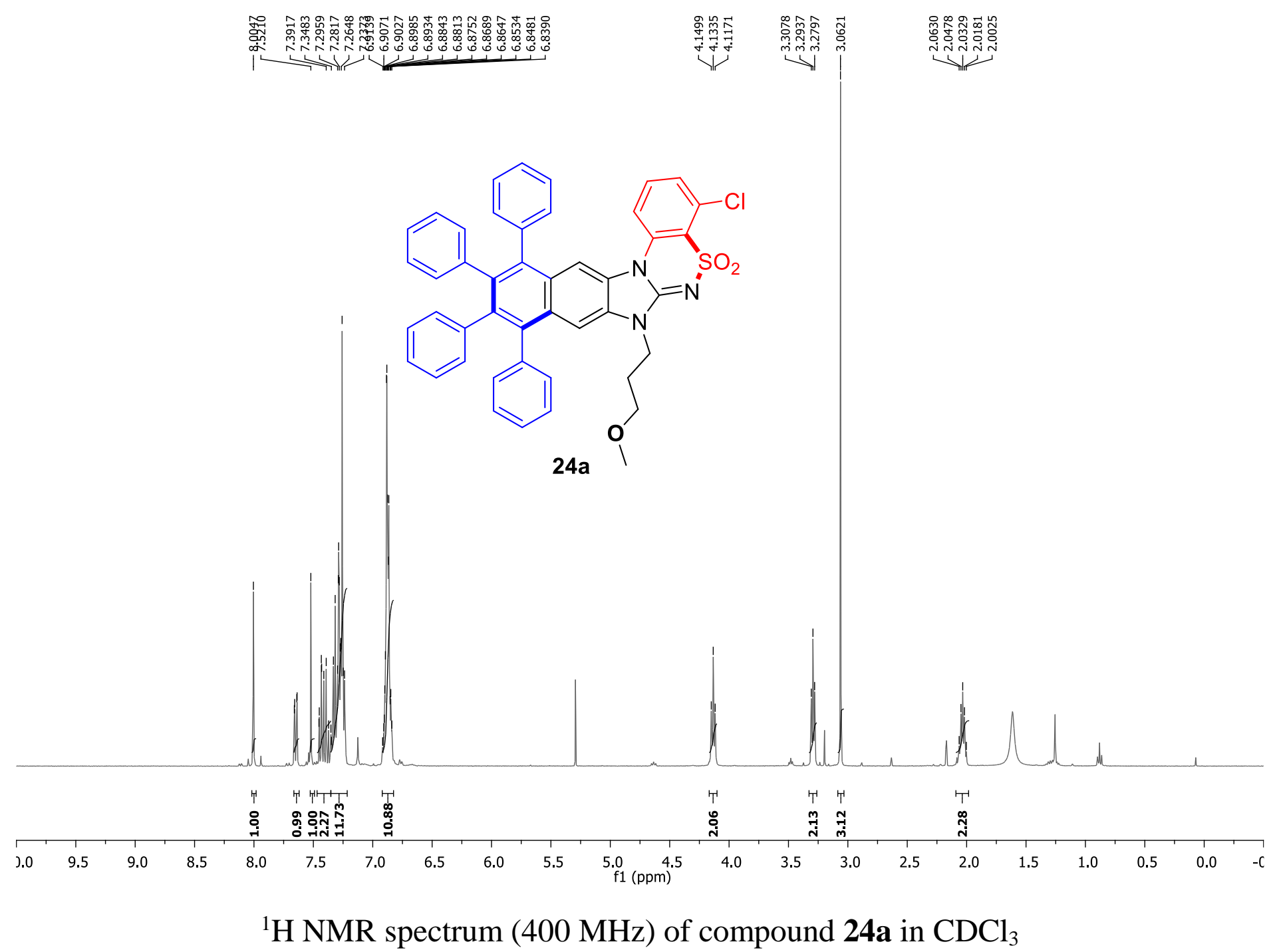



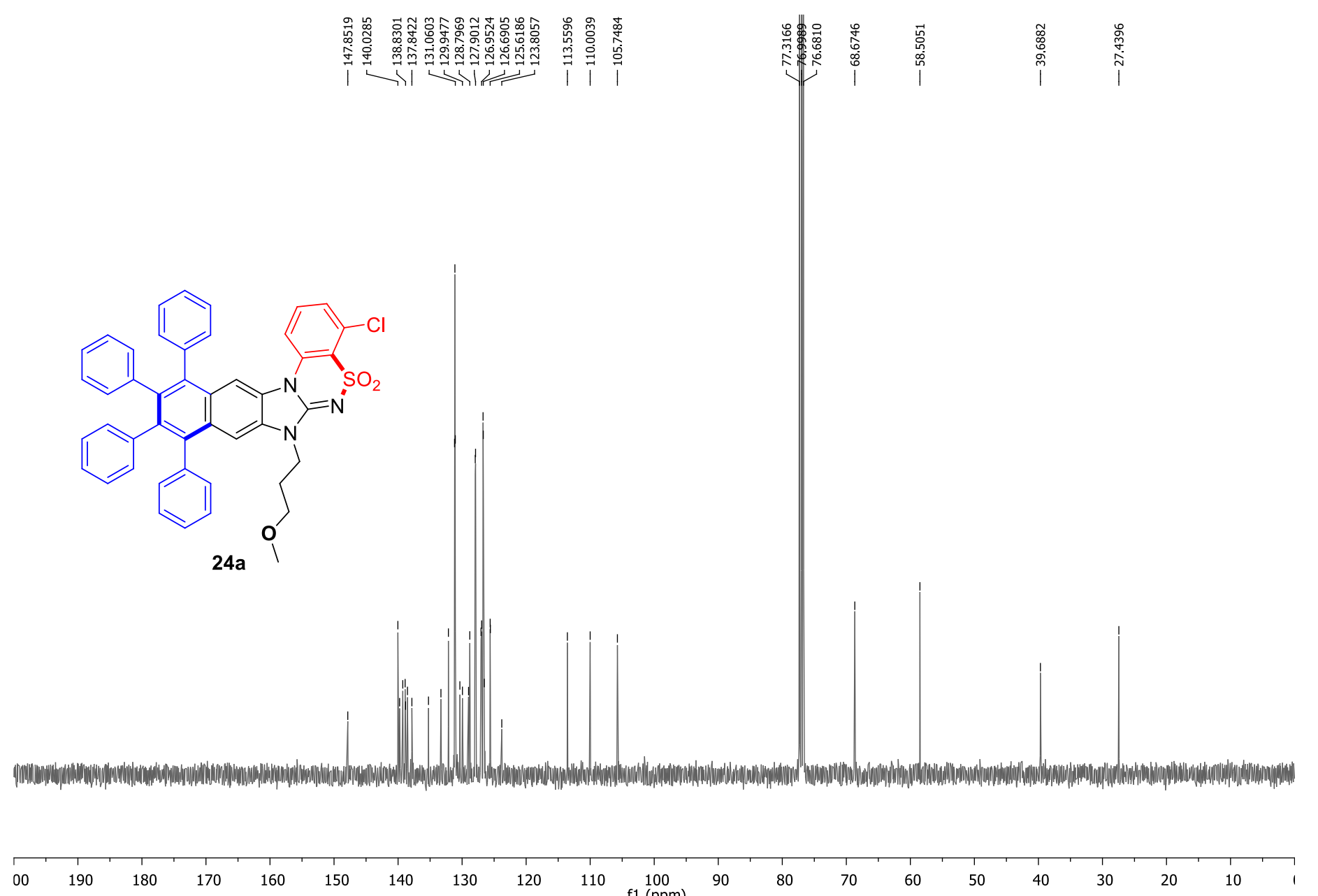

${ }^{13} \mathrm{C}$ NMR spectrum (101 MHz) of compound $\mathbf{2 4 a}$ in $\mathrm{CDCl}_{3}$ 


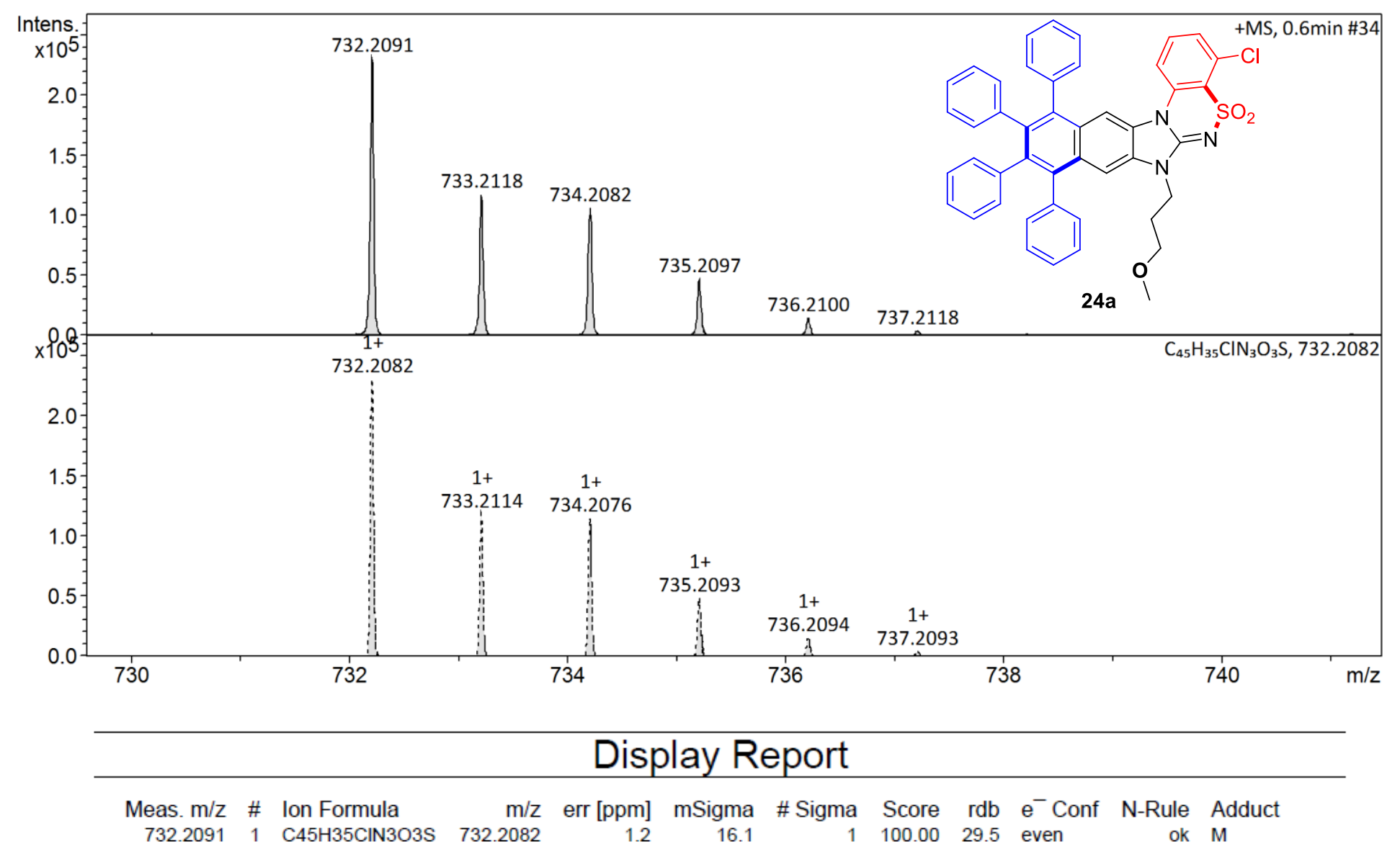

HRMS spectrum (ESI) of compound 24a 
Figure S1: ORTEP diagram of compound 9j.
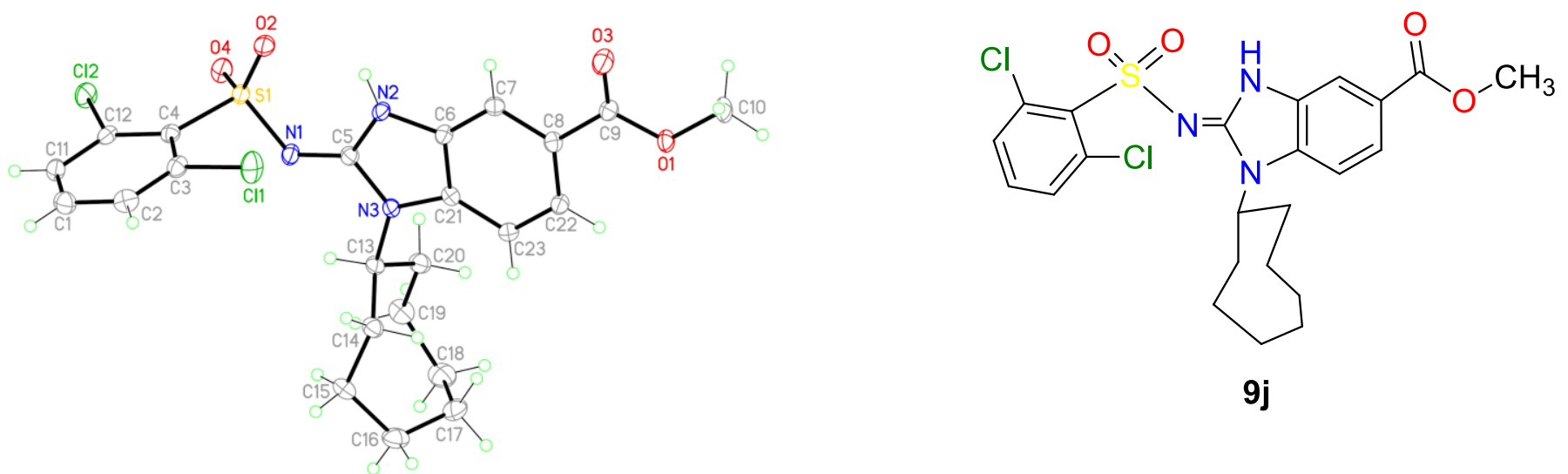

9j

Table 1. Crystal data and structure refinement for 101201LT_0m.

Identification code

Empirical formula

Formula weight

Temperature

Wavelength

Crystal system

Space group

Unit cell dimensions

Volume 1012011t_0m

C23 H25 Cl2 N3 O4 S

510.42

100(2) K

$0.71073 \AA$

Monoclinic

P 1 21/n 1

$\mathrm{a}=12.0780(5) \AA$

$\alpha=90^{\circ}$.

$\beta=93.831(2)^{\circ}$.

$\gamma=90^{\circ}$. 
Density (calculated)

Absorption coefficient

$\mathrm{F}(000)$

Crystal size

Theta range for data collection

Index ranges

Reflections collected

Independent reflections

Completeness to theta $=26.41^{\circ}$

Absorption correction

Max. and min. transmission

Refinement method

Data / restraints / parameters

Goodness-of-fit on $\mathrm{F}^{2}$

Final $\mathrm{R}$ indices [I $>2 \operatorname{sigma}(\mathrm{I})]$

$\mathrm{R}$ indices (all data)

Largest diff. peak and hole
4

$1.474 \mathrm{Mg} / \mathrm{m}^{3}$

$0.410 \mathrm{~mm}^{-1}$

1064

$0.18 \times 0.12 \times 0.10 \mathrm{~mm}^{3}$

1.96 to $26.41^{\circ}$.

$-15<=\mathrm{h}<=13,-13<=\mathrm{k}<=13,-22<=\mathrm{l}<=15$

19431

$4656[\mathrm{R}($ int $)=0.0271]$

$98.8 \%$

Semi-empirical from equivalents

0.9486 and 0.8793

Full-matrix least-squares on $\mathrm{F}^{2}$

4656 / 0 / 299

1.139

$\mathrm{R} 1=0.0310, \mathrm{wR} 2=0.0891$

$\mathrm{R} 1=0.0369, \mathrm{wR} 2=0.1070$

0.403 and -0.404 e. $\AA^{-3}$ 
Table 2. Atomic coordinates ( $\left.\times 10^{4}\right)$ and equivalent isotropic displacement parameters $\left(\AA^{2} \times 10^{3}\right)$ for 101201LT_0m. U(eq) is defined as one third of the trace of the orthogonalized $\mathrm{U}^{\mathrm{ij}}$ tensor.

\begin{tabular}{|c|c|c|c|c|}
\hline & $\mathrm{x}$ & $\mathrm{y}$ & $\mathrm{z}$ & $\mathrm{U}(\mathrm{eq})$ \\
\hline $\mathrm{S}(1)$ & $-414(1)$ & $3237(1)$ & $8267(1)$ & $16(1)$ \\
\hline $\mathrm{Cl}(2)$ & $-1378(1)$ & $2437(1)$ & $9912(1)$ & $25(1)$ \\
\hline $\mathrm{Cl}(1)$ & $-500(1)$ & $6247(1)$ & 7936(1) & $29(1)$ \\
\hline $\mathrm{O}(1)$ & $3112(1)$ & $5390(1)$ & $4190(1)$ & $18(1)$ \\
\hline $\mathrm{O}(2)$ & $-1109(1)$ & $3399(1)$ & $7600(1)$ & $22(1)$ \\
\hline $\mathrm{O}(3)$ & $1253(1)$ & $5289(1)$ & $4124(1)$ & $27(1)$ \\
\hline $\mathrm{O}(4)$ & $-398(1)$ & 1996(1) & 8591(1) & $21(1)$ \\
\hline $\mathrm{N}(1)$ & $837(1)$ & $3692(1)$ & $8197(1)$ & $17(1)$ \\
\hline $\mathrm{N}(2)$ & $690(1)$ & $4138(2)$ & $6884(1)$ & $16(1)$ \\
\hline $\mathrm{N}(3)$ & $2282(1)$ & $4442(1)$ & $7521(1)$ & $16(1)$ \\
\hline $\mathrm{C}(1)$ & $-1519(2)$ & $6195(2)$ & $9948(1)$ & $24(1)$ \\
\hline $\mathrm{C}(2)$ & $-1191(2)$ & $6576(2)$ & $9269(1)$ & $23(1)$ \\
\hline $\mathrm{C}(3)$ & $-890(1)$ & $5665(2)$ & $8772(1)$ & $19(1)$ \\
\hline$C(4)$ & $-891(1)$ & $4347(2)$ & $8927(1)$ & $16(1)$ \\
\hline$C(5)$ & $1204(1)$ & $4065(2)$ & $7566(1)$ & $15(1)$ \\
\hline$C(6)$ & 1441(1) & $4557(2)$ & $6390(1)$ & $16(1)$ \\
\hline $\mathrm{C}(7)$ & $1319(1)$ & $4737(2)$ & $5640(1)$ & $17(1)$ \\
\hline $\mathrm{C}(8)$ & $2252(1)$ & $5145(2)$ & $5295(1)$ & $16(1)$ \\
\hline $\mathrm{C}(9)$ & $2134(1)$ & $5288(2)$ & $4482(1)$ & $17(1)$ \\
\hline $\mathrm{C}(10)$ & $3074(2)$ & $5423(2)$ & 3395(1) & $23(1)$ \\
\hline
\end{tabular}




$\begin{array}{lrrrr}\mathrm{C}(11) & -1551(2) & 4914(2) & 10117(1) & 21(1) \\ \mathrm{C}(12) & -1253(1) & 3996(2) & 9613(1) & 17(1) \\ \mathrm{C}(13) & 3119(1) & 4438(2) & 8151(1) & 16(1) \\ \mathrm{C}(14) & 3502(2) & 5818(2) & 8308(1) & 19(1) \\ \mathrm{C}(15) & 4212(2) & 6001(2) & 9033(1) & 22(1) \\ \mathrm{C}(16) & 5465(2) & 6080(2) & 8959(1) & 26(1) \\ \mathrm{C}(17) & 5965(2) & 5112(2) & 8452(1) & 28(1) \\ \mathrm{C}(18) & 5931(2) & 3712(2) & 8675(1) & 30(1) \\ \mathrm{C}(19) & 4785(2) & 3082(2) & 8686(1) & 24(1) \\ \mathrm{C}(20) & 3975(1) & 3392(2) & 8023(1) & 20(1) \\ \mathrm{C}(21) & 2454(1) & 4752(2) & 6792(1) & 15(1) \\ \mathrm{C}(22) & 3266(1) & 5365(2) & 5696(1) & 17(1) \\ \mathrm{C}(23) & 3379(1) & 5172(2) & 6451(1) & 18(1) \\ \end{array}$


Table 3. Bond lengths $[\AA]$ and angles $\left[^{\circ}\right]$ for $101201 \mathrm{LT} \_0 \mathrm{~m}$.

\begin{tabular}{ll}
\hline $\mathrm{S}(1)-\mathrm{O}(4)$ & $1.4258(13)$ \\
$\mathrm{S}(1)-\mathrm{O}(2)$ & $1.4429(12)$ \\
$\mathrm{S}(1)-\mathrm{N}(1)$ & $1.5971(14)$ \\
$\mathrm{S}(1)-\mathrm{C}(4)$ & $1.7943(17)$ \\
$\mathrm{Cl}(2)-\mathrm{C}(12)$ & $1.7280(18)$ \\
$\mathrm{Cl}(1)-\mathrm{C}(3)$ & $1.7374(17)$ \\
$\mathrm{O}(1)-\mathrm{C}(9)$ & $1.331(2)$ \\
$\mathrm{O}(1)-\mathrm{C}(10)$ & $1.4504(19)$ \\
$\mathrm{O}(3)-\mathrm{C}(9)$ & $1.211(2)$ \\
$\mathrm{N}(1)-\mathrm{C}(5)$ & $1.320(2)$ \\
$\mathrm{N}(2)-\mathrm{C}(5)$ & $1.357(2)$ \\
$\mathrm{N}(2)-\mathrm{C}(6)$ & $1.392(2)$ \\
$\mathrm{N}(2)-\mathrm{H}(2)$ & 0.8800 \\
$\mathrm{~N}(3)-\mathrm{C}(5)$ & $1.369(2)$ \\
$\mathrm{N}(3)-\mathrm{C}(21)$ & $1.398(2)$ \\
$\mathrm{N}(3)-\mathrm{C}(13)$ & $1.479(2)$ \\
$\mathrm{C}(1)-\mathrm{C}(11)$ & $1.376(3)$ \\
$\mathrm{C}(1)-\mathrm{C}(2)$ & $1.384(3)$ \\
$\mathrm{C}(1)-\mathrm{H}(1)$ & 0.9500 \\
$\mathrm{C}(2)-\mathrm{C}(3)$ & $1.382(3)$ \\
$\mathrm{C}(2)-\mathrm{H}(2 \mathrm{~A})$ & 0.9500 \\
$\mathrm{C}(3)-\mathrm{C}(4)$ & $1.406(2)$ \\
$\mathrm{C}(4)-\mathrm{C}(12)$ & $1.404(2)$ \\
& \\
& \\
&
\end{tabular}




$\begin{array}{ll}\mathrm{C}(6)-\mathrm{C}(7) & 1.380(2) \\ \mathrm{C}(6)-\mathrm{C}(21) & 1.399(2) \\ \mathrm{C}(7)-\mathrm{C}(8) & 1.394(2) \\ \mathrm{C}(7)-\mathrm{H}(7) & 0.9500 \\ \mathrm{C}(8)-\mathrm{C}(22) & 1.404(2) \\ \mathrm{C}(8)-\mathrm{C}(9) & 1.490(2) \\ \mathrm{C}(10)-\mathrm{H}(10 A) & 0.9800 \\ \mathrm{C}(10)-\mathrm{H}(10 \mathrm{~B}) & 0.9800 \\ \mathrm{C}(10)-\mathrm{H}(10 \mathrm{C}) & 0.9800 \\ \mathrm{C}(11)-\mathrm{C}(12) & 1.392(2) \\ \mathrm{C}(11)-\mathrm{H}(11) & 0.9500 \\ \mathrm{C}(13)-\mathrm{C}(20) & 1.534(2) \\ \mathrm{C}(13)-\mathrm{C}(14) & 1.535(2) \\ \mathrm{C}(13)-\mathrm{H}(13) & 1.0000 \\ \mathrm{C}(14)-\mathrm{C}(15) & 1.540(2) \\ \mathrm{C}(14)-\mathrm{H}(14 \mathrm{~A}) & 0.9900 \\ \mathrm{C}(14)-\mathrm{H}(14 \mathrm{~B}) & 0.9900 \\ \mathrm{C}(15)-\mathrm{C}(16) & 1.530(3) \\ \mathrm{C}(15)-\mathrm{H}(15 \mathrm{~A}) & 0.9900 \\ \mathrm{C}(15)-\mathrm{H}(15 \mathrm{~B}) & 0.9900 \\ \mathrm{C}(16)-\mathrm{C}(17) & 1.523(3) \\ \mathrm{C}(16)-\mathrm{H}(16 \mathrm{~A}) & 0.9900 \\ \mathrm{C}(16)-\mathrm{H}(16 \mathrm{~B}) & 0.9900 \\ \mathrm{C}(17)-\mathrm{C}(18) & 1.520(3) \\ \mathrm{C}(17)-\mathrm{H}(17 \mathrm{~A}) & 0.9900 \\ & \end{array}$




$\begin{array}{lc}\mathrm{C}(17)-\mathrm{H}(17 \mathrm{~B}) & 0.9900 \\ \mathrm{C}(18)-\mathrm{C}(19) & 1.534(3) \\ \mathrm{C}(18)-\mathrm{H}(18 \mathrm{~A}) & 0.9900 \\ \mathrm{C}(18)-\mathrm{H}(18 \mathrm{~B}) & 0.9900 \\ \mathrm{C}(19)-\mathrm{C}(20) & 1.538(2) \\ \mathrm{C}(19)-\mathrm{H}(19 A) & 0.9900 \\ \mathrm{C}(19)-\mathrm{H}(19 \mathrm{~B}) & 0.9900 \\ \mathrm{C}(20)-\mathrm{H}(20 A) & 0.9900 \\ \mathrm{C}(20)-\mathrm{H}(20 \mathrm{~B}) & 0.9900 \\ \mathrm{C}(21)-\mathrm{C}(23) & 1.387(2) \\ \mathrm{C}(22)-\mathrm{C}(23) & 1.389(2) \\ \mathrm{C}(22)-\mathrm{H}(22) & 0.9500 \\ \mathrm{C}(23)-\mathrm{H}(23) & 0.9500 \\ & \\ \mathrm{O}(4)-\mathrm{S}(1)-\mathrm{O}(2) & 116.62(8) \\ \mathrm{O}(4)-\mathrm{S}(1)-\mathrm{N}(1) & 108.54(8) \\ \mathrm{O}(2)-\mathrm{S}(1)-\mathrm{N}(1) & 113.41(7) \\ \mathrm{O}(4)-\mathrm{S}(1)-\mathrm{C}(4) & 107.83(8) \\ \mathrm{O}(2)-\mathrm{S}(1)-\mathrm{C}(4) & 107.21(8) \\ \mathrm{N}(1)-\mathrm{S}(1)-\mathrm{C}(4) & 102.08(8) \\ \mathrm{C}(9)-\mathrm{O}(1)-\mathrm{C}(10) & 115.62(13) \\ \mathrm{C}(5)-\mathrm{N}(1)-\mathrm{S}(1) & 122.18(12) \\ \mathrm{C}(5)-\mathrm{N}(2)-\mathrm{C}(6) & 109.56(14) \\ \mathrm{C}(5)-\mathrm{N}(2)-\mathrm{H}(2) & 125.2 \\ \mathrm{C}(6)-\mathrm{N}(2)-\mathrm{H}(2) & 125.2 \\ & \end{array}$




$\begin{array}{ll}\mathrm{C}(5)-\mathrm{N}(3)-\mathrm{C}(21) & 109.04(13) \\ \mathrm{C}(5)-\mathrm{N}(3)-\mathrm{C}(13) & 123.71(14) \\ \mathrm{C}(21)-\mathrm{N}(3)-\mathrm{C}(13) & 127.17(14) \\ \mathrm{C}(11)-\mathrm{C}(1)-\mathrm{C}(2) & 119.67(17) \\ \mathrm{C}(11)-\mathrm{C}(1)-\mathrm{H}(1) & 120.2 \\ \mathrm{C}(2)-\mathrm{C}(1)-\mathrm{H}(1) & 120.2 \\ \mathrm{C}(3)-\mathrm{C}(2)-\mathrm{C}(1) & 119.55(17) \\ \mathrm{C}(3)-\mathrm{C}(2)-\mathrm{H}(2 \mathrm{~A}) & 120.2 \\ \mathrm{C}(1)-\mathrm{C}(2)-\mathrm{H}(2 \mathrm{~A}) & 120.2 \\ \mathrm{C}(2)-\mathrm{C}(3)-\mathrm{C}(4) & 122.68(16) \\ \mathrm{C}(2)-\mathrm{C}(3)-\mathrm{Cl}(1) & 115.73(14) \\ \mathrm{C}(4)-\mathrm{C}(3)-\mathrm{Cl}(1) & 121.59(13) \\ \mathrm{C}(12)-\mathrm{C}(4)-\mathrm{C}(3) & 116.11(15) \\ \mathrm{C}(12)-\mathrm{C}(4)-\mathrm{S}(1) & 124.26(13) \\ \mathrm{C}(3)-\mathrm{C}(4)-\mathrm{S}(1) & 119.61(13) \\ \mathrm{N}(1)-\mathrm{C}(5)-\mathrm{N}(2) & 131.18(16) \\ \mathrm{N}(1)-\mathrm{C}(5)-\mathrm{N}(3) & 120.94(15) \\ \mathrm{N}(2)-\mathrm{C}(5)-\mathrm{N}(3) & 107.86(14) \\ \mathrm{C}(7)-\mathrm{C}(6)-\mathrm{N}(2) & 130.84(15) \\ \mathrm{C}(7)-\mathrm{C}(6)-\mathrm{C}(21) & 122.24(15) \\ \mathrm{N}(2)-\mathrm{C}(6)-\mathrm{C}(21) & 106.89(14) \\ \mathrm{C}(6)-\mathrm{C}(7)-\mathrm{C}(8) & 116.97(15) \\ \mathrm{C}(6)-\mathrm{C}(7)-\mathrm{H}(7) & 121.5 \\ \mathrm{C}(8)-\mathrm{C}(7)-\mathrm{H}(7) & 121.5 \\ \mathrm{C}(7)-\mathrm{C}(8)-\mathrm{C}(22) & 121.17(15) \\ & \end{array}$




$\begin{array}{ll}\mathrm{C}(7)-\mathrm{C}(8)-\mathrm{C}(9) & 117.09(15) \\ \mathrm{C}(22)-\mathrm{C}(8)-\mathrm{C}(9) & 121.69(15) \\ \mathrm{O}(3)-\mathrm{C}(9)-\mathrm{O}(1) & 123.77(15) \\ \mathrm{O}(3)-\mathrm{C}(9)-\mathrm{C}(8) & 123.97(16) \\ \mathrm{O}(1)-\mathrm{C}(9)-\mathrm{C}(8) & 112.25(14) \\ \mathrm{O}(1)-\mathrm{C}(10)-\mathrm{H}(10 A) & 109.5 \\ \mathrm{O}(1)-\mathrm{C}(10)-\mathrm{H}(10 \mathrm{~B}) & 109.5 \\ \mathrm{H}(10 \mathrm{~A})-\mathrm{C}(10)-\mathrm{H}(10 \mathrm{~B}) & 109.5 \\ \mathrm{O}(1)-\mathrm{C}(10)-\mathrm{H}(10 \mathrm{C}) & 109.5 \\ \mathrm{H}(10 \mathrm{~A})-\mathrm{C}(10)-\mathrm{H}(10 \mathrm{C}) & 109.5 \\ \mathrm{H}(10 \mathrm{~B})-\mathrm{C}(10)-\mathrm{H}(10 \mathrm{C}) & 109.5 \\ \mathrm{C}(1)-\mathrm{C}(11)-\mathrm{C}(12) & 120.71(17) \\ \mathrm{C}(1)-\mathrm{C}(11)-\mathrm{H}(11) & 119.6 \\ \mathrm{C}(12)-\mathrm{C}(11)-\mathrm{H}(11) & 119.6 \\ \mathrm{C}(11)-\mathrm{C}(12)-\mathrm{C}(4) & 121.24(16) \\ \mathrm{C}(11)-\mathrm{C}(12)-\mathrm{Cl}(2) & 114.17(13) \\ \mathrm{C}(4)-\mathrm{C}(12)-\mathrm{Cl}(2) & 124.59(14) \\ \mathrm{N}(3)-\mathrm{C}(13)-\mathrm{C}(20) & 108.39(13) \\ \mathrm{N}(3)-\mathrm{C}(13)-\mathrm{C}(14) & 108.95(14) \\ \mathrm{C}(20)-\mathrm{C}(13)-\mathrm{C}(14) & 119.97(15) \\ \mathrm{N}(3)-\mathrm{C}(13)-\mathrm{H}(13) & 106.2 \\ \mathrm{C}(20)-\mathrm{C}(13)-\mathrm{H}(13) & 106.2 \\ \mathrm{C}(14)-\mathrm{C}(13)-\mathrm{H}(13) & 106.2 \\ \mathrm{C}(13)-\mathrm{C}(14)-\mathrm{C}(15) & 114.89(14) \\ \mathrm{C}(13)-\mathrm{C}(14)-\mathrm{H}(14 \mathrm{~A}) & 108.5 \\ & \end{array}$




\begin{tabular}{|c|c|}
\hline $\mathrm{C}(15)-\mathrm{C}(14)-\mathrm{H}(14 \mathrm{~A})$ & 108.5 \\
\hline $\mathrm{C}(13)-\mathrm{C}(14)-\mathrm{H}(14 \mathrm{~B})$ & 108.5 \\
\hline $\mathrm{C}(15)-\mathrm{C}(14)-\mathrm{H}(14 \mathrm{~B})$ & 108.5 \\
\hline $\mathrm{H}(14 \mathrm{~A})-\mathrm{C}(14)-\mathrm{H}(14 \mathrm{~B})$ & 107.5 \\
\hline$C(16)-C(15)-C(14)$ & $115.38(15)$ \\
\hline $\mathrm{C}(16)-\mathrm{C}(15)-\mathrm{H}(15 \mathrm{~A})$ & 108.4 \\
\hline $\mathrm{C}(14)-\mathrm{C}(15)-\mathrm{H}(15 \mathrm{~A})$ & 108.4 \\
\hline $\mathrm{C}(16)-\mathrm{C}(15)-\mathrm{H}(15 \mathrm{~B})$ & 108.4 \\
\hline $\mathrm{C}(14)-\mathrm{C}(15)-\mathrm{H}(15 \mathrm{~B})$ & 108.4 \\
\hline $\mathrm{H}(15 \mathrm{~A})-\mathrm{C}(15)-\mathrm{H}(15 \mathrm{~B})$ & 107.5 \\
\hline$C(17)-C(16)-C(15)$ & $116.88(15)$ \\
\hline $\mathrm{C}(17)-\mathrm{C}(16)-\mathrm{H}(16 \mathrm{~A})$ & 108.1 \\
\hline $\mathrm{C}(15)-\mathrm{C}(16)-\mathrm{H}(16 \mathrm{~A})$ & 108.1 \\
\hline $\mathrm{C}(17)-\mathrm{C}(16)-\mathrm{H}(16 \mathrm{~B})$ & 108.1 \\
\hline $\mathrm{C}(15)-\mathrm{C}(16)-\mathrm{H}(16 \mathrm{~B})$ & 108.1 \\
\hline $\mathrm{H}(16 \mathrm{~A})-\mathrm{C}(16)-\mathrm{H}(16 \mathrm{~B})$ & 107.3 \\
\hline$C(18)-C(17)-C(16)$ & $117.26(17)$ \\
\hline $\mathrm{C}(18)-\mathrm{C}(17)-\mathrm{H}(17 \mathrm{~A})$ & 108.0 \\
\hline $\mathrm{C}(16)-\mathrm{C}(17)-\mathrm{H}(17 \mathrm{~A})$ & 108.0 \\
\hline $\mathrm{C}(18)-\mathrm{C}(17)-\mathrm{H}(17 \mathrm{~B})$ & 108.0 \\
\hline $\mathrm{C}(16)-\mathrm{C}(17)-\mathrm{H}(17 \mathrm{~B})$ & 108.0 \\
\hline $\mathrm{H}(17 \mathrm{~A})-\mathrm{C}(17)-\mathrm{H}(17 \mathrm{~B})$ & 107.2 \\
\hline $\mathrm{C}(17)-\mathrm{C}(18)-\mathrm{C}(19)$ & $117.21(16)$ \\
\hline $\mathrm{C}(17)-\mathrm{C}(18)-\mathrm{H}(18 \mathrm{~A})$ & 108.0 \\
\hline $\mathrm{C}(19)-\mathrm{C}(18)-\mathrm{H}(18 \mathrm{~A})$ & 108.0 \\
\hline
\end{tabular}




\begin{tabular}{|c|c|}
\hline $\mathrm{C}(17)-\mathrm{C}(18)-\mathrm{H}(18 \mathrm{~B})$ & 108.0 \\
\hline $\mathrm{C}(19)-\mathrm{C}(18)-\mathrm{H}(18 \mathrm{~B})$ & 108.0 \\
\hline $\mathrm{H}(18 \mathrm{~A})-\mathrm{C}(18)-\mathrm{H}(18 \mathrm{~B})$ & 107.2 \\
\hline$C(18)-C(19)-C(20)$ & $115.25(16)$ \\
\hline $\mathrm{C}(18)-\mathrm{C}(19)-\mathrm{H}(19 \mathrm{~A})$ & 108.5 \\
\hline $\mathrm{C}(20)-\mathrm{C}(19)-\mathrm{H}(19 \mathrm{~A})$ & 108.5 \\
\hline $\mathrm{C}(18)-\mathrm{C}(19)-\mathrm{H}(19 \mathrm{~B})$ & 108.5 \\
\hline $\mathrm{C}(20)-\mathrm{C}(19)-\mathrm{H}(19 \mathrm{~B})$ & 108.5 \\
\hline $\mathrm{H}(19 \mathrm{~A})-\mathrm{C}(19)-\mathrm{H}(19 \mathrm{~B})$ & 107.5 \\
\hline$C(13)-C(20)-C(19)$ & $115.49(14)$ \\
\hline $\mathrm{C}(13)-\mathrm{C}(20)-\mathrm{H}(20 \mathrm{~A})$ & 108.4 \\
\hline $\mathrm{C}(19)-\mathrm{C}(20)-\mathrm{H}(20 \mathrm{~A})$ & 108.4 \\
\hline $\mathrm{C}(13)-\mathrm{C}(20)-\mathrm{H}(20 \mathrm{~B})$ & 108.4 \\
\hline $\mathrm{C}(19)-\mathrm{C}(20)-\mathrm{H}(20 \mathrm{~B})$ & 108.4 \\
\hline$H(20 A)-C(20)-H(20 B)$ & 107.5 \\
\hline $\mathrm{C}(23)-\mathrm{C}(21)-\mathrm{N}(3)$ & $132.52(15)$ \\
\hline$C(23)-C(21)-C(6)$ & $120.83(15)$ \\
\hline $\mathrm{N}(3)-\mathrm{C}(21)-\mathrm{C}(6)$ & $106.65(14)$ \\
\hline $\mathrm{C}(23)-\mathrm{C}(22)-\mathrm{C}(8)$ & $121.23(15)$ \\
\hline $\mathrm{C}(23)-\mathrm{C}(22)-\mathrm{H}(22)$ & 119.4 \\
\hline $\mathrm{C}(8)-\mathrm{C}(22)-\mathrm{H}(22)$ & 119.4 \\
\hline$C(21)-C(23)-C(22)$ & $117.55(15)$ \\
\hline $\mathrm{C}(21)-\mathrm{C}(23)-\mathrm{H}(23)$ & 121.2 \\
\hline $\mathrm{C}(22)-\mathrm{C}(23)-\mathrm{H}(23)$ & 121.2 \\
\hline
\end{tabular}


Symmetry transformations used to generate equivalent atoms:

Table 4. Anisotropic displacement parameters $\left(\AA^{2} \times 10^{3}\right)$ for 101201LT_0m. The anisotropic displacement factor exponent takes the form: $-2 \pi^{2}\left[h^{2} a^{* 2} U^{11}+\ldots+2 h k a^{*} b^{*} U^{12}\right]$

\begin{tabular}{|c|c|c|c|c|c|c|}
\hline & $\mathrm{U}^{11}$ & $\mathrm{U}^{22}$ & $\mathrm{U}^{33}$ & $\mathrm{U}^{23}$ & $\mathrm{U}^{13}$ & $\mathrm{U}^{12}$ \\
\hline$S(1)$ & $14(1)$ & $18(1)$ & $14(1)$ & $1(1)$ & $2(1)$ & $0(1)$ \\
\hline $\mathrm{Cl}(2)$ & $33(1)$ & $21(1)$ & $21(1)$ & $4(1)$ & $8(1)$ & $-6(1)$ \\
\hline $\mathrm{Cl}(1)$ & $39(1)$ & $22(1)$ & $26(1)$ & $10(1)$ & $12(1)$ & $4(1)$ \\
\hline $\mathrm{O}(1)$ & $17(1)$ & $25(1)$ & $12(1)$ & $2(1)$ & $3(1)$ & 1(1) \\
\hline $\mathrm{O}(2)$ & $16(1)$ & $34(1)$ & $16(1)$ & $1(1)$ & $-1(1)$ & $-3(1)$ \\
\hline $\mathrm{O}(3)$ & $17(1)$ & $46(1)$ & $17(1)$ & $6(1)$ & $0(1)$ & $0(1)$ \\
\hline $\mathrm{O}(4)$ & $22(1)$ & $17(1)$ & $25(1)$ & $1(1)$ & $7(1)$ & $1(1)$ \\
\hline $\mathrm{N}(1)$ & $13(1)$ & $25(1)$ & $14(1)$ & $2(1)$ & $2(1)$ & $1(1)$ \\
\hline $\mathrm{N}(2)$ & $12(1)$ & $24(1)$ & $14(1)$ & $1(1)$ & $1(1)$ & $-2(1)$ \\
\hline $\mathrm{N}(3)$ & $14(1)$ & $22(1)$ & $12(1)$ & $-1(1)$ & $1(1)$ & $-1(1)$ \\
\hline $\mathrm{C}(1)$ & $20(1)$ & $26(1)$ & $24(1)$ & $-6(1)$ & $2(1)$ & $3(1)$ \\
\hline$C(2)$ & $21(1)$ & $19(1)$ & $28(1)$ & $1(1)$ & $-1(1)$ & $3(1)$ \\
\hline$C(3)$ & $17(1)$ & $22(1)$ & $19(1)$ & $4(1)$ & $2(1)$ & $0(1)$ \\
\hline$C(4)$ & $12(1)$ & $18(1)$ & $17(1)$ & 1(1) & $0(1)$ & 1(1) \\
\hline$C(5)$ & $14(1)$ & $17(1)$ & $15(1)$ & $-1(1)$ & $1(1)$ & 1(1) \\
\hline$C(6)$ & $14(1)$ & $17(1)$ & $17(1)$ & $0(1)$ & $3(1)$ & $0(1)$ \\
\hline$C(7)$ & $15(1)$ & $20(1)$ & $16(1)$ & $0(1)$ & $-1(1)$ & $0(1)$ \\
\hline $\mathrm{C}(8)$ & $17(1)$ & $17(1)$ & $16(1)$ & $0(1)$ & $2(1)$ & $2(1)$ \\
\hline
\end{tabular}




\begin{tabular}{lllllll}
$\mathrm{C}(9)$ & $16(1)$ & $17(1)$ & $17(1)$ & $2(1)$ & $2(1)$ & $0(1)$ \\
$\mathrm{C}(10)$ & $23(1)$ & $34(1)$ & $12(1)$ & $3(1)$ & $4(1)$ & $2(1)$ \\
$\mathrm{C}(11)$ & $18(1)$ & $28(1)$ & $18(1)$ & $-1(1)$ & $4(1)$ & $-1(1)$ \\
$\mathrm{C}(12)$ & $13(1)$ & $19(1)$ & $19(1)$ & $2(1)$ & $1(1)$ & $-3(1)$ \\
$\mathrm{C}(13)$ & $14(1)$ & $23(1)$ & $12(1)$ & $-1(1)$ & $-2(1)$ & $0(1)$ \\
$\mathrm{C}(14)$ & $20(1)$ & $21(1)$ & $16(1)$ & $-2(1)$ & $-1(1)$ & $0(1)$ \\
$\mathrm{C}(15)$ & $25(1)$ & $23(1)$ & $16(1)$ & $-4(1)$ & $-2(1)$ & $0(1)$ \\
$\mathrm{C}(16)$ & $26(1)$ & $29(1)$ & $22(1)$ & $0(1)$ & $-6(1)$ & $-4(1)$ \\
$\mathrm{C}(17)$ & $16(1)$ & $41(1)$ & $26(1)$ & $-1(1)$ & $-1(1)$ & $-5(1)$ \\
$\mathrm{C}(18)$ & $21(1)$ & $36(1)$ & $30(1)$ & $-5(1)$ & $-6(1)$ & $6(1)$ \\
$\mathrm{C}(19)$ & $25(1)$ & $23(1)$ & $24(1)$ & $1(1)$ & $-2(1)$ & $6(1)$ \\
$\mathrm{C}(20)$ & $18(1)$ & $22(1)$ & $20(1)$ & $-4(1)$ & $-1(1)$ & $1(1)$ \\
$\mathrm{C}(21)$ & $17(1)$ & $16(1)$ & $13(1)$ & $-1(1)$ & $1(1)$ & $0(1)$ \\
$\mathrm{C}(22)$ & $16(1)$ & $18(1)$ & $17(1)$ & $0(1)$ & $4(1)$ & $-1(1)$ \\
$\mathrm{C}(23)$ & $15(1)$ & $22(1)$ & $17(1)$ & $-2(1)$ & $0(1)$ & $-2(1)$ \\
& & & & & & \\
\hline
\end{tabular}


Table 5. Hydrogen coordinates ( $\left.\mathrm{x} 10^{4}\right)$ and isotropic displacement parameters $\left(\AA^{2} \mathrm{x} 10^{3}\right)$ for 101201LT_0m.

\begin{tabular}{|c|c|c|c|c|}
\hline & $\mathrm{x}$ & $\mathrm{y}$ & $\mathrm{z}$ & $\mathrm{U}(\mathrm{eq})$ \\
\hline $\mathrm{H}(2)$ & -11 & 3950 & 6770 & 20 \\
\hline $\mathrm{H}(1)$ & -1720 & 6816 & 10295 & 28 \\
\hline $\mathrm{H}(2 \mathrm{~A})$ & -1174 & 7459 & 9146 & 27 \\
\hline $\mathrm{H}(7)$ & 630 & 4589 & 5371 & 20 \\
\hline $\mathrm{H}(10 \mathrm{~A})$ & 2717 & 4643 & 3197 & 34 \\
\hline $\mathrm{H}(10 \mathrm{~B})$ & 3831 & 5476 & 3234 & 34 \\
\hline $\mathrm{H}(10 \mathrm{C})$ & 2649 & 6171 & 3216 & 34 \\
\hline $\mathrm{H}(11)$ & -1779 & 4652 & 10582 & 25 \\
\hline $\mathrm{H}(13)$ & 2720 & 4161 & 8587 & 20 \\
\hline $\mathrm{H}(14 \mathrm{~A})$ & 2838 & 6372 & 8317 & 23 \\
\hline $\mathrm{H}(14 \mathrm{~B})$ & 3936 & 6111 & 7899 & 23 \\
\hline $\mathrm{H}(15 \mathrm{~A})$ & 3970 & 6796 & 9270 & 26 \\
\hline $\mathrm{H}(15 \mathrm{~B})$ & 4063 & 5280 & 9364 & 26 \\
\hline $\mathrm{H}(16 \mathrm{~A})$ & 5639 & 6948 & 8783 & 31 \\
\hline $\mathrm{H}(16 \mathrm{~B})$ & 5841 & 5982 & 9455 & 31 \\
\hline $\mathrm{H}(17 \mathrm{~A})$ & 5576 & 5196 & 7960 & 34 \\
\hline $\mathrm{H}(17 \mathrm{~B})$ & 6751 & 5349 & 8404 & 34 \\
\hline $\mathrm{H}(18 \mathrm{~A})$ & 6306 & 3631 & 9172 & 36 \\
\hline $\mathrm{H}(18 \mathrm{~B})$ & 6373 & 3218 & 8336 & 36 \\
\hline
\end{tabular}




\begin{tabular}{lllll}
$\mathrm{H}(19 \mathrm{~A})$ & 4442 & 3352 & 9138 & 29 \\
$\mathrm{H}(19 \mathrm{~B})$ & 4887 & 2143 & 8712 & 29 \\
$\mathrm{H}(20 \mathrm{~A})$ & 4413 & 3658 & 7610 & 24 \\
$\mathrm{H}(20 \mathrm{~B})$ & 3574 & 2599 & 7873 & 24 \\
$\mathrm{H}(22)$ & 3886 & 5651 & 5448 & 21 \\
$\mathrm{H}(23)$ & 4065 & 5323 & 6723 & 22 \\
\hline
\end{tabular}


Figure S2. ORTEP diagram of compound 11.

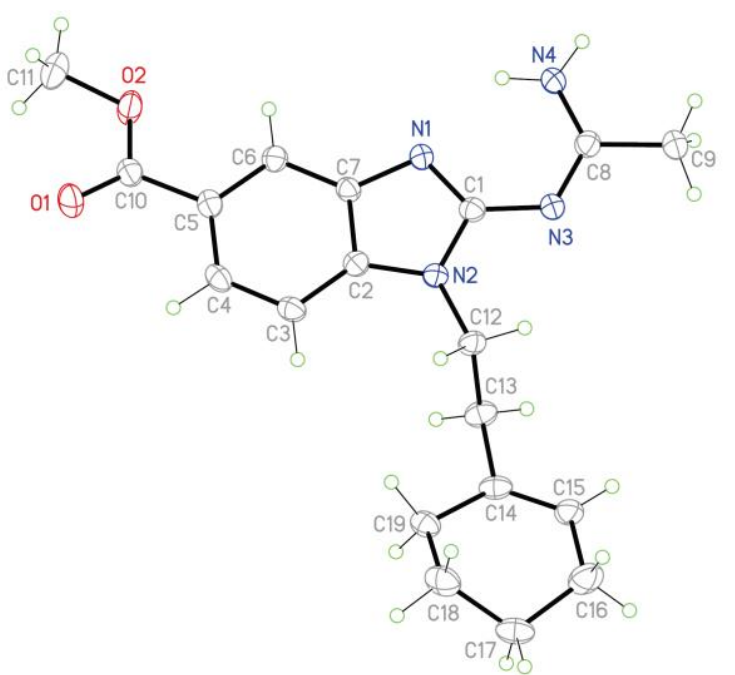

Table 1. Crystal data and structure refinement for MO_110117LT_0M.

Identification code

Empirical formula

Formula weight

Temperature

Wavelength

Crystal system

Space group

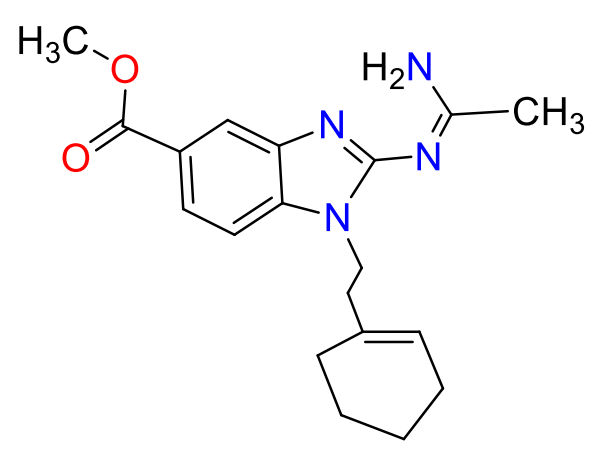

11 
Unit cell dimensions

Volume

Z

Density (calculated)

Absorption coefficient

$\mathrm{F}(000)$

Crystal size

Theta range for data collection

Index ranges

Reflections collected

Independent reflections

Completeness to theta $=25.242^{\circ}$

Absorption correction

Max. and min. transmission

Refinement method

Data / restraints / parameters

Goodness-of-fit on $\mathrm{F}^{2}$

Final $\mathrm{R}$ indices $[\mathrm{I}>2 \operatorname{sigma}(\mathrm{I})]$

$\mathrm{R}$ indices (all data)

Extinction coefficient

Largest diff. peak and hole

$\begin{array}{ll}\mathrm{a}=8.8601(3) \AA & \alpha=108.5220(10)^{\circ} . \\ \mathrm{b}=13.2927(4) \AA & \beta=103.9720(10)^{\circ} . \\ \mathrm{c}=16.7504(6) \AA & \gamma=95.7580(10)^{\circ} .\end{array}$

1781.33(10) $\AA^{3}$

2

$1.269 \mathrm{Mg} / \mathrm{m}^{3}$

$0.085 \mathrm{~mm}^{-1}$

728

$0.25 \times 0.20 \times 0.20 \mathrm{~mm}^{3}$

1.340 to $26.482^{\circ}$.

$-11<=\mathrm{h}<=11,-16<=\mathrm{k}<=16,-21<=\mathrm{l}<=20$

28825

$7314[\mathrm{R}($ int $)=0.0380]$

$99.9 \%$

Semi-empirical from equivalents

0.9486 and 0.8371

Full-matrix least-squares on $\mathrm{F}^{2}$

7314 / 24 / 489

1.030

$\mathrm{R} 1=0.0459, \mathrm{wR} 2=0.1149$

$\mathrm{R} 1=0.0633, \mathrm{wR} 2=0.1244$

$\mathrm{n} / \mathrm{a}$

0.448 and -0.347 e. $\AA^{-3}$ 
Table 2. Atomic coordinates $\left(\times 10^{4}\right)$ and equivalent isotropic displacement parameters $\left(\AA^{2} \times 10^{3}\right)$ for MO_110117LT_0M. U(eq) is defined as one third of the trace of the orthogonalized $\mathrm{U}^{\mathrm{ij}}$ tensor.

\begin{tabular}{|c|c|c|c|c|}
\hline & $\mathrm{x}$ & $\mathrm{y}$ & $\mathrm{z}$ & $\mathrm{U}(\mathrm{eq})$ \\
\hline $\mathrm{C}(1)$ & 7194(2) & $9142(1)$ & $6712(1)$ & 19(1) \\
\hline $\mathrm{C}(2)$ & $5277(2)$ & $8706(1)$ & $5456(1)$ & $20(1)$ \\
\hline $\mathrm{C}(3)$ & $3951(2)$ & $8586(1)$ & $4765(1)$ & $22(1)$ \\
\hline $\mathrm{C}(4)$ & $3843(2)$ & 7833(1) & $3956(1)$ & $22(1)$ \\
\hline $\mathrm{C}(5)$ & $5016(2)$ & 7211(1) & $3834(1)$ & $21(1)$ \\
\hline $\mathrm{C}(6)$ & $6328(2)$ & 7320(1) & $4536(1)$ & $21(1)$ \\
\hline $\mathrm{C}(7)$ & $6452(2)$ & $8077(1)$ & $5352(1)$ & $20(1)$ \\
\hline $\mathrm{C}(8)$ & $9300(2)$ & 9504(1) & 7948(1) & $22(1)$ \\
\hline $\mathrm{C}(9)$ & $10036(2)$ & $10103(2)$ & $8909(1)$ & $32(1)$ \\
\hline $\mathrm{C}(10)$ & $4865(2)$ & $6456(1)$ & $2940(1)$ & $23(1)$ \\
\hline $\mathrm{C}(11)$ & $5898(2)$ & $5051(2)$ & 2104(1) & $35(1)$ \\
\hline $\mathrm{C}(12)$ & $4961(2)$ & $10218(1)$ & $6737(1)$ & $22(1)$ \\
\hline $\mathrm{C}(13)$ & $3598(2)$ & $9805(1)$ & 7032(1) & $25(1)$ \\
\hline $\mathrm{C}(14)$ & $2530(50)$ & $10630(30)$ & $7188(15)$ & $23(2)$ \\
\hline $\mathrm{C}(15)$ & $2764(13)$ & $11385(11)$ & 7992(8) & $24(2)$ \\
\hline $\mathrm{C}(16)$ & $1865(15)$ & 12301(11) & $8143(6)$ & $36(1)$ \\
\hline $\mathrm{C}(17)$ & $341(4)$ & 12068(3) & $7408(2)$ & $32(1)$ \\
\hline $\mathrm{C}(18)$ & $674(4)$ & $11647(3)$ & $6519(2)$ & $33(1)$ \\
\hline $\mathrm{C}(19)$ & $1366(10)$ & $10636(6)$ & $6389(6)$ & $24(1)$ \\
\hline $\mathrm{C}\left(14^{\prime}\right)$ & $2580(80)$ & $10660(50)$ & $7160(30)$ & $23(2)$ \\
\hline
\end{tabular}




$\begin{array}{lcccc}\mathrm{C}\left(15^{\prime}\right) & 2960(20) & 11480(20) & 7949(15) & 24(2) \\ \mathrm{C}\left(16^{\prime}\right) & 1800(30) & 12220(19) & 8165(10) & 36(1) \\ \mathrm{C}\left(17^{\prime}\right) & 952(7) & 12395(5) & 7334(4) & 32(1) \\ \mathrm{C}\left(18^{\prime}\right) & 90(7) & 11325(5) & 6602(4) & 33(1) \\ \mathrm{C}\left(19^{\prime}\right) & 1122(18) & 10500(11) & 6451(12) & 24(1) \\ \mathrm{N}(1) & 7634(2) & 8359(1) & 6140(1) & 21(1) \\ \mathrm{N}(2) & 5768(2) & 9377(1) & 6327(1) & 20(1) \\ \mathrm{N}(3) & 7938(2) & 9714(1) & 7579(1) & 22(1) \\ \mathrm{N}(4) & 10072(2) & 8785(1) & 7551(1) & 22(1) \\ \mathrm{O}(1) & 3924(2) & 6426(1) & 2270(1) & 32(1) \\ \mathrm{O}(2) & 5911(2) & 5793(1) & 2948(1) & 30(1) \\ \mathrm{C}(20) & 54(2) & 3412(1) & 927(1) & 24(1) \\ \mathrm{C}(21) & 2133(2) & 2735(1) & 798(1) & 22(1) \\ \mathrm{C}(22) & 3341(2) & 2291(1) & 506(1) & 23(1) \\ \mathrm{C}(23) & 4539(2) & 2089(1) & 1110(1) & 22(1) \\ \mathrm{C}(24) & 4527(2) & 2322(1) & 1988(1) & 23(1) \\ \mathrm{C}(25) & 3340(2) & 2772(1) & 2290(1) & 22(1) \\ \mathrm{C}(26) & 2145(2) & 2970(1) & 1679(1) & 20(1) \\ \mathrm{C}(27) & -2057(2) & 3804(2) & 40(1) & 32(1) \\ \mathrm{C}(28) & -3569(2) & 4233(2) & -51(1) & 44(1) \\ \mathrm{C}(29) & 5862(2) & 1612(1) & 839(1) & 24(1) \\ \mathrm{C}(30) & 6936(2) & 933(2) & -339(1) & 33(1) \\ \mathrm{C}(31) & 450(2) & 3901(1) & 2576(1) & 22(1) \\ \mathrm{C}(32) & 1355(2) & 5066(1) & 3030(1) & 33(1) \\ \mathrm{C}(33) & 1587(5) & 5558(7) & 4002(4) & 20(1) \\ & & & & 5217\end{array}$




\begin{tabular}{lclcl}
$\mathrm{C}(34)$ & $2844(11)$ & $5256(9)$ & $4626(3)$ & $27(1)$ \\
$\mathrm{C}(35)$ & $2696(3)$ & $5586(2)$ & $5565(2)$ & $31(1)$ \\
$\mathrm{C}(36)$ & $2339(3)$ & $6721(2)$ & $5857(2)$ & $30(1)$ \\
$\mathrm{C}(37)$ & $771(13)$ & $6767(9)$ & $5255(5)$ & $30(1)$ \\
$\mathrm{C}(38)$ & $620(20)$ & $6206(12)$ & $4300(5)$ & $24(1)$ \\
$\mathrm{C}\left(33^{\prime}\right)$ & $1260(20)$ & $5500(20)$ & $3982(14)$ & $20(1)$ \\
$\mathrm{C}\left(34^{\prime}\right)$ & $2670(30)$ & $5240(30)$ & $4530(11)$ & $27(1)$ \\
$\mathrm{C}\left(35^{\prime}\right)$ & $3279(9)$ & $6029(7)$ & $5490(5)$ & $31(1)$ \\
$\mathrm{C}\left(36^{\prime}\right)$ & $1865(10)$ & $6271(8)$ & $5813(6)$ & $30(1)$ \\
$\mathrm{C}\left(37^{\prime}\right)$ & $850(40)$ & $6850(30)$ & $5293(16)$ & $30(1)$ \\
$\mathrm{C}\left(38^{\prime}\right)$ & $580(60)$ & $6310(40)$ & $4318(16)$ & $24(1)$ \\
$\mathrm{N}(5)$ & $816(2)$ & $3012(1)$ & $337(1)$ & $27(1)$ \\
$\mathrm{N}(6)$ & $805(2)$ & $3391(1)$ & $1748(1)$ & $21(1)$ \\
$\mathrm{N}(7)$ & $-1323(2)$ & $3798(1)$ & $819(1)$ & $30(1)$ \\
$\mathrm{N}(8)$ & $-1596(2)$ & $3474(1)$ & $-684(1)$ & $38(1)$ \\
$\mathrm{O}(3)$ & $5693(1)$ & $1390(1)$ & $-19(1)$ & $30(1)$ \\
$\mathrm{O}(4)$ & $6965(1)$ & $1429(1)$ & $1327(1)$ & $33(1)$ \\
& & & & \\
\hline
\end{tabular}


Table 3. Bond lengths $[\AA]$ and angles $\left[^{\circ}\right]$ for MO_110117LT_0M.

\begin{tabular}{ll}
\hline $\mathrm{C}(1)-\mathrm{N}(1)$ & $1.334(2)$ \\
$\mathrm{C}(1)-\mathrm{N}(3)$ & $1.364(2)$ \\
$\mathrm{C}(1)-\mathrm{N}(2)$ & $1.386(2)$ \\
$\mathrm{C}(2)-\mathrm{N}(2)$ & $1.379(2)$ \\
$\mathrm{C}(2)-\mathrm{C}(3)$ & $1.391(2)$ \\
$\mathrm{C}(2)-\mathrm{C}(7)$ & $1.408(2)$ \\
$\mathrm{C}(3)-\mathrm{C}(4)$ & $1.380(2)$ \\
$\mathrm{C}(3)-\mathrm{H}(3 \mathrm{~A})$ & 0.9500 \\
$\mathrm{C}(4)-\mathrm{C}(5)$ & $1.405(2)$ \\
$\mathrm{C}(4)-\mathrm{H}(4 \mathrm{~A})$ & 0.9500 \\
$\mathrm{C}(5)-\mathrm{C}(6)$ & $1.397(2)$ \\
$\mathrm{C}(5)-\mathrm{C}(10)$ & $1.479(2)$ \\
$\mathrm{C}(6)-\mathrm{C}(7)$ & $1.387(2)$ \\
$\mathrm{C}(6)-\mathrm{H}(6 \mathrm{~A})$ & 0.9500 \\
$\mathrm{C}(7)-\mathrm{N}(1)$ & $1.386(2)$ \\
$\mathrm{C}(8)-\mathrm{N}(3)$ & $1.316(2)$ \\
$\mathrm{C}(8)-\mathrm{N}(4)$ & $1.331(2)$ \\
$\mathrm{C}(8)-\mathrm{C}(9)$ & $1.492(2)$ \\
$\mathrm{C}(9)-\mathrm{H}(9 \mathrm{~A})$ & 0.9800 \\
$\mathrm{C}(9)-\mathrm{H}(9 \mathrm{~B})$ & 0.9800 \\
$\mathrm{C}(9)-\mathrm{H}(9 \mathrm{C})$ & 0.9800 \\
$\mathrm{C}(10)-\mathrm{O}(1)$ & $1.213(2)$ \\
$\mathrm{C}(10)-\mathrm{O}(2)$ & $1.341(2)$ \\
&
\end{tabular}




$\begin{array}{ll}\mathrm{C}(11)-\mathrm{O}(2) & 1.441(2) \\ \mathrm{C}(11)-\mathrm{H}(11 \mathrm{~A}) & 0.9800 \\ \mathrm{C}(11)-\mathrm{H}(11 \mathrm{~B}) & 0.9800 \\ \mathrm{C}(11)-\mathrm{H}(11 \mathrm{C}) & 0.9800 \\ \mathrm{C}(12)-\mathrm{N}(2) & 1.4642(19) \\ \mathrm{C}(12)-\mathrm{C}(13) & 1.527(2) \\ \mathrm{C}(12)-\mathrm{H}(12 \mathrm{~A}) & 0.9900 \\ \mathrm{C}(12)-\mathrm{H}(12 \mathrm{~B}) & 0.9900 \\ \mathrm{C}(13)-\mathrm{C}(14) & 1.51(2) \\ \mathrm{C}(13)-\mathrm{C}(14) & 1.517(12) \\ \mathrm{C}(13)-\mathrm{H}(13 \mathrm{~A}) & 0.9661 \\ \mathrm{C}(13)-\mathrm{H}(13 \mathrm{~B}) & 0.9637 \\ \mathrm{C}(14)-\mathrm{C}(15) & 1.355(9) \\ \mathrm{C}(14)-\mathrm{C}(19) & 1.484(13) \\ \mathrm{C}(15)-\mathrm{C}(16) & 1.509(8) \\ \mathrm{C}(15)-\mathrm{H}(15 \mathrm{~A}) & 0.9500 \\ \mathrm{C}(16)-\mathrm{C}(17) & 1.519(9) \\ \mathrm{C}(16)-\mathrm{H}(16 \mathrm{~A}) & 0.9900 \\ \mathrm{C}(16)-\mathrm{H}(16 \mathrm{~B}) & 0.9900 \\ \mathrm{C}(17)-\mathrm{C}(18) & 1.528(5) \\ \mathrm{C}(17)-\mathrm{H}(17 \mathrm{~A}) & 0.9900 \\ \mathrm{C}(17)-\mathrm{H}(17 \mathrm{~B}) & 0.9900 \\ \mathrm{C}(18)-\mathrm{C}(19) & 1.507(7) \\ \mathrm{C}(18)-\mathrm{H}(18 \mathrm{~A}) & 0.9900 \\ \mathrm{C}(18)-\mathrm{H}(18 \mathrm{~B}) & 0.9900 \\ & \end{array}$




$\begin{array}{ll}\mathrm{C}(19)-\mathrm{H}(19 \mathrm{~A}) & 0.9900 \\ \mathrm{C}(19)-\mathrm{H}(19 \mathrm{~B}) & 0.9900 \\ \mathrm{C}\left(14^{\prime}\right)-\mathrm{C}\left(15^{\prime}\right) & 1.354(13) \\ \mathrm{C}\left(14^{\prime}\right)-\mathrm{C}\left(19^{\prime}\right) & 1.478(17) \\ \mathrm{C}\left(15^{\prime}\right)-\mathrm{C}\left(16^{\prime}\right) & 1.519(13) \\ \mathrm{C}\left(15^{\prime}\right)-\mathrm{H}(15 \mathrm{~B}) & 0.9500 \\ \mathrm{C}\left(16^{\prime}\right)-\mathrm{C}\left(17^{\prime}\right) & 1.515(13) \\ \mathrm{C}\left(16^{\prime}\right)-\mathrm{H}(16 \mathrm{C}) & 0.9900 \\ \mathrm{C}\left(16^{\prime}\right)-\mathrm{H}(16 \mathrm{D}) & 0.9900 \\ \mathrm{C}\left(17^{\prime}\right)-\mathrm{C}\left(18^{\prime}\right) & 1.527(8) \\ \mathrm{C}\left(17^{\prime}\right)-\mathrm{H}(17 \mathrm{C}) & 0.9900 \\ \mathrm{C}\left(17^{\prime}\right)-\mathrm{H}(17 \mathrm{D}) & 0.9900 \\ \mathrm{C}\left(18^{\prime}\right)-\mathrm{C}\left(19^{\prime}\right) & 1.497(11) \\ \mathrm{C}\left(18^{\prime}\right)-\mathrm{H}(18 \mathrm{C}) & 0.9900 \\ \mathrm{C}\left(18^{\prime}\right)-\mathrm{H}(18 \mathrm{D}) & 0.9900 \\ \mathrm{C}\left(19^{\prime}\right)-\mathrm{H}(19 \mathrm{C}) & 0.9900 \\ \mathrm{C}\left(19^{\prime}\right)-\mathrm{H}(19 \mathrm{D}) & 0.9900 \\ \mathrm{~N}(4)-\mathrm{H}(4 \mathrm{~B}) & 0.8800 \\ \mathrm{~N}(4)-\mathrm{H}(4 \mathrm{C}) & 0.8800 \\ \mathrm{C}(20)-\mathrm{N}(5) & 1.332(2) \\ \mathrm{C}(20)-\mathrm{N}(7) & 1.365(2) \\ \mathrm{C}(20)-\mathrm{N}(6) & 1.387(2) \\ \mathrm{C}(21)-\mathrm{N}(5) & 1.387(2) \\ \mathrm{C}(21)-\mathrm{C}(22) & 1.389(2) \\ \mathrm{C}(21)-\mathrm{C}(26) & 1.405(2) \\ & \end{array}$




$\begin{array}{ll}\mathrm{C}(22)-\mathrm{C}(23) & 1.391(2) \\ \mathrm{C}(22)-\mathrm{H}(22 \mathrm{~A}) & 0.9500 \\ \mathrm{C}(23)-\mathrm{C}(24) & 1.405(2) \\ \mathrm{C}(23)-\mathrm{C}(29) & 1.485(2) \\ \mathrm{C}(24)-\mathrm{C}(25) & 1.383(2) \\ \mathrm{C}(24)-\mathrm{H}(24 \mathrm{~A}) & 0.9500 \\ \mathrm{C}(25)-\mathrm{C}(26) & 1.394(2) \\ \mathrm{C}(25)-\mathrm{H}(25 \mathrm{~A}) & 0.9500 \\ \mathrm{C}(26)-\mathrm{N}(6) & 1.3766(19) \\ \mathrm{C}(27)-\mathrm{N}(7) & 1.314(2) \\ \mathrm{C}(27)-\mathrm{N}(8) & 1.329(2) \\ \mathrm{C}(27)-\mathrm{C}(28) & 1.502(2) \\ \mathrm{C}(28)-\mathrm{H}(28 \mathrm{~A}) & 0.9800 \\ \mathrm{C}(28)-\mathrm{H}(28 \mathrm{~B}) & 0.9800 \\ \mathrm{C}(28)-\mathrm{H}(28 \mathrm{C}) & 0.9800 \\ \mathrm{C}(29)-\mathrm{O}(4) & 1.213(2) \\ \mathrm{C}(29)-\mathrm{O}(3) & 1.339(2) \\ \mathrm{C}(30)-\mathrm{O}(3) & 1.4432(19) \\ \mathrm{C}(30)-\mathrm{H}(30 \mathrm{~A}) & 0.9800 \\ \mathrm{C}(30)-\mathrm{H}(30 \mathrm{~B}) & 0.9800 \\ \mathrm{C}(30)-\mathrm{H}(30 \mathrm{C}) & 0.9800 \\ \mathrm{C}(31)-\mathrm{N}(6) & 1.462(2) \\ \mathrm{C}(31)-\mathrm{C}(32) & 1.526(2) \\ \mathrm{C}(31)-\mathrm{H}(31 \mathrm{~A}) & 0.9900 \\ \mathrm{C}(31)-\mathrm{H}(31 \mathrm{~B}) & 0.9900 \\ & \\ & \end{array}$




$\begin{array}{ll}\mathrm{C}(32)-\mathrm{C}(33) & 1.502(7) \\ \mathrm{C}(32)-\mathrm{C}\left(33^{\prime}\right) & 1.54(2) \\ \mathrm{C}(32)-\mathrm{H}(32 \mathrm{~A}) & 0.9590 \\ \mathrm{C}(32)-\mathrm{H}(32 \mathrm{~B}) & 0.9709 \\ \mathrm{C}(33)-\mathrm{C}(38) & 1.341(6) \\ \mathrm{C}(33)-\mathrm{C}(34) & 1.512(5) \\ \mathrm{C}(34)-\mathrm{C}(35) & 1.536(7) \\ \mathrm{C}(34)-\mathrm{H}(34 \mathrm{~A}) & 0.9900 \\ \mathrm{C}(34)-\mathrm{H}(34 \mathrm{~B}) & 0.9900 \\ \mathrm{C}(35)-\mathrm{C}(36) & 1.522(4) \\ \mathrm{C}(35)-\mathrm{H}(35 \mathrm{~A}) & 0.9900 \\ \mathrm{C}(35)-\mathrm{H}(35 \mathrm{~B}) & 0.9900 \\ \mathrm{C}(36)-\mathrm{C}(37) & 1.526(7) \\ \mathrm{C}(36)-\mathrm{H}(36 \mathrm{~A}) & 0.9900 \\ \mathrm{C}(36)-\mathrm{H}(36 \mathrm{~B}) & 0.9900 \\ \mathrm{C}(37)-\mathrm{C}(38) & 1.501(5) \\ \mathrm{C}(37)-\mathrm{H}(37 \mathrm{~A}) & 0.9900 \\ \mathrm{C}(37)-\mathrm{H}(37 \mathrm{~B}) & 0.9900 \\ \mathrm{C}(38)-\mathrm{H}(38 \mathrm{~A}) & 0.9500 \\ \mathrm{C}\left(33^{\prime}\right)-\mathrm{C}\left(38^{\prime}\right) & 1.331(15) \\ \mathrm{C}\left(33^{\prime}\right)-\mathrm{C}\left(34^{\prime}\right) & 1.494(15) \\ \mathrm{C}\left(34^{\prime}\right)-\mathrm{C}\left(35^{\prime}\right) & 1.541(15) \\ \mathrm{C}\left(34^{\prime}\right)-\mathrm{H}(34 \mathrm{C}) & 0.9900 \\ \mathrm{C}\left(34^{\prime}\right)-\mathrm{H}(34 \mathrm{D}) & 0.9900 \\ \mathrm{C}\left(35^{\prime}\right)-\mathrm{C}\left(36^{\prime}\right) & 1.504(10) \\ & \end{array}$




$\begin{array}{ll}\mathrm{C}\left(35^{\prime}\right)-\mathrm{H}(35 \mathrm{C}) & 0.9900 \\ \mathrm{C}\left(35^{\prime}\right)-\mathrm{H}(35 \mathrm{D}) & 0.9900 \\ \mathrm{C}\left(36^{\prime}\right)-\mathrm{C}\left(37^{\prime}\right) & 1.536(16) \\ \mathrm{C}\left(36^{\prime}\right)-\mathrm{H}(36 \mathrm{C}) & 0.9900 \\ \mathrm{C}\left(36^{\prime}\right)-\mathrm{H}(36 \mathrm{D}) & 0.9900 \\ \mathrm{C}\left(37^{\prime}\right)-\mathrm{C}\left(38^{\prime}\right) & 1.508(15) \\ \mathrm{C}\left(37^{\prime}\right)-\mathrm{H}(37 \mathrm{C}) & 0.9900 \\ \mathrm{C}\left(37^{\prime}\right)-\mathrm{H}(37 \mathrm{D}) & 0.9900 \\ \mathrm{C}\left(38^{\prime}\right)-\mathrm{H}(38 \mathrm{~B}) & 0.9500 \\ \mathrm{~N}(8)-\mathrm{H}(8 \mathrm{~A}) & 0.8800 \\ \mathrm{~N}(8)-\mathrm{H}(8 \mathrm{~B}) & 0.8800 \\ & \\ \mathrm{~N}(1)-\mathrm{C}(1)-\mathrm{N}(3) & 129.57(14) \\ \mathrm{N}(1)-\mathrm{C}(1)-\mathrm{N}(2) & 112.09(14) \\ \mathrm{N}(3)-\mathrm{C}(1)-\mathrm{N}(2) & 118.35(14) \\ \mathrm{N}(2)-\mathrm{C}(2)-\mathrm{C}(3) & 132.38(15) \\ \mathrm{N}(2)-\mathrm{C}(2)-\mathrm{C}(7) & 105.42(13) \\ \mathrm{C}(3)-\mathrm{C}(2)-\mathrm{C}(7) & 122.20(15) \\ \mathrm{C}(4)-\mathrm{C}(3)-\mathrm{C}(2) & 117.07(15) \\ \mathrm{C}(4)-\mathrm{C}(3)-\mathrm{H}(3 \mathrm{~A}) & 121.5 \\ \mathrm{C}(2)-\mathrm{C}(3)-\mathrm{H}(3 \mathrm{~A}) & 121.5 \\ \mathrm{C}(3)-\mathrm{C}(4)-\mathrm{C}(5) & 121.60(15) \\ \mathrm{C}(3)-\mathrm{C}(4)-\mathrm{H}(4 \mathrm{~A}) & 119.2 \\ \mathrm{C}(5)-\mathrm{C}(4)-\mathrm{H}(4 \mathrm{~A}) & 119.2 \\ \mathrm{C}(6)-\mathrm{C}(5)-\mathrm{C}(4) & 120.98(15) \\ & \\ & \\ & \\ & \end{array}$




$\begin{array}{ll}\mathrm{C}(6)-\mathrm{C}(5)-\mathrm{C}(10) & 120.20(14) \\ \mathrm{C}(4)-\mathrm{C}(5)-\mathrm{C}(10) & 118.80(14) \\ \mathrm{C}(7)-\mathrm{C}(6)-\mathrm{C}(5) & 117.92(14) \\ \mathrm{C}(7)-\mathrm{C}(6)-\mathrm{H}(6 \mathrm{~A}) & 121.0 \\ \mathrm{C}(5)-\mathrm{C}(6)-\mathrm{H}(6 \mathrm{~A}) & 121.0 \\ \mathrm{~N}(1)-\mathrm{C}(7)-\mathrm{C}(6) & 129.46(14) \\ \mathrm{N}(1)-\mathrm{C}(7)-\mathrm{C}(2) & 110.32(14) \\ \mathrm{C}(6)-\mathrm{C}(7)-\mathrm{C}(2) & 120.21(14) \\ \mathrm{N}(3)-\mathrm{C}(8)-\mathrm{N}(4) & 126.33(15) \\ \mathrm{N}(3)-\mathrm{C}(8)-\mathrm{C}(9) & 117.28(14) \\ \mathrm{N}(4)-\mathrm{C}(8)-\mathrm{C}(9) & 116.36(15) \\ \mathrm{C}(8)-\mathrm{C}(9)-\mathrm{H}(9 \mathrm{~A}) & 109.5 \\ \mathrm{C}(8)-\mathrm{C}(9)-\mathrm{H}(9 \mathrm{~B}) & 109.5 \\ \mathrm{H}(9 \mathrm{~A})-\mathrm{C}(9)-\mathrm{H}(9 \mathrm{~B}) & 109.5 \\ \mathrm{C}(8)-\mathrm{C}(9)-\mathrm{H}(9 \mathrm{C}) & 109.5 \\ \mathrm{H}(9 \mathrm{~A})-\mathrm{C}(9)-\mathrm{H}(9 \mathrm{C}) & 109.5 \\ \mathrm{H}(9 \mathrm{~B})-\mathrm{C}(9)-\mathrm{H}(9 \mathrm{C}) & 109.5 \\ \mathrm{O}(1)-\mathrm{C}(10)-\mathrm{O}(2) & 122.88(15) \\ \mathrm{O}(1)-\mathrm{C}(10)-\mathrm{C}(5) & 125.14(16) \\ \mathrm{O}(2)-\mathrm{C}(10)-\mathrm{C}(5) & 111.98(14) \\ \mathrm{O}(2)-\mathrm{C}(11)-\mathrm{H}(11 \mathrm{~A}) & 109.5 \\ \mathrm{O}(2)-\mathrm{C}(11)-\mathrm{H}(11 \mathrm{~B}) & 109.5 \\ \mathrm{H}(11 \mathrm{~A})-\mathrm{C}(11)-\mathrm{H}(11 \mathrm{~B}) & 109.5 \\ \mathrm{O}(2)-\mathrm{C}(11)-\mathrm{H}(11 \mathrm{C}) & 109.5 \\ \mathrm{H}(11 \mathrm{~A})-\mathrm{C}(11)-\mathrm{H}(11 \mathrm{C}) & 109.5 \\ & \end{array}$




$\begin{array}{ll}\mathrm{H}(11 \mathrm{~B})-\mathrm{C}(11)-\mathrm{H}(11 \mathrm{C}) & 109.5 \\ \mathrm{~N}(2)-\mathrm{C}(12)-\mathrm{C}(13) & 114.16(13) \\ \mathrm{N}(2)-\mathrm{C}(12)-\mathrm{H}(12 \mathrm{~A}) & 108.7 \\ \mathrm{C}(13)-\mathrm{C}(12)-\mathrm{H}(12 \mathrm{~A}) & 108.7 \\ \mathrm{~N}(2)-\mathrm{C}(12)-\mathrm{H}(12 \mathrm{~B}) & 108.7 \\ \mathrm{C}(13)-\mathrm{C}(12)-\mathrm{H}(12 \mathrm{~B}) & 108.7 \\ \mathrm{H}(12 \mathrm{~A})-\mathrm{C}(12)-\mathrm{H}(12 \mathrm{~B}) & 107.6 \\ \mathrm{C}(14)-\mathrm{C}(13)-\mathrm{C}(12) & 107(4) \\ \mathrm{C}(14)-\mathrm{C}(13)-\mathrm{C}(12) & 111(2) \\ \mathrm{C}(14)-\mathrm{C}(13)-\mathrm{H}(13 \mathrm{~A}) & 106.6 \\ \mathrm{C}(14)-\mathrm{C}(13)-\mathrm{H}(13 \mathrm{~A}) & 104.6 \\ \mathrm{C}(12)-\mathrm{C}(13)-\mathrm{H}(13 \mathrm{~A}) & 110.7 \\ \mathrm{C}(14)-\mathrm{C}(13)-\mathrm{H}(13 \mathrm{~B}) & 113.2 \\ \mathrm{C}(14)-\mathrm{C}(13)-\mathrm{H}(13 \mathrm{~B}) & 111.9 \\ \mathrm{C}(12)-\mathrm{C}(13)-\mathrm{H}(13 \mathrm{~B}) & 110.8 \\ \mathrm{H}(13 \mathrm{~A})-\mathrm{C}(13)-\mathrm{H}(13 \mathrm{~B}) & 108.0 \\ \mathrm{C}(15)-\mathrm{C}(14)-\mathrm{C}(19) & 122.2(10) \\ \mathrm{C}(15)-\mathrm{C}(14)-\mathrm{C}(13) & 121.5(8) \\ \mathrm{C}(19)-\mathrm{C}(14)-\mathrm{C}(13) & 115.8(12) \\ \mathrm{C}(14)-\mathrm{C}(15)-\mathrm{C}(16) & 122.7(9) \\ \mathrm{C}(14)-\mathrm{C}(15)-\mathrm{H}(15 \mathrm{~A}) & 118.7 \\ \mathrm{C}(16)-\mathrm{C}(15)-\mathrm{H}(15 \mathrm{~A}) & 118.7 \\ \mathrm{C}(15)-\mathrm{C}(16)-\mathrm{C}(17) & 112.5(7) \\ \mathrm{C}(15)-\mathrm{C}(16)-\mathrm{H}(16 \mathrm{~A}) & 109.1 \\ \mathrm{C}(17)-\mathrm{C}(16)-\mathrm{H}(16 \mathrm{~A}) & 109.1 \\ & \end{array}$




$\begin{array}{ll}\mathrm{C}(15)-\mathrm{C}(16)-\mathrm{H}(16 \mathrm{~B}) & 109.1 \\ \mathrm{C}(17)-\mathrm{C}(16)-\mathrm{H}(16 \mathrm{~B}) & 109.1 \\ \mathrm{H}(16 \mathrm{~A})-\mathrm{C}(16)-\mathrm{H}(16 \mathrm{~B}) & 107.8 \\ \mathrm{C}(16)-\mathrm{C}(17)-\mathrm{C}(18) & 109.6(6) \\ \mathrm{C}(16)-\mathrm{C}(17)-\mathrm{H}(17 \mathrm{~A}) & 109.8 \\ \mathrm{C}(18)-\mathrm{C}(17)-\mathrm{H}(17 \mathrm{~A}) & 109.8 \\ \mathrm{C}(16)-\mathrm{C}(17)-\mathrm{H}(17 \mathrm{~B}) & 109.8 \\ \mathrm{C}(18)-\mathrm{C}(17)-\mathrm{H}(17 \mathrm{~B}) & 109.8 \\ \mathrm{H}(17 \mathrm{~A})-\mathrm{C}(17)-\mathrm{H}(17 \mathrm{~B}) & 108.2 \\ \mathrm{C}(19)-\mathrm{C}(18)-\mathrm{C}(17) & 112.8(4) \\ \mathrm{C}(19)-\mathrm{C}(18)-\mathrm{H}(18 \mathrm{~A}) & 109.0 \\ \mathrm{C}(17)-\mathrm{C}(18)-\mathrm{H}(18 \mathrm{~A}) & 109.0 \\ \mathrm{C}(19)-\mathrm{C}(18)-\mathrm{H}(18 B) & 109.0 \\ \mathrm{C}(17)-\mathrm{C}(18)-\mathrm{H}(18 \mathrm{~B}) & 109.0 \\ \mathrm{H}(18 \mathrm{~A})-\mathrm{C}(18)-\mathrm{H}(18 \mathrm{~B}) & 107.8 \\ \mathrm{C}(14)-\mathrm{C}(19)-\mathrm{C}(18) & 114.4(8) \\ \mathrm{C}(14)-\mathrm{C}(19)-\mathrm{H}(19 \mathrm{~A}) & 108.7 \\ \mathrm{C}(18)-\mathrm{C}(19)-\mathrm{H}(19 \mathrm{~A}) & 108.7 \\ \mathrm{C}(14)-\mathrm{C}(19)-\mathrm{H}(19 B) & 108.7 \\ \mathrm{C}(18)-\mathrm{C}(19)-\mathrm{H}(19 \mathrm{~B}) & 108.7 \\ \mathrm{H}(19 \mathrm{~A})-\mathrm{C}(19)-\mathrm{H}(19 \mathrm{~B}) & 107.6 \\ \mathrm{C}\left(15^{\prime}\right)-\mathrm{C}\left(14^{\prime}\right)-\mathrm{C}\left(19^{\prime}\right) & 122.5(16) \\ \mathrm{C}\left(15^{\prime}\right)-\mathrm{C}\left(14^{\prime}\right)-\mathrm{C}(13) & 119.3(14) \\ \mathrm{C}\left(19^{\prime}\right)-\mathrm{C}\left(14^{\prime}\right)-\mathrm{C}(13) & 117.9(17) \\ \mathrm{C}\left(14^{\prime}\right)-\mathrm{C}\left(15^{\prime}\right)-\mathrm{C}\left(16^{\prime}\right) & 120.5(17) \\ & \end{array}$




\begin{tabular}{|c|c|}
\hline $\mathrm{C}\left(14^{\prime}\right)-\mathrm{C}\left(15^{\prime}\right)-\mathrm{H}(15 \mathrm{~B})$ & 119.7 \\
\hline $\mathrm{C}\left(16^{\prime}\right)-\mathrm{C}\left(15^{\prime}\right)-\mathrm{H}(15 \mathrm{~B})$ & 119.7 \\
\hline $\mathrm{C}\left(17^{\prime}\right)-\mathrm{C}\left(16^{\prime}\right)-\mathrm{C}\left(15^{\prime}\right)$ & $108.5(13)$ \\
\hline $\mathrm{C}\left(17^{\prime}\right)-\mathrm{C}\left(16^{\prime}\right)-\mathrm{H}(16 \mathrm{C})$ & 110.0 \\
\hline $\mathrm{C}\left(15^{\prime}\right)-\mathrm{C}\left(16^{\prime}\right)-\mathrm{H}(16 \mathrm{C})$ & 110.0 \\
\hline $\mathrm{C}\left(17^{\prime}\right)-\mathrm{C}\left(16^{\prime}\right)-\mathrm{H}(16 \mathrm{D})$ & 110.0 \\
\hline $\mathrm{C}\left(15^{\prime}\right)-\mathrm{C}\left(16^{\prime}\right)-\mathrm{H}(16 \mathrm{D})$ & 110.0 \\
\hline$H(16 C)-C\left(16^{\prime}\right)-H(16 D)$ & 108.4 \\
\hline $\mathrm{C}\left(16^{\prime}\right)-\mathrm{C}\left(17^{\prime}\right)-\mathrm{C}\left(18^{\prime}\right)$ & $111.4(10)$ \\
\hline $\mathrm{C}\left(16^{\prime}\right)-\mathrm{C}\left(17^{\prime}\right)-\mathrm{H}(17 \mathrm{C})$ & 109.4 \\
\hline $\mathrm{C}\left(18^{\prime}\right)-\mathrm{C}\left(17^{\prime}\right)-\mathrm{H}(17 \mathrm{C})$ & 109.4 \\
\hline $\mathrm{C}\left(16^{\prime}\right)-\mathrm{C}\left(17^{\prime}\right)-\mathrm{H}(17 \mathrm{D})$ & 109.4 \\
\hline $\mathrm{C}\left(18^{\prime}\right)-\mathrm{C}\left(17^{\prime}\right)-\mathrm{H}(17 \mathrm{D})$ & 109.4 \\
\hline $\mathrm{H}(17 \mathrm{C})-\mathrm{C}\left(17^{\prime}\right)-\mathrm{H}(17 \mathrm{D})$ & 108.0 \\
\hline $\mathrm{C}\left(19^{\prime}\right)-\mathrm{C}\left(18^{\prime}\right)-\mathrm{C}\left(17^{\prime}\right)$ & $113.2(8)$ \\
\hline $\mathrm{C}\left(19^{\prime}\right)-\mathrm{C}\left(18^{\prime}\right)-\mathrm{H}(18 \mathrm{C})$ & 108.9 \\
\hline $\mathrm{C}\left(17^{\prime}\right)-\mathrm{C}\left(18^{\prime}\right)-\mathrm{H}(18 \mathrm{C})$ & 108.9 \\
\hline $\mathrm{C}\left(19^{\prime}\right)-\mathrm{C}\left(18^{\prime}\right)-\mathrm{H}(18 \mathrm{D})$ & 108.9 \\
\hline $\mathrm{C}\left(17^{\prime}\right)-\mathrm{C}\left(18^{\prime}\right)-\mathrm{H}(18 \mathrm{D})$ & 108.9 \\
\hline $\mathrm{H}(18 \mathrm{C})-\mathrm{C}\left(18^{\prime}\right)-\mathrm{H}(18 \mathrm{D})$ & 107.7 \\
\hline $\mathrm{C}\left(14^{\prime}\right)-\mathrm{C}\left(19^{\prime}\right)-\mathrm{C}\left(18^{\prime}\right)$ & $117.5(12)$ \\
\hline $\mathrm{C}\left(14^{\prime}\right)-\mathrm{C}\left(19^{\prime}\right)-\mathrm{H}(19 \mathrm{C})$ & 107.9 \\
\hline $\mathrm{C}\left(18^{\prime}\right)-\mathrm{C}\left(19^{\prime}\right)-\mathrm{H}(19 \mathrm{C})$ & 107.9 \\
\hline $\mathrm{C}\left(14^{\prime}\right)-\mathrm{C}\left(19^{\prime}\right)-\mathrm{H}(19 \mathrm{D})$ & 107.9 \\
\hline $\mathrm{C}\left(18^{\prime}\right)-\mathrm{C}\left(19^{\prime}\right)-\mathrm{H}(19 \mathrm{D})$ & 107.9 \\
\hline
\end{tabular}




$\begin{array}{ll}\mathrm{H}(19 \mathrm{C})-\mathrm{C}(19))-\mathrm{H}(19 \mathrm{D}) & 107.2 \\ \mathrm{C}(1)-\mathrm{N}(1)-\mathrm{C}(7) & 105.13(13) \\ \mathrm{C}(2)-\mathrm{N}(2)-\mathrm{C}(1) & 107.04(13) \\ \mathrm{C}(2)-\mathrm{N}(2)-\mathrm{C}(12) & 125.42(13) \\ \mathrm{C}(1)-\mathrm{N}(2)-\mathrm{C}(12) & 127.44(13) \\ \mathrm{C}(8)-\mathrm{N}(3)-\mathrm{C}(1) & 118.77(14) \\ \mathrm{C}(8)-\mathrm{N}(4)-\mathrm{H}(4 \mathrm{~B}) & 120.0 \\ \mathrm{C}(8)-\mathrm{N}(4)-\mathrm{H}(4 \mathrm{C}) & 120.0 \\ \mathrm{H}(4 \mathrm{~B})-\mathrm{N}(4)-\mathrm{H}(4 \mathrm{C}) & 120.0 \\ \mathrm{C}(10)-\mathrm{O}(2)-\mathrm{C}(11) & 116.29(14) \\ \mathrm{N}(5)-\mathrm{C}(20)-\mathrm{N}(7) & 129.26(15) \\ \mathrm{N}(5)-\mathrm{C}(20)-\mathrm{N}(6) & 112.25(14) \\ \mathrm{N}(7)-\mathrm{C}(20)-\mathrm{N}(6) & 118.47(14) \\ \mathrm{N}(5)-\mathrm{C}(21)-\mathrm{C}(22) & 129.44(15) \\ \mathrm{N}(5)-\mathrm{C}(21)-\mathrm{C}(26) & 110.35(14) \\ \mathrm{C}(22)-\mathrm{C}(21)-\mathrm{C}(26) & 120.21(14) \\ \mathrm{C}(21)-\mathrm{C}(22)-\mathrm{C}(23) & 117.91(15) \\ \mathrm{C}(21)-\mathrm{C}(22)-\mathrm{H}(22 \mathrm{~A}) & 121.0 \\ \mathrm{C}(23)-\mathrm{C}(22)-\mathrm{H}(22 \mathrm{~A}) & 121.0 \\ \mathrm{C}(22)-\mathrm{C}(23)-\mathrm{C}(24) & 121.13(15) \\ \mathrm{C}(22)-\mathrm{C}(23)-\mathrm{C}(29) & 120.54(15) \\ \mathrm{C}(24)-\mathrm{C}(23)-\mathrm{C}(29) & 118.33(14) \\ \mathrm{C}(25)-\mathrm{C}(24)-\mathrm{C}(23) & 121.69(15) \\ \mathrm{C}(25)-\mathrm{C}(24)-\mathrm{H}(24 \mathrm{~A}) & 119.2 \\ \mathrm{C}(23)-\mathrm{C}(24)-\mathrm{H}(24 \mathrm{~A}) & 119.2 \\ & \end{array}$




$\begin{array}{ll}\mathrm{C}(24)-\mathrm{C}(25)-\mathrm{C}(26) & 116.64(15) \\ \mathrm{C}(24)-\mathrm{C}(25)-\mathrm{H}(25 \mathrm{~A}) & 121.7 \\ \mathrm{C}(26)-\mathrm{C}(25)-\mathrm{H}(25 \mathrm{~A}) & 121.7 \\ \mathrm{~N}(6)-\mathrm{C}(26)-\mathrm{C}(25) & 132.00(15) \\ \mathrm{N}(6)-\mathrm{C}(26)-\mathrm{C}(21) & 105.59(13) \\ \mathrm{C}(25)-\mathrm{C}(26)-\mathrm{C}(21) & 122.42(14) \\ \mathrm{N}(7)-\mathrm{C}(27)-\mathrm{N}(8) & 126.77(16) \\ \mathrm{N}(7)-\mathrm{C}(27)-\mathrm{C}(28) & 117.28(15) \\ \mathrm{N}(8)-\mathrm{C}(27)-\mathrm{C}(28) & 115.95(16) \\ \mathrm{C}(27)-\mathrm{C}(28)-\mathrm{H}(28 \mathrm{~A}) & 109.5 \\ \mathrm{C}(27)-\mathrm{C}(28)-\mathrm{H}(28 \mathrm{~B}) & 109.5 \\ \mathrm{H}(28 \mathrm{~A})-\mathrm{C}(28)-\mathrm{H}(28 \mathrm{~B}) & 109.5 \\ \mathrm{C}(27)-\mathrm{C}(28)-\mathrm{H}(28 \mathrm{C}) & 109.5 \\ \mathrm{H}(28 \mathrm{~A})-\mathrm{C}(28)-\mathrm{H}(28 \mathrm{C}) & 109.5 \\ \mathrm{H}(28 \mathrm{~B})-\mathrm{C}(28)-\mathrm{H}(28 \mathrm{C}) & 109.5 \\ \mathrm{O}(4)-\mathrm{C}(29)-\mathrm{O}(3) & 123.23(15) \\ \mathrm{O}(4)-\mathrm{C}(29)-\mathrm{C}(23) & 124.67(16) \\ \mathrm{O}(3)-\mathrm{C}(29)-\mathrm{C}(23) & 112.10(14) \\ \mathrm{O}(3)-\mathrm{C}(30)-\mathrm{H}(30 \mathrm{~A}) & 109.5 \\ \mathrm{O}(3)-\mathrm{C}(30)-\mathrm{H}(30 \mathrm{~B}) & 109.5 \\ \mathrm{H}(30 \mathrm{~A})-\mathrm{C}(30)-\mathrm{H}(30 \mathrm{~B}) & 109.5 \\ \mathrm{O}(3)-\mathrm{C}(30)-\mathrm{H}(30 \mathrm{C}) & 109.5 \\ \mathrm{H}(30 \mathrm{~A})-\mathrm{C}(30)-\mathrm{H}(30 \mathrm{C}) & 109.5 \\ \mathrm{H}(30 \mathrm{~B})-\mathrm{C}(30)-\mathrm{H}(30 \mathrm{C}) & 109.5 \\ \mathrm{~N}(6)-\mathrm{C}(31)-\mathrm{C}(32) & 110.73(14) \\ & \end{array}$




$\begin{array}{ll}\text { N(6)-C(31)-H(31A) } & 109.5 \\ \mathrm{C}(32)-\mathrm{C}(31)-\mathrm{H}(31 \mathrm{~A}) & 109.5 \\ \mathrm{~N}(6)-\mathrm{C}(31)-\mathrm{H}(31 \mathrm{~B}) & 109.5 \\ \mathrm{C}(32)-\mathrm{C}(31)-\mathrm{H}(31 \mathrm{~B}) & 109.5 \\ \mathrm{H}(31 \mathrm{~A})-\mathrm{C}(31)-\mathrm{H}(31 \mathrm{~B}) & 108.1 \\ \mathrm{C}(33)-\mathrm{C}(32)-\mathrm{C}(31) & 116.4(3) \\ \mathrm{C}(31)-\mathrm{C}(32)-\mathrm{C}\left(33{ }^{\prime}\right) & 109.5(10) \\ \mathrm{C}(33)-\mathrm{C}(32)-\mathrm{H}(32 \mathrm{~A}) & 113.5 \\ \mathrm{C}(31)-\mathrm{C}(32)-\mathrm{H}(32 \mathrm{~A}) & 110.2 \\ \mathrm{C}\left(33^{\prime}\right)-\mathrm{C}(32)-\mathrm{H}(32 \mathrm{~A}) & 111.2 \\ \mathrm{C}(33)-\mathrm{C}(32)-\mathrm{H}(32 \mathrm{~B}) & 98.8 \\ \mathrm{C}(31)-\mathrm{C}(32)-\mathrm{H}(32 \mathrm{~B}) & 109.4 \\ \mathrm{C}\left(33^{\prime}\right)-\mathrm{C}(32)-\mathrm{H}(32 \mathrm{~B}) & 109.0 \\ \mathrm{H}(32 \mathrm{~A})-\mathrm{C}(32)-\mathrm{H}(32 \mathrm{~B}) & 107.5 \\ \mathrm{C}(38)-\mathrm{C}(33)-\mathrm{C}(32) & 119.8(5) \\ \mathrm{C}(38)-\mathrm{C}(33)-\mathrm{C}(34) & 121.1(5) \\ \mathrm{C}(32)-\mathrm{C}(33)-\mathrm{C}(34) & 119.1(5) \\ \mathrm{C}(33)-\mathrm{C}(34)-\mathrm{C}(35) & 113.7(5) \\ \mathrm{C}(33)-\mathrm{C}(34)-\mathrm{H}(34 \mathrm{~A}) & 108.8 \\ \mathrm{C}(35)-\mathrm{C}(34)-\mathrm{H}(34 \mathrm{~A}) & 108.8 \\ \mathrm{C}(33)-\mathrm{C}(34)-\mathrm{H}(34 \mathrm{~B}) & 108.8 \\ \mathrm{C}(35)-\mathrm{C}(34)-\mathrm{H}(34 \mathrm{~B}) & 108.8 \\ \mathrm{H}(34 \mathrm{~A})-\mathrm{C}(34)-\mathrm{H}(34 \mathrm{~B}) & 107.7 \\ \mathrm{C}(36)-\mathrm{C}(35)-\mathrm{C}(34) & 110.6(4) \\ \mathrm{C}(36)-\mathrm{C}(35)-\mathrm{H}(35 \mathrm{~A}) & 109.5 \\ & \\ & \end{array}$




$\begin{array}{ll}\mathrm{C}(34)-\mathrm{C}(35)-\mathrm{H}(35 \mathrm{~A}) & 109.5 \\ \mathrm{C}(36)-\mathrm{C}(35)-\mathrm{H}(35 \mathrm{~B}) & 109.5 \\ \mathrm{C}(34)-\mathrm{C}(35)-\mathrm{H}(35 \mathrm{~B}) & 109.5 \\ \mathrm{H}(35 \mathrm{~A})-\mathrm{C}(35)-\mathrm{H}(35 \mathrm{~B}) & 108.1 \\ \mathrm{C}(35)-\mathrm{C}(36)-\mathrm{C}(37) & 110.4(4) \\ \mathrm{C}(35)-\mathrm{C}(36)-\mathrm{H}(36 \mathrm{~A}) & 109.6 \\ \mathrm{C}(37)-\mathrm{C}(36)-\mathrm{H}(36 \mathrm{~A}) & 109.6 \\ \mathrm{C}(35)-\mathrm{C}(36)-\mathrm{H}(36 \mathrm{~B}) & 109.6 \\ \mathrm{C}(37)-\mathrm{C}(36)-\mathrm{H}(36 \mathrm{~B}) & 109.6 \\ \mathrm{H}(36 \mathrm{~A})-\mathrm{C}(36)-\mathrm{H}(36 \mathrm{~B}) & 108.1 \\ \mathrm{C}(38)-\mathrm{C}(37)-\mathrm{C}(36) & 112.3(6) \\ \mathrm{C}(38)-\mathrm{C}(37)-\mathrm{H}(37 \mathrm{~A}) & 109.1 \\ \mathrm{C}(36)-\mathrm{C}(37)-\mathrm{H}(37 \mathrm{~A}) & 109.1 \\ \mathrm{C}(38)-\mathrm{C}(37)-\mathrm{H}(37 \mathrm{~B}) & 109.1 \\ \mathrm{C}(36)-\mathrm{C}(37)-\mathrm{H}(37 \mathrm{~B}) & 109.1 \\ \mathrm{H}(37 \mathrm{~A})-\mathrm{C}(37)-\mathrm{H}(37 \mathrm{~B}) & 107.9 \\ \mathrm{C}(33)-\mathrm{C}(38)-\mathrm{C}(37) & 124.5(6) \\ \mathrm{C}(33)-\mathrm{C}(38)-\mathrm{H}(38 \mathrm{~A}) & 117.8 \\ \mathrm{C}(37)-\mathrm{C}(38)-\mathrm{H}(38 \mathrm{~A}) & 117.8 \\ \mathrm{C}\left(38^{\prime}\right)-\mathrm{C}\left(33^{\prime}\right)-\mathrm{C}\left(34^{\prime}\right) & 122.0(18) \\ \mathrm{C}\left(38^{\prime}\right)-\mathrm{C}\left(33^{\prime}\right)-\mathrm{C}(32) & 126(2) \\ \mathrm{C}\left(34^{\prime}\right)-\mathrm{C}\left(33^{\prime}\right)-\mathrm{C}(32) & 106.1(15) \\ \mathrm{C}\left(33^{\prime}\right)-\mathrm{C}\left(34^{\prime}\right)-\mathrm{C}\left(35^{\prime}\right) & 114.2(15) \\ \mathrm{C}\left(33^{\prime}\right)-\mathrm{C}\left(34^{\prime}\right)-\mathrm{H}(34 \mathrm{C}) & 108.7 \\ \mathrm{C}\left(35^{\prime}\right)-\mathrm{C}\left(34^{\prime}\right)-\mathrm{H}(34 \mathrm{C}) & 108.7 \\ & \end{array}$




$\begin{array}{ll}\mathrm{C}\left(33^{\prime}\right)-\mathrm{C}\left(34^{\prime}\right)-\mathrm{H}(34 \mathrm{D}) & 108.7 \\ \mathrm{C}\left(35^{\prime}\right)-\mathrm{C}\left(34^{\prime}\right)-\mathrm{H}(34 \mathrm{D}) & 108.7 \\ \mathrm{H}(34 \mathrm{C})-\mathrm{C}\left(34^{\prime}\right)-\mathrm{H}(34 \mathrm{D}) & 107.6 \\ \mathrm{C}\left(36^{\prime}\right)-\mathrm{C}\left(35^{\prime}\right)-\mathrm{C}\left(34^{\prime}\right) & 108.0(12) \\ \mathrm{C}\left(36^{\prime}\right)-\mathrm{C}\left(35^{\prime}\right)-\mathrm{H}(35 \mathrm{C}) & 110.1 \\ \mathrm{C}\left(34^{\prime}\right)-\mathrm{C}\left(35^{\prime}\right)-\mathrm{H}(35 \mathrm{C}) & 110.1 \\ \mathrm{C}\left(36^{\prime}\right)-\mathrm{C}\left(35^{\prime}\right)-\mathrm{H}(35 \mathrm{D}) & 110.1 \\ \mathrm{C}\left(34^{\prime}\right)-\mathrm{C}\left(35^{\prime}\right)-\mathrm{H}(35 \mathrm{D}) & 110.1 \\ \mathrm{H}(35 \mathrm{C})-\mathrm{C}\left(35^{\prime}\right)-\mathrm{H}(35 \mathrm{D}) & 108.4 \\ \mathrm{C}\left(35^{\prime}\right)-\mathrm{C}\left(36^{\prime}\right)-\mathrm{C}\left(37^{\prime}\right) & 111.5(14) \\ \mathrm{C}\left(35^{\prime}\right)-\mathrm{C}\left(36^{\prime}\right)-\mathrm{H}(36 \mathrm{C}) & 109.3 \\ \mathrm{C}\left(37^{\prime}\right)-\mathrm{C}\left(36^{\prime}\right)-\mathrm{H}(36 \mathrm{C}) & 109.3 \\ \mathrm{C}\left(35^{\prime}\right)-\mathrm{C}\left(36^{\prime}\right)-\mathrm{H}(36 \mathrm{D}) & 109.3 \\ \mathrm{C}\left(37^{\prime}\right)-\mathrm{C}\left(36^{\prime}\right)-\mathrm{H}(36 \mathrm{D}) & 109.3 \\ \mathrm{H}(36 \mathrm{C})-\mathrm{C}\left(36^{\prime}\right)-\mathrm{H}(36 \mathrm{D}) & 108.0 \\ \mathrm{C}\left(38^{\prime}\right)-\mathrm{C}\left(37^{\prime}\right)-\mathrm{C}\left(36^{\prime}\right) & 111.0(16) \\ \mathrm{C}\left(38^{\prime}\right)-\mathrm{C}\left(37^{\prime}\right)-\mathrm{H}(37 \mathrm{C}) & 109.4 \\ \mathrm{C}\left(36^{\prime}\right)-\mathrm{C}\left(37^{\prime}\right)-\mathrm{H}(37 \mathrm{C}) & 109.4 \\ \mathrm{C}\left(38^{\prime}\right)-\mathrm{C}\left(37^{\prime}\right)-\mathrm{H}(37 \mathrm{D}) & 109.4 \\ \mathrm{C}\left(36^{\prime}\right)-\mathrm{C}\left(37^{\prime}\right)-\mathrm{H}(37 \mathrm{D}) & 109.4 \\ \mathrm{H}(37 \mathrm{C})-\mathrm{C}\left(37^{\prime}\right)-\mathrm{H}(37 \mathrm{D}) & 108.0 \\ \mathrm{C}\left(33^{\prime}\right)-\mathrm{C}\left(38^{\prime}\right)-\mathrm{C}\left(37^{\prime}\right) & 122.8(18) \\ \mathrm{C}\left(33^{\prime}\right)-\mathrm{C}\left(38^{\prime}\right)-\mathrm{H}(38 \mathrm{~B}) & 118.6 \\ \mathrm{C}\left(37^{\prime}\right)-\mathrm{C}\left(38^{\prime}\right)-\mathrm{H}(38 \mathrm{~B}) & 118.6 \\ \mathrm{C}(20)-\mathrm{N}(5)-\mathrm{C}(21) & 104.95(13) \\ & \end{array}$




$\begin{array}{ll}\mathrm{C}(26)-\mathrm{N}(6)-\mathrm{C}(20) & 106.85(13) \\ \mathrm{C}(26)-\mathrm{N}(6)-\mathrm{C}(31) & 124.76(13) \\ \mathrm{C}(20)-\mathrm{N}(6)-\mathrm{C}(31) & 127.43(13) \\ \mathrm{C}(27)-\mathrm{N}(7)-\mathrm{C}(20) & 118.91(15) \\ \mathrm{C}(27)-\mathrm{N}(8)-\mathrm{H}(8 \mathrm{~A}) & 120.0 \\ \mathrm{C}(27)-\mathrm{N}(8)-\mathrm{H}(8 \mathrm{~B}) & 120.0 \\ \mathrm{H}(8 \mathrm{~A})-\mathrm{N}(8)-\mathrm{H}(8 \mathrm{~B}) & 120.0 \\ \mathrm{C}(29)-\mathrm{O}(3)-\mathrm{C}(30) & 115.91(13)\end{array}$

Symmetry transformations used to generate equivalent atoms: 
Table 4. Anisotropic displacement parameters $\left(\AA^{2} \mathrm{x} 10^{3}\right)$ for MO_110117LT_0M. The anisotropic displacement factor exponent takes the form: $-2 \pi^{2}\left[h^{2} a^{* 2} U^{11}+\ldots+2 h k a^{*} b^{*} U^{12}\right]$

\begin{tabular}{|c|c|c|c|c|c|c|}
\hline & $\mathrm{U}^{11}$ & $\mathrm{U}^{22}$ & $\mathrm{U}^{33}$ & $\mathrm{U}^{23}$ & $\mathrm{U}^{13}$ & $\mathrm{U}^{12}$ \\
\hline $\mathrm{C}(1)$ & $18(1)$ & $21(1)$ & $20(1)$ & $9(1)$ & $6(1)$ & $4(1)$ \\
\hline$C(2)$ & $20(1)$ & $21(1)$ & $20(1)$ & $9(1)$ & $6(1)$ & $3(1)$ \\
\hline$C(3)$ & $18(1)$ & $27(1)$ & $24(1)$ & $13(1)$ & $5(1)$ & $6(1)$ \\
\hline $\mathrm{C}(4)$ & $19(1)$ & $28(1)$ & $21(1)$ & $13(1)$ & $2(1)$ & $2(1)$ \\
\hline $\mathrm{C}(5)$ & $22(1)$ & $22(1)$ & $19(1)$ & $9(1)$ & $5(1)$ & $1(1)$ \\
\hline$C(6)$ & $21(1)$ & $22(1)$ & $20(1)$ & $9(1)$ & $7(1)$ & $5(1)$ \\
\hline $\mathrm{C}(7)$ & $18(1)$ & $23(1)$ & $19(1)$ & $9(1)$ & $4(1)$ & $4(1)$ \\
\hline $\mathrm{C}(8)$ & $22(1)$ & $22(1)$ & $21(1)$ & $7(1)$ & $7(1)$ & $3(1)$ \\
\hline $\mathrm{C}(9)$ & $26(1)$ & $39(1)$ & $21(1)$ & $2(1)$ & $2(1)$ & $10(1)$ \\
\hline $\mathrm{C}(10)$ & $21(1)$ & $26(1)$ & $20(1)$ & $10(1)$ & $5(1)$ & $0(1)$ \\
\hline $\mathrm{C}(11)$ & $45(1)$ & $30(1)$ & $24(1)$ & 1(1) & $12(1)$ & $5(1)$ \\
\hline $\mathrm{C}(12)$ & $23(1)$ & $20(1)$ & $23(1)$ & $7(1)$ & $8(1)$ & $9(1)$ \\
\hline$C(13)$ & $27(1)$ & $24(1)$ & $31(1)$ & $13(1)$ & $13(1)$ & $11(1)$ \\
\hline$C(14)$ & $21(2)$ & $25(2)$ & $33(1)$ & $17(2)$ & $14(2)$ & $8(2)$ \\
\hline$C(15)$ & $23(3)$ & $31(2)$ & $24(1)$ & $13(1)$ & $8(2)$ & $11(2)$ \\
\hline$C(16)$ & $41(1)$ & $36(2)$ & $35(1)$ & $12(1)$ & $16(1)$ & $21(1)$ \\
\hline $\mathrm{C}(17)$ & $25(2)$ & $33(2)$ & $46(2)$ & $20(1)$ & $12(2)$ & $15(2)$ \\
\hline$C(18)$ & $28(2)$ & $42(2)$ & $37(1)$ & $24(1)$ & $6(1)$ & $14(2)$ \\
\hline$C(19)$ & $20(3)$ & $28(2)$ & $27(2)$ & $14(1)$ & $7(2)$ & $3(2)$ \\
\hline$C\left(14^{\prime}\right)$ & $21(2)$ & $25(2)$ & $33(1)$ & $17(2)$ & $14(2)$ & $8(2)$ \\
\hline
\end{tabular}




$\begin{array}{lllllll}\mathrm{C}\left(15^{\prime}\right) & 23(3) & 31(2) & 24(1) & 13(1) & 8(2) & 11(2) \\ \mathrm{C}\left(16^{\prime}\right) & 41(1) & 36(2) & 35(1) & 12(1) & 16(1) & 21(1) \\ \mathrm{C}\left(17^{\prime}\right) & 25(2) & 33(2) & 46(2) & 20(1) & 12(2) & 15(2) \\ \mathrm{C}\left(18^{\prime}\right) & 28(2) & 42(2) & 37(1) & 24(1) & 6(1) & 14(2) \\ \mathrm{C}\left(19^{\prime}\right) & 20(3) & 28(2) & 27(2) & 14(1) & 7(2) & 3(2) \\ \mathrm{N}(1) & 20(1) & 24(1) & 17(1) & 6(1) & 4(1) & 6(1) \\ \mathrm{N}(2) & 18(1) & 22(1) & 19(1) & 7(1) & 6(1) & 7(1) \\ \mathrm{N}(3) & 20(1) & 24(1) & 19(1) & 6(1) & 4(1) & 6(1) \\ \mathrm{N}(4) & 20(1) & 26(1) & 19(1) & 6(1) & 3(1) & 7(1) \\ \mathrm{O}(1) & 30(1) & 43(1) & 19(1) & 10(1) & 2(1) & 5(1) \\ \mathrm{O}(2) & 37(1) & 29(1) & 18(1) & 4(1) & 6(1) & 9(1) \\ \mathrm{C}(20) & 23(1) & 30(1) & 17(1) & 4(1) & 4(1) & 10(1) \\ \mathrm{C}(21) & 20(1) & 25(1) & 18(1) & 4(1) & 1(1) & 6(1) \\ \mathrm{C}(22) & 21(1) & 27(1) & 18(1) & 3(1) & 3(1) & 8(1) \\ \mathrm{C}(23) & 18(1) & 22(1) & 22(1) & 5(1) & 3(1) & 6(1) \\ \mathrm{C}(24) & 21(1) & 24(1) & 23(1) & 10(1) & 1(1) & 6(1) \\ \mathrm{C}(25) & 23(1) & 24(1) & 19(1) & 9(1) & 4(1) & 5(1) \\ \mathrm{C}(26) & 19(1) & 19(1) & 20(1) & 5(1) & 5(1) & 5(1) \\ \mathrm{C}(27) & 28(1) & 45(1) & 21(1) & 6(1) & 6(1) & 20(1) \\ \mathrm{C}(28) & 38(1) & 74(2) & 24(1) & 13(1) & 8(1) & 37(1) \\ \mathrm{C}(29) & 20(1) & 25(1) & 25(1) & 7(1) & 3(1) & 6(1) \\ \mathrm{C}(30) & 24(1) & 40(1) & 32(1) & 5(1) & 10(1) & 16(1) \\ \mathrm{C}(31) & 24(1) & 26(1) & 18(1) & 7(1) & 8(1) & 8(1) \\ \mathrm{C}(32) & 54(1) & 24(1) & 24(1) & 7(1) & 18(1) & 5(1) \\ \mathrm{C}(33) & 17(2) & 18(1) & 23(1) & 6(1) & 9(2) & -3(2) \\ & & & & & & 5236\end{array}$




\begin{tabular}{lllllll}
$\mathrm{C}(34)$ & $23(2)$ & $28(1)$ & $29(2)$ & $10(2)$ & $7(1)$ & $6(1)$ \\
$\mathrm{C}(35)$ & $31(1)$ & $33(2)$ & $29(1)$ & $15(1)$ & $4(1)$ & $4(1)$ \\
$\mathrm{C}(36)$ & $36(2)$ & $28(2)$ & $21(1)$ & $6(1)$ & $6(1)$ & $-1(1)$ \\
$\mathrm{C}(37)$ & $30(1)$ & $26(2)$ & $27(1)$ & $1(1)$ & $9(1)$ & $5(1)$ \\
$\mathrm{C}(38)$ & $28(1)$ & $20(3)$ & $23(1)$ & $7(1)$ & $5(1)$ & $5(2)$ \\
$\mathrm{C}\left(33^{\prime}\right)$ & $17(2)$ & $18(1)$ & $23(1)$ & $6(1)$ & $9(2)$ & $-3(2)$ \\
$\mathrm{C}\left(34^{\prime}\right)$ & $23(2)$ & $28(1)$ & $29(2)$ & $10(2)$ & $7(1)$ & $6(1)$ \\
$\mathrm{C}\left(35^{\prime}\right)$ & $31(1)$ & $33(2)$ & $29(1)$ & $15(1)$ & $4(1)$ & $4(1)$ \\
$\mathrm{C}\left(36^{\prime}\right)$ & $36(2)$ & $28(2)$ & $21(1)$ & $6(1)$ & $6(1)$ & $-1(1)$ \\
$\mathrm{C}\left(37^{\prime}\right)$ & $30(1)$ & $26(2)$ & $27(1)$ & $1(1)$ & $9(1)$ & $5(1)$ \\
$\mathrm{C}\left(38^{\prime}\right)$ & $28(1)$ & $20(3)$ & $23(1)$ & $7(1)$ & $5(1)$ & $5(2)$ \\
$\mathrm{N}(5)$ & $22(1)$ & $38(1)$ & $17(1)$ & $5(1)$ & $3(1)$ & $15(1)$ \\
$\mathrm{N}(6)$ & $20(1)$ & $27(1)$ & $17(1)$ & $6(1)$ & $4(1)$ & $9(1)$ \\
$\mathrm{N}(7)$ & $26(1)$ & $43(1)$ & $19(1)$ & $7(1)$ & $5(1)$ & $20(1)$ \\
$\mathrm{N}(8)$ & $32(1)$ & $63(1)$ & $19(1)$ & $12(1)$ & $6(1)$ & $30(1)$ \\
$\mathrm{O}(3)$ & $23(1)$ & $40(1)$ & $25(1)$ & $6(1)$ & $6(1)$ & $16(1)$ \\
$\mathrm{O}(4)$ & $25(1)$ & $45(1)$ & $32(1)$ & $16(1)$ & $6(1)$ & $19(1)$ \\
& & & & & & \\
\hline
\end{tabular}


Table 5. Hydrogen coordinates ( $\mathrm{x} 10^{4}$ ) and isotropic displacement parameters $\left(\AA^{2} \mathrm{x} 10^{3}\right)$ for MO_110117LT_0M.

\begin{tabular}{|c|c|c|c|c|}
\hline & $\mathrm{x}$ & y & $\mathrm{z}$ & $\mathrm{U}(\mathrm{eq})$ \\
\hline $\mathrm{H}(3 \mathrm{~A})$ & 3155 & 9005 & 4846 & 27 \\
\hline $\mathrm{H}(4 \mathrm{~A})$ & 2954 & 7733 & 3469 & 27 \\
\hline $\mathrm{H}(6 \mathrm{~A})$ & 7111 & 6889 & 4457 & 25 \\
\hline $\mathrm{H}(9 \mathrm{~A})$ & 11038 & 9877 & 9103 & 47 \\
\hline $\mathrm{H}(9 \mathrm{~B})$ & 10238 & 10881 & 9023 & 47 \\
\hline $\mathrm{H}(9 \mathrm{C})$ & 9315 & 9943 & 9233 & 47 \\
\hline $\mathrm{H}(11 \mathrm{~A})$ & 6703 & 4609 & 2187 & 52 \\
\hline $\mathrm{H}(11 \mathrm{~B})$ & 4851 & 4581 & 1814 & 52 \\
\hline $\mathrm{H}(11 \mathrm{C})$ & 6133 & 5458 & 1739 & 52 \\
\hline $\mathrm{H}(12 \mathrm{~A})$ & 4543 & 10571 & 6314 & 26 \\
\hline $\mathrm{H}(12 \mathrm{~B})$ & 5746 & 10775 & 7255 & 26 \\
\hline $\mathrm{H}(13 \mathrm{~A})$ & 3993 & 9728 & 7596 & 30 \\
\hline $\mathrm{H}(13 \mathrm{~B})$ & 3019 & 9109 & 6615 & 30 \\
\hline $\mathrm{H}(15 \mathrm{~A})$ & 3526 & 11340 & 8481 & 29 \\
\hline $\mathrm{H}(16 \mathrm{~A})$ & 2548 & 12967 & 8192 & 43 \\
\hline $\mathrm{H}(16 \mathrm{~B})$ & 1609 & 12432 & 8707 & 43 \\
\hline $\mathrm{H}(17 \mathrm{~A})$ & -93 & 12737 & 7466 & 38 \\
\hline $\mathrm{H}(17 \mathrm{~B})$ & -455 & 11523 & 7449 & 38 \\
\hline $\mathrm{H}(18 \mathrm{~A})$ & -326 & 11494 & 6044 & 40 \\
\hline
\end{tabular}




$\begin{array}{lrrrr}\mathrm{H}(18 B) & 1421 & 12216 & 6471 & 40 \\ \mathrm{H}(19 A) & 490 & 10006 & 6185 & 29 \\ \mathrm{H}(19 B) & 1890 & 10549 & 5919 & 29 \\ \mathrm{H}(15 B) & 3957 & 11591 & 8369 & 29 \\ \mathrm{H}(16 \mathrm{C}) & 1029 & 11889 & 8391 & 43 \\ \mathrm{H}(16 \mathrm{D}) & 2383 & 12921 & 8623 & 43 \\ \mathrm{H}(17 \mathrm{C}) & 1729 & 12768 & 7134 & 38 \\ \mathrm{H}(17 \mathrm{D}) & 177 & 12865 & 7462 & 38 \\ \mathrm{H}(18 \mathrm{C}) & -828 & 11029 & 6755 & 40 \\ \mathrm{H}(18 \mathrm{D}) & -323 & 11461 & 6050 & 40 \\ \mathrm{H}(19 \mathrm{C}) & 476 & 9786 & 6325 & 29 \\ \mathrm{H}(19 \mathrm{D}) & 1432 & 10470 & 5915 & 29 \\ \mathrm{H}(4 \mathrm{~B}) & 9673 & 8394 & 6988 & 27 \\ \mathrm{H}(4 \mathrm{C}) & 10981 & 8699 & 7851 & 27 \\ \mathrm{H}(22 \mathrm{~A}) & 3349 & 2131 & -87 & 28 \\ \mathrm{H}(24 \mathrm{~A}) & 5356 & 2167 & 2383 & 28 \\ \mathrm{H}(25 \mathrm{~A}) & 3338 & 2938 & 2885 & 27 \\ \mathrm{H}(28 \mathrm{~A}) & -3999 & 4196 & -659 & 66 \\ \mathrm{H}(28 B) & -4340 & 3798 & 98 & 66 \\ \mathrm{H}(28 \mathrm{C}) & -3356 & 4986 & 347 & 66 \\ \mathrm{H}(30 \mathrm{~A}) & 6701 & 801 & -970 & 50 \\ \mathrm{H}(30 \mathrm{~B}) & 7949 & 1439 & -25 & 50 \\ \mathrm{H}(30 \mathrm{C}) & 7003 & 249 & -239 & 50 \\ \mathrm{H}(31 \mathrm{~A}) & -703 & 3890 & 2969 & 27 \\ \mathrm{H}(31 \mathrm{~B}) & & 2459 & 27 \\ & & & 5239\end{array}$




$\begin{array}{lrlll}\mathrm{H}(32 \mathrm{~A}) & 933 & 5507 & 2709 & 40 \\ \mathrm{H}(32 B) & 2458 & 5090 & 3040 & 40 \\ \mathrm{H}(34 \mathrm{~A}) & 3900 & 5606 & 4646 & 32 \\ \mathrm{H}(34 \mathrm{~B}) & 2778 & 4464 & 4392 & 32 \\ \mathrm{H}(35 \mathrm{~A}) & 1836 & 5068 & 5586 & 37 \\ \mathrm{H}(35 \mathrm{~B}) & 3698 & 5563 & 5972 & 37 \\ \mathrm{H}(36 \mathrm{~A}) & 3205 & 7241 & 5842 & 36 \\ \mathrm{H}(36 \mathrm{~B}) & 2281 & 6930 & 6470 & 36 \\ \mathrm{H}(37 \mathrm{~A}) & -115 & 6425 & 5403 & 36 \\ \mathrm{H}(37 \mathrm{~B}) & 686 & 7533 & 5360 & 36 \\ \mathrm{H}(38 \mathrm{~A}) & -208 & 6318 & 3878 & 29 \\ \mathrm{H}(34 \mathrm{C}) & 3536 & 5244 & 4254 & 32 \\ \mathrm{H}(34 \mathrm{D}) & 2380 & 4501 & 4529 & 32 \\ \mathrm{H}(35 \mathrm{C}) & 3956 & 5699 & 5859 & 37 \\ \mathrm{H}(35 \mathrm{D}) & 3917 & 6704 & 5523 & 37 \\ \mathrm{H}(36 \mathrm{C}) & 2231 & 6730 & 6444 & 36 \\ \mathrm{H}(36 \mathrm{D}) & 1213 & 5587 & 5754 & 36 \\ \mathrm{H}(37 \mathrm{C}) & -188 & 6848 & 5421 & 36 \\ \mathrm{H}(37 \mathrm{D}) & 1382 & 7616 & 5482 & 36 \\ \mathrm{H}(38 B) & -112 & 6563 & 3926 & 29 \\ \mathrm{H}(8 \mathrm{~A}) & -715 & 3218 & -670 & 45 \\ \mathrm{H}(8 B) & -2172 & 3511 & -1178 & 45\end{array}$


Figure S3. ORTEP diagram of compound 8a.
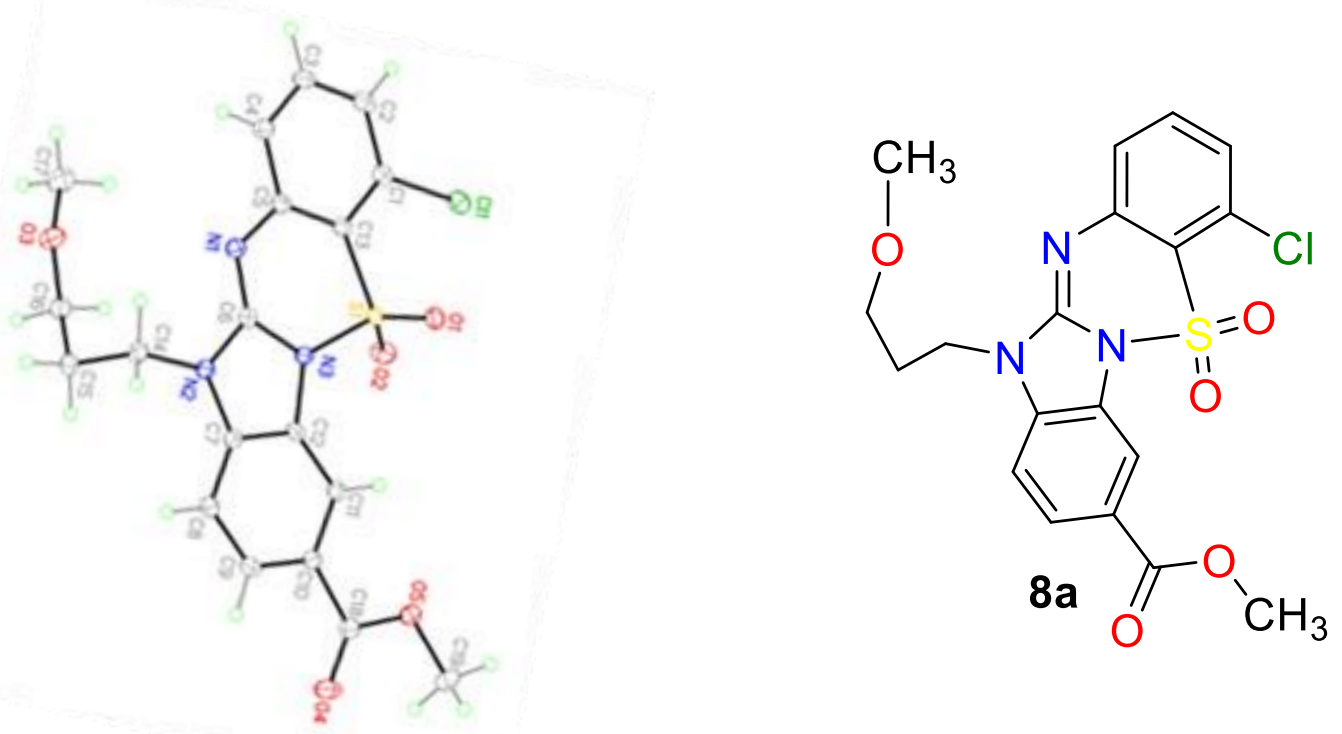

Table 1. Crystal data and structure refinement for 1009401t_0m.

Identification code

Empirical formula

Formula weight

Temperature

Wavelength

Crystal system

Space group 1009401t_0m

C19 H18 Cl N3 O5 S

435.87

100(2) K

$0.71073 \AA$

Monoclinic

P 1 21/c 1 
Unit cell dimensions

Volume

Z

Density (calculated)

Absorption coefficient

$\mathrm{F}(000)$

Crystal size

Theta range for data collection

Index ranges

Reflections collected

Independent reflections

Completeness to theta $=26.42^{\circ}$

Absorption correction

Max. and min. transmission

Refinement method

Data / restraints / parameters

Goodness-of-fit on $\mathrm{F}^{2}$

Final $\mathrm{R}$ indices [I>2sigma(I)]

$\mathrm{R}$ indices (all data)

Largest diff. peak and hole

$$
\begin{array}{ll}
\mathrm{a}=10.4885(3) \AA & \alpha=90^{\circ} . \\
\mathrm{b}=12.1708(4) \AA & \beta=97.7460(10)^{\circ} . \\
\mathrm{c}=14.9547(4) \AA & \gamma=90^{\circ} .
\end{array}
$$

1891.60(10) $\AA^{3}$

4

$1.531 \mathrm{Mg} / \mathrm{m}^{3}$

$0.351 \mathrm{~mm}^{-1}$

904

$0.27 \times 0.25 \times 0.15 \mathrm{~mm}^{3}$

1.96 to $26.42^{\circ}$.

$-13<=\mathrm{h}<=13,-14<=\mathrm{k}<=15,-18<=\mathrm{l}<=11$

16702

$3868[\mathrm{R}($ int $)=0.0403]$

$99.5 \%$

Semi-empirical from equivalents

0.9486 and 0.8890

Full-matrix least-squares on $\mathrm{F}^{2}$

3868 / 0 / 264

1.158

$\mathrm{R} 1=0.0296, \mathrm{wR} 2=0.0887$

$\mathrm{R} 1=0.0372, w \mathrm{R} 2=0.1047$

0.433 and -0.774 e. $\AA^{-3}$ 
Table 2. Atomic coordinates ( $\times 10^{4}$ ) and equivalent isotropic displacement parameters $\left(\AA^{2} \times 10^{3}\right)$ for 1009401t_0m. U(eq) is defined as one third of the trace of the orthogonalized $\mathrm{U}^{\mathrm{ij}}$ tensor.

\begin{tabular}{|c|c|c|c|c|}
\hline & $\mathrm{x}$ & $\mathrm{y}$ & $\mathrm{z}$ & $\mathrm{U}(\mathrm{eq})$ \\
\hline$C(1)$ & $13(2)$ & $2621(1)$ & $1453(1)$ & $14(1)$ \\
\hline$C(2)$ & $895(2)$ & 1833(1) & $1788(1)$ & $16(1)$ \\
\hline $\mathrm{C}(3)$ & $2102(2)$ & $2163(1)$ & $2208(1)$ & $17(1)$ \\
\hline $\mathrm{C}(4)$ & $2419(2)$ & $3260(1)$ & $2282(1)$ & $17(1)$ \\
\hline $\mathrm{C}(5)$ & $1548(2)$ & $4087(1)$ & 1942(1) & $13(1)$ \\
\hline$C(6)$ & $1229(1)$ & $5929(1)$ & 1693(1) & $12(1)$ \\
\hline $\mathrm{C}(7)$ & $524(1)$ & $7618(1)$ & $1242(1)$ & $12(1)$ \\
\hline $\mathrm{C}(8)$ & $397(2)$ & $8737(1)$ & 1082(1) & $14(1)$ \\
\hline $\mathrm{C}(9)$ & $-776(2)$ & $9092(1)$ & 631(1) & $14(1)$ \\
\hline $\mathrm{C}(10)$ & $-1772(1)$ & $8363(1)$ & $340(1)$ & $13(1)$ \\
\hline $\mathrm{C}(11)$ & $-1643(2)$ & $7233(1)$ & $506(1)$ & $13(1)$ \\
\hline $\mathrm{C}(12)$ & $-484(2)$ & 6893(1) & $964(1)$ & $12(1)$ \\
\hline $\mathrm{C}(13)$ & $317(1)$ & $3747(1)$ & 1519(1) & $13(1)$ \\
\hline$C(14)$ & $2812(1)$ & $7461(1)$ & 2049(1) & $14(1)$ \\
\hline $\mathrm{C}(15)$ & $3700(2)$ & $7653(1)$ & $1337(1)$ & $17(1)$ \\
\hline$C(16)$ & $4170(2)$ & $6595(1)$ & $967(1)$ & $18(1)$ \\
\hline $\mathrm{C}(17)$ & $5155(2)$ & 4923(2) & $1446(1)$ & $28(1)$ \\
\hline $\mathrm{C}(18)$ & $-2956(2)$ & $8830(1)$ & $-180(1)$ & $16(1)$ \\
\hline $\mathrm{C}(19)$ & $-5040(2)$ & $8462(2)$ & $-921(1)$ & $24(1)$ \\
\hline $\mathrm{Cl}(1)$ & $-1499(1)$ & $2185(1)$ & $960(1)$ & $17(1)$ \\
\hline
\end{tabular}




\begin{tabular}{lrrrr}
$\mathrm{N}(1)$ & $1979(1)$ & $5158(1)$ & $2045(1)$ & $14(1)$ \\
$\mathrm{N}(2)$ & $1551(1)$ & $7017(1)$ & $1674(1)$ & $12(1)$ \\
$\mathrm{N}(3)$ & $-28(1)$ & $5841(1)$ & $1256(1)$ & $12(1)$ \\
$\mathrm{O}(1)$ & $-1758(1)$ & $4750(1)$ & $1860(1)$ & $16(1)$ \\
$\mathrm{O}(2)$ & $-1470(1)$ & $4599(1)$ & $270(1)$ & $17(1)$ \\
$\mathrm{O}(3)$ & $4935(1)$ & $6030(1)$ & $1680(1)$ & $20(1)$ \\
$\mathrm{O}(4)$ & $-3081(1)$ & $9782(1)$ & $-411(1)$ & $26(1)$ \\
$\mathrm{O}(5)$ & $-3880(1)$ & $8072(1)$ & $-382(1)$ & $18(1)$ \\
$\mathrm{S}(1)$ & $-905(1)$ & $4698(1)$ & $1191(1)$ & $11(1)$ \\
\hline
\end{tabular}


Table 3. Bond lengths $[\AA]$ and angles $\left[^{\circ}\right]$ for $1009401 t \_0 m$.

\begin{tabular}{ll}
\hline $\mathrm{C}(1)-\mathrm{C}(2)$ & $1.380(2)$ \\
$\mathrm{C}(1)-\mathrm{C}(13)$ & $1.408(2)$ \\
$\mathrm{C}(1)-\mathrm{Cl}(1)$ & $1.7385(16)$ \\
$\mathrm{C}(2)-\mathrm{C}(3)$ & $1.395(2)$ \\
$\mathrm{C}(2)-\mathrm{H}(2)$ & 0.9500 \\
$\mathrm{C}(3)-\mathrm{C}(4)$ & $1.377(2)$ \\
$\mathrm{C}(3)-\mathrm{H}(3)$ & 0.9500 \\
$\mathrm{C}(4)-\mathrm{C}(5)$ & $1.408(2)$ \\
$\mathrm{C}(4)-\mathrm{H}(4)$ & 0.9500 \\
$\mathrm{C}(5)-\mathrm{N}(1)$ & $1.381(2)$ \\
$\mathrm{C}(5)-\mathrm{C}(13)$ & $1.420(2)$ \\
$\mathrm{C}(6)-\mathrm{N}(1)$ & $1.291(2)$ \\
$\mathrm{C}(6)-\mathrm{N}(2)$ & $1.368(2)$ \\
$\mathrm{C}(6)-\mathrm{N}(3)$ & $1.3943(19)$ \\
$\mathrm{C}(7)-\mathrm{C}(8)$ & $1.386(2)$ \\
$\mathrm{C}(7)-\mathrm{N}(2)$ & $1.3874(19)$ \\
$\mathrm{C}(7)-\mathrm{C}(12)$ & $1.396(2)$ \\
$\mathrm{C}(8)-\mathrm{C}(9)$ & $1.390(2)$ \\
$\mathrm{C}(8)-\mathrm{H}(8)$ & 0.9500 \\
$\mathrm{C}(9)-\mathrm{C}(10)$ & $1.395(2)$ \\
$\mathrm{C}(9)-\mathrm{H}(9)$ & 0.9500 \\
$\mathrm{C}(10)-\mathrm{C}(11)$ & $1.401(2)$ \\
$\mathrm{C}(10)-\mathrm{C}(18)$ & $1.486(2)$ \\
&
\end{tabular}




$\begin{array}{ll}\mathrm{C}(11)-\mathrm{C}(12) & 1.376(2) \\ \mathrm{C}(11)-\mathrm{H}(11) & 0.9500 \\ \mathrm{C}(12)-\mathrm{N}(3) & 1.416(2) \\ \mathrm{C}(13)-\mathrm{S}(1) & 1.7470(15) \\ \mathrm{C}(14)-\mathrm{N}(2) & 1.4679(19) \\ \mathrm{C}(14)-\mathrm{C}(15) & 1.524(2) \\ \mathrm{C}(14)-\mathrm{H}(14 \mathrm{~A}) & 0.9900 \\ \mathrm{C}(14)-\mathrm{H}(14 \mathrm{~B}) & 0.9900 \\ \mathrm{C}(15)-\mathrm{C}(16) & 1.510(2) \\ \mathrm{C}(15)-\mathrm{H}(15 \mathrm{~A}) & 0.9900 \\ \mathrm{C}(15)-\mathrm{H}(15 \mathrm{~B}) & 0.9900 \\ \mathrm{C}(16)-\mathrm{O}(3) & 1.4221(19) \\ \mathrm{C}(16)-\mathrm{H}(16 \mathrm{~A}) & 0.9900 \\ \mathrm{C}(16)-\mathrm{H}(16 \mathrm{~B}) & 0.9900 \\ \mathrm{C}(17)-\mathrm{O}(3) & 1.418(2) \\ \mathrm{C}(17)-\mathrm{H}(17 \mathrm{~A}) & 0.9800 \\ \mathrm{C}(17)-\mathrm{H}(17 \mathrm{~B}) & 0.9800 \\ \mathrm{C}(17)-\mathrm{H}(17 \mathrm{C}) & 0.9800 \\ \mathrm{C}(18)-\mathrm{O}(4) & 1.210(2) \\ \mathrm{C}(18)-\mathrm{O}(5) & 1.343(2) \\ \mathrm{C}(19)-\mathrm{O}(5) & 1.4461(19) \\ \mathrm{C}(19)-\mathrm{H}(19 \mathrm{~A}) & 0.9800 \\ \mathrm{C}(19)-\mathrm{H}(19 \mathrm{~B}) & 0.9800 \\ \mathrm{C}(19)-\mathrm{H}(19 \mathrm{C}) & 0.9800 \\ \mathrm{~N}(3)-\mathrm{S}(1) & 1.6627(13) \\ & \end{array}$




$\begin{array}{ll}\mathrm{O}(1)-\mathrm{S}(1) & 1.4300(12) \\ \mathrm{O}(2)-\mathrm{S}(1) & 1.4293(11) \\ & \\ \mathrm{C}(2)-\mathrm{C}(1)-\mathrm{C}(13) & 121.12(15) \\ \mathrm{C}(2)-\mathrm{C}(1)-\mathrm{Cl}(1) & 118.07(13) \\ \mathrm{C}(13)-\mathrm{C}(1)-\mathrm{Cl}(1) & 120.80(12) \\ \mathrm{C}(1)-\mathrm{C}(2)-\mathrm{C}(3) & 119.14(15) \\ \mathrm{C}(1)-\mathrm{C}(2)-\mathrm{H}(2) & 120.4 \\ \mathrm{C}(3)-\mathrm{C}(2)-\mathrm{H}(2) & 120.4 \\ \mathrm{C}(4)-\mathrm{C}(3)-\mathrm{C}(2) & 120.69(15) \\ \mathrm{C}(4)-\mathrm{C}(3)-\mathrm{H}(3) & 119.7 \\ \mathrm{C}(2)-\mathrm{C}(3)-\mathrm{H}(3) & 119.7 \\ \mathrm{C}(3)-\mathrm{C}(4)-\mathrm{C}(5) & 121.77(15) \\ \mathrm{C}(3)-\mathrm{C}(4)-\mathrm{H}(4) & 119.1 \\ \mathrm{C}(5)-\mathrm{C}(4)-\mathrm{H}(4) & 119.1 \\ \mathrm{~N}(1)-\mathrm{C}(5)-\mathrm{C}(4) & 116.54(14) \\ \mathrm{N}(1)-\mathrm{C}(5)-\mathrm{C}(13) & 126.09(14) \\ \mathrm{C}(4)-\mathrm{C}(5)-\mathrm{C}(13) & 117.37(15) \\ \mathrm{N}(1)-\mathrm{C}(6)-\mathrm{N}(2) & 125.04(14) \\ \mathrm{N}(1)-\mathrm{C}(6)-\mathrm{N}(3) & 128.34(15) \\ \mathrm{N}(2)-\mathrm{C}(6)-\mathrm{N}(3) & 106.61(13) \\ \mathrm{C}(8)-\mathrm{C}(7)-\mathrm{N}(2) & 130.45(14) \\ \mathrm{C}(8)-\mathrm{C}(7)-\mathrm{C}(12) & 121.16(14) \\ \mathrm{N}(2)-\mathrm{C}(7)-\mathrm{C}(12) & 108.39(14) \\ \mathrm{C}(7)-\mathrm{C}(8)-\mathrm{C}(9) & 116.61(14) \\ & \end{array}$




$\begin{array}{ll}\mathrm{C}(7)-\mathrm{C}(8)-\mathrm{H}(8) & 121.7 \\ \mathrm{C}(9)-\mathrm{C}(8)-\mathrm{H}(8) & 121.7 \\ \mathrm{C}(8)-\mathrm{C}(9)-\mathrm{C}(10) & 122.05(15) \\ \mathrm{C}(8)-\mathrm{C}(9)-\mathrm{H}(9) & 119.0 \\ \mathrm{C}(10)-\mathrm{C}(9)-\mathrm{H}(9) & 119.0 \\ \mathrm{C}(9)-\mathrm{C}(10)-\mathrm{C}(11) & 121.21(14) \\ \mathrm{C}(9)-\mathrm{C}(10)-\mathrm{C}(18) & 117.27(14) \\ \mathrm{C}(11)-\mathrm{C}(10)-\mathrm{C}(18) & 121.49(14) \\ \mathrm{C}(12)-\mathrm{C}(11)-\mathrm{C}(10) & 116.18(14) \\ \mathrm{C}(12)-\mathrm{C}(11)-\mathrm{H}(11) & 121.9 \\ \mathrm{C}(10)-\mathrm{C}(11)-\mathrm{H}(11) & 121.9 \\ \mathrm{C}(11)-\mathrm{C}(12)-\mathrm{C}(7) & 122.77(15) \\ \mathrm{C}(11)-\mathrm{C}(12)-\mathrm{N}(3) & 131.67(14) \\ \mathrm{C}(7)-\mathrm{C}(12)-\mathrm{N}(3) & 105.56(13) \\ \mathrm{C}(1)-\mathrm{C}(13)-\mathrm{C}(5) & 119.91(14) \\ \mathrm{C}(1)-\mathrm{C}(13)-\mathrm{S}(1) & 118.33(12) \\ \mathrm{C}(5)-\mathrm{C}(13)-\mathrm{S}(1) & 121.35(12) \\ \mathrm{N}(2)-\mathrm{C}(14)-\mathrm{C}(15) & 113.15(12) \\ \mathrm{N}(2)-\mathrm{C}(14)-\mathrm{H}(14 \mathrm{~A}) & 108.9 \\ \mathrm{C}(15)-\mathrm{C}(14)-\mathrm{H}(14 \mathrm{~A}) & 108.9 \\ \mathrm{~N}(2)-\mathrm{C}(14)-\mathrm{H}(14 \mathrm{~B}) & 108.9 \\ \mathrm{C}(15)-\mathrm{C}(14)-\mathrm{H}(14 \mathrm{~B}) & 108.9 \\ \mathrm{H}(14 \mathrm{~A})-\mathrm{C}(14)-\mathrm{H}(14 \mathrm{~B}) & 107.8 \\ \mathrm{C}(16)-\mathrm{C}(15)-\mathrm{C}(14) & 112.71(14) \\ \mathrm{C}(16)-\mathrm{C}(15)-\mathrm{H}(15 \mathrm{~A}) & 109.1 \\ & \end{array}$




$\begin{array}{ll}\mathrm{C}(14)-\mathrm{C}(15)-\mathrm{H}(15 \mathrm{~A}) & 109.1 \\ \mathrm{C}(16)-\mathrm{C}(15)-\mathrm{H}(15 \mathrm{~B}) & 109.1 \\ \mathrm{C}(14)-\mathrm{C}(15)-\mathrm{H}(15 \mathrm{~B}) & 109.1 \\ \mathrm{H}(15 \mathrm{~A})-\mathrm{C}(15)-\mathrm{H}(15 \mathrm{~B}) & 107.8 \\ \mathrm{O}(3)-\mathrm{C}(16)-\mathrm{C}(15) & 108.45(13) \\ \mathrm{O}(3)-\mathrm{C}(16)-\mathrm{H}(16 \mathrm{~A}) & 110.0 \\ \mathrm{C}(15)-\mathrm{C}(16)-\mathrm{H}(16 \mathrm{~A}) & 110.0 \\ \mathrm{O}(3)-\mathrm{C}(16)-\mathrm{H}(16 \mathrm{~B}) & 110.0 \\ \mathrm{C}(15)-\mathrm{C}(16)-\mathrm{H}(16 \mathrm{~B}) & 110.0 \\ \mathrm{H}(16 \mathrm{~A})-\mathrm{C}(16)-\mathrm{H}(16 \mathrm{~B}) & 108.4 \\ \mathrm{O}(3)-\mathrm{C}(17)-\mathrm{H}(17 \mathrm{~A}) & 109.5 \\ \mathrm{O}(3)-\mathrm{C}(17)-\mathrm{H}(17 \mathrm{~B}) & 109.5 \\ \mathrm{H}(17 \mathrm{~A})-\mathrm{C}(17)-\mathrm{H}(17 \mathrm{~B}) & 109.5 \\ \mathrm{O}(3)-\mathrm{C}(17)-\mathrm{H}(17 \mathrm{C}) & 109.5 \\ \mathrm{H}(17 \mathrm{~A})-\mathrm{C}(17)-\mathrm{H}(17 \mathrm{C}) & 109.5 \\ \mathrm{H}(17 \mathrm{~B})-\mathrm{C}(17)-\mathrm{H}(17 \mathrm{C}) & 109.5 \\ \mathrm{O}(4)-\mathrm{C}(18)-\mathrm{O}(5) & 123.09(15) \\ \mathrm{O}(4)-\mathrm{C}(18)-\mathrm{C}(10) & 124.50(15) \\ \text { O(5)-C(18)-C(10) } & 112.41(14) \\ \mathrm{O}(5)-\mathrm{C}(19)-\mathrm{H}(19 \mathrm{~A}) & 109.5 \\ \mathrm{O}(5)-\mathrm{C}(19)-\mathrm{H}(19 \mathrm{~B}) & 109.5 \\ \mathrm{H}(19 \mathrm{~A})-\mathrm{C}(19)-\mathrm{H}(19 \mathrm{~B}) & 109.5 \\ \mathrm{O}(5)-\mathrm{C}(19)-\mathrm{H}(19 \mathrm{C}) & 109.5 \\ \mathrm{H}(19 \mathrm{~A})-\mathrm{C}(19)-\mathrm{H}(19 \mathrm{C}) & 109.5 \\ \mathrm{H}(19 \mathrm{~B})-\mathrm{C}(19)-\mathrm{H}(19 \mathrm{C}) & 109.5 \\ & \end{array}$




$\begin{array}{lc}\mathrm{C}(6)-\mathrm{N}(1)-\mathrm{C}(5) & 117.92(13) \\ \mathrm{C}(6)-\mathrm{N}(2)-\mathrm{C}(7) & 109.95(13) \\ \mathrm{C}(6)-\mathrm{N}(2)-\mathrm{C}(14) & 124.09(13) \\ \mathrm{C}(7)-\mathrm{N}(2)-\mathrm{C}(14) & 125.95(13) \\ \mathrm{C}(6)-\mathrm{N}(3)-\mathrm{C}(12) & 109.48(12) \\ \mathrm{C}(6)-\mathrm{N}(3)-\mathrm{S}(1) & 124.95(11) \\ \mathrm{C}(12)-\mathrm{N}(3)-\mathrm{S}(1) & 125.26(10) \\ \mathrm{C}(17)-\mathrm{O}(3)-\mathrm{C}(16) & 111.65(13) \\ \mathrm{C}(18)-\mathrm{O}(5)-\mathrm{C}(19) & 115.29(13) \\ \mathrm{O}(2)-\mathrm{S}(1)-\mathrm{O}(1) & 117.39(7) \\ \mathrm{O}(2)-\mathrm{S}(1)-\mathrm{N}(3) & 106.35(7) \\ \mathrm{O}(1)-\mathrm{S}(1)-\mathrm{N}(3) & 108.39(7) \\ \mathrm{O}(2)-\mathrm{S}(1)-\mathrm{C}(13) & 114.06(7) \\ \mathrm{O}(1)-\mathrm{S}(1)-\mathrm{C}(13) & 109.72(7) \\ \mathrm{N}(3)-\mathrm{S}(1)-\mathrm{C}(13) & 99.12(7)\end{array}$

Symmetry transformations used to generate equivalent atoms: 
Table 4. Anisotropic displacement parameters $\left(\AA^{2} \mathrm{x} 10^{3}\right)$ for 1009401t_0m. The anisotropic displacement factor exponent takes the form: $-2 \pi^{2}\left[h^{2} a^{* 2} U^{11}+\ldots+2 h k a^{*} b^{*} U^{12}\right]$

\begin{tabular}{|c|c|c|c|c|c|c|}
\hline & $\mathrm{U}^{11}$ & $\mathrm{U}^{22}$ & $\mathrm{U}^{33}$ & $\mathrm{U}^{23}$ & $\mathrm{U}^{13}$ & $\mathrm{U}^{12}$ \\
\hline $\mathrm{C}(1)$ & $17(1)$ & $14(1)$ & 11(1) & $-1(1)$ & $4(1)$ & $0(1)$ \\
\hline $\mathrm{C}(2)$ & $24(1)$ & $11(1)$ & $15(1)$ & $1(1)$ & $7(1)$ & $2(1)$ \\
\hline$C(3)$ & $20(1)$ & $15(1)$ & $17(1)$ & $5(1)$ & $6(1)$ & $6(1)$ \\
\hline $\mathrm{C}(4)$ & $15(1)$ & $18(1)$ & $17(1)$ & $4(1)$ & $3(1)$ & $2(1)$ \\
\hline$C(5)$ & $16(1)$ & $14(1)$ & $12(1)$ & $1(1)$ & $4(1)$ & $1(1)$ \\
\hline$C(6)$ & $12(1)$ & $14(1)$ & $10(1)$ & $-2(1)$ & $2(1)$ & $-1(1)$ \\
\hline$C(7)$ & $12(1)$ & $14(1)$ & $10(1)$ & $0(1)$ & $2(1)$ & 1(1) \\
\hline $\mathrm{C}(8)$ & $15(1)$ & $13(1)$ & $13(1)$ & $-2(1)$ & $2(1)$ & $-2(1)$ \\
\hline $\mathrm{C}(9)$ & $18(1)$ & $12(1)$ & $14(1)$ & $2(1)$ & $4(1)$ & 1(1) \\
\hline $\mathrm{C}(10)$ & $14(1)$ & $14(1)$ & $12(1)$ & $1(1)$ & $3(1)$ & 1(1) \\
\hline $\mathrm{C}(11)$ & $13(1)$ & $14(1)$ & $12(1)$ & $0(1)$ & $2(1)$ & $-1(1)$ \\
\hline$C(12)$ & $16(1)$ & $10(1)$ & $10(1)$ & $0(1)$ & $4(1)$ & $0(1)$ \\
\hline$C(13)$ & $15(1)$ & $13(1)$ & $11(1)$ & $1(1)$ & $3(1)$ & $2(1)$ \\
\hline$C(14)$ & $12(1)$ & $15(1)$ & $16(1)$ & $-3(1)$ & $-2(1)$ & $-1(1)$ \\
\hline$C(15)$ & $13(1)$ & $17(1)$ & $21(1)$ & $2(1)$ & $0(1)$ & $-3(1)$ \\
\hline$C(16)$ & $15(1)$ & $21(1)$ & $16(1)$ & $2(1)$ & $2(1)$ & $-1(1)$ \\
\hline$C(17)$ & $22(1)$ & $22(1)$ & $39(1)$ & $0(1)$ & $5(1)$ & $5(1)$ \\
\hline $\mathrm{C}(18)$ & $15(1)$ & $16(1)$ & $16(1)$ & $2(1)$ & $2(1)$ & $1(1)$ \\
\hline$C(19)$ & $15(1)$ & $31(1)$ & $24(1)$ & $8(1)$ & $-5(1)$ & $2(1)$ \\
\hline $\mathrm{Cl}(1)$ & $20(1)$ & $13(1)$ & $18(1)$ & $-2(1)$ & $2(1)$ & $-3(1)$ \\
\hline
\end{tabular}




\begin{tabular}{lllllll}
$\mathrm{N}(1)$ & $13(1)$ & $13(1)$ & $16(1)$ & $2(1)$ & $0(1)$ & $0(1)$ \\
$\mathrm{N}(2)$ & $11(1)$ & $12(1)$ & $13(1)$ & $0(1)$ & $-1(1)$ & $-1(1)$ \\
$\mathrm{N}(3)$ & $11(1)$ & $10(1)$ & $13(1)$ & $2(1)$ & $0(1)$ & $0(1)$ \\
$\mathrm{O}(1)$ & $16(1)$ & $14(1)$ & $21(1)$ & $1(1)$ & $7(1)$ & $0(1)$ \\
$\mathrm{O}(2)$ & $20(1)$ & $16(1)$ & $14(1)$ & $-1(1)$ & $-4(1)$ & $-2(1)$ \\
$\mathrm{O}(3)$ & $18(1)$ & $20(1)$ & $22(1)$ & $1(1)$ & $-1(1)$ & $5(1)$ \\
$\mathrm{O}(4)$ & $22(1)$ & $17(1)$ & $36(1)$ & $9(1)$ & $-4(1)$ & $2(1)$ \\
$\mathrm{O}(5)$ & $14(1)$ & $20(1)$ & $19(1)$ & $5(1)$ & $-3(1)$ & $0(1)$ \\
$\mathrm{S}(1)$ & $12(1)$ & $10(1)$ & $13(1)$ & $0(1)$ & $1(1)$ & $-1(1)$ \\
& & & & & & \\
\hline
\end{tabular}


Table 5. Hydrogen coordinates ( $\left.\mathrm{x} 10^{4}\right)$ and isotropic displacement parameters $\left(\AA^{2} \mathrm{x} 10^{3}\right)$ for 1009401t_0m.

\begin{tabular}{|c|c|c|c|c|}
\hline & $\mathrm{x}$ & $\mathrm{y}$ & $\mathrm{z}$ & $\mathrm{U}(\mathrm{eq})$ \\
\hline $\mathrm{H}(2)$ & 684 & 1074 & 1733 & 20 \\
\hline $\mathrm{H}(3)$ & 2712 & 1626 & 2447 & 20 \\
\hline $\mathrm{H}(4)$ & 3248 & 3465 & 2570 & 20 \\
\hline $\mathrm{H}(8)$ & 1077 & 9235 & 1270 & 16 \\
\hline $\mathrm{H}(9)$ & -902 & 9855 & 517 & 17 \\
\hline $\mathrm{H}(11)$ & -2318 & 6730 & 314 & 15 \\
\hline $\mathrm{H}(14 \mathrm{~A})$ & 3232 & 6943 & 2508 & 17 \\
\hline $\mathrm{H}(14 \mathrm{~B})$ & 2684 & 8165 & 2356 & 17 \\
\hline $\mathrm{H}(15 \mathrm{~A})$ & 3235 & 8085 & 835 & 21 \\
\hline $\mathrm{H}(15 \mathrm{~B})$ & 4451 & 8090 & 1606 & 21 \\
\hline $\mathrm{H}(16 \mathrm{~A})$ & 3429 & 6134 & 719 & 21 \\
\hline $\mathrm{H}(16 \mathrm{~B})$ & 4687 & 6756 & 475 & 21 \\
\hline $\mathrm{H}(17 \mathrm{~A})$ & 4328 & 4547 & 1295 & 41 \\
\hline $\mathrm{H}(17 \mathrm{~B})$ & 5660 & 4553 & 1958 & 41 \\
\hline $\mathrm{H}(17 \mathrm{C})$ & 5628 & 4906 & 924 & 41 \\
\hline $\mathrm{H}(19 \mathrm{~A})$ & -5440 & 9030 & -588 & 36 \\
\hline $\mathrm{H}(19 \mathrm{~B})$ & -5641 & 7848 & -1054 & 36 \\
\hline $\mathrm{H}(19 \mathrm{C})$ & -4824 & 8770 & -1487 & 36 \\
\hline
\end{tabular}


Figure S4. ORTEP diagram of compound 10a.
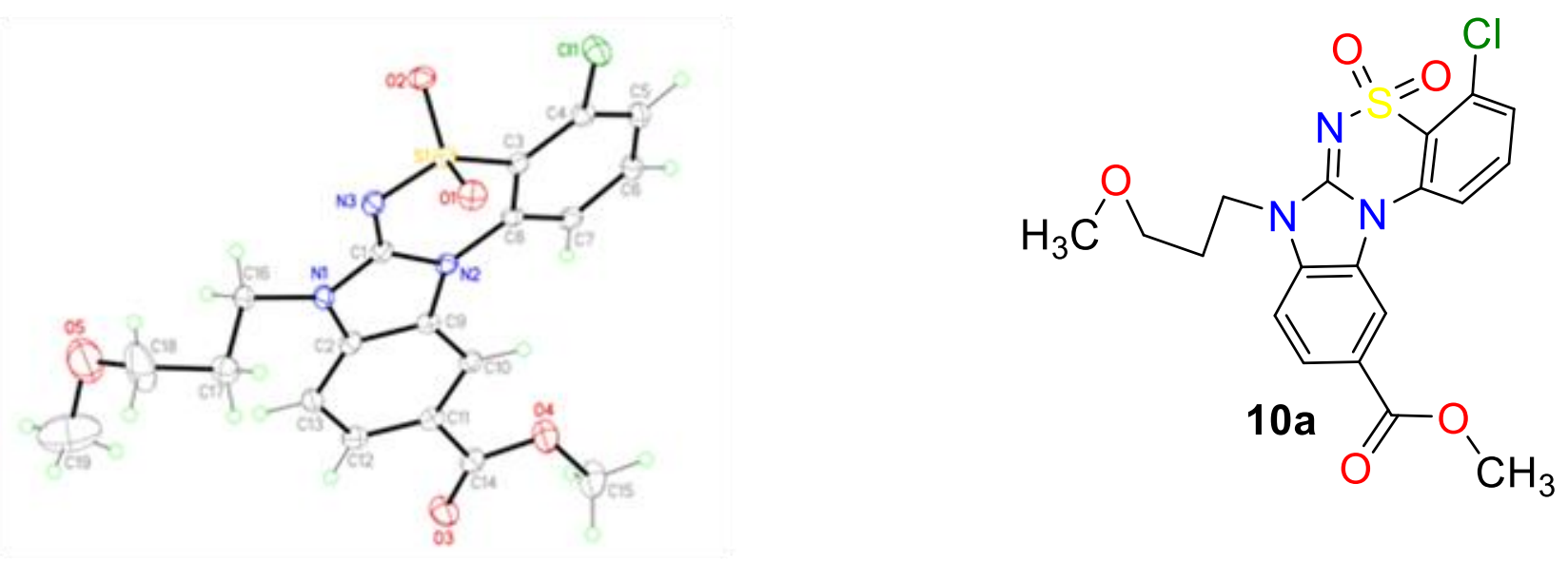

Table 1. Crystal data and structure refinement for 110208LT_0m.

Identification code

1102081t_0m

$\mathrm{C} 19 \mathrm{H} 18 \mathrm{ClN} 3 \mathrm{O} 5 \mathrm{~S}$

435.87

100(2) K

$0.71073 \AA$

Monoclinic

P 1 21/n 1

Space group

$\mathrm{a}=5.2273(2) \AA$

$\alpha=90^{\circ}$. 
Volume

Z

Density (calculated)

Absorption coefficient

$\mathrm{F}(000)$

Crystal size

Theta range for data collection

Index ranges

Reflections collected

Independent reflections

Completeness to theta $=26.37^{\circ}$ Absorption correction

Max. and min. transmission

Refinement method

Data / restraints / parameters

Goodness-of-fit on $\mathrm{F}^{2}$

Final R indices [I $>2 \operatorname{sigma}(\mathrm{I})]$

$\mathrm{R}$ indices (all data)

Largest diff. peak and hole

$\begin{array}{ll}\mathrm{b}=23.0379(11) \AA & \beta=95.5580(10)^{\circ} . \\ \mathrm{c}=15.5364(8) \AA & \gamma=90^{\circ} .\end{array}$

1862.19(15) $\AA^{3}$

4

$1.555 \mathrm{Mg} / \mathrm{m}^{3}$

$0.357 \mathrm{~mm}^{-1}$

904

$0.20 \times 0.02 \times 0.02 \mathrm{~mm}^{3}$

1.59 to $26.37^{\circ}$

$-6<=\mathrm{h}<=3,-28<=\mathrm{k}<=28,-18<=\mathrm{l}<=19$

16224

3807 [R(int) $=0.0327]$

$99.8 \%$

Semi-empirical from equivalents

0.9486 and 0.8964

Full-matrix least-squares on $\mathrm{F}^{2}$

3807 / 0 / 264

1.130

$\mathrm{R} 1=0.0531, \mathrm{wR} 2=0.1469$

$\mathrm{R} 1=0.0697, \mathrm{wR} 2=0.1745$

2.608 and -0.450 e. $\AA^{-3}$ 
Table 2. Atomic coordinates ( $\times 10^{4}$ ) and equivalent isotropic displacement parameters $\left(\AA^{2} \times 10^{3}\right)$ for 110208LT_0m. U(eq) is defined as one third of the trace of the orthogonalized $\mathrm{U}^{\mathrm{ij}}$ tensor.

\begin{tabular}{|c|c|c|c|c|}
\hline & $\mathrm{x}$ & $\mathrm{y}$ & $\mathrm{z}$ & $\mathrm{U}(\mathrm{eq})$ \\
\hline $\mathrm{C}(1)$ & $785(6)$ & 1591(1) & $6388(2)$ & $15(1)$ \\
\hline$C(2)$ & $3801(6)$ & $980(1)$ & $6006(2)$ & $16(1)$ \\
\hline$C(3)$ & $-2162(6)$ & $2455(1)$ & $5456(2)$ & $15(1)$ \\
\hline $\mathrm{C}(4)$ & $-3797(6)$ & $2828(1)$ & $4942(2)$ & $17(1)$ \\
\hline$C(5)$ & $-3791(6)$ & $2843(1)$ & $4053(2)$ & 19(1) \\
\hline$C(6)$ & $-2063(6)$ & $2500(1)$ & $3662(2)$ & 19(1) \\
\hline$C(7)$ & $-355(6)$ & $2145(1)$ & $4154(2)$ & $17(1)$ \\
\hline $\mathrm{C}(8)$ & $-409(5)$ & 2113(1) & $5047(2)$ & $15(1)$ \\
\hline $\mathrm{C}(9)$ & $3133(6)$ & $1343(1)$ & $5292(2)$ & $14(1)$ \\
\hline$C(10)$ & $4398(6)$ & $1289(1)$ & $4550(2)$ & $15(1)$ \\
\hline $\mathrm{C}(11)$ & $6297(6)$ & $859(1)$ & $4548(2)$ & $16(1)$ \\
\hline$C(12)$ & $6941(6)$ & $507(1)$ & $5264(2)$ & $18(1)$ \\
\hline$C(13)$ & $5715(6)$ & $563(1)$ & $6013(2)$ & $18(1)$ \\
\hline$C(14)$ & $7683(6)$ & $764(1)$ & $3769(2)$ & $18(1)$ \\
\hline$C(15)$ & $8278(8)$ & $1105(2)$ & $2371(2)$ & $31(1)$ \\
\hline$C(16)$ & $2329(6)$ & $850(1)$ & $7498(2)$ & $18(1)$ \\
\hline$C(17)$ & $4717(7)$ & $984(2)$ & $8100(2)$ & $24(1)$ \\
\hline $\mathrm{C}(18)$ & $4643(11)$ & $649(2)$ & $8946(3)$ & $53(1)$ \\
\hline$C(19)$ & $6840(11)$ & $-230(3)$ & $8761(4)$ & $76(2)$ \\
\hline $\mathrm{N}(1)$ & $2298(5)$ & $1135(1)$ & $6654(2)$ & $16(1)$ \\
\hline
\end{tabular}




\begin{tabular}{lrrrr}
$\mathrm{N}(2)$ & $1189(5)$ & $1718(1)$ & $5543(2)$ & $14(1)$ \\
$\mathrm{N}(3)$ & $-814(5)$ & $1835(1)$ & $6872(2)$ & $17(1)$ \\
$\mathrm{O}(5)$ & $4436(7)$ & $45(2)$ & $8873(2)$ & $55(1)$ \\
$\mathrm{O}(1)$ & $-1092(4)$ & $2912(1)$ & $6984(2)$ & $22(1)$ \\
$\mathrm{O}(2)$ & $-4978(4)$ & $2327(1)$ & $6744(2)$ & $20(1)$ \\
$\mathrm{O}(3)$ & $9359(5)$ & $409(1)$ & $3717(2)$ & $26(1)$ \\
$\mathrm{O}(4)$ & $6857(5)$ & $1120(1)$ & $3123(2)$ & $26(1)$ \\
$\mathrm{S}(1)$ & $-2346(1)$ & $2413(1)$ & $6586(1)$ & $14(1)$ \\
$\mathrm{Cl}(1)$ & $-5898(2)$ & $3290(1)$ & $5401(1)$ & $24(1)$ \\
& & & & \\
\hline
\end{tabular}


Table 3. Bond lengths $[\AA]$ and angles $\left[^{\circ}\right]$ for $110208 \mathrm{LT} \_0 \mathrm{~m}$.

\begin{tabular}{ll}
\hline $\mathrm{C}(1)-\mathrm{N}(3)$ & $1.305(4)$ \\
$\mathrm{C}(1)-\mathrm{N}(1)$ & $1.354(4)$ \\
$\mathrm{C}(1)-\mathrm{N}(2)$ & $1.382(4)$ \\
$\mathrm{C}(2)-\mathrm{N}(1)$ & $1.382(4)$ \\
$\mathrm{C}(2)-\mathrm{C}(13)$ & $1.386(4)$ \\
$\mathrm{C}(2)-\mathrm{C}(9)$ & $1.405(4)$ \\
$\mathrm{C}(3)-\mathrm{C}(8)$ & $1.406(4)$ \\
$\mathrm{C}(3)-\mathrm{C}(4)$ & $1.406(4)$ \\
$\mathrm{C}(3)-\mathrm{S}(1)$ & $1.770(3)$ \\
$\mathrm{C}(4)-\mathrm{C}(5)$ & $1.381(4)$ \\
$\mathrm{C}(4)-\mathrm{Cl}(1)$ & $1.732(3)$ \\
$\mathrm{C}(5)-\mathrm{C}(6)$ & $1.384(5)$ \\
$\mathrm{C}(5)-\mathrm{H}(5)$ & 0.9500 \\
$\mathrm{C}(6)-\mathrm{C}(7)$ & $1.385(4)$ \\
$\mathrm{C}(6)-\mathrm{H}(6)$ & 0.9500 \\
$\mathrm{C}(7)-\mathrm{C}(8)$ & $1.393(4)$ \\
$\mathrm{C}(7)-\mathrm{H}(7)$ & 0.9500 \\
$\mathrm{C}(8)-\mathrm{N}(2)$ & $1.412(4)$ \\
$\mathrm{C}(9)-\mathrm{C}(10)$ & $1.388(4)$ \\
$\mathrm{C}(9)-\mathrm{N}(2)$ & $1.417(4)$ \\
$\mathrm{C}(10)-\mathrm{C}(11)$ & $1.403(4)$ \\
$\mathrm{C}(10)-\mathrm{H}(10)$ & 0.9500 \\
$\mathrm{C}(11)-\mathrm{C}(12)$ & $1.391(4)$ \\
&
\end{tabular}




$\begin{array}{ll}\mathrm{C}(11)-\mathrm{C}(14) & 1.486(4) \\ \mathrm{C}(12)-\mathrm{C}(13) & 1.388(5) \\ \mathrm{C}(12)-\mathrm{H}(12) & 0.9500 \\ \mathrm{C}(13)-\mathrm{H}(13) & 0.9500 \\ \mathrm{C}(14)-\mathrm{O}(3) & 1.208(4) \\ \mathrm{C}(14)-\mathrm{O}(4) & 1.335(4) \\ \mathrm{C}(15)-\mathrm{O}(4) & 1.443(4) \\ \mathrm{C}(15)-\mathrm{H}(15 \mathrm{~A}) & 0.9800 \\ \mathrm{C}(15)-\mathrm{H}(15 \mathrm{~B}) & 0.9800 \\ \mathrm{C}(15)-\mathrm{H}(15 \mathrm{C}) & 0.9800 \\ \mathrm{C}(16)-\mathrm{N}(1) & 1.467(4) \\ \mathrm{C}(16)-\mathrm{C}(17) & 1.518(4) \\ \mathrm{C}(16)-\mathrm{H}(16 \mathrm{~A}) & 0.9900 \\ \mathrm{C}(16)-\mathrm{H}(16 \mathrm{~B}) & 0.9900 \\ \mathrm{C}(17)-\mathrm{C}(18) & 1.528(6) \\ \mathrm{C}(17)-\mathrm{H}(17 \mathrm{~A}) & 0.9900 \\ \mathrm{C}(17)-\mathrm{H}(17 \mathrm{~B}) & 0.9900 \\ \mathrm{C}(18)-\mathrm{O}(5) & 1.399(6) \\ \mathrm{C}(18)-\mathrm{H}(18 \mathrm{~A}) & 0.9900 \\ \mathrm{C}(18)-\mathrm{H}(18 \mathrm{~B}) & 0.9900 \\ \mathrm{C}(19)-\mathrm{O}(5) & 1.433(7) \\ \mathrm{C}(19)-\mathrm{H}(19 \mathrm{~A}) & 0.9800 \\ \mathrm{C}(19)-\mathrm{H}(19 \mathrm{~B}) & 0.9800 \\ \mathrm{C}(19)-\mathrm{H}(19 \mathrm{C}) & 0.9800 \\ \mathrm{~N}(3)-\mathrm{S}(1) & 1.594(3) \\ & \end{array}$




$\begin{array}{ll}\mathrm{O}(1)-\mathrm{S}(1) & 1.434(2) \\ \mathrm{O}(2)-\mathrm{S}(1) & 1.435(2) \\ & \\ \mathrm{N}(3)-\mathrm{C}(1)-\mathrm{N}(1) & 123.2(3) \\ \mathrm{N}(3)-\mathrm{C}(1)-\mathrm{N}(2) & 128.4(3) \\ \mathrm{N}(1)-\mathrm{C}(1)-\mathrm{N}(2) & 108.3(3) \\ \mathrm{N}(1)-\mathrm{C}(2)-\mathrm{C}(13) & 129.3(3) \\ \mathrm{N}(1)-\mathrm{C}(2)-\mathrm{C}(9) & 107.8(3) \\ \mathrm{C}(13)-\mathrm{C}(2)-\mathrm{C}(9) & 122.8(3) \\ \mathrm{C}(8)-\mathrm{C}(3)-\mathrm{C}(4) & 118.3(3) \\ \mathrm{C}(8)-\mathrm{C}(3)-\mathrm{S}(1) & 121.2(2) \\ \mathrm{C}(4)-\mathrm{C}(3)-\mathrm{S}(1) & 120.5(2) \\ \mathrm{C}(5)-\mathrm{C}(4)-\mathrm{C}(3) & 121.5(3) \\ \mathrm{C}(5)-\mathrm{C}(4)-\mathrm{Cl}(1) & 117.4(2) \\ \mathrm{C}(3)-\mathrm{C}(4)-\mathrm{Cl}(1) & 121.1(2) \\ \mathrm{C}(4)-\mathrm{C}(5)-\mathrm{C}(6) & 119.3(3) \\ \mathrm{C}(4)-\mathrm{C}(5)-\mathrm{H}(5) & 120.4 \\ \mathrm{C}(6)-\mathrm{C}(5)-\mathrm{H}(5) & 120.4 \\ \mathrm{C}(5)-\mathrm{C}(6)-\mathrm{C}(7) & 120.6(3) \\ \mathrm{C}(5)-\mathrm{C}(6)-\mathrm{H}(6) & 119.7 \\ \mathrm{C}(7)-\mathrm{C}(6)-\mathrm{H}(6) & 119.7 \\ \mathrm{C}(6)-\mathrm{C}(7)-\mathrm{C}(8) & 120.4(3) \\ \mathrm{C}(6)-\mathrm{C}(7)-\mathrm{H}(7) & 119.8 \\ \mathrm{C}(8)-\mathrm{C}(7)-\mathrm{H}(7) & 119.8 \\ \mathrm{C}(7)-\mathrm{C}(8)-\mathrm{C}(3) & 119.8(3) \\ & \\ & \end{array}$




$\begin{array}{ll}\mathrm{C}(7)-\mathrm{C}(8)-\mathrm{N}(2) & 120.7(3) \\ \mathrm{C}(3)-\mathrm{C}(8)-\mathrm{N}(2) & 119.4(3) \\ \mathrm{C}(10)-\mathrm{C}(9)-\mathrm{C}(2) & 120.0(3) \\ \mathrm{C}(10)-\mathrm{C}(9)-\mathrm{N}(2) & 134.0(3) \\ \mathrm{C}(2)-\mathrm{C}(9)-\mathrm{N}(2) & 105.9(3) \\ \mathrm{C}(9)-\mathrm{C}(10)-\mathrm{C}(11) & 117.3(3) \\ \mathrm{C}(9)-\mathrm{C}(10)-\mathrm{H}(10) & 121.3 \\ \mathrm{C}(11)-\mathrm{C}(10)-\mathrm{H}(10) & 121.3 \\ \mathrm{C}(12)-\mathrm{C}(11)-\mathrm{C}(10) & 121.7(3) \\ \mathrm{C}(12)-\mathrm{C}(11)-\mathrm{C}(14) & 117.8(3) \\ \mathrm{C}(10)-\mathrm{C}(11)-\mathrm{C}(14) & 120.5(3) \\ \mathrm{C}(13)-\mathrm{C}(12)-\mathrm{C}(11) & 121.4(3) \\ \mathrm{C}(13)-\mathrm{C}(12)-\mathrm{H}(12) & 119.3 \\ \mathrm{C}(11)-\mathrm{C}(12)-\mathrm{H}(12) & 119.3 \\ \mathrm{C}(2)-\mathrm{C}(13)-\mathrm{C}(12) & 116.7(3) \\ \mathrm{C}(2)-\mathrm{C}(13)-\mathrm{H}(13) & 121.6 \\ \mathrm{C}(12)-\mathrm{C}(13)-\mathrm{H}(13) & 121.6 \\ \mathrm{O}(3)-\mathrm{C}(14)-\mathrm{O}(4) & 123.3(3) \\ \mathrm{O}(3)-\mathrm{C}(14)-\mathrm{C}(11) & 124.6(3) \\ \mathrm{O}(4)-\mathrm{C}(14)-\mathrm{C}(11) & 112.0(3) \\ \mathrm{O}(4)-\mathrm{C}(15)-\mathrm{H}(15 \mathrm{~A}) & 109.5 \\ \mathrm{O}(4)-\mathrm{C}(15)-\mathrm{H}(15 \mathrm{~B}) & 109.5 \\ \mathrm{H}(15 \mathrm{~A})-\mathrm{C}(15)-\mathrm{H}(15 \mathrm{~B}) & 109.5 \\ \mathrm{O}(4)-\mathrm{C}(15)-\mathrm{H}(15 \mathrm{C}) & 109.5 \\ \mathrm{H}(15 \mathrm{~A})-\mathrm{C}(15)-\mathrm{H}(15 \mathrm{C}) & 109.5 \\ & \\ & \end{array}$




$\begin{array}{ll}\mathrm{H}(15 \mathrm{~B})-\mathrm{C}(15)-\mathrm{H}(15 \mathrm{C}) & 109.5 \\ \mathrm{~N}(1)-\mathrm{C}(16)-\mathrm{C}(17) & 113.4(3) \\ \mathrm{N}(1)-\mathrm{C}(16)-\mathrm{H}(16 \mathrm{~A}) & 108.9 \\ \mathrm{C}(17)-\mathrm{C}(16)-\mathrm{H}(16 \mathrm{~A}) & 108.9 \\ \mathrm{~N}(1)-\mathrm{C}(16)-\mathrm{H}(16 \mathrm{~B}) & 108.9 \\ \mathrm{C}(17)-\mathrm{C}(16)-\mathrm{H}(16 \mathrm{~B}) & 108.9 \\ \mathrm{H}(16 \mathrm{~A})-\mathrm{C}(16)-\mathrm{H}(16 \mathrm{~B}) & 107.7 \\ \mathrm{C}(16)-\mathrm{C}(17)-\mathrm{C}(18) & 109.8(3) \\ \mathrm{C}(16)-\mathrm{C}(17)-\mathrm{H}(17 \mathrm{~A}) & 109.7 \\ \mathrm{C}(18)-\mathrm{C}(17)-\mathrm{H}(17 \mathrm{~A}) & 109.7 \\ \mathrm{C}(16)-\mathrm{C}(17)-\mathrm{H}(17 \mathrm{~B}) & 109.7 \\ \mathrm{C}(18)-\mathrm{C}(17)-\mathrm{H}(17 \mathrm{~B}) & 109.7 \\ \mathrm{H}(17 \mathrm{~A})-\mathrm{C}(17)-\mathrm{H}(17 \mathrm{~B}) & 108.2 \\ \mathrm{O}(5)-\mathrm{C}(18)-\mathrm{C}(17) & 116.2(4) \\ \mathrm{O}(5)-\mathrm{C}(18)-\mathrm{H}(18 \mathrm{~A}) & 108.2 \\ \mathrm{C}(17)-\mathrm{C}(18)-\mathrm{H}(18 \mathrm{~A}) & 108.2 \\ \mathrm{O}(5)-\mathrm{C}(18)-\mathrm{H}(18 \mathrm{~B}) & 108.2 \\ \mathrm{C}(17)-\mathrm{C}(18)-\mathrm{H}(18 \mathrm{~B}) & 108.2 \\ \mathrm{H}(18 \mathrm{~A})-\mathrm{C}(18)-\mathrm{H}(18 \mathrm{~B}) & 107.4 \\ \mathrm{O}(5)-\mathrm{C}(19)-\mathrm{H}(19 \mathrm{~A}) & 109.5 \\ \mathrm{O}(5)-\mathrm{C}(19)-\mathrm{H}(19 \mathrm{~B}) & 109.5 \\ \mathrm{H}(19 \mathrm{~A})-\mathrm{C}(19)-\mathrm{H}(19 \mathrm{~B}) & 109.5 \\ \mathrm{O}(5)-\mathrm{C}(19)-\mathrm{H}(19 \mathrm{C}) & 109.5 \\ \mathrm{H}(19 \mathrm{~A})-\mathrm{C}(19)-\mathrm{H}(19 \mathrm{C}) & 109.5 \\ \mathrm{H}(19 \mathrm{~B})-\mathrm{C}(19)-\mathrm{H}(19 \mathrm{C}) & 109.5 \\ & \end{array}$




$\begin{array}{ll}\mathrm{C}(1)-\mathrm{N}(1)-\mathrm{C}(2) & 109.6(3) \\ \mathrm{C}(1)-\mathrm{N}(1)-\mathrm{C}(16) & 125.1(3) \\ \mathrm{C}(2)-\mathrm{N}(1)-\mathrm{C}(16) & 125.3(3) \\ \mathrm{C}(1)-\mathrm{N}(2)-\mathrm{C}(8) & 121.2(2) \\ \mathrm{C}(1)-\mathrm{N}(2)-\mathrm{C}(9) & 108.2(2) \\ \mathrm{C}(8)-\mathrm{N}(2)-\mathrm{C}(9) & 130.1(3) \\ \mathrm{C}(1)-\mathrm{N}(3)-\mathrm{S}(1) & 122.1(2) \\ \mathrm{C}(18)-\mathrm{O}(5)-\mathrm{C}(19) & 112.8(4) \\ \mathrm{C}(14)-\mathrm{O}(4)-\mathrm{C}(15) & 116.1(3) \\ \mathrm{O}(1)-\mathrm{S}(1)-\mathrm{O}(2) & 116.26(14) \\ \mathrm{O}(1)-\mathrm{S}(1)-\mathrm{N}(3) & 110.65(14) \\ \mathrm{O}(2)-\mathrm{S}(1)-\mathrm{N}(3) & 107.49(14) \\ \mathrm{O}(1)-\mathrm{S}(1)-\mathrm{C}(3) & 108.54(14) \\ \mathrm{O}(2)-\mathrm{S}(1)-\mathrm{C}(3) & 108.85(14) \\ \mathrm{N}(3)-\mathrm{S}(1)-\mathrm{C}(3) & 104.39(14)\end{array}$

Symmetry transformations used to generate equivalent atoms: 
Table 4. Anisotropic displacement parameters $\left(\AA^{2} \mathrm{x} 10^{3}\right)$ for 110208LT_0m. The anisotropic displacement factor exponent takes the form: $-2 \pi^{2}\left[h^{2} a^{* 2} U^{11}+\ldots+2 h k a^{*} b^{*} U^{12}\right]$

\begin{tabular}{|c|c|c|c|c|c|c|}
\hline & $\mathrm{U}^{11}$ & $\mathrm{U}^{22}$ & $\mathrm{U}^{33}$ & $\mathrm{U}^{23}$ & $\mathrm{U}^{13}$ & $\mathrm{U}^{12}$ \\
\hline $\mathrm{C}(1)$ & $15(1)$ & $18(1)$ & $13(2)$ & $0(1)$ & $1(1)$ & $-4(1)$ \\
\hline$C(2)$ & $17(1)$ & $16(2)$ & $15(2)$ & $-1(1)$ & $2(1)$ & $-3(1)$ \\
\hline $\mathrm{C}(3)$ & $14(1)$ & $17(1)$ & $13(2)$ & $0(1)$ & $1(1)$ & $-3(1)$ \\
\hline$C(4)$ & $17(1)$ & $18(2)$ & $18(2)$ & $-1(1)$ & $4(1)$ & $0(1)$ \\
\hline$C(5)$ & $20(2)$ & $22(2)$ & $16(2)$ & $4(1)$ & $2(1)$ & $2(1)$ \\
\hline$C(6)$ & $20(2)$ & $25(2)$ & $13(2)$ & $4(1)$ & $2(1)$ & $1(1)$ \\
\hline$C(7)$ & $16(1)$ & $22(2)$ & $14(2)$ & $0(1)$ & $5(1)$ & $0(1)$ \\
\hline$C(8)$ & $12(1)$ & $16(1)$ & $15(2)$ & $0(1)$ & $0(1)$ & $-3(1)$ \\
\hline $\mathrm{C}(9)$ & $13(1)$ & $15(1)$ & $16(2)$ & $-2(1)$ & $2(1)$ & $-1(1)$ \\
\hline$C(10)$ & $15(1)$ & $16(1)$ & $15(2)$ & $-1(1)$ & 1(1) & $-2(1)$ \\
\hline $\mathrm{C}(11)$ & $15(1)$ & $17(2)$ & $18(2)$ & $-2(1)$ & $4(1)$ & $-3(1)$ \\
\hline $\mathrm{C}(12)$ & $16(1)$ & $16(2)$ & $23(2)$ & $-2(1)$ & $2(1)$ & $0(1)$ \\
\hline$C(13)$ & $19(2)$ & $16(1)$ & $17(2)$ & $2(1)$ & $0(1)$ & $0(1)$ \\
\hline$C(14)$ & $18(2)$ & $17(2)$ & $19(2)$ & $-1(1)$ & $3(1)$ & $-2(1)$ \\
\hline$C(15)$ & $43(2)$ & $32(2)$ & $22(2)$ & $5(2)$ & $18(2)$ & $13(2)$ \\
\hline$C(16)$ & $20(2)$ & $21(2)$ & $14(2)$ & $4(1)$ & $2(1)$ & $0(1)$ \\
\hline$C(17)$ & $24(2)$ & $28(2)$ & $18(2)$ & $-3(1)$ & $-1(1)$ & $0(1)$ \\
\hline $\mathrm{C}(18)$ & $76(3)$ & $43(2)$ & $34(2)$ & $8(2)$ & $-28(2)$ & $-10(2)$ \\
\hline$C(19)$ & $46(3)$ & $100(5)$ & $85(5)$ & $-35(4)$ & $20(3)$ & $-6(3)$ \\
\hline $\mathrm{N}(1)$ & $17(1)$ & $18(1)$ & $12(1)$ & $3(1)$ & $2(1)$ & $1(1)$ \\
\hline
\end{tabular}




\begin{tabular}{lllllll}
$\mathrm{N}(2)$ & $13(1)$ & $17(1)$ & $12(1)$ & $-1(1)$ & $2(1)$ & $0(1)$ \\
$\mathrm{N}(3)$ & $18(1)$ & $19(1)$ & $14(1)$ & $1(1)$ & $4(1)$ & $1(1)$ \\
$\mathrm{O}(5)$ & $60(2)$ & $51(2)$ & $52(2)$ & $12(2)$ & $-5(2)$ & $4(2)$ \\
$\mathrm{O}(1)$ & $26(1)$ & $21(1)$ & $19(1)$ & $-5(1)$ & $-2(1)$ & $-4(1)$ \\
$\mathrm{O}(2)$ & $15(1)$ & $29(1)$ & $16(1)$ & $0(1)$ & $3(1)$ & $0(1)$ \\
$\mathrm{O}(3)$ & $27(1)$ & $29(1)$ & $23(1)$ & $2(1)$ & $8(1)$ & $12(1)$ \\
$\mathrm{O}(4)$ & $33(1)$ & $27(1)$ & $19(1)$ & $3(1)$ & $12(1)$ & $12(1)$ \\
$\mathrm{S}(1)$ & $14(1)$ & $18(1)$ & $11(1)$ & $-2(1)$ & $2(1)$ & $-1(1)$ \\
$\mathrm{Cl}(1)$ & $27(1)$ & $27(1)$ & $20(1)$ & $0(1)$ & $5(1)$ & $11(1)$ \\
& & & & & & \\
\hline
\end{tabular}

Table 5. Hydrogen coordinates ( x $10^{4}$ ) and isotropic displacement parameters $\left(\AA^{2} \mathrm{x} 10^{3}\right)$ for 110208LT_0m.

\begin{tabular}{lrrrr}
\hline & $x$ & $y$ & U(eq) \\
& & & \\
\hline & & & & \\
$H(5)$ & -4960 & 3086 & 3714 & 23 \\
$H(6)$ & -2048 & 2509 & 3051 & 23 \\
$H(7)$ & 861 & 1921 & 3879 & 21 \\
$H(10)$ & 3995 & 1534 & 4064 & 18 \\
$H(12)$ & 8247 & 222 & 5240 & 22
\end{tabular}




\begin{tabular}{lrrrr}
$\mathrm{H}(13)$ & 6165 & 328 & 6507 & 21 \\
$\mathrm{H}(15 \mathrm{~A})$ & 10099 & 1181 & 2547 & 47 \\
$\mathrm{H}(15 \mathrm{~B})$ & 7608 & 1403 & 1959 & 47 \\
$\mathrm{H}(15 \mathrm{C})$ & 8093 & 722 & 2099 & 47 \\
$\mathrm{H}(16 \mathrm{~A})$ & 2213 & 425 & 7409 & 22 \\
$\mathrm{H}(16 \mathrm{~B})$ & 795 & 973 & 7778 & 22 \\
$\mathrm{H}(17 \mathrm{~A})$ & 4802 & 1406 & 8222 & 28 \\
$\mathrm{H}(17 \mathrm{~B})$ & 6269 & 874 & 7821 & 28 \\
$\mathrm{H}(18 \mathrm{~A})$ & 6227 & 740 & 9324 & 63 \\
$\mathrm{H}(18 B)$ & 3169 & 793 & 9240 & 63 \\
$\mathrm{H}(19 \mathrm{~A})$ & 7427 & -110 & 8208 & 114 \\
$\mathrm{H}(19 B)$ & 6618 & -653 & 8765 & 114 \\
$\mathrm{H}(19 \mathrm{C})$ & 8118 & -117 & 9234 & 114 \\
& & & & \\
\hline
\end{tabular}


Figure S5. ORTEP diagram of compound 21a.

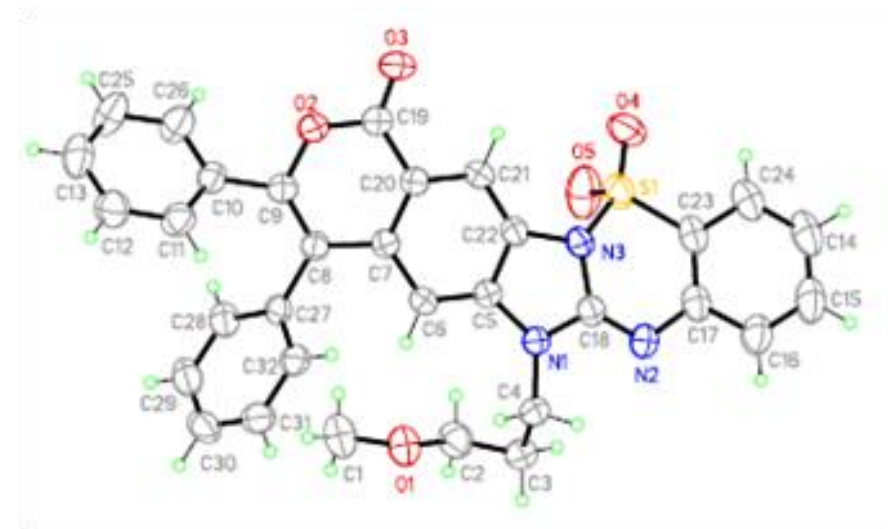

Table 1. Crystal data and structure refinement for 151022_0M.

Identification code

Empirical formula

Formula weight

Temperature

Wavelength

Crystal system

Space group

Unit cell dimensions 151022_0m

C32 H25 N3 O5 S

563.61

296(2) K

$0.71073 \AA$

Triclinic

P - 1

$\begin{array}{ll}\mathrm{a}=10.3849(4) \AA & \alpha=91.474(2)^{\circ} . \\ \mathrm{b}=11.4904(5) \AA & \beta=97.590(2)^{\circ} . \\ \mathrm{c}=12.6969(5) \AA & \gamma=115.976(2)^{\circ} .\end{array}$

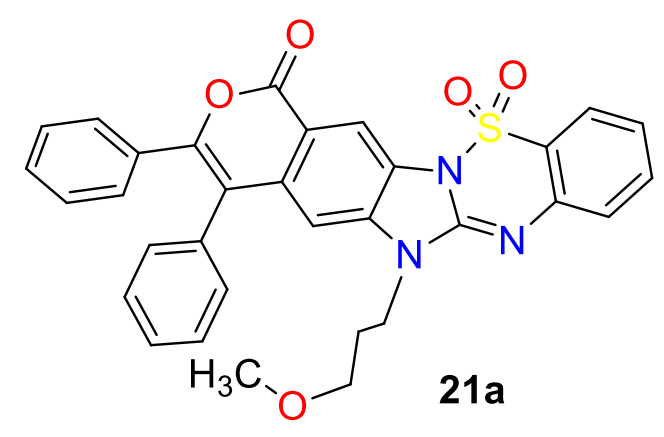

21a 
Volume

$\mathrm{Z}$

Density (calculated)

Absorption coefficient

$\mathrm{F}(000)$

Crystal size

Theta range for data collection

Index ranges

Reflections collected

Independent reflections

Completeness to theta $=25.242^{\circ}$

Absorption correction

Max. and min. transmission

Refinement method

Data / restraints / parameters

Goodness-of-fit on $\mathrm{F}^{2}$

Final R indices [I>2sigma(I)]

$\mathrm{R}$ indices (all data)

Extinction coefficient

Largest diff. peak and hole
1344.14(10) $\AA^{3}$

2

$1.393 \mathrm{Mg} / \mathrm{m}^{3}$

$0.169 \mathrm{~mm}^{-1}$

588

0.17 x $0.11 \times 0.08 \mathrm{~mm}^{3}$

1.625 to $26.403^{\circ}$.

$-12<=\mathrm{h}<=11,-14<=\mathrm{k}<=14,-15<=\mathrm{l}<=15$

20937

$5483[\mathrm{R}(\mathrm{int})=0.0327]$

$99.9 \%$

Semi-empirical from equivalents

0.9485 and 0.9025

Full-matrix least-squares on $\mathrm{F}^{2}$

5483 / 0 / 371

1.044

$\mathrm{R} 1=0.0417, \mathrm{wR} 2=0.1018$

$\mathrm{R} 1=0.0601, \mathrm{wR} 2=0.1134$

$\mathrm{n} / \mathrm{a}$

0.272 and -0.399 e. $\AA^{-3}$ 
Table 2. Atomic coordinates ( $\left.\mathrm{x} 10^{4}\right)$ and equivalent isotropic displacement parameters $\left(\AA^{2} \mathrm{x} 10^{3}\right)$ for 151022_0M. U(eq) is defined as one third of the trace of the orthogonalized $U^{i j}$ tensor.

\begin{tabular}{|c|c|c|c|c|}
\hline & $\mathrm{x}$ & $\mathrm{y}$ & $\mathrm{z}$ & $\mathrm{U}(\mathrm{eq})$ \\
\hline$S(1)$ & $5788(1)$ & $8427(1)$ & 10340(1) & $47(1)$ \\
\hline $\mathrm{O}(1)$ & 10290(1) & $6240(1)$ & $8592(1)$ & $54(1)$ \\
\hline $\mathrm{O}(2)$ & $3056(1)$ & $3658(1)$ & $6089(1)$ & $46(1)$ \\
\hline $\mathrm{O}(3)$ & $2168(1)$ & $4780(2)$ & $6902(1)$ & $63(1)$ \\
\hline $\mathrm{O}(4)$ & $4254(2)$ & $7852(2)$ & $10278(1)$ & $70(1)$ \\
\hline $\mathrm{O}(5)$ & $6417(2)$ & $9327(2)$ & $9594(1)$ & $75(1)$ \\
\hline $\mathrm{N}(1)$ & $7924(1)$ & $6503(1)$ & 10143(1) & $37(1)$ \\
\hline $\mathrm{N}(2)$ & $8393(2)$ & $8066(2)$ & 11559(1) & $45(1)$ \\
\hline $\mathrm{N}(3)$ & $6246(1)$ & $7209(1)$ & $10212(1)$ & $39(1)$ \\
\hline $\mathrm{C}(1)$ & $10360(2)$ & $6304(2)$ & $7495(2)$ & $64(1)$ \\
\hline $\mathrm{C}(2)$ & $10654(2)$ & $7460(2)$ & $9138(2)$ & $52(1)$ \\
\hline $\mathrm{C}(3)$ & $10592(2)$ & $7285(2)$ & $10299(2)$ & $50(1)$ \\
\hline $\mathrm{C}(4)$ & $9160(2)$ & $6241(2)$ & $10520(1)$ & $42(1)$ \\
\hline $\mathrm{C}(5)$ & $6856(2)$ & $5887(2)$ & $9259(1)$ & $34(1)$ \\
\hline$C(6)$ & $6761(2)$ & $5036(2)$ & $8439(1)$ & $34(1)$ \\
\hline$C(7)$ & $5535(2)$ & $4571(2)$ & $7637(1)$ & $34(1)$ \\
\hline $\mathrm{C}(8)$ & $5382(2)$ & $3745(2)$ & $6686(1)$ & $34(1)$ \\
\hline $\mathrm{C}(9)$ & $4156(2)$ & $3322(2)$ & $5959(1)$ & $38(1)$ \\
\hline$C(10)$ & $3765(2)$ & $2512(2)$ & 4938(1) & $39(1)$ \\
\hline $\mathrm{C}(11)$ & $4149(2)$ & $1508(2)$ & 4811(2) & $48(1)$ \\
\hline
\end{tabular}




\begin{tabular}{lrrrr}
$\mathrm{C}(12)$ & $3773(2)$ & $772(2)$ & $3848(2)$ & $57(1)$ \\
$\mathrm{C}(13)$ & $3019(2)$ & $1030(2)$ & $2986(2)$ & $62(1)$ \\
$\mathrm{C}(14)$ & $7055(3)$ & $10497(2)$ & $13143(2)$ & $62(1)$ \\
$\mathrm{C}(15)$ & $8236(3)$ & $10316(2)$ & $13571(2)$ & $66(1)$ \\
$\mathrm{C}(16)$ & $8655(2)$ & $9514(2)$ & $13044(2)$ & $57(1)$ \\
$\mathrm{C}(17)$ & $7897(2)$ & $8855(2)$ & $12048(2)$ & $45(1)$ \\
$\mathrm{C}(18)$ & $7601(2)$ & $7340(2)$ & $10711(1)$ & $38(1)$ \\
$\mathrm{C}(19)$ & $3154(2)$ & $4503(2)$ & $6911(1)$ & $43(1)$ \\
$\mathrm{C}(20)$ & $4448(2)$ & $4976(2)$ & $7717(1)$ & $36(1)$ \\
$\mathrm{C}(21)$ & $4565(2)$ & $5862(2)$ & $8541(1)$ & $38(1)$ \\
$\mathrm{C}(22)$ & $5783(2)$ & $6317(2)$ & $9293(1)$ & $35(1)$ \\
$\mathrm{C}(23)$ & $6694(2)$ & $9054(2)$ & $11621(1)$ & $44(1)$ \\
$\mathrm{C}(24)$ & $6282(2)$ & $9871(2)$ & $12167(2)$ & $53(1)$ \\
$\mathrm{C}(25)$ & $2618(3)$ & $2007(2)$ & $3099(2)$ & $64(1)$ \\
$\mathrm{C}(26)$ & $2979(2)$ & $2746(2)$ & $4066(2)$ & $54(1)$ \\
$\mathrm{C}(27)$ & $6604(2)$ & $3475(2)$ & $6462(1)$ & $35(1)$ \\
$\mathrm{C}(28)$ & $7218(2)$ & $3902(2)$ & $5554(1)$ & $44(1)$ \\
$\mathrm{C}(29)$ & $8318(2)$ & $3627(2)$ & $5296(2)$ & $54(1)$ \\
$\mathrm{C}(30)$ & $8835(2)$ & $2943(2)$ & $5954(2)$ & $57(1)$ \\
$\mathrm{C}(31)$ & $8255(2)$ & $2531(2)$ & $6866(2)$ & $51(1)$ \\
$\mathrm{C}(32)$ & $7142(2)$ & $2788(2)$ & $7122(1)$ & $39(1)$ \\
& & & & \\
\hline
\end{tabular}


Table 3. Bond lengths $[\AA]$ and angles $\left[^{\circ}\right]$ for $151022 \_0 \mathrm{M}$.

\begin{tabular}{ll}
\hline $\mathrm{S}(1)-\mathrm{O}(5)$ & $1.4200(15)$ \\
$\mathrm{S}(1)-\mathrm{O}(4)$ & $1.4225(14)$ \\
$\mathrm{S}(1)-\mathrm{N}(3)$ & $1.6736(14)$ \\
$\mathrm{S}(1)-\mathrm{C}(23)$ & $1.7303(19)$ \\
$\mathrm{O}(1)-\mathrm{C}(1)$ & $1.407(2)$ \\
$\mathrm{O}(1)-\mathrm{C}(2)$ & $1.417(2)$ \\
$\mathrm{O}(2)-\mathrm{C}(19)$ & $1.369(2)$ \\
$\mathrm{O}(2)-\mathrm{C}(9)$ & $1.384(2)$ \\
$\mathrm{O}(3)-\mathrm{C}(19)$ & $1.198(2)$ \\
$\mathrm{N}(1)-\mathrm{C}(18)$ & $1.367(2)$ \\
$\mathrm{N}(1)-\mathrm{C}(5)$ & $1.390(2)$ \\
$\mathrm{N}(1)-\mathrm{C}(4)$ & $1.466(2)$ \\
$\mathrm{N}(2)-\mathrm{C}(18)$ & $1.285(2)$ \\
$\mathrm{N}(2)-\mathrm{C}(17)$ & $1.392(2)$ \\
$\mathrm{N}(3)-\mathrm{C}(18)$ & $1.407(2)$ \\
$\mathrm{N}(3)-\mathrm{C}(22)$ & $1.415(2)$ \\
$\mathrm{C}(1)-\mathrm{H}(1)$ & 0.9600 \\
$\mathrm{C}(1)-\mathrm{H}(19)$ & 0.9600 \\
$\mathrm{C}(1)-\mathrm{H}(20)$ & 0.9600 \\
$\mathrm{C}(2)-\mathrm{C}(3)$ & $1.499(3)$ \\
$\mathrm{C}(2)-\mathrm{H}(21)$ & 0.9700 \\
$\mathrm{C}(2)-\mathrm{H}(22)$ & 0.9700 \\
$\mathrm{C}(3)-\mathrm{C}(4)$ & $1.515(2)$ \\
&
\end{tabular}




$\begin{array}{ll}\mathrm{C}(3)-\mathrm{H}(18) & 0.9700 \\ \mathrm{C}(3)-\mathrm{H}(17) & 0.9700 \\ \mathrm{C}(4)-\mathrm{H}(23) & 0.9700 \\ \mathrm{C}(4)-\mathrm{H}(24) & 0.9700 \\ \mathrm{C}(5)-\mathrm{C}(6) & 1.374(2) \\ \mathrm{C}(5)-\mathrm{C}(22) & 1.407(2) \\ \mathrm{C}(6)-\mathrm{C}(7) & 1.406(2) \\ \mathrm{C}(6)-\mathrm{H}(16) & 0.9300 \\ \mathrm{C}(7)-\mathrm{C}(20) & 1.410(2) \\ \mathrm{C}(7)-\mathrm{C}(8) & 1.463(2) \\ \mathrm{C}(8)-\mathrm{C}(9) & 1.354(2) \\ \mathrm{C}(8)-\mathrm{C}(27) & 1.491(2) \\ \mathrm{C}(9)-\mathrm{C}(10) & 1.476(2) \\ \mathrm{C}(10)-\mathrm{C}(11) & 1.388(3) \\ \mathrm{C}(10)-\mathrm{C}(26) & 1.391(2) \\ \mathrm{C}(11)-\mathrm{C}(12) & 1.376(3) \\ \mathrm{C}(11)-\mathrm{H}(10) & 0.9300 \\ \mathrm{C}(12)-\mathrm{C}(13) & 1.376(3) \\ \mathrm{C}(12)-\mathrm{H}(9) & 0.9300 \\ \mathrm{C}(13)-\mathrm{C}(25) & 1.368(3) \\ \mathrm{C}(13)-\mathrm{H}(2) & 0.9300 \\ \mathrm{C}(14)-\mathrm{C}(24) & 1.373(3) \\ \mathrm{C}(14)-\mathrm{C}(15) & 1.377(3) \\ \mathrm{C}(14)-\mathrm{H}(3) & 0.9300 \\ \mathrm{C}(15)-\mathrm{C}(16) & 1.372(3) \\ & \\ & \end{array}$




$\begin{array}{ll}\mathrm{C}(15)-\mathrm{H}(26) & 0.9300 \\ \mathrm{C}(16)-\mathrm{C}(17) & 1.399(3) \\ \mathrm{C}(16)-\mathrm{H}(25) & 0.9300 \\ \mathrm{C}(17)-\mathrm{C}(23) & 1.408(3) \\ \mathrm{C}(19)-\mathrm{C}(20) & 1.455(2) \\ \mathrm{C}(20)-\mathrm{C}(21) & 1.396(2) \\ \mathrm{C}(21)-\mathrm{C}(22) & 1.364(2) \\ \mathrm{C}(21)-\mathrm{H}(6) & 0.9300 \\ \mathrm{C}(23)-\mathrm{C}(24) & 1.393(3) \\ \mathrm{C}(24)-\mathrm{H}(4) & 0.9300 \\ \mathrm{C}(25)-\mathrm{C}(26) & 1.384(3) \\ \mathrm{C}(25)-\mathrm{H}(8) & 0.9300 \\ \mathrm{C}(26)-\mathrm{H}(7) & 0.9300 \\ \mathrm{C}(27)-\mathrm{C}(28) & 1.389(2) \\ \mathrm{C}(27)-\mathrm{C}(32) & 1.391(2) \\ \mathrm{C}(28)-\mathrm{C}(29) & 1.386(3) \\ \mathrm{C}(28)-\mathrm{H}(15) & 0.9300 \\ \mathrm{C}(29)-\mathrm{C}(30) & 1.375(3) \\ \mathrm{C}(29)-\mathrm{H}(14) & 0.9300 \\ \mathrm{C}(30)-\mathrm{C}(31) & 1.375(3) \\ \mathrm{C}(30)-\mathrm{H}(13) & 0.9300 \\ \mathrm{C}(31)-\mathrm{C}(32) & 1.386(2) \\ \mathrm{C}(31)-\mathrm{H}(12) & 0.9300 \\ \mathrm{C}(32)-\mathrm{H}(11) & 0.9300 \\ & \end{array}$




$\begin{array}{ll}\mathrm{O}(5)-\mathrm{S}(1)-\mathrm{O}(4) & 117.90(10) \\ \mathrm{O}(5)-\mathrm{S}(1)-\mathrm{N}(3) & 108.47(9) \\ \mathrm{O}(4)-\mathrm{S}(1)-\mathrm{N}(3) & 106.95(8) \\ \mathrm{O}(5)-\mathrm{S}(1)-\mathrm{C}(23) & 110.55(9) \\ \mathrm{O}(4)-\mathrm{S}(1)-\mathrm{C}(23) & 112.64(9) \\ \mathrm{N}(3)-\mathrm{S}(1)-\mathrm{C}(23) & 98.33(8) \\ \mathrm{C}(1)-\mathrm{O}(1)-\mathrm{C}(2) & 112.98(16) \\ \mathrm{C}(19)-\mathrm{O}(2)-\mathrm{C}(9) & 123.27(13) \\ \mathrm{C}(18)-\mathrm{N}(1)-\mathrm{C}(5) & 110.23(13) \\ \mathrm{C}(18)-\mathrm{N}(1)-\mathrm{C}(4) & 122.17(14) \\ \mathrm{C}(5)-\mathrm{N}(1)-\mathrm{C}(4) & 127.32(14) \\ \mathrm{C}(18)-\mathrm{N}(2)-\mathrm{C}(17) & 117.63(15) \\ \mathrm{C}(18)-\mathrm{N}(3)-\mathrm{C}(22) & 108.37(13) \\ \mathrm{C}(18)-\mathrm{N}(3)-\mathrm{S}(1) & 120.68(12) \\ \mathrm{C}(22)-\mathrm{N}(3)-\mathrm{S}(1) & 124.20(11) \\ \mathrm{O}(1)-\mathrm{C}(1)-\mathrm{H}(1) & 109.5 \\ \mathrm{O}(1)-\mathrm{C}(1)-\mathrm{H}(19) & 109.5 \\ \mathrm{H}(1)-\mathrm{C}(1)-\mathrm{H}(19) & 109.5 \\ \mathrm{O}(1)-\mathrm{C}(1)-\mathrm{H}(20) & 109.5 \\ \mathrm{H}(1)-\mathrm{C}(1)-\mathrm{H}(20) & 109.5 \\ \mathrm{H}(19)-\mathrm{C}(1)-\mathrm{H}(20) & 109.5 \\ \mathrm{O}(1)-\mathrm{C}(2)-\mathrm{C}(3) & 108.34(17) \\ \mathrm{O}(1)-\mathrm{C}(2)-\mathrm{H}(21) & 110.0 \\ \mathrm{C}(3)-\mathrm{C}(2)-\mathrm{H}(21) & 110.0 \\ \mathrm{O}(1)-\mathrm{C}(2)-\mathrm{H}(22) & 110.0 \\ & \end{array}$




$\begin{array}{ll}\mathrm{C}(3)-\mathrm{C}(2)-\mathrm{H}(22) & 110.0 \\ \mathrm{H}(21)-\mathrm{C}(2)-\mathrm{H}(22) & 108.4 \\ \mathrm{C}(2)-\mathrm{C}(3)-\mathrm{C}(4) & 114.03(15) \\ \mathrm{C}(2)-\mathrm{C}(3)-\mathrm{H}(18) & 108.7 \\ \mathrm{C}(4)-\mathrm{C}(3)-\mathrm{H}(18) & 108.7 \\ \mathrm{C}(2)-\mathrm{C}(3)-\mathrm{H}(17) & 108.7 \\ \mathrm{C}(4)-\mathrm{C}(3)-\mathrm{H}(17) & 108.7 \\ \mathrm{H}(18)-\mathrm{C}(3)-\mathrm{H}(17) & 107.6 \\ \mathrm{~N}(1)-\mathrm{C}(4)-\mathrm{C}(3) & 113.12(15) \\ \mathrm{N}(1)-\mathrm{C}(4)-\mathrm{H}(23) & 109.0 \\ \mathrm{C}(3)-\mathrm{C}(4)-\mathrm{H}(23) & 109.0 \\ \mathrm{~N}(1)-\mathrm{C}(4)-\mathrm{H}(24) & 109.0 \\ \mathrm{C}(3)-\mathrm{C}(4)-\mathrm{H}(24) & 109.0 \\ \mathrm{H}(23)-\mathrm{C}(4)-\mathrm{H}(24) & 107.8 \\ \mathrm{C}(6)-\mathrm{C}(5)-\mathrm{N}(1) & 130.88(15) \\ \mathrm{C}(6)-\mathrm{C}(5)-\mathrm{C}(22) & 121.57(14) \\ \mathrm{N}(1)-\mathrm{C}(5)-\mathrm{C}(22) & 107.52(14) \\ \mathrm{C}(5)-\mathrm{C}(6)-\mathrm{C}(7) & 118.20(15) \\ \mathrm{C}(5)-\mathrm{C}(6)-\mathrm{H}(16) & 120.9 \\ \mathrm{C}(7)-\mathrm{C}(6)-\mathrm{H}(16) & 120.9 \\ \mathrm{C}(6)-\mathrm{C}(7)-\mathrm{C}(20) & 118.90(15) \\ \mathrm{C}(6)-\mathrm{C}(7)-\mathrm{C}(8) & 122.58(14) \\ \mathrm{C}(20)-\mathrm{C}(7)-\mathrm{C}(8) & 118.42(14) \\ \mathrm{C}(9)-\mathrm{C}(8)-\mathrm{C}(7) & 118.84(15) \\ \mathrm{C}(9)-\mathrm{C}(8)-\mathrm{C}(27) & 120.00(15) \\ & \end{array}$




$\begin{array}{ll}\mathrm{C}(7)-\mathrm{C}(8)-\mathrm{C}(27) & 120.89(13) \\ \mathrm{C}(8)-\mathrm{C}(9)-\mathrm{O}(2) & 121.75(15) \\ \mathrm{C}(8)-\mathrm{C}(9)-\mathrm{C}(10) & 128.88(16) \\ \mathrm{O}(2)-\mathrm{C}(9)-\mathrm{C}(10) & 109.34(13) \\ \mathrm{C}(11)-\mathrm{C}(10)-\mathrm{C}(26) & 117.96(17) \\ \mathrm{C}(11)-\mathrm{C}(10)-\mathrm{C}(9) & 122.37(16) \\ \mathrm{C}(26)-\mathrm{C}(10)-\mathrm{C}(9) & 119.66(16) \\ \mathrm{C}(12)-\mathrm{C}(11)-\mathrm{C}(10) & 121.09(18) \\ \mathrm{C}(12)-\mathrm{C}(11)-\mathrm{H}(10) & 119.5 \\ \mathrm{C}(10)-\mathrm{C}(11)-\mathrm{H}(10) & 119.5 \\ \mathrm{C}(11)-\mathrm{C}(12)-\mathrm{C}(13) & 120.3(2) \\ \mathrm{C}(11)-\mathrm{C}(12)-\mathrm{H}(9) & 119.8 \\ \mathrm{C}(13)-\mathrm{C}(12)-\mathrm{H}(9) & 119.8 \\ \mathrm{C}(25)-\mathrm{C}(13)-\mathrm{C}(12) & 119.5(2) \\ \mathrm{C}(25)-\mathrm{C}(13)-\mathrm{H}(2) & 120.3 \\ \mathrm{C}(12)-\mathrm{C}(13)-\mathrm{H}(2) & 120.3 \\ \mathrm{C}(24)-\mathrm{C}(14)-\mathrm{C}(15) & 119.6(2) \\ \mathrm{C}(24)-\mathrm{C}(14)-\mathrm{H}(3) & 120.2 \\ \mathrm{C}(15)-\mathrm{C}(14)-\mathrm{H}(3) & 120.2 \\ \mathrm{C}(16)-\mathrm{C}(15)-\mathrm{C}(14) & 121.4(2) \\ \mathrm{C}(16)-\mathrm{C}(15)-\mathrm{H}(26) & 119.3 \\ \mathrm{C}(14)-\mathrm{C}(15)-\mathrm{H}(26) & 119.3 \\ \mathrm{C}(15)-\mathrm{C}(16)-\mathrm{C}(17) & 121.0(2) \\ \mathrm{C}(15)-\mathrm{C}(16)-\mathrm{H}(25) & 119.5 \\ \mathrm{C}(17)-\mathrm{C}(16)-\mathrm{H}(25) & 119.5 \\ & \end{array}$




$\begin{array}{ll}\mathrm{N}(2)-\mathrm{C}(17)-\mathrm{C}(16) & 117.62(18) \\ \mathrm{N}(2)-\mathrm{C}(17)-\mathrm{C}(23) & 125.48(17) \\ \mathrm{C}(16)-\mathrm{C}(17)-\mathrm{C}(23) & 116.90(18) \\ \mathrm{N}(2)-\mathrm{C}(18)-\mathrm{N}(1) & 124.96(15) \\ \mathrm{N}(2)-\mathrm{C}(18)-\mathrm{N}(3) & 127.88(16) \\ \mathrm{N}(1)-\mathrm{C}(18)-\mathrm{N}(3) & 107.13(14) \\ \mathrm{O}(3)-\mathrm{C}(19)-\mathrm{O}(2) & 117.07(16) \\ \mathrm{O}(3)-\mathrm{C}(19)-\mathrm{C}(20) & 126.12(18) \\ \mathrm{O}(2)-\mathrm{C}(19)-\mathrm{C}(20) & 116.81(15) \\ \mathrm{C}(21)-\mathrm{C}(20)-\mathrm{C}(7) & 122.61(15) \\ \mathrm{C}(21)-\mathrm{C}(20)-\mathrm{C}(19) & 116.79(15) \\ \mathrm{C}(7)-\mathrm{C}(20)-\mathrm{C}(19) & 120.58(16) \\ \mathrm{C}(22)-\mathrm{C}(21)-\mathrm{C}(20) & 116.99(15) \\ \mathrm{C}(22)-\mathrm{C}(21)-\mathrm{H}(6) & 121.5 \\ \mathrm{C}(20)-\mathrm{C}(21)-\mathrm{H}(6) & 121.5 \\ \mathrm{C}(21)-\mathrm{C}(22)-\mathrm{C}(5) & 121.65(16) \\ \mathrm{C}(21)-\mathrm{C}(22)-\mathrm{N}(3) & 131.69(15) \\ \mathrm{C}(5)-\mathrm{C}(22)-\mathrm{N}(3) & 106.65(14) \\ \mathrm{C}(24)-\mathrm{C}(23)-\mathrm{C}(17) & 121.45(18) \\ \mathrm{C}(24)-\mathrm{C}(23)-\mathrm{S}(1) & 117.22(16) \\ \mathrm{C}(17)-\mathrm{C}(23)-\mathrm{S}(1) & 121.00(14) \\ \mathrm{C}(14)-\mathrm{C}(24)-\mathrm{C}(23) & 119.7(2) \\ \mathrm{C}(14)-\mathrm{C}(24)-\mathrm{H}(4) & 120.1 \\ \mathrm{C}(23)-\mathrm{C}(24)-\mathrm{H}(4) & 120.1 \\ \mathrm{C}(13)-\mathrm{C}(25)-\mathrm{C}(26) & 120.64(19) \\ & \end{array}$




\begin{tabular}{ll}
$\mathrm{C}(13)-\mathrm{C}(25)-\mathrm{H}(8)$ & 119.7 \\
$\mathrm{C}(26)-\mathrm{C}(25)-\mathrm{H}(8)$ & 119.7 \\
$\mathrm{C}(25)-\mathrm{C}(26)-\mathrm{C}(10)$ & $120.50(19)$ \\
$\mathrm{C}(25)-\mathrm{C}(26)-\mathrm{H}(7)$ & 119.8 \\
$\mathrm{C}(10)-\mathrm{C}(26)-\mathrm{H}(7)$ & 119.8 \\
$\mathrm{C}(28)-\mathrm{C}(27)-\mathrm{C}(32)$ & $118.32(16)$ \\
$\mathrm{C}(28)-\mathrm{C}(27)-\mathrm{C}(8)$ & $119.23(15)$ \\
$\mathrm{C}(32)-\mathrm{C}(27)-\mathrm{C}(8)$ & $122.44(15)$ \\
$\mathrm{C}(29)-\mathrm{C}(28)-\mathrm{C}(27)$ & $120.98(18)$ \\
$\mathrm{C}(29)-\mathrm{C}(28)-\mathrm{H}(15)$ & 119.5 \\
$\mathrm{C}(27)-\mathrm{C}(28)-\mathrm{H}(15)$ & 119.5 \\
$\mathrm{C}(30)-\mathrm{C}(29)-\mathrm{C}(28)$ & $120.01(19)$ \\
$\mathrm{C}(30)-\mathrm{C}(29)-\mathrm{H}(14)$ & 120.0 \\
$\mathrm{C}(28)-\mathrm{C}(29)-\mathrm{H}(14)$ & 120.0 \\
$\mathrm{C}(29)-\mathrm{C}(30)-\mathrm{C}(31)$ & $119.77(18)$ \\
$\mathrm{C}(29)-\mathrm{C}(30)-\mathrm{H}(13)$ & 120.1 \\
$\mathrm{C}(31)-\mathrm{C}(30)-\mathrm{H}(13)$ & 120.1 \\
$\mathrm{C}(30)-\mathrm{C}(31)-\mathrm{C}(32)$ & $120.52(19)$ \\
$\mathrm{C}(30)-\mathrm{C}(31)-\mathrm{H}(12)$ & 119.7 \\
$\mathrm{C}(32)-\mathrm{C}(31)-\mathrm{H}(12)$ & 119.7 \\
$\mathrm{C}(31)-\mathrm{C}(32)-\mathrm{C}(27)$ & $120.38(17)$ \\
$\mathrm{C}(31)-\mathrm{C}(32)-\mathrm{H}(11)$ & 119.8 \\
$\mathrm{C}(27)-\mathrm{C}(32)-\mathrm{H}(11)$ & 119.8 \\
\hline
\end{tabular}

Symmetry transformations used to generate equivalent atoms: 
Table 4. Anisotropic displacement parameters $\left(\AA^{2} \mathrm{x} 10^{3}\right)$ for $151022 \_0 \mathrm{M}$. The anisotropic displacement factor exponent takes the form: $-2 \pi^{2}\left[h^{2} a^{* 2} U^{11}+\ldots+2 h k a^{*} b^{*} U^{12}\right]$

\begin{tabular}{|c|c|c|c|c|c|c|}
\hline & $\mathrm{U}^{11}$ & $\mathrm{U}^{22}$ & $\mathrm{U}^{33}$ & $\mathrm{U}^{23}$ & $\mathrm{U}^{13}$ & $\mathrm{U}^{12}$ \\
\hline $\mathrm{S}(1)$ & $59(1)$ & $47(1)$ & $45(1)$ & $-1(1)$ & $9(1)$ & $32(1)$ \\
\hline $\mathrm{O}(1)$ & $63(1)$ & $52(1)$ & $48(1)$ & $-1(1)$ & $13(1)$ & $25(1)$ \\
\hline $\mathrm{O}(2)$ & $40(1)$ & $58(1)$ & $43(1)$ & $-10(1)$ & $-4(1)$ & $28(1)$ \\
\hline $\mathrm{O}(3)$ & $47(1)$ & $91(1)$ & $62(1)$ & $-16(1)$ & $-8(1)$ & $46(1)$ \\
\hline $\mathrm{O}(4)$ & $55(1)$ & $86(1)$ & $81(1)$ & $-24(1)$ & $0(1)$ & $46(1)$ \\
\hline $\mathrm{O}(5)$ & $128(1)$ & $58(1)$ & $49(1)$ & $14(1)$ & $23(1)$ & $48(1)$ \\
\hline $\mathrm{N}(1)$ & $36(1)$ & $43(1)$ & $34(1)$ & $-6(1)$ & $1(1)$ & $21(1)$ \\
\hline $\mathrm{N}(2)$ & $48(1)$ & $49(1)$ & $38(1)$ & $-9(1)$ & $1(1)$ & $23(1)$ \\
\hline $\mathrm{N}(3)$ & $40(1)$ & $42(1)$ & $39(1)$ & $-6(1)$ & $5(1)$ & $23(1)$ \\
\hline $\mathrm{C}(1)$ & $77(2)$ & $66(2)$ & $54(1)$ & $0(1)$ & $24(1)$ & $32(1)$ \\
\hline $\mathrm{C}(2)$ & $48(1)$ & $48(1)$ & $57(1)$ & $-3(1)$ & 11(1) & $17(1)$ \\
\hline $\mathrm{C}(3)$ & $40(1)$ & $63(1)$ & $46(1)$ & $-8(1)$ & $-1(1)$ & $24(1)$ \\
\hline $\mathrm{C}(4)$ & $45(1)$ & $53(1)$ & $35(1)$ & $0(1)$ & $0(1)$ & $29(1)$ \\
\hline$C(5)$ & $33(1)$ & $36(1)$ & $33(1)$ & $2(1)$ & $4(1)$ & $16(1)$ \\
\hline$C(6)$ & $34(1)$ & $38(1)$ & $35(1)$ & $1(1)$ & $6(1)$ & $20(1)$ \\
\hline$C(7)$ & $34(1)$ & $35(1)$ & $32(1)$ & $3(1)$ & $6(1)$ & $15(1)$ \\
\hline $\mathrm{C}(8)$ & $36(1)$ & $36(1)$ & $32(1)$ & $2(1)$ & $5(1)$ & $16(1)$ \\
\hline $\mathrm{C}(9)$ & $40(1)$ & $42(1)$ & $37(1)$ & $3(1)$ & $5(1)$ & $21(1)$ \\
\hline $\mathrm{C}(10)$ & $40(1)$ & $39(1)$ & $36(1)$ & $0(1)$ & $3(1)$ & $16(1)$ \\
\hline $\mathrm{C}(11)$ & $48(1)$ & $44(1)$ & $49(1)$ & $-2(1)$ & $-1(1)$ & $20(1)$ \\
\hline
\end{tabular}




\begin{tabular}{|c|c|c|c|c|c|c|}
\hline $\mathrm{C}(12)$ & $58(1)$ & $49(1)$ & $62(1)$ & $-13(1)$ & $3(1)$ & $24(1)$ \\
\hline$C(13)$ & $72(1)$ & $57(1)$ & $45(1)$ & $-12(1)$ & $7(1)$ & $18(1)$ \\
\hline $\mathrm{C}(14)$ & $74(1)$ & $49(1)$ & $64(1)$ & $-10(1)$ & $28(1)$ & $22(1)$ \\
\hline$C(15)$ & $76(2)$ & $59(1)$ & $49(1)$ & $-16(1)$ & $14(1)$ & $18(1)$ \\
\hline$C(16)$ & $62(1)$ & $55(1)$ & $45(1)$ & $-9(1)$ & $6(1)$ & $20(1)$ \\
\hline $\mathrm{C}(17)$ & $50(1)$ & $39(1)$ & $41(1)$ & $-3(1)$ & $13(1)$ & $14(1)$ \\
\hline $\mathrm{C}(18)$ & $40(1)$ & $39(1)$ & $37(1)$ & $0(1)$ & $8(1)$ & $19(1)$ \\
\hline C(19) & $39(1)$ & $53(1)$ & $40(1)$ & $-2(1)$ & $3(1)$ & $24(1)$ \\
\hline $\mathrm{C}(20)$ & $34(1)$ & $42(1)$ & $34(1)$ & $4(1)$ & $5(1)$ & $19(1)$ \\
\hline $\mathrm{C}(21)$ & $36(1)$ & $46(1)$ & $39(1)$ & $2(1)$ & $8(1)$ & $23(1)$ \\
\hline $\mathrm{C}(22)$ & $37(1)$ & $36(1)$ & $34(1)$ & $1(1)$ & $9(1)$ & $18(1)$ \\
\hline $\mathrm{C}(23)$ & $52(1)$ & $38(1)$ & $43(1)$ & $1(1)$ & $18(1)$ & $17(1)$ \\
\hline$C(24)$ & $62(1)$ & $45(1)$ & $57(1)$ & $0(1)$ & $24(1)$ & $23(1)$ \\
\hline $\mathrm{C}(25)$ & $84(2)$ & $62(1)$ & $38(1)$ & $-1(1)$ & $-9(1)$ & $29(1)$ \\
\hline$C(26)$ & $65(1)$ & $49(1)$ & $45(1)$ & $-2(1)$ & $-5(1)$ & $28(1)$ \\
\hline $\mathrm{C}(27)$ & $35(1)$ & $34(1)$ & $34(1)$ & $-5(1)$ & $4(1)$ & $14(1)$ \\
\hline $\mathrm{C}(28)$ & $47(1)$ & $44(1)$ & $40(1)$ & $-1(1)$ & $10(1)$ & $17(1)$ \\
\hline$C(29)$ & $46(1)$ & $57(1)$ & $49(1)$ & $-11(1)$ & $16(1)$ & $12(1)$ \\
\hline$C(30)$ & $40(1)$ & $62(1)$ & $66(1)$ & $-25(1)$ & $4(1)$ & $23(1)$ \\
\hline $\mathrm{C}(31)$ & $46(1)$ & $49(1)$ & $58(1)$ & $-15(1)$ & $-8(1)$ & $27(1)$ \\
\hline$C(32)$ & $39(1)$ & $37(1)$ & $39(1)$ & $-5(1)$ & $0(1)$ & $17(1)$ \\
\hline
\end{tabular}


Table 5. Hydrogen coordinates ( $\left.\mathrm{x} 10^{4}\right)$ and isotropic displacement parameters $\left(\AA^{2} \mathrm{x} 10^{3}\right)$ for 151022_0M.

\begin{tabular}{|c|c|c|c|c|}
\hline & $\mathrm{x}$ & $\mathrm{y}$ & $\mathrm{z}$ & $\mathrm{U}(\mathrm{eq})$ \\
\hline $\mathrm{H}(1)$ & 9747 & 6672 & 7181 & 97 \\
\hline $\mathrm{H}(19)$ & 10039 & 5445 & 7158 & 97 \\
\hline $\mathrm{H}(20)$ & 11343 & 6839 & 7398 & 97 \\
\hline $\mathrm{H}(21)$ & 9975 & 7788 & 8853 & 63 \\
\hline $\mathrm{H}(22)$ & 11621 & 8083 & 9045 & 63 \\
\hline $\mathrm{H}(18)$ & 10774 & 8104 & 10669 & 60 \\
\hline $\mathrm{H}(17)$ & 11362 & 7067 & 10590 & 60 \\
\hline $\mathrm{H}(23)$ & 8994 & 5413 & 10176 & 51 \\
\hline $\mathrm{H}(24)$ & 9218 & 6168 & 11283 & 51 \\
\hline $\mathrm{H}(16)$ & 7487 & 4775 & 8415 & 41 \\
\hline $\mathrm{H}(10)$ & 4668 & 1330 & 5385 & 57 \\
\hline $\mathrm{H}(9)$ & 4030 & 97 & 3780 & 69 \\
\hline $\mathrm{H}(2)$ & 2784 & 544 & 2331 & 75 \\
\hline $\mathrm{H}(3)$ & 6783 & 11041 & 13514 & 75 \\
\hline $\mathrm{H}(26)$ & 8760 & 10745 & 14232 & 79 \\
\hline $\mathrm{H}(25)$ & 9456 & 9408 & 13353 & 68 \\
\hline $\mathrm{H}(6)$ & 3845 & 6129 & 8576 & 46 \\
\hline $\mathrm{H}(4)$ & 5486 & 9992 & 11871 & 64 \\
\hline $\mathrm{H}(8)$ & 2099 & 2177 & 2519 & 77 \\
\hline
\end{tabular}




\begin{tabular}{lllll}
$\mathrm{H}(7)$ & 2693 & 3403 & 4133 & 64 \\
$\mathrm{H}(15)$ & 6887 & 4380 & 5112 & 53 \\
$\mathrm{H}(14)$ & 8705 & 3905 & 4677 & 65 \\
$\mathrm{H}(13)$ & 9576 & 2761 & 5784 & 68 \\
$\mathrm{H}(12)$ & 8612 & 2075 & 7315 & 61 \\
$\mathrm{H}(11)$ & 6753 & 2500 & 7738 & 47 \\
\hline
\end{tabular}


